\title{
Neuronal mechanisms of odor classification in the Drosophila antennal lobe: an optical imaging study
}

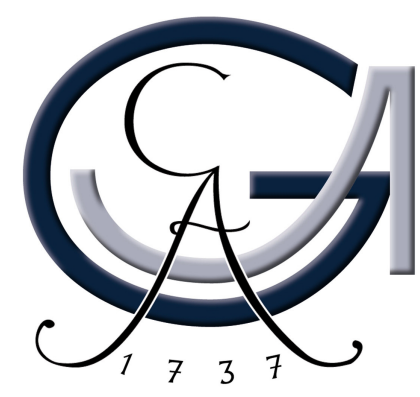

\author{
Dissertation \\ for the award of the degree \\ "Doctor rerum naturalium" \\ of the Georg-August-Universität Göttingen
}

within the doctoral program

"Theoretical and Computational Neuroscience (PTCN)"

of the Georg-August-University School of Science (GAUSS)

\author{
submitted by \\ Shubham Dipt \\ from \\ Patna, India
}

Göttingen, 2015 



\section{Thesis Committee Members}

Prof. Dr. André Fiala (Supervisor)

Department of Molecular Neurobiology of Behavior

Georg-August-University Göttingen

Prof. Dr. Marc Timme

Department of Network Dynamics

Max-Planck-Institute for Dynamics and Self-Organization

Prof. Dr. Dr. Detlev Schild

Department of Neurophysiology and Cellular Biophysics

University Medical Center Göttingen

\section{Members of the Examination Board}

Prof. Dr. André Fiala (Supervisor, Reviewer)

Department of Molecular Neurobiology of Behavior

Georg-August-University Göttingen

Prof. Dr. Marc Timme (Reviewer)

Department of Network Dynamics

Max-Planck-Institute for Dynamics and Self-Organization

Prof. Dr. Dr. Detlev Schild

Department of Neurophysiology and Cellular Biophysics

University Medical Center Göttingen

Prof. Dr. Martin Göpfert

Department of Cellular Neurobiology

Georg-August-University Göttingen

Prof. Dr. Florentin Wörgötter

Third Institute of Physics (Biophysics)

Georg-August-University Göttingen

Prof. Dr. Ernst Wimmer

Department of Developmental Biology

Georg-August-University Göttingen

Thesis submission:

February $28^{\text {th }}, 2015$ 


\section{Declaration of academic honesty}

I hereby declare that this $\mathrm{PhD}$ thesis entitled "Neuronal mechanisms of odor classification in the Drosophila antennal lobe: an optical imaging study" has been written independently and with no sources and aids other than quoted within texts, references and acknowledgments.

Shubham Dipt

Göttingen, February $28^{\text {th }}, 2015$ 


\section{Contents}

1. Introduction 9

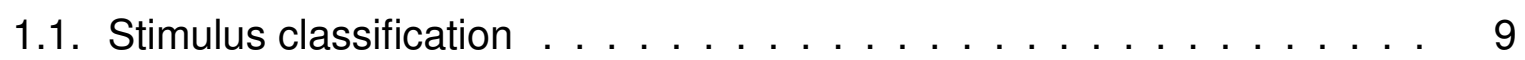

1.2. Primary olfactory circuits . . . . . . . . . . . . . 10

1.3. Odor processing by neuronal circuits . . . . . . . . . . . . 12

1.4. Drosophila melanogaster as a model organism . . . . . . . . . . 13

1.4.1. Binary gene expression systems . . . . . . . . . . . 13

1.4.2. Monitoring neuronal activity by in vivo $\mathrm{Ca}^{2+}$ imaging . . . . . 16

1.4.3. The olfactory system of Drosophila . . . . . . . . . . . . . 19

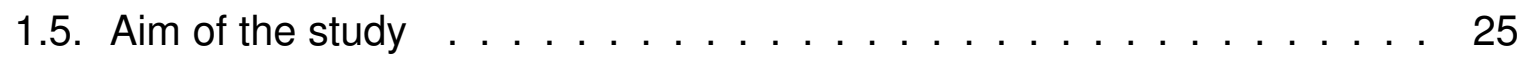

2. Materials and Methods 26

2.1. Materials . . . . . . . . . . . . . . . . . . 26

2.1.1. Drosophila melanogaster strains . . . . . . . . . . . 26

2.1.2. Media . . . . . . . . . . . . . . . . 26

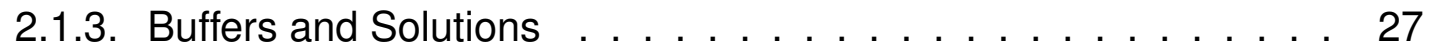

2.1.4. $\mathrm{Ca}^{2+}$ imaging set-up . . . . . . . . . . . . . 28

2.1.5. Chemical substances . . . . . . . . . . . . . . 30

2.1.6. Consumables . . . . . . . . . . . . . . . . . 30

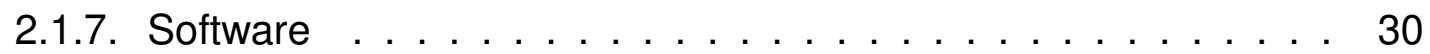

2.2. Methods . . . . . . . . . . . . . . . . . 31

2.2.1. Fly husbandry $\ldots \ldots \ldots \ldots \ldots$

2.2.2. In-vivo $\mathrm{Ca}^{2+}$ imaging . . . . . . . . . . . . . . 31

3. Results 49

3.1. $\mathrm{Ca}^{2+}$ activity of individual glomeruli to different odors and their concen-

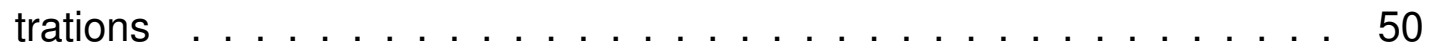

3.1.1. Pentyl acetate $(\mathrm{PA}) \ldots \ldots \ldots \ldots \ldots$

3.1.2. 3-octanol (3-Oct) . . . . . . . . . . . . . . . 56 
3.1.3. 4-methylcyclohexanol $(\mathrm{MCH}) \ldots \ldots \ldots$. . . . . . . . 61

3.1.4. Odor similarity at the level of olfactory sensory neurons and projection neurons . . . . . . . . . . . . . . 66

3.2. Classification of odor stimuli . . . . . . . . . . . . . . 68

3.2.1. Glomerular representations of binary odor mixtures . . . . . 68

3.2.2. Transitions between glomerular representations of binary odor mixtures . . . . . . . . . . . . . . 70

3.2.3. Odortopic aspects of odor classification . . . . . . . . 73

3.3. Possible mechanisms of classification of odor stimuli . . . . . . . 78

3.3.1. Concentration dependence of $\mathrm{Ca}^{2+}$ activity patterns of individual

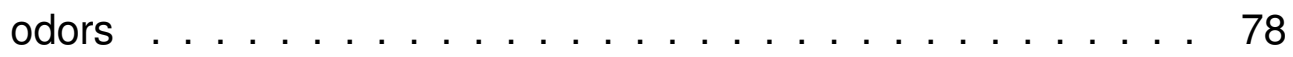

3.3.2. Involvement of lateral inhibition in transition of odor representations in odor morphing . . . . . . . . . . . . . 81

4. Discussion 102

4.1. Neuronal coding of odor perception . . . . . . . . . . . . . 102

4.2. How does $\mathrm{Ca}^{2+}$ imaging relate to membrane depolarization? . . . . 103

4.3. Transformation of odor responses from OSNs to PNs . . . . . . . . . 104

4.4. Temporal coding of odor mixtures . . . . . . . . . . . . 106

4.5. GABAergic inhibition and other possible mechanisms for classification of odors . . . . . . . . . . . . . . . . . . . . . 107

4.6. Future perspectives . . . . . . . . . . . . . . 109

$\begin{array}{ll}\text { 5. Summary } & 110\end{array}$

$\begin{array}{ll}\text { 6. Acknowledgements } & 131\end{array}$

A. The codes for data analysis 133

A.1. Image J plugins (Java) $\ldots \ldots \ldots \ldots$

A.1.1. TwoPhoton Batch Metamorph StackBuild . . . . . . . . . . 133

A.1.2. GCamP MetaMorph StackBuild . . . . . . . . . . . . . . . 136

A.1.3. TwoPhoton Batch Matlab StackBuild Processing . . . . . . . 141

A.1.4. MatlabTif StackBuild . . . . . . . . . . . . . . . . . . . . 145

A.1.5. Matlab Evaluate Journal . . . . . . . . . . . . . . . . . . 147

A.1.6. TwoPhoton Batch Z StackBuild . . . . . . . . . . . . 152

A.1.7. TwoPhoton Batch Processing . . . . . . . . . . . . . . 155 


\section{Contents}

A.1.8. GCamP Align Single Stack . . . . . . . . . . . . . . 159

A.1.9. Fo GCamP Evaluate Journal . . . . . . . . . . . . . . . . . . . 164

A.2. Matlab . . . . . . . . . . . . . . . . . . . . . . . . . 169

A.2.1. Batch Alignment GFP . . . . . . . . . . . . . . . . . . . . . 169

A.3. Microsoft Excel VBA . . . . . . . . . . . . . . . . . . . . . . . . . 171

A.3.1. TwoPhotonCalculation macro . . . . . . . . . . . . . . . . 171

$\begin{array}{ll}\text { B. Contributions to other studies } & 173\end{array}$

C. Abbreviations $\quad 244$

$\begin{array}{ll}\text { Curriculum vitae } & 245\end{array}$ 


\section{List of Figures}

1.1. Binary expression systems in Drosophila . . . . . . . . . . . . 14

1.2. Schematic illustration of different genetically encoded calcium indicators 18

2.1. Photograph of the olfactometer . . . . . . . . . . . . 29

2.2. Scheme of the fly preparation . . . . . . . . . . . . 32

2.3. Scheme of the imaging set-up . . . . . . . . . . . 33

2.4. Schematic illustration of the olfactometer and its control . . . . . . . 35

2.5. The macro for scanning protocol $\ldots \ldots \ldots \ldots$

2.6. LabVIEW user interface . . . . . . . . . . . . . . . 38

2.7. Identified glomeruli in the three focal planes of the antennal lobe $\ldots 46$

3.1. Odor evoked $\mathrm{Ca}^{2+}$ activity to different concentrations of pentyl acetate in OSNs and PNs . . . . . . . . . . . . . . . . 50

3.2. Dynamics of pentyl acetate-evoked $\mathrm{Ca}^{2+}$ activity in selected glomeruli . 52

3.3. Temporal distribution of peak glomerular responses . . . . . . . 53

3.4. Concentration dependence of pentyl acetate-evoked $\mathrm{Ca}^{2+}$ activity in the antennal lobe . . . . . . . . . . . . . . . . 55

3.5. Odor-evoked $\mathrm{Ca}^{2+}$ activity to different concentrations of 3-octanol in OSNs

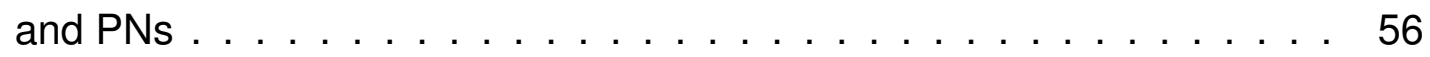

3.6. Dynamics of 3-octanol-evoked $\mathrm{Ca}^{2+}$ activity in selected glomeruli $\ldots . .57$

3.7. Temporal distribution of peak glomerular responses . . . . . . . 58

3.8. Concentration-dependence of 3-octanol-evoked $\mathrm{Ca}^{2+}$ activity in the an-

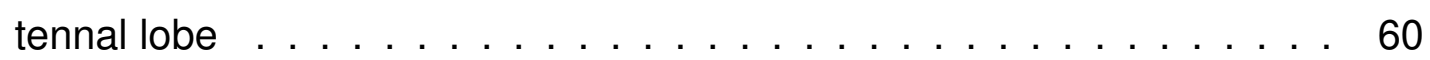

3.9. Odor-evoked $\mathrm{Ca}^{2+}$ activity to different concentrations of 4-methylcyclohexanol in OSNs and PNs . . . . . . . . . . . . . . . . . . . . . . 61

3.10. Dynamics of 4-methylcyclohexanol-evoked $\mathrm{Ca}^{2+}$ activity in selected glomeruli 62

3.11. Temporal distribution of peak glomerular responses 63 
3.12. Concentration-dependence of 4-methylcyclohexanol-evoked $\mathrm{Ca}^{2+}$ activity in the antennal lobe . . . . . . . . . . . . . . . . . . . 65

3.13. Similarity between the $\mathrm{Ca}^{2+}$ activity patterns of different odors . . . . . . 67

3.14. Odor-evoked $\mathrm{Ca}^{2+}$ activity to different binary odor mixtures in OSNs and PNs . . . . . . . . . . . . . . . . . . . 70

3.15. Dynamics of odor-evoked $\mathrm{Ca}^{2+}$ activity patterns to different binary odor mixtures . . . . . . . . . . . . . . . . . . . 73

3.16. Odortopic differences in odor-evoked $\mathrm{Ca}^{2+}$ activity patterns of different odor mixtures at different time points . . . . . . . . . . . . . . . . . 75

3.17. Odortopic differences in odor-evoked $\mathrm{Ca}^{2+}$ activity patterns of different odor mixtures at odor offset . . . . . . . . . . . . . . . . . . . . . 78

3.18. Odortopic differences in odor-evoked $\mathrm{Ca}^{2+}$ activity patterns of different concentrations . . . . . . . . . . . . . . . . . . . 80

3.19. Odor-evoked $\mathrm{Ca}^{2+}$ activity to different binary odor mixtures of $\mathrm{MCH} / 3-$ Oct in OSNs and PNs during the three stages of PTX application . . . .

3.20. Dynamics of odor-evoked $\mathrm{Ca}^{2+}$ activity patterns to $\mathrm{MCH} / 3-O c t$ odor mixture in three different stages of PTX application . . . . . . . . . . . . . .

3.21. Odortopic differences in odor-evoked $\mathrm{Ca}^{2+}$ activity patterns of different odor mixtures of $\mathrm{MCH} / 3-O c t$ at different stages of PTX application . . .

3.22. Odortopic differences in odor-evoked $\mathrm{Ca}^{2+}$ activity patterns of $\mathrm{MCH} / 3-$ Oct odor mixtures at different stages of PTX application in OSNs and PNs at odor offset

3.23. Odor-evoked $\mathrm{Ca}^{2+}$ activity to different binary odor mixtures of $\mathrm{MCH} / \mathrm{PA}$ in OSNs and PNs during the three stages of PTX application

3.24. Dynamics of odor-evoked $\mathrm{Ca}^{2+}$ activity patterns to $\mathrm{MCH} / \mathrm{PA}$ odor mixture in three different stages of PTX application . . . . . . . . . . . . . .

3.25. Odortopic differences in odor-evoked $\mathrm{Ca}^{2+}$ activity patterns of different odor mixtures of MCH/PA at different stages of PTX application . . . . .

3.26. Odortopic differences in odor-evoked $\mathrm{Ca}^{2+}$ activity patterns of $\mathrm{MCH} / \mathrm{PA}$ odor mixtures at different stages of PTX application in OSNs and PNs

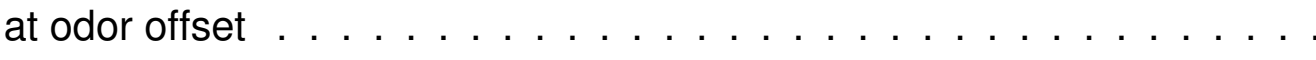

3.27. Odor-evoked $\mathrm{Ca}^{2+}$ activity to different binary odor mixtures of 3-Oct/PA in OSNs and PNs during the three stages of PTX application . . . . . .

3.28. Dynamics of odor-evoked $\mathrm{Ca}^{2+}$ activity patterns to 3-Oct/PA odor mixture in three different stages of PTX application . . . . . . . . . . . . 


\section{List of Figures}

3.29. Odortopic differences in odor-evoked $\mathrm{Ca}^{2+}$ activity patterns of different odor mixtures of 3-Oct/PA at different stages of PTX application . . . . 100

3.30. Odortopic differences in odor-evoked $\mathrm{Ca}^{2+}$ activity patterns of 3-Oct/PA odor mixtures at different stages of PTX application in OSNs and PNs

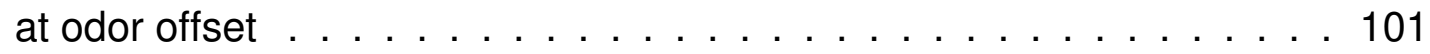




\section{Introduction}

\subsection{Stimulus classification}

Every organism needs to interact with its external environment, and this relies on the sensation and perception of physical and chemical sensory stimuli. Some of these stimuli can be beneficial for the organism whereas some can harm it. These stimuli are therefore processed by the biological system, starting from the sensory system, so that the organism can elicit an adequate response. These responses can vary from food search, escape strategies from harmful substances to selection of mating partners. In its simplest form, this stimulus-behavior reaction can be observed already in microorganisms such as bacteria. Bacteria are sensitive to their both chemical and non-chemical environment [Hu and Tu, 2014]. They exhibit chemotactic or thermotactic behavior depending on the concentration of harmful chemicals or heat in their surrounding [Wadhams and Armitage, 2004; Paster and Ryu, 2008]. Considering the vast repertoire of thermal and chemical stimuli, in terms of their ability to elicit tactic behavior, either of two simple outcomes is decided, i.e., moving towards it or away from it. Such a simple biological system requires to categorize many different stimuli into two simple categories eliciting these particular behaviors. However, this becomes more complicated in higher organisms which can form several such categories underlying a variety of behaviors. These categories become more necessary in higher organisms because they experience a much higher order of different stimuli or even mixtures of stimuli, so they need to combine them perceptually in order to elicit a relatively limited number of behaviors. Such a phenomenon is called categorical perception [Harnad, $1987,2003]$. This happens when any stimuli is changed along a continuum, their perception is not experienced as gradual but instead they are experienced as instances of discrete categories at the sensory level.

According to categorical perception, even human beings tend to visualize their surrounding in terms of the categories that they have formed from the various sensory 


\section{Introduction}

stimuli [Goldstone and Hendrickson, 2009]. As one of the classical examples of categorical perception of colors, on observing a rainbow, we tend to see seven distinct bands of color although on physical terms it consists of a continuous and full range of visible wavelengths of light [Bornstein and Korda, 1984; Pos and Albertazzi, 2010]. These categories, at least for humans, are not just based on the outputs of perceptual systems without any feedback. Instead they have been or they can be customized to a certain behavioral output either at an evolutionary timescale or at the timescale of individual learning [Livingston et al., 1998].

Analogous behaviors relying on categorical perception have also been found across different species [Ehret and Haack, 1981; Nelson and Marler, 1989; May et al., 1989; Wyttenbach et al., 1996; Baugh et al., 2008]. This phenomenon is now considered to be a ubiquitous feature of all perceptual systems. Relatively linear sensory stimuli are transformed by categorical perception into relatively nonlinear representations [Goldstone and Hendrickson, 2009]. In the extreme case, this transformation can be a step function where the perception is unaffected by the changes in the sensory stimuli until they reach a certain threshold. At the point of this threshold, an abrupt transition occurs in the perception. Categorization helps the animals to elicit a stable and appropriate behavior for a certain range of variation in sensory stimuli, and these variations are deemphasized as noise.

In olfaction, animals perceive different odors as complex mixtures of diverse chemical components. These mixtures can vary in their relative composition of their individual components. However, for the animal to exhibit a stable response to such odor stimuli, small variations in the relative composition of the mixture components are often suppressed. This can be achieved by categorizing odor stimuli. This process of categorization occurring in the olfactory system of the animals and the possible mechanisms behind it are the subjects of this thesis.

\subsection{Primary olfactory circuits}

Olfaction is one of the most important senses for most animals. The primary task of the olfactory system is to decipher various odor signals. The organization of the olfactory system is surprisingly similar across different species, ranging from the structure 


\section{Introduction}

of the olfactory receptor proteins to the neuronal organization of the olfactory system, implying an existence of an optimal solution for detecting and discriminating odors in nature [Ache and Young, 2005]. In the very first step, the odorant molecules bind to the olfactory receptor proteins. In vertebrates, these proteins belong to the family of $G$ protein-coupled receptors (GPCRs), seven-transmembrane domain proteins that activate G protein-based signaling pathways [Buck and Axel, 1991]. In mammals, each olfactory sensory neuron expresses exclusively one of the alleles of one of the many functional receptor genes [Chess et al., 1994; Serizawa et al., 2000]. This one receptor one neuron principle ensures a segregation of receptor responses in different neurons. Moreover, each receptor can bind multiple odorants and each odorant can activate multiple receptors [Malnic et al., 1999]. This combinatorial coding helps the animals to discriminate a vast number of odorants. Such similar principle can also be found in the fruit fly, Drosophila melanogaster, where, besides a specific olfactory receptor, an additional receptor Or83b is broadly expressed with the specific receptors [Larsson et al., 2004; Goldman et al., 2005]. However, in nematode worms (Caenorhabditis elegans), their 32 chemosensory neurons express multiple odorant receptors in each neuron [Troemel et al., 1995].

From studies on Drosophila melanogaster, their olfactory sensory neurons have been found to vary in their odor tuning profiles, which means some neurons respond to only few of the odorants, while others respond to many odorants [Hallem and Carlson, 2006]. The odorants also vary in their number of neurons they activate. In different species, including Drosophila, the olfactory receptors can also mediate both inhibition and activation of the neurons [Bruyne et al., 2001; Kang and Caprio, 1995; Sanhueza et al., 2000; Duchamp-Viret et al., 1999]. The olfactory information is then carried by these neurons to the first "relay center" of the olfactory system. This first odor information processing center is called olfactory bulb in vertebrates, antennal lobe in insects and olfactory lobe in crustaceans [Mori et al., 1999; Hansson and Anton, 2000; Sandeman et al., 1993]. In all cases, it consists of several spherical structures called glomeruli, where the sensory neurons branch and synapse onto second-order olfactory neurons, in insects projection neurons (PNs) and local interneurons (LNs) [Hansson and Anton, 2000]. In mammals, they synapse onto mitral/tufted and periglomerular cells [Pinching and Powell, 1971; Tolbert and Hildebrand, 1981]. The mammals' mitral/tufted cells and the insects' projection neurons then further carry the information from these glomeruli to higher brain centers, i.e., the olfactory cortex in mammals 


\section{Introduction}

(the olfactory tubercle, the piriform cortex, the cortical nucleus of the amygdala, and the entorhinal area) [Lledo et al., 2005] and the lateral protocerebrum and the corpora pedunculata (mushroom bodies) in arthropods [Heisenberg, 1998].

\subsection{Odor processing by neuronal circuits}

The odors are encoded as a combinatorial code of the receptors they activate or inhibit. The olfactory sensory neurons expressing the same receptor proteins converge onto one or very few glomeruli, both in mammals and in insects [Mombaerts et al., 1996; Dynes and Ngai, 1998; Vosshall et al., 2000]. Hence, these glomeruli act as a functional and structural units of the first olfactory relay center of the brain. The activity across the population of glomeruli of the sensory neurons, therefore, reflects the combinatorial code of the receptors to some degree. Optical imaging studies have shown that odors evoke stereotypic glomerular activity patterns [Rubin and Katz, 1999; Belluscio and Katz, 2001; Friedrich and Korsching, 1997; Joerges et al., 1997; Vickers et al., 1998; Wang et al., 2003]. Single odorants and their concentrations are found to elicit unique and complex activity patterns where each glomerulus responds differently to a given odorant [Hallem and Carlson, 2006]. These odor representations in the sensory neurons undergo a transformation when they are further carried to the second order olfactory neurons, in insects the projection neurons [Wilson et al., 2004; Yaksi et al., 2009; Sosulski et al., 2011]. One of the mechanisms behind this transformation relies on GABAergic inhibition by local interneurons present in the first olfactory relay center of the brain [Sachse and Galizia, 2002; Wilson and Laurent, 2005; Poo and Isaacson, 2009]. Many studies have shown that GABAergic inhibition is involved in odor processing in the brain [Yokoi et al., 1995; Olsen and Wilson, 2008; Silbering et al., 2008; Isaacson, 2010; Acebes et al., 2011], but how this processing helps in determining odor perception of the animals in terms of behavioral olfactory responses is not clear.

In this thesis, I investigate whether GABAergic inhibition is involved in classifying odor representations. In the natural environment, odors are mostly intermittent and consist of mixtures of chemicals, which makes it difficult for the animals to decipher relevant odor signals. The brain has to extract the relevant information from the continuous stream of different sensory stimuli for appropriate behavior. Therefore, to elicit 


\section{Introduction}

a stable response, varying stimuli are classified into distinct classes. Such classes could be potentially achieved by transforming the sensory representations into discrete representations [Niessing and Friedrich, 2010]. I first aimed at testing whether odor classification occurs at the first olfactory processing center of the brain, i.e., the antennal lobe $(A L)$ of Drosophila. Since the transformation from sensory neurons to projection neurons in AL is influenced by GABAergic inhibition, I here also investigate the role of GABA in potential odor classification.

\subsection{Drosophila melanogaster as a model organism}

The fruit fly Drosophila melanogaster is one of the standard model organisms to investigate key questions in the field of biology [Arias, 2008; Stephenson and Metcalfe, 2013]. Since the discovery of the first gene mutation, the white gene, in 1910 [Morgan, 1910], several genetic tools have been engineered which gave an upper hand to the fruit flies to be used extensively in research [Spradling, 1997], e.g., the balancer chromosomes to prevent genetic recombination [Rubin and Spradling, 1982], binary expression systems for cell specific expression [Brand and Perrimon, 1993], or neuronal manipulation by temperature or light [Venken et al., 2011]. Apart from these technical advantages Drosophila offers, it also has a much simpler nervous system than mammals. The Drosophila brain consists of only $\sim 10^{5}$ neurons, much less compared to the number of neurons in mammals $\left(10^{8}-10^{11}\right.$ neurons) [Chiang et al., 2011; Herculano-Houzel and Lent, 2005]. In spite of this simplicity, complex neuronal circuits and behaviors can also be found in flies, e.g., learning and memory [Waddell and Quinn, 2001; Fiala, 2007], circadian rhythms [Hendricks et al., 2000; Tataroglu and Emery, 2014] or aggression [Asahina et al., 2014]. In terms of neuromodulators and neurotransmitters, flies also share many similarities with vertebrates. Specific neuronal subsets can be precisely targeted and manipulated to study the functional role of those neurons. This is conveniently nowadays achieved by the binary expression systems.

\subsubsection{Binary gene expression systems}

A major advancement in Drosophila genetics occurred in 1982 when Spradling and Rubin developed P-element-mediated transformation of Drosophila germline cells to generate transgenic fly strains [Spradling and Rubin, 1982]. However, presently the 


\section{Introduction}

main advantage of Drosophila comes from the binary GAL4-UAS system developed by Brand and Perrimon [1993]. Using the GAL4-UAS system, different transgenes can be expressed in different cells without creating new transgenic lines for each combination. This system consists of two transgenic lines: the "driver strain" which contains a cell specific enhancer sequence controlling the expression of an yeast protein GAL4, and the "effector strain" which contains an upstream activator sequence (UAS) control-

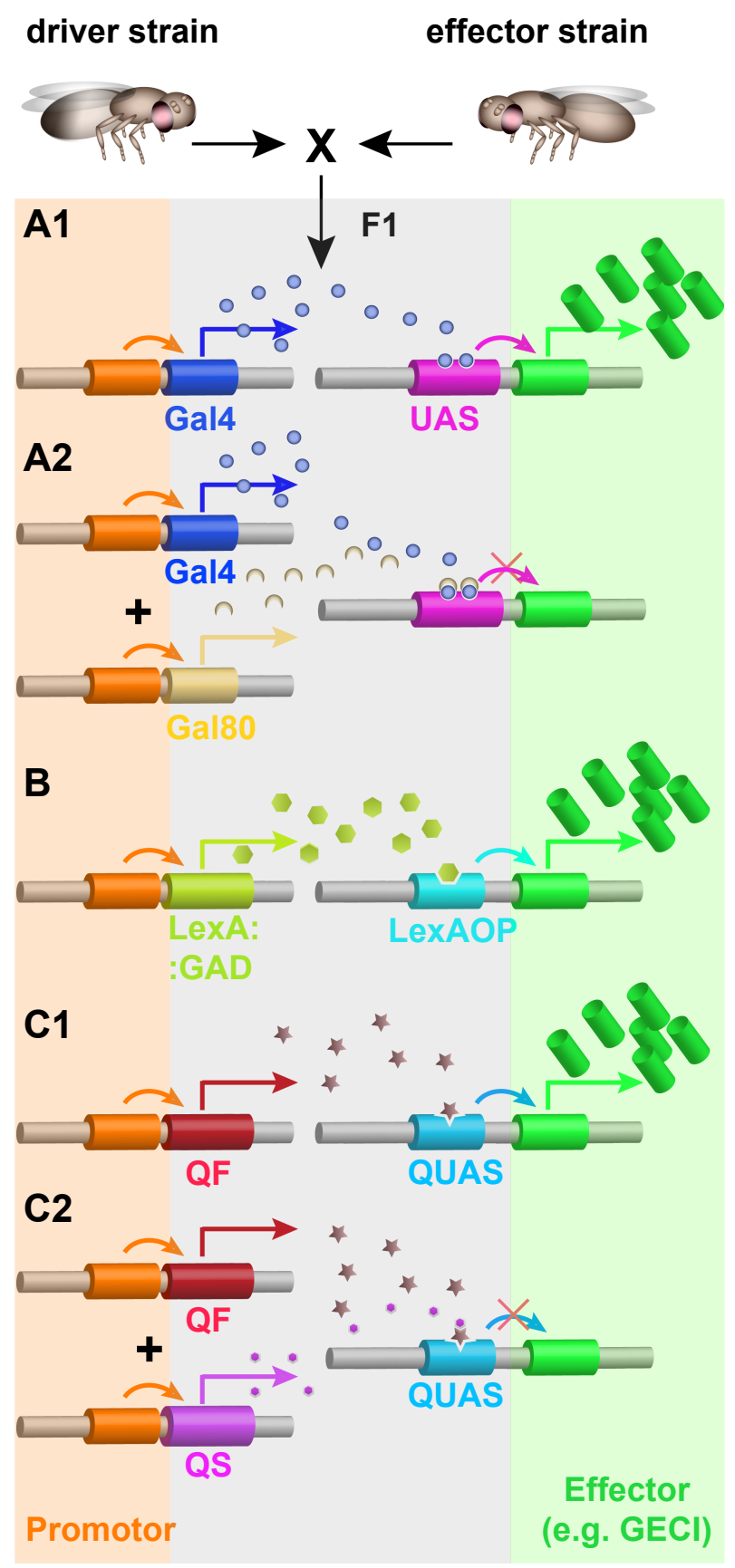

Figure 1.1 Binary expression systems in Drosophila.

(A1) In the GAL4-UAS system, one fly strain called the driver strain which, contains the GAL4 DNA under the control of a genomic enhancer or promoter sequence, is crossed to another strain called the effector strain, in which the gene of interest is coupled to an upstream activator sequence (UAS). In the F1 generation, the effector gene is expressed in those specific cells determined by the driver strain. (A2) Gene expression can be repressed by GAL80, which blocks the activation domain of GAL4. (B) In the LexA/LexAOP system, the driver strain contains the LexA DNA which, when crossed to the effector strain, activates transcription of the effector gene by binding to a LexAOP activation sequence in specific cells. (C1) Like the other two systems, the $Q$ binary expression system consists of a driver strain, which expresses the transcription factor QF under the control of a genomic enhancer, and an effector strain, which has the gene of interest coupled to a QUAS sequence. On crossing the driver and the effector strain, the cells having the QF will activate the expression of the gene of interest by binding to the QUAS activation domain. (C2) Similar to GAL80, the gene expression can be repressed in the Q-system by the coexpression of the inhibitor QS.

Figure from Riemensperger et al. [2012]. 


\section{Introduction}

ling the expression of the gene of interest. The GAL4 DNA is expressed only in specific cells in the driver strain, and UAS is present in all the cells in the effector strain. However the presence of the UAS DNA itself does not have any effect. The gene of interest can only be expressed when the GAL4 binds to the UAS sequence. So, on crossing the driver strain with the effector strain, the specific cells containing the GAL4 will bind to the UAS, thereby leading to the expression of the gene of interest (Figure 1.1 A1). In this way, different genes of interest can be expressed in the same specific cells by altering the effector strain and, similarly, the same gene can be expressed in different specific cells by changing the driver strain [Duffy, 2002].

Further advancements have been made for the GAL4-UAS system by the introduction of GAL80, especially GAL80 ${ }^{\text {ts }}$, which enables one to control the expression of the gene temporally [Zeidler et al., 2004]. GAL80 blocks the activation domain of GAl4, thereby repressing gene expression (Figure 1.1 A2). However, the temperature sensitive form of GAL80 $\left(\right.$ GAL80 $\left.{ }^{\text {ts }}\right)$ can be deactivated at $29^{\circ} \mathrm{C}$. This property of GAL80 ${ }^{\text {ts }}$ gives us the advantage to induce the gene expression in a time-specific manner. In conclusion, the GAI4-UAS system provides a convenient tool to control the expression of any gene of interest both spatially and temporally in Drosophila.

Similar to the GAL4-UAS system, the alternative independent binary system LexA/ LexAOP was developed [Szüts and Bienz, 2000; Lai and Lee, 2006]. The effector strain contains the gene of interest under the control of the regulatory sequence LexAOP. The gene expression only takes place when the LexA present in the specific cells of the driver strain binds to LexAOP, thereby activating the transcription of the gene (Figure 1.1 B). There are two kinds of LexA, one which is sensitive to GAL80 and one which is not. The sensitive version uses a part of the GAL4 called GAD, whereas the insensitive form uses a viral promoter called VP16 which cannot be blocked by GAL80 [Lai and Lee, 2006].

Recently, there was a third binary system introduced called the $Q$ system [Potter et al., 2010]. Similar to UAS, gene expression is controlled by the regulatory sequence QUAS and the transcription is only activated when the transcription factor QF binds to the QUAS (Figure 1.1 C1). This system also has an inhibitor similar to GAL80 called QS, which prevents binding of QF to QUAS (Figure 1.1 C2). The QS inhibition can also be blocked by feeding quinic acid to the flies [Potter et al., 2010]. 


\section{Introduction}

The advent of multiple binary systems enabled us with the possibility of combining any two systems in a single fly. By this way, two different genes of interest can be expressed in two distinct subsets of cells, thereby opening a plethora of options for genetic manipulation. With the help of these binary systems, the expression of genetically encoded calcium sensors can also be targeted to specific neurons to observe their calcium dynamics as a correlate of neuronal activity [Riemensperger et al., 2012].

\subsubsection{Monitoring neuronal activity by in vivo $\mathrm{Ca}^{2+}$ imaging}

To understand the functional role of neurons and their responses to certain stimuli, the first step required is to monitor and record their activity. This can be achieved by electrophysiologically recording their membrane potential. However, electrophysiology is mostly restricted to single neurons or small populations of neurons surrounding an extracellular electrode. Non-invasive optical imaging methods provide powerful tools to monitor the activity of several neurons simultaneously [Griesbeck, 2004]. Since 1973, when neuronal activity was first recorded with the help of voltage sensitive dyes [Salzberg et al., 1973], tremendous progress has been made in the field of optical imaging [Mutoh and Knöpfel, 2013]. Optical calcium imaging is one such method for monitoring neuronal activity [Tsien, 1988; Mao et al., 2001; Stosiek et al., 2003]. It is based on the principle that any depolarizing signal in a living neurons is mostly associated with $\mathrm{Ca}^{2+}$ influx through various types of $\mathrm{Ca}^{2+}$ channels expressed in neurons [Tsien and Tsien, 1990; Berridge et al., 2000]. Such $\mathrm{Ca}^{2+}$ signals are important for chemical synaptic transmission in neurons [Yuste and Denk, 1995; Neher, 1998; Südhof, 2000].

Most of the fluorescent $\mathrm{Ca}^{2+}$ sensors are generally expressed in the cytosol of the neurons. During the resting state, the intracellular $\mathrm{Ca}^{2+}$ concentration is generally around 10-100 nM, which rises drastically 10-100 times during the depolarization of neuron [Berridge et al., 2000]. At any moment, the $\mathrm{Ca}^{2+}$ concentration reflected by a cytosolic $\mathrm{Ca}^{2+}$ sensor is an integrated effect of multiple factors such as the $\mathrm{Ca}^{2+}$ influx rate, efflux rate as well as the $\mathrm{Ca}^{2+}$ exchange with internal stores such as endoplasmic reticulum [Berridge, 1998] and mitochondria [Duchen, 1999]. In the presynapses, as the action potential "arrives", the $\mathrm{Ca}^{2+}$ influx is mainly driven by voltage-gated $\mathrm{Ca}^{2+}$ channels, causing micro- or nanodomains of high $\mathrm{Ca}^{2+}$ concentration around the channels [Neher and Sakaba, 2008]. But in the postsynapses, the cytosolic sensors detect 


\section{Introduction}

$\mathrm{Ca}^{2+}$ potentially from ligated-gated ionotropic receptors, internal $\mathrm{Ca}^{2+}$ stores or from the voltage-gated $\mathrm{Ca}^{2+}$ channels present at the postsynaptic membrane. $\mathrm{Ca}^{2+}$ concentration in the mammalian axons get influenced by several kinds of proteins involved in the homeostasis of the intracellular $\mathrm{Ca}^{2+}$ such as $\mathrm{Ca}^{2+}$ pumps, $\mathrm{Ca}^{2+}$-dependent ion channels and $\mathrm{Na}^{+}-\mathrm{Ca}^{2+}$ exchangers [Waxman and Ritchie, 1993]. Moreover, the $\mathrm{Ca}^{2+}$ influx in the axons is also mediated by the axonal voltage-gated $\mathrm{Ca}^{2+}$ channels [Callewaert et al., 1996; Bacskai et al., 1995]. Therefore, any $\mathrm{Ca}^{2+}$ signal obtained from a cytosolic $\mathrm{Ca}^{2+}$ sensor is an integrated effect resulting from various sources of $\mathrm{Ca}^{2+}$ present in the neuron.

A major advancement in the field of $\mathrm{Ca}^{2+}$ imaging came with the advent of genetically encoded $\mathrm{Ca}^{2+}$ indicators (GECl) [Pérez Koldenkova and Nagai, 2013]. These sensors provided an additional property to be genetically expressed in specific neurons, and Drosophila served perfectly as one of the best organisms for this purpose due to their different binary expression systems [Fiala et al., 2002]. The first genetically encoded $\mathrm{Ca}^{2+}$ indicator was named "Cameleon" which was developed in 1997 by Miyawaki et al. [1997]. It consists of two fluorescent proteins, ECFP (enhanced cyan fluorescent protein) and EYFP (enhanced yellow fluorescent protein), which are connected by a $\mathrm{Ca}^{2+}$ sensitive protein called calmodulin and a M13 domain (Figure 1.2 A). In the absence of $\mathrm{Ca}^{2+}$ ions, illuminating the sensor with light of $\sim 440 \mathrm{~nm}$ wavelength leads to the excitation of ECFP, which results in an emission of $\sim 480 \mathrm{~nm}$ wavelength light. However, in the presence of $\mathrm{Ca}^{2+}$ ions, due to its binding to calmodulin, a conformational change occurs in the sensor which brings the ECFP and EYFP proteins in closer proximity. Due to the reduced distance between ECFP and EYFP, and the resulting Förster resonance energy transfer (FRET) effect, the energy of the excited ECFP gets transferred to EYFP, leading to an enhanced emission of light of $\sim 535 \mathrm{~nm}$ wavelength. The $\mathrm{Ca}^{2+}$ concentration can henceforth be determined by the ratio of 480 and $530 \mathrm{~nm}$ wavelength of light. A similar sensor, TN-XL, was also made which used troponin $\mathrm{C}$ sequence as the $\mathrm{Ca}^{2+}$ binding component independent of the additional peptide like M13 [Griesbeck, 2004] (Figure 1.2 B).

Based on the same principle, improved versions of ratiometric $\mathrm{Ca}^{2+}$ sensors have been developed in subsequent years [Guerrero and Isacoff, 2001; Knöpfel et al., 2006; Pérez Koldenkova and Nagai, 2013]. In contrast to the ratiometric sensors, an onefluorescent protein sensor was also developed, called GCaMP, in 2001 with a better 


\section{Introduction}

signal to nose ratio [Nakai et al., 2001]. GCaMP was based on a circularly permuted enhanced GFP protein (cpEGFP) which was connected to the $\mathrm{Ca}^{2+}$ sensitive domain calmodulin at the $\mathrm{C}$ terminus and the $\mathrm{M} 13$ domain at the $\mathrm{N}$ terminus (Figure 1.2 $\mathrm{C}$ ). The fluorescence of GCaMP further increases on binding to $\mathrm{Ca}^{2+}$ ions due to the conformational change by the $\mathrm{Ca}^{2+}$-calmodulin-M13 interaction [Nakai et al., 2001]. Recent versions of GCaMP have even added the possibility of measuring $\mathrm{Ca}^{2+}$ dynamics in neuronal subregions (GCaMP1.6, [Ohkura et al., 2005]) and also with higher sensitivity for detecting even single action potentials (GCaMP3, [Tian et al., 2009]). The affinity of GCaMP3 for $\mathrm{Ca}^{2+}\left(\mathrm{K}_{\mathrm{D}}=660 \pm 19 \mathrm{nM}\right)$ is higher than the earlier variants of GCaMP and has been used in this thesis for monitoring the neuronal activity. However, further developments have been made to GCaMP in recent years with varying properties like dynamic range and excitation-emission wavelengths [Zhao et al., 2011; Akerboom et al., 2012; Ohkura et al., 2012; Chen et al., 2013; Badura et al., 2014]. The red and blue one-fluorescent protein sensors, for example, enable us to do multicolor imaging, and their combination helps us to measure the activity of different sub-compartments of cells or different populations of neurons at the same time in the same organism
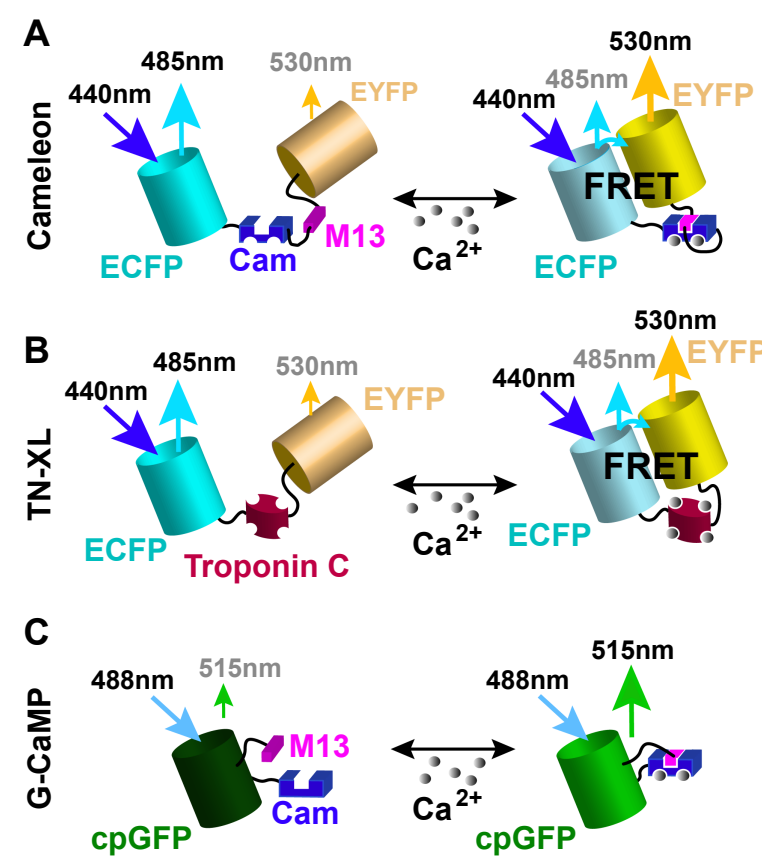

Figure 1.2 Schematic illustration of different genetically encoded calcium indicators.

(A) FRET-based Cameleon sensor: Two fluorescent proteins (ECFP and EYFP) are connected by a $\mathrm{Ca}^{2+}$ sensitive domain called calmodulin (Cam) and a calmodulin binding peptide (M13). In the presence of $\mathrm{Ca}^{2+}$, a conformational change due to the $\mathrm{Ca}^{2+}-\mathrm{Cam}-\mathrm{M} 13$ interaction, brings ECFP and EYFP in a proximity close enough for FRET. (B) Troponin based TN-XL sensor: It functions similar to Cameleon, except that the Cam-M13 domains are replaced by the $\mathrm{Ca}^{2+}$ binding domain of troponin. (C) Onefluorescent protein GCaMP sensor: It consists of a circularly permuted variant of GFP connected to Cam and M13. On binding to $\mathrm{Ca}^{2+}$ ions, a conformational change occurs in the barrel structure of GFP due to the $\mathrm{Ca}^{2+}-\mathrm{Cam}-\mathrm{M} 13$ interaction which leads to an increase in fluorescence emission.

Figure from Riemensperger et al. [2012]. 


\section{Introduction}

[Lindenburg and Merkx, 2012]. Depending on the requirements of the experiments, different $\mathrm{Ca}^{2+}$ sensors can potentially be used nowadays [Pérez Koldenkova and $\mathrm{Na}-$ gai, 2013].

In conclusion, the possibilities for the genetic manipulation in Drosophila and for monitoring the neuronal activity make this animal an advantageous model organism to understand the function of complex neuronal circuits.

\subsubsection{The olfactory system of Drosophila}

\subsubsection{Peripheral odor detection}

Odor sensing in flies occurs when volatile odorant molecules bind to olfactory receptors (ORs) present on the dendrites of the olfactory sensory neurons. The olfactory sensory neurons (OSNs) are located on the third segment of the antennae and the maxillary palps [Su et al., 2009]. There are about 1200 neurons per antenna and 120 neurons per maxillary palp [Stocker et al., 1990; Bruyne et al., 1999], which are housed in hair-like structures called olfactory sensilla [Shanbhag et al., 1999; Bruyne et al., 2001]. Each sensillum contains between one and four OSNs. These sensilla are insulated from each other by the sensillum lymph secreted by the supporting cells surrounding the OSNs. Based on their morphology, sensilla are subdivided into three major classes: basiconic, trichoid and coeloconic [Shanbhag et al., 1999]. Basiconic sensilla are characterized by their long and thick structures. They contain between two and four neurons and they mainly cover receptors responding to food odorants. Coeloconic sensilla are thick and small and contain between two to three neurons. They do not only respond to food odorants, but also to humidity [Yao et al., 2005]. Trichoid sensilla are highly sensitive to pheromones and can contain up to three neurons. All the three classes of sensilla can be found on the antennae, whereas the maxillary palps contain only basiconic sensilla [Riesgo-Escovar et al., 1997].

The OSNs in the sensilla are activated when odorant molecules bind to the ORs [Clyne et al., 1999; Vosshall et al., 1999]. The ORs in insects are seven transmembrane domain proteins, but unlike ORs in mammals, they are evolutionary unrelated to G protein coupled receptors (GPCR) [Vosshall et al., 1999; Benton et al., 2006]. Each OSN usually expresses only one kind of OR together with a broadly expressed co- 


\section{Introduction}

receptor Or83b or Orco [Larsson et al., 2004; Vosshall and Stocker, 2007]. The signal transduction of the olfactory receptors is still currently under investigation and there is no clear consensus about it [Ronderos and Smith, 2009; Nakagawa and Vosshall, 2009]. One study suggests that Or83b forms a heteromeric ion channel with the OR [Sato et al., 2008] while another study indicates that Or83b forms the ion channel itself [Wicher et al., 2008]. In addition, the odor response could also be modulated by Or83b via the cAMP signaling cascade [Wicher et al., 2008; Nakagawa and Vosshall, 2009].

In coeloconic sensilla, only one OR (Or35a) is found to be co-expressed with Or83b [Su et al., 2009]. The other OSNs present in coeloconic sensilla, however, do not express either Or83b or any members of the Or family [Couto et al., 2005; Yao et al., 2005]. They were instead found to express a different class of chemosensory receptors called ionotropic receptors (IRs) [Benton et al., 2009]. These receptors do not co-express Or83b. Instead they are ion channels and are directly activated by the ligand.

The odor response spectrum of each OSN depends on the OR that it expresses [Dobritsa et al., 2003]. Several ligands were identified for each specific OR [Dobritsa et al., 2003; Hallem et al., 2004; Fishilevich and Vosshall, 2005; Couto et al., 2005; Hallem and Carlson, 2006; Galizia et al., 2010]. The axons of OSNs project from the antennae and the maxillary palps to the first relay center of the Drosophila brain, the antennal lobe $(A L)$. All OSNs expressing one kind of chemosensory receptor converge to a single discrete structures called glomeruli [Couto et al., 2005; Fishilevich and Vosshall, 2005]. The AL consists of 56 such glomeruli, each of which receives input only from a particular type of OSNs [Tanaka et al., 2012]. Some studies have also reported the presence of neurons other than OSNs terminating in the $A L$, like thermal sensor neurons, neurons of unknown sensory modalities in arista and antenna and, internal thermal sensor neurons [Stocker et al., 1990; Hamada et al., 2008; Gallio et al., 2011]. In the AL glomeruli, OSNs synapse onto dendrites of projection neurons (PNs) as well as local interneurons (LNs) [Vosshall and Stocker, 2007]. The odor information is first processed in $A L$ and then carried by the $P N s$ to the higher brain centers namely the mushroom body (MB) and the lateral horn ( $\mathrm{LH})$. 


\section{Introduction}

\subsubsection{Odor processing in the antennal lobe}

The odors are encoded in terms of a combinatorial code of the ORs they activate, and this information is carried by the OSNs to the AL. All the OSNs target their specific glomerulus (based on the receptors they express), in many cases both in the ipsilateral and the contralateral side [Stocker et al., 1990], except the OSNs expressing Gr21a which only target the ipsilateral V glomerulus [Vosshall et al., 2000; Scott et al., 2001].

In recent years, several studies have been performed to characterize the responses of OSNs to large and chemically diverse types of odor stimuli [Bruyne et al., 1999, 2001; Hallem et al., 2004; Yao et al., 2005; Hallem and Carlson, 2006; Su et al., 2011; Martelli et al., 2013; Mathew et al., 2013]. These studies have helped us to some degree to understand the mechanism how odors are encoded in OSNs [Wilson, 2013]. Based on the responses to multiple ligands, the OSNs can be divided into classes of different odor "tuning width", i.e., the broader the tuning width is, the larger the number of odorants is that an OSN responds to. Some OSN types are broadly tuned, while some are narrowly tuned [Hallem and Carlson, 2006]. As the concentration of the odor is increased, the OSNs show increases in their firing rates, and more OSNs get recruited [Hallem and Carlson, 2006]. Therefore, the concentration is likely to be encoded by the number of OSNs it activates as well as by the magnitude of response in each OSN. Spikes can even be found in the absence of any ligand. The OSNs, on average, fire spontaneously at the rate of $\sim 8$ spikes/s [Bruyne et al., 1999, 2001]. There are also some ligands which are inhibitory in nature, i.e., they decrease the firing rate of OSNs to below their spontaneous firing rate [Bruyne et al., 1999; Hallem and Carlson, 2006; Silbering et al., 2011; Nagel and Wilson, 2011]. On presenting an odor for long duration, adaptation of OSNs occurs, which is reflected by the weakening of responses to subsequent stimuli [Bruyne et al., 1999]. In terms of the dynamics of OSN activity, the spike rates rise sharply during odor onset and after the odor offset, they reach below their spontaneous rates. However each OSN has their unique dynamics depending on the odor as well its concentration [Schuckel and French, 2008; Schuckel et al., 2008, 2009; Nagel and Wilson, 2011; Kim et al., 2011]. The response diversity of OSNs reflects the diversity of the ORs they express and the affinity of ORs towards different ligands.

OSNs form excitatory synapses onto PNs in their individually defined glomeruli. The 


\section{Introduction}

OSN-PN synapses are cholinergic, and the PNs, like the mitral cells in the mammalian olfactory bulb, are mainly post-synaptic in the AL [Stocker et al., 1990; Kazama and Wilson, 2008]. There are also "sister" PNs found in glomeruli which show highly correlated patterns of activity [Kazama and Wilson, 2009]. There are several studies on the relation between the activities of the OSNs and the PNs [Bhandawat et al., 2007; Ng et al., 2002; Schlief and Wilson, 2007; Silbering et al., 2008; Wang et al., 2003; Wilson et al., 2004]. Compared to OSNs, PNs are less variable in terms of trial-to-trial spike responses to the same stimulus [Bhandawat et al., 2007]. PNs also show more transient responses, i.e., the spike frequency peaks and decays relatively quickly when compared to OSNs [Bhandawat et al., 2007; Wilson et al., 2004]. In terms of odor tuning, PNs are less selective to odors compared to OSNs. PNs show high sensitivity to weak inputs from OSNs and low sensitivity to strong inputs, indicating a nonlinear transformation of olfactory input from OSNs [Olsen et al., 2010]. These differences have been partly explained by the degree of convergence of OSNs onto PNs. There are only about 150 PNs per brain hemisphere, i.e., about 3 neurons per glomerulus, whereas on average 25 OSNs innervate one glomerulus [Stocker et al., 1990; Laissue et al., 1999; Abbott, 2008]. This convergence makes PNs highly sensitive to weak OSN inputs as well as less variable because they integrate the combined responses of many OSNs. The transient responses of PNs and the nonlinear relationship between OSNs and PNs, on other hand, could be explained by the property of OSN-PN synapses which show short-term depression due to the high vesicular release probability, i.e., fast depletion of synaptic vesicles [Kazama and Wilson, 2008; Wilson, 2013]. The PNs also show spontaneous activity in the absence of any ligand. But this is mainly due to the fluctuating inputs coming from OSNs [Gouwens and Wilson, 2009; Kazama and Wilson, 2009]. Most of the PNs are cholinergic [Yasuyama and Salvaterra, 1999] and they release acetylcholine in higher brain centers as well as from presynapses in the AL [Kazama and Wilson, 2008; Ng et al., 2002; Wilson et al., 2004; Yaksi and Wilson, 2010].

The PNs are mainly unilateral, mostly uniglomerular, and they terminate on both the MB and the LH; however there also exist another kinds of PNs [Tanaka et al., 2012]. One PN not only arborizes in the ipsilateral glomerulus but also in the contralateral glomerulus, thereby having bilateral projections. There is another type of PN (from the glomerulus DA4m), which, other than projecting to $\mathrm{MB}$ and $\mathrm{LH}$, also projects to the posterior lateral protocerebrum (PLP). Another kind of PN show multiglomeru- 


\section{Introduction}

lar projections without the formation of any glomerular arborization in the $\mathrm{AL}$. One of such multiglomerular PNs also project to the PLP, in addition to MB and LH. Some uniglomerular as well as multiglomerular PNs project only to the $\mathrm{LH}$. These various types of PNs have been very well characterized in Tanaka et al. [2012]. Other than the cholinergic PNs, GABAergic PNs also exist and have been recently characterized in Drosophila [Strutz et al., 2014]. These neurons are both uniglomerular and multiglomerular.

The glomeruli in the $\mathrm{AL}$ are also interconnected by several populations of $\mathrm{LNs}$. These LNs lack axons, most of them are inhibitory and release GABA [Ng et al., 2002; Wilson et al., 2004]. Several studies have investigated the role of LNs in odor coding [Silbering and Galizia, 2007; Olsen and Wilson, 2008; Tanaka et al., 2009; Acebes et al., 2011]. For example, due to the presence of this interglomerular inhibitory interactions, when a mixture of two odors is presented to the fly, reduced activity in some PNs are observed in comparison to their responses to the individual components of the mixture [Silbering and Galizia, 2007; Olsen et al., 2010]. Therefore, not necessarily all PNs show activity if their corresponding OSNs are activated [Olsen and Wilson, 2008]. The lateral inhibition is mainly presynaptic at the OSN axon terminal [Olsen and Wilson, 2008; Root et al., 2008]; however, inhibition is not restricted to only OSNs but can also affect PNs [Wilson and Laurent, 2005; Yaksi and Wilson, 2010] as well as LNs themselves [Yaksi and Wilson, 2010; Huang et al., 2010]. The inhibition tends to increase as the concentration of the odorant increases [Silbering et al., 2008; Olsen et al., 2010]. The magnitude of inhibition has been shown previously to correlate positively with the total activity of entire OSN population, thereby mediating gain control on PNs [Olsen and Wilson, 2008; Olsen et al., 2010]. However, the inhibition is not global in the AL, but rather spatially specific and patchy [Ng et al., 2002; Silbering and Galizia, 2007]. This could not be explained morphologically by the innervation patterns of LNs, because most of them innervate all glomeruli [Chou et al., 2010; Seki et al., 2010]. Only a small number of LNs are highly specific, and $11 \%$ of all LNs innervate less than half of all the glomeruli [Chou et al., 2010]. Moreover, the spatial pattern of inhibition has been reported to be invariant to odors [Wilson and Laurent, 2005; Chou et al., 2010]. These discrepancies could recently be resolved by Hong and Wilson [2015] who show that all the GABAergic LNs are activated even by the activation of single glomerulus and the LN activity in the glomeruli are invariant to odors. However, the PNs in the glomeruli are differentially affected by LNs due to their varying sensitivity to GABA. As a sum- 


\section{Introduction}

mary from these studies, it can be concluded that lateral inhibition appears to be odor invariant and affects PNs differently in different glomeruli in a stereotypic way.

Odor evoked activity in OSNs and PNs in a glomerulus tends to inhibit the activity in other glomeruli by GABAergic LNs, but at the same time, other glomeruli become activated. This phenomenon is mediated by excitatory LNs (eLNs) [Olsen et al., 2007; Root et al., 2007; Shang et al., 2007]. In other words, both activation and inhibition can be observed in different glomeruli from the activity of a single glomerulus. These excitatory inputs to PNs are mediated via electrical connections by gap junctions between eLNs and PN, and not by nicotinic acetylcholine receptors [Huang et al., 2010; Yaksi and Wilson, 2010]. However, eLNs themselves receive cholinergic inputs from both OSNs and PNs [Huang et al., 2010; Yaksi and Wilson, 2010]. On mutating a gap junction subunit, some PNs show a reduced response, while some show an enhanced response [Yaksi and Wilson, 2010]. The reduction is explained by electrical synapses between PNs and eLNs, whereas the increase in responses is explained by the involvement of GABAergic LNs by mixed synapses (both chemical and electrical) between eLNs and GABAergic LNs [Yaksi and Wilson, 2010]. In conclusion, eLNs can mediate both excitation and inhibition in PNs depending on the odor as well as the glomerulus. However, the functional consequences of their network is still not clear.

In recent years, another population of $L N s$ were found in the $A L$ which were glutamatergic [Chou et al., 2010; Das et al., 2011]. They account for about one-third of the LNs in the AL. These neurons are also not odor selective and are broadly tuned, similar to other LNs. These are inhibitory neurons and suppress the activity of both PNs and GABAergic LNs via glutamate-gated chloride channels [Liu and Wilson, 2013], thereby mediating both excitation and inhibition in PNs. However, the functional role of glutamate as well as the reason behind a system of two inhibitory neurotransmitters are poorly understood.

In spite of the neuronal complexity of the $A L$, it serves a model to figure out the mechanisms of complex odor coding because of the immense advantages of the genetic tools, options of neuronal manipulation as well as the accessibility of monitoring the activity of specific subsets of neurons. This organism has been, therefore, used in this thesis to analyze odor classification. 


\section{Introduction}

\subsection{Aim of the study}

It is important for animals to react to odor stimuli with appropriate behavioral responses. But the natural environment often consist of a plethora of potentially relevant volatile chemicals, and it is very unlikely that each and every kind of odor stimulus is of equal importance for the animal. Therefore, animals classify these odor stimuli into meaningful categories. This study uses Drosophila melanogaster as a model organism to investigate, firstly, whether there is any odor classification in the first olfactory processing center $(\mathrm{AL})$ of the brain at all, and secondly, if so, it is aimed at determining the neuronal mechanism behind this odor classification.

In order to visualize potential classification of odors, one possible way is to stimulate the olfactory system of Drosophila with a certain odor stimulus, and then varying that stimulus gradually. This can be achieved by using binary odor mixtures in a way that one odor is gradually "morphed" into another [Niessing and Friedrich, 2010; Niessing, 2012; Khan et al., 2008]. Here, three odors are chosen namely pentyl acetate (PA), 3-octanol (3-Oct) and 4-methylcyclohexanol ( $\mathrm{MCH})$. The choice of the odors is based on previous studies indicating different levels of similarity in chemical structure as well as in terms of behavioral perception [Haddad et al., 2008; Niewalda et al., 2011; Barth et al., 2014] . Using these three odors, three different combinations of binary mixtures are prepared. The odor stimulus of the binary mixtures is varied by changing the relative concentrations of their chemical components. The AL network represents the neuronal circuitry to be investigated as a potential site mediating odor classification. This is accomplished using optical $\mathrm{Ca}^{2+}$ imaging. Odor evoked $\mathrm{Ca}^{2+}$ activity in individual glomeruli can be monitored at the level of both OSNs and PNs. These glomerular activity patterns can further be evaluated to detect and visualize different types of representation of different odor stimuli. Any odor classification would be observed as an abrupt transition in the odor representations for a linearly varying stimuli. The next step is to determine underlying mechanisms of the antennal lobe network in mediating such potential odor classification. The interglomerular interactions is primarily influenced by the LNs. Since many of the LNs are GABAergic, it is hypothesized that GABA might play a role in classifying odor representations. Therefore as a next step, the involvement of GABA is subsequently investigated. 


\section{Materials and Methods}

\subsection{Materials}

\subsubsection{Drosophila melanogaster strains}

For the $\mathrm{Ca}^{2+}$ imaging experiments, the $\mathrm{Ca}^{2+}$ sensor GCaMP3 [Tian et al., 2009] was used. To express GCaMP3 specifically in olfactory sensory neurons or the projection neurons, flies carrying the DNA construct for GCaMP3 under the control of UAS was used. These flies were crossed with the driver strain Or83b-Gal4 [Larsson et al., 2004] or GH146-Gal4 [Stocker et al., 1997] and were then made homozygous (+;UAS GCaMP3.0;Or83b - GAL4 and +;GH146 - GAL4;UAS - GCaMP3.0) to monitor the $\mathrm{Ca}^{2+}$ dynamics in the cytosol of the olfactory sensory neurons and the projection neurons respectively.

\subsubsection{Media}

Fly food

To prepare $10 \mathrm{I}$ of the fly food medium the following ingredients were used:

$\begin{array}{lc}\text { Agar (Carl Roth GmbH) } & 102.5 \mathrm{~g} \\ \text { Ethanol (Merck) } & 50 \mathrm{ml} \\ \text { Nipagin (Sigma-Aldrich) } & 15 \mathrm{~g} \\ \text { Propionic acid (Carl Roth GmbH) } & 63 \mathrm{ml} \\ \text { Soy flour (Obermühle Rosdorf) } & 100 \mathrm{~g} \\ \text { Corn flour (Obermühle Rosdorf) } & 800 \mathrm{~g} \mathrm{in} \mathrm{2} \mathrm{I} \mathrm{of} \mathrm{water} \\ \text { Malt (Obermühle Rosdorf) } & 800 \mathrm{~g} \text { in 1 I of water } \\ \text { Treacle (Obermühle Rosdorf) } & 220 \mathrm{~g} \text { in 1 I of water } \\ \text { Yeast (Carl Roth GmbH) } & 180 \mathrm{~g} \text { in 1 I of water }\end{array}$




\section{Materials and Methods}

The agar is first added to $5 \mathrm{I}$ of water and dissolved by heating to $99.5^{\circ} \mathrm{C}$. Malt solution, corn flour solution, soy flour, treacle solution and yeast solution are then added and boiled for two hours. After that, the solution is left for cooling until it reaches $55-60{ }^{\circ} \mathrm{C}$. Ethanol, nipagin and propionic acid are then added and mixed. The food is poured into plastic vials and stored at $4{ }^{\circ} \mathrm{C}$.

\subsubsection{Buffers and Solutions}

\section{Ringer's solution}

Ringer's solution is isotonic to the fly's hemolymph, which helps to keep the dissected brain tissue alive for a longer period of time. 1 I of the solution is prepared by dissolving the following chemicals in $1 \mathrm{I}$ of $\mathrm{ddH}_{2} \mathrm{O}$ :

$\begin{array}{lc}\text { Hepes (Carl Roth GmbH) } & 5 \mathrm{mM} \\ \mathrm{NaCl} \text { (AppliChem) } & 130 \mathrm{mM} \\ \mathrm{KCl} \text { (Carl Roth GmbH) } & 5 \mathrm{mM} \\ \left.\mathrm{MgCl}_{2} \text { (Carl Roth } \mathrm{GmbH}\right) & 2 \mathrm{mM} \\ \left.\mathrm{CaCl}_{2} \text { (Carl Roth } \mathrm{GmbH}\right) & 2 \mathrm{mM} \\ \text { Sucrose (Carl Roth } \mathrm{GmbH}) & 36 \mathrm{mM}\end{array}$

The $\mathrm{pH}$ of the solution is adjusted to 7.3 and its osmolarity is adjusted to $290-310$ mOsmol. The solution is stored at $-20{ }^{\circ} \mathrm{C}$ until usage.

\section{Low-melting Agarose solution (1.5\%)}

$0.15 \mathrm{~g}$ of low-melting agarose (Sigma-Aldrich), having congealing temperature of 26 - $30{ }^{\circ} \mathrm{C}$, is added to $10 \mathrm{ml}$ of Ringer's solution and heated in a microwave for a short time so that the agarose dissolves.

\section{Picrotoxin solution}

$0.603 \mathrm{~g}$ of picrotoxin (Sigma-Aldrich) was dissolved in $10 \mathrm{ml}$ of dimethyl sulfoxide (Carl Roth $\mathrm{GmbH}$ ) to make a stock solution of $100 \mathrm{mM}$. Before every experiment, the stock solution was further diluted to $10 \mu \mathrm{M}$ in Ringer's solution. 


\section{Materials and Methods}

\subsection{4. $\mathrm{Ca}^{2+}$ imaging set-up}

\section{In vivo fly preparation}

The following materials were required for in vivo imaging of the fly's brain :

\section{Name}

Microscope slides

Stainless steel metal mesh (diameter of wire $0.3 \mathrm{~mm}$ )

Transparent adhesive tape

$0.1 \mathrm{~mm}$ breakable razor blades

Blade holder

Forceps

Syringe

Syringe needle $(0.5 \mathrm{~mm} \times 25 \mathrm{~mm})$

Low melting agarose

Ringer's solution

A binocular stereomicroscope
Company

VWR International

VWR International

tesa SE

Martor KG, Solingen

Fine Science Tools

Fine Science Tools

B. Braun, Melsungen

Terumo Corporation

Sigma-Aldrich

Olympus
Article \#

631-1550

510-0472

35010

10053-13

11252-23

39346-81-1

SZX12

\section{Widefield microscope}

Widefield microscopy was performed using a fluorescence microscope (Zeiss Axioscope 2 FS). It was equipped with a xenon lamp (Lambda DG-4, Sutter Instrument), a 20x/1.0 NA water immersion objective and different band-pass filter sets. Images were acquired using a 14 bit CCD camera (Coolsnap $\mathrm{HQ}$, Photometrics) installed on the microscope and the acquisition was controlled by the software MetaFluor (Visitron Systems, Puchheim, Germany).

\section{Two-Photon microscope}

For $\mathrm{Ca}^{2+}$ imaging experiments, a two-photon microscope (LSM 7 MP, Zeiss) was used. The two-photon microscope was equipped with the following: 


\section{Materials and Methods}

a. Mode-locked Ti-sapphire Chameleon Vision II laser generating femtosecond pulses of infrared light tuned to 680-1080 nm (Coherent)

b. Photomultiplier detectors (PMT) (Zeiss LSM BiG)

c. Band-pass filter (Zeiss 1768-670)

d. Zeiss CCD camera-AxioCam MRm

e. Zeiss W Plan-Apochromat, 20x/1.0 NA DIC VIS-IR water immersion objective

f. ZEN software for image acquisition

\section{Olfactometer}

A custom-made olfactometer was built to stimulate the flies with different odors. Airborne odorants were provided at a constant airflow rate of $1 \mathrm{ml} / \mathrm{s}$. The air was pumped into the olfactometer by an external aquarium pump. The air was then directed into different channels depending on the open/close state of individual valves (12 V two-way solenoid valves, The Lee Company). There were 8 channels, each having two separate valves and a different odor vial (of total volume $20 \mathrm{ml}$ ) connected to it. These valves were controlled by a computer using a custom-made interface written in LabVIEW. The air in all channels was guided to one common exit, at which a pipette tip was connected to direct the air-flow to fly's antennae. Only during the time of odor stimulation, the air is guided through an odor and, in the rest of the cases, the air is blown through an empty odor vial. To avoid contamination, the air (with or without odor) was continuously removed by an exhaust tube positioned behind the fly preparation set-up.

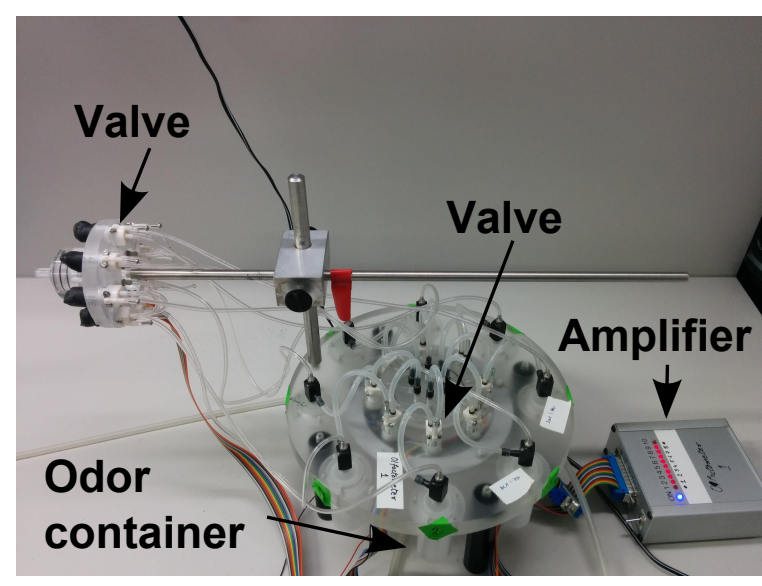

Figure 2.1. Photograph of the olfactometer.

There are 8 different odors channels. Each channel has 2 valves, one at the beginning and the other at the end of the channel. The channels converge into a common exit for odor delivery. The valves are connected to an amplifier which receives inputs from a computer. The air flow in each channel is prodiced by an external air pump. 


\section{Materials and Methods}

\subsubsection{Chemical substances}

\author{
Name \\ 3-Octanol \\ 4-Methylcyclohexanol \\ Pentyl acetate \\ Mineral Oil \\ Dimethyl sulfoxide \\ Picrotoxin \\ Potassium chloride
}

\section{Company}

Sigma

Sigma

Sigma

Sigma

Roth

Sigma

Roth
Catalogue \#

W358126

153095

109584

M5904

4720.1

P1675

6781.3

\subsubsection{Consumables}

Name

Cover slips

Falcon tubes

Forceps

Food vial

Microscope slides

Microtubes

Pipette Tips

Serological pipette
Company

Thermoscientific

Sarstedt

Fine Science Tools

Greiner bio-one

Roth

Sarstedt

Sarstedt

Roth
Catalogue \#

L40931

62.547254

11252-20

205101, 217101

0656

$72.691,72.690$

$70.760002,70.762$

N242.1

\subsubsection{Software}

Name

MetaMorph 7.7

MetaFluor 7.5

ImageJ

ZEN 2011

MATLAB (R2011b)

LabVIEW 2012

Microsoft Excel 2010

\section{Company}

Molecular Devices, LLC.

Molecular Devices, LLC.

National Institutes of Health

Zeiss

The MathWorks

National Instruments

Microsoft 


\section{Materials and Methods}

\subsection{Methods}

\subsubsection{Fly husbandry}

The flies were raised on standard fly food under regulated conditions of temperature $\left(25^{\circ} \mathrm{C}\right)$, humidity $(60 \%)$ and light/dark cycles of $12 \mathrm{~h} / 12 \mathrm{~h}$ in a climate chamber. Different sizes of plastic vials were used depending on the number of flies required for the experiments. For all experiments, 3-5 days old female flies were used.

\subsubsection{In-vivo $\mathrm{Ca}^{2+}$ imaging}

\subsubsection{Fly preparation}

\section{The fly holder}

The fly had to be positioned such that it was completely immobilized. A fly holder was constructed using adhesive tape, a microscope slide and a fine metal mesh (Figure 2.2). The metal mesh was placed over the microscope slide and was fixed using adhesive tape. Several more layers of tape were put on the slide until the thickness of the layers was similar to the height of a fly. Using a splint of a razor blade and a blade holder, a small chamber of about the dimensions of a fly $\left(\sim 3 \times 1 \times 1.5 \mathrm{~mm}^{3}\right)$ was cut into the layers of tape. A narrow passage was cut through the tape layers so that a syringe needle could be placed there for odor application. This set-up was used for the preparation and for optical $\mathrm{Ca}^{2+}$ imaging.

\section{In vivo fly preparation}

A fly expressing the $\mathrm{Ca}^{2+}$ sensor was placed in the chamber of the fly holder (Figure 2.2). It was sealed and restrained with a piece of adhesive tape. Using a splint of a razor blade and a blade holder, a small rectangular window was cut through the tape just over the fly's head. A drop of Ringer's solution was then put on the tape above the fly's head. To remove the cuticle of the head capsule, a fine pair of forceps was used and its one end was poked through the ocelli. By grabbing the cuticle with the forceps, it was ripped off from the head capsule. After removing the cuticle, fat tissue and trachea inside the brain were removed. The drop of Ringer's solution containing the cuticle and tissue debris over the fly head was replaced with a fresh drop of $1.5 \%$ lowmelting agarose solution. The agarose was added to prevent the brain from moving 


\section{Materials and Methods}
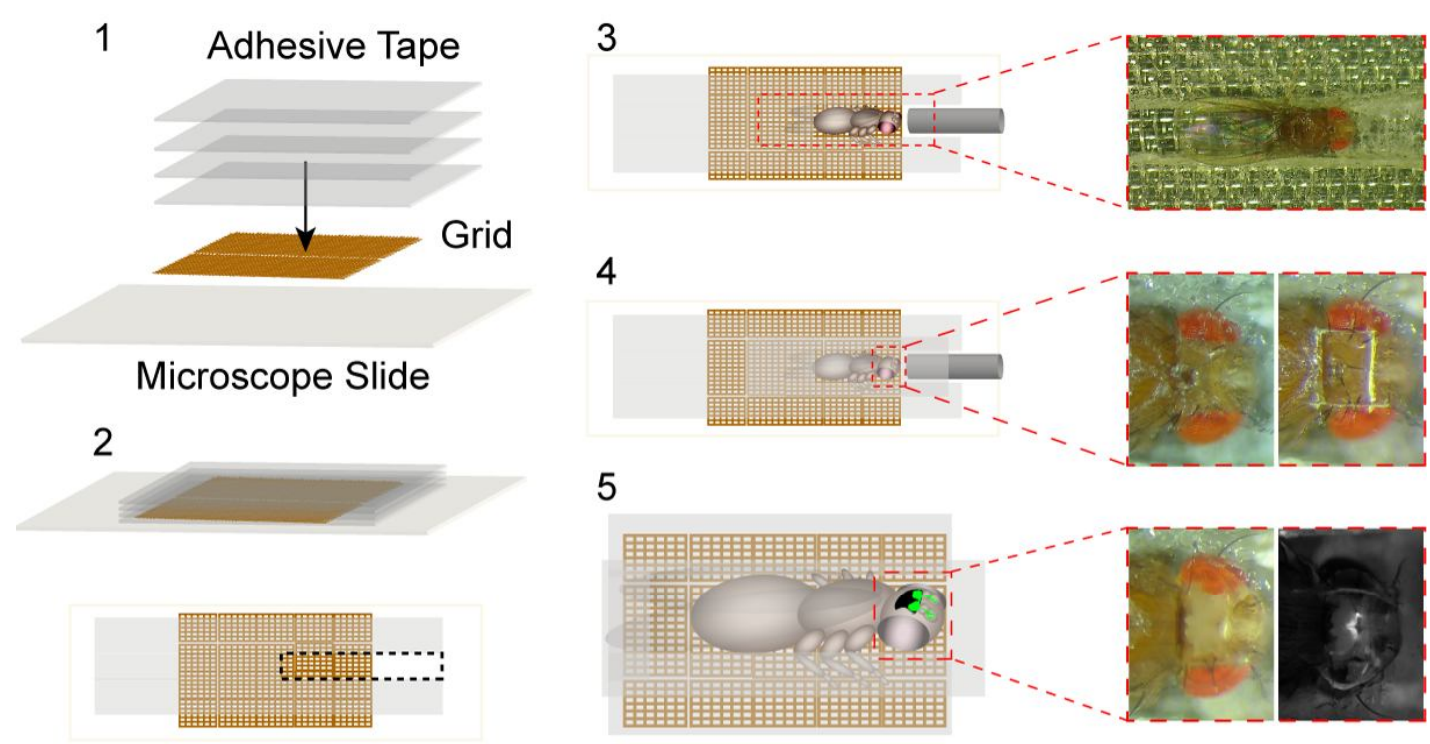

Figure 2.2. Fly preparation for optical imaging.

(1) A metal grid was placed on a microscope slide followed by multiple layers of adhesive tape. (2) A chamber with a passage was cut through the layers of the adhesive tape. (3) A fly was placed within the chamber and a syringe needle was kept in front of its antennae for odor delivery. (4) The fly was then fixed with an another piece of adhesive tape over it. A small rectangular hole was cut through the tape exactly over the fly head. (5) The cuticle over the head was cautiously removed to provide optical access to the brain. The right panels depict the actual images of the preparation during different stages.

Figure from Dipt et al. [2014].

during image acquisition. After the solidification of the agarose, a drop of Ringer's solution was again added over it (Figure 2.2).

\subsubsection{Image acquisition}

The fly preparation was positioned under a $20 \mathrm{X}$ water immersion objective with a numerical aperture of 1.0. For locating the regions of interest, a xenon lamp and a GFP filter set (bandpass: 500-550 nm) was used. After finding the structures of interest, the laser was tuned to $920 \mathrm{~nm}$ wavelength for two-photon excitation of the $\mathrm{Ca}^{2+}$ sensor GCaMP3. Emission and excitation light were separated using a dichroic mirror $(690 \mathrm{~nm}$ long pass) and the emission light was detected by a photomultiplier using an another 


\section{Materials and Methods}

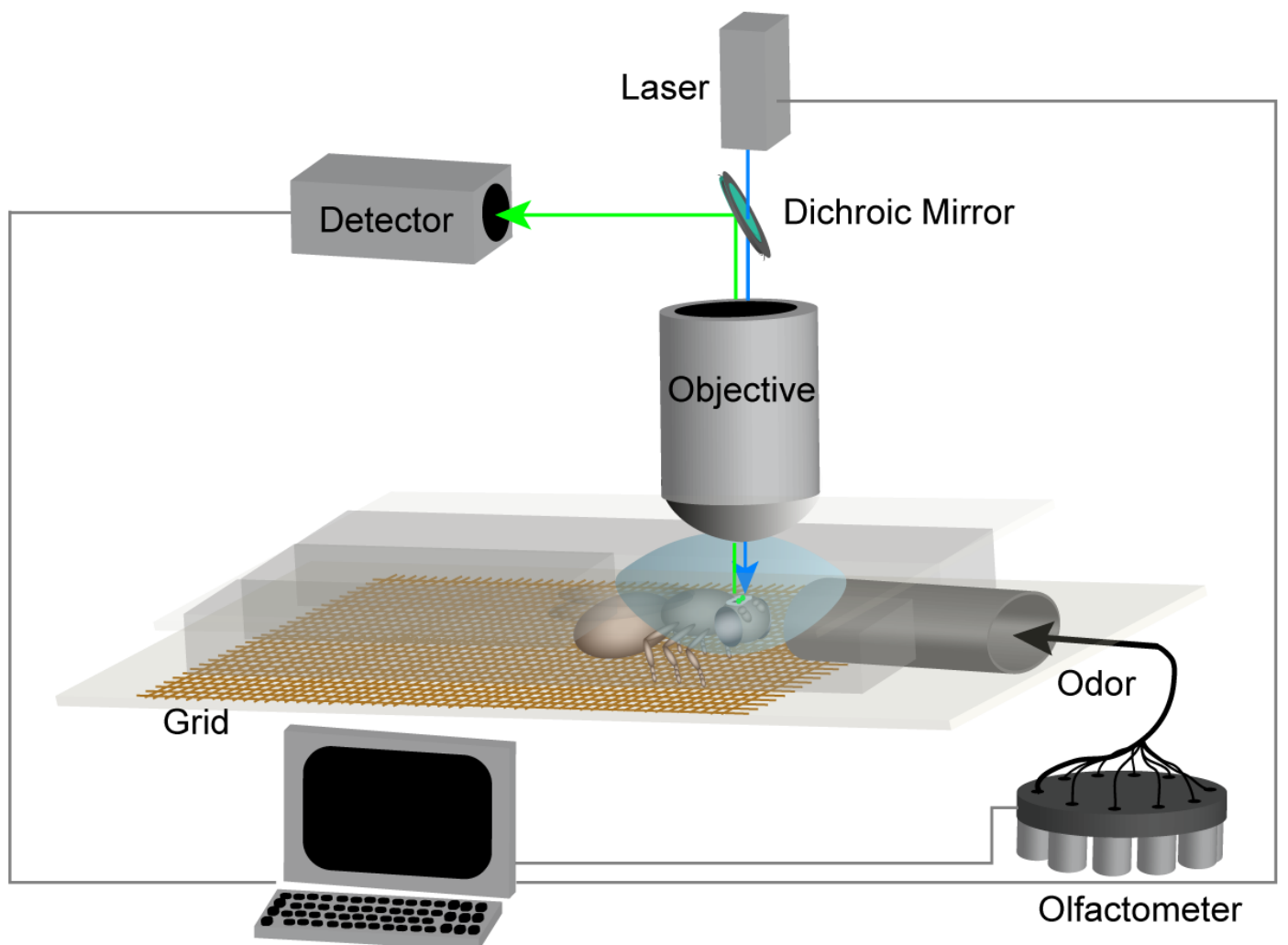

Figure 2.3. Imaging set-up.

A fly was placed in the fly holder with Ringer's solution covering its brain. The fly holder was kept under an objective and the odor was delivered by the olfactometer via a syringe needle directed towards fly's antennae. The fluorophore expressed in the fly's brain was excited by a laser. The emitted light was detected by a photomultiplier. Image acquisition and the olfactometer were externally controlled by a computer using a custom made user-interface.

Figure from Dipt et al. [2014].

set of GFP band-pass filter (bandpass: $500-550 \mathrm{~nm}$ ) (Figure 2.3). The microscope, the laser and the image acquisition were controlled by the software ZEN. For every stimulus, 85 images were acquired at a frame rate of $5 \mathrm{~Hz}$. The odor stimulus was applied for $2 \mathrm{~s}$ from the beginning of the 25th image to the beginning of the 35th image. Images 1 to 24 were used to determine the baseline fluorescence, and images 35 to 85 were used to compare the dynamics of the $\mathrm{Ca}^{2+}$ signal. The images were saved in MetaFluor format. 


\section{Materials and Methods}

\subsubsection{Olfactometer control}

The olfactometer had 8 odor channels, each connected with a different odor vial. Every channel was equipped with two $12 \mathrm{~V}$ two-way valves, one connected at the beginning and the other one connected at the end to prevent any back-flow of odor. The two valves at every channel were triggered by a single common input. Therefore, there were 8 trigger inputs required for 16 valves in 8 channels. The olfactometer was connected to a computer by a parallel (Line Printer Terminal) port cable to control these input triggers. The LPT (Line Printer Terminal) port had 25 pins, out of which 8 could be controlled, called the data pins (pin nr. 2 - 9), and each data pin had its specific ground pin. The LPT port pins sent $5 \mathrm{~V}$ trigger pulses which were amplified to $12 \mathrm{~V}$ to be used as trigger inputs for the $12 \mathrm{~V}$ valves. These 8 data pins together with their ground pins were used as the 8 trigger inputs for the valves by connecting them via a LPT port cable. An amplifier was connected between the olfactometer and the LPT port cable to amplify the $5 \mathrm{~V}$ pulses to $12 \mathrm{~V}$ triggers for the valves. The valves opened on receiving an input signal of $12 \mathrm{~V}$ (Figure 2.4).

\subsubsection{Odor stimulation protocol}

The olfactometer had eight odor channels, out of which one of them was always connected to an empty odor cup. During the image acquisition of 85 images, from the 1st image to the 24th image, the empty channel was kept open. At the beginning of the 25th image, the empty channel was closed and the odor channel was opened. The odor stimulus was applied for $2 \mathrm{~s}$. At the beginning of the 35th image, the odor channel was closed and the empty channel was again opened simultaneously. This complete protocol was repeated for every odor stimulus.

\subsubsection{Scanning trigger \& scanning protocol}

To synchronize the scanning protocol of the microscope with the odor stimulation, the start of the image acquisition was based on an external trigger of $5 \mathrm{~V}$. There were four input triggers and output triggers installed on the microscope computer. Out of the four input triggers "Trigger 1" was used as an external trigger. This trigger was provided by a second computer connected by a 25 pins LPT port cable. To create a scanning protocol, a macro (Visual Basic for Applications (VBA)) package was installed in the ZEN software. Using this package, a macro was written involving the following subroutines: 


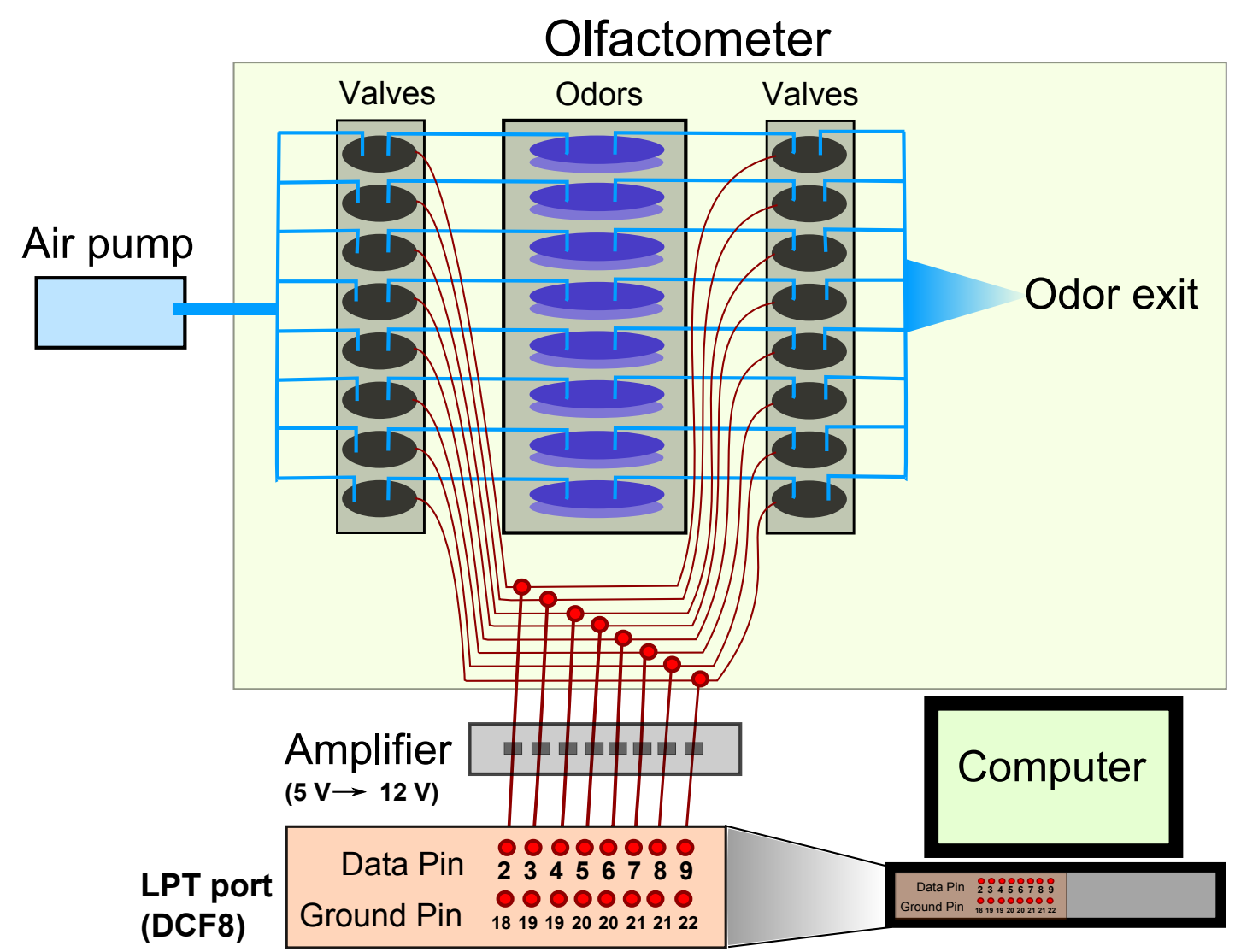

Figure 2.4. Schematic illustration of the olfactometer and its control.

The olfactometer is connected to an air pump which blows in air to the first set of 8 valves via tubes. Each valve is connected to a single odorant-container, which is further connected to an another valve. Therefore, there are two separate valves for each odorcontainer at its opposite ends. On opening the two valves of each odor-container, the air from the pump is guided through the container, taking the odor with it, and then goes out from the olfactometer. Each pair of valves is controlled by a common trigger input of $12 \mathrm{~V}$. The valves open when a $12 \mathrm{~V}$ input is provided. The triggers are provided by a computer through its LPT port having base address DCF8. The LPT port has 8 data pins and each data pin has its specific ground pin. The data pin together with its respective ground pin can provide an output of $5 \mathrm{~V}$. In order to control 8 pairs of valves, which require a $12 \mathrm{~V}$ input each, the $5 \mathrm{~V}$ outputs from the 8 data pins of the LPT port are amplified to $12 \mathrm{~V}$ using an amplifier. The $5 \mathrm{~V}$ outputs from the LPT port are controlled by a computer using a custom made user-interface. 


\section{Materials and Methods}

Time series, Delay, Repeat, Concatenate and Image display.

\begin{tabular}{|l|l|}
\hline Time series & \begin{tabular}{l} 
This subroutine takes all the parameters for scanning \\
previously set in the ZEN software. The start of the time \\
series was dependent on "Trigger In 1". The number of \\
images to be taken is set to 85. \\
It provides a pause in scanning and the interval was set to \\
20 s. \\
\hline Delay
\end{tabular}$\quad \begin{array}{l}\text { It determines the number of time series to be taken. The } \\
\text { number of repetitions is dependent on the number of } \\
\text { stimuli. }\end{array}$ \\
\hline Repeat & $\begin{array}{l}\text { After every time series, all the images are concatenated to } \\
\text { the previously obtained set of images. }\end{array}$ \\
\hline Image display & $\begin{array}{l}\text { It displays all the concatenated images from all the time } \\
\text { series finished by that time. }\end{array}$ \\
\hline
\end{tabular}

On running the macro, image acquisition was initiated by the microscope after receiving an external trigger. 85 images of the sample were acquired. The series of images was then displayed on the screen and the scanning stopped for $20 \mathrm{~s}$. This protocol was repeated several times depending on the number of stimuli, and the images were acquired on receiving the external trigger followed by a pause of $20 \mathrm{~s}$. After every series of scans, the images were first added to the previous set of images and then displayed on the screen (Figure 2.5).

\subsubsection{LabVIEW user-interface}

A custom made user-interface was written using LabVIEW (Olafctometer-MACROSHOCK) which controlled the olfactometer as well as the scanning protocol by the two parallel LPT ports installed on the computer. These parallel ports had base addresses named as ECF8 and DCF8 (Figure 2.6).

ECF8 parallel port: The ECF8 LPT port communicated with the microscope via input "Trigger 1". This trigger was generated by pin nr. 3 as a $5 \mathrm{~V}$ TTL pulse, having 


\section{Materials and Methods}

transition from "High" state to "Low" state. The pin nr. 3 was set on a "High" state whenever this LabVIEW program was started. The imaging protocol (the scanning as well as the odor stimulation protocols) could only be initiated by pressing the "Start Experiment" button, which immediately set the pin nr. 3 to "Low" state, thereby generating the "Trigger 1 " input.

DCF8 parallel port: This parallel port was used to control the channels of the olfactometer by pin $\mathrm{nr}$. 2-9. These pins were initially set to "Low" state and the triggers for the odor channels were generated by changing the respective pin to "High" state.

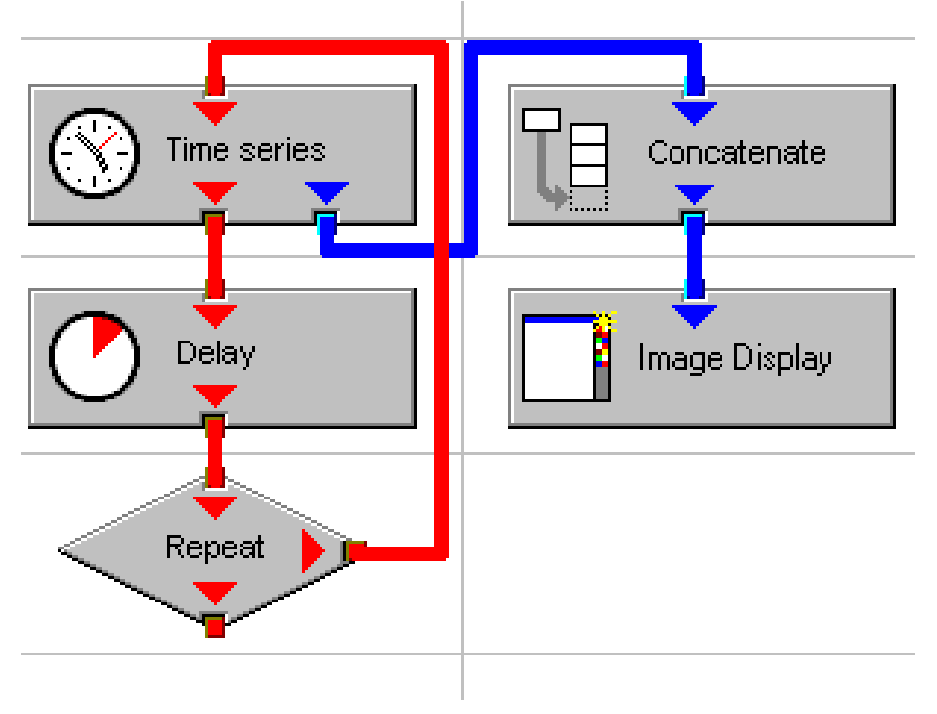

Figure 2.5. The ZEN VBA macro.

The macro consists of five subroutines starting with "Time series". The "Time series" starts the image acquisition for every odor stimulus on receiving an external trigger. It takes up all the parameters of image acquisition determined before in the ZEN software. The next subroutine "Delay" halts the program for $20 \mathrm{~s}$. Depending on the number of odor stimuli, this whole sequence of subroutines are repeated by the "Repeat" subroutine. In parallel, all the images acquired during each "Time series" is concatenated to the previously acquired set of images by the "Concatenate" subroutine. All the images concatenated till the present moment is displayed on the screen by "Image Display". 


\section{Materials and Methods}

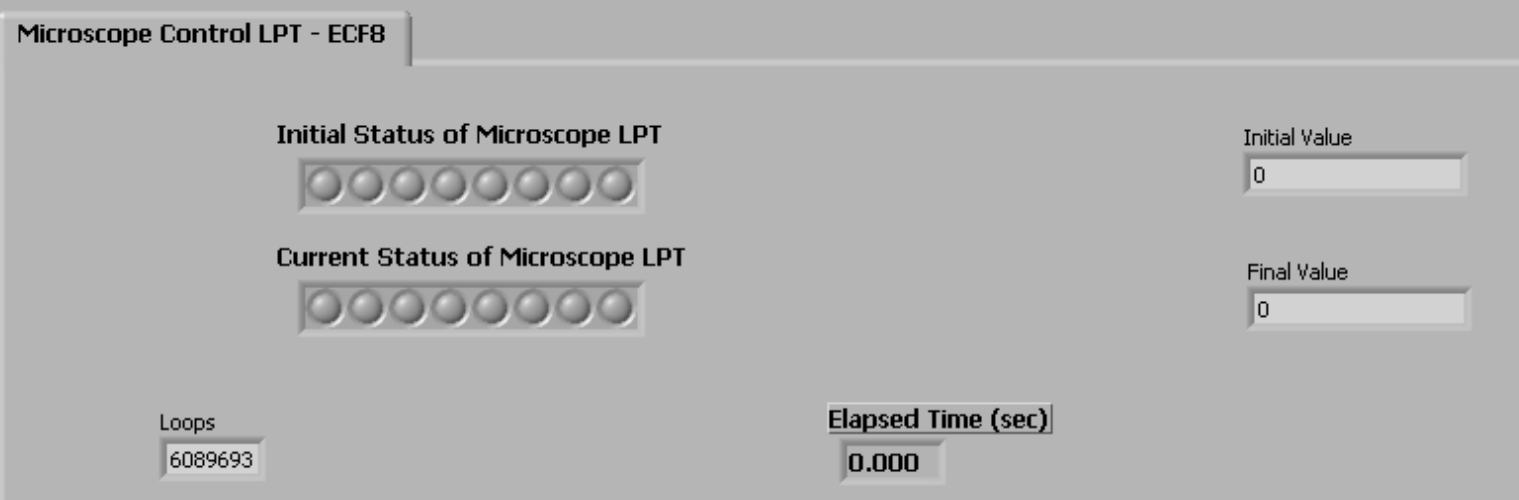

\section{Start Experiment}

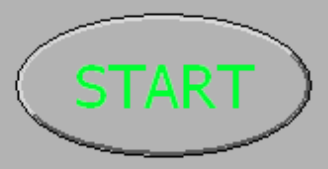

Shock Status (J)
Current Status of olfactometer

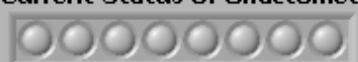

Onset 1

t) 25

Offset 1

강 35

Onset 2

ㄱ) 25

register write value 1

Cycle Number

0

Cases

0

Shock1

(

Shock2

Figure 2.6. Olfactometer-MACRO-SHOCK (LabVIEW user interface) .

This program was used to control the olfactometer and the scanning by the microscope. The upper panel of the program displays the settings of the ECF8 parallel port. The lower panel controls the DCF8 parallel port which is used for the odor stimulus protocol. Each row separated by lines defines the protocol for one single stimulus termed as "Cases". The protocol is initiated by the "Start" button and can be halted anytime by the "Stop" button. More details are explained in the section 2.2.2.6. 


\section{Materials and Methods}

\section{Details of the user-interface :}

\section{Control buttons/switches}

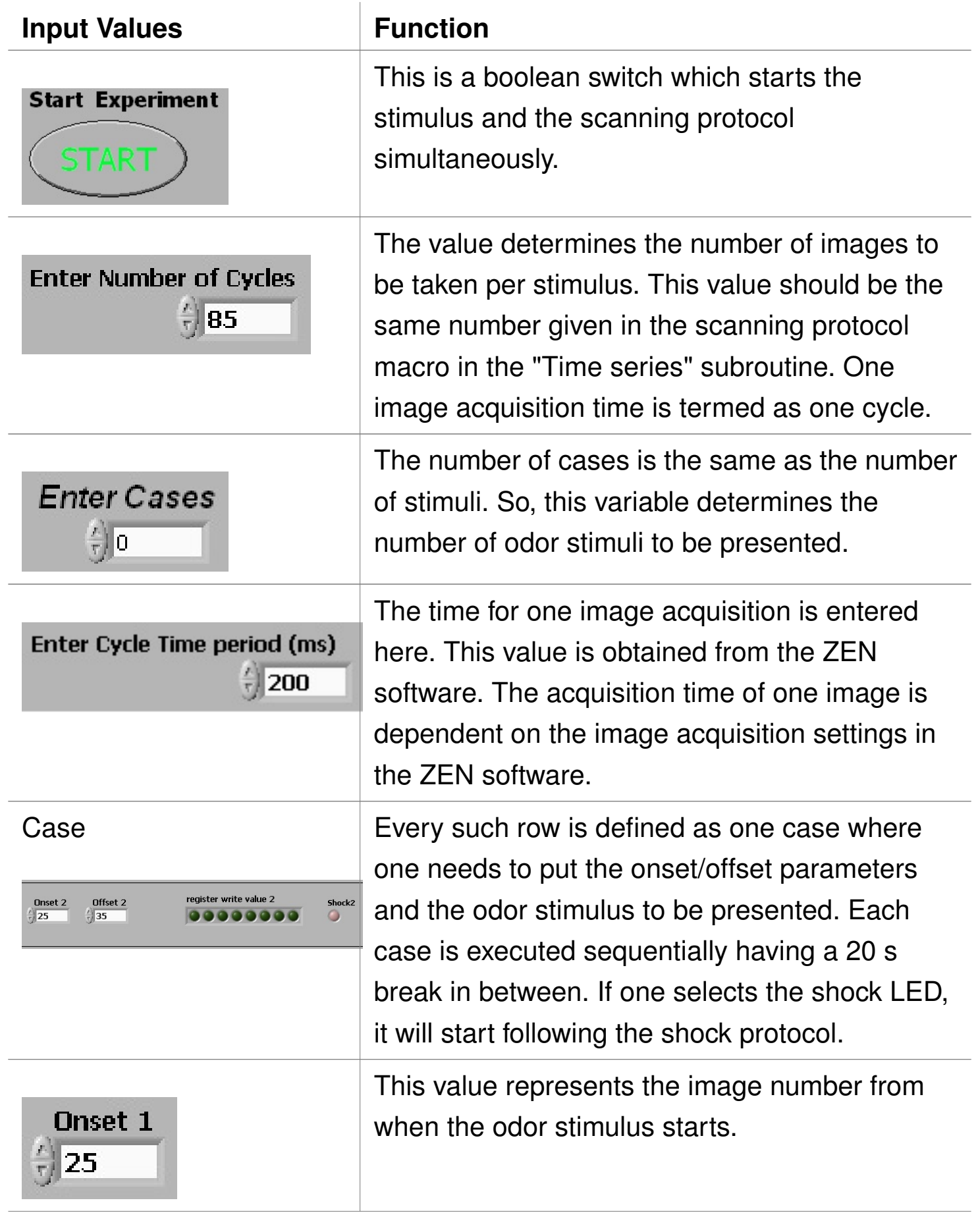




\section{Materials and Methods}

\begin{tabular}{|c|c|}
\hline$\frac{\text { Offset } 1}{35}$ & $\begin{array}{l}\text { This value represents the image number when } \\
\text { the odor stimulus ends. }\end{array}$ \\
\hline $\begin{array}{l}\text { register write value } 1 \\
00000000\end{array}$ & $\begin{array}{l}\text { This determines the status of the channels of } \\
\text { the olfactometer from the onset cycle until the } \\
\text { offset cycle during a particular "Case", which is } \\
\text { the number shown here. Each LED represents a } \\
\text { specific odor channel in the olfactometer. }\end{array}$ \\
\hline Shock1 & $\begin{array}{l}\text { The status of this LED determines whether in } \\
\text { this case the "Shock Protocol" will be followed. }\end{array}$ \\
\hline $\begin{array}{l}\text { st口p } \\
\text { 5TणP }\end{array}$ & $\begin{array}{l}\text { This switch stops the odor stimulus protocol } \\
\text { immediately. }\end{array}$ \\
\hline
\end{tabular}

\section{Indicators}

\begin{tabular}{|c|c|}
\hline Display & Function \\
\hline Current Status of Dlfactometer & $\begin{array}{l}\text { This displays the current status of the ( } 2 \text { valves } \\
\text { x } 8 \text { channels) valves in the olfactometer. Each } \\
\text { LED represents the two valves of one specific } \\
\text { channel of the olfactometer. }\end{array}$ \\
\hline $\begin{array}{l}\text { Cycle Number } \\
\mathbf{0}\end{array}$ & This displays the image number being taken. \\
\hline
\end{tabular}




\section{Materials and Methods}

\begin{tabular}{|c|c|}
\hline Cases & This displays the current case being executed. \\
\hline 0 & \\
\hline Shock Status & $\begin{array}{l}\text { This LED glows when a shock is being delivered } \\
\text { to the fly. }\end{array}$ \\
\hline $\begin{array}{l}\text { Initial Status of Microscope LPT } \\
00000000000\end{array}$ & $\begin{array}{l}\text { This displays the initial status (i.e. before } \\
\text { pressing the "Start Experiment" button) of the } \\
\text { ECF8 LPT parallel port used for triggering the } \\
\text { scanning protocol. Each LED represents the } \\
\text { state of the data pins 2-9. }\end{array}$ \\
\hline $\begin{array}{l}\text { Current Status of Microscope LPT } \\
000000000\end{array}$ & $\begin{array}{l}\text { This displays the current status of the ECF8 } \\
\text { LPT parallel port used for triggering the } \\
\text { scanning protocol. Each LED represents the } \\
\text { state of the data pins } 2-9 \text {. }\end{array}$ \\
\hline $\begin{array}{l}\text { Initial Value } \\
0\end{array}$ & $\begin{array}{l}\text { This displays the initial status (i.e. before } \\
\text { pressing the "Start Experiment" button) of the } \\
\text { ECF8 LPT parallel port in terms of a 8-bit value. }\end{array}$ \\
\hline $\begin{array}{l}\text { Final Value } \\
0\end{array}$ & $\begin{array}{l}\text { This displays the current status of the ECF8 } \\
\text { LPT parallel port in terms of a 8-bit value. }\end{array}$ \\
\hline $\begin{array}{l}\text { Loops } \\
6089693\end{array}$ & $\begin{array}{l}\text { This displays the loop number of the program; } \\
\text { i.e., the number of times the whole program has } \\
\text { been executed. }\end{array}$ \\
\hline$\frac{\text { Elapsed Time (sec) }}{0.000}$ & This displays the time elapsed by the program. \\
\hline
\end{tabular}




\section{Materials and Methods}

Protocol : The scanning protocol is first prepared using the VBA macro. The "Repeat" value from the macro is given in the "Enter Cases" and the number of images set in the "Time series" is given in "Enter Number of Cycles". The "Onset" and "Offset" of all the cases are set to 25 and 35, respectively. The odor channels are selected for every case by clicking the corresponding LEDs in "register write value \#". The scan time is obtained from the ZEN software and the same value is entered in "Enter Cycle Time period(ms)". After setting all these values, the VBA macro is initiated followed by pressing the "Start Experiment" button.

\subsubsection{Experimental design}

The regions of interest was focused on the AL of the fly's brain. Three focal planes were selected in order to cover the maximum number of glomeruli in the $A L$. The odors were diluted in mineral oil in required concentrations at a total volume of $2 \mathrm{ml}$.

\subsubsection{Data analysis}

\section{Image processing}

The images obtained from the ZEN software were saved in MetaFluor format. These images were processed by the following methods:

\section{TwoPhoton Batch Metamorph StackBuild}

This plugin was created in ImageJ software for converting all the images in MetaFluor format to a single stickfigure animator file (.stk) file (A.1.1). This plugin used another plugin (A.1.2) which it used to process every folder containing the images. A new folder named "AlignteStacks" was created where the stickfigure animator file called "GFP.stk" was saved.

\begin{tabular}{|l|l|}
\hline Input & Value \\
\hline Path: & $\begin{array}{l}\text { The path of the parent folder of the folders containing } \\
\text { the images was entered here. }\end{array}$ \\
\hline $\begin{array}{l}\text { Number of slices for } \\
\text { each stack }\end{array}$ & $\begin{array}{l}\text { The number of images in each folder was given here. } \\
\text { The number was kept the same for every folder in the } \\
\text { parent folder. }\end{array}$ \\
\hline
\end{tabular}




\section{Materials and Methods}

Prefix:

The common prefix of the names of all the folders in the parent folder was entered here. The plugin processed only the folders having the names starting with the "Prefix" characters.

\section{Batch Alignment GFP}

This Matlab script was used for aligning the images taken in one measurement (A.2.1). The mean of the first three images was taken as a template image and all the other images were aligned to it. The algorithm of the alignment was based on Guizar-Sicairos et al. [2008]. This script generated a series of images (.tif) aligned to the reference image in a new folder called "GFP_Matlab_Aligned".

\begin{tabular}{|l|l|}
\hline Input & Value \\
\hline $\begin{array}{l}\text { Number of images for } \\
\text { mean reference image } \\
\text { for alignment : }\end{array}$ & $\begin{array}{l}\text { It required the number of images needed to make a } \\
\text { reference image. }\end{array}$ \\
\hline Number of GROUPS: & $\begin{array}{l}\text { The number of parent folders containing folders with } \\
\text {.stk files was entered here. }\end{array}$ \\
\hline GROUP number \# & $\begin{array}{l}\text { It required the names of each parent folder as } \\
\text { described above. }\end{array}$ \\
\hline Location of the files: & The path of such parent folders was entered here. \\
\hline
\end{tabular}

\section{TwoPhoton Batch Matlab StackBuild Processing}

This ImageJ plugin (A.1.3) which was based on two other plugins namely MatlabTif StackBuild (A.1.4) and Matlab Evaluate Journal (A.1.5). MatlabTif StackBuild plugin created a single file (.stk) from the aligned .tif images generated by the Batch Alignment GFP. This file was then saved as "GFP_Matlab_Aligned.stk" in the folder "AlignteStacks". Matlab Evaluate Journal used this file to create false-color coded images to display the regions of high $\mathrm{Ca}^{2+}$ activity in warm colors and low $\mathrm{Ca}^{2+}$ activity in cold 


\section{Materials and Methods}

colors. The false color coded images were generated by $\frac{F_{i}-F_{0}}{F_{0}} \times 100+1000$, where $F_{0}$ was the mean of a given number of images before odor onset and $F_{i}$ was the mean of the same number of images during the peak of the $\mathrm{Ca}^{2+}$ signal. 1000 was added to the images to set the minimum pixel value (i.e. 0 ) to 1000 so that inhibition of $\mathrm{Ca}^{2+}$ signals could be observed and such pixel values would come below 1000 . Noise in these images were reduced by applying a median filter of 5 pixels.

\begin{tabular}{|l|l|}
\hline Input & Value \\
\hline Path : & $\begin{array}{l}\text { It required the path of the parent folder containing the } \\
\text { folder with the images. }\end{array}$ \\
\hline $\begin{array}{l}\text { Number of slices for } \\
\text { each stack }\end{array}$ & $\begin{array}{l}\text { The number of images present in every folder was } \\
\text { entered here. }\end{array}$ \\
\hline $\begin{array}{l}\text { Number of slices used } \\
\text { for averaging when } \\
\text { evaluating }\end{array}$ & $\begin{array}{l}\text { The number of images required for creating } F_{0} \text { and } F_{i} \\
\text { images was entered here. }\end{array}$ \\
\hline Prefix: & $\begin{array}{l}\text { The common prefix of the names of all the folders in } \\
\text { the parent folder was entered here. The plugin } \\
\text { processed only those folders having the names starting } \\
\text { with the "Prefix" characters. }\end{array}$ \\
\hline Journal: & $\begin{array}{l}\text { This required the information about the image numbers } \\
\text { which should be used for creating } F_{0} \text { and } F_{i} \text { images. } \\
\text { The journal used was: } 7 \text { Conc0 } 2027 \text { Conc1 } 105112 \\
\text { Conc25 } 190197 \text { Conc50 } 275282 \text { Conc75 } 360367 \\
\text { Conc99 } 445452 \text { Conc100 } 530537 \text { (where the first } \\
\text { number determined the number of stimuli presented } \\
\text { during the acquisition of images; Conc0 was the name } \\
\text { given to the first stimulus; } 20 \text { and } 27 \text { indicated that } 20 \text { th } \\
\text { and } 27 \text { th image would be taken as the first image to be } \\
\text { averaged to create } F_{0} \text { and } F_{i} \text { images respectively. The } \\
\text { same was repeated for every stimulus). }\end{array}$ \\
\hline
\end{tabular}




\section{Materials and Methods}

\section{Defining the regions of interest (ROI)}

The regions of interest were drawn around the glomeruli of the AL. Three different focal planes were selected to cover most glomeruli (Figure 2.7). However, due to variability in the orientation of the brain during the fly preparation and the imaging process, there were differences in the focal planes. 14 glomeruli (namely DA1, DA4, DC1, DL1, DL5, DM1, DM2, DM3, DM4, DM6, VA1, VC1, VC2 and VM2) could be identified in all flies measured. The identification was done with the help of a 3D model of the AL [Laissue et al., 1999] with labeled glomeruli available online at http://www.flybrain.org [Armstrong et al., 1995]. In cases of small glomeruli, where the boundaries between them were not distinctly visible, the boundaries of the ROls' were defined by comparing the baseline activity in very small regions. Moreover, these boundaries were verified when these glomeruli were activated during the odor stimulus.

\section{Evaluation of the $\mathrm{Ca}^{2+}$ activity}

The "GFP_Matlab_Aligned.stk" files were opened using a software called MetaMorph. This software was used to draw ROls and transfer the values of average intensity of the ROls to a Microsoft Excel file. The fluorescence intensity values $\left(F_{i}\right.$, where $\mathrm{i}$ is a time point) of 5 images before odor onset were averaged to obtain a baseline fluorescence value as $F_{0}$. To calculate the change in fluorescence caused by an odor stimulus, $F_{0}$ was subtracted from all fluorescence intensity values $\left(F_{i}\right)$, thereby producing $\Delta F$ at all time points. This difference in intensity $(\Delta F)$ was subsequently divided by the baseline fluorescence $\left(F_{0}\right)$ in order to normalize for different baseline fluorescence levels of GCaMP3 in different ROls and across flies: $\frac{\Delta F}{F_{0}}=\frac{F_{i}-F_{0}}{F_{0}}$. This evaluation was then repeated for every stimulus using a custom-written Excel Visual Basic for Applications (VBA) program (see A.3.1).

\section{Principal Component Analysis}

The principal component analysis was performed using Matlab. It reduces the dimensionality of data while retaining most of the variation. This is done by transforming the data into a new set of dimensions called principal components, such that the variation along those dimensions are maximal [Ringner, 2008]. 


\section{Materials and Methods}

A
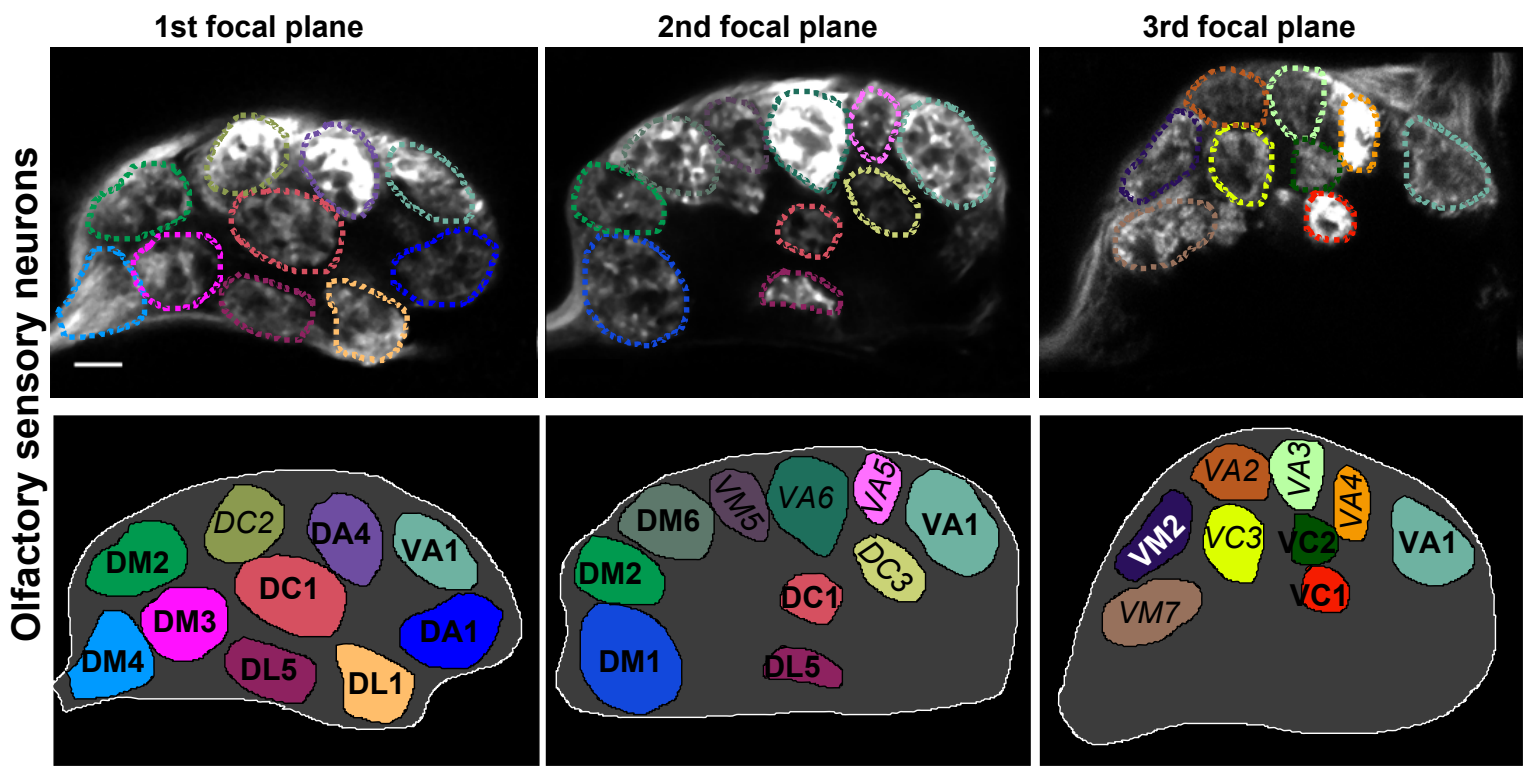

B
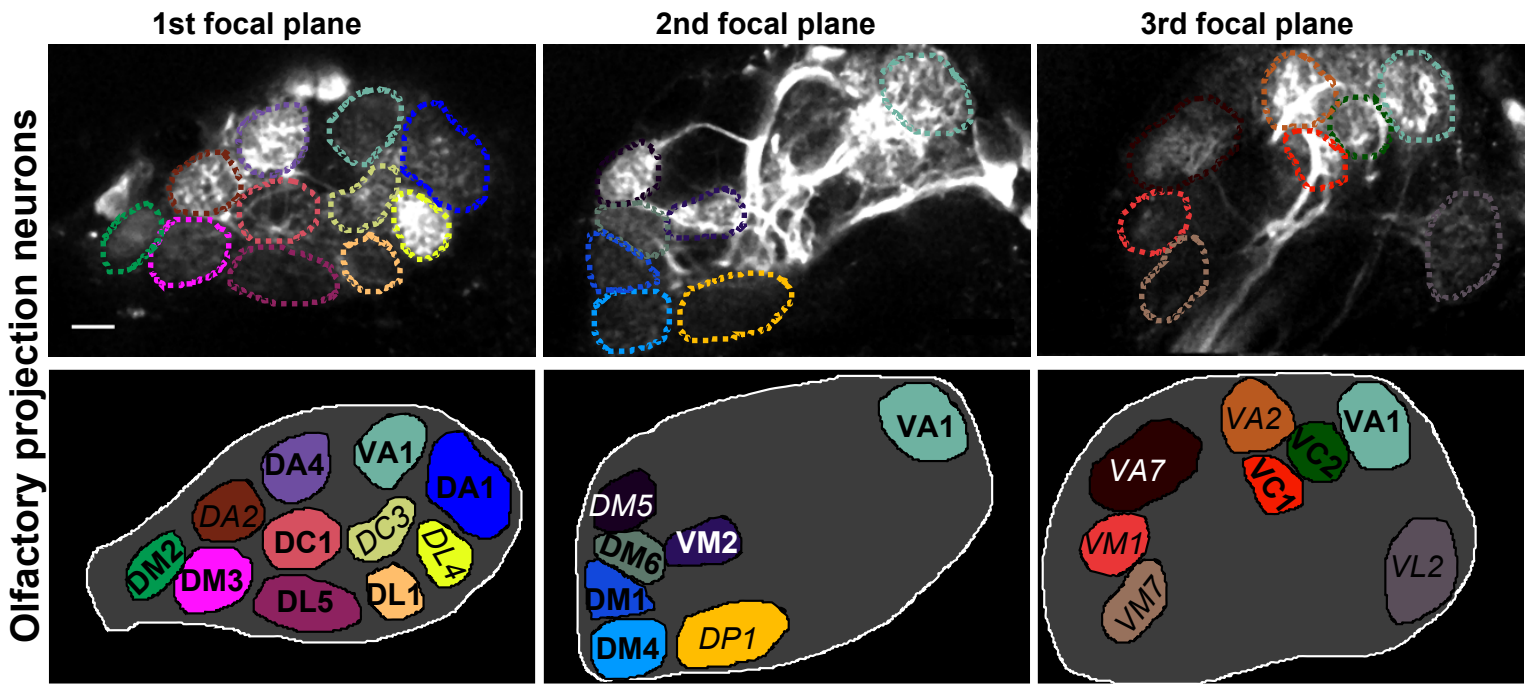

Figure 2.7. Identified glomeruli in the three focal planes of the antennal lobe.

(A) Upper panel: The three focal planes in the antennal lobe of a single fly with genotype +;UAS - GCaMP3.0; Or $83 b-G A L 4$ measured using two-photon microscopy. This fly expresses GCaMP3 in the olfactory sensory neurons and the images depict the basal fluorescence of GCaMP3. The dotted lines indicate the boundaries of different glomeruli present in these focal planes. The glomeruli labeled in bold and not in italics could be identified in all the flies for all experiments and were therefore used for the analysis. Scale bar: $10 \mu \mathrm{m}$. Lower panel: A schematic diagram of the antennal lobe with different glomeruli labeled in different colors. (B) Similar to $(A)$, this panel shows the three focal planes of a single fly with genotype +;GH146-GAL4;UAS-GCaMP3.0. This fly expresses GCaMP3 in olfactory projection neurons and the images depict the basal fluorescence of GCaMP3. Scale bar: $10 \mu \mathrm{m}$. 


\section{Materials and Methods}

\section{Correlation Analysis}

The correlation maps were constructed by calculating the Pearson's correlation coefficients between odor evoked activity patterns [Niessing and Friedrich, 2010]. The $\frac{\Delta F}{F_{0}}$ calculated in every glomerulus for a certain odor stimulus was taken as observations. These observations together were then taken as a vector which represented the activity pattern of that stimulus. The same procedure was repeated for other stimuli. The similarity of the activity patterns of any two stimuli were then compared by determining the Pearson's correlation coefficient between their vectors using Matlab as follows :

$$
r=\frac{\operatorname{cov}(X, Y)}{\sigma_{X} \sigma_{Y}}=\frac{n \sum_{i=1}^{n} x_{i} y_{i}-\sum_{i=1}^{n} x_{i} \sum_{i=1}^{n} y_{i}}{\sqrt{n \sum_{i=1}^{n} x_{i}^{2}-\left(\sum_{i=1}^{n} x_{i}\right)^{2}} \sqrt{n \sum_{i=1}^{n} y_{i}^{2}-\left(\sum_{i=1}^{n} y_{i}\right)^{2}}}
$$

where

$r=$ Pearson's correlation coefficient,

$X$ and $Y$ are two vectors,

$c o v$ is the covariance,

$\sigma$ is the standard deviation,

$n=$ number of observations in each vector,

$x_{i}=$ the $i$ 'th observation of the vector $x$,

$y_{i}=$ the $i$ 'th observation of the vector $y$.

The significance of the correlation coefficients were determined by the $p$-values obtained by t statistics in Matlab for testing the hypothesis of no correlation.

\section{Angular distances}

The angular distances were calculated to determine differences in odor evoked activity patterns [Parnas et al., 2013]. Each glomerulus was taken as one dimension and the odor stimuli were then represented as a vector in this multidimensional space based on the $\frac{\Delta F}{F_{0}}$ values in every glomerulus. Such vectors were then generated for all the odor stimuli. The angular distances between any two activity patterns of different odor stimuli were defined as the angle between their corresponding vectors. They were calculated in Matlab using the following : 


\section{Materials and Methods}

$$
\theta=\cos ^{-1}\left\{\frac{X . Y}{\|X\|\|Y\|}\right\}
$$

where;

$X, Y=$ the vectors representing odor stimuli,

$X . Y=$ the dot product of two vectors i.e. $X$ and $Y$.

\subsubsection{Pharmacology}

A stock solution of picrotoxin (PTX) was prepared by dissolving it in dimethyl sulfoxide (DMSO) at a concentration of $100 \mathrm{mM}$. The solution was always kept protected from light at $-20^{\circ} \mathrm{C}$. For the experiments, the stock solution was further diluted in Ringer's solution to achieve a final concentration of $10 \mu \mathrm{M}$. This solution was always freshly prepared before the experiments. The $\mathrm{Ca}^{2+}$ activity of the flies was measured in three stages: before the application of PTX, during the application of PTX and after washing off the PTX. After recording the odor evoked $\mathrm{Ca}^{2+}$ activity in the first stage, the PTX solution was applied to the fly's brain and was left for 45 min to penetrate the brain tissue. The $\mathrm{Ca}^{2+}$ activity to the odor stimuli was then again recorded. In the third stage, for washing off the PTX, the brain was flushed with fresh Ringer's solution several times for another $45 \mathrm{~min}$. After the wash, $\mathrm{Ca}^{2+}$ activity was then again recorded. 


\section{Results}

In order to test whether or not the antennal lobe network mediates classification of odor representations, gradually varying odor stimuli were presented to the fly. These stimuli comprised of different binary mixtures with varying relative composition of their components. Any classification of odors would be reflected in an abrupt transition of a linearly varying odor stimulus. To find such non-linear transformation of odor representations in the AL, three types of binary mixtures were prepared from the combinations of three distinct odors, namely pentyl acetate (PA), 3-octanol (3-Oct) and 4-methylcyclohexanol $(\mathrm{MCH})$. These odors have been previously used in several studies regarding odor coding and behavior in Drosophila [Bruyne et al., 2001; Hallem et al., 2004; Knaden et al., 2012; Barth et al., 2014]. Moreover, these three odors have different degrees of similarity to each other in terms of chemical structure [Haddad et al., 2008] as well as behavioral perception [Niewalda et al., 2011; Barth et al., 2014].

Using any two of the three odors, seven different binary mixtures, with varying ratios of components, were made as follows: $100 \%-0 \%, 99 \%-1 \%, 75 \%-25 \%, 50 \%-50 \%$, $25 \%-75 \%, 1 \%-99 \%$ and $0 \%-100 \%$. An odor concentration of $10^{-2}$ diluted in mineral oil was termed as $100 \%$, and the other concentrations were accordingly made as $99 \%$, $75 \%, 50 \%, 25 \%$ and $1 \%$ on a linear scale. Linear scale was chosen so that the total odor content was kept constant in all the binary mixtures. However, to make sure that the chosen set of binary mixtures evoked different odor representations in the AL especially in OSNs, one of the prerequisites was that the individual odors of the binary mixture itself did not evoke a same odor representation across the selected range of concentrations, i.e., from $1 \%$ to $100 \%$. Therefore, the first step was to investigate the concentration dependency of odor representations of single odors. This was achieved by recording the $\mathrm{Ca}^{2+}$ activity in individual glomeruli in the $\mathrm{AL}$ for different concentrations of PA, 3-Oct and $\mathrm{MCH}$. 


\section{Results}

\section{1. $\mathrm{Ca}^{2+}$ activity of individual glomeruli to different odors and their concentrations}

\subsubsection{Pentyl acetate (PA)}

On presenting pentyl acetate as an odor stimulus, 50\% of the 14 glomeruli showed strong responses in both OSNs and PNs (Figure 3.1). Every responding glomerulus had its own unique dynamics to different concentrations of PA which are described in following sections.

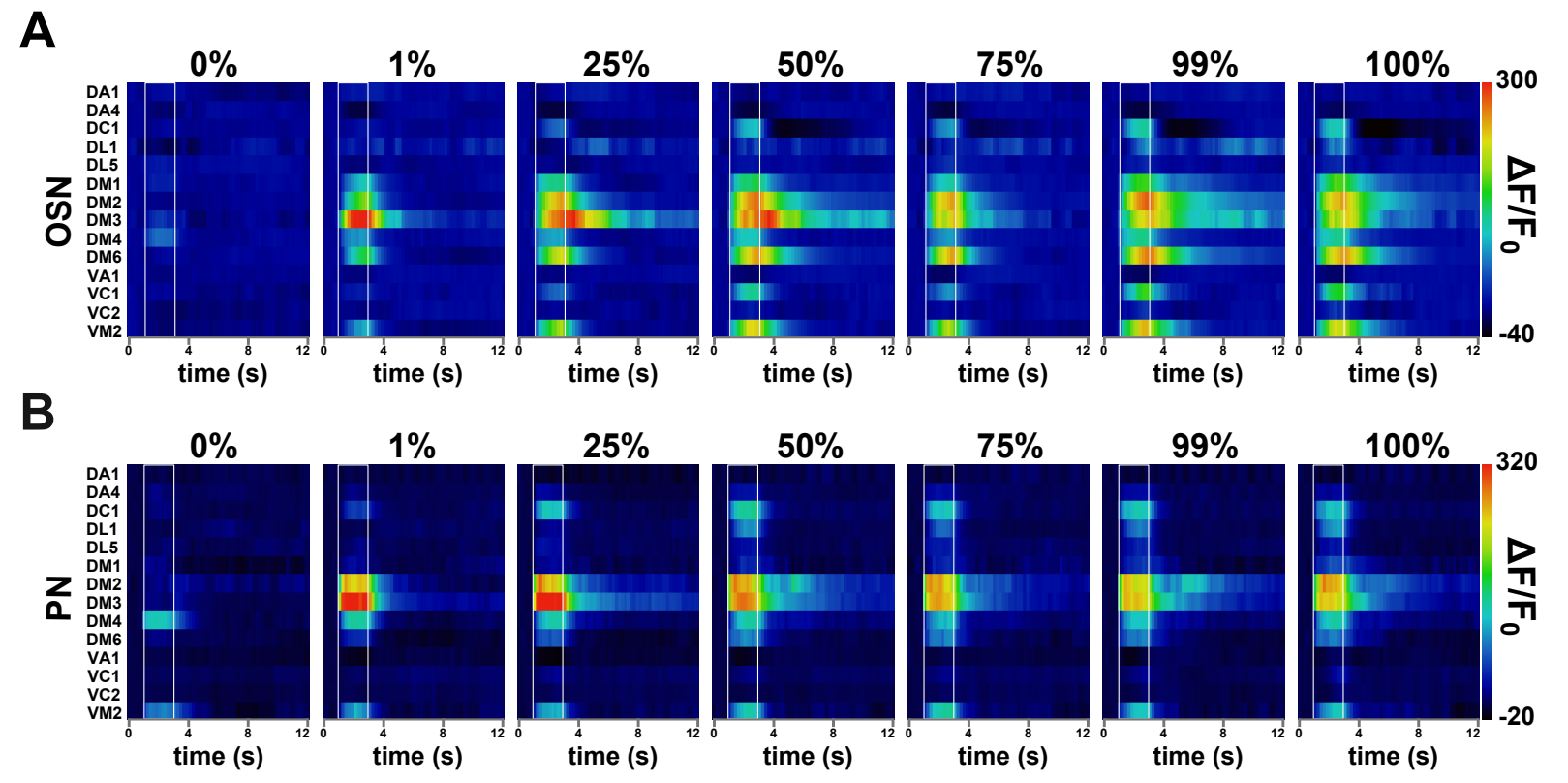

Figure 3.1. Odor evoked $\mathrm{Ca}^{2+}$ activity to different concentrations of pentyl acetate in OSNs and PNs.

The figure shows the glomerular activity map of different concentrations of pentyl acetate in OSNs (A) and PNs (B). The activity map was determined by the $\frac{\Delta F}{F_{0}}$ values obtained for the 14 glomeruli. The white frame indicates the duration of the odor stimulus which was applied for $2 \mathrm{~s}$. High $\mathrm{Ca}^{2+}$ activity is shown in warm colors and low activity in cold colors. Every row in each map represents the activity of single glomerulus indicated on the extreme left. Different glomeruli responded differently to varying concentrations of PA in both OSNs and PNs. In OSNs, stronger activity could be observed in more glomeruli as the concentration increased. Therefore each concentration evoked a distinct glomerular activity pattern in OSNs. In PNs, few glomeruli showed changes with increasing concentrations. $n=5$ flies. 


\section{Results}

\subsubsection{1. $\mathrm{Ca}^{2+}$ activity in OSNs}

The glomerulus DM3 showed the strongest response in OSNs. The increase in concentration of PA was characterized by the increase in responses in glomeruli DC1, DM2, DM6, VC1 and VM2. The glomerular activity patterns evoked by PA at low concentrations were a subset of the activity patterns at higher concentrations. However, DA4 and VA1 showed an inhibition in activity across all the concentrations (Figure 3.2). The activity in DC1 went below the baseline after the odor offset, thereby showing a post stimulus inhibition. In case of DM3, as concentration increased, the amplitude of the signal dropped, but at the same time the decay period of the signal increased. In other glomeruli like DM6 and DM1, the decay period became longer with increasing concentrations. Overall, from the activities in all the glomeruli, differences in the PA representation of different concentrations was observed to be complex and not just restricted to one property of the dynamics.

Interestingly, in terms of temporal aspects, on comparing the time points of the peak responses of all the responding glomeruli to each concentration, it was found that the majority of them showed a maximum response at odor offset in OSNs (Figure 3.3). Therefore, although there were differences in activity across all glomeruli, most of them however still showed the same time point of their peak responses in spite of their different amplitudes of $\mathrm{Ca}^{2+}$ activity.

\subsubsection{2. $\mathrm{Ca}^{2+}$ activity in PNs}

In case of PNs, the responses in the glomeruli were comparatively more transient than OSNs. The glomerulus DL1 showed a prominent signal from $50 \%$ concentration unlike in OSNs. There was a strong decrease in $\mathrm{Ca}^{2+}$ signals in glomeruli DM1, DM6 and $\mathrm{VC} 1$ when compared to OSNs, indicating a presence of inhibition. The glomerulus VA1 again showed a similar inhibition in activity as in OSNs across all concentrations; but, DA4 on other hand did not show any inhibition. The increase in PA concentrations was not strongly reflected in terms of increase in strength of the signals in the glomeruli (Figure 3.2) especially after 25\%, however in case of DM2, prolonged responses were observed with increasing concentrations. So similar to OSNs, the odor representations in PNs did not show a linear change with concentrations, but rather it was complicated because the changes in $\mathrm{Ca}^{2+}$ activity with concentrations varied across all glomeruli. But unlike OSNs, on comparing the time point of maximum signal 


\section{Results}

in all glomeruli to all concentrations, different glomeruli showed different time points of their peak responses, thereby giving an even distribution of glomeruli, especially after the first quarter $(0.5 \mathrm{~s})$ of the odor stimulus. This indicated a stronger role of temporal variability in PNs. (Figure 3.3).

\section{A}

\section{Olfactory sensory neurons}

DC1
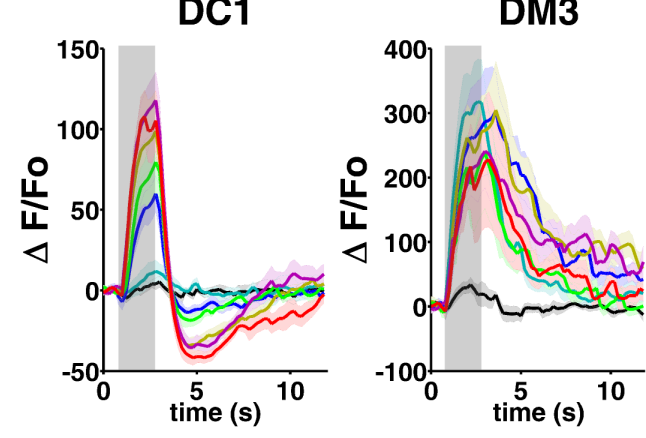

DM4

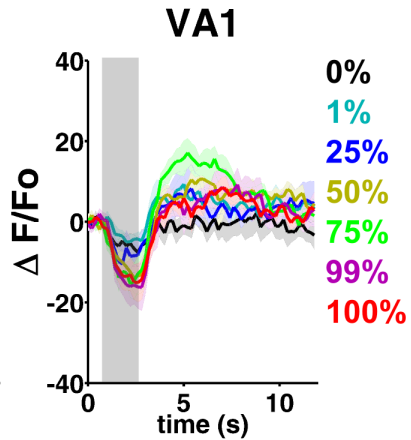

B

\section{Olfactory projection neurons}
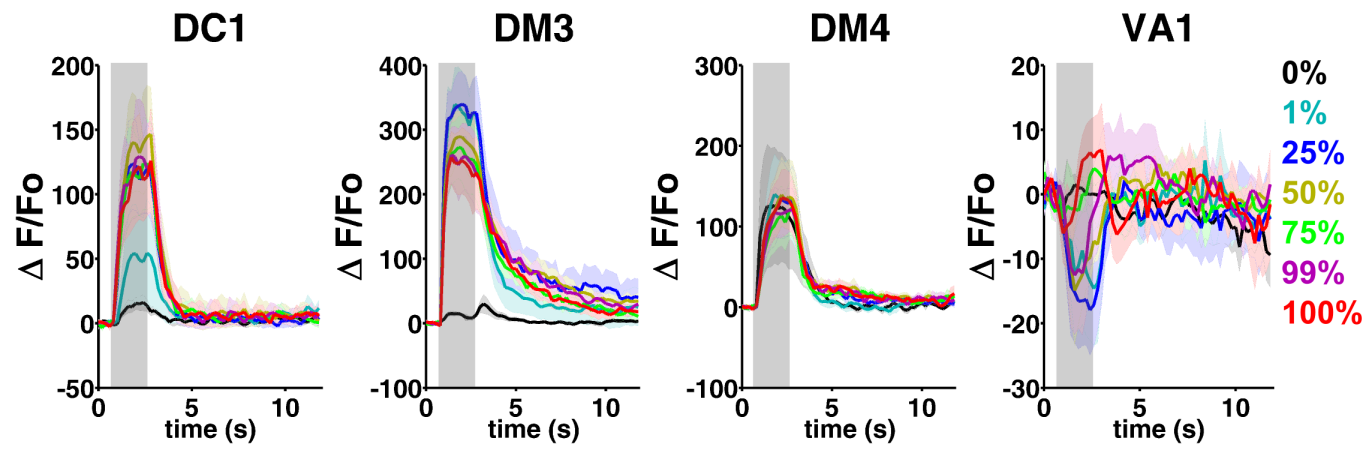

Figure 3.2. Dynamics of pentyl acetate-evoked $\mathrm{Ca}^{2+}$ activity in selected glomeruli. Comparison of $\mathrm{Ca}^{2+}$ activity dynamics of different groups of OSNs (A) and PNs (B) to different concentrations of pentyl acetate. The grey shaded region indicates the duration of the odor stimulus. Different glomeruli showed different dynamics with increasing odor concentrations, both in OSNs and PNs. In OSNs, DC1 and DM4 showed an increase in activity with increasing odor concentrations. DM3 showed a decrease in amplitude, but an increase in the decay period of the signal. VA1 showed an inhibition in both OSNs and PNs. In PNs, beyond $50 \%$ of odor concentration, all the concentrations showed similar $\mathrm{Ca}^{2+}$ dynamics in DC1, DM3 and DM4. Lines indicate mean activity of 5 flies and the shaded region around them indicate \pm SEM. 


\section{Results}

\subsubsection{Concentration-dependent global $\mathrm{Ca}^{2+}$ activity}

In terms of individual glomeruli in OSNs and PNs, changes in odor representations with concentrations seemed complex. Therefore, in the next step the overall activity measured in the 14 glomeruli was calculated to observe the dependence of global $A L$ activity on different concentrations of PA (Figure 3.4 A). It was observed that, as concentration of PA increased, the total $\mathrm{Ca}^{2+}$ activity also increased in OSNs, but in PNs, all the concentrations were found to evoke similar total activity. Therefore, in terms of total activity, PNs were relatively more invariant to PA concentrations in comparison to OSNs. However, to determine whether the total activity was influenced by the number of glomeruli recruited at each odor concentration, the number of responding glomeruli was evaluated. This was determined based on the criteria that a responding glomerulus should evoke $\mathrm{Ca}^{2+}$ activity higher or lower than mean $\pm(3 \times$ standard deviation $)$ of the pre-stimulus spontaneous $\mathrm{Ca}^{2+}$ activity. All the concentrations showed an equal number of responding glomeruli in both OSNs and PNs (Figure 3.4 B).

From the results above, it could be concluded that the complexity in the differences in the PA representations of different concentrations mainly lay in the activities and their dynamics in individual glomeruli. Subsequently, this was further verified by determining the PA representations of different concentrations in terms of the maximum odor evoked $\mathrm{Ca}^{2+}$ activities in the 14 glomeruli (Figure $3.4 \mathrm{C}$ ). Different glomeruli showed different dependencies on PA concentrations in terms of maximum $\mathrm{Ca}^{2+}$ activity. For
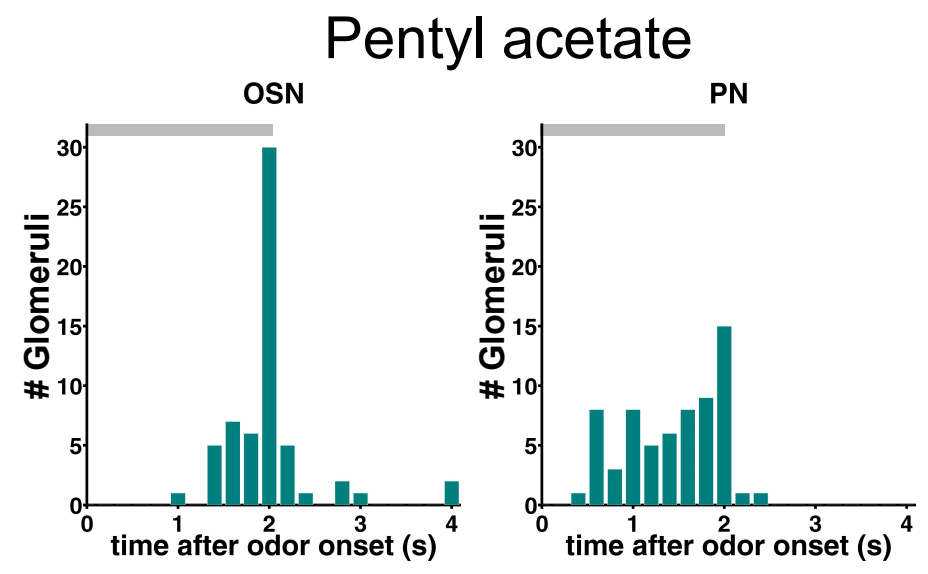

Figure 3.3 Temporal distribution of peak glomerular responses.

This figure shows histograms of the peak response time points of all responding glomeruli across all concentrations in OSNs and PNs. The grey bar indicates the duration of the odor stimulus. Most of the glomeruli in OSNs showed peak responses during the odor offset. In PNs, different glomeruli showed different time points of their peak response. $\mathrm{n}$ (\# responding glomeruli $x \#$ odor stimuli $)=60$ (OSN) and 65 (PN). 


\section{Results}

A
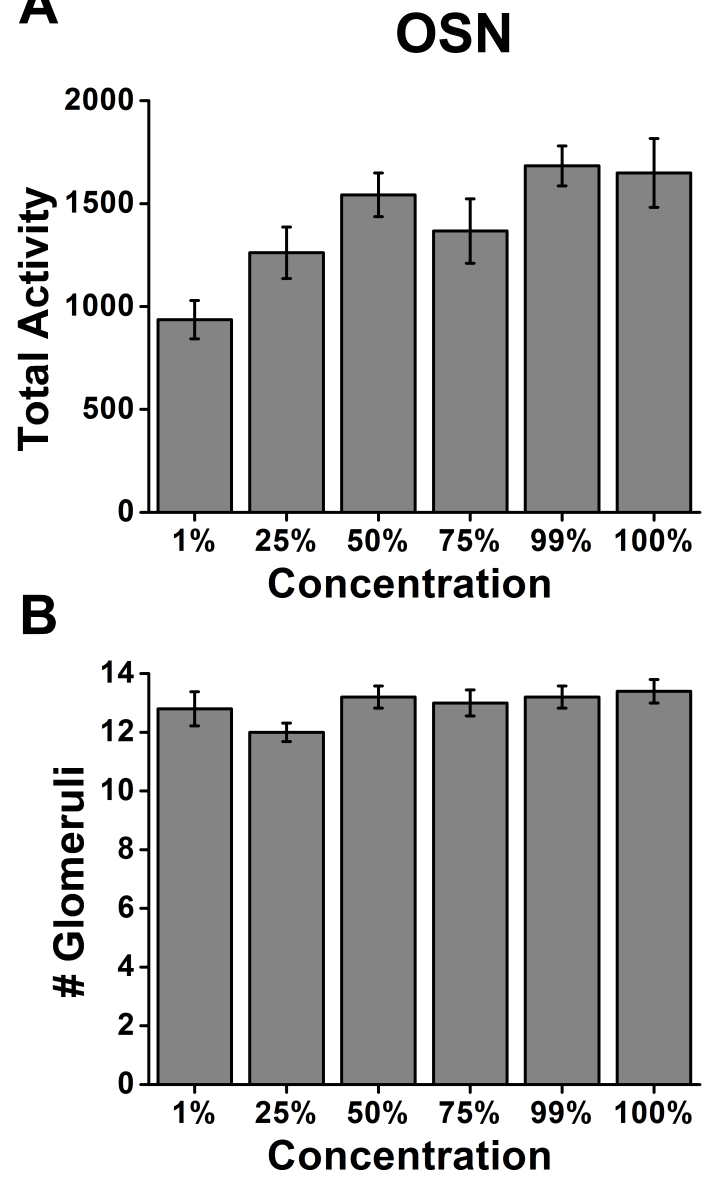

C

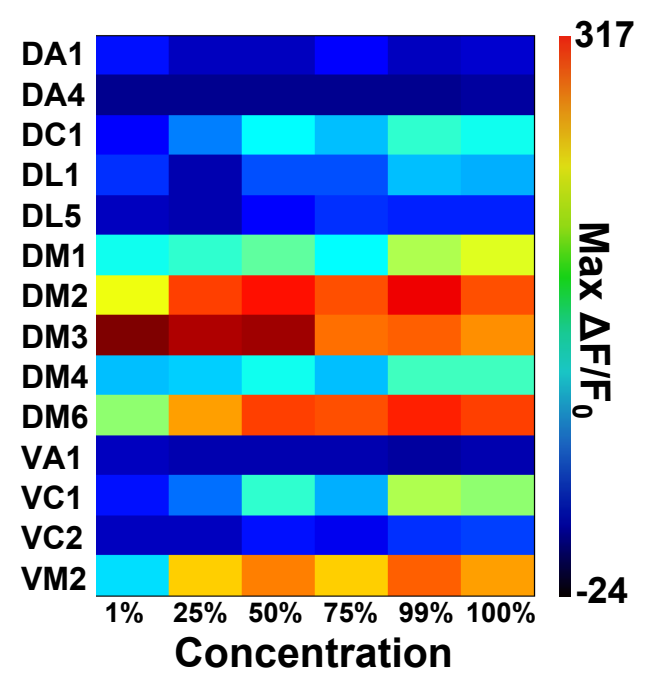

PN
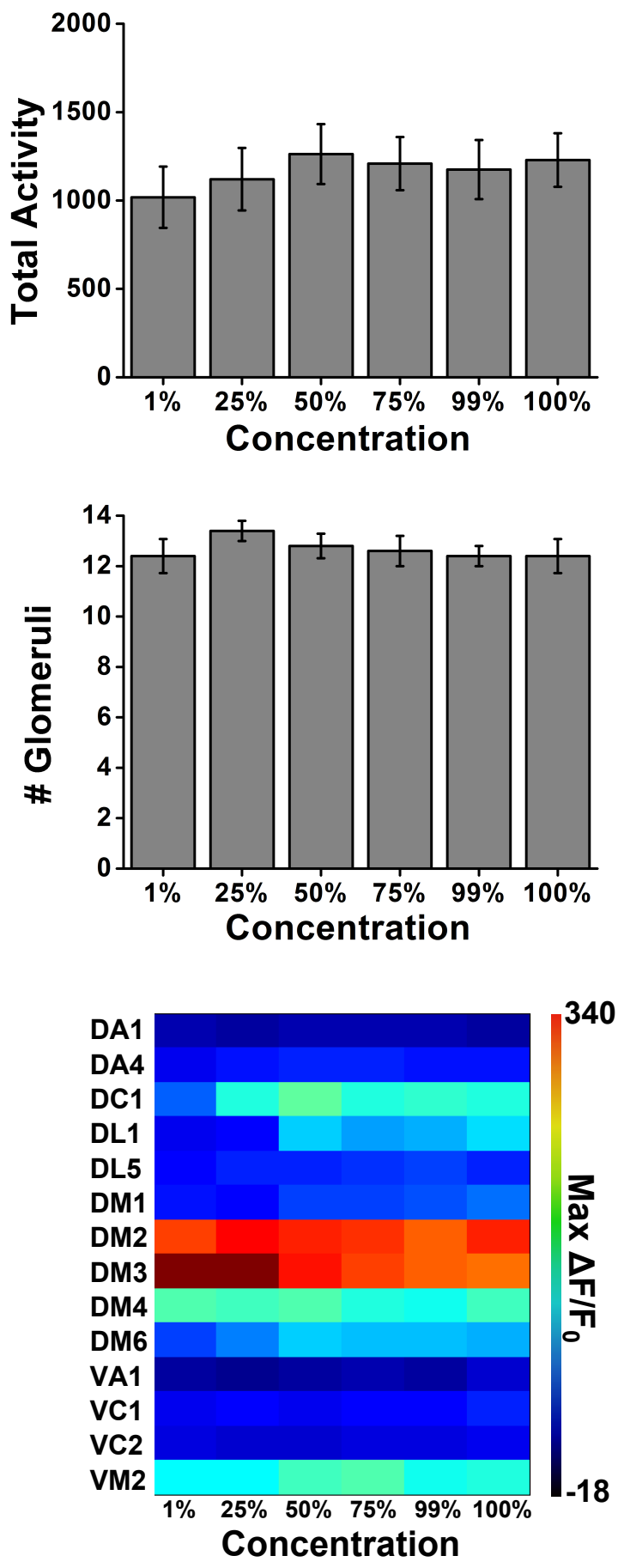


\section{Results}

Figure 3.4. Concentration dependence of pentyl acetate-evoked $\mathrm{Ca}^{2+}$ activity in the antennal lobe.

(A) Comparison of total activity of the antennal lobe across its 14 glomeruli in OSNs and PNs in response to different concentrations of pentyl acetate. The total activity was determined by the sum of maximum $\mathrm{Ca}^{2+}$ activity evoked in each glomerulus for each individual fly. In OSNs, total activity was different for different concentrations. In PNs, all the concentration evoked same total activity. Bars indicate means of the maximum total activity of 5 flies \pm SEM. (B) Number of glomeruli responding to different concentrations of pentyl acetate. Any glomerulus showing higher or lower response than mean $\pm(3 \times$ standard deviation) of the pre-stimulus spontaneous $\mathrm{Ca}^{2+}$ activity was taken as a responding glomerulus. 12 out of 14 glomeruli showed responses to all concentrations of pentyl acetate in both OSNs and PNs. No differences could be found in terms of the number of glomeruli recruited at each concentration in both OSNs and PNs. Bars indicate means of the number of responding glomeruli of 5 flies \pm SEM. (C) Maximum $\mathrm{Ca}^{2+}$ activity evoked by different concentrations of pentyl acetate in each glomerulus. Different glomeruli were differentially sensitive to various pentyl acetate concentrations. In OSNs, glomeruli like DC1, DM1 and VC1 showed an increase in activity with increasing odor concentration. In PNs, such concentration dependence was not observed in different glomeruli. However, DC1, DL1 and DM6 showed a sharp increase in activity beyond a certain concentration. High activity is shown in warm colors and low activity in cold colors.

example, DC1 and VC1 showed a greater dependency on the used range of concentrations than DM2 in OSNs. However, such a gradual increase in activity with concentration was not observed in PNs. But an abrupt increase in activity across different concentrations could be observed in some glomeruli in PNs, e.g., DL1 and DM6 which showed a drastic increase in their activities from $25 \%$ to $50 \%$, but beyond $50 \%$ their activity remained the same. Similarly, DC1 responded weakly to $1 \%$, but a strong response was observed beyond $25 \%$. No change in the maximum responses of DM2 and DM4 was observed across different concentrations. These variations across glomeruli in both OSNs and PNs made the overall odor representation of different concentrations of PA distinct. 


\section{Results}

\subsubsection{3-octanol (3-Oct)}

This compound is an alcohol with eight carbon atoms and it is in some aspects similar to pentyl acetate in terms of chemical structure. This similarity is also reflected in the glomerular activity patterns evoked by 3-Oct (Figure 3.5). Across different concentrations, differences in the glomerular activity patterns could be observed which are described in the following sections.

A
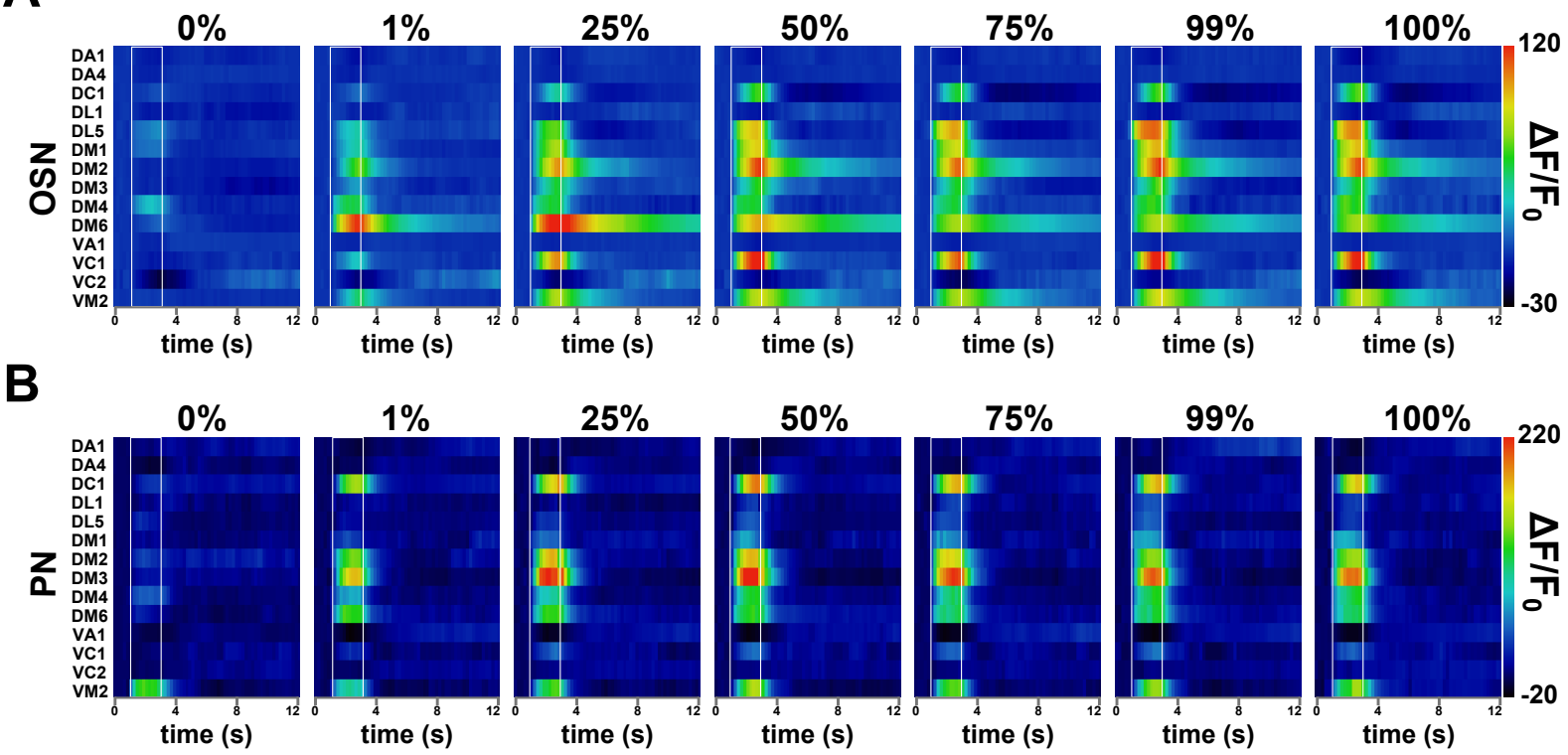

$75 \%$
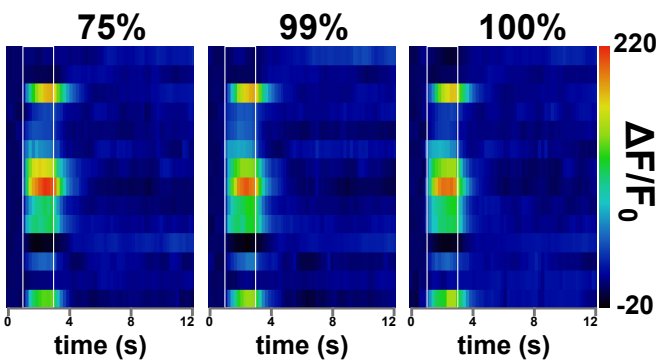

Figure 3.5. Odor-evoked $\mathrm{Ca}^{2+}$ activity to different concentrations of 3-octanol in OSNs and PNs.

The figure shows the glomerular activity map of different concentrations of 3-octanol in OSNs (A) and PNs (B). The activity map was determined by the $\frac{\Delta F}{F_{0}}$ values obtained for the 14 glomeruli. The white frame indicates the duration of the odor stimulus. $\mathrm{High} \mathrm{Ca}^{2+}$ activity is shown in warm colors and low activity in cold colors. Every row in each map represents the activity of single glomerulus indicated on the extreme left. Different glomeruli responded differently to varying concentrations of 3-Oct in OSNs. Responses in some glomeruli, like DL5 and DM2, became stronger with increase in concentrations, which made the glomerular activity pattern of each concentration distinct. In PNs, a similar concentration dependency was not observed, and especially beyond $25 \%$, the glomerular activity patterns of different concentrations did not drastically change. $n=6$ flies.

\subsubsection{1. $\mathrm{Ca}^{2+}$ activity in OSNs}

Out of the 14 glomeruli, nine glomeruli responded strongly to 3-Oct. The glomeruli DM6 and VC1 showed the strongest response. Most of the glomeruli showed an 
A

B
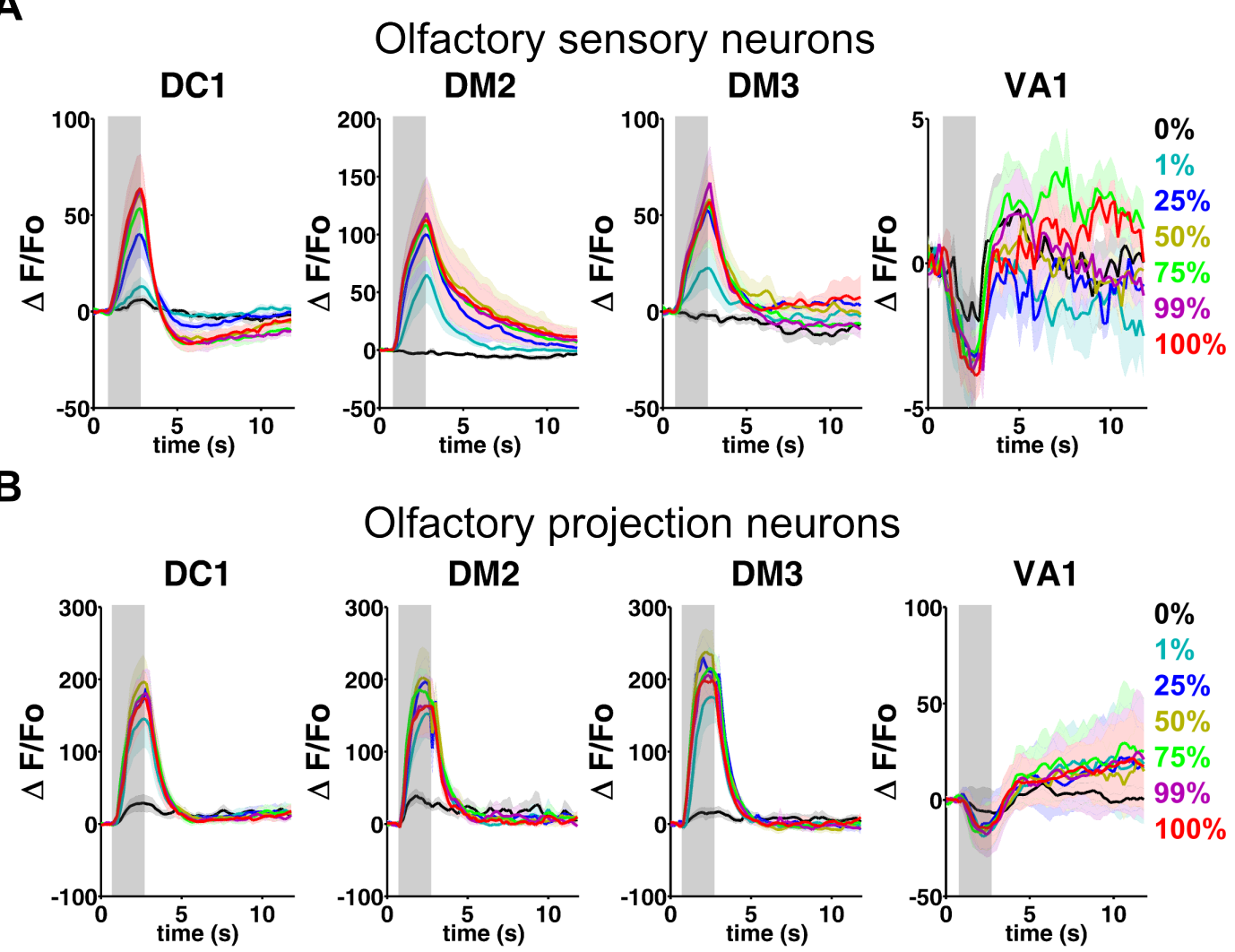

Olfactory projection neurons
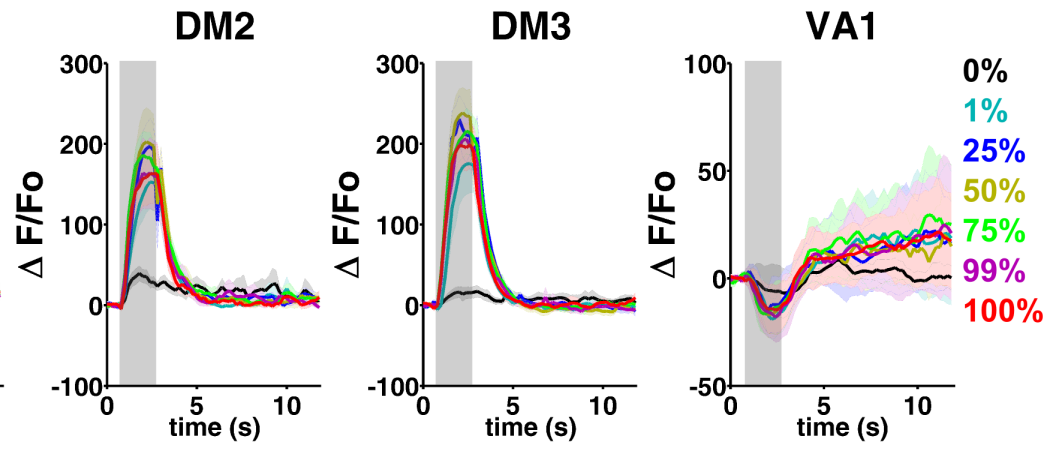

Figure 3.6. Dynamics of 3-octanol-evoked $\mathrm{Ca}^{2+}$ activity in selected glomeruli. Comparison of response dynamics of different groups of OSNs (A) and PNs (B) to different concentrations of 3-octanol. The grey-shaded region indicates the duration of the odor stimulus. Different dynamics could be observed in different glomeruli in both OSNs and PNs. In PNs, all the concentrations showed similar dynamics in each glomerulus, unlike in OSNs. In OSNs, different concentrations evoked different $\mathrm{Ca}^{2+}$ dynamics. The glomerulus VA1 showed inhibition in both OSNs and PNs. Lines indicate mean $\mathrm{Ca}^{2+}$ activity of 6 flies and the shaded region around them indicates \pm SEM.

increase in the amplitude of the signal with increase in concentration, e.g., DL5, DM1 and DM2. A prolonged response was observed in DM2, DM6 and VM2, and the decay period became longer with increasing concentrations. In case of DM2 and DM6, the $\mathrm{Ca}^{2+}$ concentration did not recover to baseline until the end of the measurement. An inhibition was observed in VC2, VA1 and DL1. Similar to PA, a post stimulation inhibition was again observed in the case of $D C 1$, which became stronger with increasing concentrations (Figure 3.6). Therefore, as observed in PA, not all the glomeruli showed the same effect with the increase in odor concentrations. However, in spite of such dif- 


\section{Results}

ferences across glomeruli, the majority of them showed peak responses at the odor offset (Figure 3.7).

\subsubsection{2. $\mathrm{Ca}^{2+}$ activity in PNs}

Similar to PA, the transient property of the responses was again observed in the PNs. The decay period of the signals were short, unlike in OSNs. The response in glomerulus DM3 in OSNs got amplified and showed the strongest response (Figure 3.6). The amplification of responses was also observed in DC1, DM2 and VM2. The inhibition in OSNs in VA1 also got stronger in PNs. DA1 and DA4 also showed inhibition, but the same was not observed in OSNs. However, the activity in these glomeruli did not considerably change from lower concentrations to higher concentrations, unlike in OSNs (Figure 3.5). But a temporal factor was again observed on comparing the dynamics of the glomerular peak responses. The time point of the peak response varied in different glomeruli. Most of the glomeruli showed peak responses in the second half of the odor stimulus (Figure 3.7).

\subsubsection{Concentration-dependent global $\mathrm{Ca}^{2+}$ activity}

The dependency of the total $\mathrm{Ca}^{2+}$ activity of the $\mathrm{AL}$ on concentration was then determined for the entire population of glomeruli. The overall $\mathrm{Ca}^{2+}$ activity in the 14 glomeruli was calculated to observe the global AL activity for different concentrations of 3-Oct
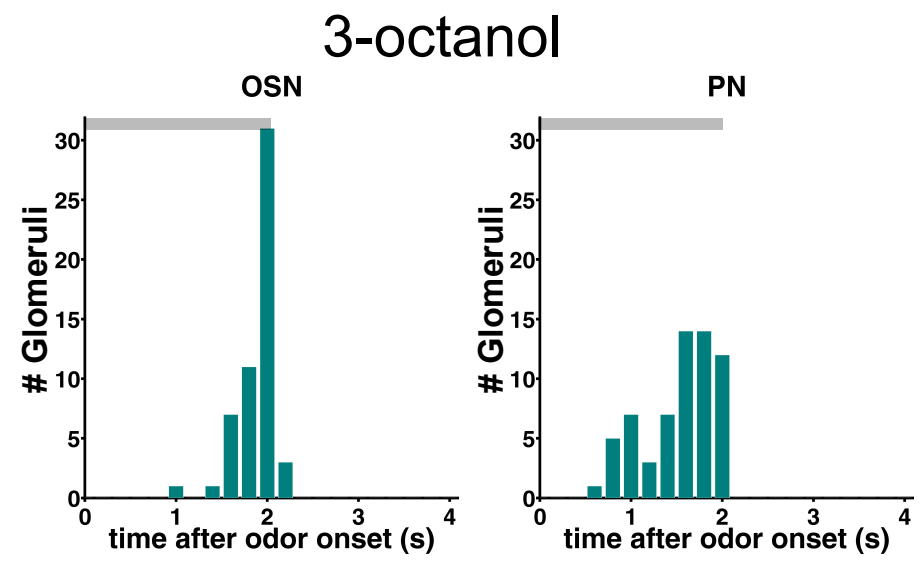

Figure 3.7 Temporal distribution of peak glomerular responses.

This figure shows histograms of the peak response time points of all responding glomeruli across all concentrations in OSNs and PNs. The grey bars indicate the duration of the odor stimulus. Most of the glomeruli in OSNs showed peak responses during the odor offset. In PNs, different glomeruli showed different time points of their peak responses. $n(\#$ responding glomeruli $x$ \# odor stimuli) $=54$ (OSN), 63 (PN). 


\section{Results}

A

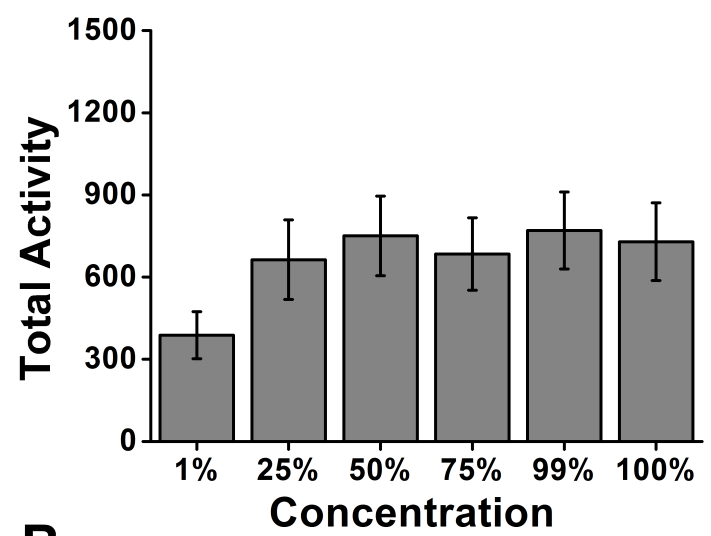

B

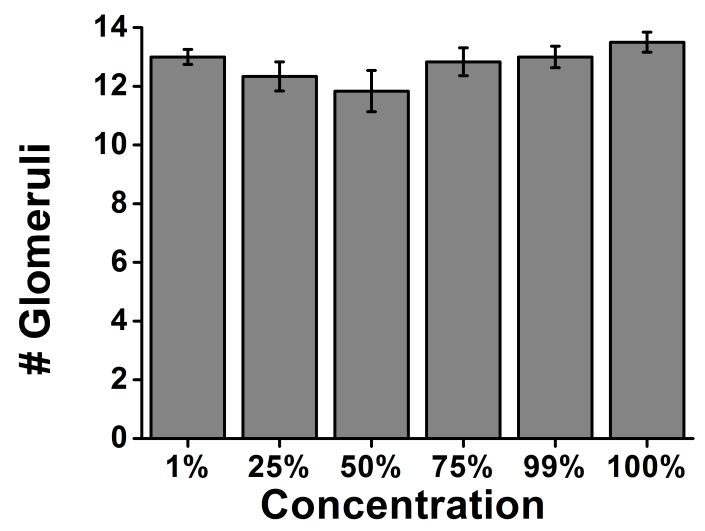

C

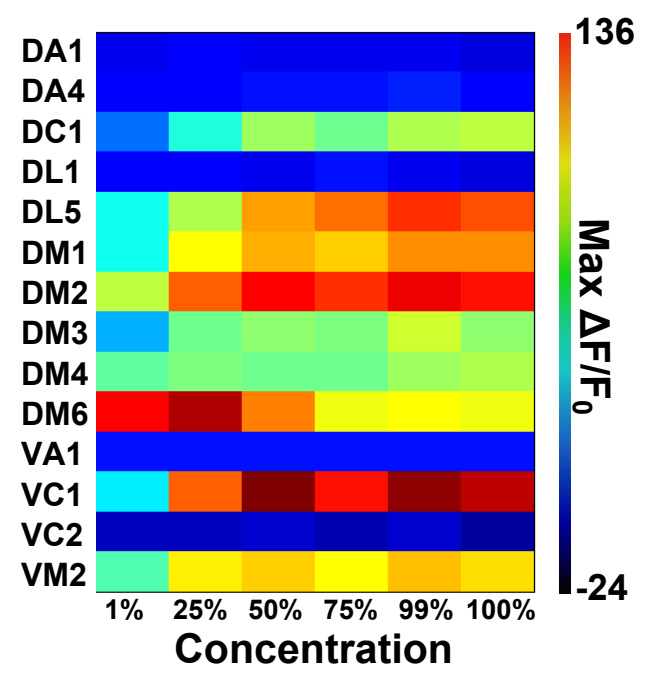

PN
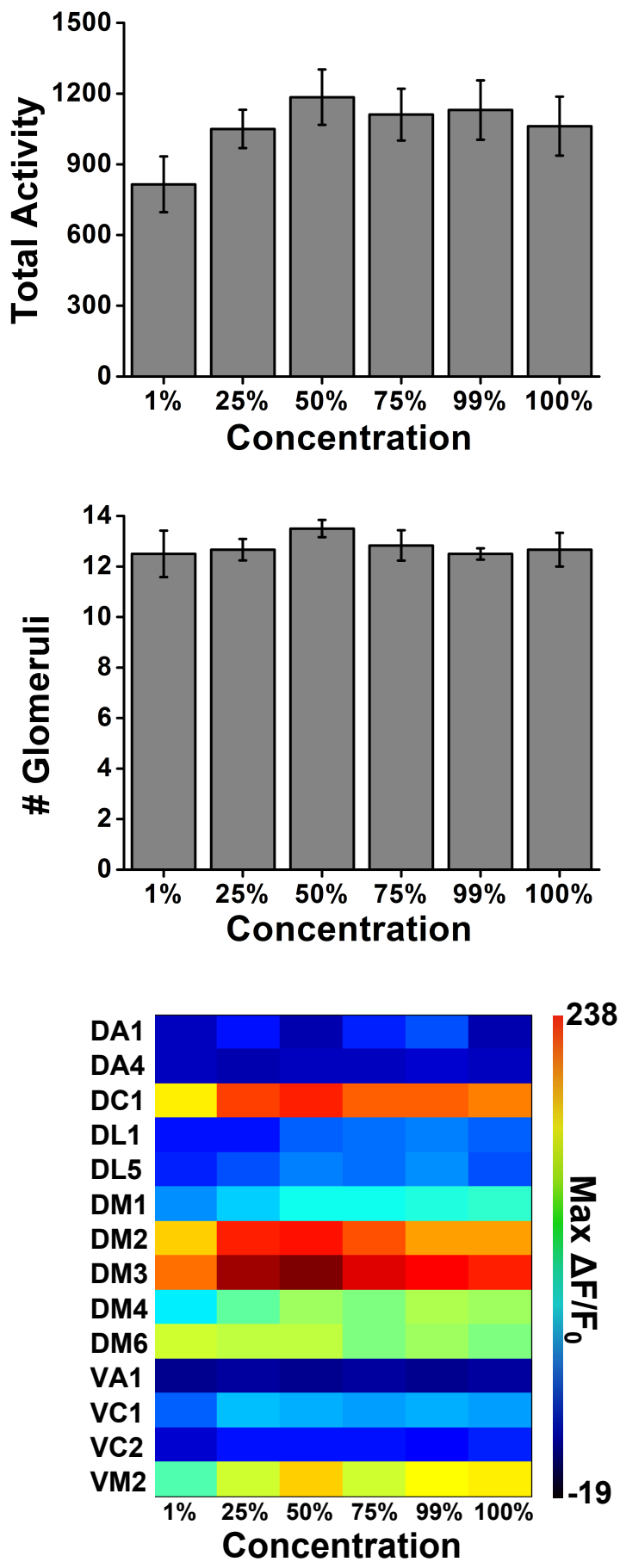


\section{Results}

Figure 3.8. Concentration-dependence of 3-octanol-evoked $\mathrm{Ca}^{2+}$ activity in the antennal lobe.

(A) Comparison of total activity of the AL across its 14 glomeruli in OSNs and PNs in response to different concentrations of 3-octanol. The total activity was determined by the sum of maximum $\mathrm{Ca}^{2+}$ activity evoked in each glomerulus for an individual fly. OSNs showed a similar dependence of total activity on concentrations as PNs. Beyond $50 \%$, no further increase in total activity was observed in both OSNs and PNs. Bars indicate means of the maximum total activity of 6 flies \pm SEM. (B) Number of glomeruli responding to different concentrations of 3-octanol. Any glomerulus showing higher or lower response than mean $\pm(3 \times$ standard deviation $)$ of the pre-stimulus spontaneous $\mathrm{Ca}^{2+}$ activity was taken as a responding glomerulus. 12 out of 14 glomeruli showed responses to all concentrations of 3-octanol. No differences were observed across concentrations in both OSNs and PNs. Bars indicate means of the number of responding glomeruli of 6 flies \pm SEM. (C) Maximum $\mathrm{Ca}^{2+}$ activity evoked by different concentrations of 3-octanol in each glomerulus. Different glomeruli are differentially sensitive to the 3-octanol concentrations. In OSNs, DL5 and DM2 showed increases in $\mathrm{Ca}^{2+}$ activity with concentrations. In terms of the combination of the 14 glomeruli, different concentrations evoked different $\mathrm{Ca}^{2+}$ activity patterns in OSNs. However in $\mathrm{PNs}$, beyond $1 \%$, the $\mathrm{Ca}^{2+}$ activity evoked in all the glomeruli remained the same across different concentrations. High $\mathrm{Ca}^{2+}$ activity is shown in warm colors and low $\mathrm{Ca}^{2+}$ activity in cold colors.

(Figure $3.8 \mathrm{~A}$ ). It was observed that above $50 \%$, all higher concentrations were found to evoke similar total activity in both OSNs and PNs. In PNs however, higher activities were observed for all concentrations in comparison to OSNs. In terms of the number of glomeruli responding to different concentrations, 12 glomeruli showed responses to all the concentrations in both OSNs and PNs (Figure 3.8 B). However, the differences observed in each glomerulus, discussed in section 3.1.2.1 and 3.1.2.2, was summarized by calculating the maximum activity in each glomerulus for each concentration (Figure $3.8 \mathrm{C}$ ). In OSNs, DC1, DL5 and DM1 showed increases in $\mathrm{Ca}^{2+}$ activity with increasing odor concentration. Taking all the 14 glomeruli into account, every concentration evoked a different glomerular activity pattern in OSNs. However in PNs, beyond $25 \%$, such differences were not observed across concentrations. 


\section{Results}

\subsubsection{4-methylcyclohexanol (MCH)}

This compound is a cyclic hydrocarbon with a hydroxyl group and is more different to 3-Oct and PA in terms of chemical structure. It also evokes different spatiotemporal activity patterns described in detail below (Figure 3.9).

A
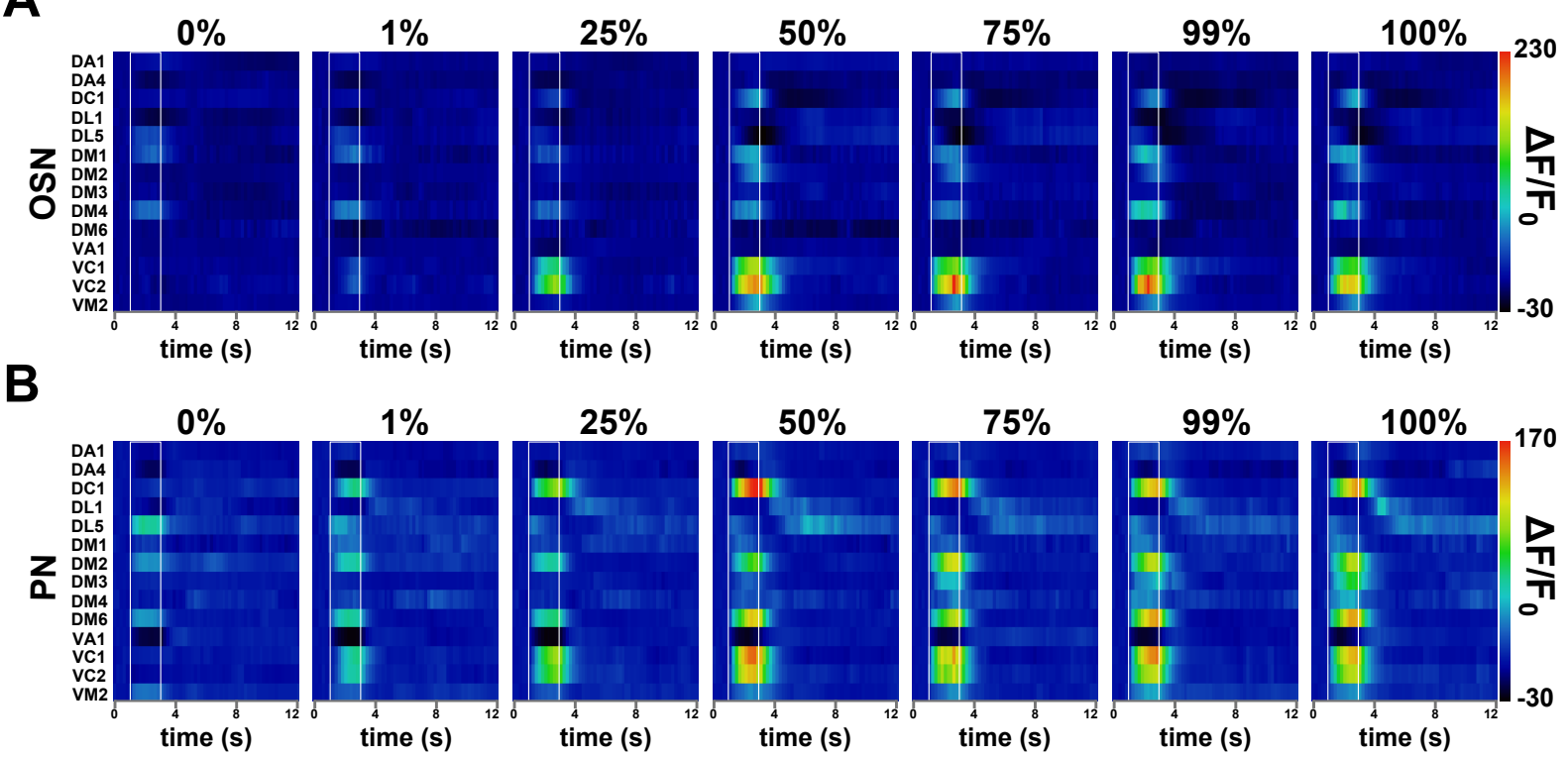

Figure 3.9. Odor-evoked $\mathrm{Ca}^{2+}$ activity to different concentrations of 4methylcyclohexanol in OSNs and PNs.

The figure shows the glomerular $\mathrm{Ca}^{2+}$ activity map of different concentrations of 4methylcyclohexanol in OSNs (A) and PNs (B). The $\mathrm{Ca}^{2+}$ activity map was determined by the $\frac{\Delta F}{F_{0}}$ values obtained for the 14 glomeruli. The white frame indicates the duration of the odor stimuli. High $\mathrm{Ca}^{2+}$ activity is shown in warm colors and low activity in cold colors. Every row in each map represents the activity of single glomerulus indicated on the extreme left. Different glomeruli responded differently to varying concentrations of $\mathrm{MCH}$ in both OSNs and PNs. In OSNs, the $\mathrm{Ca}^{2+}$ activity became stronger in most of the glomeruli with increasing concentrations. Therefore, each concentration evoked a distinct glomerular activity pattern in OSNs. In PNs, a similar concentration dependency of the glomerular $\mathrm{Ca}^{2+}$ activity patterns was not observed. However, some glomeruli like DM3 and DM4 responded differently to different concentrations. $n=6$ flies.

\subsubsection{1. $\mathrm{Ca}^{2+}$ activity in OSNs}

The strongest responses were obtained in the ventral glomeruli, namely VC2 and VC1. The responses in most of the responding glomeruli increased with increasing concentrations, e.g., DM2, DC1 and VC2. DC1, similar to the other two odors, and they again 


\section{Results}

showed a post stimulus inhibition after odor offset. Inhibition was observed in DL1 and VA1 (Figure 3.10). DL5 also showed inhibition, but only during the odor offset. In the case of $\mathrm{MCH}$, none of the 14 glomeruli showed any prolonged response. On comparing the time point of peak glomeruli response, the distribution did not differ between OSNs and PNs (Figure 3.11).

A

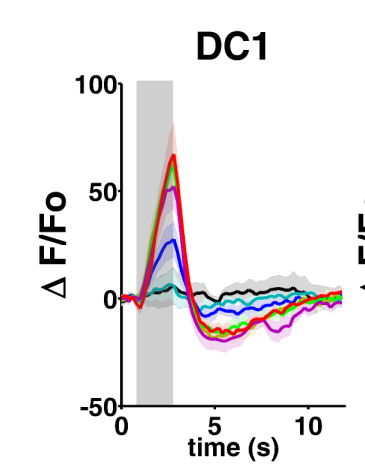

Olfactory sensory neurons

B
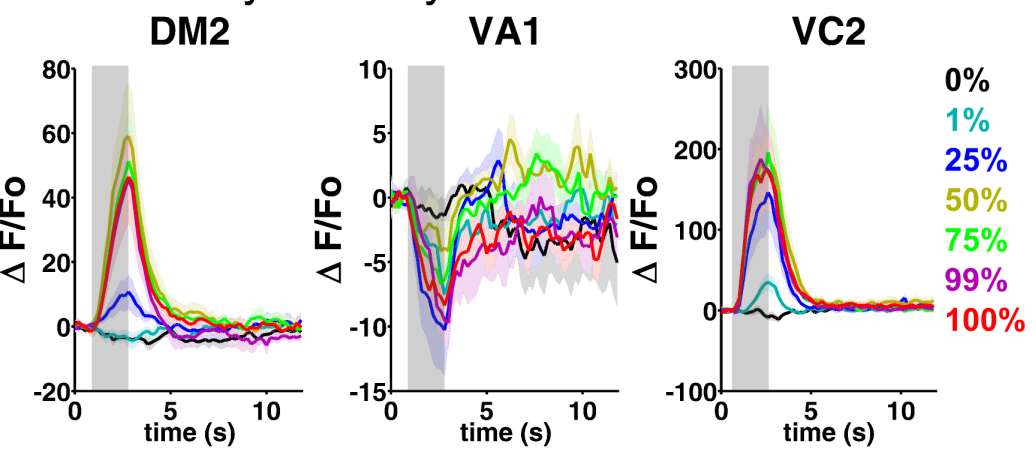

Olfactory projection neurons
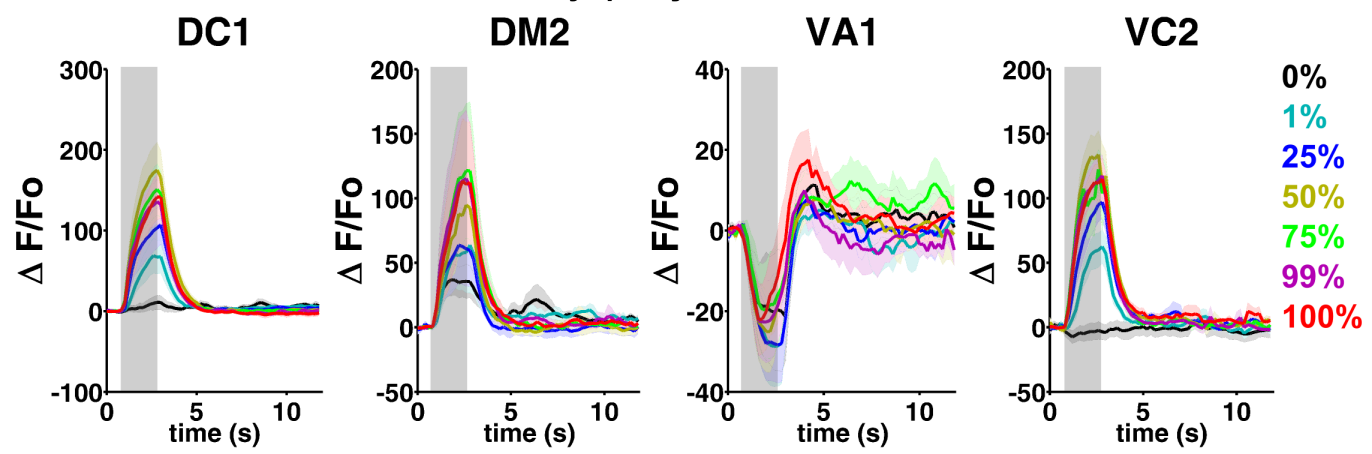

Figure 3.10. Dynamics of 4-methylcyclohexanol-evoked $\mathrm{Ca}^{2+}$ activity in selected glomeruli.

Comparison of response dynamics of different OSNs (A) and PNs (B) to different concentrations of 4-methylcyclohexanol. The grey shaded region indicates the duration of odor stimulus. In both OSNs and PNs, DC1, DM2 and VC2 showed different $\mathrm{Ca}^{2+}$ dynamics with different concentrations. Inhibition was found in VA1 glomerulus in both OSNs and PNs. Lines indicate mean activity of 6 flies and the shaded region around them indicates \pm SEM.

\subsubsection{2. $\mathrm{Ca}^{2+}$ activity in PNs}

In PNs, strong responses were observed in DC1, DM2, DM3, DM6, VC1 and VC2. In all these glomeruli, the signal got amplified from OSNs to PNs. Inhibition also got 


\section{Results}

strengthened in VA1 (Figure 3.10). Both DA4 and VA1 showed inhibition across all concentrations of $\mathrm{MCH}$. Similar to OSNs, DL5 showed an inhibited activity during the odor offset, but in PNs, this was followed by an overshoot of $\mathrm{Ca}^{2+}$ concentration above baseline. The increase in odor concentration was reflected in an increase in activity, mainly in DM2, DM6 and DM3. DC1, VC1 and VC2, however did not show any further increase in signals beyond $25 \%$ odor concentration.

\subsubsection{Concentration-dependent global $\mathrm{Ca}^{2+}$ activity}

The overall activity from the 14 glomeruli was calculated to observe the dependence of global AL activity on different concentrations of $\mathrm{MCH}$ (Figure 3.12 A). It was observed that as odor concentration increased, there was increase in the total activity in both OSNs and PNs. Beyond 50\% concentration, all concentrations showed similar overall activities. In terms of the number of glomeruli recruited at each concentration, 12 glomeruli showed responses to all the concentrations of $\mathrm{MCH}$ (Figure 3.12 B). In the next step, odor evoked glomerular activity pattern for each concentration was determined by calculating the maximum response in each glomerulus (Figure $3.12 \mathrm{C}$ ). Some glomeruli, like DM1 and DM4, showed an increase in $\mathrm{Ca}^{2+}$ activity with increasing odor concentration in OSNs, while others showed an abrupt increase beyond a certain odor concentration. In PNs too, $\mathrm{Ca}^{2+}$ activities in glomeruli like DM2 and DM6,
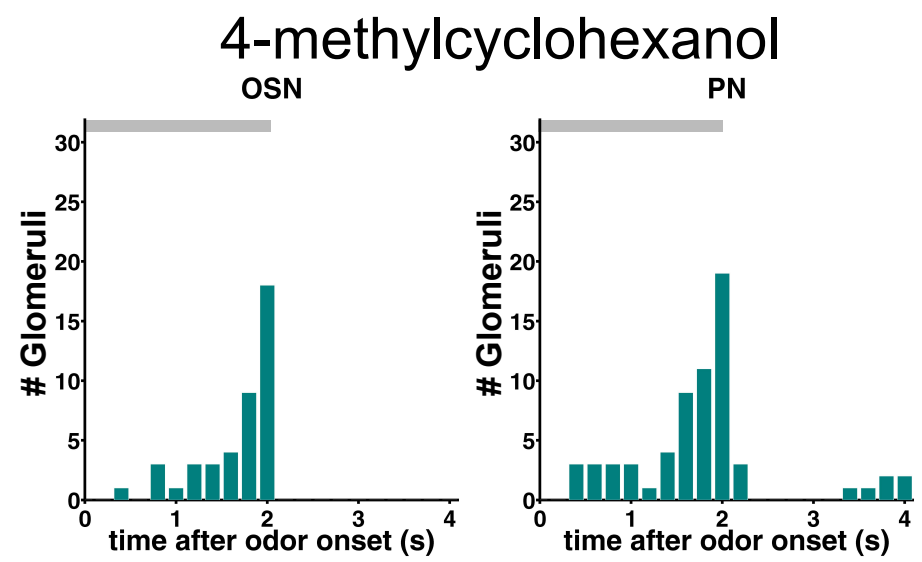

Figure 3.11 Temporal distribution of peak glomerular responses.

This figure shows histograms of the peak response time points of all responding glomeruli across all odor concentrations in OSNs and PNs. The grey bars indicate the duration of the odor stimulus. The distribution of the peak response time points of all the glomeruli was observed to be similar between OSNs and PNs. Moreover, unlike PA and 3-Oct, most of the glomeruli in OSNs did not show their peak response during odor offset. $\mathrm{n}$ (\# responding glomeruli $\mathrm{x}$ $\#$ odor stimuli) $=42(\mathrm{OSN}), 65$ (PN). 


\section{Results}

A
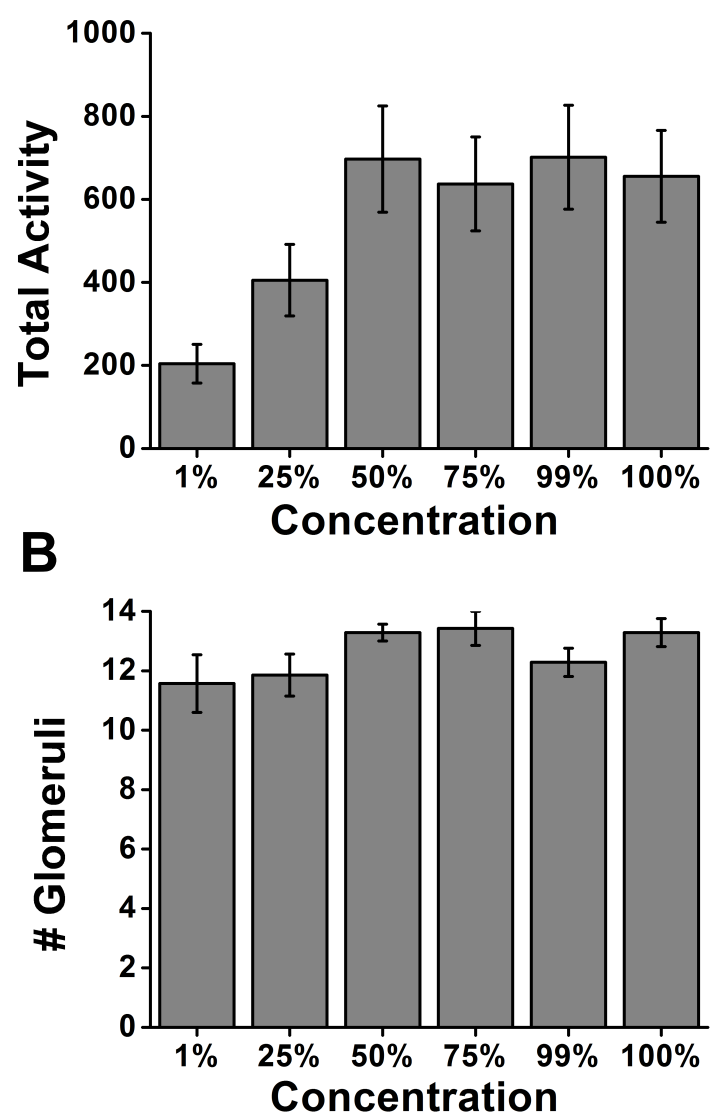

C

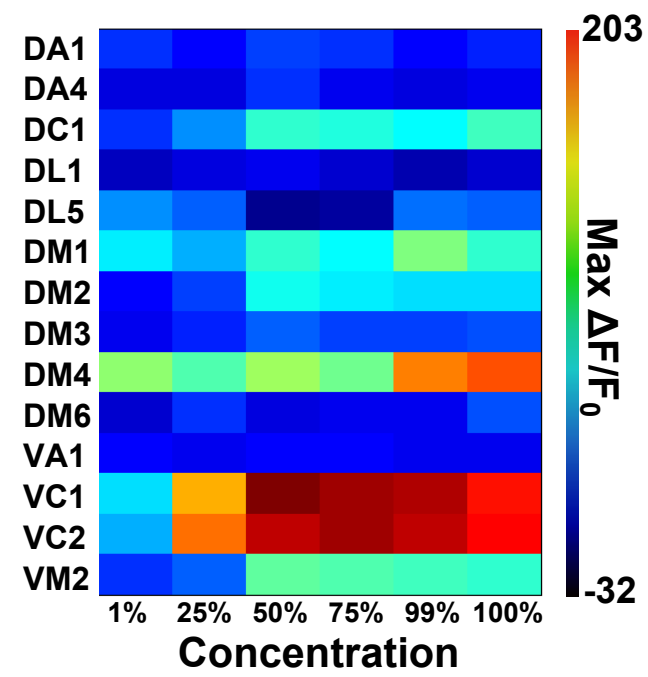

PN
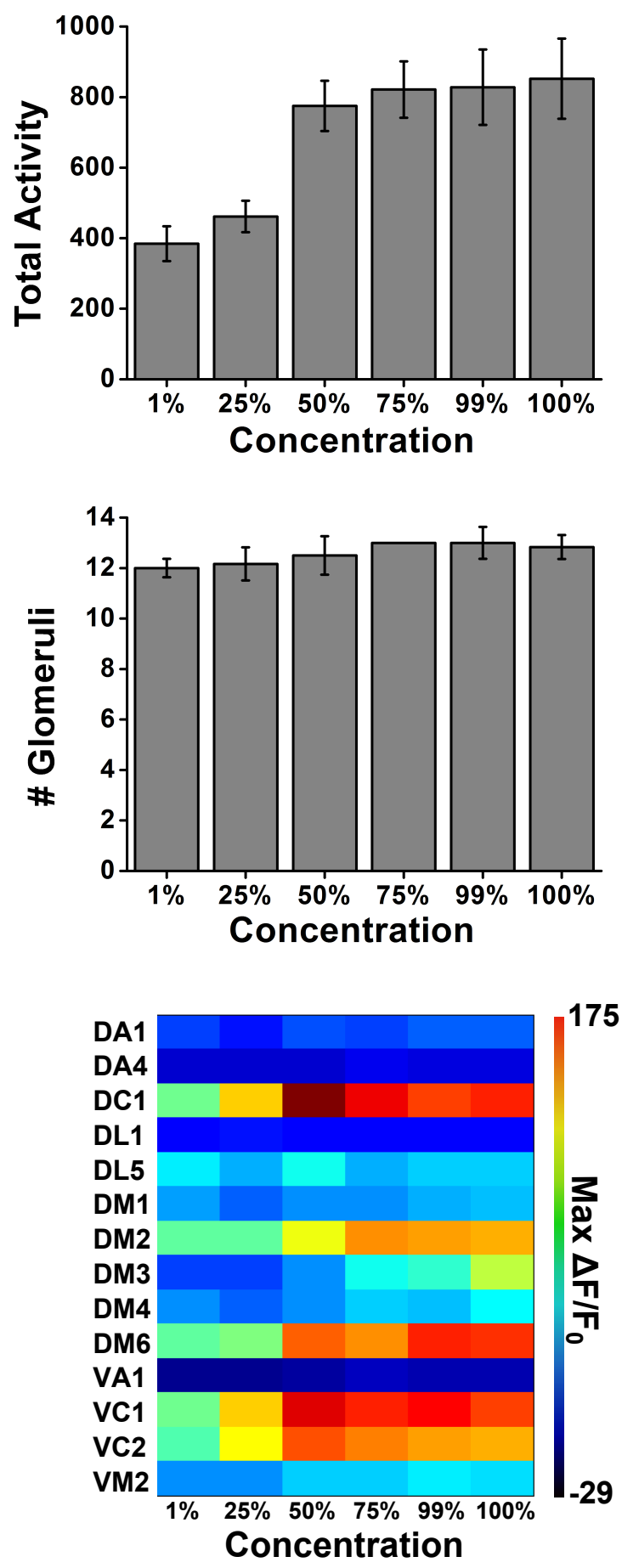


\section{Results}

Figure 3.12. Concentration-dependence of 4-methylcyclohexanol-evoked $\mathrm{Ca}^{2+}$ activity in the antennal lobe.

(A) Comparison of total $\mathrm{Ca}^{2+}$ activity of the $\mathrm{AL}$ across its 14 glomeruli in OSNs and PNs in response to different concentrations of 4-methylcyclohexanol. The total activity was determined by the sum of maximum $\mathrm{Ca}^{2+}$ activity evoked in each glomerulus for an individual fly. OSNs show a similar dependence on concentrations as PNs. Above $50 \%$ concentration, no increase in the total activity was observed in OSNs as well as PNs. Bars indicate means of the maximum total activity of 6 flies \pm SEM. (B) Number of glomeruli responding to different concentrations of 4-methylcyclohexanol. Any glomerulus showing higher or lower response than mean $\pm(3 \times$ standard deviation) of the pre-stimulus spontaneous $\mathrm{Ca}^{2+}$ activity was taken as a responding glomerulus. 12 out of 14 glomeruli showed responses to all concentrations of 4-methylcyclohexanol. No differences across concentrations were found in OSNs and PNs. Bars indicate means of the number of responding glomeruli of 6 flies \pm SEM. (C) Maximum $\mathrm{Ca}^{2+}$ activity evoked by different concentrations of 4-methylcyclohexanol in each glomerulus. Different glomeruli are differentially sensitive to the 4-methylcyclohexanol concentrations in both OSNs and PNs. Therefore, the activity patterns of different concentrations, in terms of the maximum $\mathrm{Ca}^{2+}$ activity in each glomerulus, were found to be distinct in both OSNs and PNs. High activity is shown in warm colors and low activity in cold colors.

showed a concentration dependence. Taking into account all such glomeruli, each

odor concentration was found to evoke different $\mathrm{Ca}^{2+}$ activity patterns in both OSNs and PNs, and these differences relied mainly on each and every responding glomerulus in a combinatorial way. 


\section{Results}

\subsubsection{Odor similarity at the level of olfactory sensory neurons and projection neurons}

In comparison to $\mathrm{MCH}, \mathrm{PA}$ and 3-Oct are more similar in some aspects of their chemical structure as well as in behavioral perception [Haddad et al., 2008; Niewalda et al., 2011]. In order to detect any odor classification using these three odors as binary mixtures, it was important to determine the degree of similarity in their odor representations in the AL. The primary reason was that the differences in the odor representations of intermediate binary mixtures would also depend on the degree of similarity between the components of that binary mixture. Therefore, the odortopic similarities between the odor representations of the three odors were determined, and this was achieved by correlation analysis. The activity in every glomeruli to a certain odor stimulus at a single time point was taken as one vector and another vectors of different odor stimuli of the same time point were similarly taken. Each of these vectors represented an activity pattern of the respective stimulus at a certain time point. A Pearson's correlation coefficient was calculated between these vectors, which gave a measure of similarity between glomerular activity patterns. Such pairwise correlations were done across all concentrations of any two different odors, thereby creating a correlation matrix. In figure 3.13, these correlation matrices were determined for different time points.

In the OSNs correlation matrices of $\mathrm{MCH}$ and 3-Oct (Figure 3.13 A), it was found that all the concentrations of $\mathrm{MCH}$ and 3-Oct were decorrelated to each other. The lowest $0 \%$ of both odors, which was the diluent mineral oil, was the only stimulus to show a high correlation with another separate measurement of mineral oil. The correlations between $\mathrm{MCH}$ and 3-Oct became weaker as the time reached odor offset. In PNs also, no strong correlation was found between $\mathrm{MCH}$ and 3-Oct. In the case of $\mathrm{MCH}$ and PA (Figure $3.13 \mathrm{~B}$ ), similar results were obtained, but PA showed even stronger decorrelation with $\mathrm{MCH}$ compared to 3-Oct in both OSNs and PNs. However, between PA and 3-Oct (Figure $3.13 \mathrm{C}$ ), a high degree of similarity was observed in both OSNs and PNs. In OSNs, this similarity was dependent on time, and the correlations increased as the time reached odor offset. The high concentrations of PA showed even higher similarity with low concentrations of 3-Oct. This was not true in the case of PNs, where PA and 3-Oct showed high correlations irrespective of any odor concentration. Moreover, it was found that, compared to OSNs, stronger correlations were obtained between PA and 3-Oct in PNs. Therefore from these results, it was concluded that PA and 3-Oct are 


\section{Results}

A

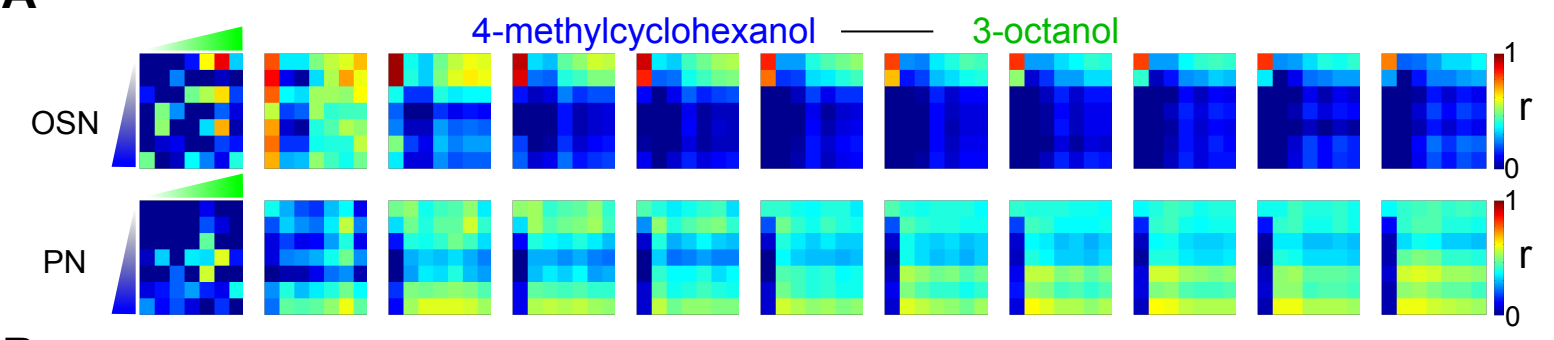

B

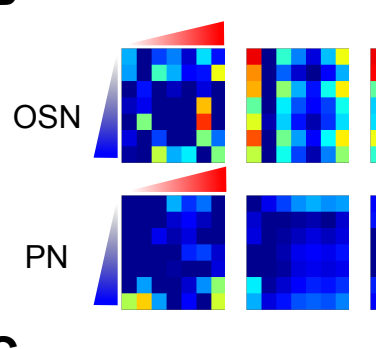

4-methylcyclohexanol
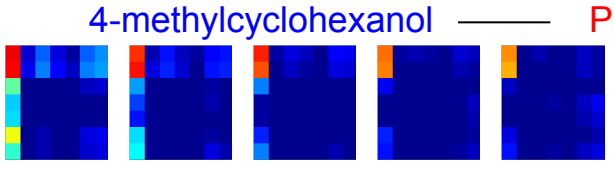

Pentyl acetate

$\mathbf{C}$
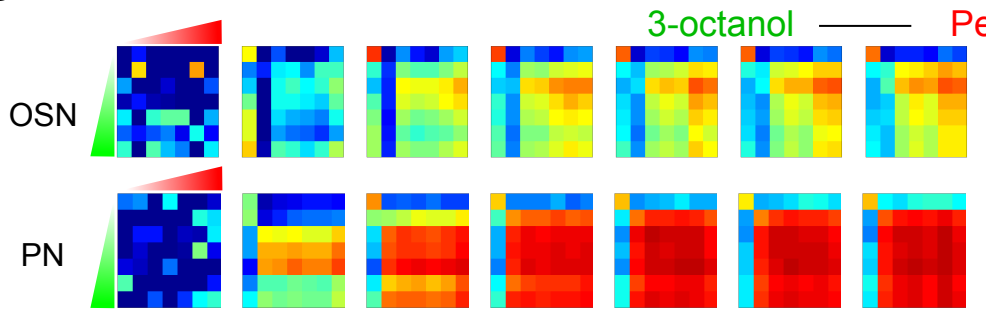

Pentyl acetate
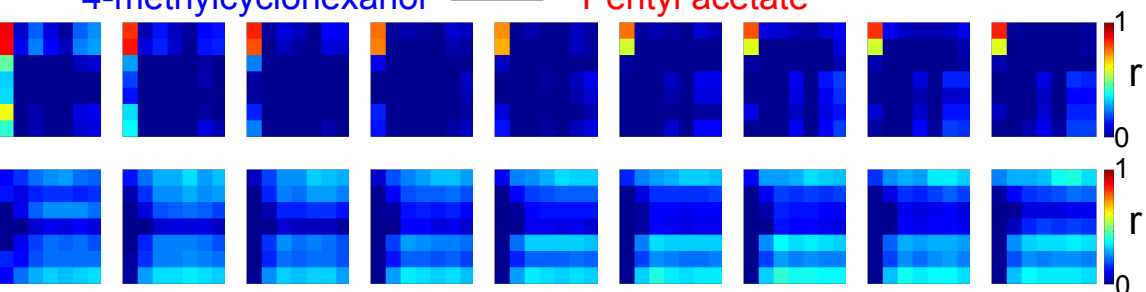

$-0.2$
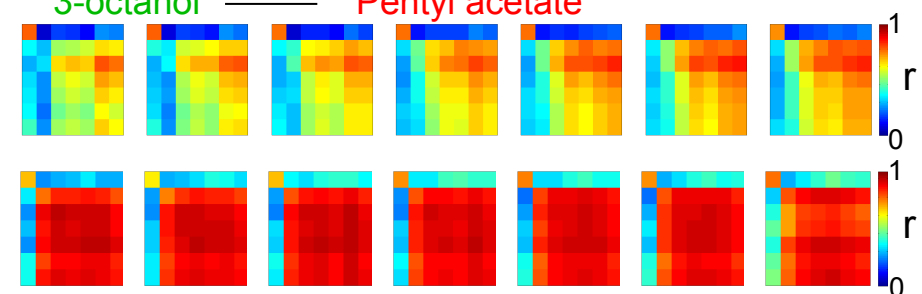

odor stimulus (s)

2.0

Figure 3.13. Similarity between the $\mathrm{Ca}^{2+}$ activity patterns of different odors.

Correlation analysis between the activity patterns of different concentrations of odors: $\mathrm{MCH} / 3-O c t(\mathbf{A}), \mathrm{MCH} / \mathrm{PA}(\mathbf{B}), 3-O c t / P A(C)$, in OSNs and PNs at different time points during the odor stimulus. The activity pattern was determined by the $\mathrm{Ca}^{2+}$ activity evoked in the 14 glomeruli. A Pearson's correlation coefficient $(r)$ was calculated between activity patterns of different concentrations for different odors. The concentrations of odors depicted here are $0 \%, 1 \%, 25 \%, 50 \%, 75 \%, 99 \%$ and $100 \%$, and they are indicated by the triangles on the extreme left. High correlation coefficients are indicated in warm colors and low coefficients in cold colors. The $\mathrm{Ca}^{2+}$ activity patterns of all concentrations of $\mathrm{MCH}$ were found to decorrelate with all the $\mathrm{Ca}^{2+}$ activity patterns of both 3-Oct and PA in OSNs and PNs. In the case of PA and 3-Oct, in OSNs, 3-Oct $\mathrm{Ca}^{2+}$ activity patterns were found to be similar to $\mathrm{PA} \mathrm{Ca}{ }^{2+}$ activity patterns. This similarity was even stronger between high concentrations of PA and low concentrations of 3-Oct. However, in PNs, the similarity between all the concentrations of PA and 3-Oct became even stronger in comparison to OSNs, as reflected by the high correlation coefficients obtained between any pair of activity patterns of different concentrations of PA and 3-Oct. 


\section{Results}

relatively more similar to each other compared to $\mathrm{MCH}$. Moreover, the activity patterns of PA and 3-Oct became even more similar in PNs relative to OSNs. This result was in accordance with the previous studies reported by Niewalda et al. [2011].

\subsection{Classification of odor stimuli}

\subsubsection{Glomerular representations of binary odor mixtures}

In order to detect any classification of odors, the three odors (PA, 3-Oct and MCH) with different levels of similarity were used for making three different combinations of binary odor mixtures. The mixtures were made in such a way such that the concentration of one component was decreased and at the same time the concentration of the other component was increased so that the total volume of the odors remained constant. The ratios of the individual components in the mixtures varied as follows: $100 \%-0 \%, 99 \%-$ $1 \%, 75 \%-25 \%, 50 \%-50 \%, 25 \%-75 \%, 1 \%-99 \%$ and $0 \%-100 \%$. These mixtures were then presented to the fly's antennae and the $\mathrm{Ca}^{2+}$ activities in the 14 glomeruli were recorded (Figure 3.14). The $\mathrm{Ca}^{2+}$ activity patterns evoked by the binary mixtures were found to resemble to either of its component odors. No new glomeruli were found to be recruited for the intermediate mixtures. 3-Oct and PA showed very similar patterns across their different binary mixtures, which was due to the fact that both the odors evoked $\mathrm{Ca}^{2+}$ activity in almost the same glomeruli. However, differences could be observed in the temporal component of the glomerular activity dynamics. In the case of $\mathrm{MCH}$ and 3-Oct, gradual changes in $\mathrm{Ca}^{2+}$ activity patterns were observed in OSNs, which could be visualized by a gradual increase in glomerular $\mathrm{Ca}^{2+}$ activity specific to 3-Oct as the concentration ratio of 3-Oct increased. In PNs, some glomeruli showed a gradual change with varying concentration ratios, but in case of the glomerulus DM3, a drastic change was observed between $99 \% \mathrm{MCH}-1 \% 3-\mathrm{Oct}$ and $75 \% \mathrm{MCH}-25 \% 3-$ Oct from a weak activity to high activity. Similar effect in the DM3 glomerulus was also found in the MCH-PA mixtures between 100\% $\mathrm{MCH}-0 \% \mathrm{PA}$ and $99 \% \mathrm{MCH}-1 \% \mathrm{PA}$. However, any odor representation depended on the activities across all the glomeruli. Therefore, to detect any odor classification, activities in all the glomeruli were needed to be compared across different binary mixtures. 


\section{Results}

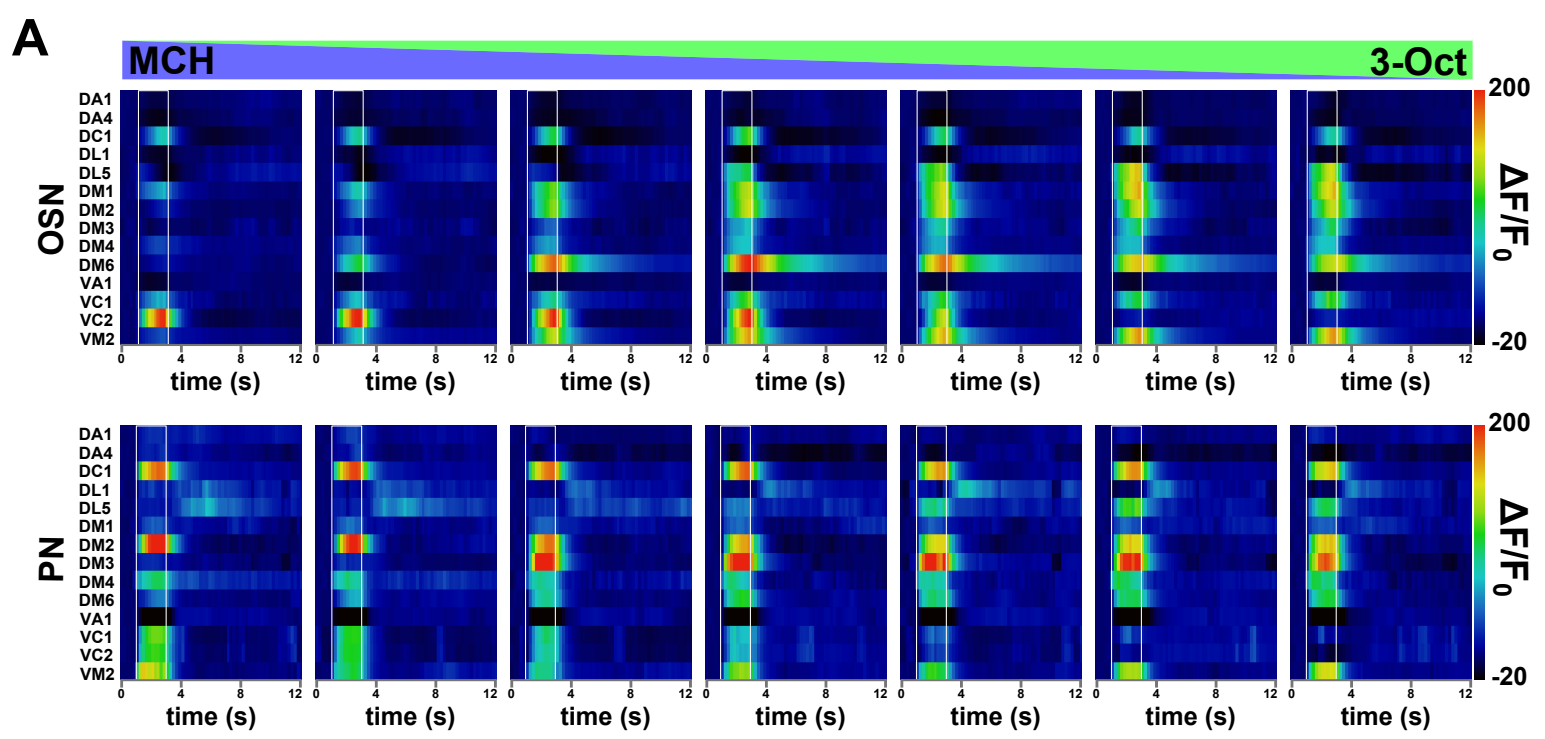

B
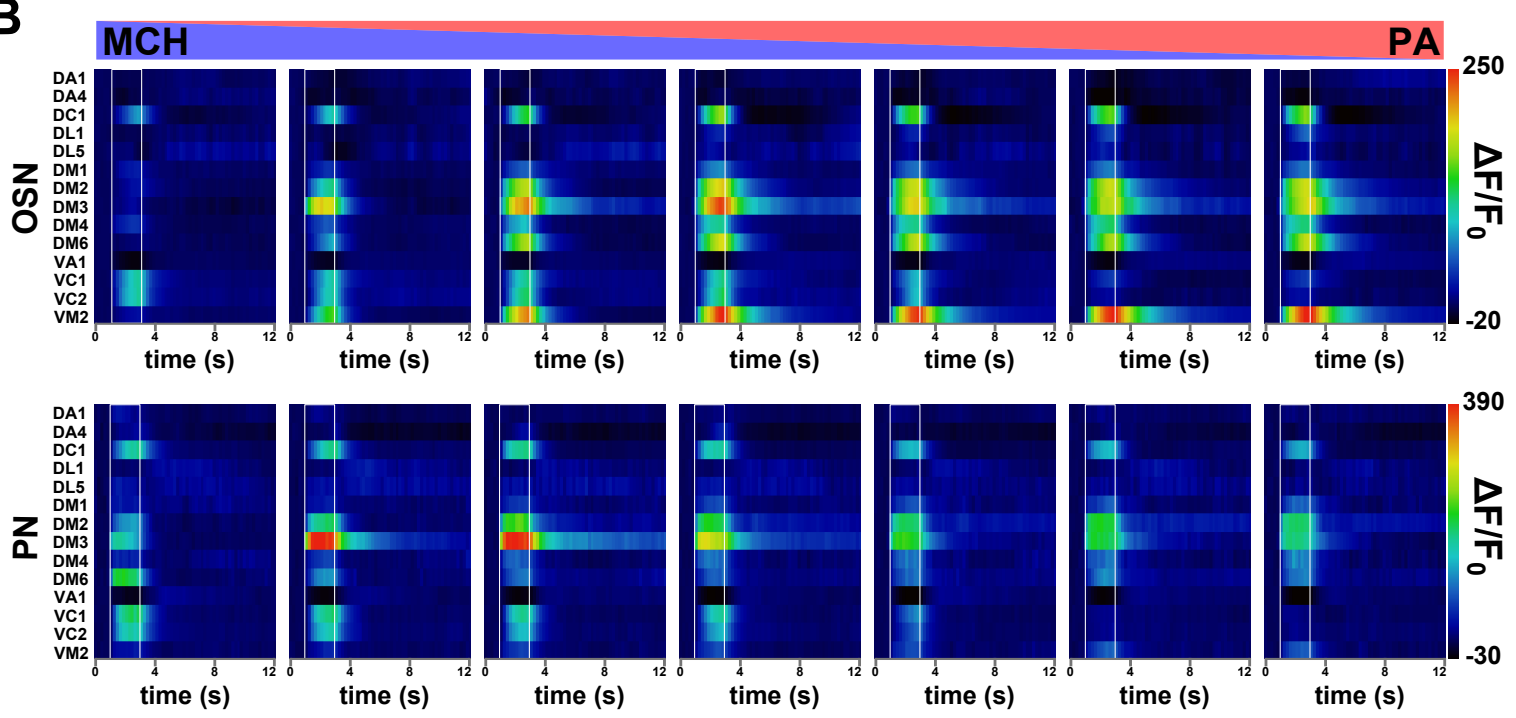

C

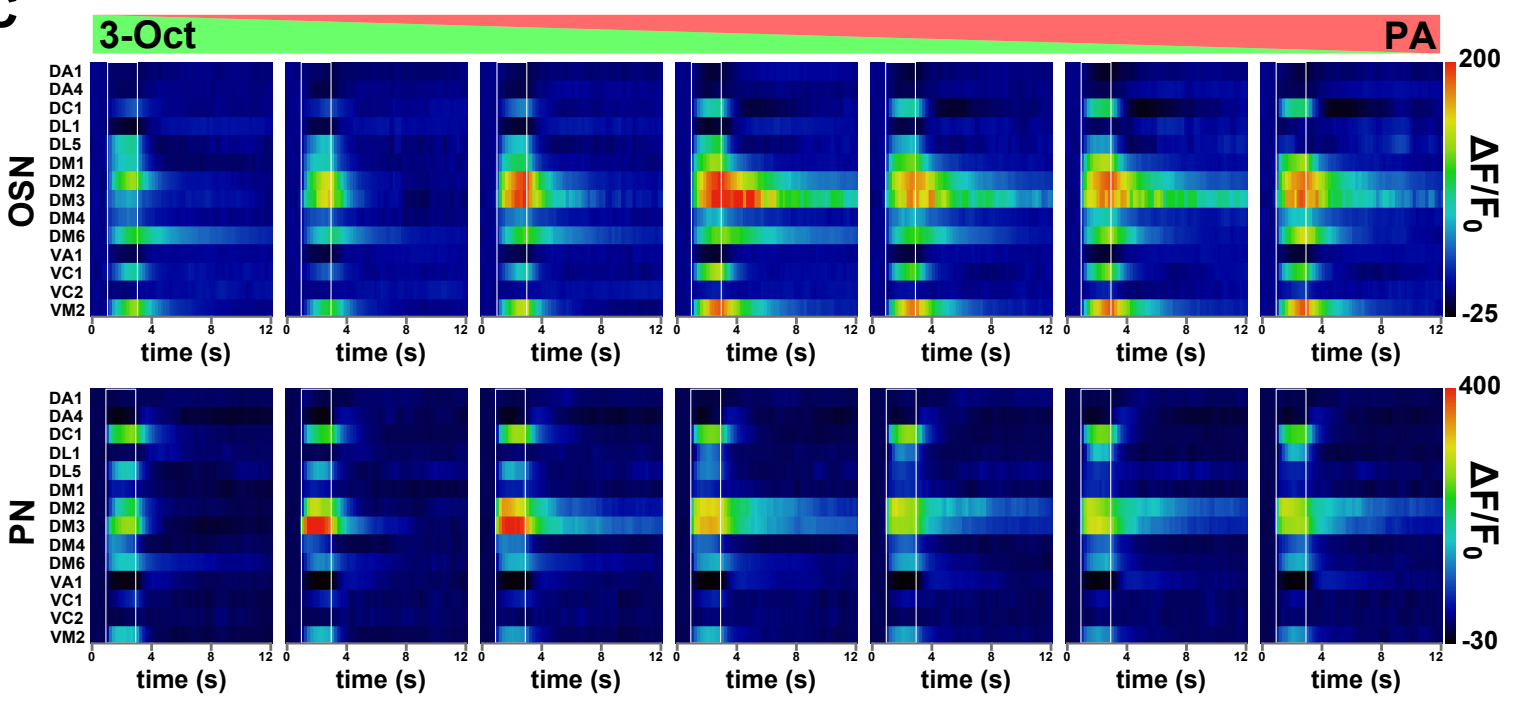




\section{Results}

Figure 3.14. Odor-evoked $\mathrm{Ca}^{2+}$ activity to different binary odor mixtures in OSNs and PNs.

Glomerular activity map of 14 glomeruli to different binary odor mixtures: $\mathrm{MCH} / 3-O c t$ (A), MCH/PA (B) and 3-Oct/PA (C). The activity map was determined by the $\frac{\Delta F}{F_{0}}$ values obtained for the 14 glomeruli. The white frames indicate the duration of the odor stimuli. In all the binary mixtures, the glomerular $\mathrm{Ca}^{2+}$ activity patterns changed from one odor to another. High $\mathrm{Ca}^{2+}$ activity is shown in warm colors and low activity in cold colors. Every row in each panel represents the activity of single glomerulus labeled at the extreme left. $\mathrm{n}=5-6$ flies.

\subsubsection{Transitions between glomerular representations of binary odor mixtures}

Each odor mixture was represented in the $\mathrm{AL}$ in terms of $\mathrm{Ca}^{2+}$ activity in every glomerulus. The fly brain then has to process the information from all the glomeruli in order to encode the properties of the odors [Sachse and Galizia, 2003; Silbering et al., 2008]. However, the differences in odor responses in individual glomeruli makes it difficult to understand the population coding of the odor mixtures. In order to visualize this multidimensional information, a mathematical algorithm called principal component analysis (PCA) was applied on the data set. It reduces the dimensionality of the data while keeping most of the variation in the data intact. This is achieved by finding new dimensions along which the variation among observations is maximal and these new dimensions are then called principal components (PC). For the data set of the odorevoked responses in 14 glomeruli, each glomerulus acted as one dimension, which consisted of the dynamics of responses to each odor stimulus, i.e., each dimension contained the $\frac{\Delta F}{F_{0}}$ values of a particular glomerulus from 5 frames before the odor onset until the odor offset as observations. The 14 dimensions from the 14 glomeruli were then reduced by PCA to first three principal components, where each single point represented the $\mathrm{Ca}^{2+}$ activity pattern at a certain time point and the trajectories represented the dynamics of these glomerular activity patterns (Figure 3.15).

On applying PCA to the data set (Figure 3.15 A), it could be observed in PNs in the case of $\mathrm{MCH} / 3-$ Oct binary mixtures that $100 \% \mathrm{MCH}-0 \% 3-\mathrm{Oct}$ and $99 \% \mathrm{MCH}-1 \%$ $3-O c t$ ended up in one subvolume, whereas the other $\mathrm{MCH} / 3-$ Oct mixtures into another subvolume. This implied that $100 \% \mathrm{MCH}-0 \% 3-$ Oct and $99 \% \mathrm{MCH}-1 \% 3-$ Oct got classified into one category and the others into another. This kind of classification was, 


\section{Results}

however, not observed strongly in the case of OSNs. Although $100 \% \mathrm{MCH}-0 \% 3-\mathrm{Oct}$, 99\% $\mathrm{MCH}-1 \% 3-$ Oct and 0\% $\mathrm{MCH}-100 \% 3-O c t$ and 1\% $\mathrm{MCH}-99 \% 3-O c t$ were found to follow similar trajectories in OSNs, but the other intermediate mixtures were found to be uniformly distributed in the PCA odor space, unlike in PNs. Therefore, it could be concluded that an odor classification of certain binary mixtures of $\mathrm{MCH} / 3-\mathrm{Oct}$ was observed in PNs which was not the case already in OSNs.

In $\mathrm{MCH} / \mathrm{PA}$ odor mixtures (Figure $3.15 \mathrm{~B}$ ), the $\mathrm{Ca}^{2+}$ activity patterns were found to be similarly distributed across the OSN odor space as the $\mathrm{MCH} / 3-\mathrm{Oct}$. The $0 \% \mathrm{MCH}$ $100 \% \mathrm{PA}$ and $1 \% \mathrm{MCH}-99 \% \mathrm{PA}$ was also similarly found to follow the same trajectory. $100 \% \mathrm{MCH}-0 \% \mathrm{PA}$ and $99 \% \mathrm{MCH}-1 \% \mathrm{PA}$ were, however, found separated in the odor space in this odor pair. In PNs on the other hand, except $100 \% \mathrm{MCH}-0 \% \mathrm{PA}$, all other intermediate mixtures ended up in a small subvolume, but each of them was found to follow different trajectories. Therefore, all the mixtures, except $100 \% \mathrm{MCH}-0 \% \mathrm{PA}$, got classified into one major distinct category as they reached odor offset. It could also be interpreted such that once PA was added to the mixture, all the mixtures got classified as one in PNs. So, it was concluded that, similar to the $\mathrm{MCH} / 3-O c t$ mixture, a stronger classification was again observed in PNs. However, this was mainly dominated by the presence of the odor PA.

A similar kind of PA dominance could also be observed in the 3-Oct/PA odor mixtures, but in this odor pair, this was observed directly at the level of OSNs (Figure 3.15 C). Except for 100\% 3-Oct-0\% PA, all intermediate mixtures were found to evoke similar spatiotemporal patterns both in OSNs and PNs. No differences were found between OSNs and PNs in terms of activity patterns of their binary mixtures. In this odor pair, odor classification already happened at the level of OSNs because of the high degree of similarity present between 3-Oct and PA and therefore, no further classification was observed in PNs as this similarity became stronger in PNs (Figure $3.13 \mathrm{C}$ ).

From the results of the binary mixtures of these three odor pairs, it could be concluded that, depending on the odor combination and the degree of similarity between any pair of odors, different kinds of odor classification of odors could be observed. In the case of $\mathrm{MCH} / 3-O c t$, specific binary mixtures got more distinctly categorized in PNs. Similar results were obtained with $\mathrm{MCH} / \mathrm{PA}$ too, but this was mainly caused by the PA dominance which resulted in a bigger cluster of all mixtures containing any amount of 


\section{Results}

A 4-methylcyclohexanol
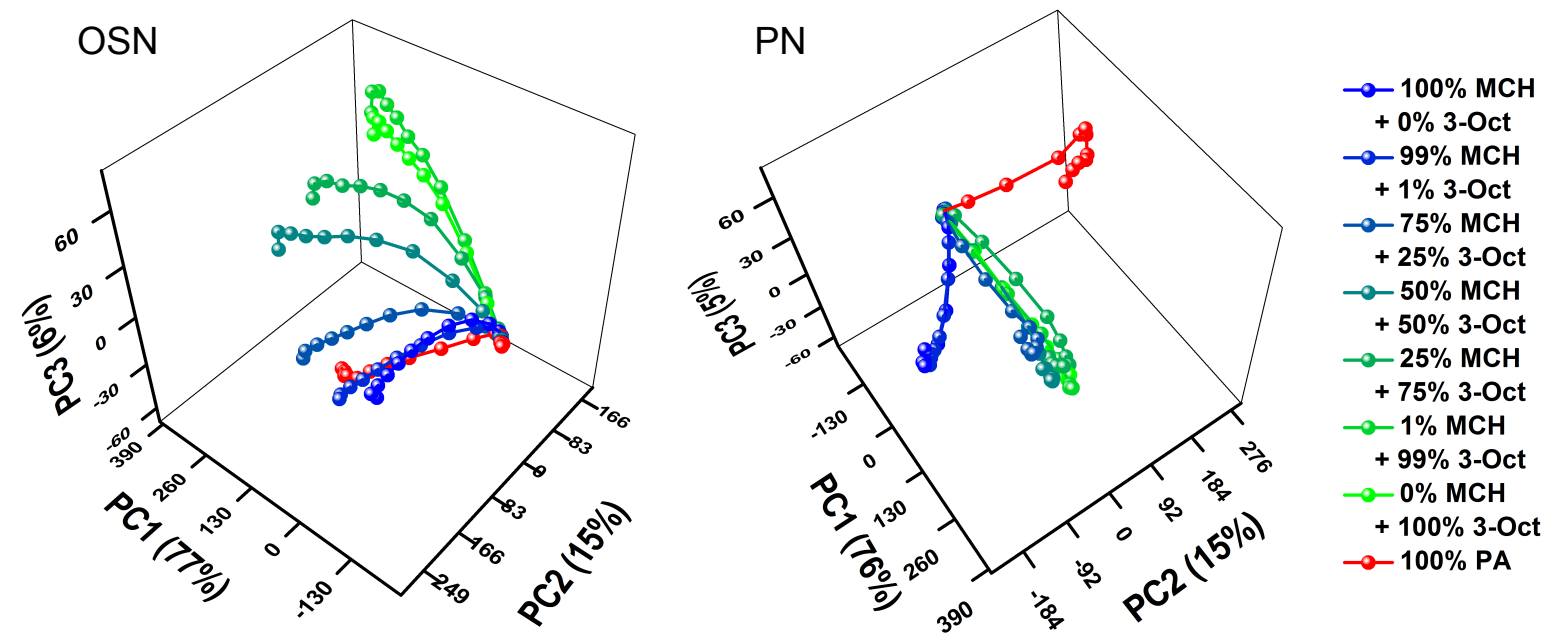

B
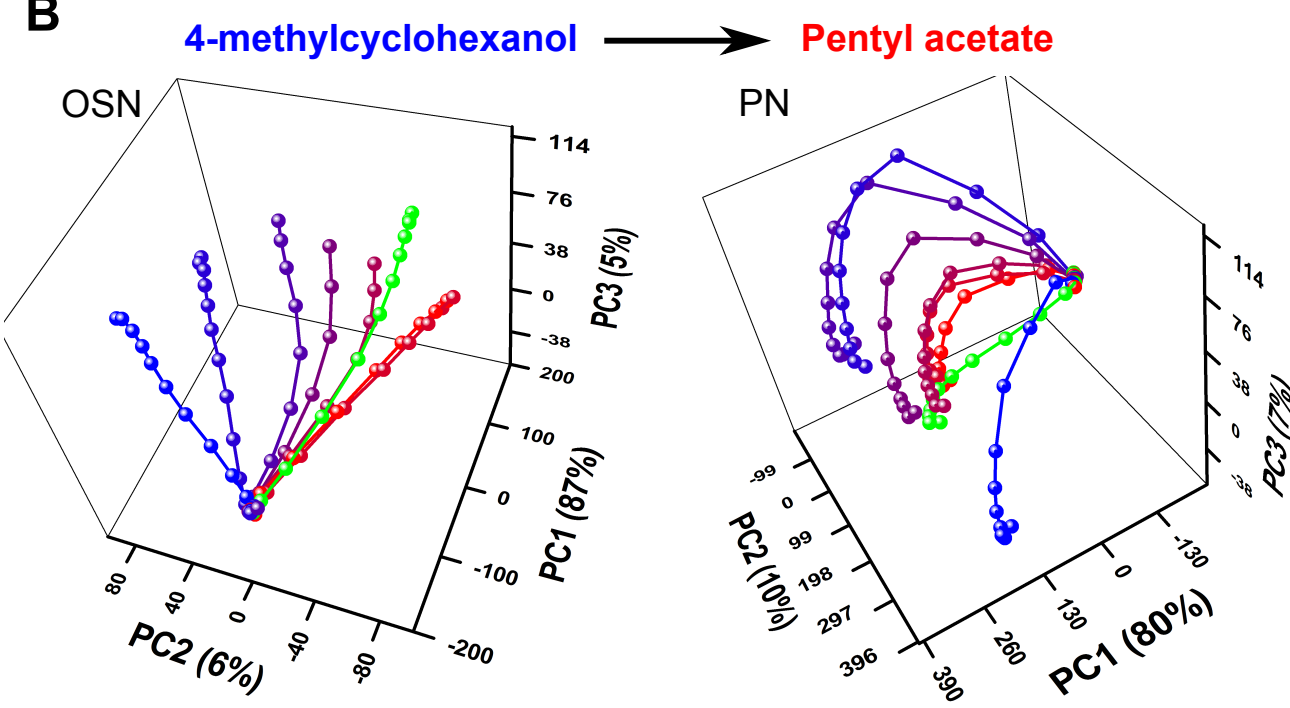

$\rightarrow 100 \% \mathrm{MCH}$ $+0 \% \mathrm{PA}$

$-99 \% \mathrm{MCH}$

$+1 \% \mathrm{PA}$

$-75 \% \mathrm{MCH}$

$+25 \%$ PA

$-50 \% \mathrm{MCH}$

$+50 \%$ PA

$+50 \%$ PA
$-25 \% \mathrm{MCH}$

$+75 \%$ PA

$\multimap 1 \% \mathrm{MCH}$

$+99 \%$ PA

$\multimap 0 \% \mathrm{MCH}$

$+100 \% \mathrm{PA}$

$-\odot-100 \% 3-$ Oct

C

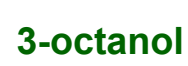

\section{Pentyl acetate}
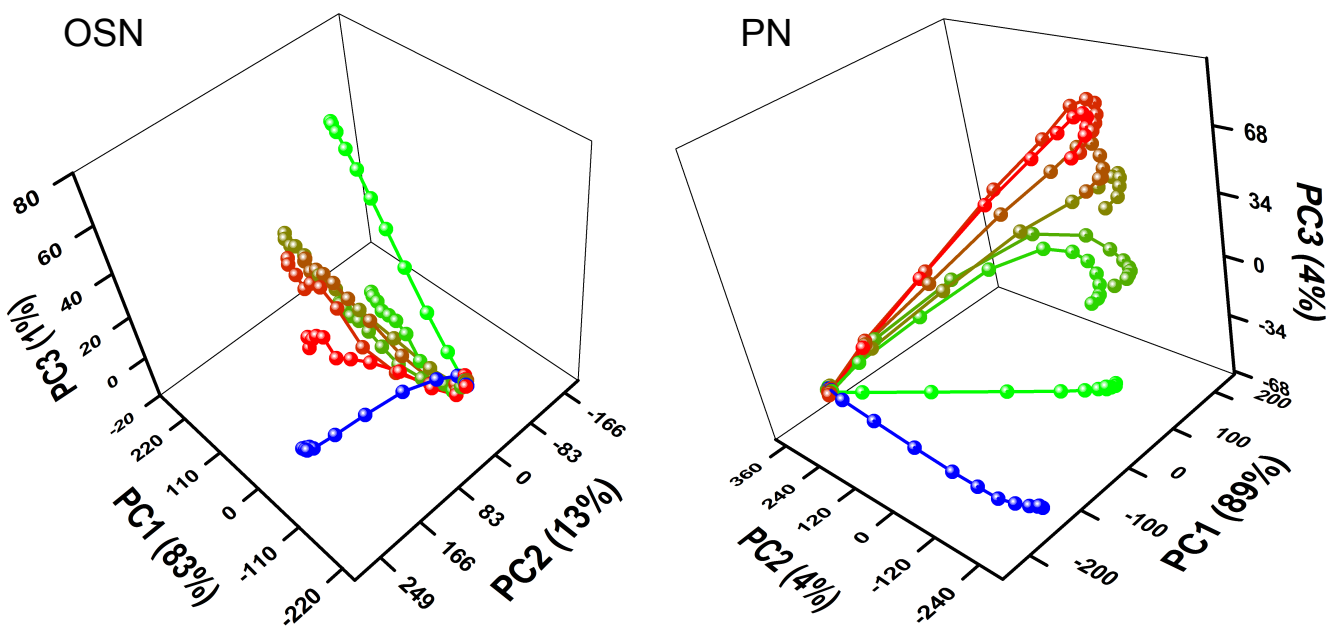

$-100 \% 3-$ Oct $+0 \%$ PA

$-99 \% 3-$ Oct $+1 \%$ PA

$-75 \%$ 3-Oct $+25 \%$ PA

$-50 \%$ 3-Oct $+50 \%$ PA

$-25 \% 3-$ Oct $+75 \%$ PA

$\multimap-1 \% 3-$ Oct

$+99 \%$ PA

$\multimap 0 \% 3-$ Oct

$+100 \%$ PA

$\multimap-100 \% \mathrm{MCH}$ 


\section{Results}

Figure 3.15. Dynamics of odor-evoked $\mathrm{Ca}^{2+}$ activity patterns to different binary odor mixtures.

Comparison of dynamics of $\mathrm{Ca}^{2+}$ activity patterns in OSNs and $\mathrm{PNs}$ in response to different binary mixtures of odors: $\mathrm{MCH} / 3-\mathrm{Oct}(\mathbf{A}), \mathrm{MCH} / \mathrm{PA}(\mathbf{B})$ and 3-Oct/PA (C) in a principal component space spanned by the first three principal components. The trajectories represent the time period from $1 \mathrm{~s}$ before the odor onset until the odor offset. The principal component analysis was done on the data set comprising of odor evoked $\mathrm{Ca}^{2+}$ activities from different time points in the 14 glomeruli. Each glomerulus represented one dimension and the 14 dimensions were then reduced to three principal components. The values given in axis labels indicate the percentage of variance covered by the first three principal components. In the case of $\mathrm{MCH} / 3-O c t$ binary mixtures, in OSNs, the odor representations were found to spread across the PCA odor space. In PNs, $100 \%$ $\mathrm{MCH}-0 \% 3-O c t$ and $99 \% \mathrm{MCH}-1 \% 3-O c t$ followed almost the same trajectory and ended up in one subvolume, while other binary mixtures formed one separate category. Hence, an odor classification was observed in $\mathrm{MCH} / 3-O c t$ binary mixtures in PNs. In MCH/PA mixtures, odor representation were found distributed uniformly in OSNs. In PNs, 100\% $\mathrm{MCH}-0 \%$ PA followed a completely different trajectory than the other binary mixtures. In $3-O c t / P A$ binary mixtures, except $100 \% 3-O c t-0 \%$ PA, all the other binary mixtures were found clustered together in OSNs. This also reflects a high degree of similarity existing between 3-Oct and PA odor representations. A similar distribution was observed in PNs, where 100\% 3-Oct-0\% PA again followed a different trajectory than the others. In both $\mathrm{MCH} / \mathrm{PA}$ (only in PNs) and 3-Oct/PA binary mixtures, a dominance of PA could be observed by the fact that all the binary mixtures having $>0 \%$ PA followed a distinctly different trajectory than the $0 \%$ PA binary mixtures.

PA > $0 \%$. The third binary mixture, which was of two similar odors (3-Oct/PA), classification already happened at the level of OSNs, which did not show any further change in PNs.

\subsubsection{Odortopic aspects of odor classification}

To understand the odortopic aspects of odor classification, the temporal and the spatial factors were needed to be dissected. This was achieved by comparing the different glomerular $\mathrm{Ca}^{2+}$ activity patterns by correlation analysis at different time points. The $\mathrm{Ca}^{2+}$ activity in each glomerulus to a particular odor mixture at a certain time point was taken as a vector. Similar vectors were created for other odor mixtures, but for the same time point. Subsequently, a correlation matrix was made based on the Pearson's correlation coefficients obtained between all possible combinations of the different binary mixtures of the same pairs of odors. Subsequently, similar correlation matrices were made for all the time points from the odor onset to odor offset for the three different 


\section{Results}

pair of odors (MCH/3-Oct, $\mathrm{MCH} / \mathrm{PA}$ and 3-Oct/PA). In the odor pair $\mathrm{MCH} / 3-O c t$ (Figure $3.16 \mathrm{~A}$ ), it could be observed in OSNs that every single odor mixture showed high correlation only to their immediate neighboring combination of odor mixture, thereby creating a linear distribution of high correlation values around the diagonal of the matrix. This pattern was independent of time in OSNs. In PNs, on other hand, two distinct clusters of high correlation were found. One cluster consisted of $100 \% \mathrm{MCH}-0 \% 3-\mathrm{Oct}$, $99 \% \mathrm{MCH}-1 \% 3-O c t$, and the other cluster consisted of the other $\mathrm{MCH} / 3-O c t$ binary mixtures. These clusters were found to be stable across time during the odor stimulus and were similar to the ones observed in the PCA analysis.

In the case of the $\mathrm{MCH} / \mathrm{PA}$ odor combination (Figure $3.16 \mathrm{~B}$ ), in OSNs, $100 \% \mathrm{MCH}-$ $0 \%$ PA remained always different from the rest of the mixtures. Strong correlations were found across any odor mixtures which consisted of $>0 \%$ of PA, especially during the odor onset. However, these correlations faded out as activity patterns reached odor offset but still no distinct clusters of high correlations were found in OSNs. $100 \%$ $\mathrm{MCH}-0 \% \mathrm{PA}$, however, remained distinct from other combinations irrespective of the time. In PNs, as time reached odor offset, the activity patterns evolved to develop into two distinct clusters of high correlation. Therefore, in PNs, with time, odortopic activity patterns of $99 \% \mathrm{MCH}-1 \% \mathrm{PA}, 75 \% \mathrm{MCH}-25 \% \mathrm{PA}, 50 \% \mathrm{MCH}-50 \% \mathrm{PA}$ and $25 \% \mathrm{MCH}-$ $75 \%$ PA became similar to each other, developing into one class of similar patterns. Another class of distinct patterns was found with $1 \% \mathrm{MCH}-99 \% \mathrm{PA}$ and $0 \% \mathrm{MCH}-100 \%$ PA. However, it should be noted that similar clusters were also found in OSNs, but with relatively low correlation coefficients compared to PNs. Moreover, such odor classification was not observed in the PCA space (Figure 3.15 B). This discrepancy could be explained by the fact that the PCA analysis also included the temporal component of glomerular activity patterns. Since these clusters were relatively unstable and formed only during the second half of the odor stimulus, they were not strongly reflected in the PCA analysis.

From the 3-Oct/PA odor mixtures (Figure $3.16 \mathrm{C}$ ), no distinct clusters of high correlation could be found in both OSNs and PNs. One fact that might explain this was the high degree of odortopic similarity already existing between 3-Oct and PA (Figure $3.13 \mathrm{C}$ ). Distinct clusters of even higher correlation were difficult to detect. Both of these odors, therefore, got classified at the level of both OSNs and PNs and this categorization was also not dependent on time. 


\section{Results}

A

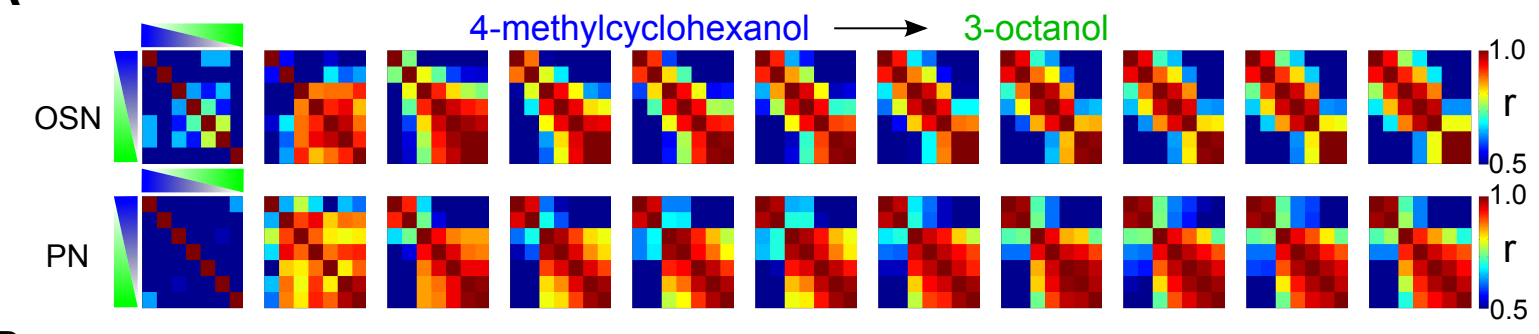

B

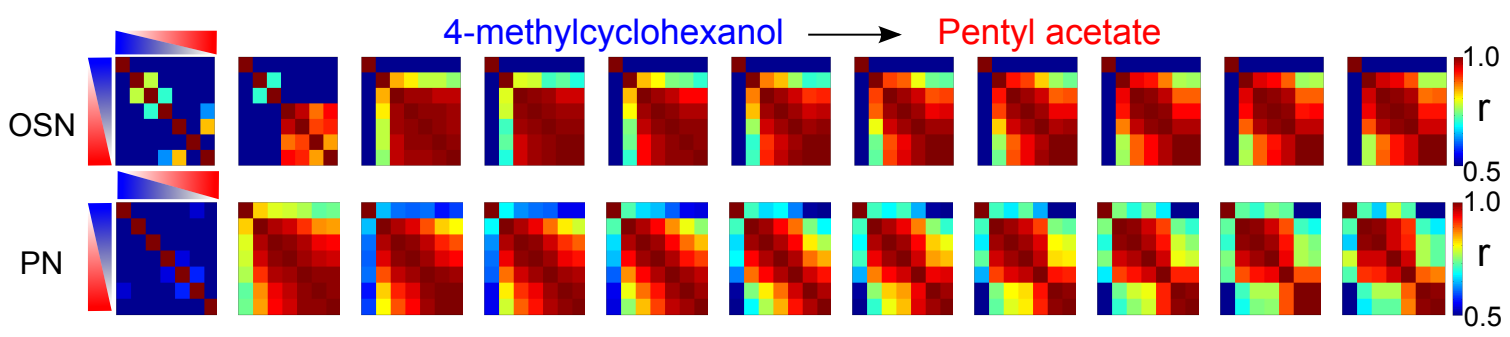

C

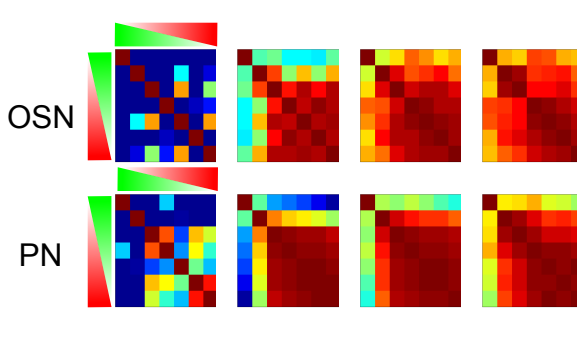

3-octanol $\longrightarrow$ Pentyl acetate
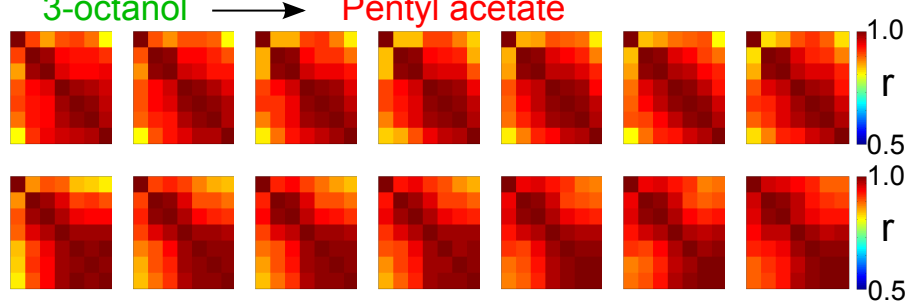

$-0.2 \quad 0.0$

odor stimulus (s)

2.0

Figure 3.16. Odortopic differences in odor-evoked $\mathrm{Ca}^{2+}$ activity patterns of different odor mixtures at different time points.

Matrices indicate the pairwise Pearson's correlations ( $r$ ) between glomerular $\mathrm{Ca}^{2+}$ activity patterns evoked by different odor mixtures at different time points: $\mathrm{MCH} / 3-\mathrm{Oct}(\mathrm{A})$, $\mathrm{MCH} / \mathrm{PA}$ (B) and 3-Oct/PA (C). High correlations are represented in warm colors and low correlations in cold colors. The triangles with color gradient at the left and top of the first correlation matrix indicates that the concentration of the one odor increases and the other odor decreases (PA indicated by red, 3-Oct by green and $\mathrm{MCH}$ by blue). In the case of $\mathrm{MCH} / 3-\mathrm{Oct}$, in OSNs, high correlation could be observed almost uniformly distributed along the diagonal of the correlation matrices. This implied that the glomerular activity patterns of binary mixtures are similar only to their neighboring closest mixture combination in terms relative concentration of components. However, in PNs, two clusters of high correlation were found, indicating a high degree of similarity among the activity patterns of certain sets of binary mixtures. Therefore, an odor classification of those sets of binary mixtures of $\mathrm{MCH} / 3-\mathrm{Oct}$ was observed in PNs. In $\mathrm{MCH} / \mathrm{PA}$ binary mixtures, no such distinct clusters of high correlation was found in OSNs. But in PNs, two such clusters could be observed which became more distinct during the odor offset. In case of 3-Oct/PA, due to the high degree of similarity existing between 3-Oct and PA, high correlation was obtained between any pair of their binary mixtures and no distinct clusters could be found. $n=5-6$ flies. 


\section{Results}

In the next step, the abrupt transitions observed across the odor mixtures were quantitatively analyzed across all the flies at the time point of odor offset. To do so, the $\mathrm{Ca}^{2+}$ activity in every glomeruli during odor offset to a certain odor stimulus was determined. The odor offset time point was chosen because by then the activity patterns of every odor stimuli seemed to be stable. This stable pattern formation could be observed by the $\mathrm{Ca}^{2+}$ activity in individual glomeruli during the odor offset (Figure 3.14). For each odor stimulus, the $\mathrm{Ca}^{2+}$ activity values during the odor offset from the 14 glomeruli were then represented as a vector for that odor stimulus in a 14 dimensional space. Similar vectors were generated for all odor stimuli. The odortopic differences were measured by calculating the angles between the vectors (i.e., angular distance). For each pair of odor combination, the $100 \%$ of the individual components were taken as reference vectors. Using these reference vectors, the angular distances of all the intermediate mixtures were subsequently calculated which then determined the odortopic differences from the $100 \%$ of the mixture components (Figure 3.17). Interestingly a linear distribution of $\mathrm{MCH} / 3-O c t$ odor mixtures were found in OSNs (Figure 3.17 A). In PNs, however, the abrupt transition observed in previous results was again reflected in a non-linear distribution of the odortopic activity patterns across flies. $100 \% \mathrm{MCH}-0 \%$ 3-Oct and 99\% $\mathrm{MCH}-1 \% 3-$ Oct showed similar angular distances from 100\% 3-Oct in PNs. The other concentrations showed almost equal angular distances from $100 \%$ $\mathrm{MCH}$. In the case of $\mathrm{MCH} / \mathrm{PA}$ odor mixtures (Figure $3.17 \mathrm{~B}$ ), no such strong nonlinearity was observed in PNs. Due to the presence of the dominant odor PA, from $100 \% \mathrm{MCH}-0 \% \mathrm{PA}$ a huge transition was observed in terms of angular distances from $100 \% \mathrm{MCH}$ in both OSNs and PNs. However, 99\% MCH-1\% PA, 75\% MCH-25\% PA, $50 \% \mathrm{MCH}-50 \% \mathrm{PA}$ and $25 \% \mathrm{MCH}-75 \% \mathrm{PA}$ showed similar angular distances from $100 \% \mathrm{MCH}$, and linear transitions between them were not observed as was found in OSNs. This reflected the formation of the same cluster observed during the odor offset in the correlation matrices of $\mathrm{MCH} / \mathrm{PA}$ binary mixtures (Figure 3.16 B). In 3-Oct/PA odor mixtures (Figure $3.17 \mathrm{C}$ ), such effects could not be detected due to the low angular distances found between 3-Oct and PA. However, those low angular distances reflected the odortopic similarity between 3-Oct and PA. From these results, it could be overall concluded that PNs, compared to OSNs, showed a non-linear change in activity patterns in the case of $\mathrm{MCH} / 3-$ Oct. The dominance of PA could be observed in $\mathrm{MCH} / \mathrm{PA}$ mixtures in both OSNs and PNs. This PA dominance overshadowed any possible formation of non-linear transition from $100 \% \mathrm{MCH}$ to $100 \% \mathrm{PA}$ in PNs. 3-Oct/PA, on other hand, similar odorants already at the level of OSNs, did not show any further 


\section{Results}

change in PNs.

A

$\mathrm{MCH} \longrightarrow 3-\mathrm{Oct}$
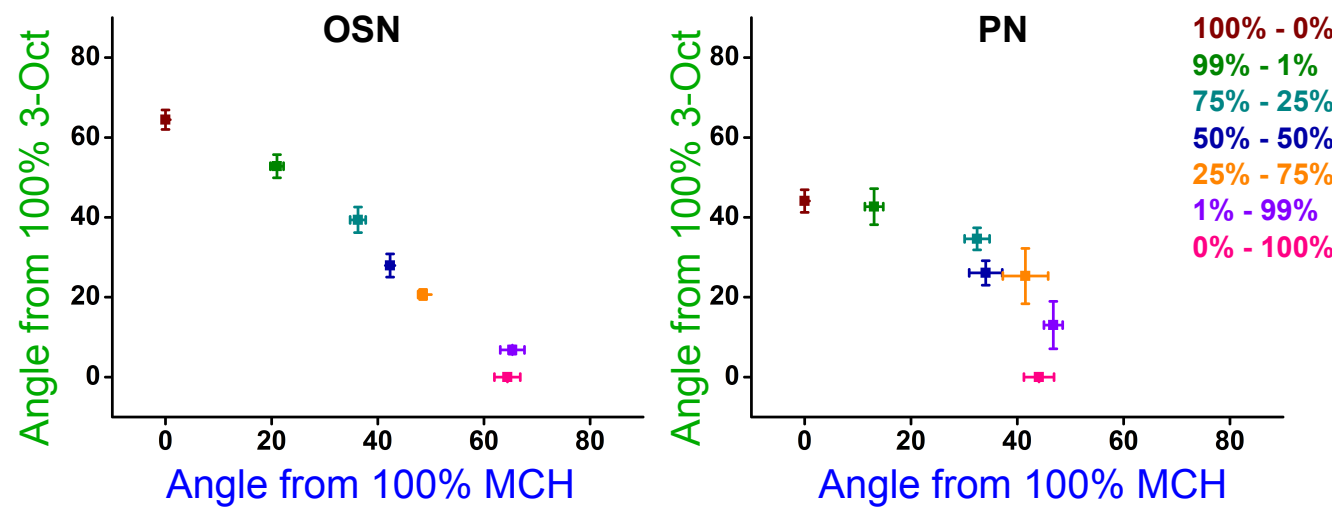

B
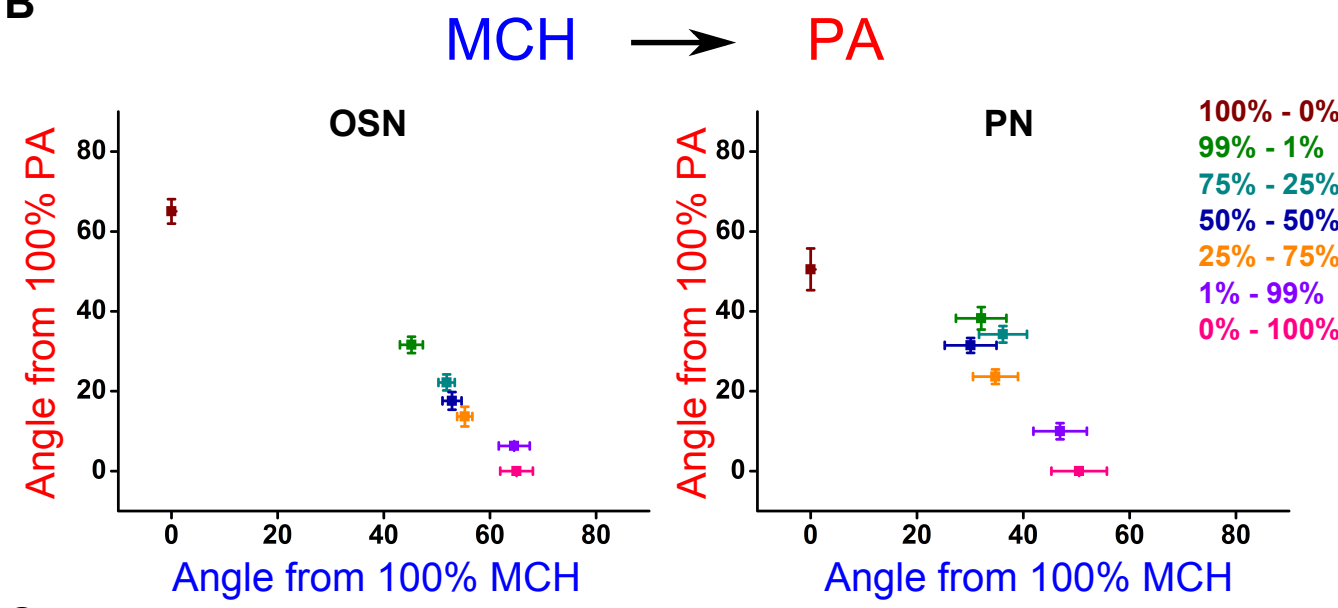

C

3-Oct $\rightarrow$ PA
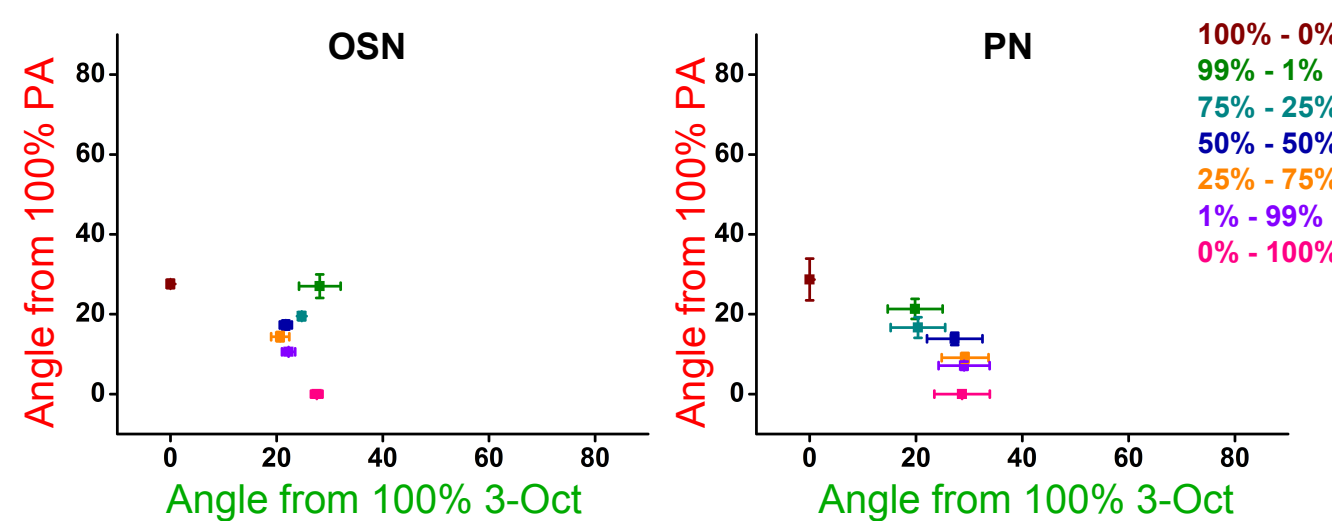


\section{Results}

Figure 3.17. Odortopic differences in odor-evoked $\mathrm{Ca}^{2+}$ activity patterns of different odor mixtures at odor offset.

Angular distances between the glomerular activity patterns from $100 \%$ of both odor components during the odor offset (MCH/3-Oct (A), $\mathrm{MCH} / \mathrm{PA}(\mathbf{B})$ and 3-Oct/PA (C)). Each odor stimulus represents a vector in the 14 dimensional odor space. This vector is defined by the odor evoked $\mathrm{Ca}^{2+}$ activities in the 14 glomeruli. The angular distances are determined by calculating the angles between each odor stimulus and the $100 \%$ concentrations of the individual components of the mixture as reference vectors. The $100 \%$ concentrations of the components are hence represented by the axes in the figure and each value represents an odor stimulus by the angles from the $100 \%$ of individual components. $\mathrm{n}=5-6$ flies. Error bars indicate \pm SEM. In MCH/3-Oct binary mixtures, odor representation were found to be changing linearly from $100 \% \mathrm{MCH}$ to $100 \% 3-$ Oct in OSNs. But in PNs, $100 \% \mathrm{MCH}-0 \% 3-O c t$ and $99 \% \mathrm{MCH}-1 \% 3-$ Oct showed similar angular distances from 100\% 3-Oct while their other mixtures showed similar angular distances from $100 \%$ $\mathrm{MCH}$, thereby creating a non-linear distribution of odor representations. In the case of $\mathrm{MCH} / \mathrm{PA}$ mixtures, the dominance of odor PA was reflected by a huge transition between $100 \% \mathrm{MCH}-0 \% \mathrm{PA}$ and $99 \% \mathrm{MCH}-1 \% \mathrm{PA}$ in both OSNs and PNs. 99\% $\mathrm{MCH}-1 \% \mathrm{PA}$, $75 \% \mathrm{MCH}-25 \% \mathrm{PA}, 50 \% \mathrm{MCH}-50 \% \mathrm{PA}$ and $25 \% \mathrm{MCH}-75 \% \mathrm{PA}$ showed similar angular distances from $100 \% \mathrm{MCH}$ in PNs which was not observed in OSNs. In the case of 3-Oct/PA binary mixtures, dominance of PA was reflected in a transition between $100 \%$ 3-Oct-0\% PA and 99\% 3-Oct-1\% PA in both OSNs and PNs. Except 100\% 3-Oct-0\% PA, all other binary mixtures showed similar angular distances from 100\% 3-Oct in OSNs as well as PNs.

\subsection{Possible mechanisms of classification of odor stimuli}

\subsubsection{Concentration dependence of $\mathrm{Ca}^{2+}$ activity patterns of individual odors}

To explain the odor classification effect observed in PNs, one hypothesis was that the odor classification might occur due to a categorization already existing in the individual components of the odor mixtures across their different concentrations. In other words, odor representations of the single odors might already form similar categories in PNs as was observed with their binary mixtures. To test this hypothesis, concentration dependence of $\mathrm{Ca}^{2+}$ activity patterns of individual odors was needed to be determined. This was achieved by calculating the Pearson's correlation coefficients between the 


\section{Results}

activity patterns of different concentrations of the same odor. The $\mathrm{Ca}^{2+}$ activity in each glomerulus to a particular odor concentration at a certain time point was taken as a vector. Similar vectors were created for other concentrations of the same odor and for the same time point. Subsequently a correlation matrix was made based on the Pearson's correlation coefficients obtained between all possible combinations between the different odor concentrations of the same odor. Similar correlation matrices were made for all the time points from the odor onset to odor offset.

In figure 3.18, these correlation matrices were determined for different time points. No strong correlation was observed between any odor concentrations before the odor onset. However, it was observed in all the three odors that the correlation coefficients across different concentrations increased from the time point of odor onset to odor offset. $0 \%$ concentration activity pattern showed complete odortopic decorrelation with the other concentrations at all time points. In PNs compared to OSNs, higher correlation was observed across all concentrations irrespective of any odor, thereby indicating a higher degree of odortopic similarity between glomerular $\mathrm{Ca}^{2+}$ activity patterns of different concentrations in PNs. In OSNs, lower correlation values were observed for $1 \%$ and $25 \%$.

On comparing these results with the odor mixtures, similar clusters of high correlation were not found in the correlation matrices of individual odors. On the contrary, PNs rather showed invariance to different concentrations of the odors and no subclusters were formed as observed in case of binary mixtures. This, therefore, failed to explain the formation of categories of different odor mixtures in terms of single odor concentrations. However, from the results of concentration dependence of $\mathrm{Ca}^{2+}$ activity patterns, it was observed that PNs showed stable pattern formation to the selected range of concentrations of different odors. So in other words, PNs generated stable network states for each odor which were resistant to the varying inputs coming from OSNs. This might imply that on presenting such odors together as a mixture in different ratios, a sharp transition would be likely to be observed between their stable states in PNs, in order to maintain the resistance to the two varying inputs. This transition would therefore lead to a classification of these intermediate mixtures to either of the stable states of their individual components. However, how this transition was mediated could not be determined. 


\section{Results}

A

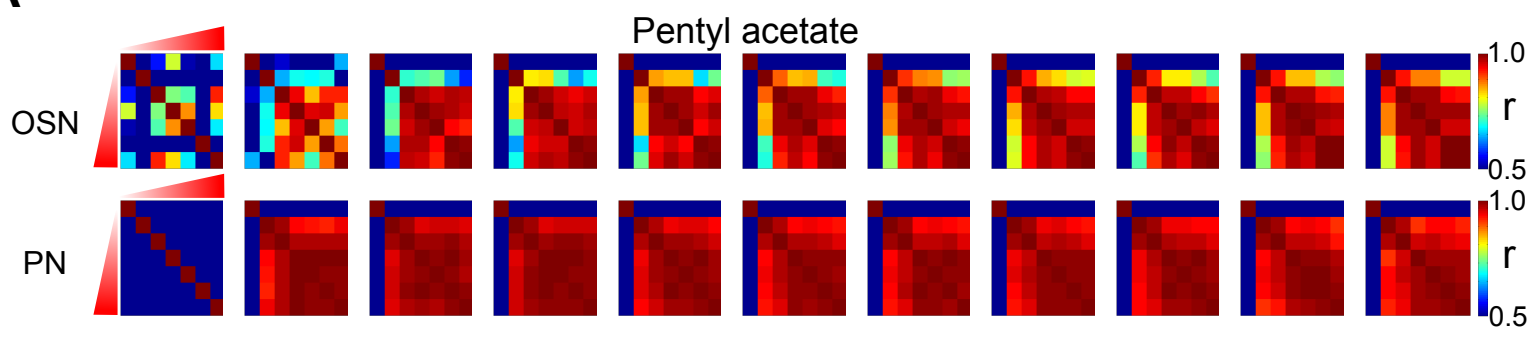

B

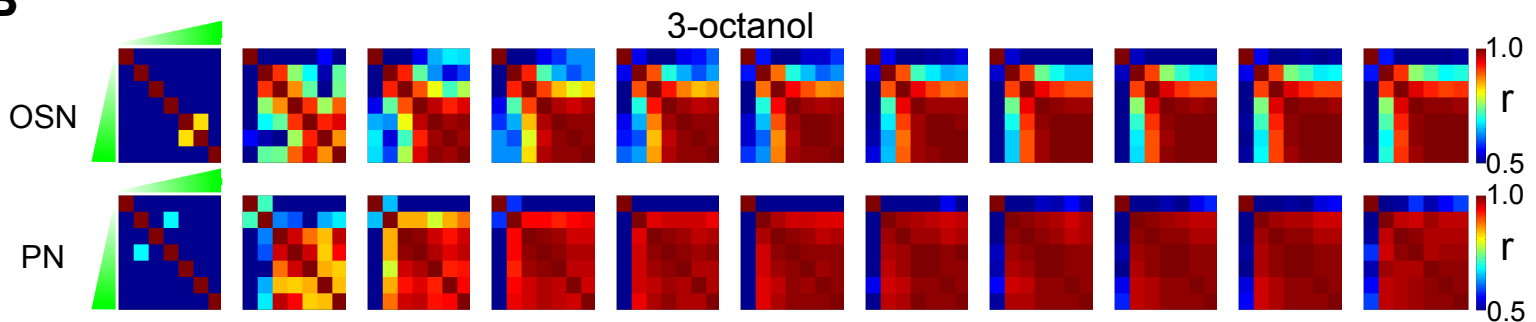

C

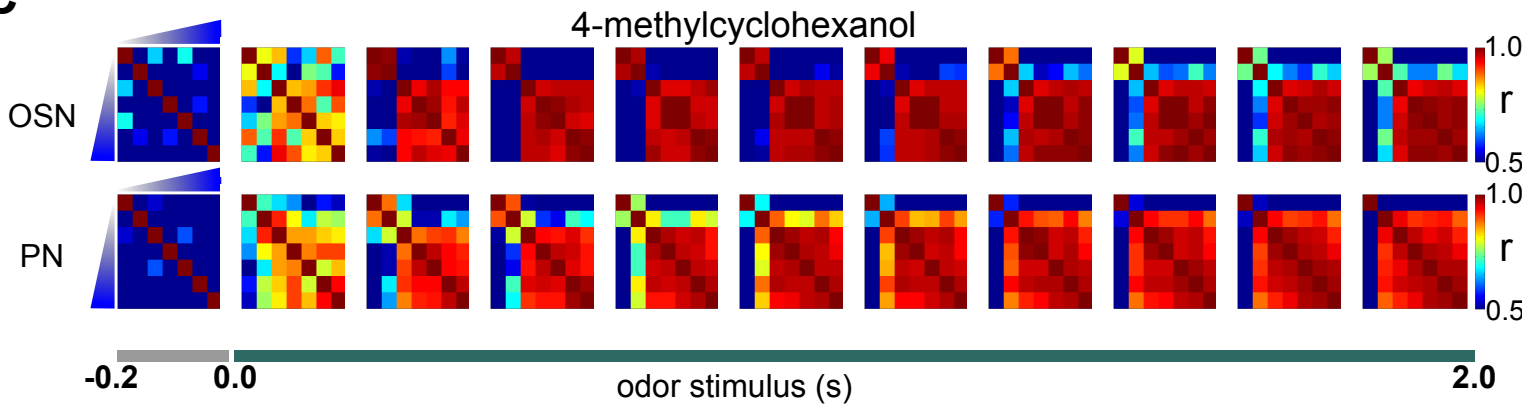

Figure 3.18. Odortopic differences in odor-evoked $\mathrm{Ca}^{2+}$ activity patterns of different concentrations.

Matrices indicate pairwise Pearson's correlations ( $r$ ) between glomerular $\mathrm{Ca}^{2+}$ activity patterns evoked by different concentrations of odors at different time points (PA (A), 3-Oct (B) and $\mathrm{MCH}(\mathbf{C})$ ). High correlations are represented in warm colors and low correlations in cold colors. The triangles with color gradient at the left and top of the first correlation matrix indicate that the concentration of the odor increases from left to right and top to bottom starting from $0 \%$ to $100 \%$. In all the three odors, relatively lower correlation coefficients were obtained for $1 \%$ and $25 \%$ concentrations in OSNs. But in PNs, all the odor representations of different concentrations became similar to each other. $n=5$ (PA), 6 (3-Oct) and $6(\mathrm{MCH})$. 


\section{Results}

\subsubsection{Involvement of lateral inhibition in transition of odor representations in odor morphing}

The glomeruli in the AL are inter-connected by GABAergic local interneurons [Stocker et al., 1997; $\mathrm{Ng}$ et al., 2002]. Some of these interneurons connect the majority of glomeruli, whereas some innervate only specific glomeruli [Chou et al., 2010; Seki et al., 2010]. These local interneurons release GABA and affect the spatiotemporal patterns in the $A L$ [Wilson and Laurent, 2005]. The $\mathrm{GABA}_{A}$-type receptor had been described before for influencing the early phase of the odor responses in PNs [Wilson and Laurent, 2005; Silbering and Galizia, 2007]. Moreover, recently it has been shown that different glomeruli were differentially sensitive to GABA and this sensitivity was stereotypic across glomeruli [Hong and Wilson, 2015]. Therefore, taking into account these facts, it was hypothesized that GABA might play a role in causing classification of different odor mixtures, and the $\mathrm{GABA}_{A}$-type receptor might be a good candidate. $\mathrm{GABA}_{A}$-type receptors are picrotoxin sensitive [Hosie et al., 1997]. In order to test this hypothesis, picrotoxin $(10 \mu \mathrm{M})$ was used to block these receptors pharmacologically to analyze a potential role of GABA. The picrotoxin (PTX) solution was therefore applied onto the fly's brain and the $\mathrm{Ca}^{2+}$ activity of the $\mathrm{AL}$ to the different odor mixtures was recorded in three phases: Before the application of PTX, During the PTX application and after washing off the PTX. This was repeated for the three odor mixtures $(\mathrm{MCH} / 3-$ Oct, $\mathrm{MCH} / \mathrm{PA}$ and $3-\mathrm{Oct} / \mathrm{PA}$ ) and the role of $\mathrm{GABA}_{\mathrm{A}}$ receptors on the classification of odors was determined.

\subsubsection{1. $\mathrm{MCH} / 3-$ Oct odor mixture}

From the glomerular activity of $\mathrm{MCH} / 3-O c t$ odor mixtures during the three stages of PTX application (Figure 3.19) it was found that there was a considerable decrease in $\mathrm{Ca}^{2+}$ activity in OSNs in the presence of PTX. However, at the same time, the glomeruli DC1 and VC1 showed an increase in activity in PNs. DM2 and DM3 on other hand showed a decrease in $\mathrm{Ca}^{2+}$ activity in PNs. After washing the brain, the $\mathrm{Ca}^{2+}$ activity in most of the glomeruli was restored. 


\section{Results}

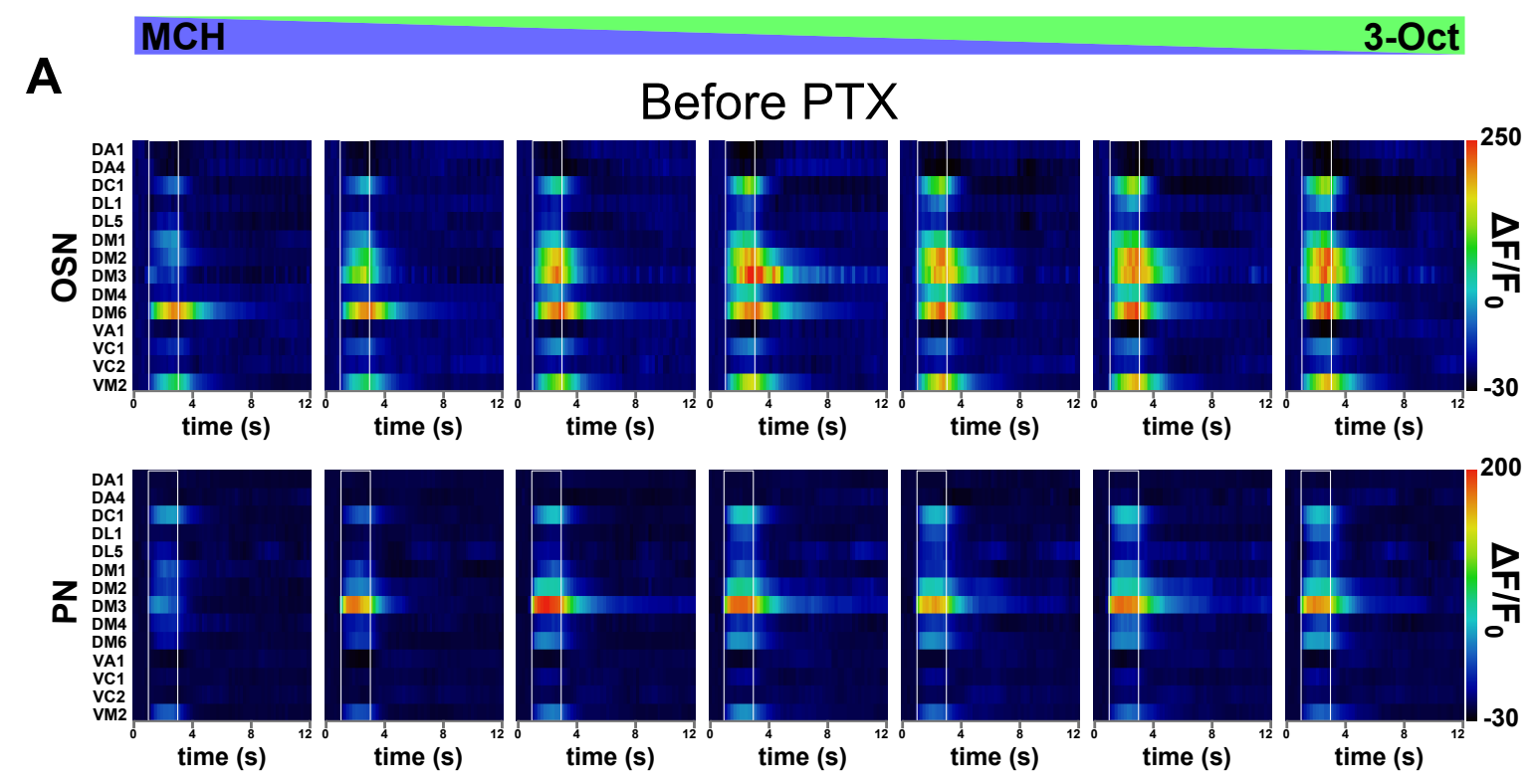

B

During PTX
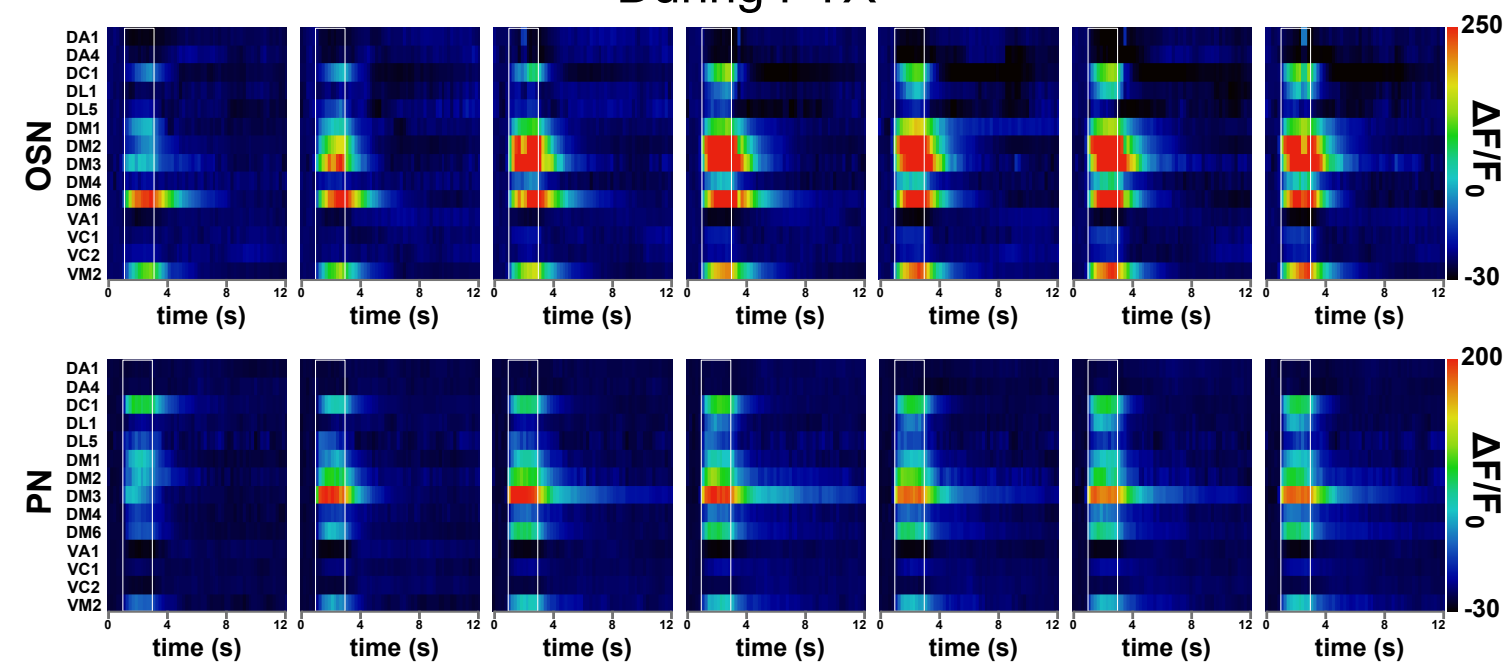

C

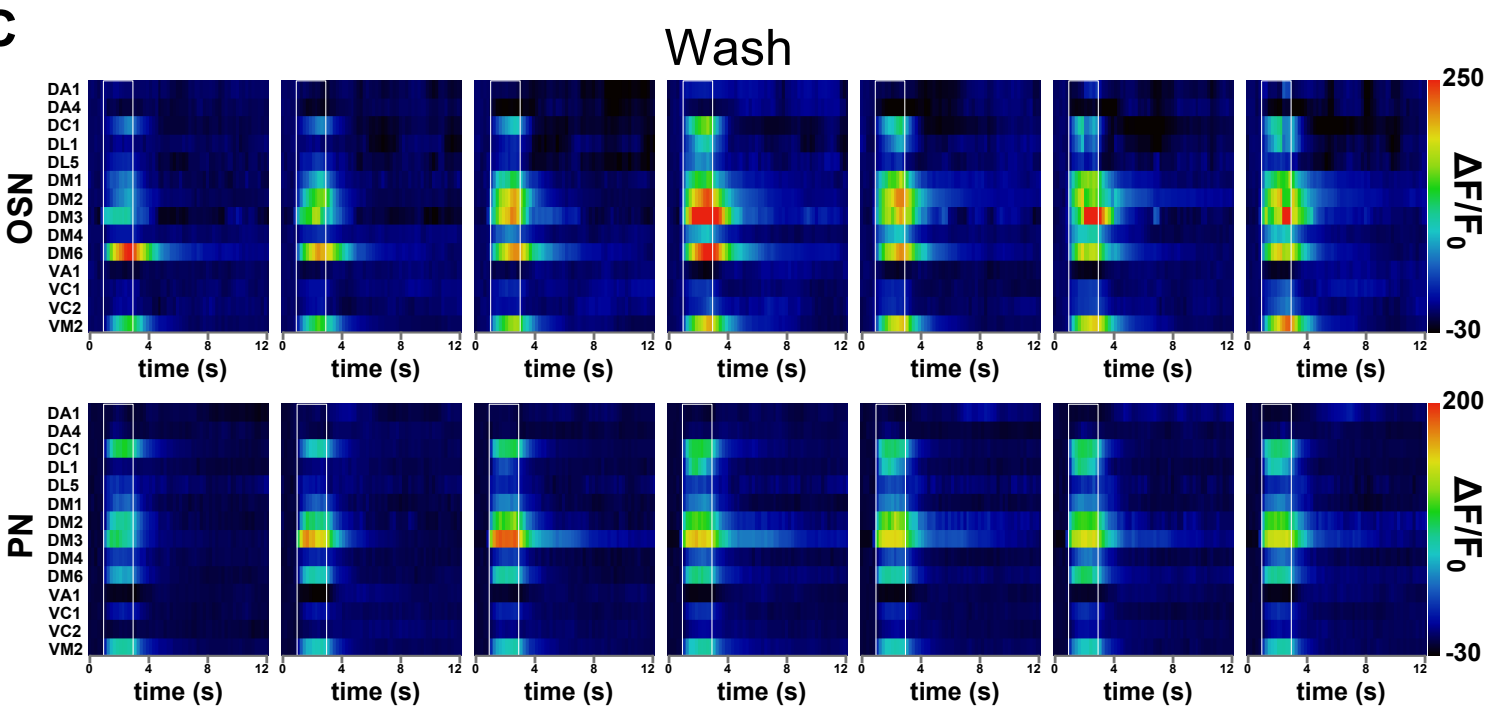




\section{Results}

Figure 3.19. Odor-evoked $\mathrm{Ca}^{2+}$ activity to different binary odor mixtures of $\mathrm{MCH} / 3-O c t$ in OSNs and PNs during the three stages of PTX application.

Glomerular activity map of 14 glomeruli to different binary odor mixtures of $\mathrm{MCH} / 3-O c t$ in three different stages of PTX application: Before PTX (A), During PTX (B) and Wash (C). The $\mathrm{Ca}^{2+}$ activity map was determined by the $\Delta F / F_{0}$ values obtained for the 14 glomeruli. The white bar indicates the duration of the odor stimulus. High $\mathrm{Ca}^{2+}$ activity is shown in warm colors and low activity in cold colors. Every row in each panel represents the $\mathrm{Ca}^{2+}$ activity of single glomerulus indicated at the extreme left. $\mathrm{n}=5$ flies.

\section{Population coding}

In order to visualize the combinatorial effects of PTX on the $\mathrm{Ca}^{2+}$ activities of the glomeruli, the data was subjected to PCA similar to the section 3.2.2. The classification of odor mixtures previously observed in the case of $\mathrm{MCH} / 3-O c t$ was again observed in PNs before the application of PTX (Figure 3.20 A). However, during the application of PTX (Figure $3.20 \mathrm{~B}$ ), this classification could not be found and instead the trajectories of the odor mixtures in the PCA space resembled that of the OSNs. The classification was almost restored after washing off the PTX (Figure 3.20 C). Therefore, it could be concluded from these results that GABA was required for causing this classification through $\mathrm{GABA}_{\mathrm{A}}$ receptors.

\section{Odortopic properties}

The odortopic aspects of the classification of the odor mixtures was observed at three different stages of PTX application. Before the application of PTX, $100 \% \mathrm{MCH}-0 \%$ $3-$ Oct and $99 \% \mathrm{MCH}-0 \% 1-$ Oct showed a higher degree of correlation, thereby forming one cluster in the correlation matrix during the odor offset (Figure 3.21). Except for $50 \% \mathrm{MCH}-50 \% 3-$ Oct, the other concentrations formed one more cluster of high correlations. During the PTX application no such clusters were found. However, after washing off the PTX from the brain, the clusters of high correlation did not return back to their native state completely.

To confirm the observations from the correlation analysis, the angular distances of the mixtures from $100 \% \mathrm{MCH}$ and $100 \% 3-$ Oct from all the flies were plotted (Figure 3.22) from different stages of PTX application. In OSNs, as observed before, the $\mathrm{Ca}^{2+}$ 


\section{Results}

\section{A

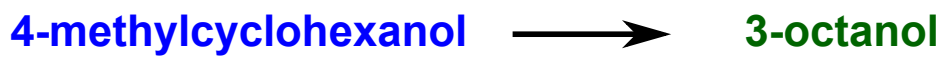

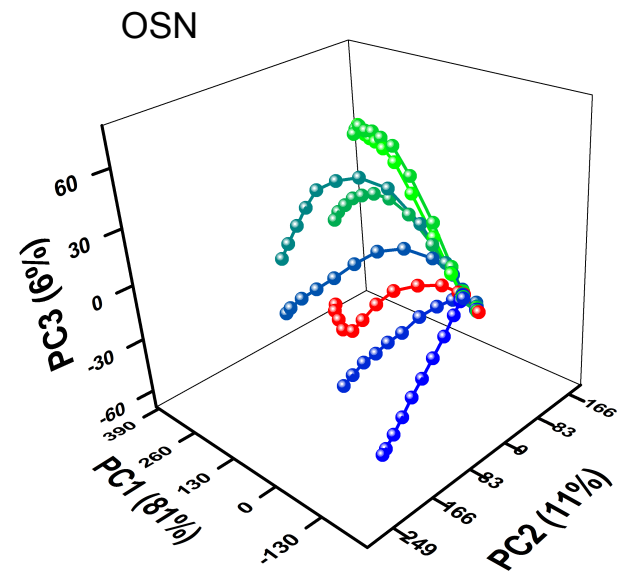

Before PTX

B
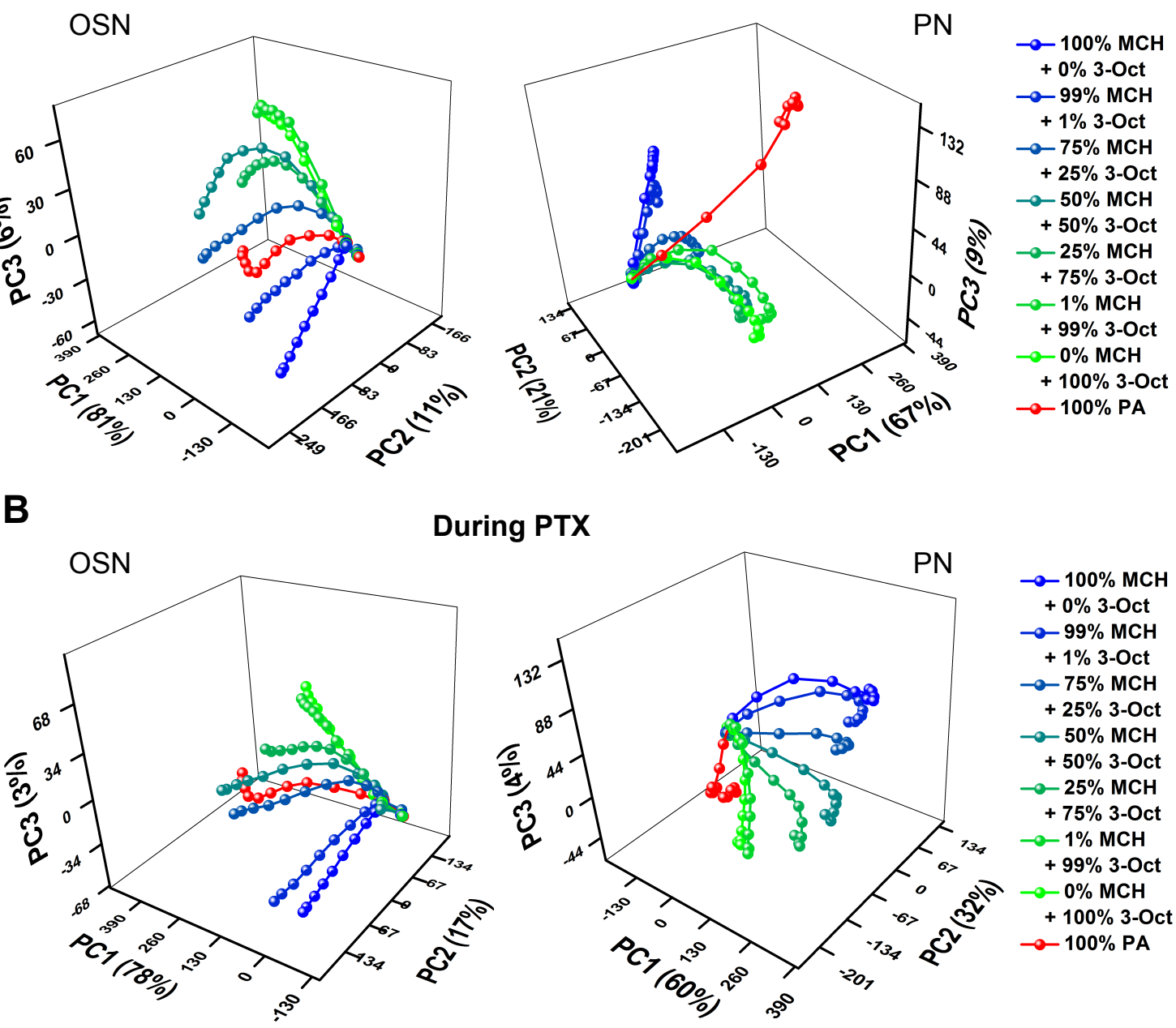

During PTX

C

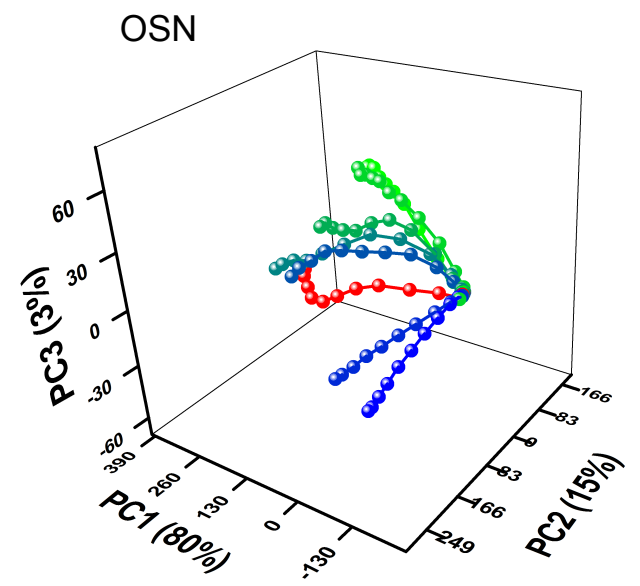

Wash

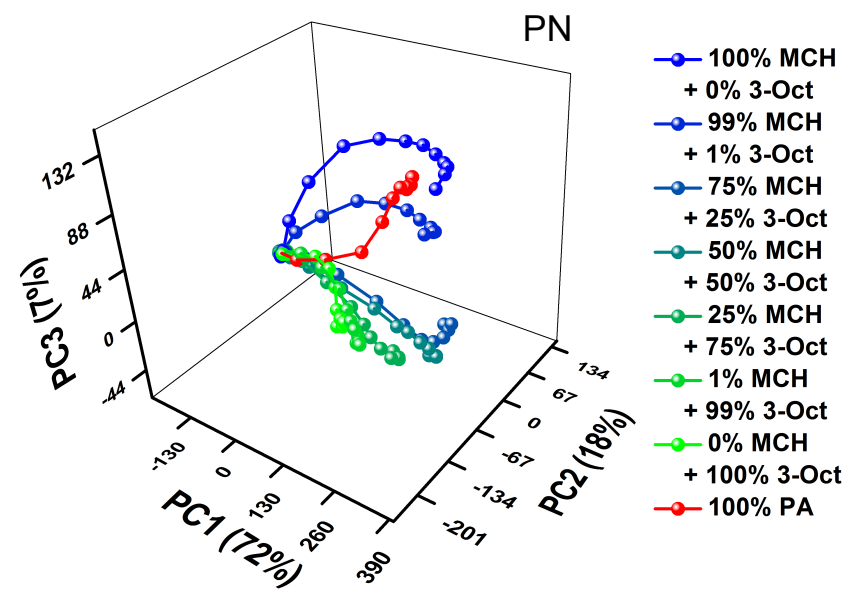




\section{Results}

Figure 3.20. Dynamics of odor-evoked $\mathrm{Ca}^{2+}$ activity patterns to $\mathrm{MCH} / 3-\mathrm{Oct}$ odor mixture in three different stages of PTX application.

Comparison of dynamics of $\mathrm{Ca}^{2+}$ activity patterns in OSNs and PNs in response to $\mathrm{MCH} / 3-O c t$ binary mixtures during three stages: Before the application of PTX (A), during the application of PTX (B) and after the wash (C) in a principal component space spanned by the first three principal components. The trajectories represent the time period from 1 second before the odor onset until the odor offset. The values given in axis labels indicate the percentage of variance covered by that axis. Before the application of PTX, the binary mixtures were distributed across the PCA odor space in OSNs. In PNs, $100 \% \mathrm{MCH}-0 \% 3-O c t$ and $99 \% \mathrm{MCH}-1 \% 3-O c t$, as observed before, followed similar trajectories in PNs and formed a distinct category, while other binary mixtures formed another distinct category. Such odor classification was, however, lost when PTX was added. Moreover, the distribution of the binary mixtures in PNs resembled that of OSNs during the PTX application. After the washing of PTX, $100 \% \mathrm{MCH}-0 \% 3-$ Oct and $99 \%$ $\mathrm{MCH}-1 \% 3-O c t$ again followed, not the same, but still similar trajectories. The other binary mixtures formed a similar cluster again as was observed before the application of PTX.

activity patterns of the odor mixtures during odor offset were linearly distributed. In PNs, on other hand, this linearity was not present, which subsequently caused the activity patterns of $100 \% \mathrm{MCH}-0 \% 3-O c t$ and $99 \% \mathrm{MCH}-1 \% 3-O c t$ to form one category which was more similar to $100 \% \mathrm{MCH}$ and the other mixtures as another category which showed similar patterns to $100 \% 3$-Oct. Interestingly, during the application of PTX (Figure $3.22 \mathrm{~B}$ ), the distribution of activity patterns of odor mixtures in PNs resembled closely to that of OSNs. Therefore, it was further confirmed that on blocking the $\mathrm{GABA}_{\mathrm{A}}$ receptors, no classification was observed in PNs across individual flies. The classification was, however, restored after washing off the PTX from the fly's brain, when the GABAergic inhibition was again restored. 


\section{Results}

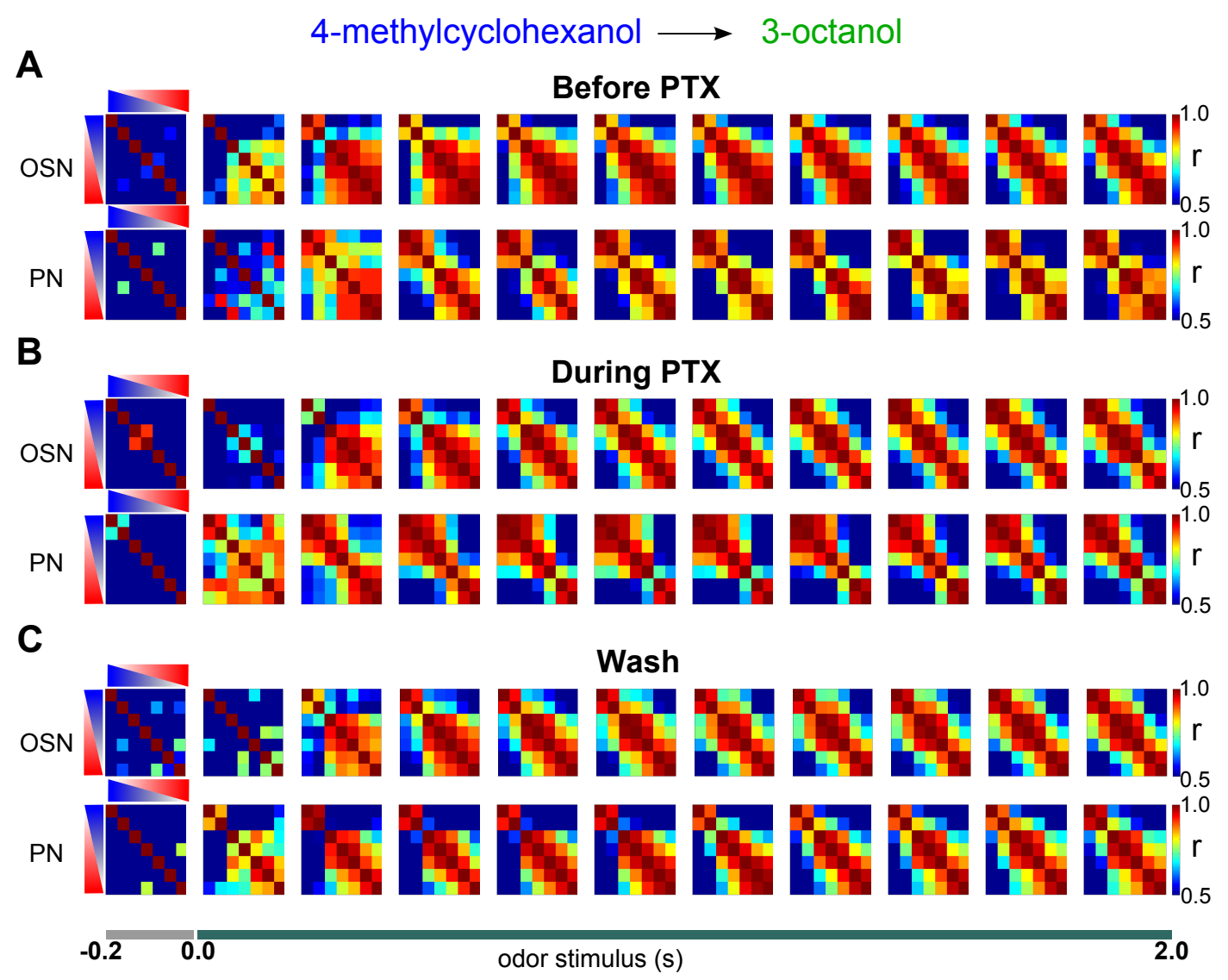

Figure 3.21. Odortopic differences in odor-evoked $\mathrm{Ca}^{2+}$ activity patterns of different odor mixtures of $\mathrm{MCH} / 3-O c t$ at different stages of PTX application.

Matrices indicate the pairwise Pearson's correlations ( $r$ ) between glomerular activity patterns evoked by different odor mixtures of $\mathrm{MCH} / 3-O c t$ at different stages of PTX application: Before PTX (A), during PTX (B) and wash (C). High correlations are represented in warm colors and low correlations in cold colors. The triangles with color gradient at the left and top of the first correlation matrix indicate that the concentration of one odor increases and that of the other odor decreases. Before the application of PTX, clusters of high correlation coefficients were found in PNs but not in OSNs. During PTX application, no such high correlation clusters, as was observed before, in PNs could be found. Even after washing the PTX, such clusters of high correlation could not be completely restored. $\mathrm{n}=5$ flies. 


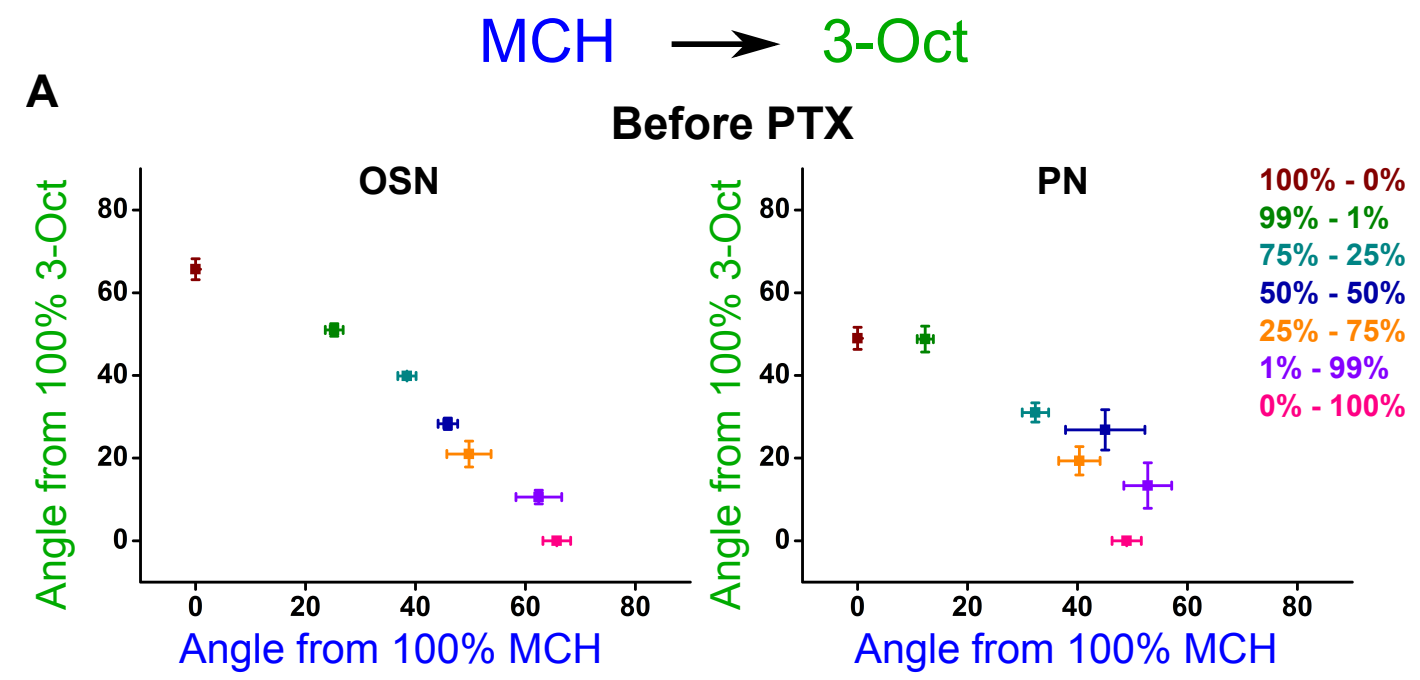

B

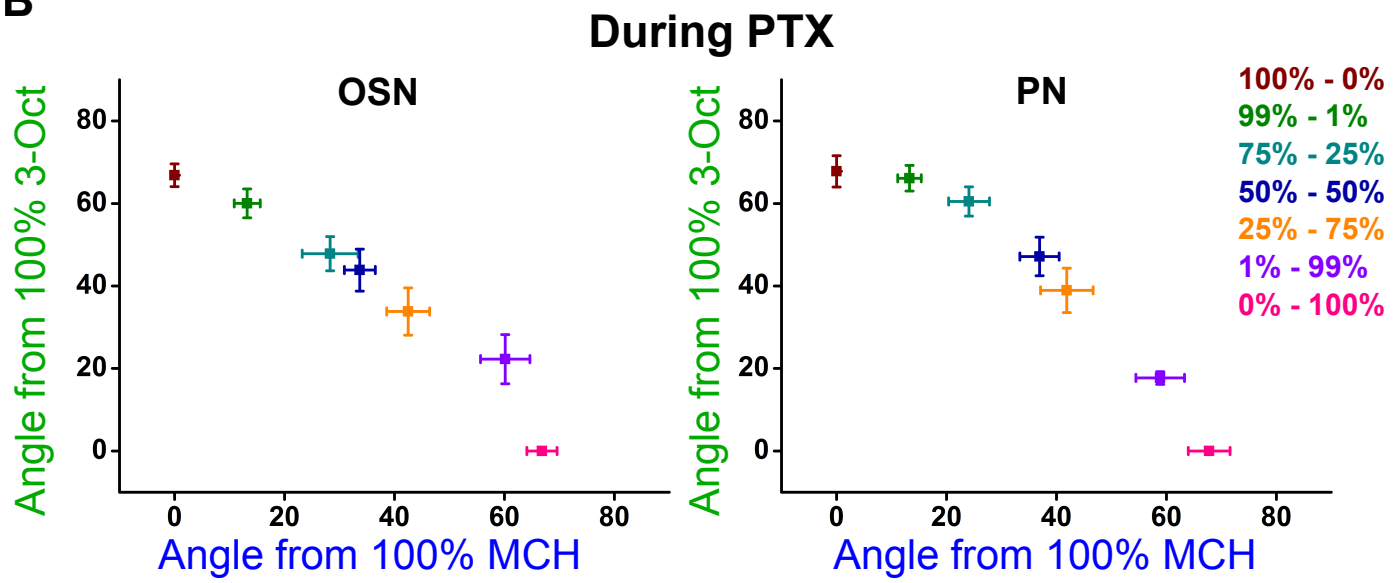

C

\section{Wash}
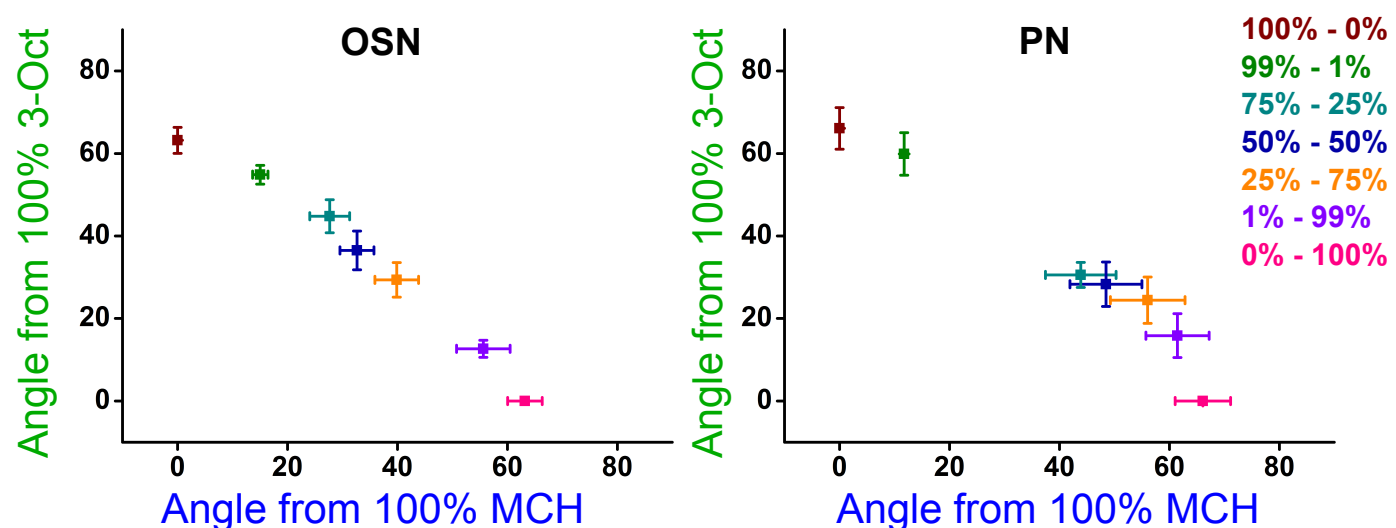


\section{Results}

Figure 3.22. Odortopic differences in odor-evoked $\mathrm{Ca}^{2+}$ activity patterns of $\mathrm{MCH} / 3-O c t$ odor mixtures at different stages of PTX application in OSNs and PNs at odor offset.

Angular distances between the glomerular activity patterns from $100 \% \mathrm{MCH}$ and $100 \%$ 3 -Oct (as reference) at odor offset. Each odor stimulus represents a vector in the $14 \mathrm{di}-$ mensional odor space. This vector is defined by the odor evoked $\mathrm{Ca}^{2+}$ activities in the 14 glomeruli. The angular distances are determined by calculating the angles between each odor stimulus and the $100 \%$ concentrations of $\mathrm{MCH}$ and 3-Oct as reference vectors. The $100 \% \mathrm{MCH}$ and $100 \% 3-O c t$ are hence represented by the axes in the figure and each value represents an odor stimulus by the angles from the $100 \% \mathrm{MCH}$ and $100 \% 3-O c t . \mathrm{n}$ $=5$ flies. Error bars indicate \pm SEM. Before the application of PTX, odor representations of binary mixtures were found to be changing linearly from $100 \% \mathrm{MCH}$ to $100 \% 3-$ Oct in OSNs. But in PNs, 100\% MCH-0\% 3-Oct and 99\% MCH-1\% 3-Oct showed similar angular distances from 100\% 3-Oct, while their other mixtures showed almost similar angular distances from $100 \% \mathrm{MCH}$, thereby creating a non-linear distribution of odor representations. But during the application of PTX, in PNs, such non-linearity is lost and instead, the distribution of the odor representations became similar to that of OSNs. No effect of PTX was observed in OSNs. After washing off the PTX, the distribution of representations in PNs became similar to the distribution observed before the application of PTX.

\subsubsection{2. $\mathrm{MCH} / \mathrm{PA}$ odor mixture}

The effects of blocking $\mathrm{GABA}_{\mathrm{A}}$ receptors was further tested with $\mathrm{MCH} / \mathrm{PA}$ mixtures, where due to $\mathrm{PA}$ dominance, all the mixtures with $\mathrm{PA} \%>0 \%$ were found to classify into one major category in PNs. In the first step, $\mathrm{Ca}^{2+}$ activity in the 14 glomeruli were measured in all the three stages of PTX application (Figure 3.23). On comparing the activities, it was found that during PTX application there was considerable increase in OSN activity in some glomeruli especially DM2, DM3, DM6 and VM2. Such strong differences were not observed in PNs.

\section{Population coding}

Taking into account these differences in glomerular $\mathrm{Ca}^{2+}$ activity, the $\mathrm{Ca}^{2+}$ activity patterns were projected into a PCA space to visualize any effect of PTX on classification of odors (Figure 3.24). Surprisingly, no changes were observed by blocking the $\mathrm{GABA}_{A}$ receptors, unlike previously found in the case of $\mathrm{MCH} / 3-O c t$. Therefore, this classification might not be mediated by GABA or more specifically by $\mathrm{GABA}_{\mathrm{A}}$ receptors. 


\section{Results}

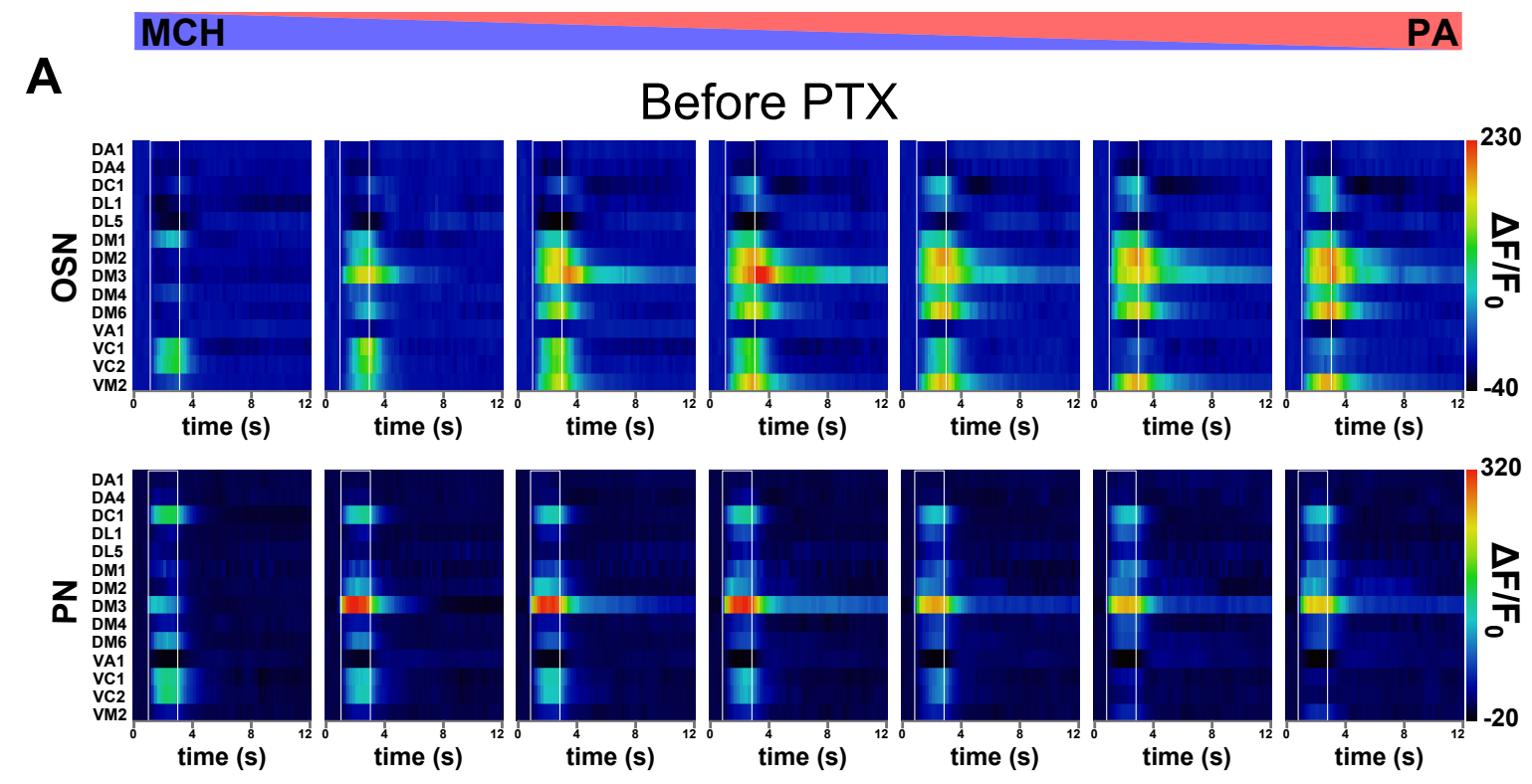

B

During PTX
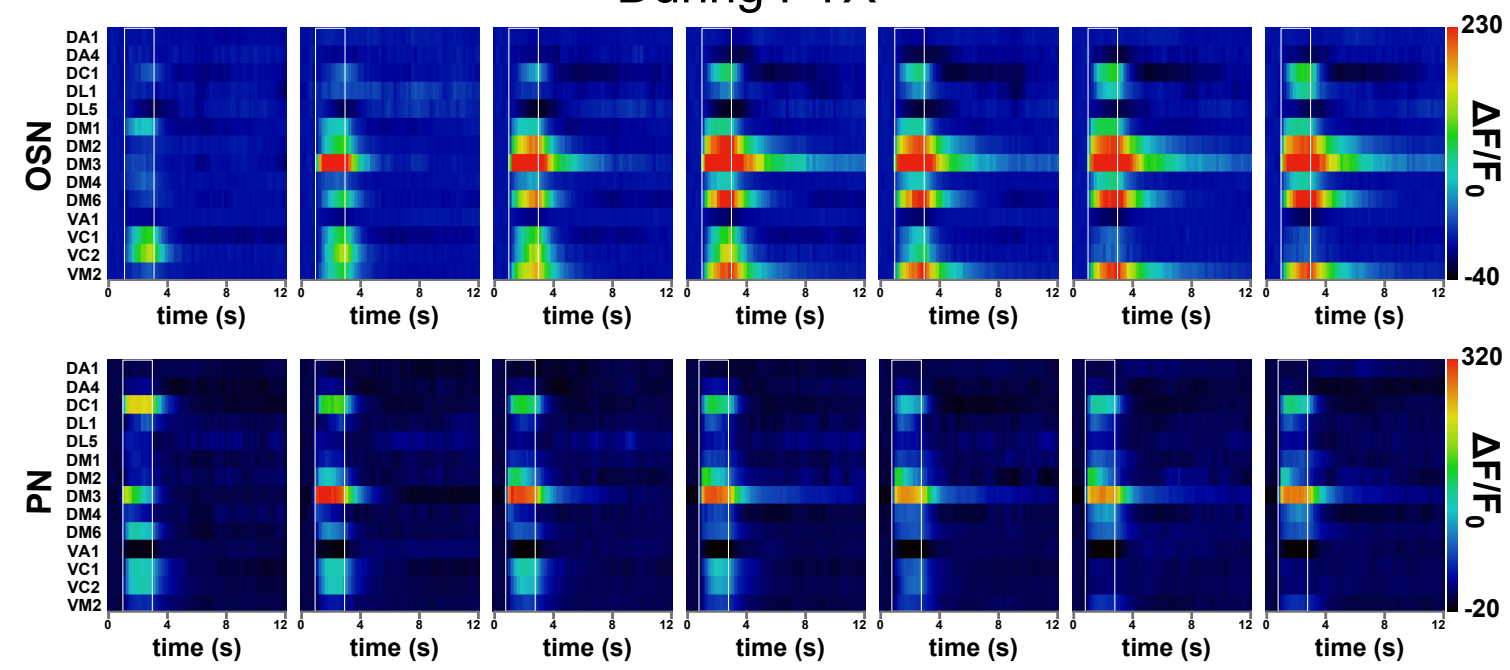

C

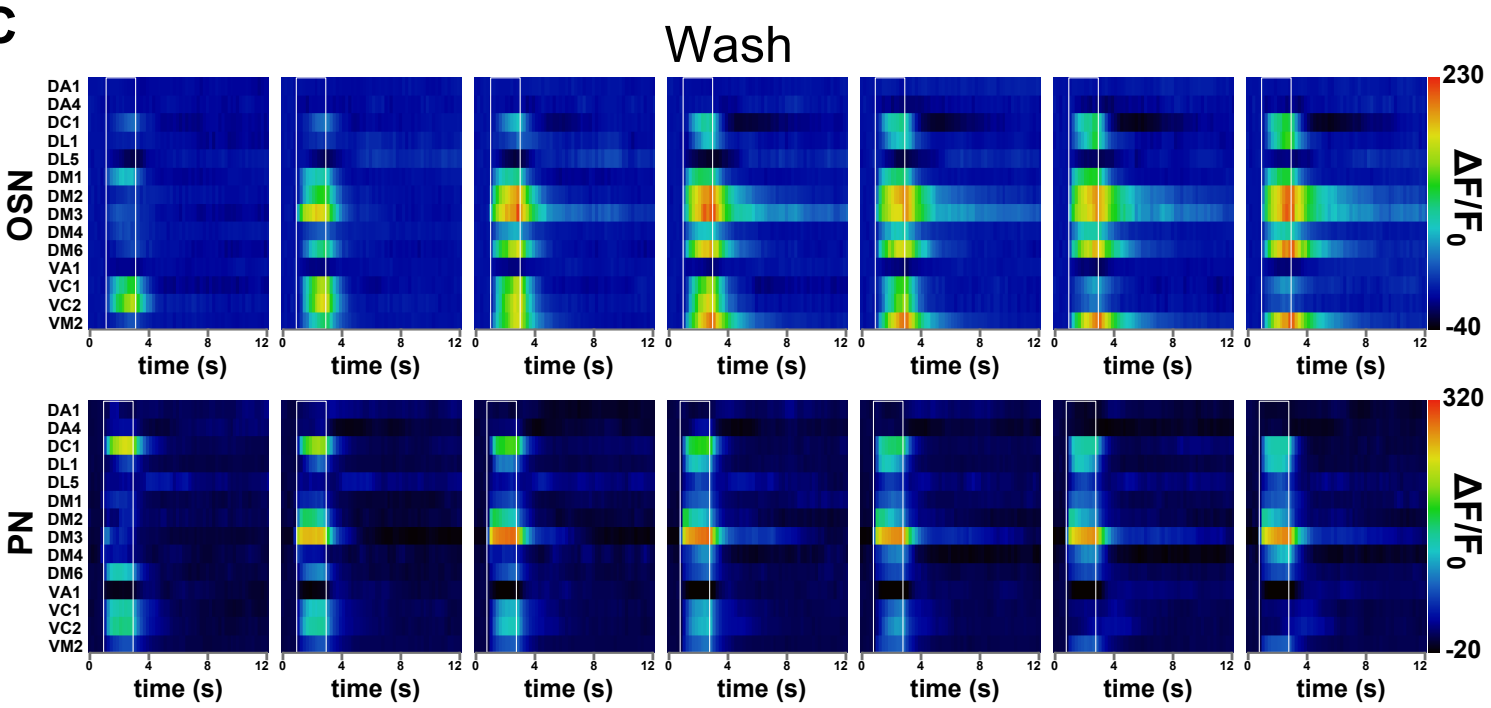




\section{Results}

Figure 3.23. Odor-evoked $\mathrm{Ca}^{2+}$ activity to different binary odor mixtures of $\mathrm{MCH} / \mathrm{PA}$ in OSNs and PNs during the three stages of PTX application.

Glomerular $\mathrm{Ca}^{2+}$ activity map of 14 glomeruli to different binary odor mixtures of $\mathrm{MCH} / \mathrm{PA}$ in three different stages of PTX application: Before PTX (A), during PTX (B) and wash (C). The $\mathrm{Ca}^{2+}$ activity map was determined by the $\Delta F / F_{0}$ values obtained for the 14 glomeruli. The white bar indicates the duration of the odor stimulus. High $\mathrm{Ca}^{2+}$ activity is shown in warm colors and low activity in cold colors. Every row in each panel represents the activity of single glomerulus labeled at the extreme left. $n=5-6$ flies.

\section{Odortopic properties}

On analyzing the odortopic differences by correlation coefficients, no differences were again observed between "before PTX" and "during PTX" (Figure 3.25). GABA $A_{A}$ receptors were not found to be involved in the classification of odors occurring due to the odor dominance of PA. This dominance was still retained on the application of PTX. This was further verified by determining the angular distances of the odor mixtures from $100 \% \mathrm{MCH}$ and $100 \% \mathrm{PA}$ (Figure 3.26). A huge difference was observed between $100 \% \mathrm{MCH}-0 \% \mathrm{PA}$ and $99 \% \mathrm{MCH}-1 \% \mathrm{PA}$. It was the same effect as observed before PTX application (Figure 3.17 B and 3.26 A). Therefore, the dominance of PA remained unaltered by $\mathrm{PTX}$, and it was concluded that $\mathrm{GABA}_{\mathrm{A}}$ receptors did not play any role in $\mathrm{MCH} / \mathrm{PA}$ odor combination. 


\section{Results}

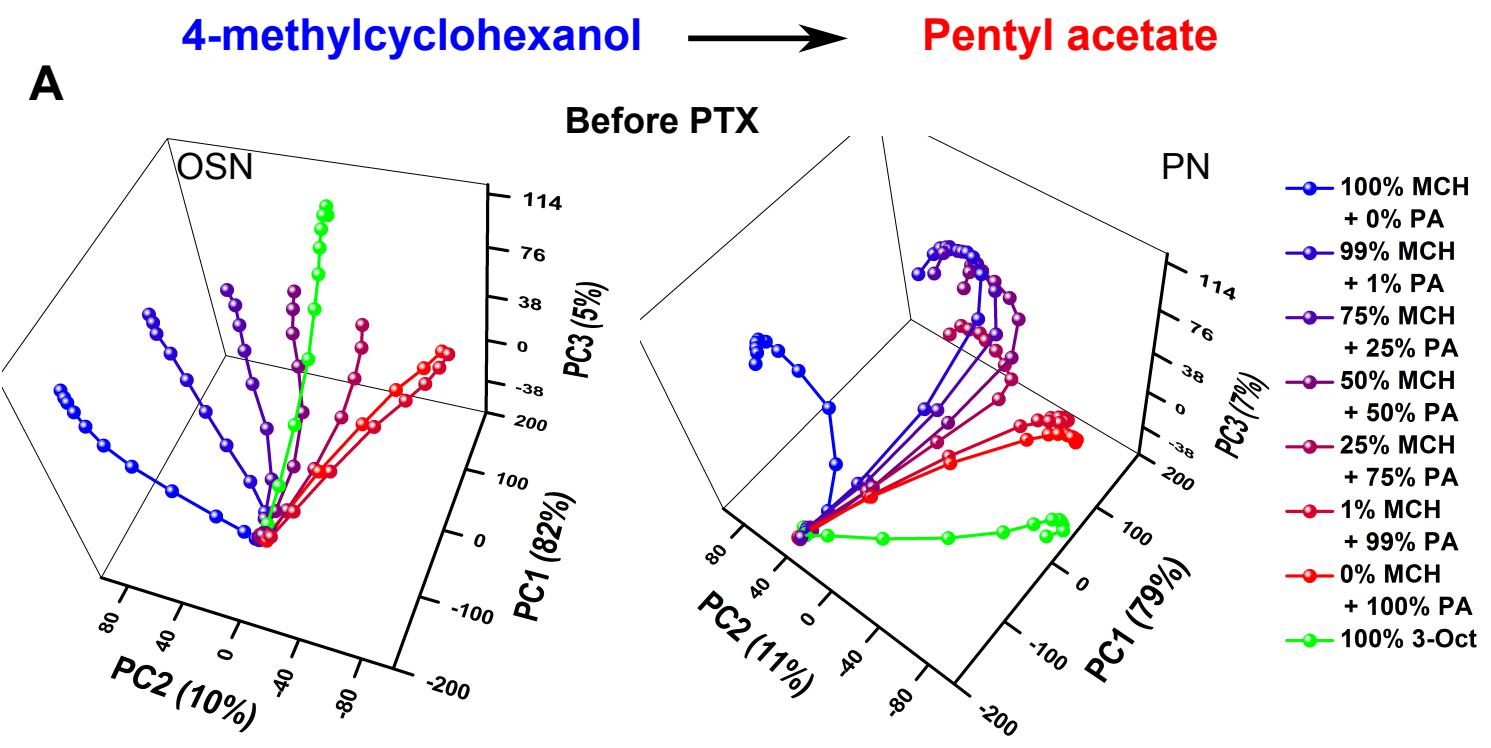

B

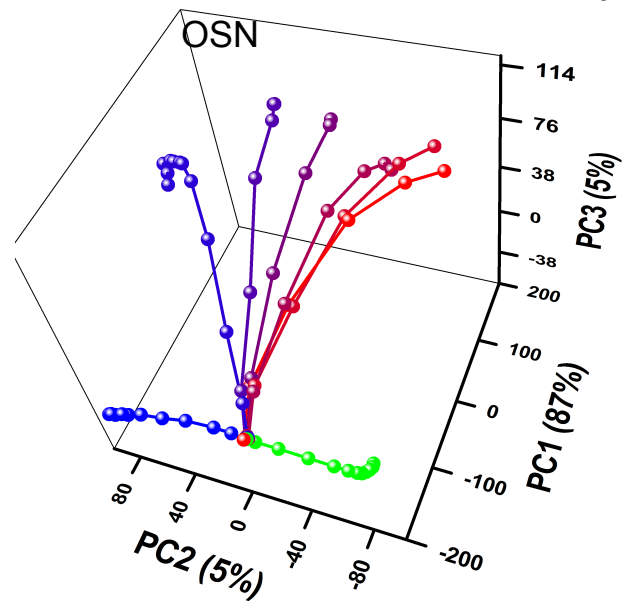

During PTX

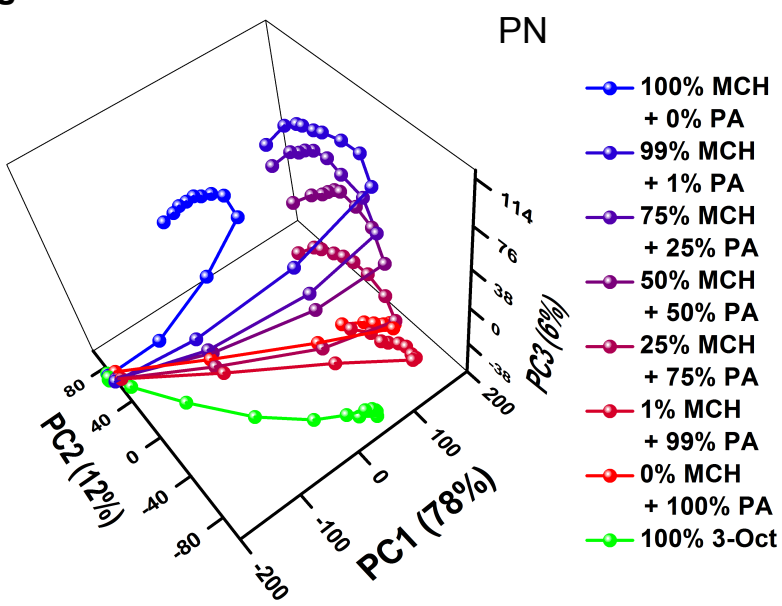

Wash
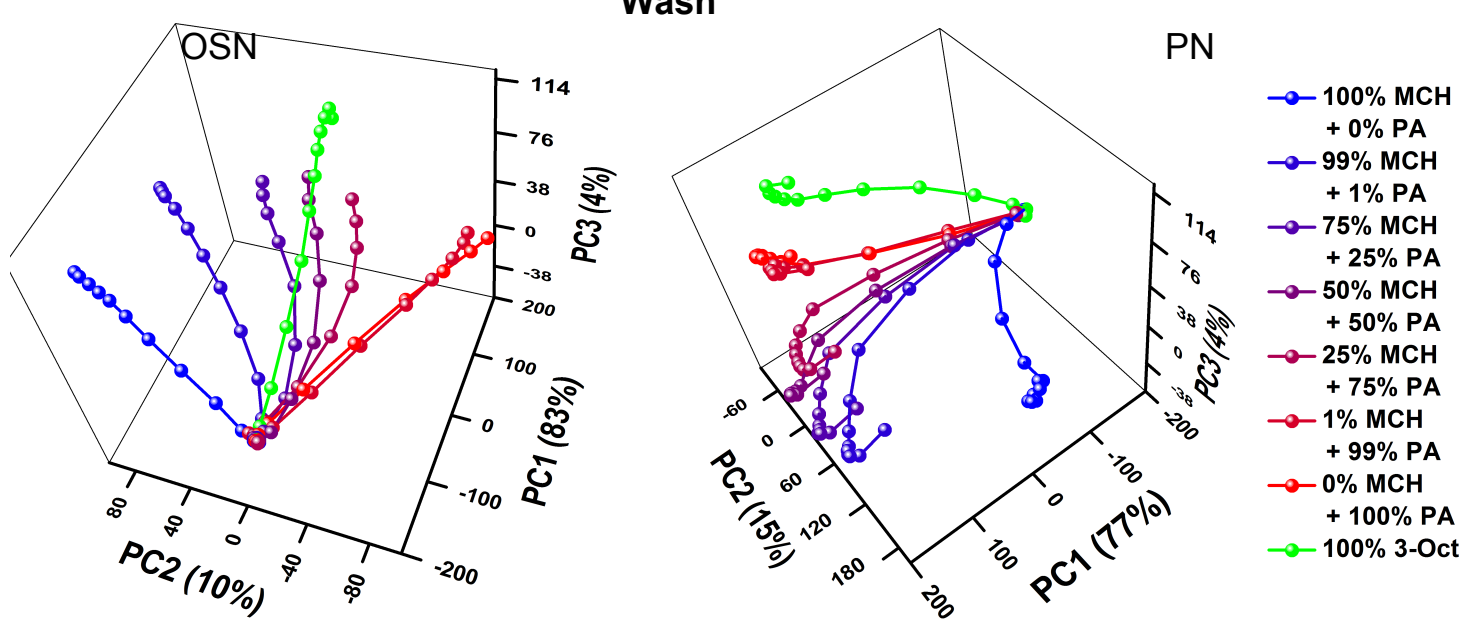


\section{Results}

Figure 3.24. Dynamics of odor-evoked $\mathrm{Ca}^{2+}$ activity patterns to $\mathrm{MCH} / \mathrm{PA}$ odor mixture in three different stages of PTX application.

Comparison of dynamics of $\mathrm{Ca}^{2+}$ activity patterns in OSNs and $\mathrm{PNs}$ in response to $\mathrm{MCH} / \mathrm{PA}$ binary mixtures during three stages: Before the application of PTX (A), during the application of PTX (B) and after the wash (C) in a principal component space spanned by the first three principal components. The trajectories represent the time period from 1 second before the odor onset until the odor offset. The values given in axis labels indicate the percentage of variance covered by that axis. No changes were observed on comparing the three stages of PTX application. 


\section{Results}

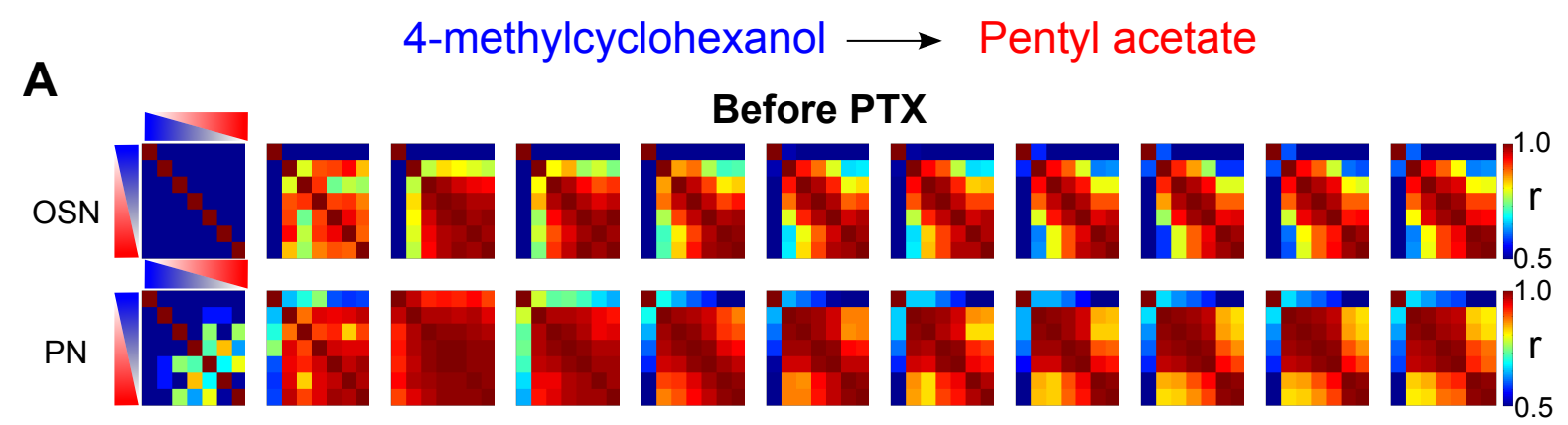

B
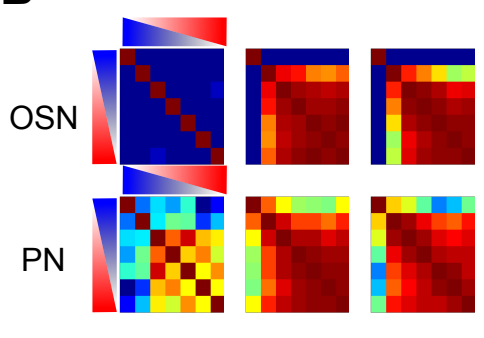

During PTX

C
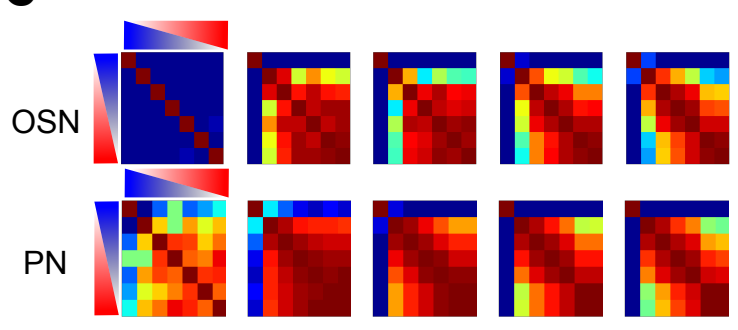

Wash
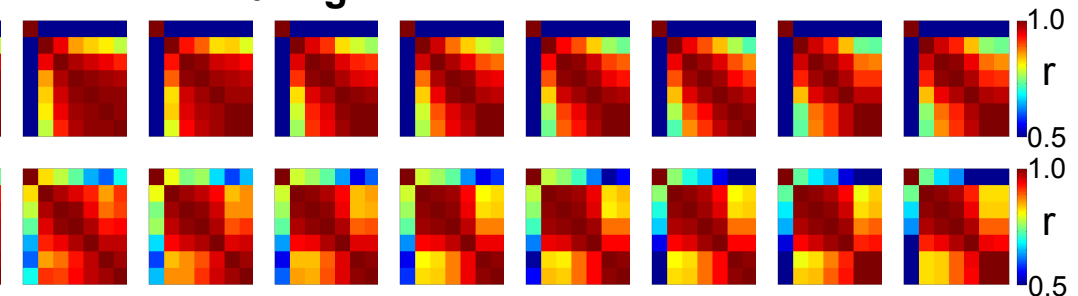

$-0.2 \quad 0.0$

odor stimulus (s)

2.0

Figure 3.25. Odortopic differences in odor-evoked $\mathrm{Ca}^{2+}$ activity patterns of different odor mixtures of MCH/PA at different stages of PTX application.

Matrices indicate the pairwise correlations between glomerular activity patterns evoked by different odor mixtures of MCH/PA at different stages of PTX application: Before PTX (A), during PTX (B) and wash (C). High correlations are represented in warm colors and low correlations in cold colors. The triangles with color gradient at the left and top of the first correlation matrix indicate that the concentration of one odor increases and that of the other odor decreases. No changes were observed on comparing the three stages of PTX application. $n=5$ flies. 


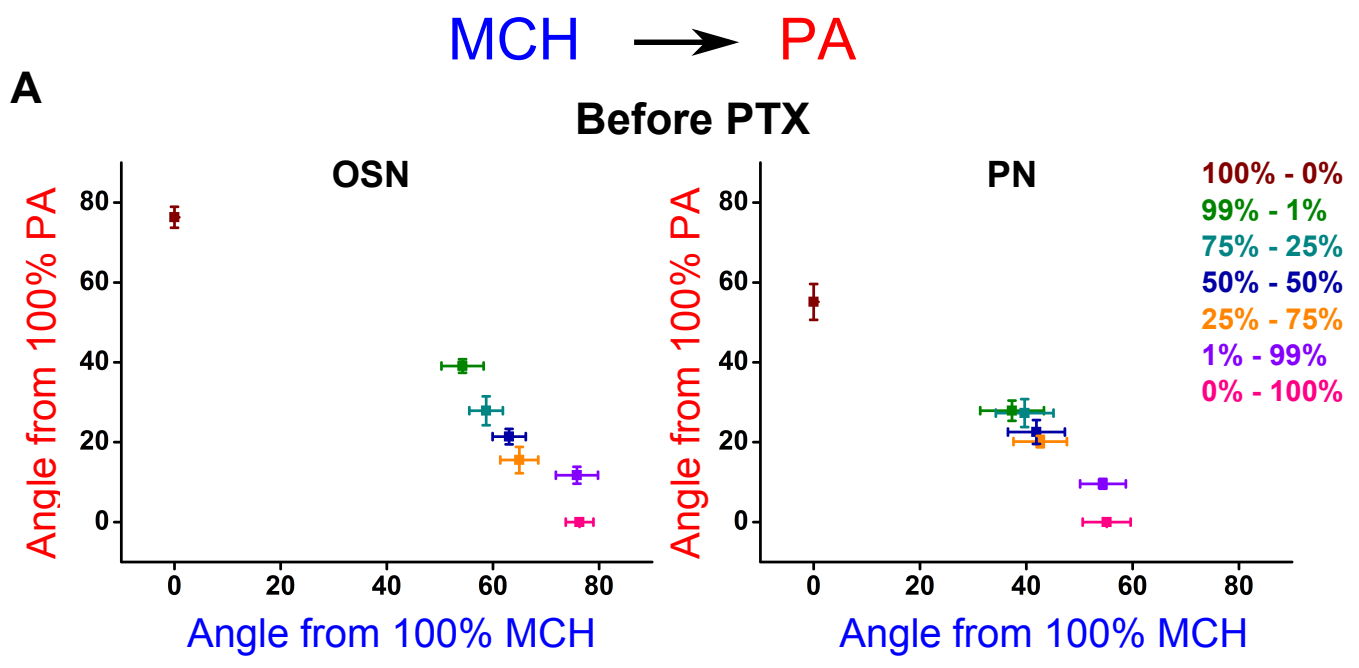

B
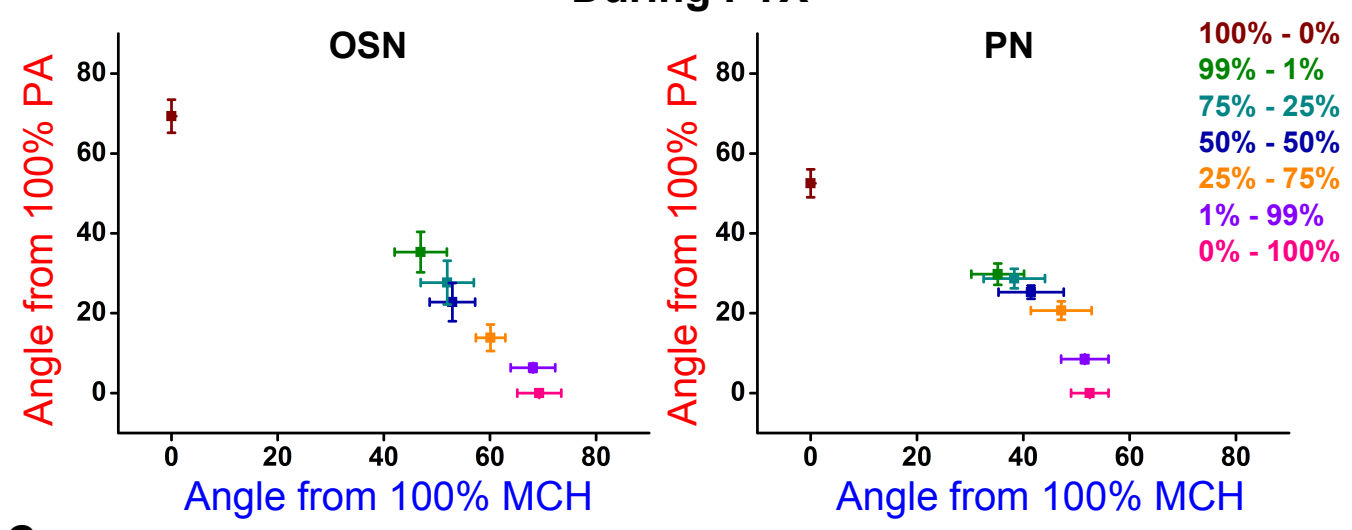

C

\section{Wash}
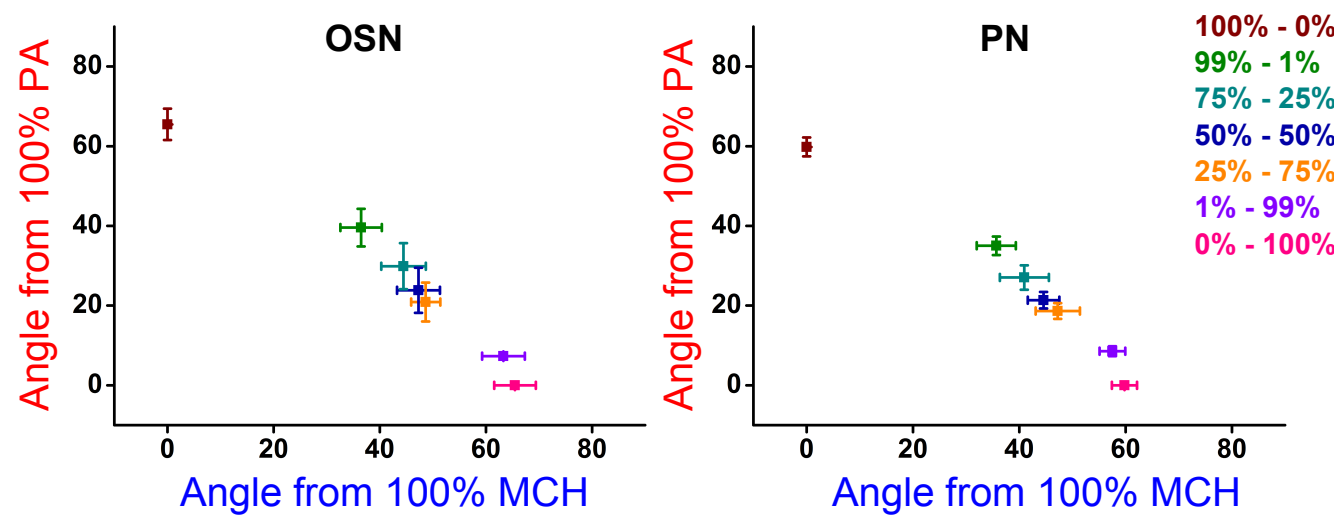

Figure 3.26. Odortopic differences in odor-evoked $\mathrm{Ca}^{2+}$ activity patterns of $\mathrm{MCH} / \mathrm{PA}$ odor mixtures at different stages of PTX application in OSNs and PNs at odor offset.

Angular distances between the glomerular activity patterns of $\mathrm{MCH} / \mathrm{PA}$ mixtures from $100 \% \mathrm{MCH}$ and $100 \% \mathrm{PA}$ (as reference) at odor offset. The axes indicate angles from the $100 \%$ concentrations of each individual component and the values are indicated in degrees. No changes were observed on comparing the three stages of PTX application. $\mathrm{n}=5$ flies. Error bars indicate \pm SEM. 


\section{Results}

\subsubsection{3-Oct/PA odor mixture}

Due to the high degree of similarity already present between 3-Oct and PA, the intermediate mixtures were already found to categorize in OSNs. However, it was interesting to know about the possible effects of blocking the $\mathrm{GABA}_{\mathrm{A}}$ receptors on an odor mixture which shows classification already in OSNs. Effects of PTX could be again observed by the increase in the $\mathrm{Ca}^{2+}$ activity of especially DM2, DM3 and DM6 glomeruli in OSNs (Figure 3.27 B). However, no effects of PTX could be found in both OSNs and PNs in both population coding and odortopic differences with regards to odor classification (Figure 3.28, 3.29 and 3.30). This implied that the classification already present in OSNs remained unaffected even after blocking the GABAergic inhibition via the $\mathrm{GABA}_{\mathrm{A}}$ receptors. 


\section{Results}

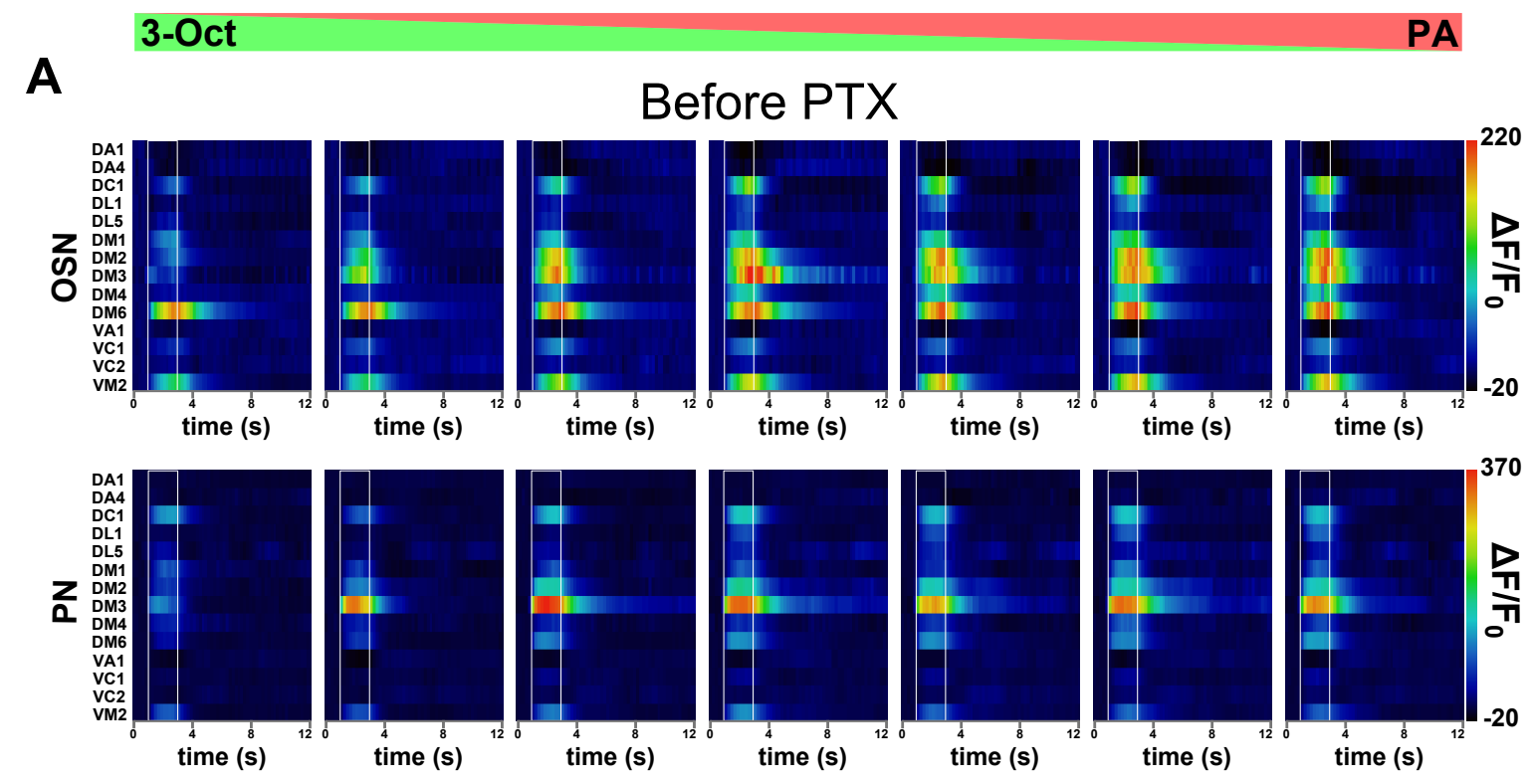

B
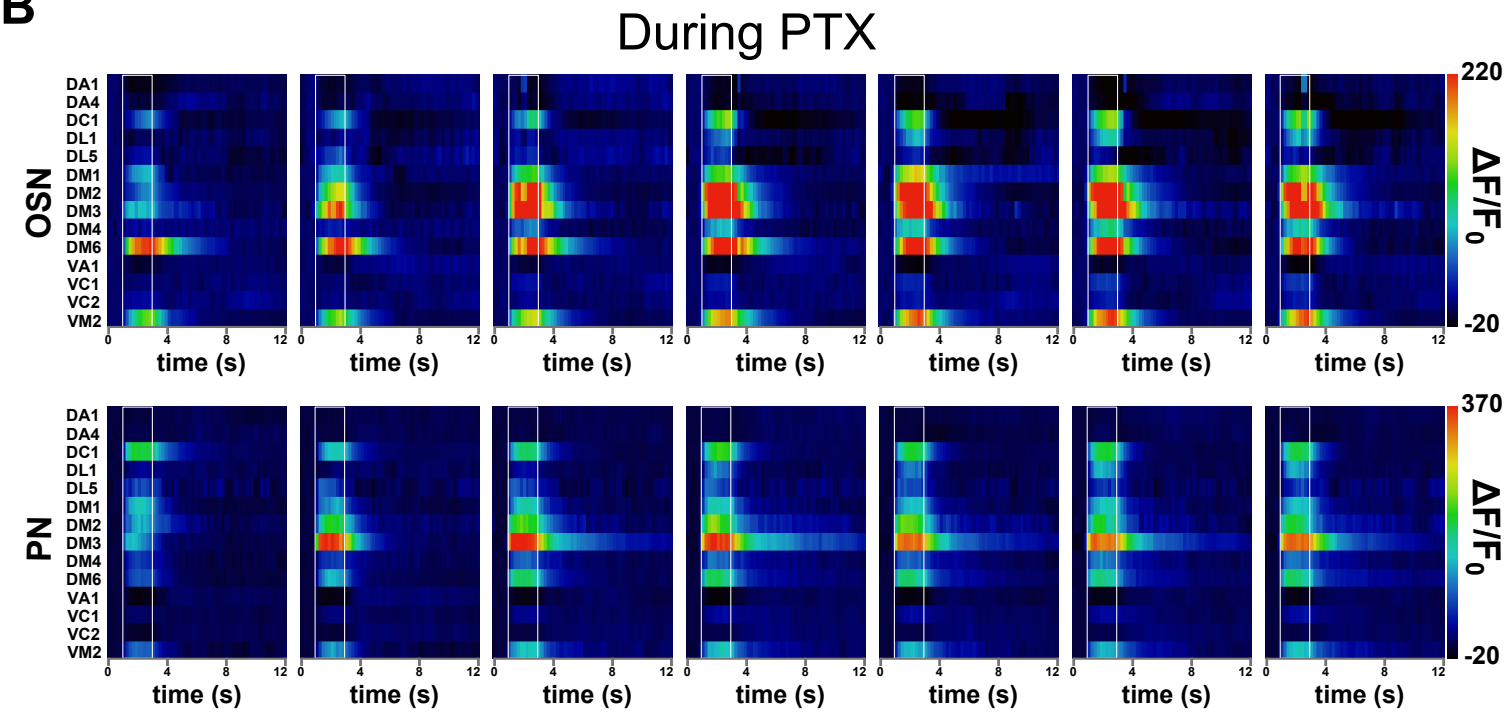

C

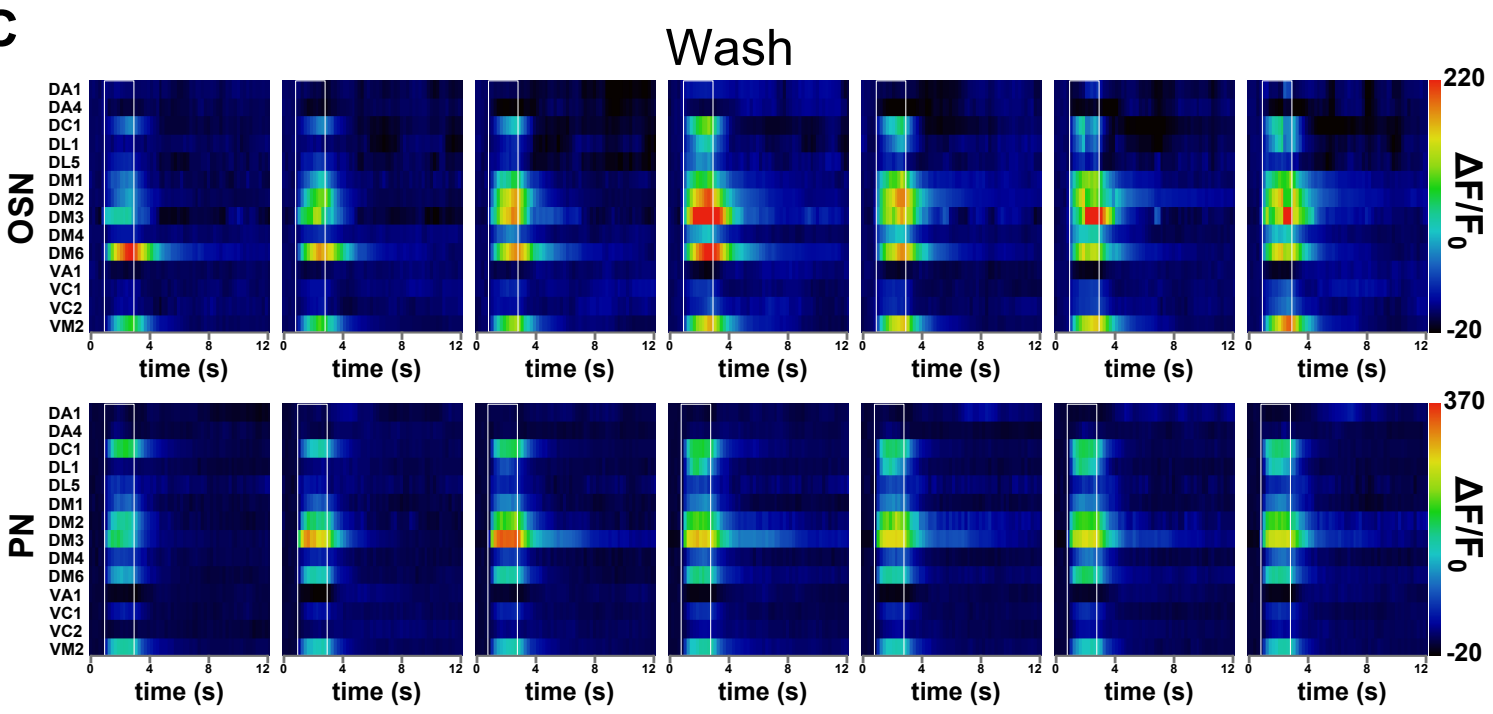




\section{Results}

Figure 3.27. Odor-evoked $\mathrm{Ca}^{2+}$ activity to different binary odor mixtures of 3Oct/PA in OSNs and PNs during the three stages of PTX application.

Glomerular $\mathrm{Ca}^{2+}$ activity map of 14 glomeruli to different binary odor mixtures of 3-Oct/PA in three different stages of PTX application: Before PTX (A), during PTX (B) and wash (C). The white bar indicates the duration of the odor stimulus. The activity map was determined by the $\Delta F / F_{0}$ values obtained for the 14 glomeruli. $\mathrm{High} \mathrm{Ca}^{2+}$ activity is shown in warm colors and low activity in cold colors. Every row in each panel represents the activity of single glomerulus labeled at the extreme left. $n=5$ flies. 


\section{Results}

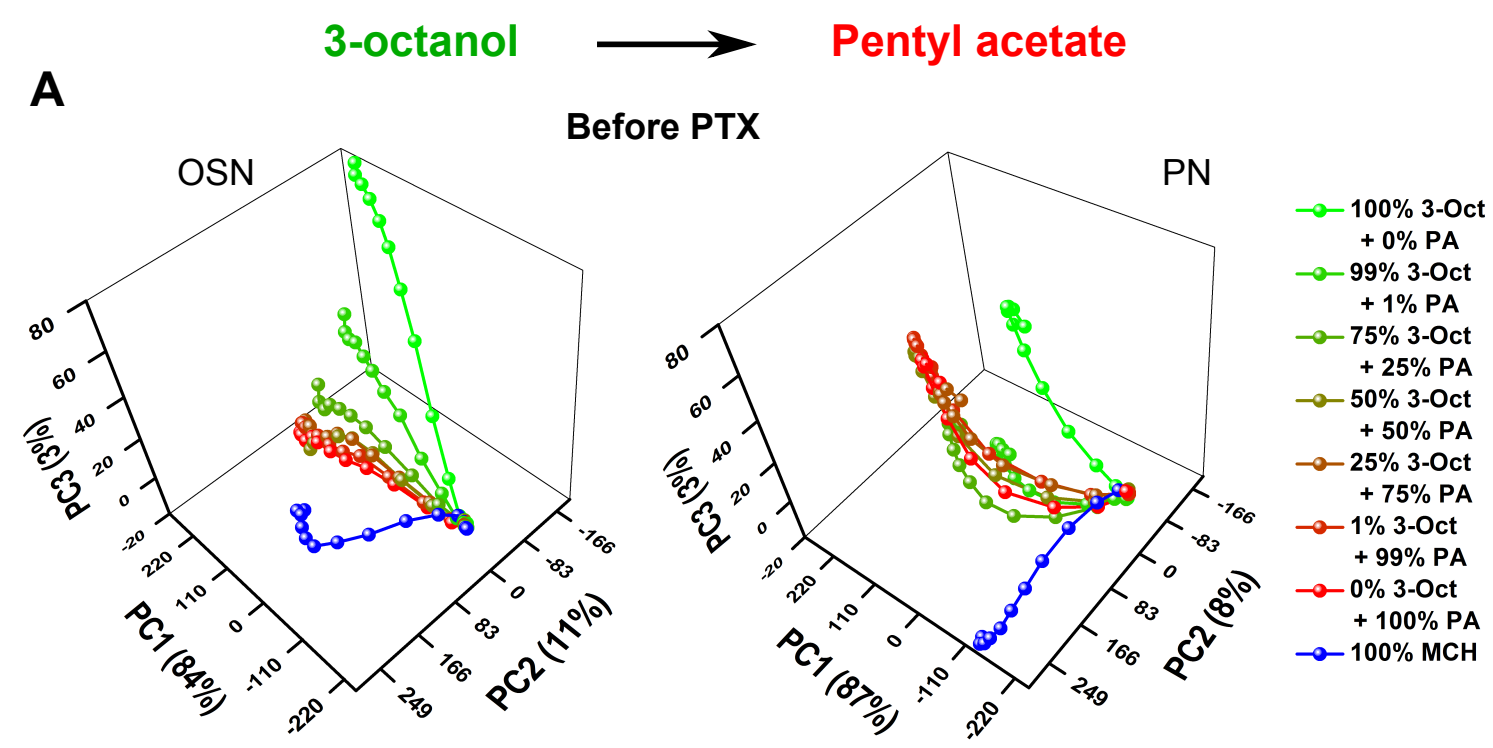

B

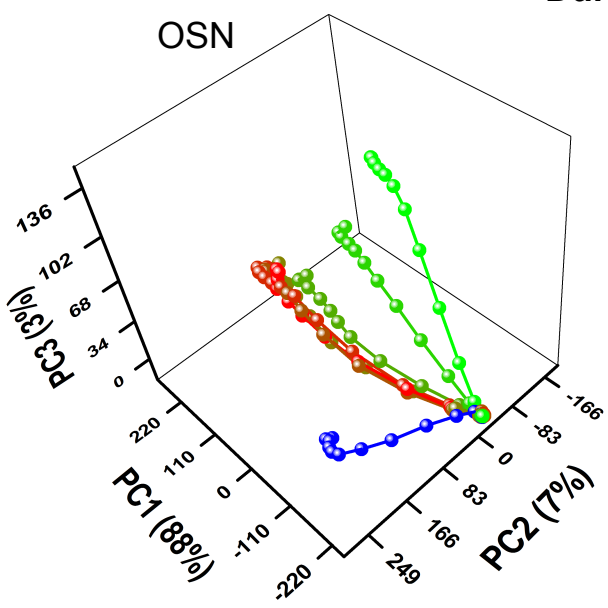

During PTX

C

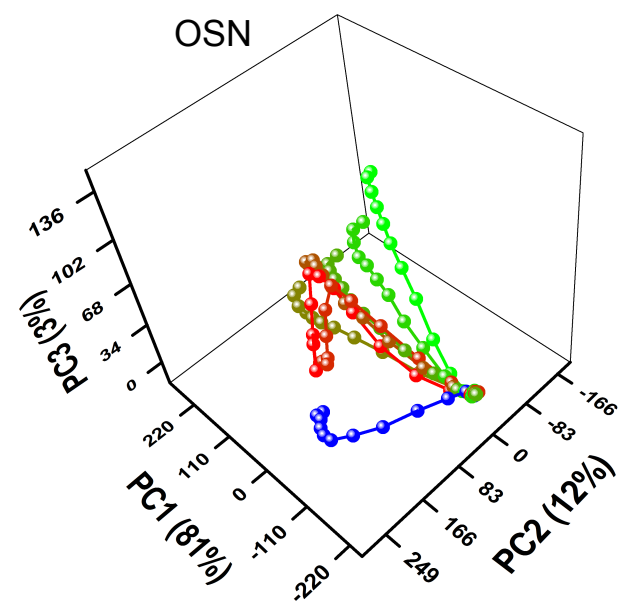

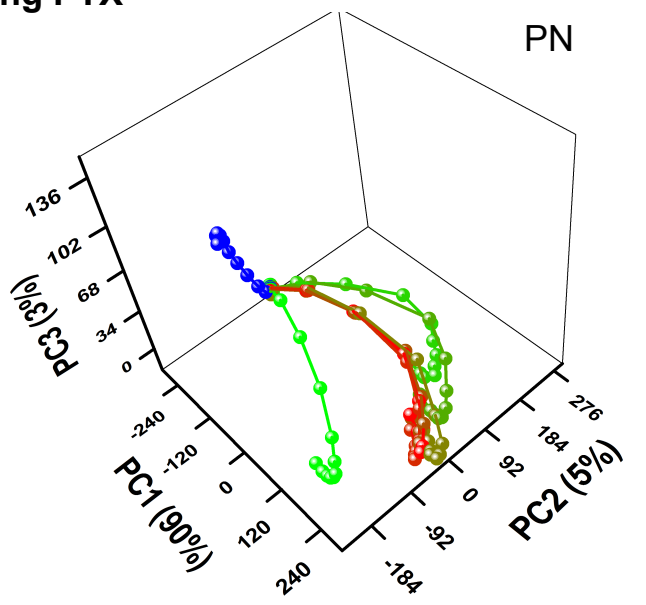

$-100 \% 3-$ Oct $+0 \% \mathrm{PA}$

$-0-99 \% 3-O c t$

$+1 \%$ PA

$-0-75 \%$ 3-Oct $+25 \%$ PA

$-0-50 \%$ 3-Oct $+50 \%$ PA

$-0-25 \% 3-O c t$ $+75 \%$ PA

$-0-1 \% 3-O c t$

$+99 \%$ PA

$-0-0 \% 3-0 c t$

$+100 \%$ PA

$-100 \% \mathrm{MCH}$

\section{Wash}

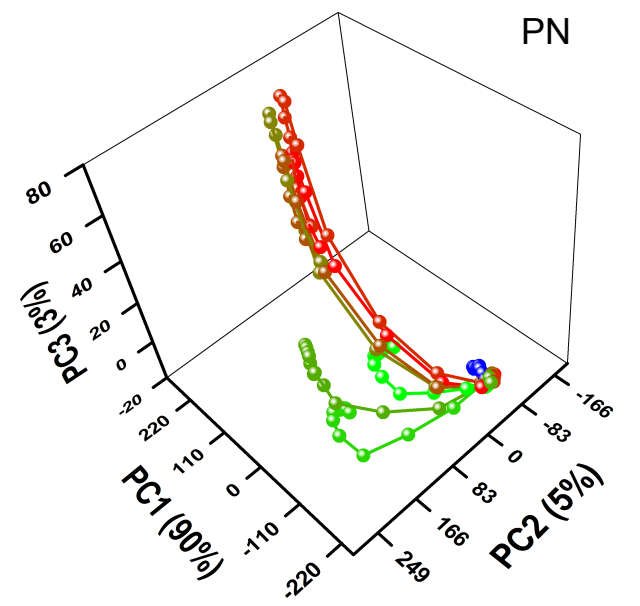

$-100 \% 3-$ Oct $+0 \% \mathrm{PA}$

$-99 \% 3-O c t$ $+1 \% \mathrm{PA}$

$-75 \% 3-O c t$ $+25 \%$ PA

$-50 \%$ 3-Oct $+50 \% \mathrm{PA}$

$-0-25 \% 3-O c t$ $+75 \%$ PA

$-1 \% 3-$ Oct $+99 \%$ PA

$-0 \% 3-0 c t$ $+100 \%$ PA $-100 \% \mathrm{MCH}$ 


\section{Results}

Figure 3.28. Dynamics of odor-evoked $\mathrm{Ca}^{2+}$ activity patterns to 3-Oct/PA odor mixture in three different stages of PTX application.

Comparison of the dynamics of $\mathrm{Ca}^{2+}$ activity patterns in OSNs and PNs in response to 3Oct/PA binary mixtures during three stages: Before the application of PTX (A), during the application of PTX (B) and after the wash (C) in a principal component space spanned by the first three principal components. The trajectories represent the time period from 1 second before the odor onset until the odor offset. The values given in axis labels indicate the percentage of variance covered by that axis. No changes were observed on comparing the three stages of PTX application. 
3. Results

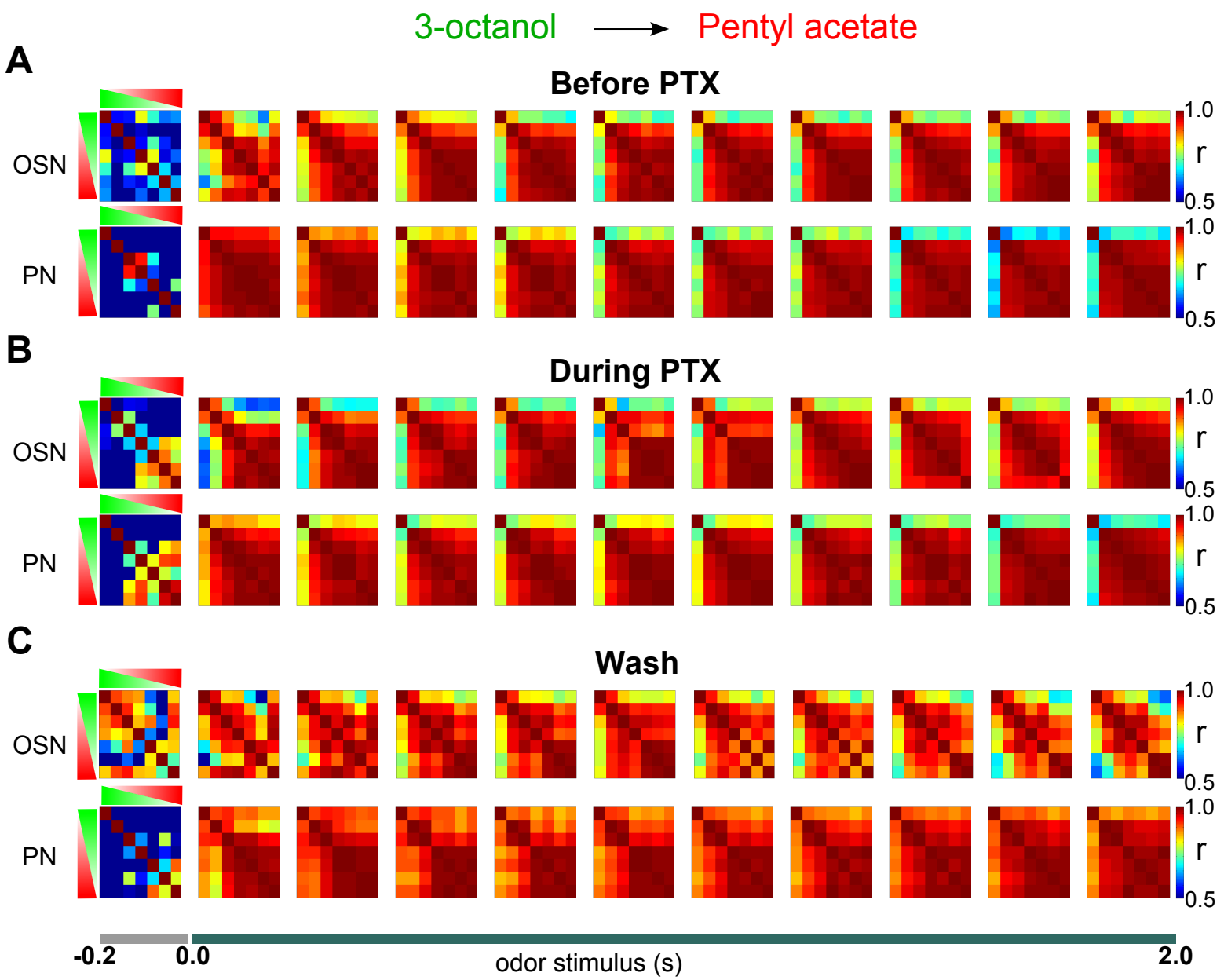

Figure 3.29. Odortopic differences in odor-evoked $\mathrm{Ca}^{2+}$ activity patterns of different odor mixtures of 3-Oct/PA at different stages of PTX application.

Matrices indicate pairwise Pearson's correlations ( $r$ ) between glomerular activity patterns evoked by different odor mixtures of 3-Oct/PA at different stages of PTX application: Before PTX (A), during PTX (B) and wash (C). High correlations are represented in warm colors and low correlations in cold colors. The triangles with color gradient at the left and top of the first correlation matrix indicate that the concentration of the one odor increases and the other odor decreases. No changes were observed on comparing the three stages of PTX application. $n=5$ flies. 


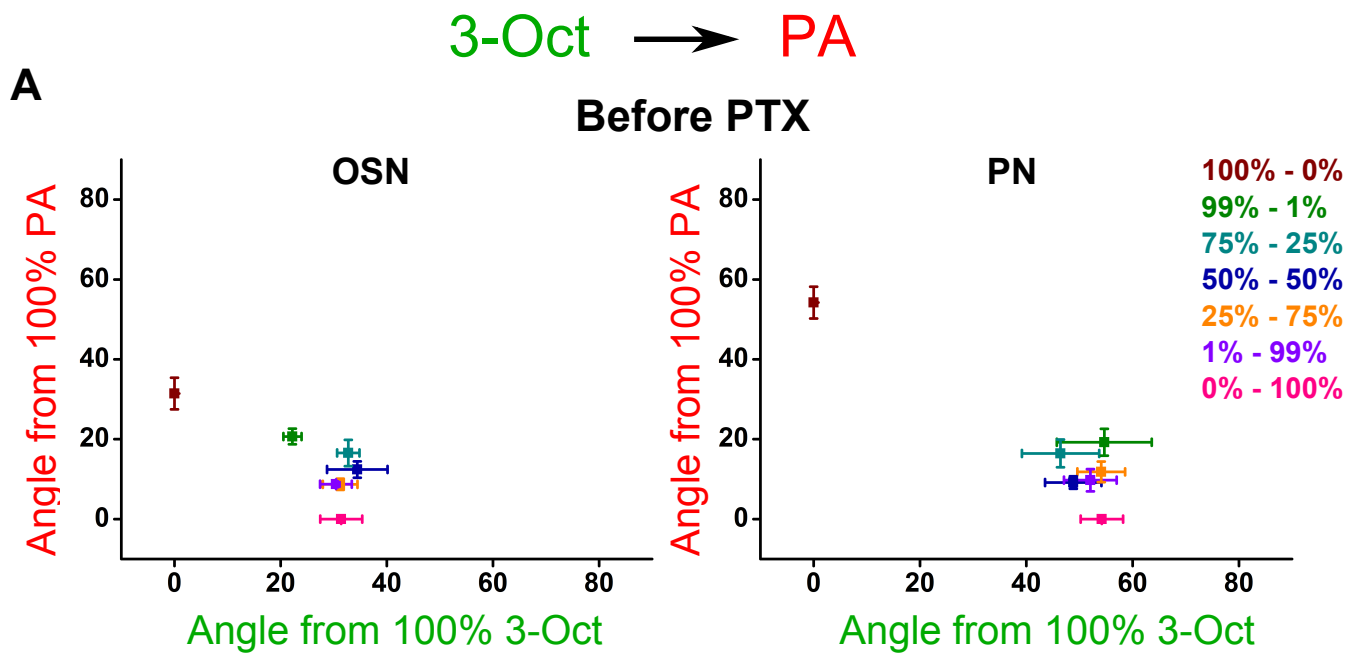

B

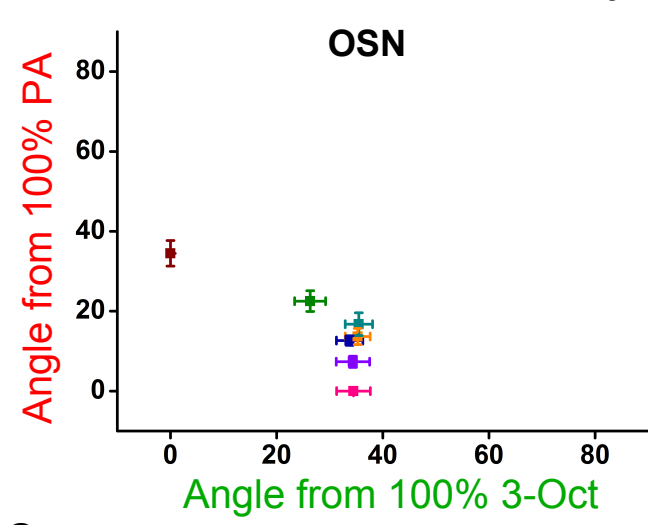

\section{During PTX}

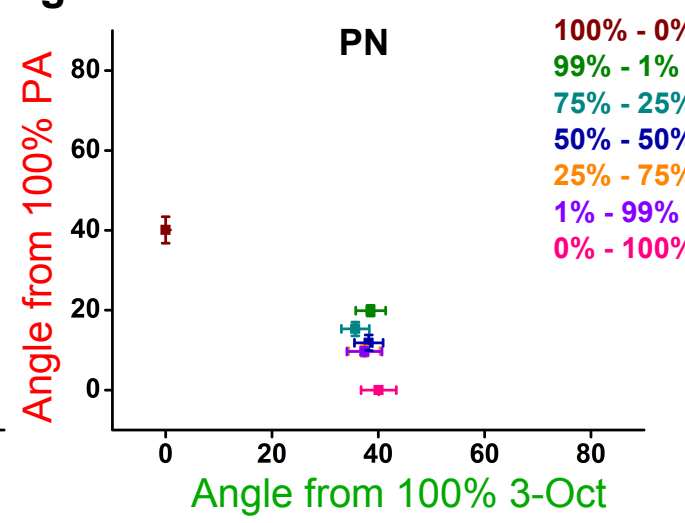

C

\section{Wash}
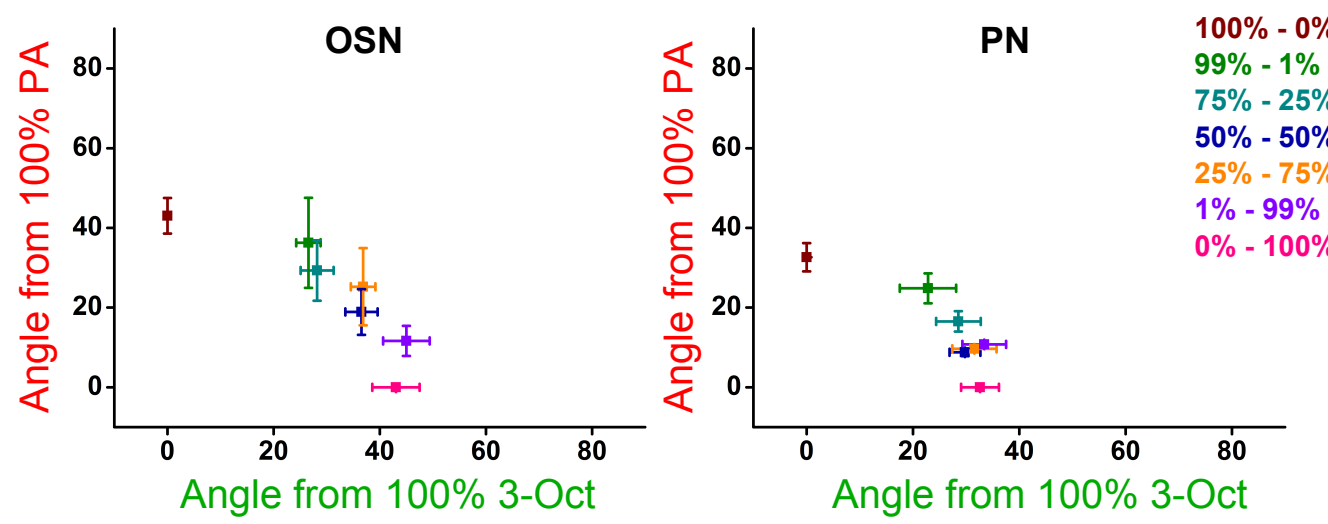

Figure 3.30. Odortopic differences in odor-evoked $\mathrm{Ca}^{2+}$ activity patterns of 3Oct/PA odor mixtures at different stages of PTX application in OSNs and PNs at odor offset.

Angular distances between the glomerular activity patterns of 3-Oct/PA mixtures from $100 \% 3-O c t$ and $100 \%$ PA (as reference) at odor offset. The axes indicate angles from the $100 \%$ concentrations of each individual component and the values are indicated in degrees. No changes were observed on comparing the three stages of PTX application. $\mathrm{n}=5$ flies. Error bars indicate \pm SEM. 


\section{Discussion}

\subsection{Neuronal coding of odor perception}

The present study showed that odor classification was observed in the AL of Drosophila. This was concluded on the basis of odor evoked $\mathrm{Ca}^{2+}$ activity patterns in the AL. But the first question was how olfactory perception is related to the measured glomerular activity patterns. This question had been previously addressed in honeybees (Apis mellifera) [Guerrieri et al., 2005] as well as in Drosophila [Niewalda et al., 2011]. The authors used several odors of various chemical structures and checked whether the perceived similarity correspond to the similarity in the neuronal activity patterns in the brain. The perceived similarity was determined by different behavioral tasks using appetitive or aversive odor conditioning. Niewalda et al. [2011] used three different paradigms to determine the perceptual similarity of odors in flies. They first conditioned the flies by pairing an odor with electric shocks and later quantified their avoidance behavior to both the trained odor and the similar odor separately. A "perceptual distance score" was then determined on comparing the behavior towards the trained and the similar odor. As a second step, they trained the flies similarly again, but tested the flies for their choice between the trained odor or similar odor. Lastly, they trained the flies by pairing one odor with shock and the novel odor explicitly not paired with shock and then tested with the choice between the trained or the novel odor. For each paradigm, "perceptual distance scores" were determined and all these scores were then combined to yield a final perceptual score, which was independent of the paradigms used. This perceptual score was found to correlate with the similarity in the glomerular activity patterns of those odors in the AL, especially in the PNs of Drosophila. Therefore taking into account of the results obtained from previous studies [Guerrieri et al., 2005; Niewalda et al., 2011], the odor classification observed with different binary mixtures in the current study should ideally reflect odor classification in terms of behavioral perception also. 


\subsection{How does $\mathrm{Ca}^{2+}$ imaging relate to membrane depolarization?}

The first and foremost requirement to understand the role of the AL network in mediating any kind of odor classification was to monitor and record the activity of several OSNs and PNs in the AL. This was achieved in this study using $\mathrm{Ca}^{2+}$ imaging. An alternative to $\mathrm{Ca}^{2+}$ imaging could have been electrophysiology. The use of electrophysiology is, however, constrained by the limited number of neurons that can be monitored at a given time. Since the AL of Drosophila consists of $\sim 1200$ OSNs and $\sim 150 \mathrm{PNs}$, it would not have been feasible to record the activity of such a huge number of neurons by electrophysiology for the present study. Optical $\mathrm{Ca}^{2+}$ imaging, on other hand provided a convenient tool to monitor the activity of neuronal network at one time [Tsien, 1988; Mao et al., 2001; Stosiek et al., 2003]. However, it allows only to measure a correlate of membrane depolarization in neurons in terms of $\mathrm{Ca}^{2+}$. It relies on the fact that the membrane depolarization in the neurons is associated with $\mathrm{Ca}^{2+}$ influx due to the activation of different types of voltage-gated $\mathrm{Ca}^{2+}$ channels expressed in the neurons [Tsien and Tsien, 1990; Berridge et al., 2000].

$\mathrm{Ca}^{2+}$ imaging heavily relies on the type of $\mathrm{Ca}^{2+}$ sensor being used to detect the neuronal activity. In recent years, many $\mathrm{Ca}^{2+}$ sensors have been developed with different properties [Chen et al., 2013]. However, a perfect $\mathrm{Ca}^{2+}$ sensor should have good response kinetics, high sensitivity as well as good signal to noise ratio. Moreover, the sensor should also be photostable and should have satisfactory baseline fluorescence. In the present study GCaMP3 was used to detect the neuronal activities of OSNs and PNs. It is photostable and with sufficient baseline fluorescence [Tian et al., 2009]. It is also capable to detect even single action potential in the neuron [Tian et al., 2009]. In terms of kinetics, there is around tens of milliseconds delay in responding to action potentials [Badura et al., 2014]. GCaMP3 is a cytosolic $\mathrm{Ca}^{2+}$ sensor and therefore, it not only responds to changes in $\mathrm{Ca}^{2+}$ concentration due to membrane depolarization but also to the $\mathrm{Ca}^{2+}$ released from internal stores like endoplasmic reticulum and mitochondria [Verkhratsky and Petersen, 1998]. The decay in the $\mathrm{Ca}^{2+}$ signal, in general, is limited by the rate at which $\mathrm{Ca}^{2+}$ is cleared and this is directly proportional to the surface to volume ratio [Badura et al., 2014]. Therefore, at the soma, it takes tenths of seconds or longer to decay, whereas in axonal boutons and dendritic spines, it takes 


\section{Discussion}

around 10-100 ms [Müller and Connor, 1991; Yasuda et al., 2004]. To convert the $\mathrm{Ca}^{2+}$ activity in terms of action potentials, deconvolution algorithms or other approaches to estimate the spike timings can be used [Vogelstein et al., 2009].

Another important parameter for the selection of $\mathrm{Ca}^{2+}$ sensors is their dissociation constant $\left(\mathrm{K}_{\mathrm{D}}\right)$. Depending on the range of $\mathrm{Ca}^{2+}$ concentration to be investigated, an appropriate sensor should be used. Generally, the $\mathrm{Ca}^{2+}$ concentration in the neurons during the resting phase varies from $10 \mathrm{nM}$ to $200 \mathrm{nM}$. But during activity, the concentration might reach 1 to $10 \mu \mathrm{M}$ or even higher [Gamble and Koch, 1987; Regehr and Tank, 1992]. A high affinity $\mathrm{Ca}^{2+}$ sensor with its $\mathrm{K}_{\mathrm{D}}$ near the resting $\mathrm{Ca}^{2+}$ concentration will allow to detect small $\mathrm{Ca}^{2+}$ transients with high signal to noise ratio, but will saturate at high $\mathrm{Ca}^{2+}$ concentrations. So, a perfect $\mathrm{Ca}^{2+}$ sensor should span the whole range of $\mathrm{Ca}^{2+}$ concentrations to be investigated. GCaMP3 has $\mathrm{K}_{\mathrm{D}}=660 \mathrm{nM}$, which is within the $\mathrm{Ca}^{2+}$ concentration range of neurons, however any saturation of GCaMP3 during the activity of OSNs and PNs in different glomeruli cannot be excluded. Therefore, whether the limitations of GCaMP3 contributes to the odor classification in PNs cannot be commented. The exact change in $\mathrm{Ca}^{2+}$ concentration in both OSNs and PNs during their activity is not known. However, the odor classification of $\mathrm{MCH} / 3-\mathrm{Oct}$ binary mixtures was no more observed after the addition of $\mathrm{GABA}_{\mathrm{A}}$ receptor antagonist (picrotoxin). So, had the limitations of GCaMP3 be the sole factor in mediating the odor classification, then such an effect of picrotoxin would not have been observed.

\subsection{Transformation of odor responses from OSNs to PNs}

Several studies have shown that the OSNs and their corresponding PNs both respond to a similar set of odor stimuli [Bhandawat et al., 2007; $\mathrm{Ng}$ et al., 2002; Schlief and Wilson, 2007; Silbering et al., 2008; Wang et al., 2003; Wilson et al., 2004]. This indicated that the major source of activation of PNs come from their respective OSNs. However, the odor responses in OSNs and PNs are not exactly the same. The odor responses in PNs are less variable in terms of spike counts in comparison to OSNs [Bhandawat et al., 2007]. In addition, PNs mainly respond to the rising phase of the OSNs response rather than the peak response of the OSNs [Bhandawat et al., 2007], which also makes their responses more transient than OSNs. PNs are also less se- 


\section{Discussion}

lective to odors compared to OSNs, thereby displaying a broader odor tuning profile than OSNs [Bhandawat et al., 2007; Wilson et al., 2004; Olsen and Wilson, 2008]. Moreover, there is a non-linear transformation function between OSNs and PNs for each glomerulus, which implies that PNs are more sensitive to weak OSN inputs and less sensitive to strong OSN inputs [Bhandawat et al., 2007; Olsen et al., 2010]. Bhandawat et al. [2007] also showed that the odor responses become more distinct and discriminable in PNs relative to OSNs.

The question then arises whether the observed odor classification of binary mixtures in PNs is mediated by such transformation of odor responses from OSNs to PNs. The above mentioned studies, however, do not explicitly exclude the involvement of any inhibition in mediating such transformations [Bhandawat et al., 2007; Wilson et al., 2004]. Nevertheless, some of these transformation could be explained by the property of OSN-PN synapses only. Each PN forms synapses with all the OSNs expressing one kind of olfactory receptor [Kazama and Wilson, 2009]. Since there are $\sim 1200$ OSNs and only $\sim 150$ PNs, there is a huge convergence of OSN inputs onto each PN. Such convergence explains the high sensitivity of PNs to weak presynaptic inputs of OSNs [Wilson, 2013]. Moreover since each PN combines the multiple inputs from many OSNs, the variability in the odor responses get reduced in PNs [Abbott, 2008; Wilson, 2013]. In addition, each OSN-PN synapse has high probability of vesicle release [Kazama and Wilson, 2008; Wilson, 2013] and therefore even a single spike in OSN releases a lot of vesicles onto the PN, which further contributes to decreasing the variability in PNs. The high probability of vesicle release also partly explains the transient nature of the PN responses because due to the high vesicular release probability, vesicles would be quickly depleted and short-term depression would be observed [Kazama and Wilson, 2008; Wilson, 2013]. The short-term depression at OSN-PN synapses not only explains the transient nature of PNs but also the non-linear relationship between the OSN-PN responses [Abbott et al., 1997; Wilson, 2013]. Moreover, it also partly explains the broad odor tuning of PNs compared to OSNs [Wilson, 2013]. In the present study, any contribution of such property of OSNPN synapse to the non-linear transformation of odor representations of binary mixtures from OSNs to PNs cannot be excluded.

In terms of population coding of odors, it is not clearly known how short-term depression might play a role in encoding the odors. But it is known that on silencing 


\section{Discussion}

the input to all other glomeruli, disinhibition in PNs is observed [Asahina et al., 2009; Olsen and Wilson, 2008], which clearly indicates a role of lateral inhibition in population coding. Moreover, Silbering and Galizia [2007] showed that on presenting binary odor mixtures to the fly, the responses of OSNs to the mixture could be predicted from the responses of its odor components. But in PNs, on mixing one odor to the second odor, an interglomerular inhibition was observed, which could be eliminated using a $\mathrm{GABA}_{A}$ receptor antagonist. This experiment clearly showed that lateral inhibition via GABA can modify the population coding of different odors in PNs. A similar effect of GABA was also observed in the present study, where GABA was shown to be involved in mediating an odor classification in PNs by influencing the population coding of the odors.

\subsection{Temporal coding of odor mixtures}

The odor mixtures are often perceived different than their individual components which are difficult to identify [Laing and Francis, 1989]. This configural processing of mixtures is believed to be done by the antennal lobe network in insects [Broome et al., 2006; Deisig et al., 2006; Silbering and Galizia, 2007] or by the olfactory bulb network in the vertebrates [Tabor et al., 2004; Johnson et al., 2010]. However, some studies have also shown the involvement of only OSNs in integrating the olfactory information of individual components of a binary mixture [Su et al., 2011; Duchamp-Viret et al., 2003; Rospars et al., 2008]. Su et al. [2011] showed that even a single OSN can encode a binary odor mixtures in their temporal dynamics and response magnitudes. Moreover, the components of the mixture themselves vary in their physiochemical properties (e.g., vapor pressure), which also affects the odor stimulus in terms of their relative concentration at a given time [Martelli et al., 2013]. OSNs are also capable to encode such properties of the binary mixture in their temporal dynamics [Su et al., 2011]. The temporal coding by OSNs also helps the flies in behavioral odor discrimination. This was shown by DasGupta and Waddell [2008], who used flies expressing only one kind of OR and tested those flies for different odors activating that particular OR. Those flies could successfully learn to discriminate such odors within a certain range of concentrations, implying the role of temporal dynamics in encoding the odor identity. Another study on locusts has shown that each OSN responds differently to different odors in terms of the temporal structure of the firing patterns and such diversity across OSNs 


\section{Discussion}

is required for encoding the spatiotemporal information of the odor in the AL [Raman et al., 2010].

However, whether the temporal coding of OSNs contributes to odor discrimination or categorization is not clearly understood. In any case, these effects could not be detected in our study because of the limitation of temporal resolution with optical imaging. The neuronal activities were recorded at the rate of $5 \mathrm{~Hz}$ compared to the requisite 20 $\mathrm{Hz}$ to observe such effects. However, differences in the temporal dynamics of individual glomeruli could still be observed with this temporal resolution (Figure 3.3), but whether the stimulus dynamics could be decoded from these glomerular dynamics of $5 \mathrm{~Hz}$ resolution is hard to conclude. In spite of this low temporal resolution, PNs convincingly showed categorization in this study although it cannot be claimed that OSNs do not contribute at all to this categorization. The fact that the classification of odors become more distinct only in PNs, and not in OSNs, could also be reasoned by the nonlinear amplification of the OSN inputs at the OSN-PN synapse [Olsen et al., 2007; Bhandawat et al., 2007], thereby leading to a greater effect in PNs.

\subsection{GABAergic inhibition and other possible mechanisms for classification of odors}

Several studies have shown that inhibition is important for odor processing in the AL, starting from contrast enhancement of odor representations [Deisig et al., 2010; Sachse and Galizia, 2002], gain control [Olsen and Wilson, 2008; Root et al., 2008; Olsen et al., 2010] to synchronization of neuronal activity [Stopfer et al., 1997]. But whether inhibition can mediate the formation of different categories of odor percepts, was not known. The present study shows that GABAergic inhibition plays a role in categorical perception. This has been shown in case of $\mathrm{MCH} / 3-O c t$ odor mixtures which shows categorization of some intermediate mixtures in PNs. On adding picrotoxin to block the $\mathrm{GABA}_{\mathrm{A}}$ receptors, the categorization is lost in some cases in PNs and instead the distribution of mixtures in PNs become similar to that of the OSNs.

The glomeruli are innervated by a population of GABAergic local interneurons. Most of these LNs innervate every glomerulus [Chou et al., 2010; Seki et al., 2010] and recently it has been found that even an activation of single glomerulus recruits LN ac- 


\section{Discussion}

tivity in all the glomeruli [Hong and Wilson, 2015]. However each glomerulus has its unique and stereotypic sensitivity to GABA [Hong and Wilson, 2015]. Therefore, each glomerulus shows different levels of inhibition. These differences in inhibition across glomeruli can either lead to an increase or a decrease in distances between two odorevoked glomerular activity patterns. This can be explained by the fact that any two glomerular activity patterns, in general, has some glomeruli showing similar activities, while others show dissimilar activities. If the "dissimilar glomeruli" are made similar in terms of activity by inhibition, the two activity patterns will become more similar. Similarly, if the similar glomeruli are made dissimilar by inhibition, the activity patterns become more distinct. Therefore, as a consequence of such differential inhibition across glomeruli, some odors become more similar in their glomerular activity patterns while some more distinct, thereby leading to categorization.

Other than inhibition, in a similar way excitation can also serve as another method of categorization. The $A L$ is also innervated by a population of excitatory local interneurons (eLNs) [Huang et al., 2010; Yaksi and Wilson, 2010]. These eLNs form electrical synapses onto both GABAergic LNs as well as PNs. Therefore, the net effect on PNs due to the eLN network can be both inhibition or activation depending on whether eLNs excite GABAergic LNs or PNs respectively. Since eLNs form electrical synapses via gap junctions, the lateral excitation by eLNs is insensitive to GABA. This could be one of the mechanism which might explain the odor dominance of PA observed in the odor representations of $\mathrm{MCH} / \mathrm{PA}$ mixtures in PNs but not in OSNs.

Recently another population of $L N$ s have been found in the AL which release glutamate as an inhibitory neurotransmitter [Liu and Wilson, 2013]. Similar to eLNs, they can also mediate inhibition or excitation in PNs but in this case, either by inhibiting PNs directly, or by inhibiting GABAergic LNs. Glutamatergic LNs mediate such inhibition via glutamate-gated chloride channels [Liu and Wilson, 2013]. These glutamate-gated chloride channels are also sensitive to picrotoxin [Cleland, 1996] and could therefore be equally contributing as GABA to the categorization of $\mathrm{MCH} / 3-O c t$ odor mixtures in PNs. However, it is still not clear how both GABA and glutamate simultaneously contribute to odor coding in the AL. 


\section{Discussion}

\subsection{Future perspectives}

In the present study, I showed that GABA was involved via $\mathrm{GABA}_{A}$ receptors in mediating odor classification in the AL. This was achieved by using picrotoxin as a $G A B A_{A}$ receptor antagonist. However, using picrotoxin has some drawbacks. The most important one is that it affects all the neurons expressing $\mathrm{GABA}_{A}$ receptors as well as recently found glutamate-gated chloride channels in the Drosophila brain. Therefore, as future experiments, genetic tools should be used to specifically downregulate such receptors or channels in local interneurons. One possible way to achieve that is by transgenic RNA interference (RNAi)-inducing lines which can block the expression of those receptors or channels in specific neurons.

Moreover, it will also be interesting to know whether the odor classification is achieved by a combinatorial effect of all the glomeruli or by just few glomeruli. This could be determined by estimating the contribution of each glomerulus to odor classification. In addition, how different concentrations of individual odors get influenced by GABA is also worth investigating. The reason is that binary mixtures consist of different concentrations of two individual odors and therefore any sort of odor classification observed with binary mixture can potentially be also influenced by its components.

Last but not the least, the odor classification inferred from the classification of odor evoked $\mathrm{Ca}^{2+}$ activity patterns in the AL, should also be verified in terms of behavioral perception. This can be accomplished by odor conditioning paradigm using different odors as described in Niewalda et al. [2011]. 


\section{Summary}

Drosophila senses diverse kinds of olfactory stimuli in the external environment. These stimuli are often not pure chemicals, but rather mixtures of many odorants. The fly olfactory system, however, needs to extract the requisite information from this complex stimulus. This is done by converting odor representations into meaningful, discrete categories in the perceptual space. The first olfactory relay system in the Drosophila brain is the AL which consists of OSNs, PNs and LNs, and these three populations of neurons interact and process the odor stimuli. To observe any transformations of odor representations in terms of odor classification, linearly varying odor stimuli were presented. This was achieved using binary mixtures such that one odor was "morphed" into another. Three binary mixtures were made from combinations of three distinct odorants ( $\mathrm{MCH}, 3-\mathrm{Oct}$ and $\mathrm{PA})$. In the first step, I determined and confirmed that each single odor-evoked distinct odor representations across its different concentrations in the AL, especially in the OSNs. A second requirement was to determine the degree of similarity between the three odors in both OSNs and PNs. From their odor representations, it was found that PA was more similar to 3-Oct compared to $\mathrm{MCH}$ in OSNs. Moreover, the similarity between PA and 3-Oct became even stronger in PNs.

After determining the prerequisites of using PA, 3-Oct and $\mathrm{MCH}$ for binary mixtures, odor-evoked $\mathrm{Ca}^{2+}$ activity patterns for the three different types of binary mixtures were determined in both OSNs and PNs. Each binary odor mixture showed a different kind of odor classification. The $\mathrm{MCH} / 3-O c t$ binary mixtures showed no odor classification in OSNs, but in PNs two distinct categories were observed. However, the same could not be found in either of the other two odor mixtures. For MCH/PA binary mixtures, no odor classification was found in OSNs. But in PNs, all mixtures having greater than $0 \%$ PA got categorized and distinctly separated from the $100 \% \mathrm{MCH}-0 \% \mathrm{PA}$. This was referred to as odor dominance of PA. The last odor mixture was of the similar odor pair, 3-Oct/PA, in which all the intermediate mixtures formed one cluster in both OSNs and PNs. This was due to the high degree of similarity already present between the 


\section{Summary}

individual odors in OSNs and PNs.

The glomeruli in the $A L$ are interconnected by a population of GABAergic LNs and they are known from previous studies to be involved in odor processing. GABA binds to two kinds of receptors: $\mathrm{GABA}_{A}$ and $\mathrm{GABA}_{B}$. $\mathrm{GABA}_{\mathrm{A}}$ receptors are known to be involved in the early phase of the odor processing and they can be pharmacologically blocked by picrotoxin. Therefore, fly brains were subjected to picrotoxin solution and its effects were observed on the three types of odor classification found with the combination of the selected odors. GABAergic inhibition mediated via $G_{A B A}$ receptors was found to be required for the odor classification of binary mixtures of $\mathrm{MCH} / 3-\mathrm{Oct}$. However, no changes were found due to picrotoxin on MCH/PA and 3-Oct/PA binary mixtures.

In conclusion, different kinds of odor classification could be observed for different odors in the AL of the fly. GABAergic inhibition has been found to play a role in mediating odor classification of different stimuli. However, not every odor classification requires GABAergic inhibition, as was observed in the case of $\mathrm{MCH} / \mathrm{PA}$ binary mixtures, thereby also suggesting an existence of other possible mechanisms for odor classification. 


\section{Bibliography}

Abbott, L. F. (2008). Theoretical neuroscience rising. Neuron, 60(3):489-495.

Abbott, L. F., Varela, J. A., Sen, K., and Nelson, S. B. (1997). Synaptic depression and cortical gain control. Science, 275(5297):220-224.

Acebes, A., Martín-Peña, A., Chevalier, V. V., Ferrús, A., Martin-Pena, A., Chevalier, V. V., and Ferrus, A. (2011). Synapse loss in olfactory local interneurons modifies perception. The Journal of Neuroscience, 31(8):2734-2745.

Ache, B. W. and Young, J. M. (2005). Olfaction: diverse species, conserved principles. Neuron, 48(3):417-430.

Akerboom, J., Chen, T. W., Wardill, T. J., Tian, L., Marvin, J. S., Mutlu, S., Calderon, N. C., Esposti, F., Borghuis, B. G., Sun, X. R., Gordus, A., Orger, M. B., Portugues, R., Engert, F., Macklin, J. J., Filosa, A., Aggarwal, A., Kerr, R. A., Takagi, R., Kracun, S., Shigetomi, E., Khakh, B. S., Baier, H., Lagnado, L., Wang, S. S. H., Bargmann, C. I., Kimmel, B. E., Jayaraman, V., Svoboda, K., Kim, D. S., Schreiter, E. R., and Looger, L. L. (2012). Optimization of a GCaMP calcium indicator for neural activity imaging. The Journal of Neuroscience, 32(40):13819-13840.

Arias, A. M. (2008). Drosophila melanogaster and the development of biology in the 20th century. Methods in Molecular Biology (Clifton, N.J.), 420:1-25.

Armstrong, J. D., Kaiser, K., Müller, A., Fischbach, K. F., Merchant, N., and Strausfeld, N. J. (1995). Flybrain, an on-line atlas and database of the Drosophila nervous system. Neuron, 15(1):17-20.

Asahina, K., Louis, M., Piccinotti, S., and Vosshall, L. B. (2009). A circuit supporting concentration-invariant odor perception in Drosophila. Journal of Biology, 8(1):9. 


\section{Bibliography}

Asahina, K., Watanabe, K., Duistermars, B., Hoopfer, E., González, C., Eyjólfsdóttir, E., Perona, P., and Anderson, D. (2014). Tachykinin-Expressing Neurons Control Male-Specific Aggressive Arousal in Drosophila. Cell, 156(1-2):221-235.

Bacskai, B. J., Wallén, P., Lev-Ram, V., Grillner, S., and Tsien, R. Y. (1995). Activityrelated calcium dynamics in lamprey motoneurons as revealed by video-rate confocal microscopy. Neuron, 14(1):19-28.

Badura, A., Sun, X. R., Giovannucci, A., Lynch, L. A., and Wang, S. S.-H. (2014). Fast calcium sensor proteins for monitoring neural activity. Neurophotonics, 1(2):025008.

Barth, J., Dipt, S., Pech, U., Hermann, M., Riemensperger, T., and Fiala, A. A. (2014). Differential associative training enhances olfactory acuity in Drosophila melanogaster. The Journal of Neuroscience, 34(5):1819-1837.

Baugh, A. T., Akre, K. L., and Ryan, M. J. (2008). Categorical perception of a natural, multivariate signal: mating call recognition in túngara frogs. Proceedings of the National Academy of Sciences of the United States of America, 105(26):8985-8988.

Belluscio, L. and Katz, L. C. (2001). Symmetry, stereotypy, and topography of odorant representations in mouse olfactory bulbs. The Journal of Neuroscience, 21(6):21132122.

Benton, R., Sachse, S., Michnick, S. W., and Vosshall, L. B. (2006). Atypical membrane topology and heteromeric function of Drosophila odorant receptors in vivo. PLOS Biology, 4(2):240-257.

Benton, R., Vannice, K. S., Gomez-Diaz, C., and Vosshall, L. B. (2009). Variant ionotropic glutamate receptors as chemosensory receptors in Drosophila. Cell, 136(1):149-162.

Berridge, M. J. (1998). Neuronal calcium signaling. Neuron, 21(1):13-26.

Berridge, M. J., Lipp, P., and Bootman, M. D. (2000). The versatility and universality of calcium signalling. Nature Reviews. Molecular Cell Biology, 1(1):11-21.

Bhandawat, V., Olsen, S. R., Gouwens, N. W., Schlief, M. L., and Wilson, R. I. (2007). Sensory processing in the Drosophila antennal lobe increases reliability and separability of ensemble odor representations. Nature Neuroscience, 10(11):1474-1482. 


\section{Bibliography}

Bornstein, M. H. and Korda, N. O. (1984). Discrimination and matching within and between hues measured by reaction times: some implications for categorical perception and levels of information processing. Psychological Research, 46(3):207-222.

Brand, A. H. and Perrimon, N. (1993). Targeted gene expression as a means of altering cell fates and generating dominant phenotypes. Development, 118(2):401-415.

Broome, B. M., Jayaraman, V., and Laurent, G. (2006). Encoding and decoding of overlapping odor sequences. Neuron, 51(4):467-482.

Bruyne, M. D., Clyne, P. J., and Carlson, J. R. (1999). Odor coding in a model olfactory organ: the Drosophila maxillary palp. The Journal of Neuroscience, 19(11):4520-32.

Bruyne, M. D., Foster, K., and Carlson, J. R. (2001). Odor coding in the Drosophila antenna. Neuron, 30(2):537-52.

Buck, L. and Axel, R. (1991). A novel multigene family may encode odorant receptors: A molecular basis for odor recognition. Cell, 65(1):175-187.

Callewaert, G., Eilers, J., and Konnerth, A. (1996). Axonal calcium entry during fast 'sodium' action potentials in rat cerebellar Purkinje neurones. The Journal of Physiology, 495(Pt 3):641-647.

Chen, T. W., Wardill, T. J., Sun, Y., Pulver, S. R., Renninger, S. L., Baohan, A., Schreiter, E. R., Kerr, R. A., Orger, M. B., Jayaraman, V., Looger, L. L., Svoboda, K., and Kim, D. S. (2013). Ultrasensitive fluorescent proteins for imaging neuronal activity. Nature, 499(7458):295-300.

Chess, A., Simon, I., Cedar, H., and Axel, R. (1994). Allelic inactivation regulates olfactory receptor gene expression. Cell, 78(5):823-834.

Chiang, A. S., Lin, C. Y., Chuang, C. C., Chang, H. M., Hsieh, C. H., Yeh, C. W., Shih, C. T., Wu, J. J., Wang, G. T., Chen, Y. C., Wu, C. C., Chen, G. Y., Ching, Y. T., Lee, P. C., Lin, C.-Y., Lin, H. H., Wu, C. C., Hsu, H. W., Huang, Y. A., Chen, J. Y., Chiang, H. J., Lu, C. F., Ni, R. F., Yeh, C. Y., and Hwang, J. K. (2011). Three-dimensional reconstruction of brain-wide wiring networks in Drosophila at single-cell resolution. Current Biology, 21(1):1-11. 


\section{Bibliography}

Chou, Y. H., Spletter, M. L., Yaksi, E., Leong, J. C. S., Wilson, R. I., and Luo, L. (2010). Diversity and wiring variability of olfactory local interneurons in the Drosophila antennal lobe. Nature Neuroscience, 13(4):439-449.

Cleland, T. A. (1996). Inhibitory glutamate receptor channels. Molecular Neurobiology, 13(2):97-136.

Clyne, P. J., Warr, C. G., Freeman, M. R., Lessing, D., Kim, J., and Carlson, J. R. (1999). A novel family of divergent seven-transmembrane proteins: candidate odorant receptors in Drosophila. Neuron, 22(2):327-338.

Couto, A., Alenius, M., and Dickson, B. J. (2005). Molecular, anatomical, and functional organization of the Drosophila olfactory system. Current Biology, 15(17):1535-1547.

Das, A., Chiang, A., Davla, S., Priya, R., Reichert, H., VijayRaghavan, K., and Rodrigues, $\mathrm{V}$. (2011). Identification and analysis of a glutamatergic local interneuron lineage in the adult Drosophila olfactory system. Neural Systems \& Circuits, 1(1):4.

DasGupta, S. and Waddell, S. (2008). Learned Odor Discrimination in Drosophila without Combinatorial Odor Maps in the Antennal Lobe. Current Biology, 18(21):16681674.

Deisig, N., Giurfa, M., Lachnit, H., and Sandoz, J.-C. (2006). Neural representation of olfactory mixtures in the honeybee antennal lobe. European Journal of Neuroscience, 24(4):1161-1174.

Deisig, N., Giurfa, M., and Sandoz, J. C. (2010). Antennal lobe processing increases separability of odor mixture representations in the honeybee. Journal of Neurophysiology, 103(4):2185-2194.

Dipt, S., Riemensperger, T., and Fiala, A. (2014). Optical Calcium Imaging Using DNAEncoded Fluorescence Sensors in Transgenic Fruit Flies, Drosophila melanogaster. In Fluorescent Protein-Based Biosensors, volume 1071 of Methods in Molecular Biology, pages 195-206. Humana Press, Totowa, NJ.

Dobritsa, A. A., Naters, W. V. D. G. V., Warr, C. G., Steinbrecht, R. A., Carlson, J. R., Haven, N., and Vic, C. (2003). Integrating the molecular and cellular basis of odor coding in the Drosophila antenna. Neuron, 37(5):827-841. 


\section{Bibliography}

Duchamp-Viret, P., Chaput, M. a., and Duchamp, A. (1999). Odor response properties of rat olfactory receptor neurons. Science, 284(5423):2171-2174.

Duchamp-Viret, P., Duchamp, A., and Chaput, M. A. (2003). Single olfactory sensory neurons simultaneously integrate the components of an odour mixture. European Journal of Neuroscience, 18(10):2690-2696.

Duchen, M. R. (1999). Contributions of mitochondria to animal physiology: from homeostatic sensor to calcium signalling and cell death. The Journal of Physiology, $516(\mathrm{Pt}$ 1):1-17.

Duffy, J. B. (2002). GAL4 system in Drosophila: a fly geneticist's Swiss army knife. Genesis, 34(1-2):1-15.

Dynes, J. L. and Ngai, J. (1998). Pathfinding of Olfactory Neuron Axons to Stereotyped Glomerular Targets Revealed by Dynamic Imaging in Living Zebrafish Embryos. Neuron, 20(6):1081-1091.

Ehret, G. and Haack, B. (1981). Categorical perception of mouse pup ultrasound by lactating females. Die Naturwissenschaften, 68(4):208-209.

Fiala, A. (2007). Olfaction and olfactory learning in Drosophila: recent progress. Current Opinion in Neurobiology, 17(6):720-726.

Fiala, A., Spall, T., Diegelmann, S., Eisermann, B., Sachse, S., Devaud, J.-M. M., Buchner, E., and Galizia, C. G. (2002). Genetically expressed cameleon in Drosophila melanogaster is used to visualize olfactory information in projection neurons. Current Biology, 12(21):1877-84.

Fishilevich, E. and Vosshall, L. B. (2005). Genetic and functional subdivision of the Drosophila antennal lobe. Current Biology, 15(17):1548-1553.

Friedrich, R. W. and Korsching, S. I. (1997). Combinatorial and Chemotopic Odorant Coding in the Zebrafish Olfactory Bulb Visualized by Optical Imaging. Neuron, 18(5):737-752.

Galizia, C. G., Münch, D., Strauch, M., Nissler, A., Ma, S., Munch, D., Strauch, M., Nissler, A., and Ma, S. (2010). Integrating heterogeneous odor response data into a common response model: A DoOR to the complete olfactome. Chemical Senses, 35(7):551-563. 


\section{Bibliography}

Gallio, M., Ofstad, T. A., Macpherson, L. J., Wang, J. W., and Zuker, C. S. (2011). The coding of temperature in the Drosophila brain. Cell, 144(4):614-624.

Gamble, E. and Koch, C. (1987). The dynamics of free calcium in dendritic spines in response to repetitive synaptic input. Science, 236(4806):1311-1315.

Goldman, A. L., Van der Goes van Naters, W., Lessing, D., Warr, C. G., and Carlson, J. R. (2005). Coexpression of two functional odor receptors in one neuron. Neuron, 45(5):661-666.

Goldstone, R. L. and Hendrickson, A. T. (2009). Categorical perception. Wiley Interdisciplinary Reviews: Cognitive Science, 1(1):69-78.

Gouwens, N. W. and Wilson, R. I. (2009). Signal propagation in Drosophila central neurons. The Journal of Neuroscience, 29(19):6239-6249.

Griesbeck, O. (2004). Fluorescent proteins as sensors for cellular functions. Current Opinion in Neurobiology, 14(5):636-641.

Guerrero, G. and Isacoff, E. (2001). Genetically encoded optical sensors of neuronal activity and cellular function. Current Opinion in Neurobiology, 11(5):601-607.

Guerrieri, F., Schubert, M., Sandoz, J. C., and Giurfa, M. (2005). Perceptual and neural olfactory similarity in honeybees. PLoS Biology, 3(4):e60.

Guizar-Sicairos, M., Thurman, S. T., and Fienup, J. R. (2008). Efficient subpixel image registration algorithms. Optics Letters, 33(2):156.

Haddad, R., Khan, R., Takahashi, Y. K., Mori, K., Harel, D., and Sobel, N. (2008). A metric for odorant comparison. Nature Methods, 5(5):425-429.

Hallem, E. A. and Carlson, J. R. (2006). Coding of odors by a receptor repertoire. Cell, 125(1):143-160.

Hallem, E. A., Ho, M. G., Carlson, J. R., and Haven, N. (2004). The molecular basis of odor coding in the Drosophila antenna. Cell, 117(2004):965-979.

Hamada, F. N., Rosenzweig, M., Kang, K., Pulver, S. R., Ghezzi, A., Jegla, T. J., and Garrity, P. A. (2008). An internal thermal sensor controlling temperature preference in Drosophila. Nature, 454(7201):217-220. 


\section{Bibliography}

Hansson, B. S. and Anton, S. (2000). Function and morphology of the antennal lobe: new developments. Annual Review of Entomology, 45:203-231.

Harnad, S. (1987). Psychophysical and cognitive aspects of categorical perception: A critical overview. Categorical perception: The groundwork of cognition, pages 1-25.

Harnad, S. (2003). Categorical Perception. Encyclopedia of cognitive science, LXVII(4):448-452.

Heisenberg, M. (1998). What do the mushroom bodies do for the insect brain? an introduction. Learning \& Memory, 5(1):1-10.

Hendricks, J. C., Finn, S. M., Panckeri, K. a., Chavkin, J., Williams, J. a., Sehgal, a., and Pack, a. I. (2000). Rest in Drosophila is a sleep-like state. Neuron, 25(1):129138.

Herculano-Houzel, S. and Lent, R. (2005). Isotropic fractionator: a simple, rapid method for the quantification of total cell and neuron numbers in the brain. The Journal of Neuroscience, 25(10):2518-2521.

Hong, E. J. and Wilson, R. I. (2015). Simultaneous Encoding of Odors by Channels with Diverse Sensitivity to Inhibition. Neuron, 85(3):573-589.

Hosie, A. M., Aronstein, K., Sattelle, D. B., and Ffrench-Constant, R. H. (1997). Molecular biology of insect neuronal GABA receptors. Trends in Neurosciences, 20(97):578-583.

Hu, B. and Tu, Y. (2014). Behaviors and strategies of bacterial navigation in chemical and nonchemical gradients. PLoS Computational Biology, 10(6):e1003672.

Huang, J., Zhang, W., Qiao, W., Hu, A., and Wang, Z. (2010). Functional connectivity and selective odor responses of excitatory local interneurons in Drosophila antennal lobe. Neuron, 67(6):1021-1033.

Isaacson, J. S. (2010). Odor representations in mammalian cortical circuits. Current Opinion in Neurobiology, 20(3):328-331.

Joerges, J., Küttner, A., Galizia, C. G., and Menzel, R. (1997). Representations of odours and odour mixtures visualized in the honeybee brain. Nature, 387(6630):285-288. 


\section{Bibliography}

Johnson, B. A., Ong, J., and Leon, M. (2010). Glomerular activity patterns evoked by natural odor objects in the rat olfactory bulb are related to patterns evoked by major odorant components. The Journal of Comparative Neurology, 518(9):1542-1555.

Kang, J. and Caprio, J. (1995). In vivo responses of single olfactory receptor neurons in the channel catfish, Ictalurus punctatus. Journal of Neurophysiology, 73(1):172177.

Kazama, H. and Wilson, R. I. (2008). Homeostatic matching and nonlinear amplification at identified central synapses. Neuron, 58(3):401-413.

Kazama, H. and Wilson, R. I. (2009). Origins of correlated activity in an olfactory circuit. Nature Neuroscience, 12(9):1136-1144.

Khan, A. G., Thattai, M., and Bhalla, U. S. (2008). Odor representations in the rat olfactory bulb change smoothly with morphing stimuli. Neuron, 57(4):571-585.

Kim, A. J., Lazar, A. A., and Slutskiy, Y. B. (2011). System identification of Drosophila olfactory sensory neurons. Journal of Computational Neuroscience, 30(1):143-161.

Knaden, M., Strutz, A., Ahsan, J., Sachse, S., and Hansson, B. S. (2012). Spatial representation of odorant valence in an insect brain. Cell Reports, 1(4):392-399.

Knöpfel, T., Díez-García, J., and Akemann, W. (2006). Optical probing of neuronal circuit dynamics: Genetically encoded versus classical fluorescent sensors. Trends in Neurosciences, 29(3):160-166.

Lai, S.-L. and Lee, T. (2006). Genetic mosaic with dual binary transcriptional systems in Drosophila. Nature Neuroscience, 9(5):703-709.

Laing, D. G. and Francis, G. W. (1989). The capacity of humans to identify odors in mixtures. Physiology \& Behavior, 46(5):809-814.

Laissue, P. P., Reiter, C., Hiesinger, P. R., Halter, S., Fischbach, K. F., and Stocker, R. F. (1999). Three-dimensional reconstruction of the antennal lobe in Drosophila melanogaster. The Journal of Comparative Neurology, 405(4):543-552.

Larsson, M. C., Domingos, A. I., Jones, W. D., Chiappe, M. E., Amrein, H., and Vosshall, L. B. (2004). Or83b encodes a broadly expressed odorant receptor essential for Drosophila olfaction. Neuron, 43(5):703-714. 


\section{Bibliography}

Lindenburg, L. and Merkx, M. (2012). Colorful calcium sensors. ChemBioChem, 13(3):349-351.

Liu, W. W. and Wilson, R. I. (2013). Glutamate is an inhibitory neurotransmitter in the Drosophila olfactory system. Proceedings of the National Academy of Sciences of the United States of America, 110(25):10294-10299.

Livingston, K. R., Andrews, J. K., and Harnad, S. (1998). Categorical perception effects induced by category learning. Journal of Experimental Psychology: Learning, Memory, and Cognition, 24(3):732-753.

Lledo, P.-M., Gheusi, G., and Vincent, J.-D. (2005). Information processing in the mammalian olfactory system. Physiological Reviews, 85(1):281-317.

Malnic, B., Hirono, J., Sato, T., and Buck, L. B. (1999). Combinatorial Receptor Codes for Odors. Cell, 96(5):713-723.

Mao, B. Q., Hamzei-Sichani, F., Aronov, D., Froemke, R. C., and Yuste, R. (2001). Dynamics of spontaneous activity in neocortical slices. Neuron, 32(5):883-898.

Martelli, C., Carlson, J. R., and Emonet, T. (2013). Intensity invariant dynamics and odor-specific latencies in olfactory receptor neuron response. The Journal of Neuroscience, 33(15):6285-6297.

Mathew, D., Martelli, C., Kelley-Swift, E., Brusalis, C., Gershow, M., Samuel, A. D. T., Emonet, T., and Carlson, J. R. (2013). Functional diversity among sensory receptors in a $<i>$ Drosophila</i> olfactory circuit. Proceedings of the National Academy of Sciences, 110(23):E2134-E2143.

May, B., Moody, D. B., and Stebbins, W. C. (1989). Categorical perception of conspecific communication sounds by Japanese macaques, Macaca fuscata. The Journal of the Acoustical Society of America, 85(2):837-847.

Miyawaki, A., Llopis, J., Heim, R., McCaffery, J. M., Adams, J. A., Ikura, M., and Tsien, R. Y. (1997). Fluorescent indicators for $\mathrm{Ca2}+$ based on green fluorescent proteins and calmodulin. Nature, 388(6645):882-887.

Mombaerts, P., Wang, F., Dulac, C., Chao, S. K., Nemes, A., Mendelsohn, M., Edmondson, J., and Axel, R. (1996). Visualizing an Olfactory Sensory Map. Cell, 87(4):675-686. 


\section{Bibliography}

Morgan, T. H. (1910). Sex limited inheritance in Drosophila. Science, 32(812):120122.

Mori, K., Nagao, H., and Yoshihara, Y. (1999). The olfactory bulb: coding and processing of odor molecule information. Science, 286(5440):711-715.

Müller, W. and Connor, J. A. (1991). Dendritic spines as individual neuronal compartments for synaptic Ca2+ responses. Nature, 354(6348):73-76.

Mutoh, H. and Knöpfel, T. (2013). Probing neuronal activities with genetically encoded optical indicators: From a historical to a forward-looking perspective. Pflügers Archiv - European Journal of Physiology, 465(3):361-371.

Nagel, K. I. and Wilson, R. I. (2011). Biophysical mechanisms underlying olfactory receptor neuron dynamics. Nature Neuroscience, 14(2):208-216.

Nakagawa, T. and Vosshall, L. B. (2009). Controversy and consensus: noncanonical signaling mechanisms in the insect olfactory system. Current Opinion in Neurobiology, 19(3):284-292.

Nakai, J., Ohkura, M., and Imoto, K. (2001). A high signal-to-noise $\mathrm{Ca}(2+)$ probe composed of a single green fluorescent protein. Nature Biotechnology, 19(2):137141.

Neher, E. (1998). Vesicle pools and Ca2+ microdomains: new tools for understanding their roles in neurotransmitter release. Neuron, 20(3):389-399.

Neher, E. and Sakaba, T. (2008). Multiple roles of calcium ions in the regulation of neurotransmitter release. Neuron, 59(6):861-872.

Nelson, D. and Marler, P. (1989). Categorical perception of a natural stimulus continuum: birdsong. Science, 244(4907):976-978.

Ng, M., Roorda, R. D., Lima, S. Q., Zemelman, B. V., Morcillo, P., and Miesenbock, G. (2002). Transmission of olfactory information between three populations of neurons in the antennal lobe of the fly. Neuron, 36(3):463-474.

Niessing, J. (2012). Optophysiological analysis of pattern classification strategies in the zebrafish olfactory bulb. PhD Thesis, University of Basel, Faculty of Science. 


\section{Bibliography}

Niessing, J. and Friedrich, R. W. (2010). Olfactory pattern classification by discrete neuronal network states. Nature, 465(7294):47-52.

Niewalda, T., Völler, T., Eschbach, C., Ehmer, J., Chou, W. C., Timme, M., Fiala, A., and Gerber, B. (2011). A combined perceptual, physico-chemical, and imaging approach to 'odour-distances' suggests a categorizing function of the Drosophila antennal lobe. PloS one, 6(9):e24300.

Ohkura, M., Matsuzaki, M., Kasai, H., Imoto, K., and Nakai, J. (2005). Genetically encoded bright $\mathrm{Ca} 2+$ probe applicable for dynamic $\mathrm{Ca} 2+$ imaging of dendritic spines. Analytical Chemistry, 77(18):5861-5869.

Ohkura, M., Sasaki, T., Sadakari, J., Gengyo-Ando, K., Kagawa-Nagamura, Y., Kobayashi, C., Ikegaya, Y., and Nakai, J. (2012). Genetically Encoded Green Fluorescent $\mathrm{Ca} 2+$ Indicators with Improved Detectability for Neuronal $\mathrm{Ca} 2+$ Signals. PLoS one, 7(12):e51286.

Olsen, S. R., Bhandawat, V., and Wilson, R. I. (2007). Excitatory interactions between olfactory processing channels in the Drosophila antennal lobe. Neuron, 54(1):89103.

Olsen, S. R., Bhandawat, V., and Wilson, R. I. (2010). Divisive normalization in olfactory population codes. Neuron, 66(2):287-299.

Olsen, S. R. and Wilson, R. I. (2008). Lateral presynaptic inhibition mediates gain control in an olfactory circuit. Nature, 452(7190):956-960.

Parnas, M., Lin, A. C., Huetteroth, W., and Miesenböck, G. (2013). Odor discrimination in Drosophila: from neural population codes to behavior. Neuron, 79(5):932-944.

Paster, E. and Ryu, W. S. (2008). The thermal impulse response of Escherichia coli. Proceedings of the National Academy of Sciences of the United States of America, 105(14):5373-5377.

Pérez Koldenkova, V. and Nagai, T. (2013). Genetically encoded Ca2+ indicators: Properties and evaluation. Biochimica et Biophysica Acta, 1833(7):1787-1797.

Pinching, A. J. and Powell, T. P. S. (1971). The neuropil of the glomeruli of the olfactory bulb. Journal of Cell Science, 9(2):347-377. 


\section{Bibliography}

Poo, C. and Isaacson, J. S. (2009). Odor representations in olfactory cortex: "sparse" coding, global inhibition, and oscillations. Neuron, 62(6):850-861.

Pos, O. D. and Albertazzi, L. (2010). It is in the nature of the colours. Seeing and Perceiving, 23(1):39-73.

Potter, C. J., Tasic, B., Russler, E. V., Liang, L., and Luo, L. (2010). The Q system: A repressible binary system for transgene expression, lineage tracing, and mosaic analysis. Cell, 141(3):536-548.

Raman, B., Joseph, J., Tang, J., and Stopfer, M. (2010). Temporally diverse firing patterns in olfactory receptor neurons underlie spatiotemporal neural codes for odors. The Journal of Neuroscience, 30(6):1994-2006.

Regehr, W. G. and Tank, D. W. (1992). Calcium concentration dynamics produced by synaptic activation of CA1 hippocampal pyramidal cells. The Journal of Neuroscience, 12(11):4202-4223.

Riemensperger, T., Pech, U., Dipt, S., and Fiala, A. (2012). Optical calcium imaging in the nervous system of Drosophila melanogaster. Biochimica et Biophysica Acta, 1820(8):1169-1178.

Riesgo-Escovar, J. R., Piekos, W. B., and Carlson, J. R. (1997). The maxillary palp of Drosophila: ultrastructure and physiology depends on the lozenge gene. Journal of Comparative Physiology. A, Sensory, neural, and behavioral physiology, 180(2):143-150.

Ringner, M. (2008). What is principal component analysis? Nature Biotechnology, 26(3):303-304.

Ronderos, D. S. and Smith, D. P. (2009). Diverse signaling mechanisms mediate volatile odorant detection in Drosophila. Fly, 3(4):290-297.

Root, C. M., Masuyama, K., Green, D. S., Enell, L. E., Nassel, D. R., Lee, C.-H., Wang, J. W., Nässel, D. R., Lee, C.-H., and Wang, J. W. (2008). A presynaptic gain control mechanism fine-tunes olfactory behavior. Neuron, 59(2):311-321.

Root, C. M., Semmelhack, J. L., Wong, A. M., Flores, J., and Wang, J. W. (2007). Propagation of olfactory information in Drosophila. Proceedings of the National Academy of Sciences of the United States of America, 104(28):11826-11831. 


\section{Bibliography}

Rospars, J. P., Lansky, P., Chaput, M., and Duchamp-Viret, P. (2008). Competitive and noncompetitive odorant interactions in the early neural coding of odorant mixtures. The Journal of Neuroscience, 28(10):2659-2666.

Rubin, B. D. and Katz, L. C. (1999). Optical Imaging of Odorant Representations in the Mammalian Olfactory Bulb. Neuron, 23(3):499-511.

Rubin, G. M. and Spradling, A. C. (1982). Genetic transformation of Drosophila with transposable element vectors. Science, 218(4570):348-353.

Sachse, S. and Galizia, C. G. (2002). Role of inhibition for temporal and spatial odor representation in olfactory output neurons: a calcium imaging study. Journal of Neurophysiology, 87(2):1106-1117.

Sachse, S. and Galizia, C. G. (2003). The coding of odour-intensity in the honeybee antennal lobe: Local computation optimizes odour representation. European Journal of Neuroscience, 18(8):2119-2132.

Salzberg, B. M., Davila, H. V., and Cohen, L. B. (1973). Optical recording of impulses in individual neurones of an invertebrate central nervous system. Nature, 246(5434):508-509.

Sandeman, D. C., Scholtz, G., and Sandeman, R. E. (1993). Brain evolution in decapod crustacea. Journal of Experimental Zoology, 265(2):112-133.

Sanhueza, M., Schmachtenberg, O., and Bacigalupo, J. (2000). Excitation, inhibition, and suppression by odors in isolated toad and rat olfactory receptor neurons. American Journal of Physiology - Cell Physiology, 279(1):C31-C39.

Sato, K., Pellegrino, M., Nakagawa, T., Nakagawa, T., Vosshall, L. B., and Touhara, K. (2008). Insect olfactory receptors are heteromeric ligand-gated ion channels. Nature, 452(7190):1002-1006.

Schlief, M. L. and Wilson, R. I. (2007). Olfactory processing and behavior downstream from highly selective receptor neurons. Nature Neuroscience, 10(5):623-630.

Schuckel, J. and French, A. S. (2008). A digital sequence method of dynamic olfactory characterization. Journal of Neuroscience Methods, 171(1):98-103. 


\section{Bibliography}

Schuckel, J., Meisner, S., Torkkeli, P. H., and French, A. S. (2008). Dynamic properties of Drosophila olfactory electroantennograms. Journal of Comparative Physiology A, 194(5):483-489.

Schuckel, J., Torkkeli, P. H. P. H., and French, A. S. (2009). Two interacting olfactory transduction mechanisms have linked polarities and dynamics in Drosophila melanogaster antennal basiconic sensilla neurons. Journal of Neurophysiology, 102(1):214-223.

Scott, K., Brady, R. J., Cravchik, a., Morozov, P., Rzhetsky, A., Zuker, C., and Axel, R. (2001). A chemosensory gene family encoding candidate gustatory and olfactory receptors in Drosophila. Cell, 104(5):661-673.

Seki, Y., Rybak, J., Wicher, D., Sachse, S., and Hansson, B. S. (2010). Physiological and morphological characterization of local interneurons in the Drosophila antennal lobe. Journal of Neurophysiology, 104(2):1007-1019.

Serizawa, S., Ishii, T., Nakatani, H., Tsuboi, A., Nagawa, F., Asano, M., Sudo, K., Sakagami, J., Sakano, H., ljiri, T., Matsuda, Y., Suzuki, M., Yamamori, T., Iwakura, Y., and Sakano, H. (2000). Mutually exclusive expression of odorant receptor transgenes. Nature Neuroscience, 3(7):687-693.

Shanbhag, S. R., Müller, B., and Steinbrecht, R. A. (1999). Atlas of olfactory organs of Drosophila melanogaster: 1. Types, external organization, innervation and distribution of olfactory sensilla. International Journal of Insect Morphology and Embryology, 28(1999):377-397.

Shang, Y., Claridge-Chang, A., Sjulson, L., Pypaert, M., and Miesenbock, G. (2007). Excitatory local circuits and their implications for olfactory processing in the fly antennal lobe. Cell, 128(3):601-612.

Silbering, A. F. and Galizia, C. G. (2007). Processing of odor mixtures in the Drosophila antennal lobe reveals both global inhibition and glomerulus-specific interactions. The Journal of Neuroscience, 27(44):11966-11977.

Silbering, A. F., Okada, R., Ito, K., and Galizia, C. G. (2008). Olfactory information processing in the Drosophila antennal lobe: anything goes? The Journal of Neuroscience, 28(49):13075-13087. 


\section{Bibliography}

Silbering, A. F., Rytz, R., Grosjean, Y., Abuin, L., Ramdya, P., Jefferis, G. S. X. E., and Benton, R. (2011). Complementary function and integrated wiring of the evolutionarily distinct Drosophila olfactory subsystems. The Journal of Neuroscience, 31(38):13357-13375.

Sosulski, D. L., Bloom, M. L., Cutforth, T., Axel, R., and Datta, S. R. (2011). Distinct representations of olfactory information in different cortical centres. Nature, 472(7342):213-216.

Spradling, A. C. (1997). Fly pushing: The theory and practice of Drosophila genetics. Trends in Genetics, 13(10):418.

Spradling, a. C. and Rubin, G. M. (1982). Transposition of cloned P elements into Drosophila germ line chromosomes. Science, 218(4570):341-347.

Stephenson, R. and Metcalfe, N. H. (2013). Drosophila melanogaster: a fly through its history and current use. The Journal of the Royal College of Physicians of Edinburgh, 43(1):70-75.

Stocker, R. F., Heimbeck, G., Gendre, N., and de Belle, J. S. (1997). Neuroblast ablation in Drosophila P[GAL4] lines reveals origins of olfactory interneurons. Journal of Neurobiology, 32(5):443-456.

Stocker, R. F., Lienhard, M. C., Borst, A., and Fischbach, K. F. (1990). Neuronal architecture of the antennal lobe in Drosophila melanogaster. Cell and Tissue Research, 262(1):9-34.

Stopfer, M., Bhagavan, S., Smith, B. H., and Laurent, G. (1997). Impaired odour discrimination on desynchronization of odour-encoding neural assemblies. Nature, 390(6655):70-74.

Stosiek, C., Garaschuk, O., Holthoff, K., and Konnerth, A. (2003). In vivo two-photon calcium imaging of neuronal networks. Proceedings of the National Academy of Sciences of the United States of America, 100(12):7319-7324.

Strutz, A., Soelter, J., Baschwitz, A., Farhan, A., Grabe, V., Rybak, J., Knaden, M., Schmuker, M., Hansson, B. S., and Sachse, S. (2014). Decoding odor quality and intensity in the Drosophila brain. eLife, 3(e04147):1-21. 


\section{Bibliography}

Su, C. Y., Martelli, C., Emonet, T., and Carlson, J. R. (2011). Temporal coding of odor mixtures in an olfactory receptor neuron. Proceedings of the National Academy of Sciences of the United States of America, 108(12):5075-5080.

Su, C. Y., Menuz, K., and Carlson, J. R. (2009). Olfactory perception: receptors, cells, and circuits. Cell, 139(1):45-59.

Südhof, T. C. (2000). The synaptic vesicle cycle revisited. Neuron, 28(2):317-320.

Szüts, D. and Bienz, M. (2000). LexA chimeras reveal the function of Drosophila Fos as a context-dependent transcriptional activator. Proceedings of the National Academy of Sciences of the United States of America, 97(10):5351-5356.

Tabor, R., Yaksi, E., Weislogel, J. M., and Friedrich, R. W. (2004). Processing of odor mixtures in the zebrafish olfactory bulb. The Journal of Neuroscience, 24(29):66116620 .

Tanaka, N. K., Endo, K., and Ito, K. (2012). Organization of antennal lobe-associated neurons in adult Drosophila melanogaster brain. Journal of Comparative Neurology, 520(18):4067-4130.

Tanaka, N. K., Ito, K., and Stopfer, M. (2009). Odor-evoked neural oscillations in Drosophila are mediated by widely branching interneurons. The Journal of $\mathrm{Neu}$ roscience, 29(26):8595-8603.

Tataroglu, O. and Emery, P. (2014). Studying circadian rhythms in Drosophila melanogaster. Methods, 68(1):140-150.

Tian, L., Hires, S. A., Mao, T., Huber, D., Chiappe, M. E., Chalasani, S. H., Petreanu, L., Akerboom, J., McKinney, S. A., Schreiter, E. R., Bargmann, C. I., Jayaraman, V., Svoboda, K., and Looger, L. L. (2009). Imaging neural activity in worms, flies and mice with improved GCaMP calcium indicators. Nature Methods, 6(12):875-881.

Tolbert, L. P. and Hildebrand, J. G. (1981). Organization and Synaptic Ultrastructure of Glomeruli in the Antennal Lobes of the Moth Manduca sexta: A Study Using Thin Sections and Freeze-Fracture. Proceedings of the Royal Society B: Biological Sciences, 213(1192):279-301. 


\section{Bibliography}

Troemel, E. R., Chou, J. H., Dwyer, N. D., Colbert, H. A., and Bargmann, C. I. (1995). Divergent seven transmembrane receptors are candidate chemosensory receptors in C. elegans. Cell, 83(2):207-218.

Tsien, R. W. and Tsien, R. Y. (1990). Calcium channels, stores, and oscillations. Annual Review of Cell Biology, 6:715-760.

Tsien, R. Y. (1988). Fluorescence measurement and photochemical manipulation of cytosolic free calcium. Trends in Neurosciences, 11(10):419-424.

Venken, K. J. T., Simpson, J. H., and Bellen, H. J. (2011). Genetic manipulation of genes and cells in the nervous system of the fruit fly. Neuron, 72(2):202-230.

Verkhratsky, A. J. and Petersen, O. H. (1998). Neuronal calcium stores. Cell Calcium, 24(5-6):333-343.

Vickers, N. J., Christensen, T. a., and Hildebrand, J. G. (1998). Combinatorial odor discrimination in the brain: Attractive and antagonist odor blends are represented in distinct combinations of uniquely identifiable glomeruli. Journal of Comparative Neurology, 400(1):35-56.

Vogelstein, J. T., Watson, B. O., Packer, A. M., Yuste, R., Jedynak, B., and Paninski, L. (2009). Spike inference from calcium imaging using sequential Monte Carlo methods. Biophysical Journal, 97(2):636-655.

Vosshall, L. B., Amrein, H., Morozov, P. S., Rzhetsky, A., and Axel, R. (1999). A spatial map of olfactory receptor expression in the Drosophila antenna. Cell, 96(5):725736.

Vosshall, L. B. and Stocker, R. F. (2007). Molecular architecture of smell and taste in Drosophila. Annual Review of Neuroscience, 30:505-533.

Vosshall, L. B., Wong, A. M., and Axel, R. (2000). An Olfactory Sensory Map in the Fly Brain. Cell, 102(2):147-159.

Waddell, S. and Quinn, W. G. (2001). What can we teach Drosophila? What can they teach us? Trends in Genetics, 17(12):719-726.

Wadhams, G. H. and Armitage, J. P. (2004). Making sense of it all: bacterial chemotaxis. Nature Reviews. Molecular Cell Biology, 5(12):1024-1037. 


\section{Bibliography}

Wang, J. W., Wong, A. M., Flores, J., Vosshall, L. B., and Axel, R. (2003). Twophoton calcium imaging reveals an odor-evoked map of activity in the fly brain. Cell, 112(2):271-282.

Waxman, S. G. and Ritchie, J. M. (1993). Molecular dissection of the myelinated axon. Annals of Neurology, 33(2):121-136.

Wicher, D., Schäfer, R., Bauernfeind, R., Stensmyr, M. C., Heller, R., Heinemann, S. H., and Hansson, B. S. (2008). Drosophila odorant receptors are both ligand-gated and cyclic-nucleotide-activated cation channels. Nature, 452(7190):1007-1011.

Wilson, R. I. (2013). Early olfactory processing in Drosophila: mechanisms and principles. Annual Review of Neuroscience, 36:217-241.

Wilson, R. I. and Laurent, G. (2005). Role of GABAergic inhibition in shaping odorevoked spatiotemporal patterns in the Drosophila antennal lobe. The Journal of Neuroscience, 25(40):9069-9079.

Wilson, R. I., Turner, G. C., and Laurent, G. (2004). Transformation of olfactory representations in the Drosophila antennal lobe. Science, 303(5656):366-370.

Wyttenbach, R. A., May, M. L., and Hoy, R. R. (1996). Categorical Perception of Sound Frequency by Crickets. Science, 273(5281):1542-1544.

Yaksi, E., von Saint Paul, F., Niessing, J., Bundschuh, S. T., and Friedrich, R. W. (2009). Transformation of odor representations in target areas of the olfactory bulb. Nature Neuroscience, 12(4):474-482.

Yaksi, E. and Wilson, R. I. (2010). Electrical coupling between olfactory glomeruli. Neuron, 67(6):1034-1047.

Yao, C. A., Ignell, R., and Carlson, J. R. (2005). Chemosensory coding by neurons in the coeloconic sensilla of the Drosophila antenna. The Journal of Neuroscience, 25(37):8359-8367.

Yasuda, R., Nimchinsky, E. A., Scheuss, V., Pologruto, T. A., Oertner, T. G., Sabatini, B. L., and Svoboda, K. (2004). Imaging calcium concentration dynamics in small neuronal compartments. Science Signaling, 2004(219):pl5-pl5. 


\section{Bibliography}

Yasuyama, K. and Salvaterra, P. M. (1999). Localization of choline acetyltransferaseexpressing neurons in Drosophila nervous system. Microscopy Research and Technique, 45(2):65-79.

Yokoi, M., Mori, K., and Nakanishi, S. (1995). Refinement of odor molecule tuning by dendrodendritic synaptic inhibition in the olfactory bulb. Proceedings of the National Academy of Sciences of the United States of America, 92(8):3371-3375.

Yuste, R. and Denk, W. (1995). Dendritic spines as basic functional units of neuronal integration. Nature, 375(6533):682-684.

Zeidler, M. P., Tan, C., Bellaiche, Y., Cherry, S., Häder, S., Gayko, U., and Perrimon, N. (2004). Temperature-sensitive control of protein activity by conditionally splicing inteins. Nature Biotechnology, 22(7):871-876.

Zhao, Y., Araki, S., Wu, J., Teramoto, T., Chang, Y.-F., Nakano, M., Abdelfattah, A. S., Fujiwara, M., Ishihara, T., Nagai, T., and Campbell, R. E. (2011). An expanded palette of genetically encoded Ca2+ indicators. Science, 333(6051):1888-1891. 


\section{Acknowledgements}

First and foremost, I would like to express my deepest appreciation and thanks to Prof. Dr. André Fiala for his continuous support and supervision throughout my study.

I would also like to thank my second reviewer, Prof. Dr. Marc Timme, and also Prof. Dr. Dr. Detlev Schild for their regular insightful comments and suggestions during the thesis committee meetings and other times.

I would also like to acknowledge the Bernstein Center for Computational Neuroscience (BCCN) for funding this entire project.

I am, no doubt also indebted to all my former and current lab members who provided helpful discussions along with a lively environment throughout the last three and a half years: Dr. Ulrike Pech, Dr. Atefeh Pooryasin, David Vasmer, Dr. Jonas Barth, Dr. Simon Kobbenbring, Dr. Thomas Riemensperger, Dr. Carlotta Martelli, Dr. Mandy Jauch, Dr. Priyanka Singh, Jutta Boeker and Sandra Schubert. Special thanks to Dr. Thomas Riemensperger for helping me with the complicated fly crosses and Jutta Boeker for making the fly food. I am also grateful to Dr. Wen-Chuang Chou for providing me help with his expertise in data analysis. I would also like to express my warm thanks to Dr. Atefeh Pooryasin, Dr. Ulrike Pech and David Vasmer for their immense support and help and most of all their friendship which made every moment of my stay in Göttingen a memorable one.

I would also like to take this opportunity to thank GGNB for their assistance and for organizing several methods and skills courses. In addition, I am also indebted to them for providing me with the bridging fund for the last month of my PhD.

Special thanks to Kanchan Agarwal for her unconditional love and also to my old friends, Ebad Kamil and Debanjan Basu, for being supportive and for enduring my in- 


\section{Acknowledgements}

sanity throughout my stay in Göttingen and before.

Last but not the least, I would also like to thank my parents for their infinite love and care, and also for being supportive at every stage of my life. 


\section{A. The codes for data analysis}

\section{A.1. ImageJ plugins (Java)}

\section{A.1.1. TwoPhoton Batch Metamorph StackBuild}

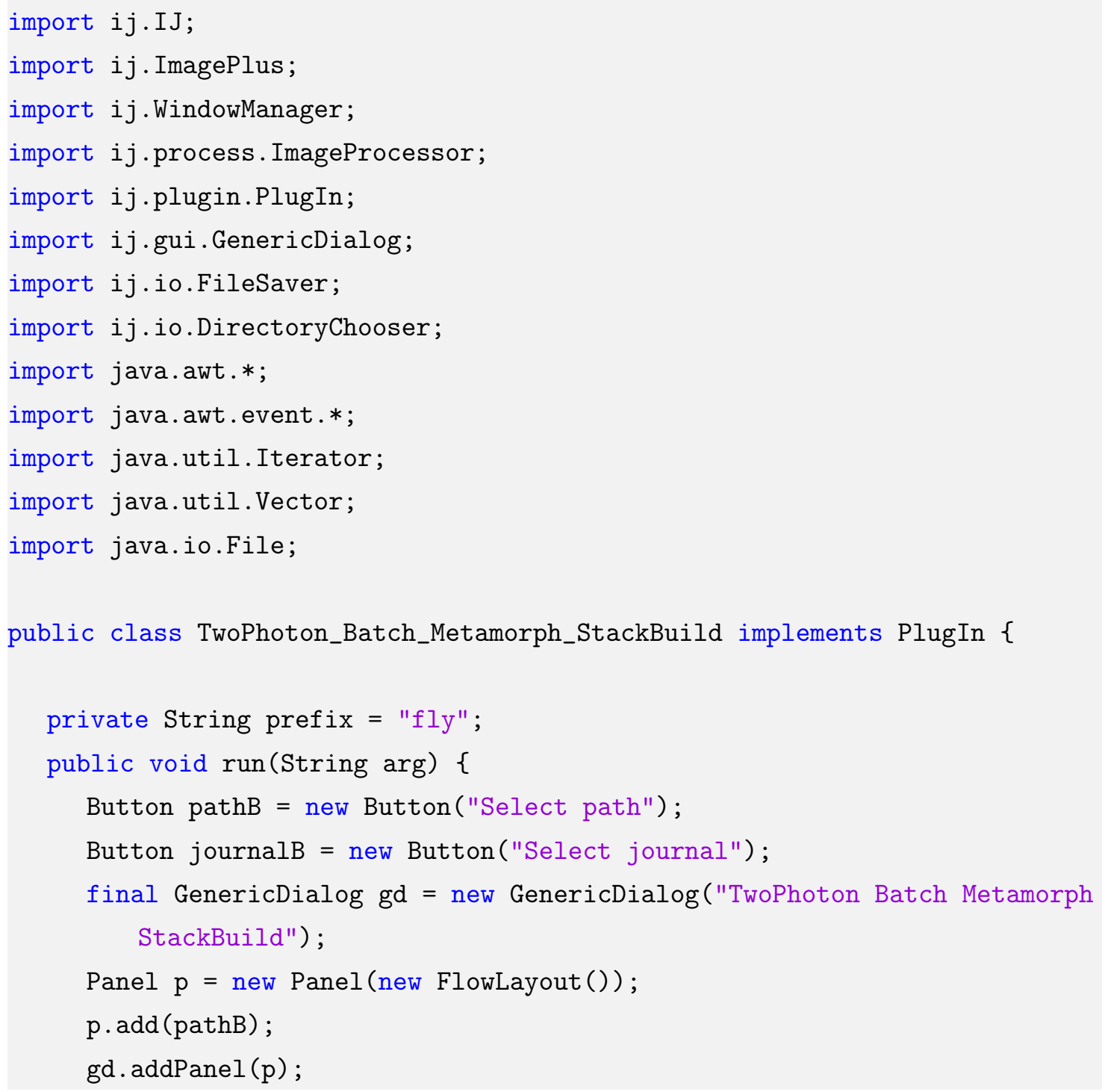




\section{A. The codes for data analysis}

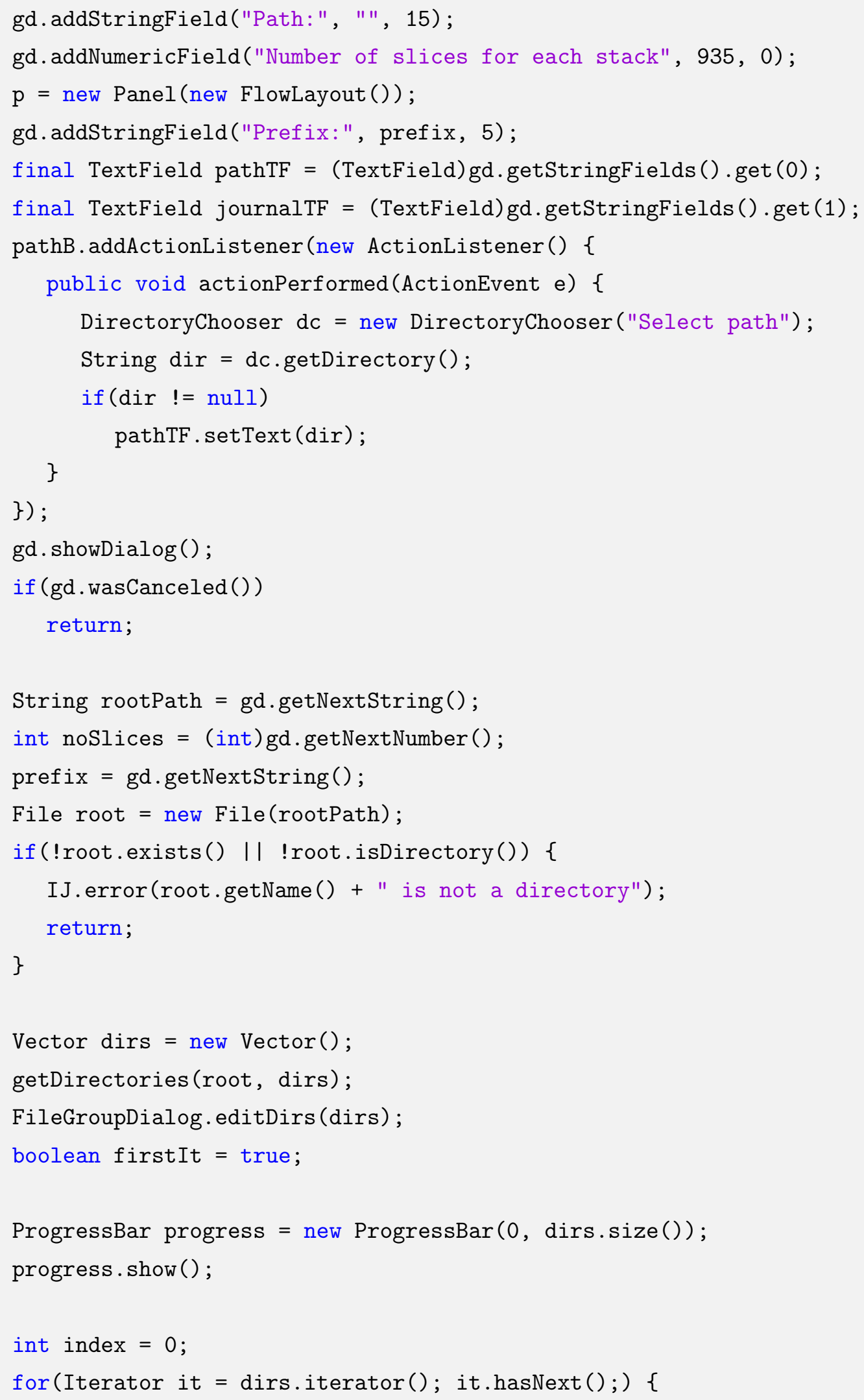




\section{A. The codes for data analysis}

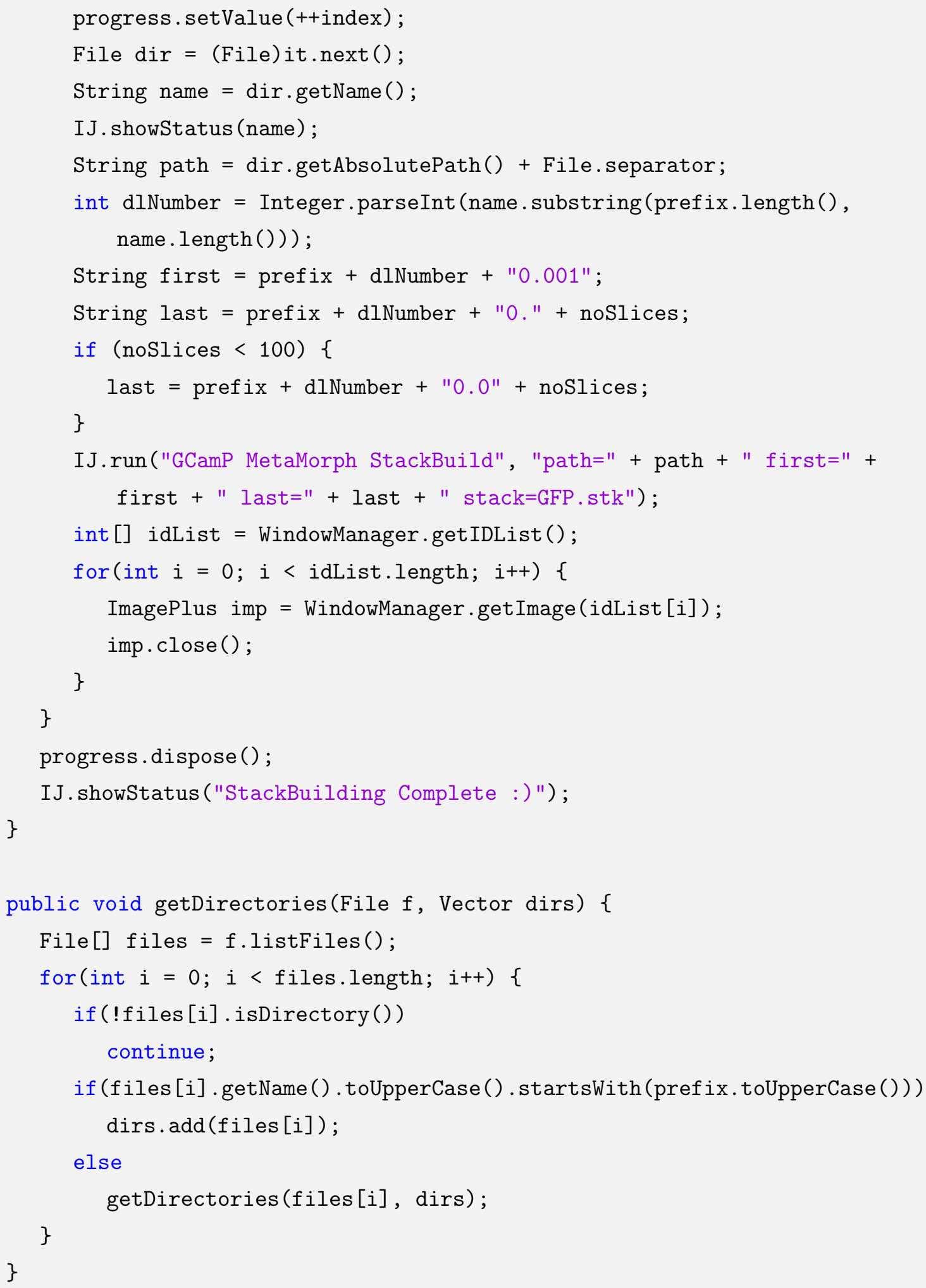




\section{A. The codes for data analysis}

\section{A.1.2. GCamP MetaMorph StackBuild}

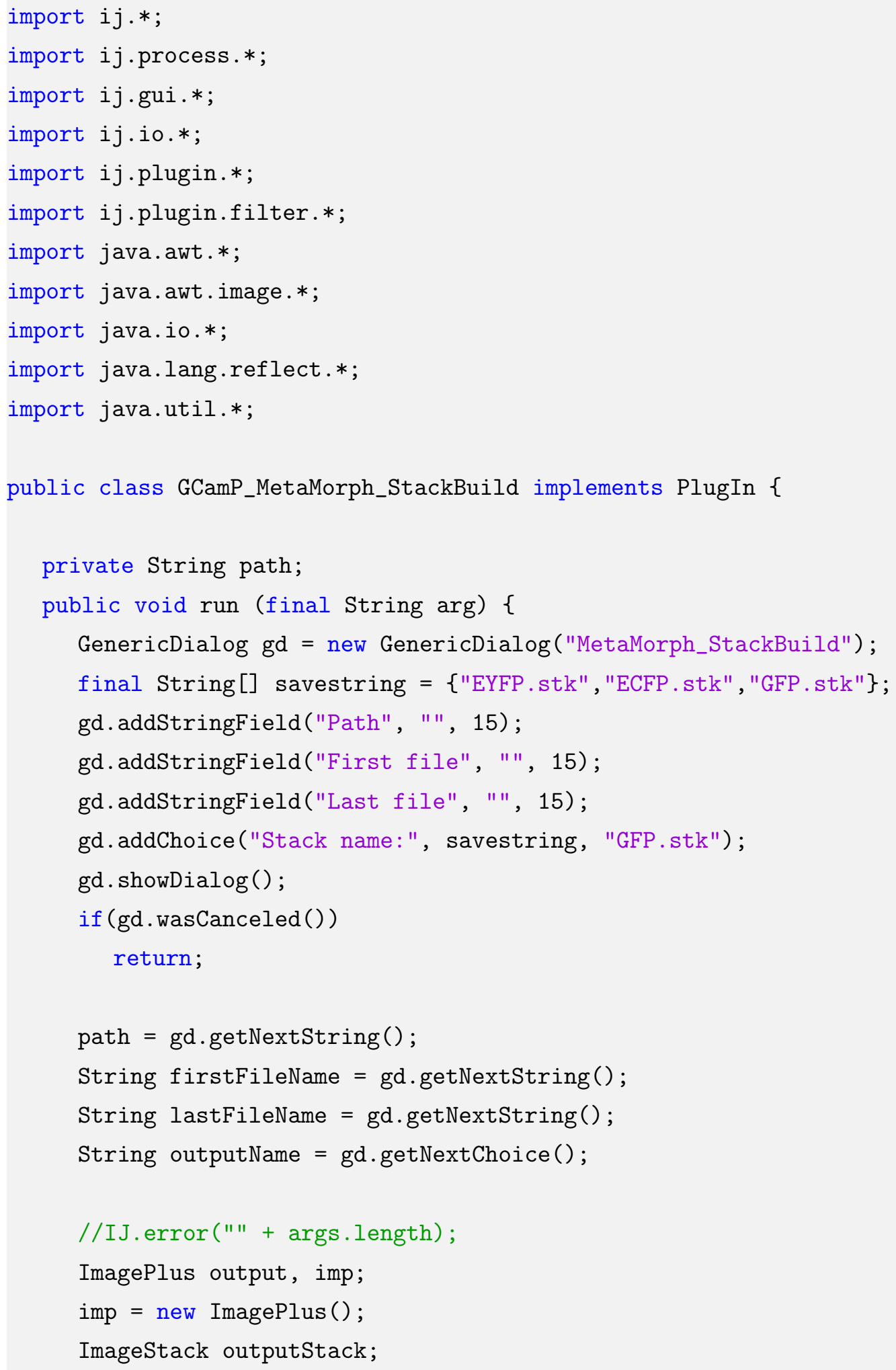




\section{A. The codes for data analysis}

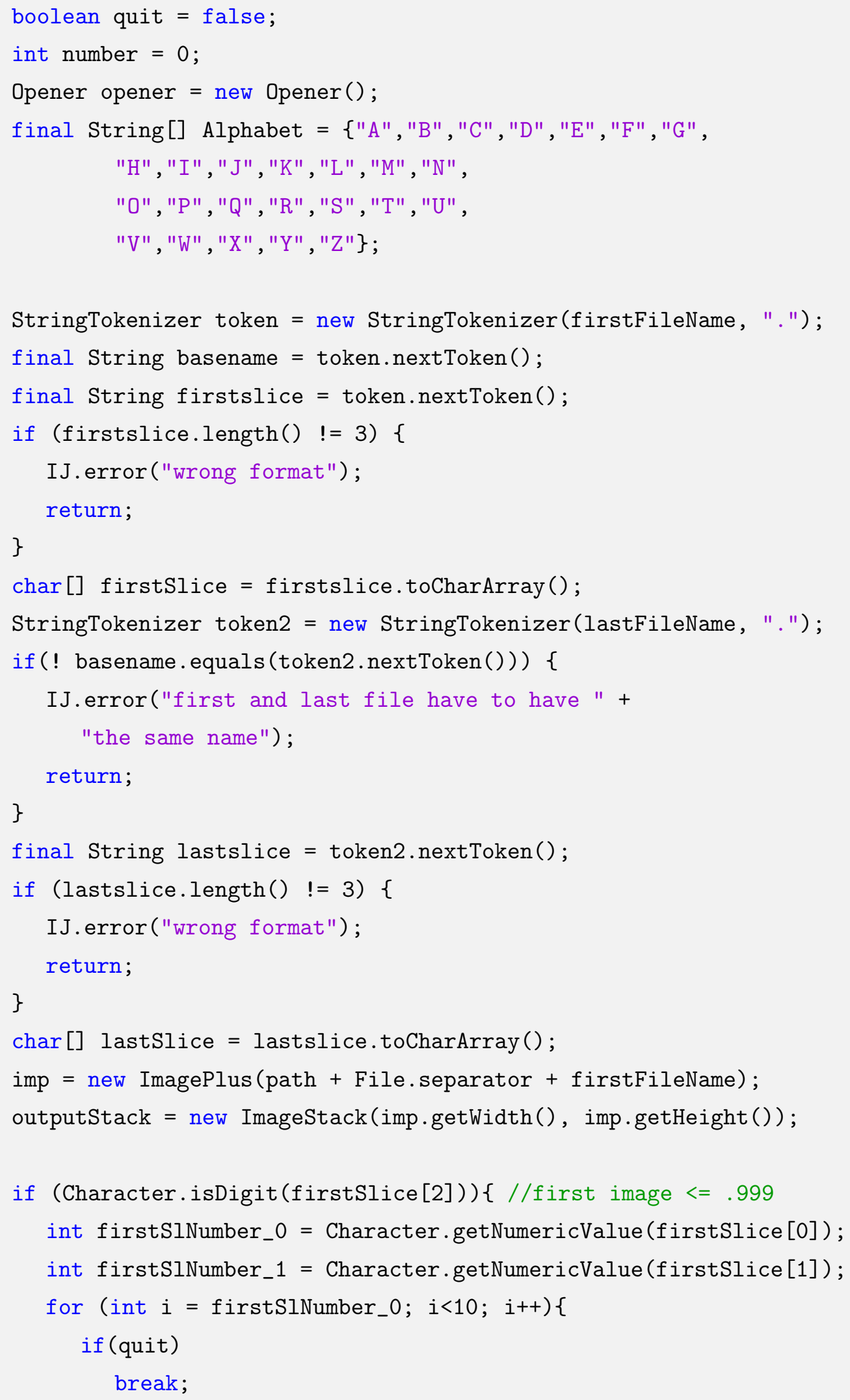




\section{A. The codes for data analysis}

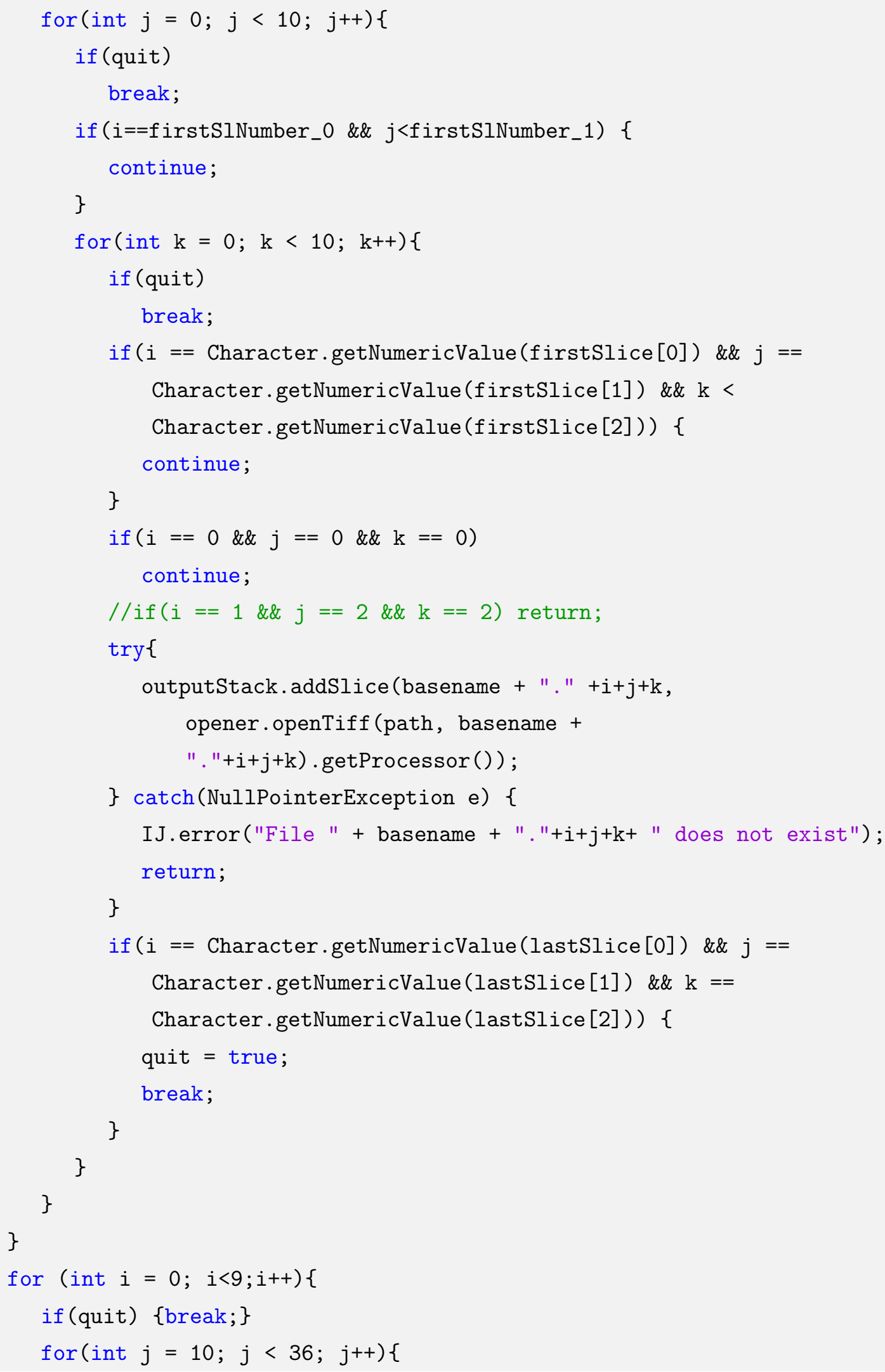




\section{A. The codes for data analysis}

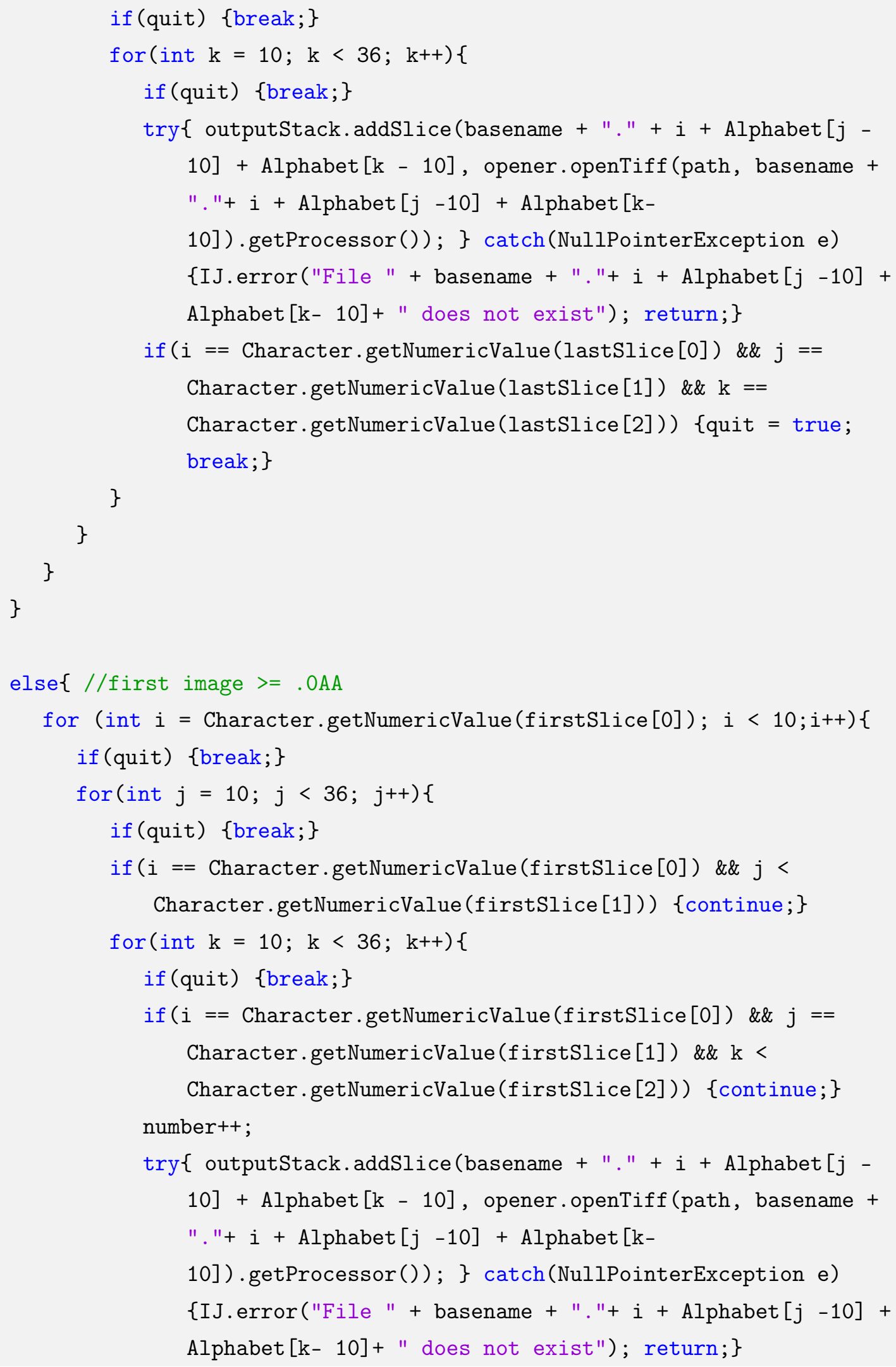




\section{A. The codes for data analysis}

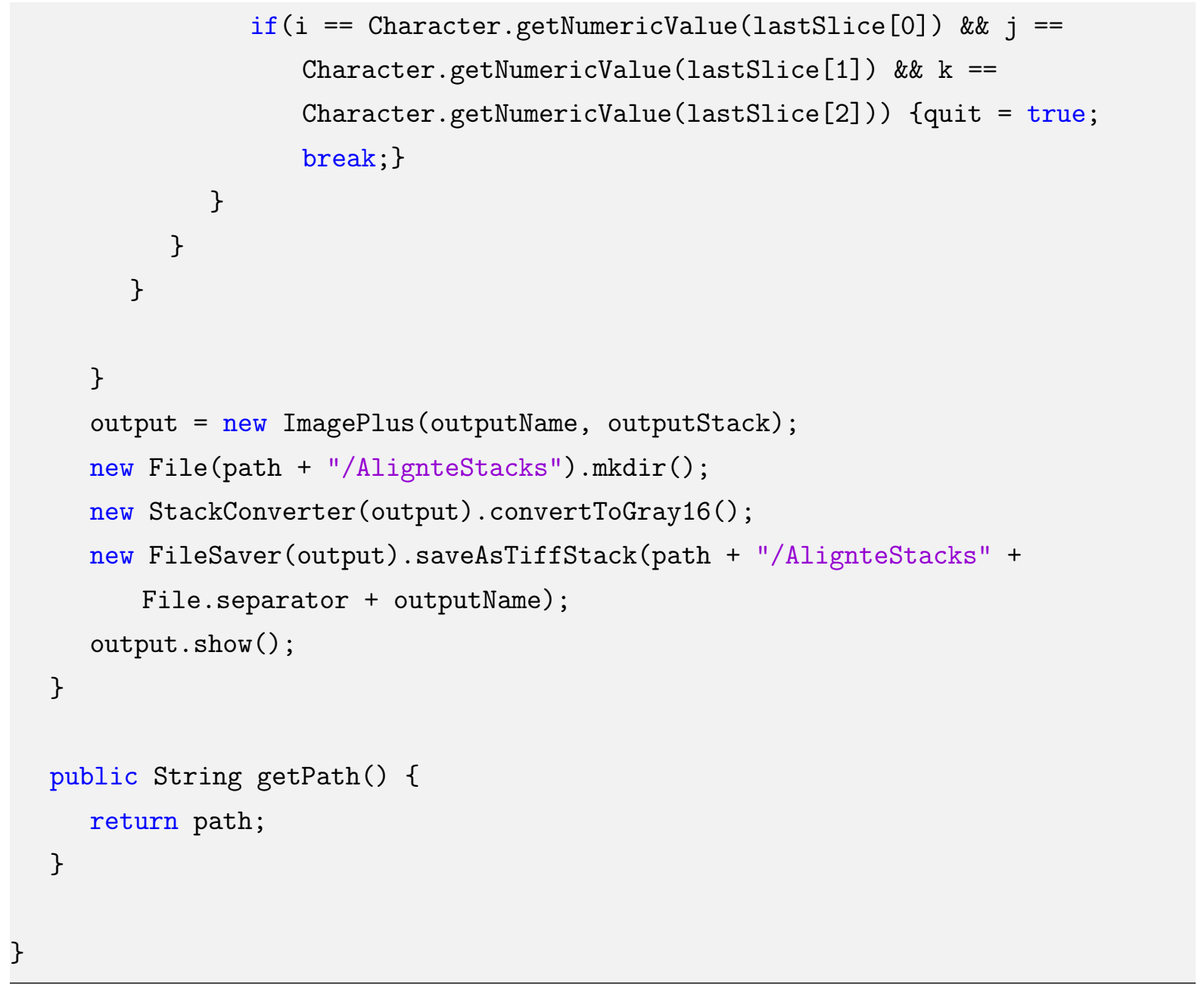




\section{A. The codes for data analysis}

\section{A.1.3. TwoPhoton Batch Matlab StackBuild Processing}

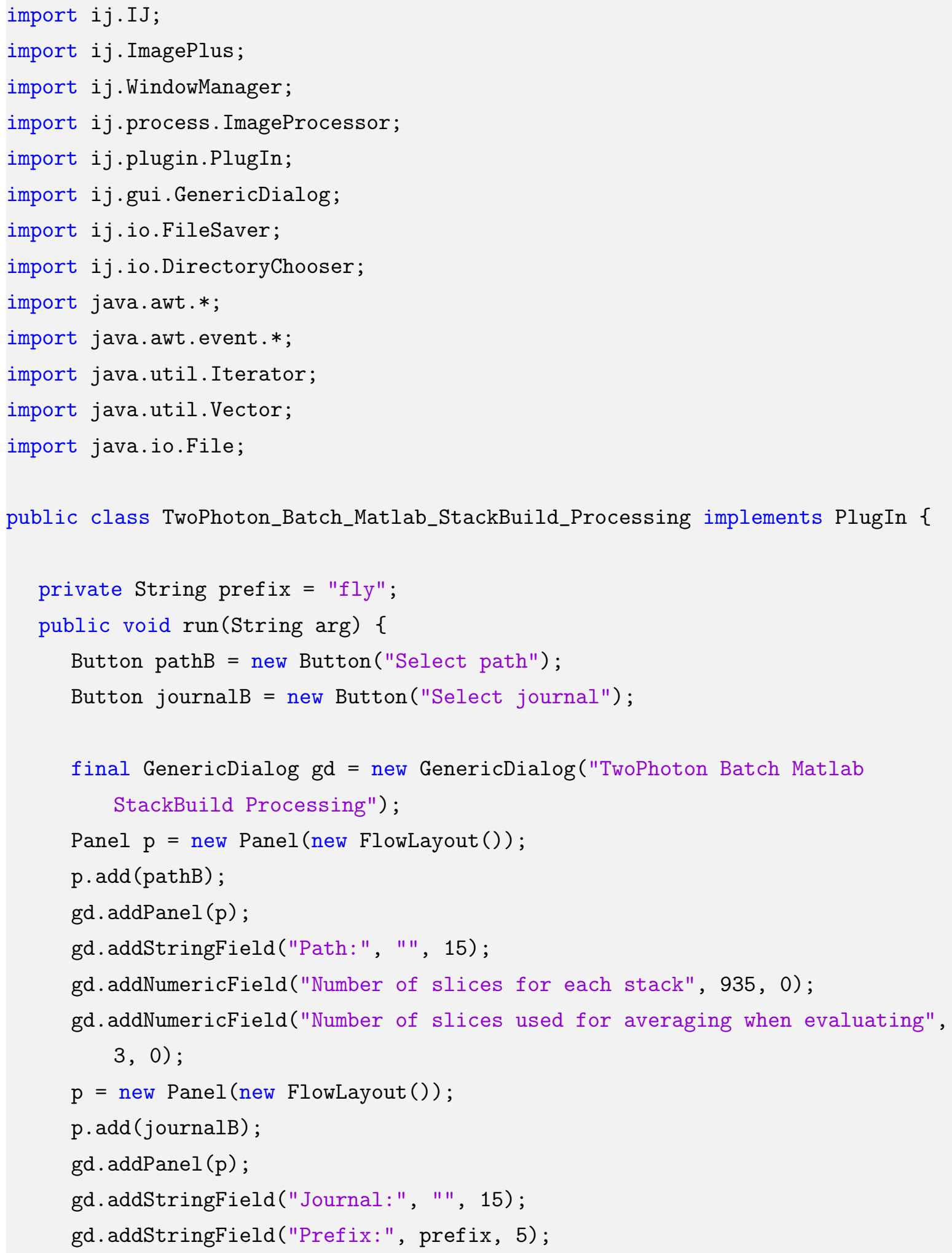




\section{A. The codes for data analysis}

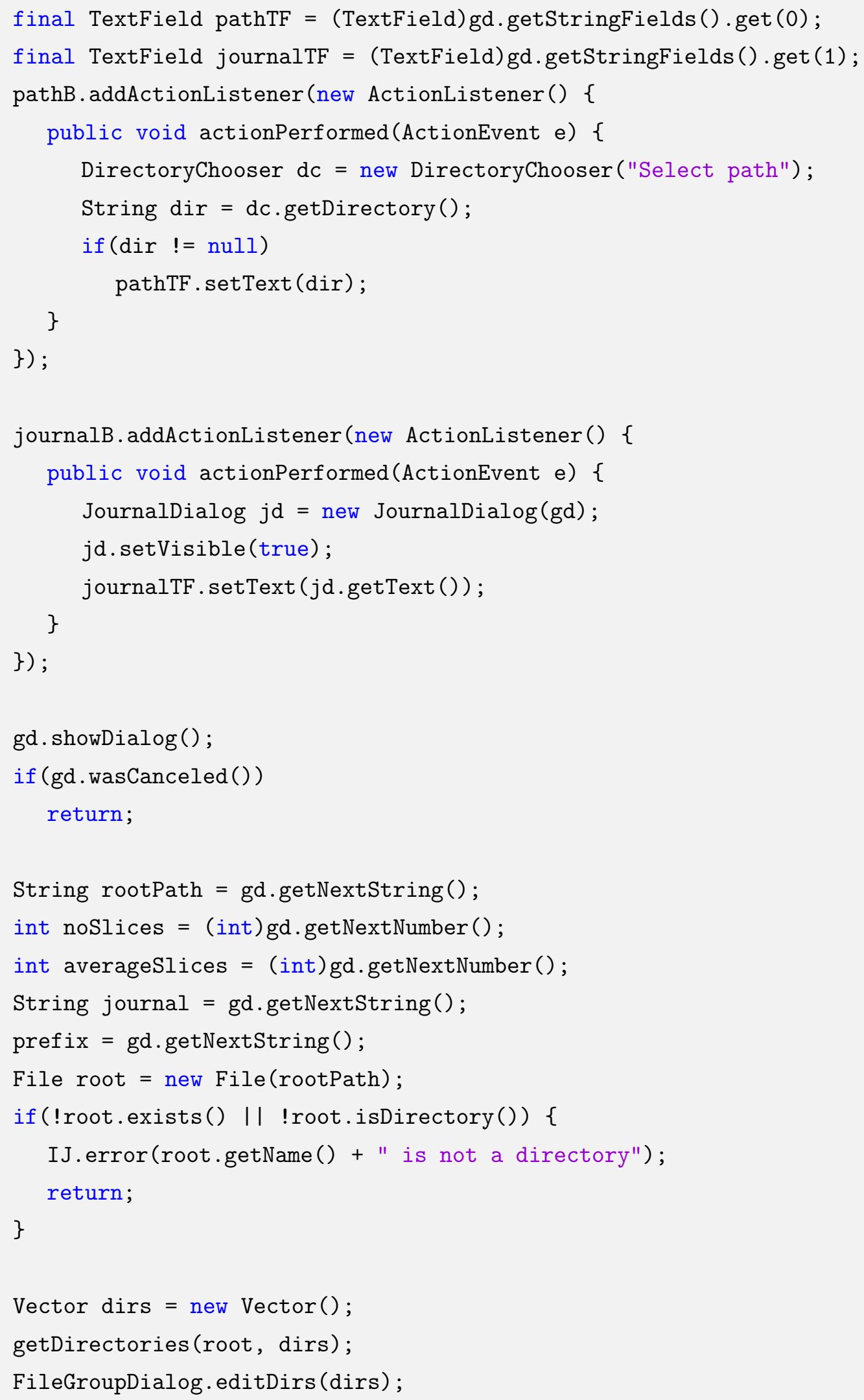




\section{A. The codes for data analysis}

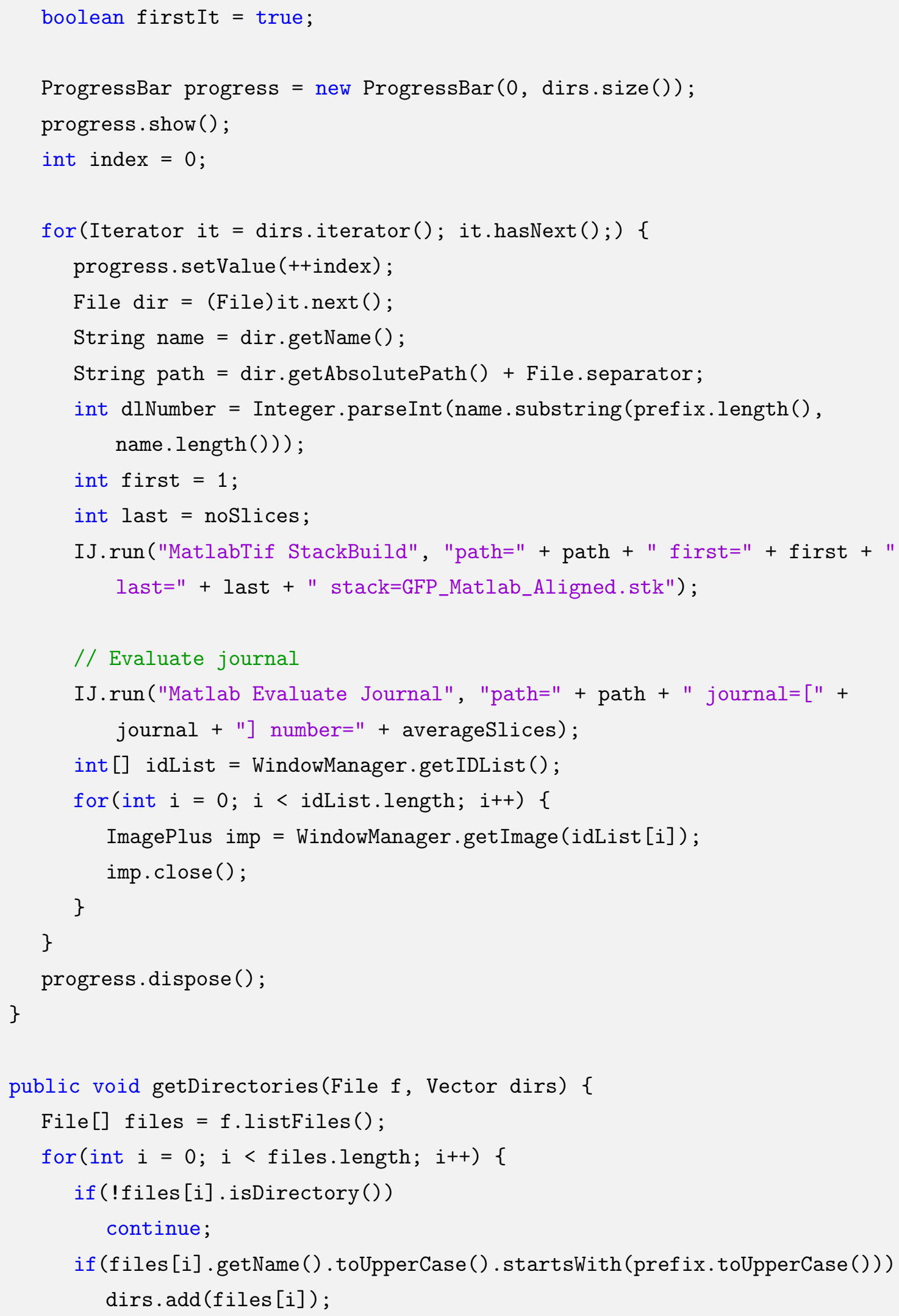




\section{A. The codes for data analysis}

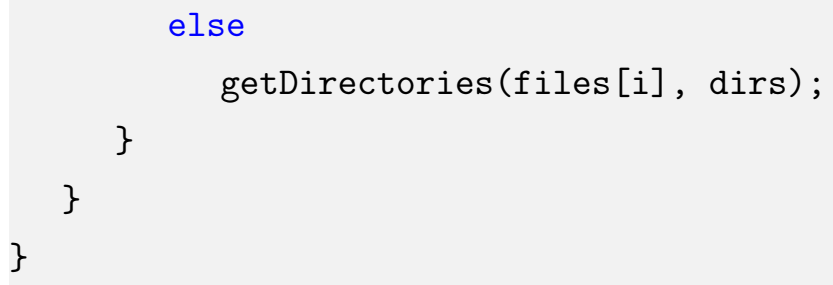




\section{A. The codes for data analysis}

\section{A.1.4. MatlabTif StackBuild}

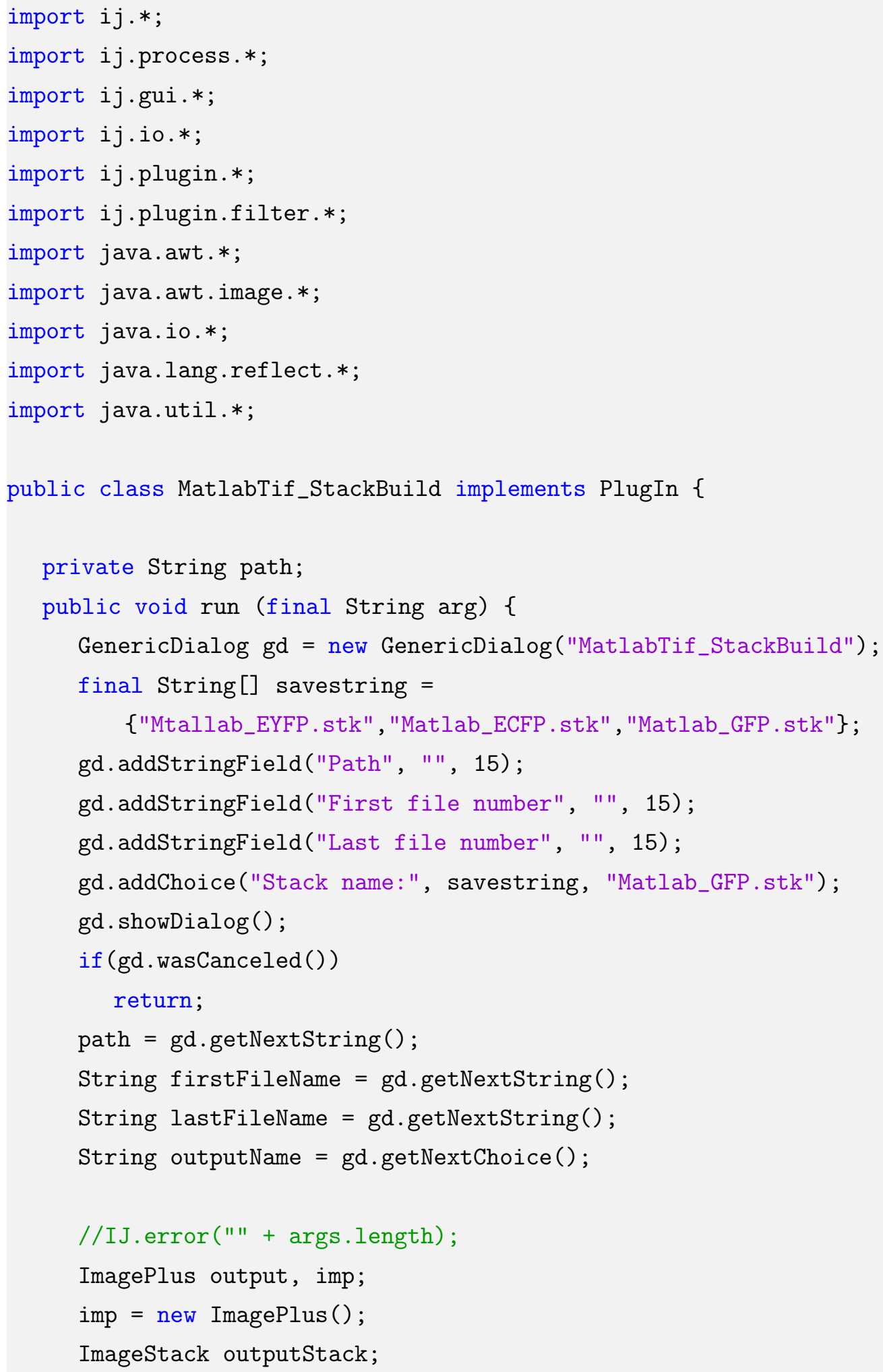




\section{A. The codes for data analysis}

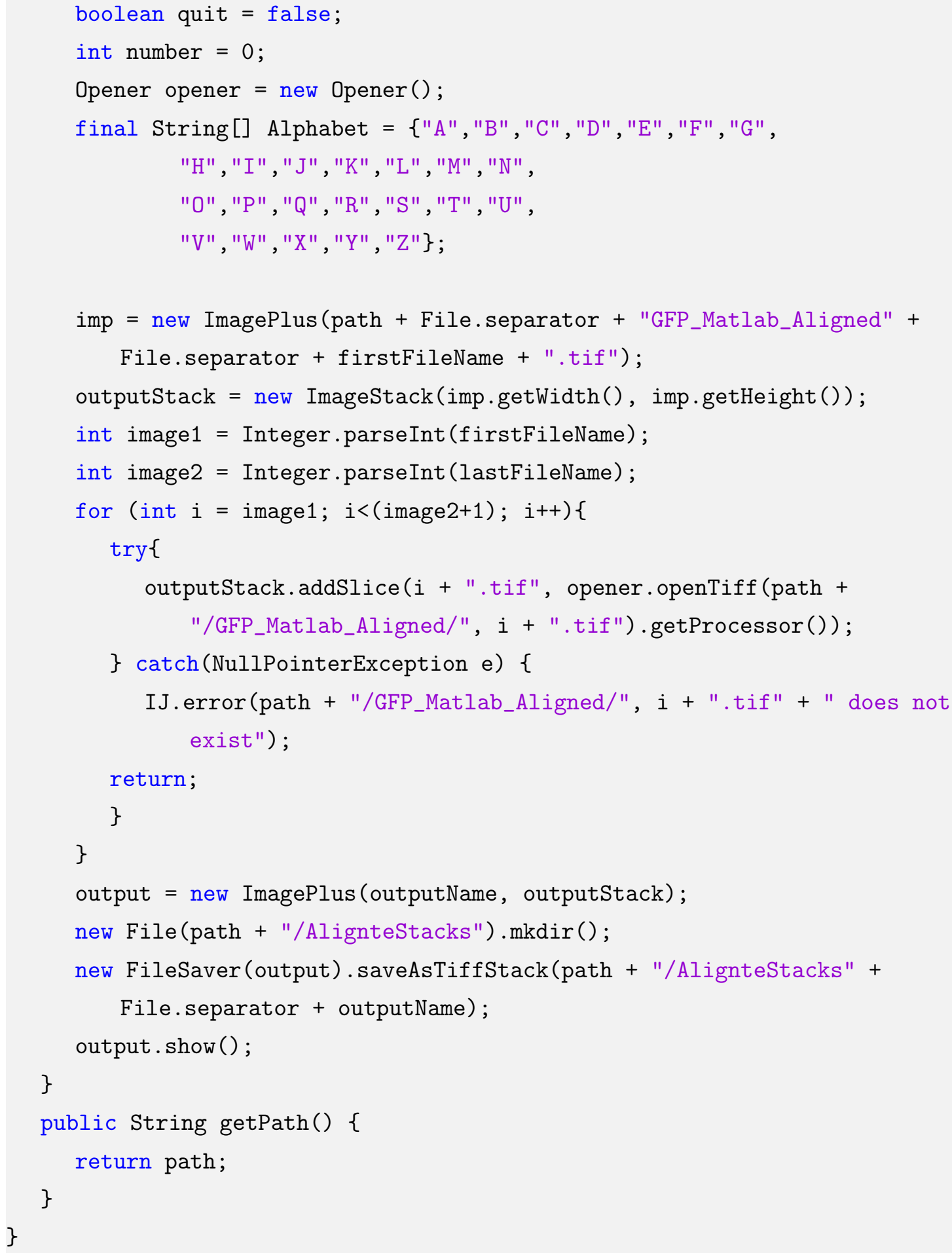




\section{A. The codes for data analysis}

\section{A.1.5. Matlab Evaluate Journal}

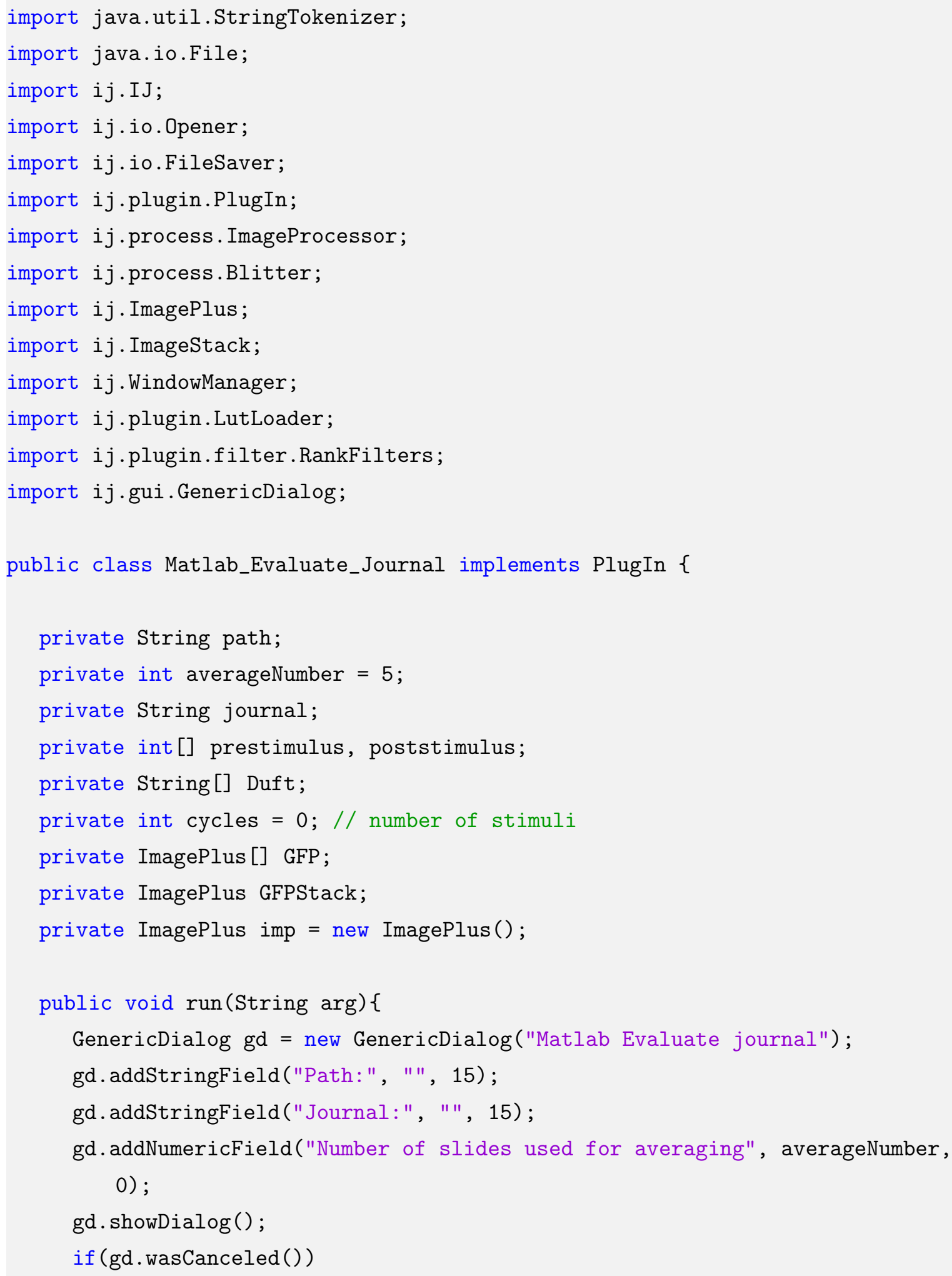




\section{A. The codes for data analysis}

return;

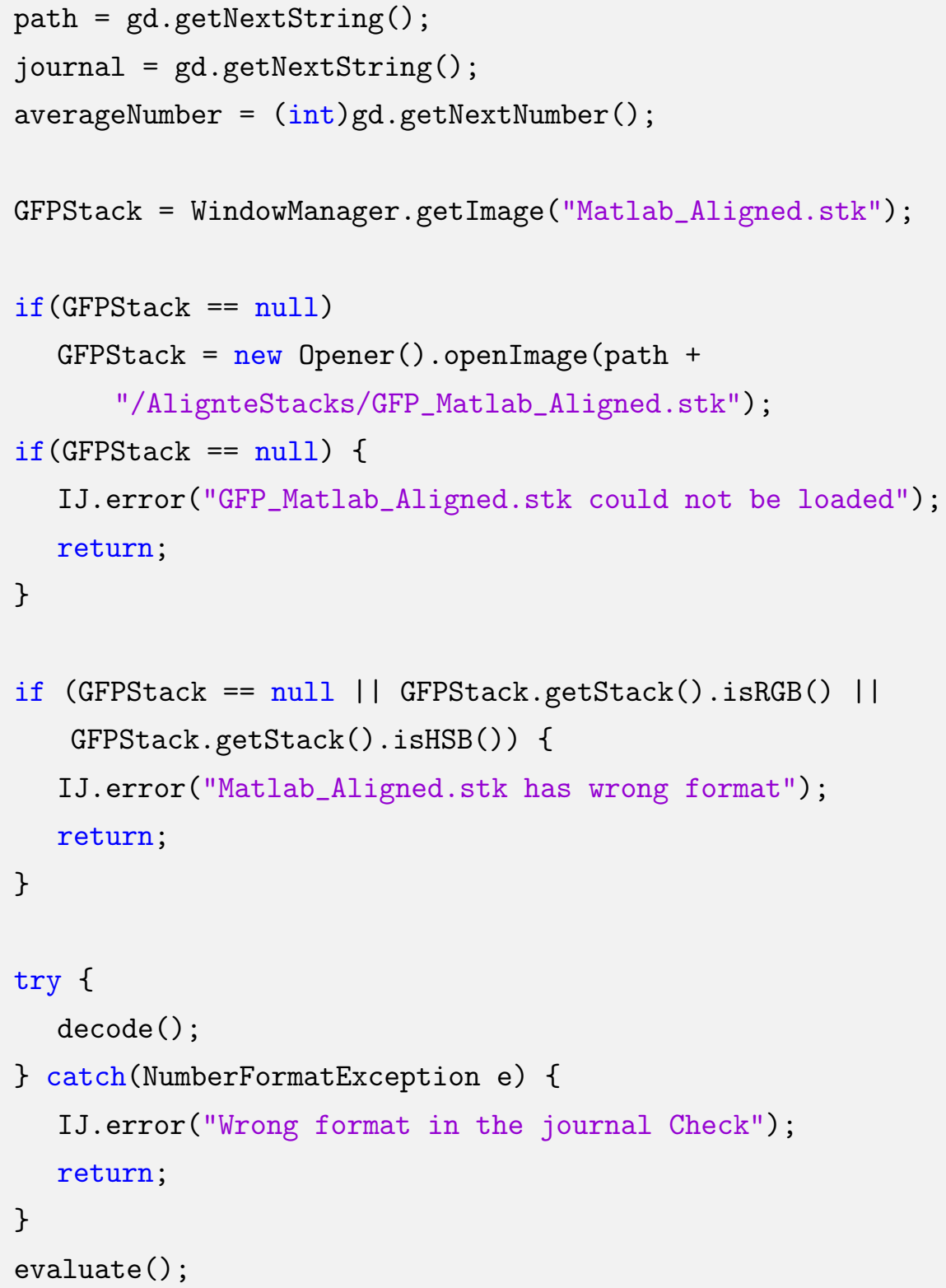




\section{A. The codes for data analysis}

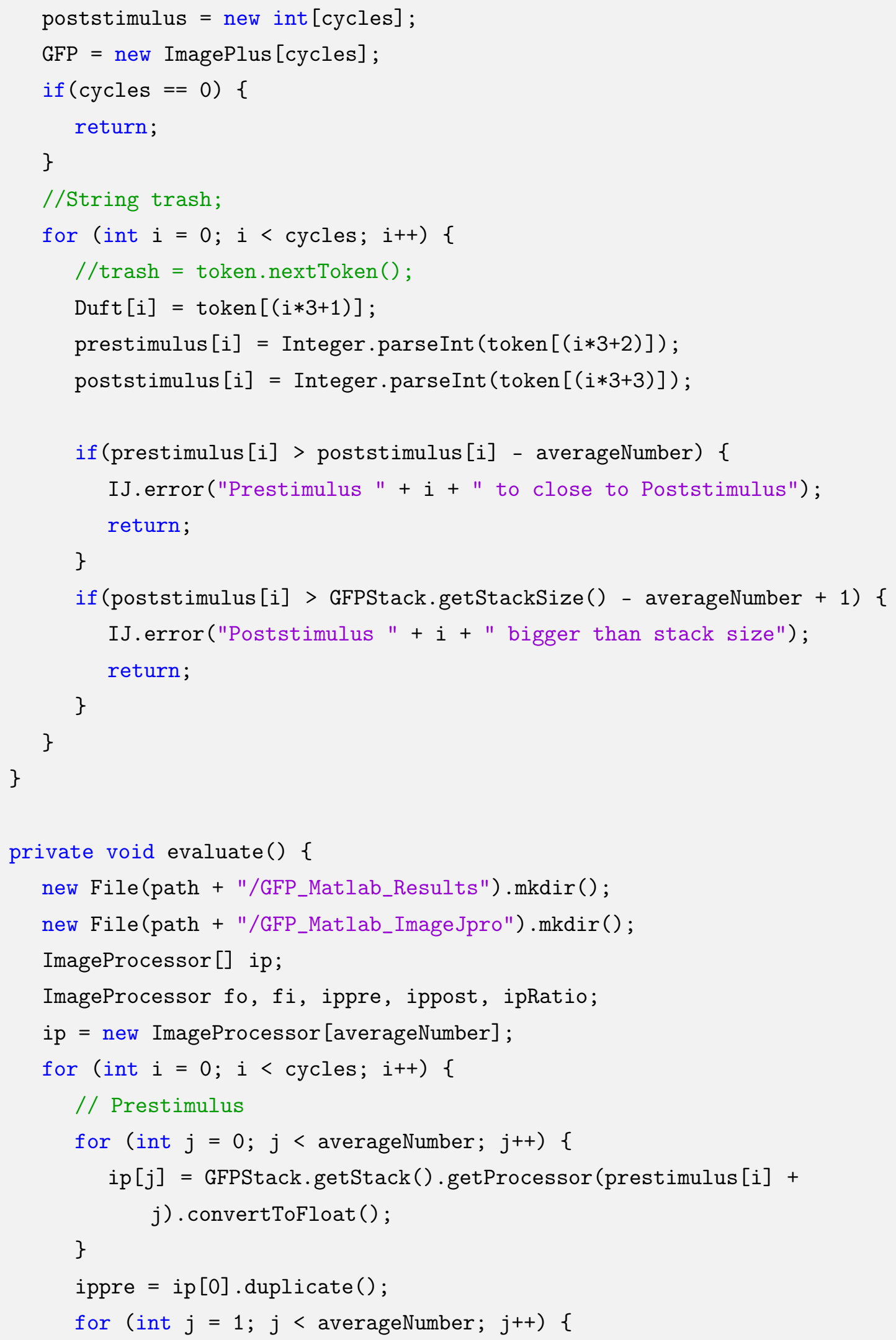




\section{A. The codes for data analysis}

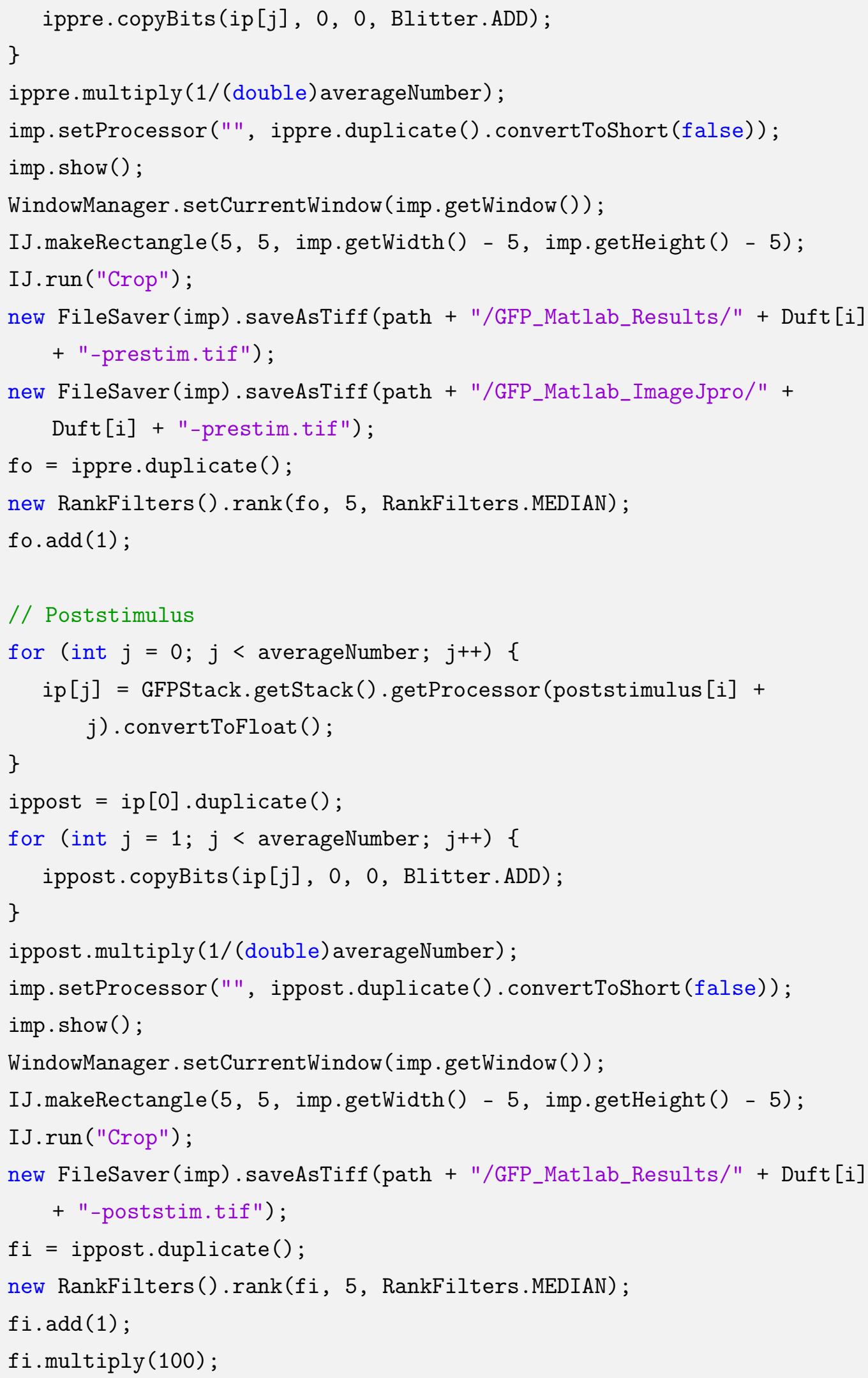




\section{A. The codes for data analysis}

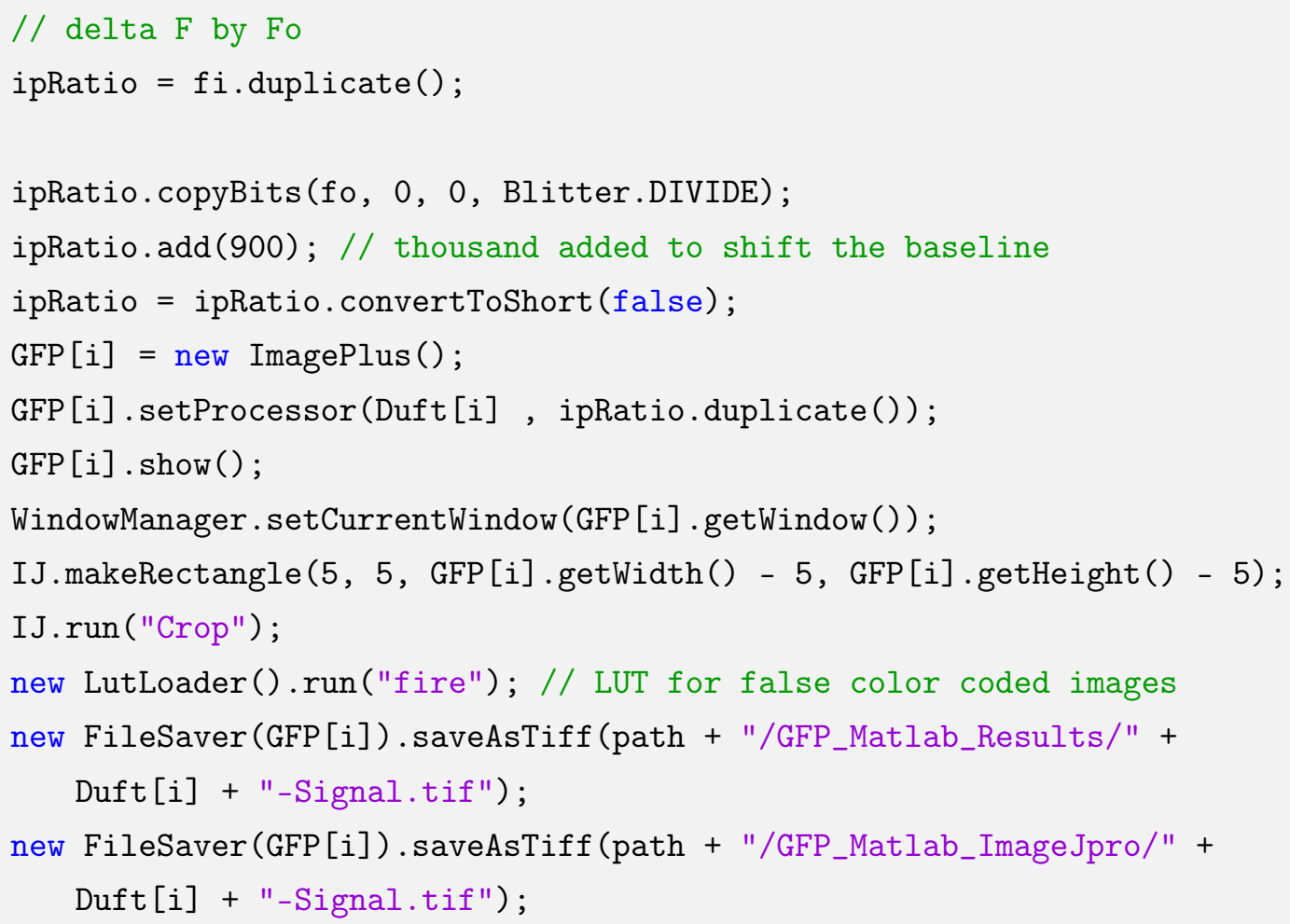




\section{A. The codes for data analysis}

\section{A.1.6. TwoPhoton Batch Z StackBuild}

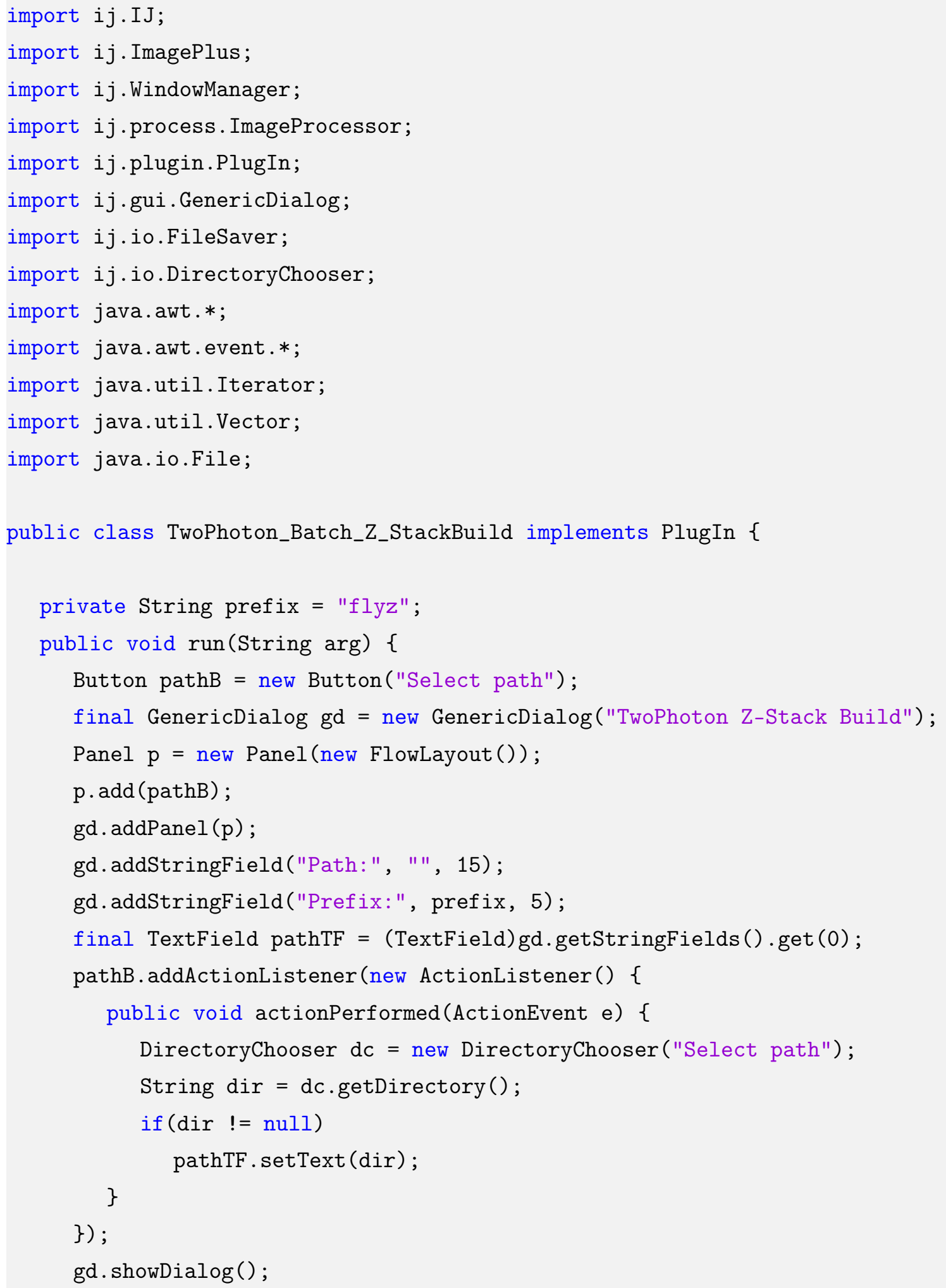




\section{A. The codes for data analysis}

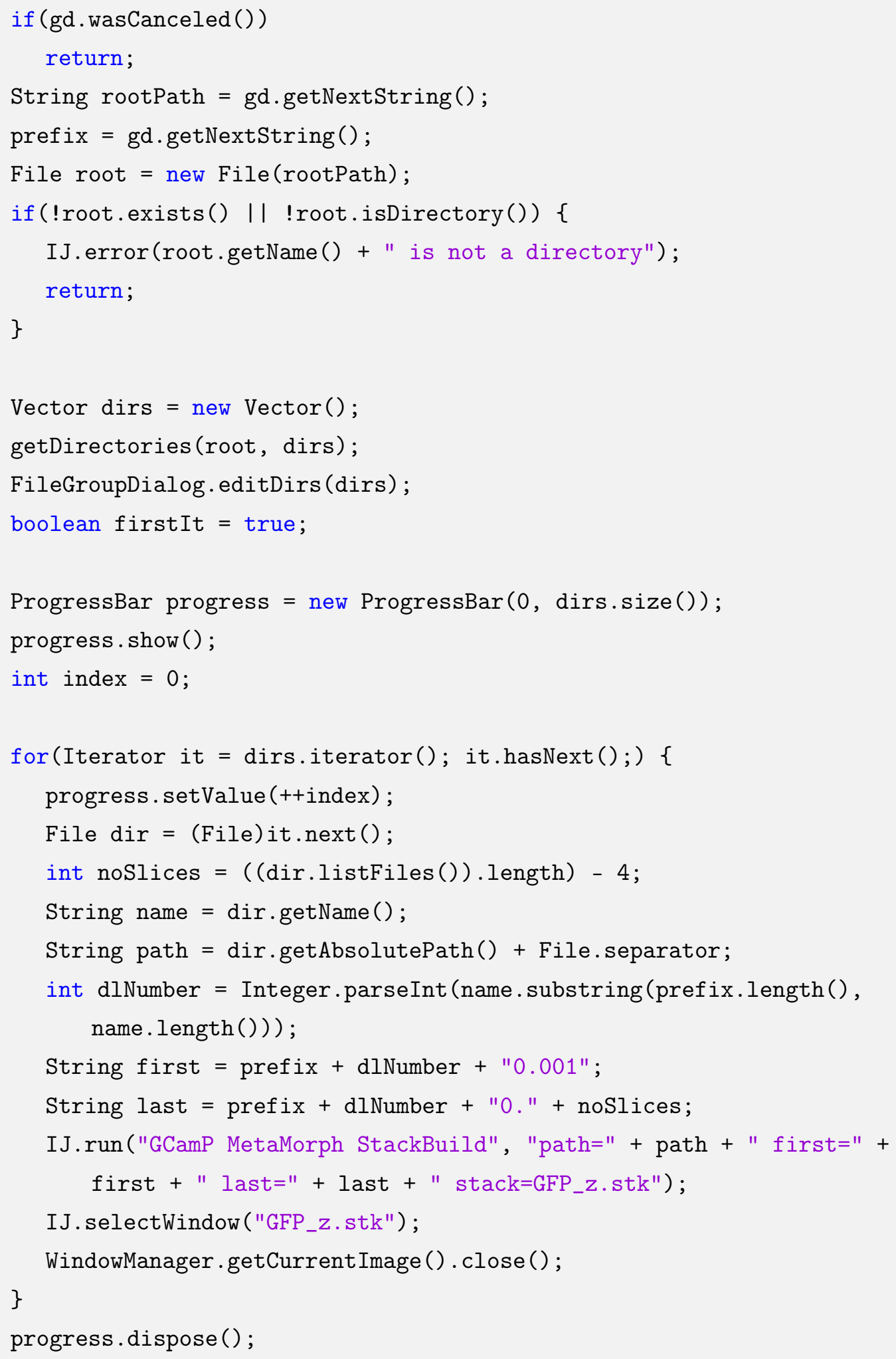




\section{A. The codes for data analysis}

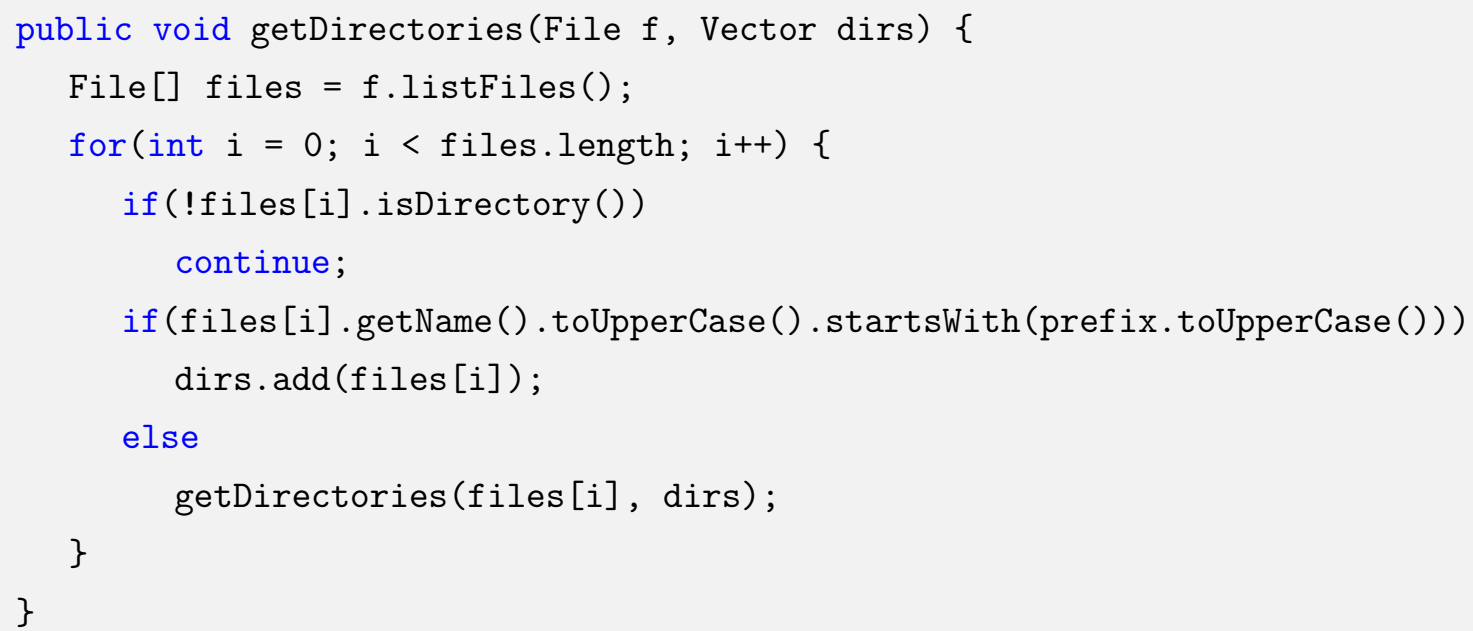




\section{A. The codes for data analysis}

\section{A.1.7. TwoPhoton Batch Processing}

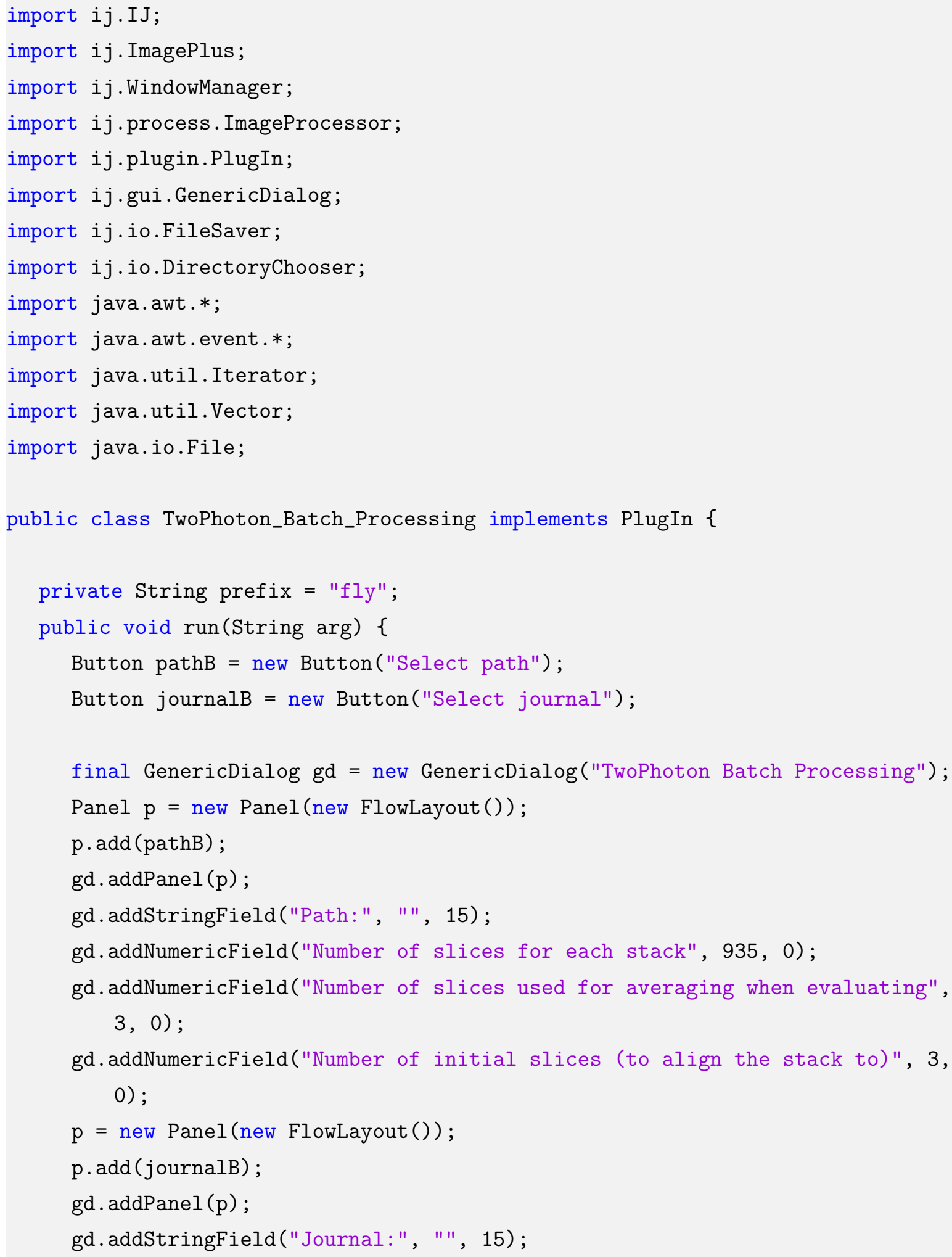




\section{A. The codes for data analysis}

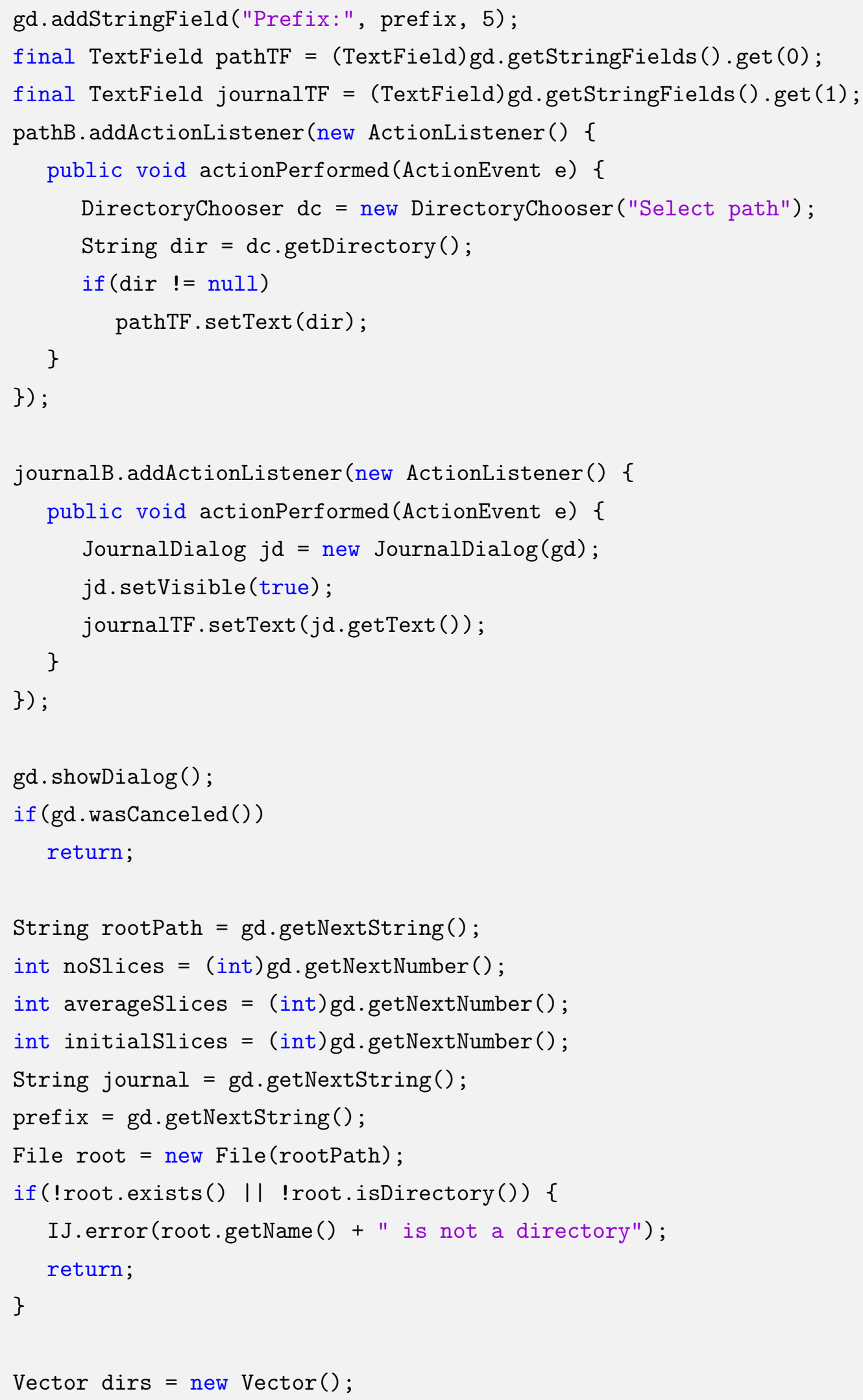




\section{A. The codes for data analysis}

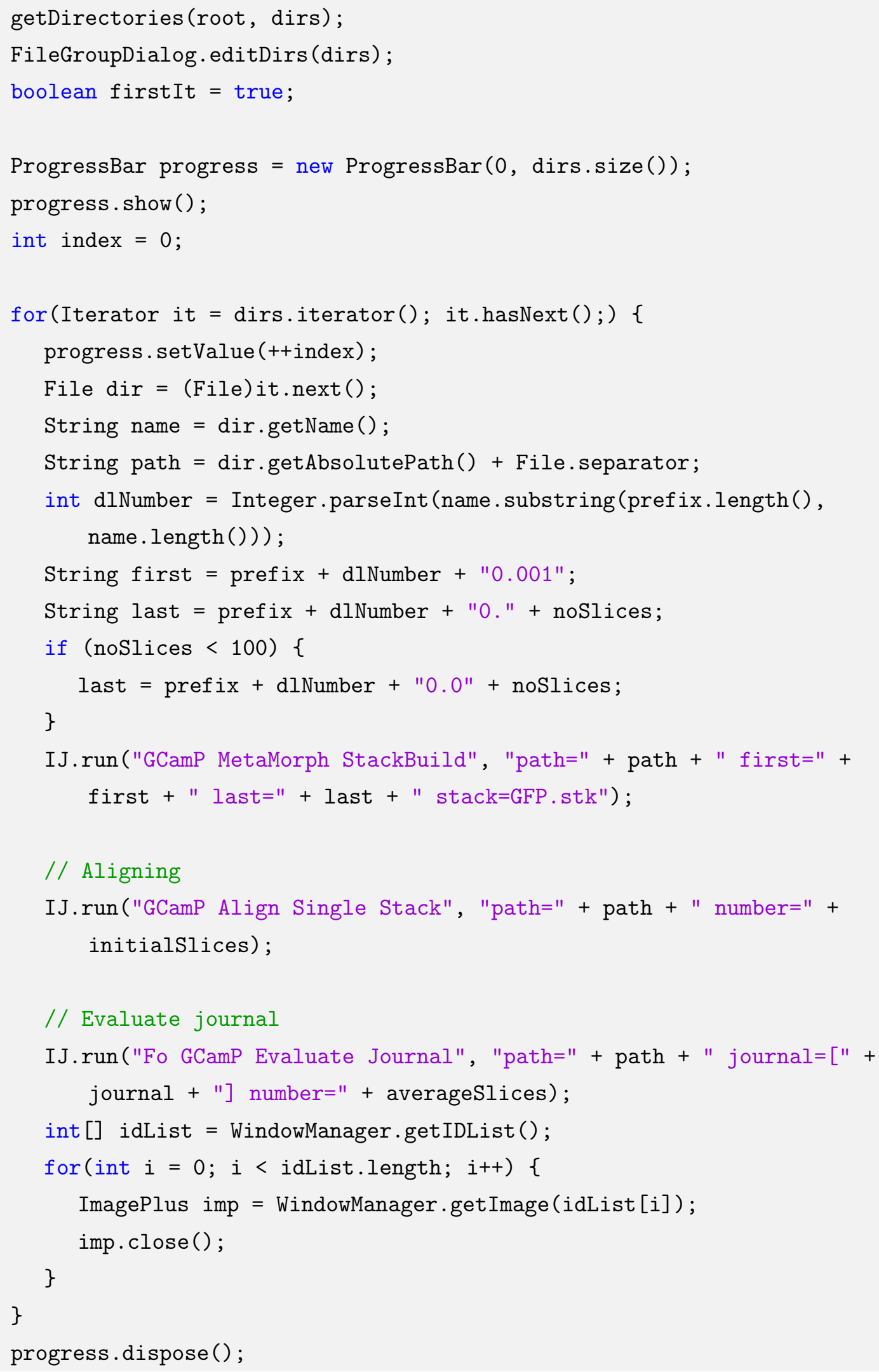




\section{A. The codes for data analysis}

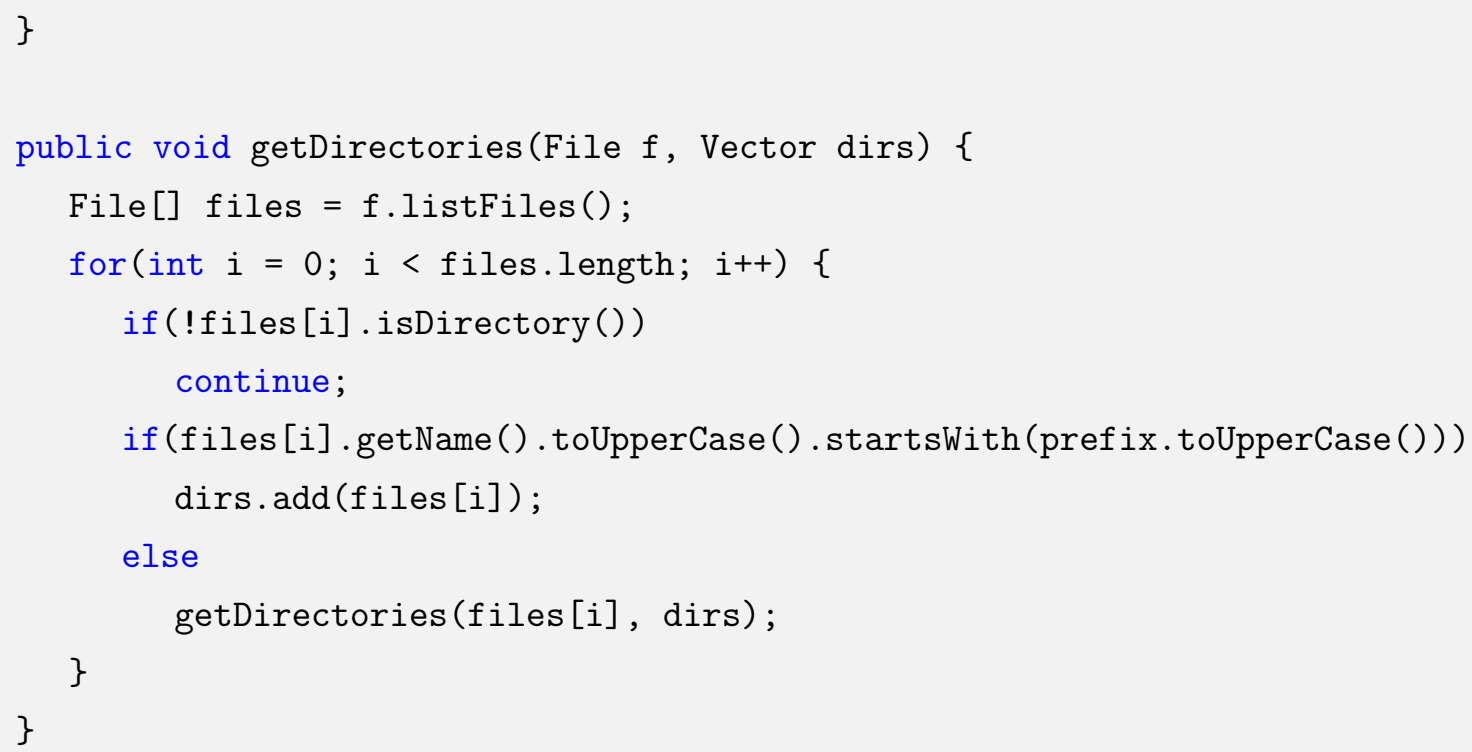




\section{A. The codes for data analysis}

\section{A.1.8. GCamP Align Single Stack}

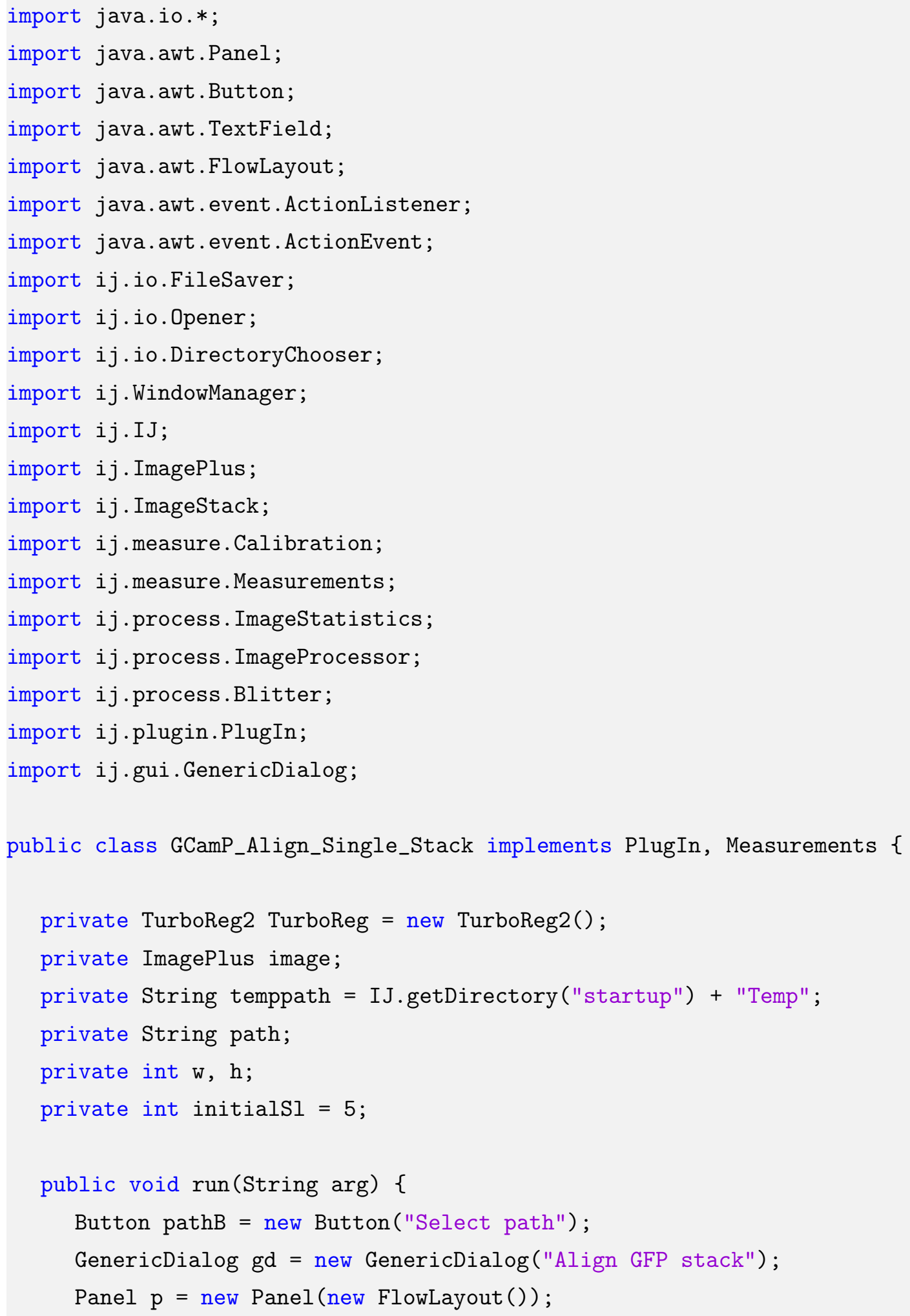




\section{A. The codes for data analysis}

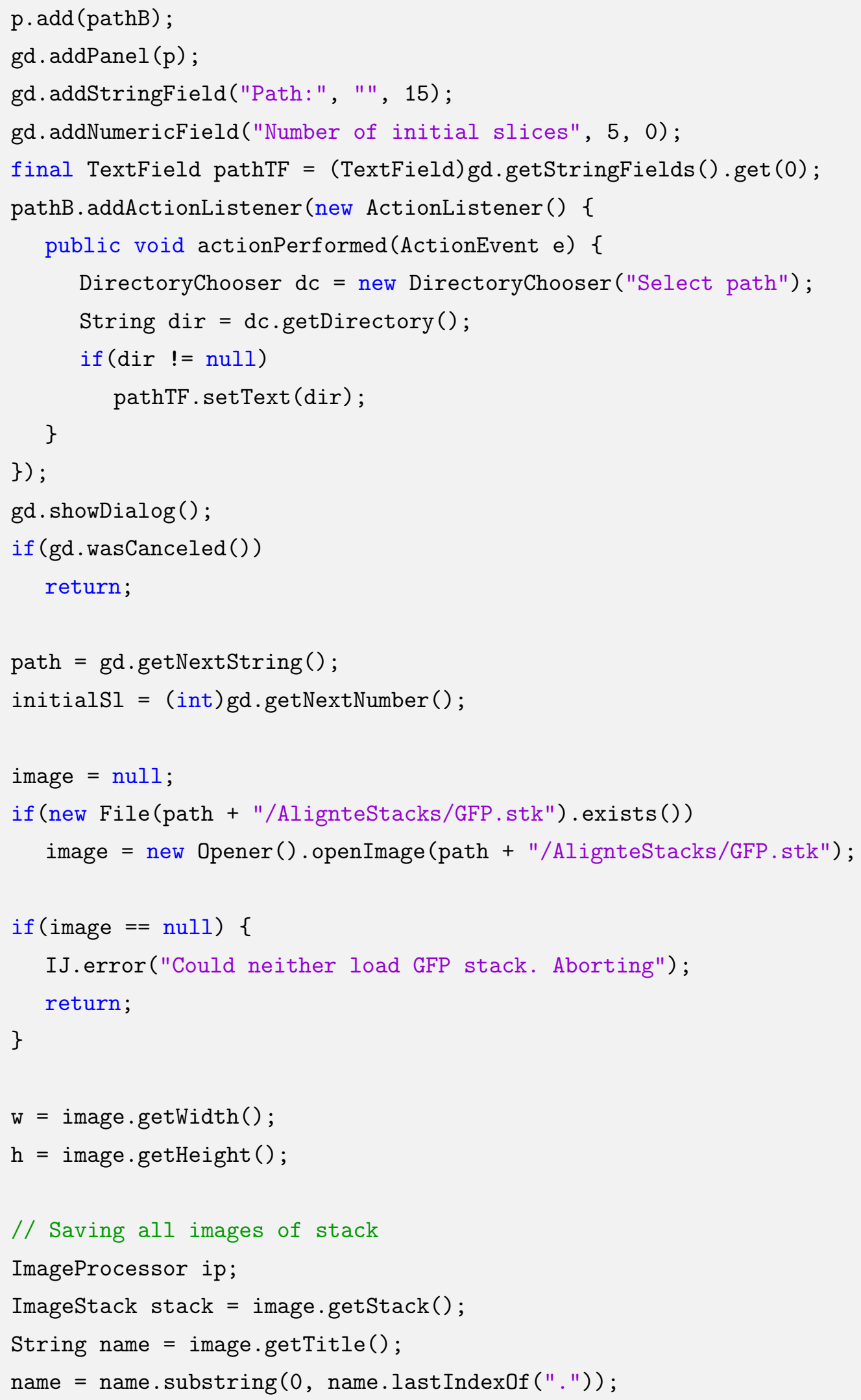




\section{A. The codes for data analysis}

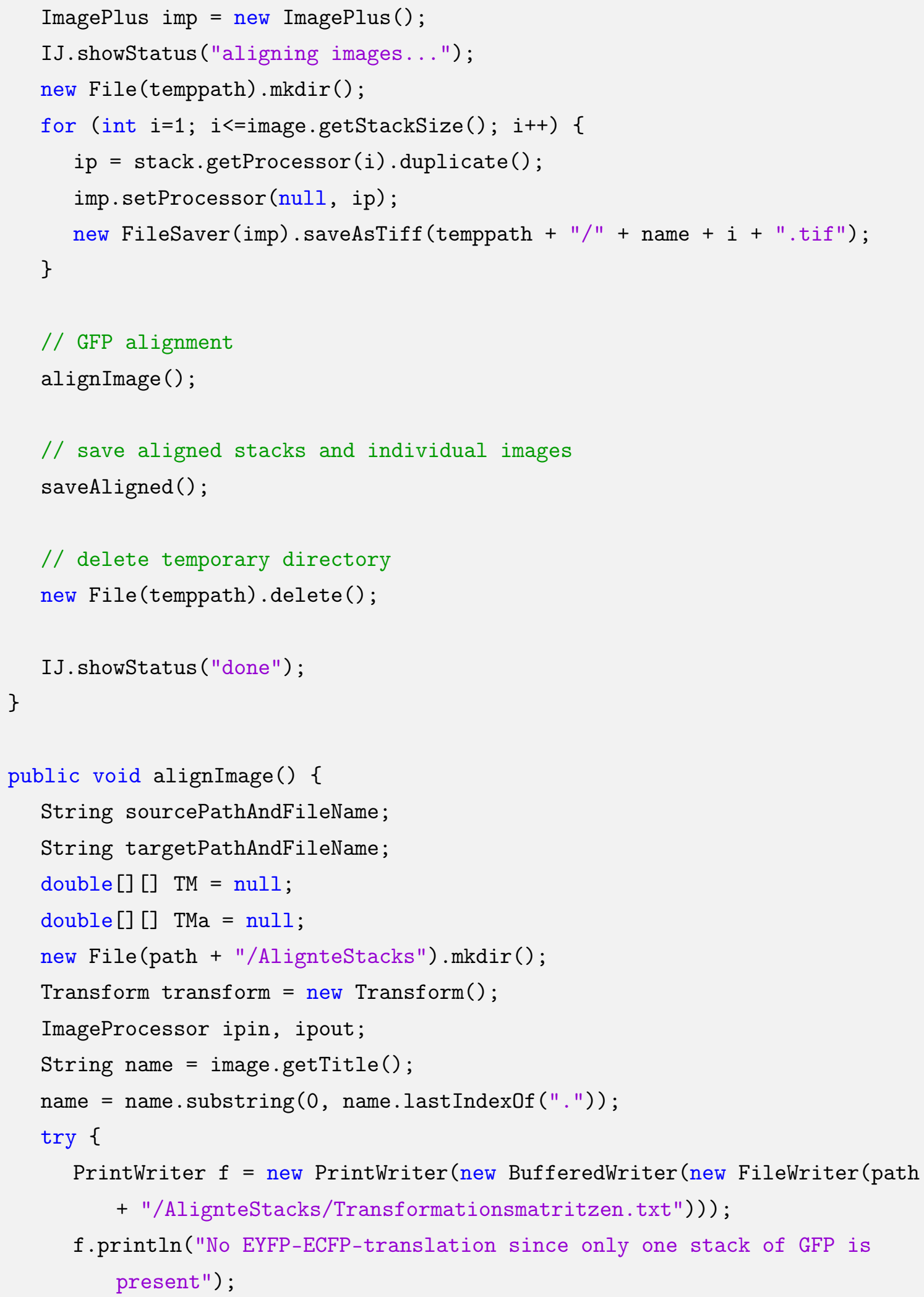




\section{A. The codes for data analysis}

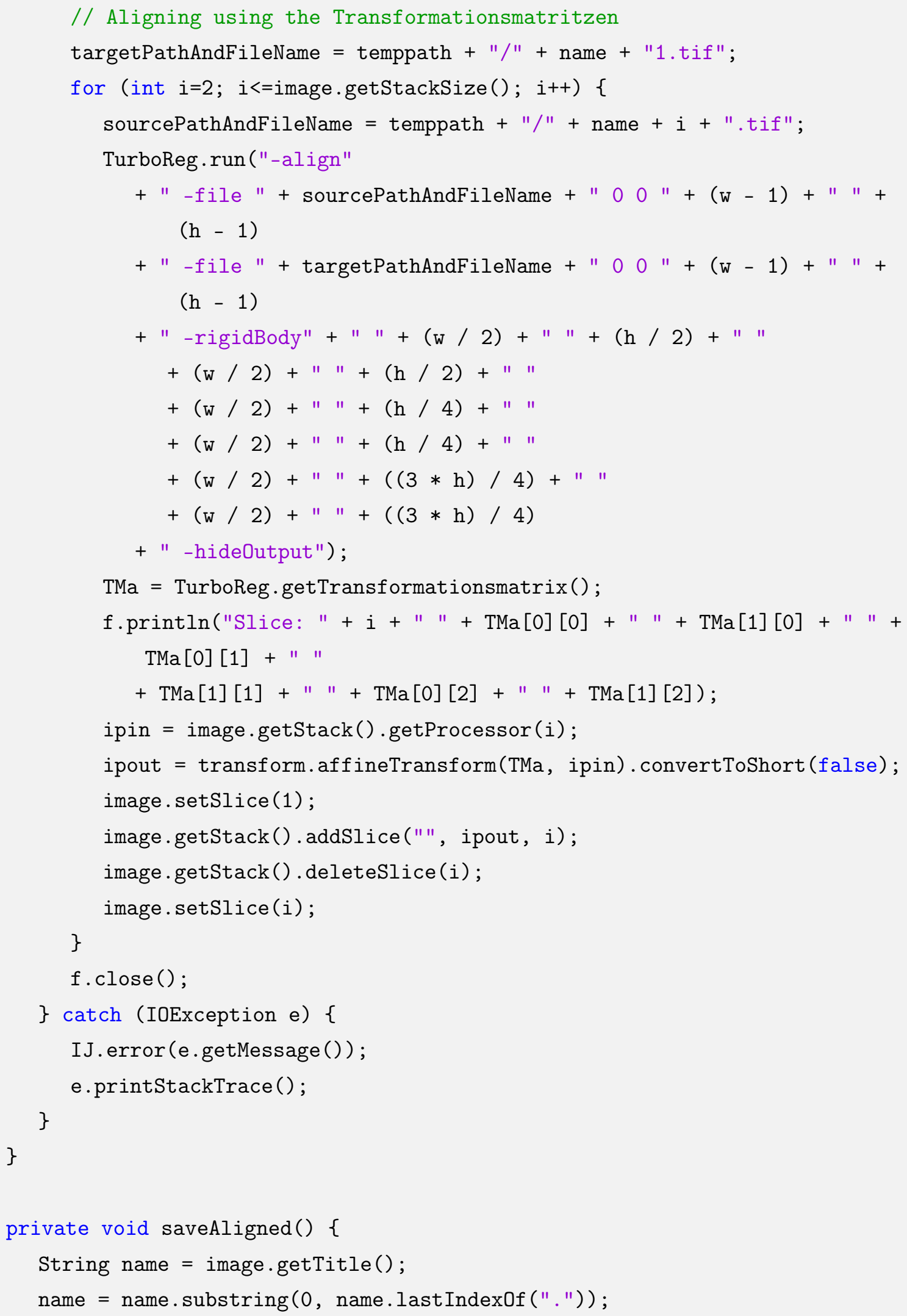




\section{A. The codes for data analysis}

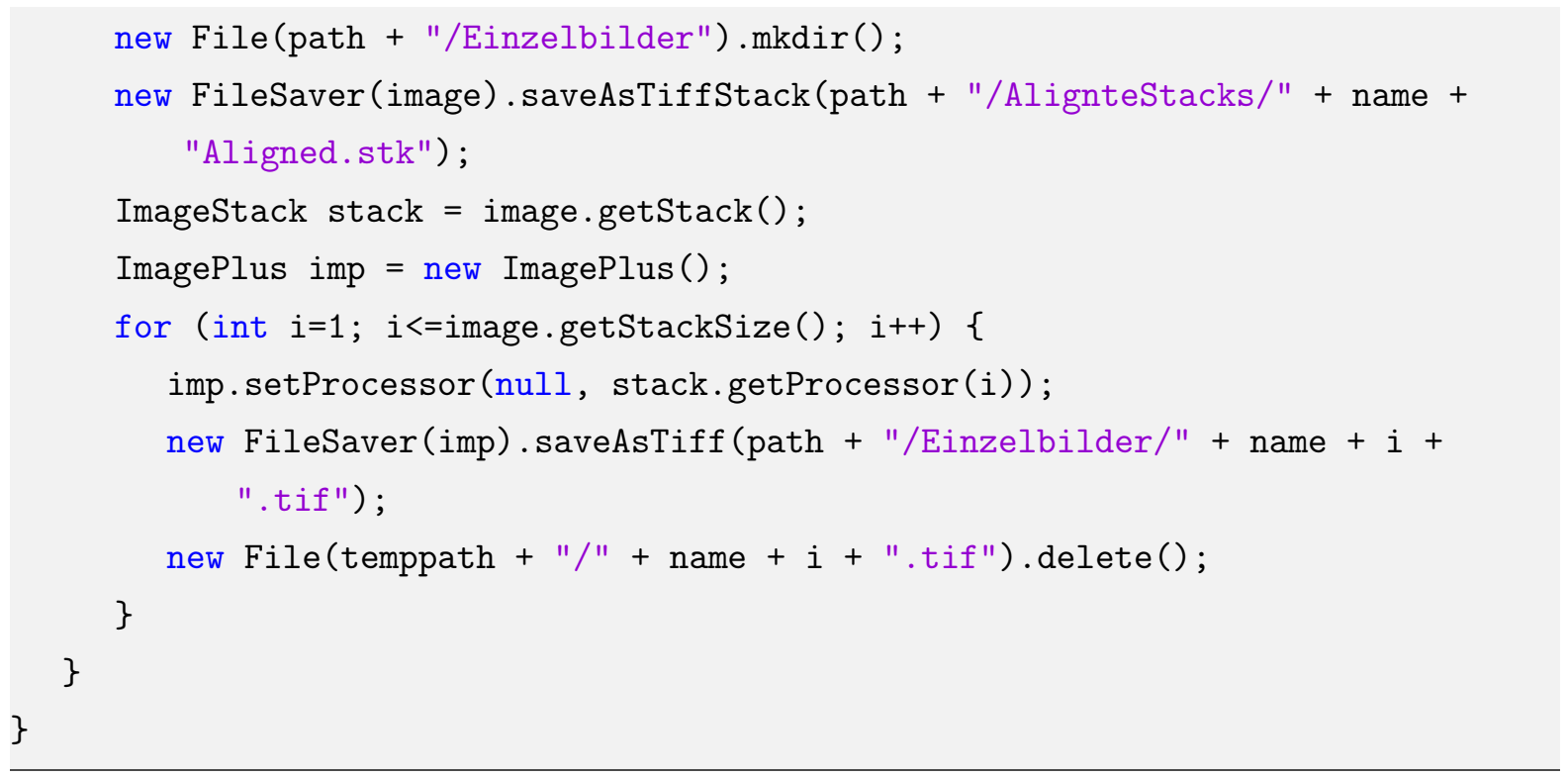




\section{A. The codes for data analysis}

\section{A.1.9. Fo GCamP Evaluate Journal}

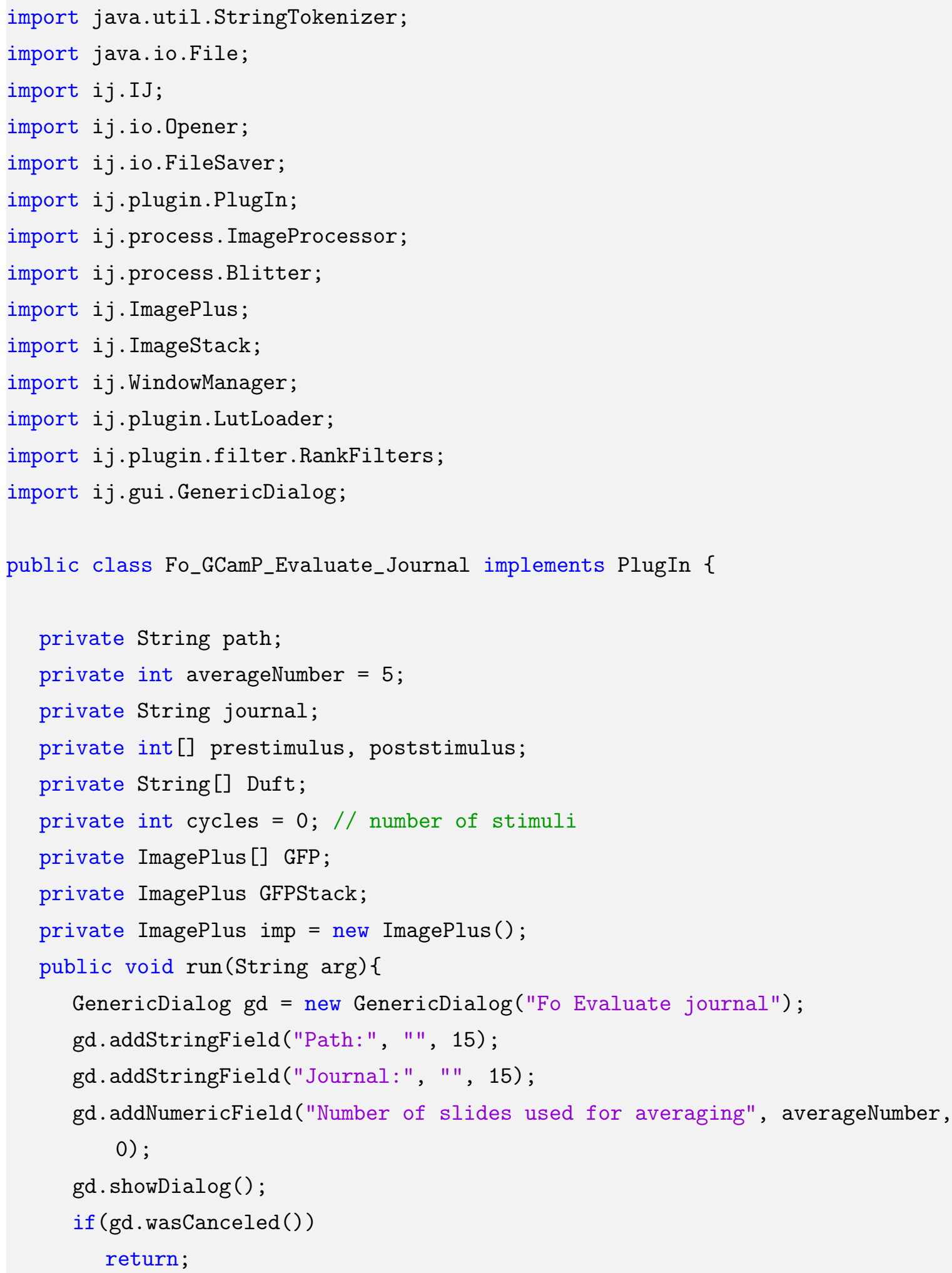




\section{A. The codes for data analysis}

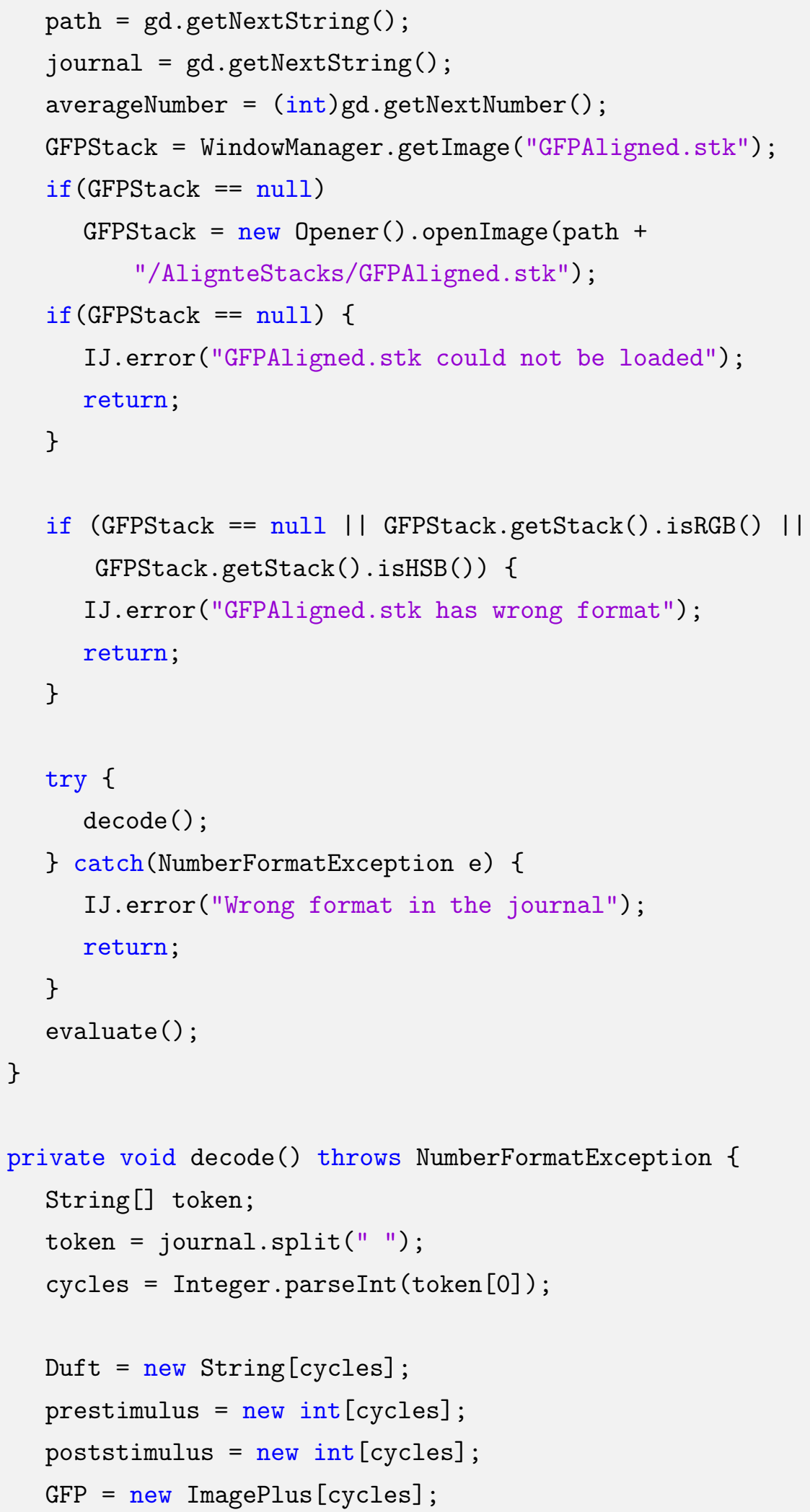




\section{A. The codes for data analysis}

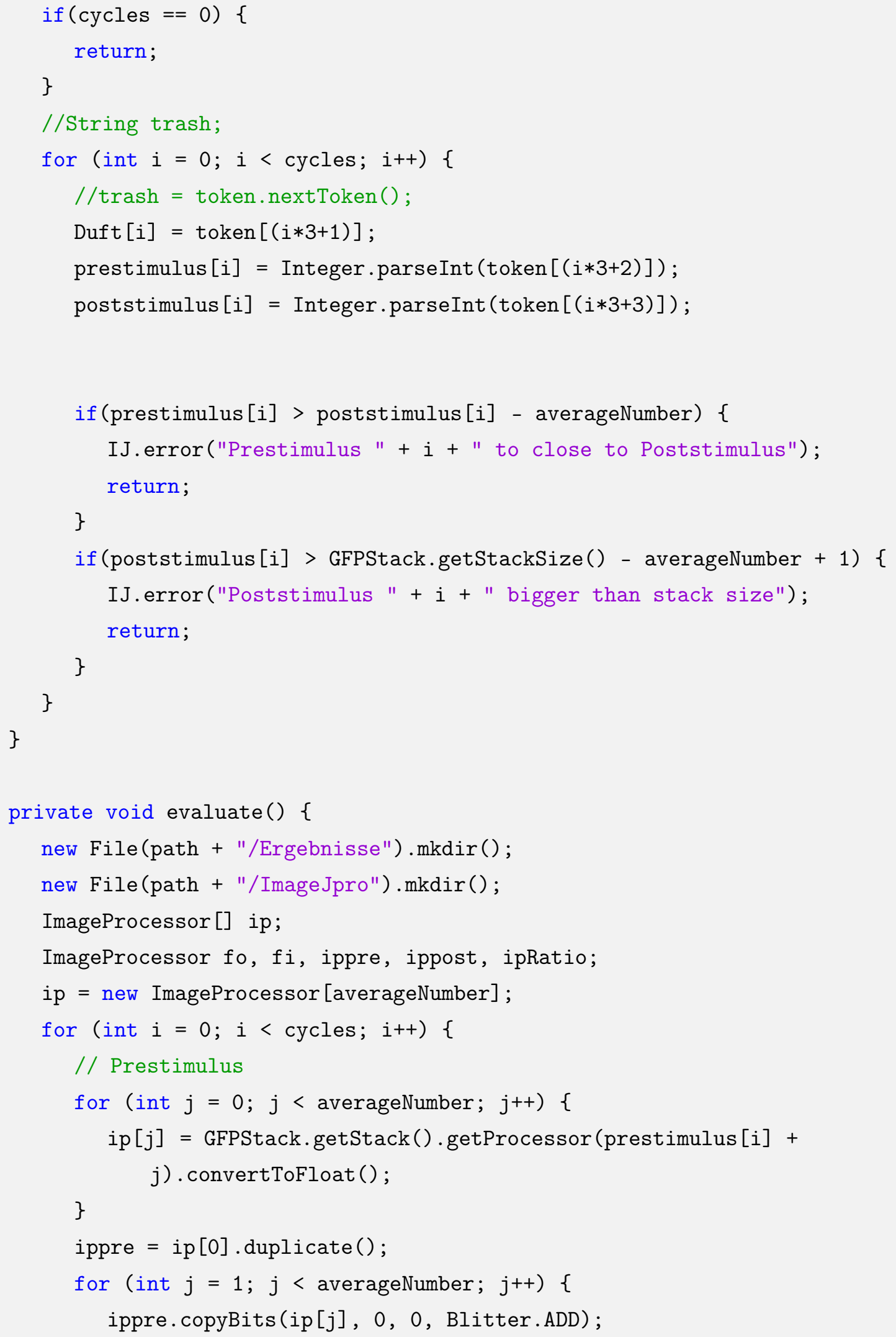




\section{A. The codes for data analysis}

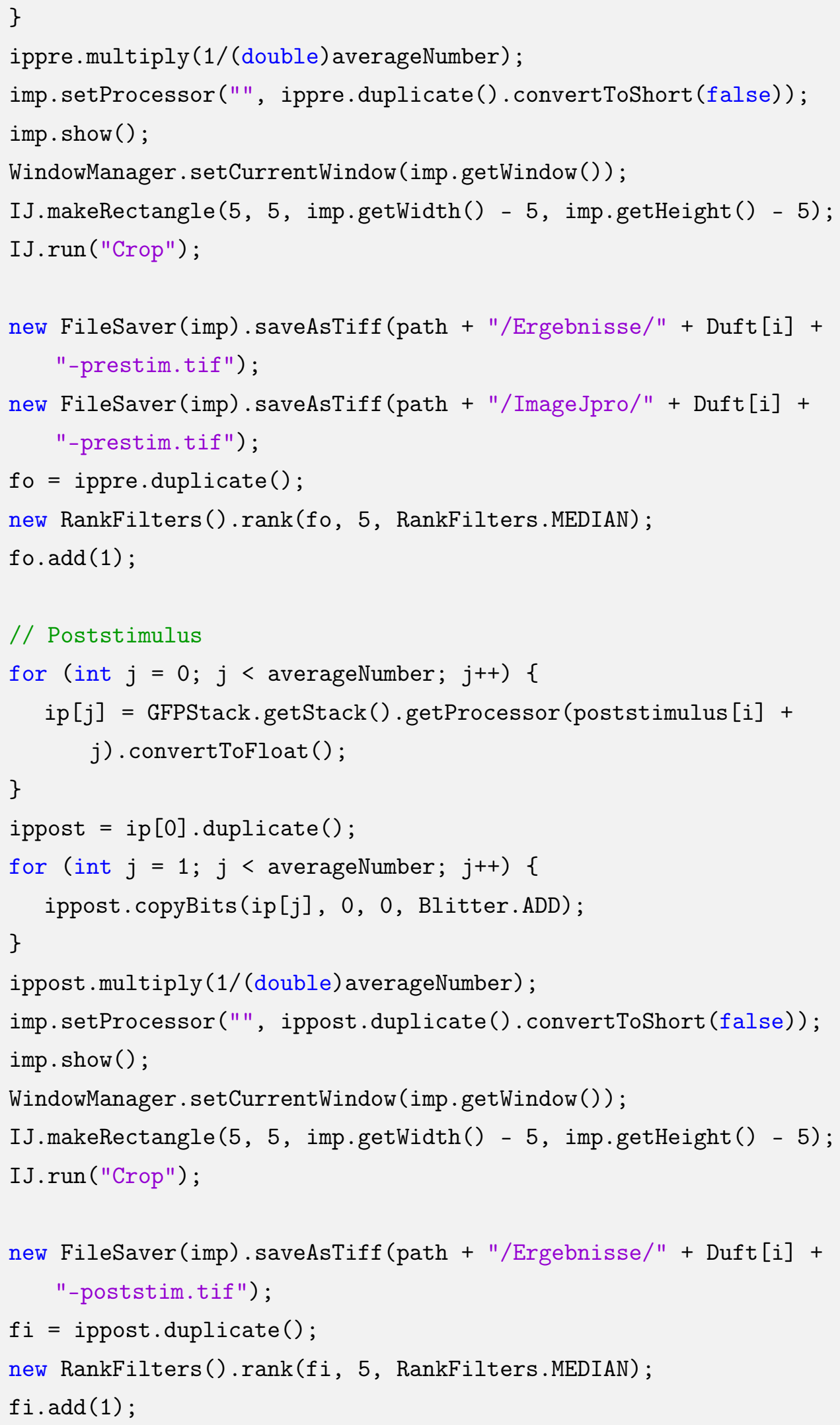




\section{A. The codes for data analysis}

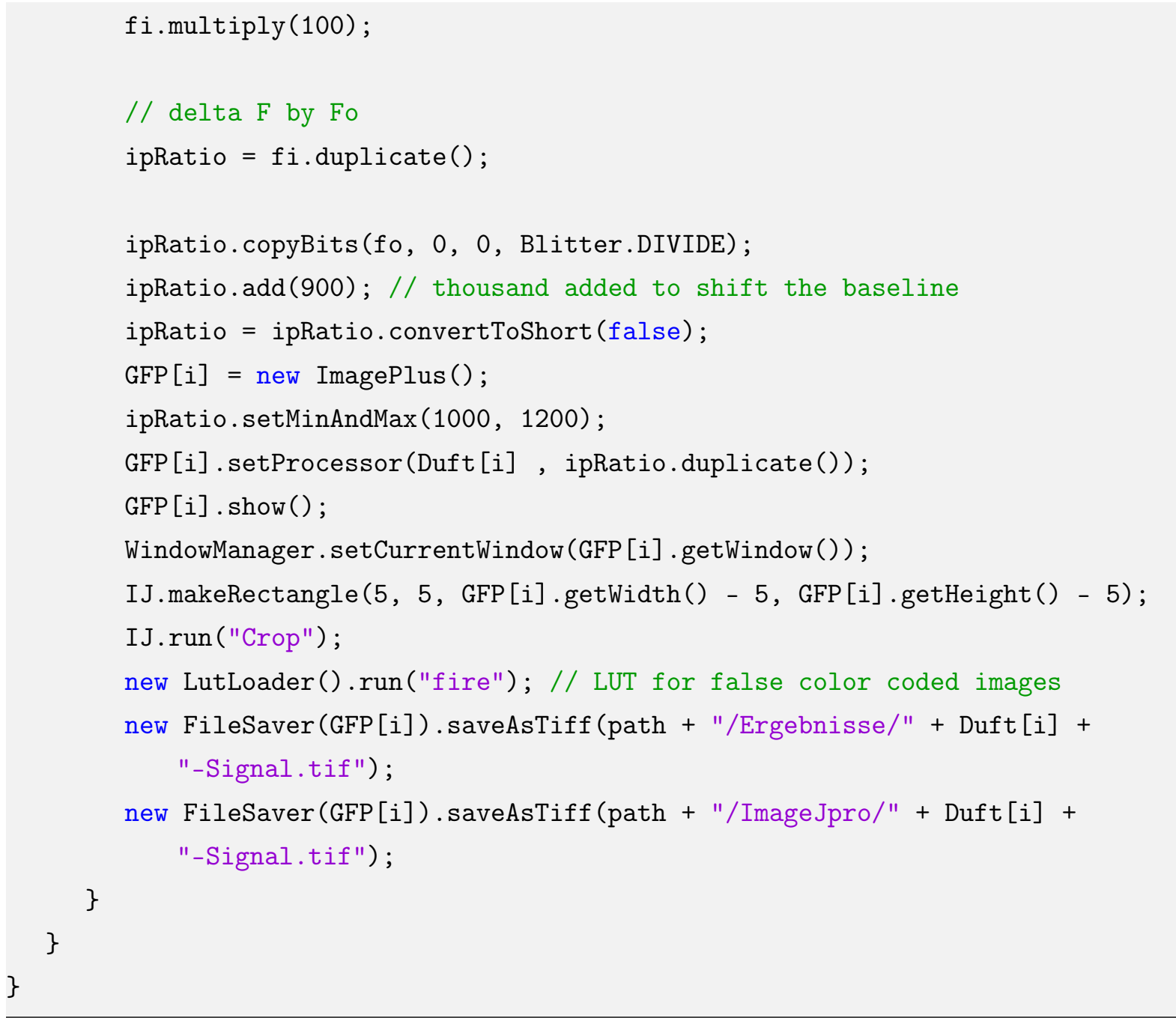




\section{A. The codes for data analysis}

\section{A.2. Matlab}

\section{A.2.1. Batch Alignment GFP}

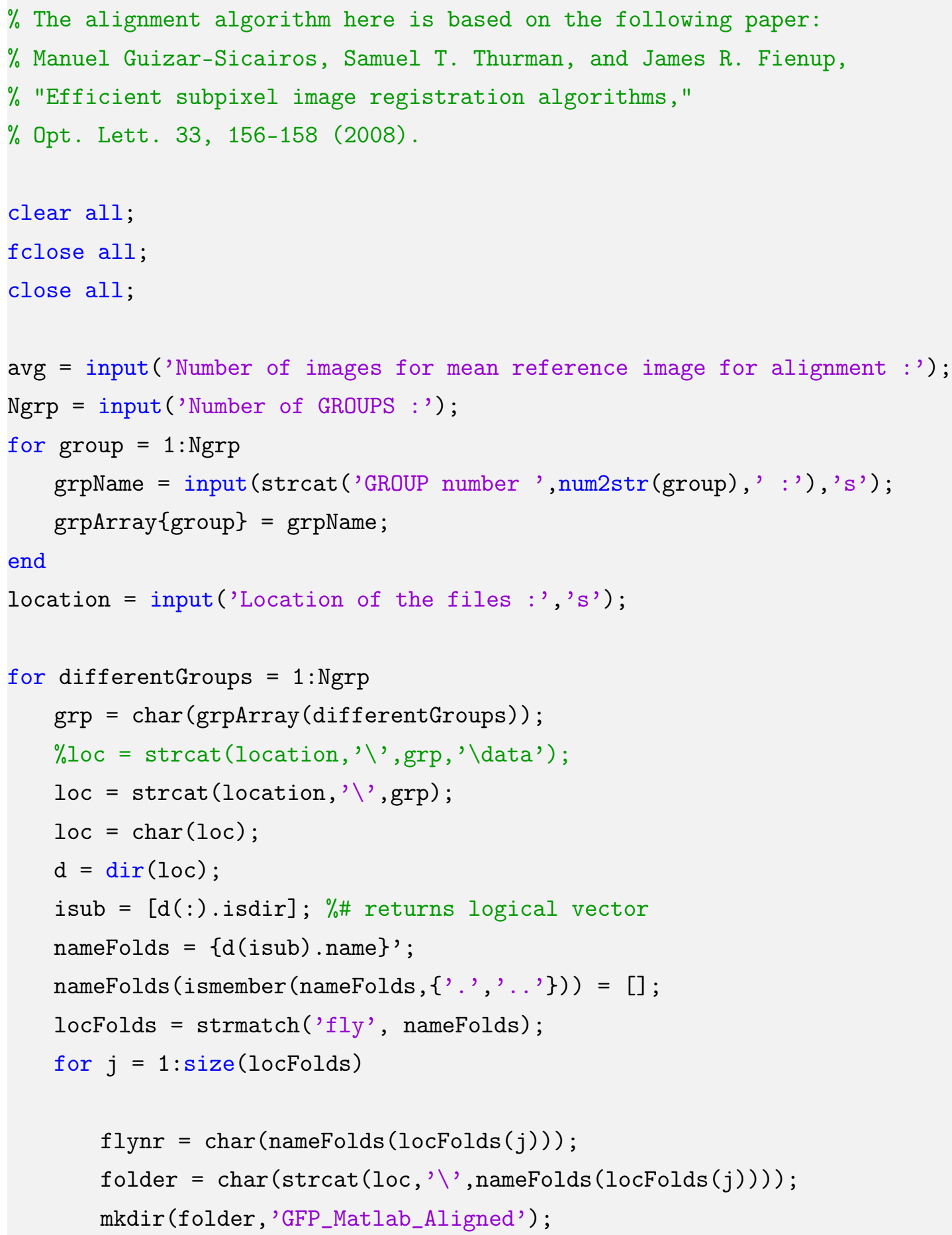




\section{A. The codes for data analysis}

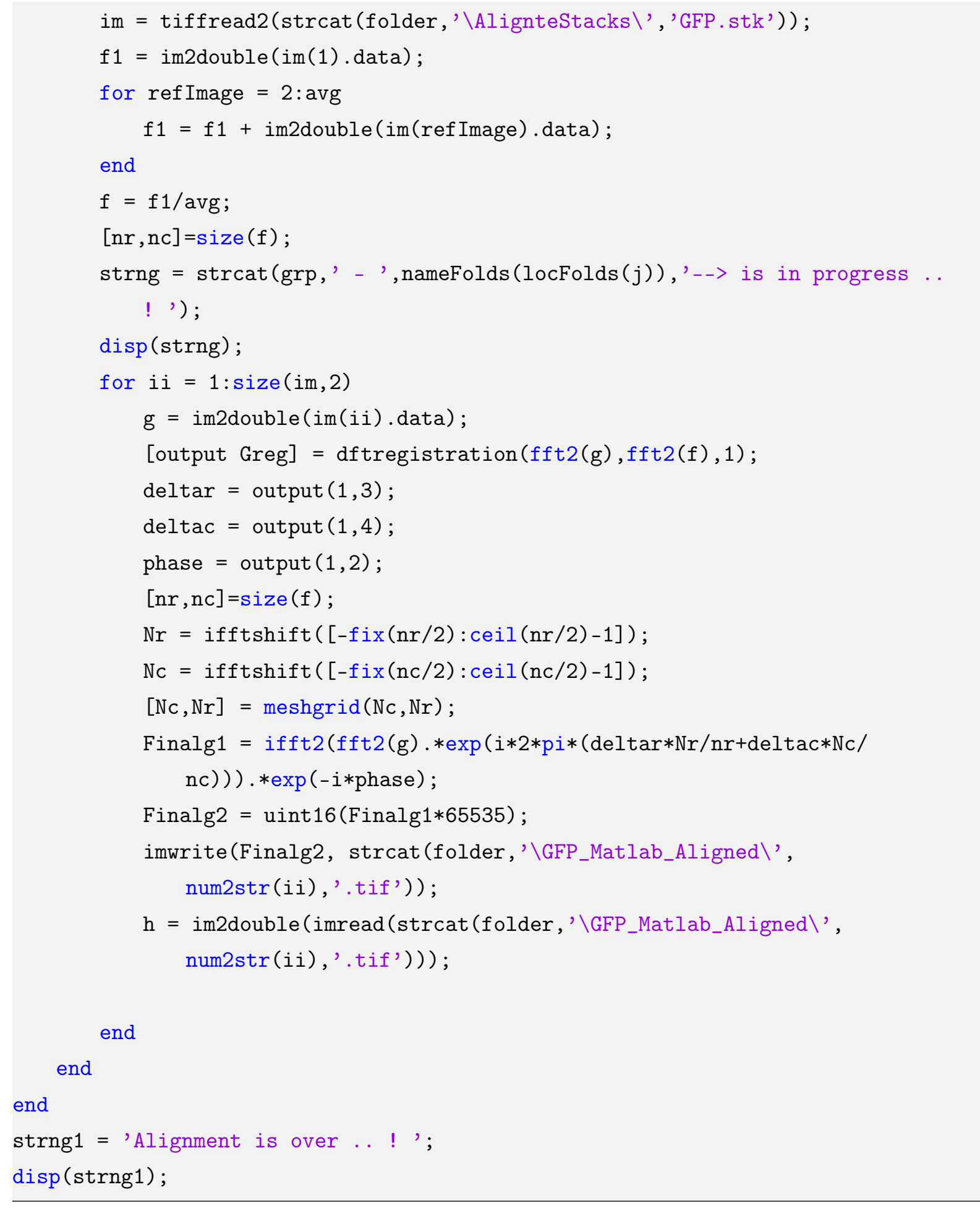




\title{
A. The codes for data analysis
}

\section{A.3. Microsoft Excel VBA}

\section{A.3.1. TwoPhotonCalculation macro}

Sub TwoPhotonCalculation()

\author{
Dim i As Integer \\ Dim num As Integer \\ Dim StartPoint As Integer \\ Dim EndPoint As Integer \\ Dim rStartPoint As String \\ Dim rEndPoint As String \\ Dim chrObj As Integer \\ Dim oldsheet As String \\ Dim rngData As Range \\ StartPoint $=25$ \\ EndPoint $=84$ \\ chrObj $=1$
}

MsgBox "Exported data should be starting from Row->5" \& vbNewLine \& "i.e.

Data numbers for calculation starts from Row->6" \& vbNewLine \& vbNewLine \& " Every measurement should be exactly 85 images" _

, Title:="Things to keep in mind :)"

num = Application. InputBox

(Prompt:="Enter the number.", -

Title:="How many measurements have you taken ? ", Type:=1)

ActiveCell.Offset (19, 0). Range("A1"). Select

For $i=1$ To num

ActiveCell.Select

ActiveCell.FormulaR1C1 = "=AVERAGE (RC [-1]:R [4]C [-1])"

ActiveCell.Offset (1, 0). Range ("A1"). Select

ActiveCell. FormulaR1C1 = "=R $[-1] \mathrm{C} "$

ActiveCell.Offset (-1, 0). Range ("A1:A2"). Select

ActiveCell.Offset (1, 0). Range("A1"). Select

Selection.AutoFill Destination:=ActiveCell.Range("A1:A59"), Type:= _ 


\section{A. The codes for data analysis}

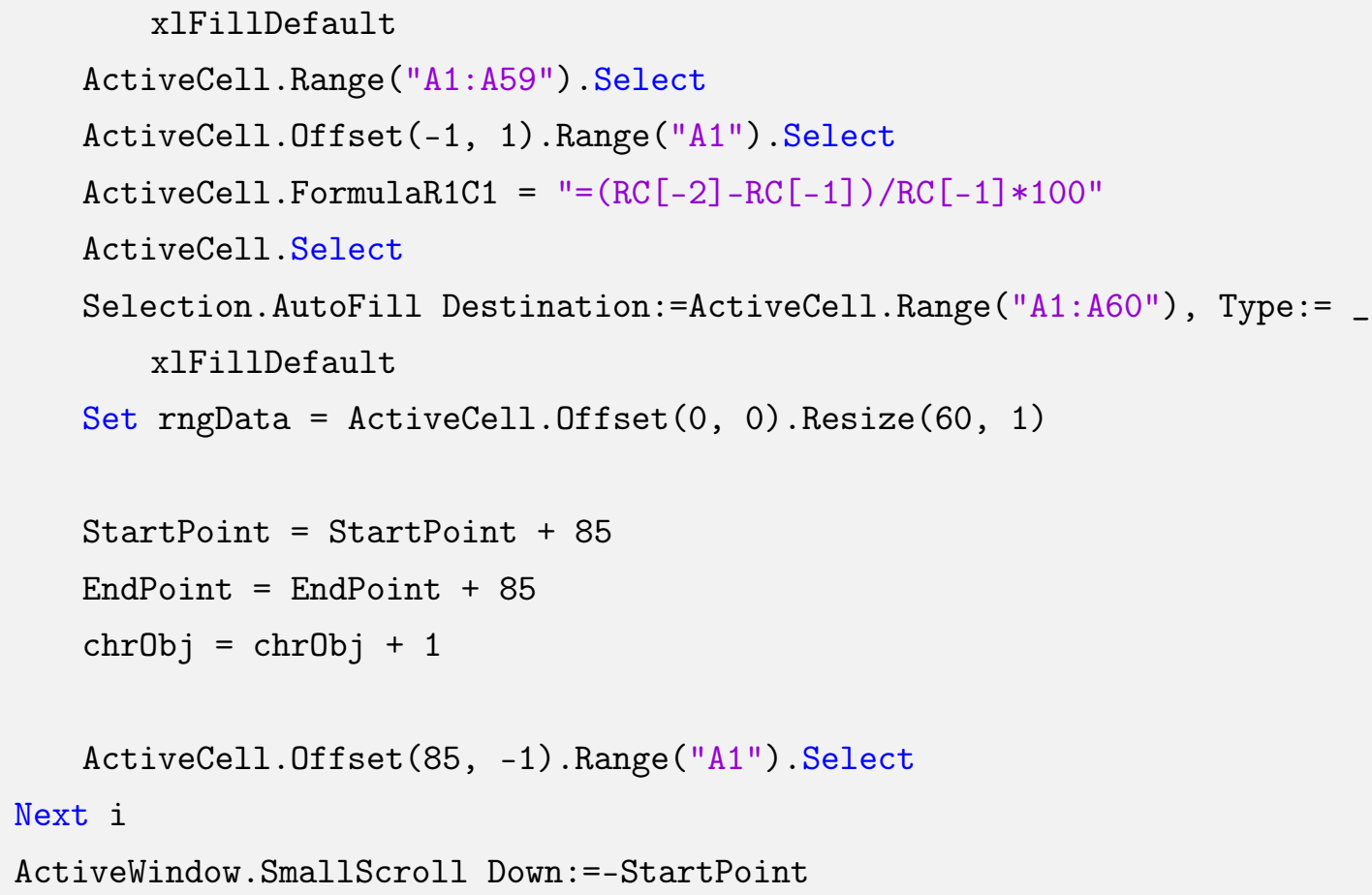




\section{B. Contributions to other studies}

Optical $\mathrm{Ca}^{2+}$ imaging in the nervous system of Drosophila melanogaster.

My contribution :

- Performed the experiments and analyzed the data for Figure 5 and Figure 6. 
Review

\title{
Optical calcium imaging in the nervous system of Drosophila melanogaster ${ }^{\text {is }}$
}

\author{
Thomas Riemensperger, Ulrike Pech, Shubham Dipt, André Fiala* \\ Molecular Neurobiology of Behavior, Johann-Friedrich-Blumenbach-Institute for Zoology and Anthropology, Georg-August-University of Goettingen, Julia-Lermontowa-Weg 3, \\ 37077 Goettingen, Germany
}

\section{A R T I C L E I N F O}

\section{Article history:}

Received 20 December 2011

Received in revised form 21 February 2012

Accepted 22 February 2012

Available online 28 February 2012

\section{Keywords:}

Drosophila

Optical calcium imaging

Genetically encoded fluorescence sensor

Calcium sensor protein

Neuronal processing

\begin{abstract}
A B S T R A C T
Background: Drosophila melanogaster is one of the best-studied model organisms in biology, mainly because of the versatility of methods by which heredity and specific expression of genes can be traced and manipulated. Sophisticated genetic tools have been developed to express transgenes in selected cell types, and these techniques can be utilized to target DNA-encoded fluorescence probes to genetically defined subsets of neurons. Neuroscientists make use of this approach to monitor the activity of restricted types or subsets of neurons in the brain and the peripheral nervous system. Since membrane depolarization is typically accompanied by an increase in intracellular calcium ions, calcium-sensitive fluorescence proteins provide favorable tools to monitor the spatio-temporal activity across groups of neurons.

Scope of review: Here we describe approaches to perform optical calcium imaging in Drosophila in consideration of various calcium sensors and expression systems. In addition, we outline by way of examples for which particular neuronal systems in Drosophila optical calcium imaging have been used. Finally, we exemplify briefly how optical calcium imaging in the brain of Drosophila can be carried out in practice.

Major conclusions and general significance: Drosophila provides an excellent model organism to combine genetic expression systems with optical calcium imaging in order to investigate principles of sensory coding, neuronal plasticity, and processing of neuronal information underlying behavior. This article is part of a Special Issue entitled Biochemical, Biophysical and Genetic Approaches to Intracellular Calcium Signaling.
\end{abstract}

(c) 2012 Elsevier B.V. All rights reserved.

\section{Introduction: visualization of neuronal activity in Drosophila}

Since many decades the fruit fly Drosophila melanogaster is a major model organism for geneticists, mainly because of its enormous fertility and fast reproduction cycle. The analysis of gene mutations represents a key approach to dissect the genetic basis of biological functions, e.g. development or behavior [1]. But Drosophila has also emerged as a major neurobiological model organism. The analysis of DNA mutations resulting in structural brain abnormalities, e.g. deficiency of distinct brain regions, has proven to be a very useful approach for the investigation of neuronal substrates controlling behavior (e.g., [2-4]). Experiments to analyze physiological mechanisms, however, have for many years remained difficult in this perfect genetic model organism due to the small size of neurons that make electrophysiological recordings of single cells challenging. Electrophysiological recordings have for a long time been restricted to preparations of neuromuscular junctions at the larval body wall (e.g., [5]) or to extracellular recordings from sensory cells (e.g., [6]). Only recently, patch clamp recording from central neurons of the Drosophila

\footnotetext{
is This article is part of a Special Issue entitled Biochemical, Biophysical and Genetic Approaches to Intracellular Calcium Signaling.

* Corresponding author. Tel.: + 49551 39177920; fax: + 4955139177921.

E-mail addresses: triemen@gwdg.de (T. Riemensperger), upech@gwdg.de (U. Pech), sdipt@gwdg.de (S. Dipt), afiala@gwdg.de (A. Fiala).
}

brain has been successfully established [7]. This represents a milestone in Drosophila neurobiology as electrophysiological recordings from single cells provide insights into the activity of individual neurons with an excellent temporal precision.

However, Drosophila sensory stimuli or motor outputs are, just as in the brains of vertebrates, often encoded as spatio-temporal activity patterns across extended populations of neurons. An odor stimulus, for instance, is represented at the periphery as combinatorial activity of many olfactory sensory neurons expressing distinct olfactory receptors [8]. Complex gustatory stimuli can be classified by the animal according to combinations of taste qualities, e.g. dependent on the activity of bitter-mediating and sweetness-mediating gustatory sensory cells [9]. Visual stimuli are transduced into retinotopic maps of activity across many photoreceptor cells, and are subsequently processed by arrays of neurons maintaining aspects of those retinotopic maps [10]. But not only encoding sensory stimuli requires a concerted activity of many neurons. Central processing events, e.g. the association of sensory stimuli with rewarding or punitive events through learning, of course also involve activity of multiple cells [8]. Also, more intrinsic influences on behavior-release, like motivation or circadian rhythms, are not entirely controlled by single cells only $[11,12]$. These examples demonstrate that besides recordings from individual neurons a method to monitor the activity across many cells is valuable. Initial approaches to visualize the activity of broader areas of nervous tissue in correlation with sensory stimulation or motor 
performance have relied on 2-deoxyglucose mapping [13-15]. Nowadays, as important as these classical experiments were, this method has inherent limitations because of a very low temporal resolution, and the researchers had no possibility to restrict the analysis to classes of neurons defined by their functionality (e.g., inhibitory or excitatory), their genetic identity or the projection and connectivity patterns of their neurites. The invention of genetically encoded fluorescence proteins designed to report cellular signaling events associated with neuronal excitation has provided the key to overcome these limitations, and in combination with genetic tools by which transgenes can be expressed in a cell-type specific manner Drosophila has turned into an excellent model organism also for studying physiological mechanisms underlying brain function [16].

A variety of different sensor proteins have been described which respond to various aspects of intracellular signaling, e.g. cytosolic calcium ion $\left(\mathrm{Ca}^{2+}\right)$ influx (reviewed in [17-19]), synaptic vesicle release [20], kinase-dependent phosphorylation [21-24] or the synthesis of second messenger molecules like cAMP [25]. Many of these sensor proteins have been successfully used in the nervous system of Drosophila. For the functional analysis of neuronal circuits, a sensor protein to monitor membrane depolarization and hyperpolarization with good spatio-temporal resolution would apparently be desirable. Unfortunately, the development of DNA-encoded fluorescence sensors reporting changes in membrane potential has not yet resulted in indicators displaying sufficiently high signal-to-noise-ratios and temporal kinetics for studies on intact brains [26]. Membrane depolarizations are, however, typically accompanied by fastly propagating intracellular $\mathrm{Ca}^{2+}$ influx via voltage-gated $\mathrm{Ca}^{2+}$ channels as well as slowly propagating regenerative $\mathrm{Ca}^{2+}$ signals deriving from the endoplasmic reticulum and mitochondrial $\mathrm{Ca}^{2+}$ reserve pools [27]. In addition, both synaptic input at dendritic structures and transmitter release at presynapses are coupled to $\mathrm{Ca}^{2+}$ influx [27]. The most widely used parameter to monitor correlates of neuronal activity in Drosophila represents intracellular $\mathrm{Ca}^{2+}$ concentration. Since the invention of genetically encoded $\mathrm{Ca}^{2+}$ indicators (GECIs) $[28,29]$ a variety of probes have been described which differ in their principal mode of action, their $\mathrm{Ca}^{2+}$ affinities, intrinsic baseline fluorescence and kinetic properties [17-19]. These GECIs have contributed enormously to the understanding of neuronal functions and their contribution to behavior in Drosophila, and since the first report of $\mathrm{Ca}^{2+}$ imaging in the Drosophila brain [30] this method has evolved to a standard technique in Drosophila neurobiology.

Here, we will first review the current status of the development of GECIs at the time of writing this article in this rapidly progressing field. Second, we will discuss genetic tools to specifically target GECIs to defined populations of neurons in Drosophila. Third, we will review which type of scientific questions concerning the function of the Drosophila brain have been addressed using optical $\mathrm{Ca}^{2+}$ imaging. And fourth, we will briefly explain on the basis of examples of our own research how optical $\mathrm{Ca}^{2+}$ imaging of neuronal activity in the Drosophila nervous system can be experimentally performed.

\section{Genetically encoded calcium sensors (GECIs)}

All GECIs rely on variants of the green fluorescent protein (GFP), and two principal strategies to develop GECIs have been pursued, FRET-based GECIs and circularly permuted GFP variants (Fig. 1). FRET-based sensors consist of two GFP variants, a donor chromophore, usually a cyan variant of GFP, and an acceptor chromophore, usually a yellow variant of GFP, both of which are fused to the terminals of a $\mathrm{Ca}^{2+}$ binding sequence. $\mathrm{Ca}^{2+}$ binding leads to a conformational change, ultimately enhancing the Förster resonance energy transfer (FRET) from donor to acceptor. If the donor chromophore is excited at $\sim 440 \mathrm{~nm}$, increase in intracellular $\mathrm{Ca}^{2+}$ can be detected by a decrease in emission intensity from the donor chromophore $(\sim 485 \mathrm{~nm})$ and an increase in emission intensity from the acceptor
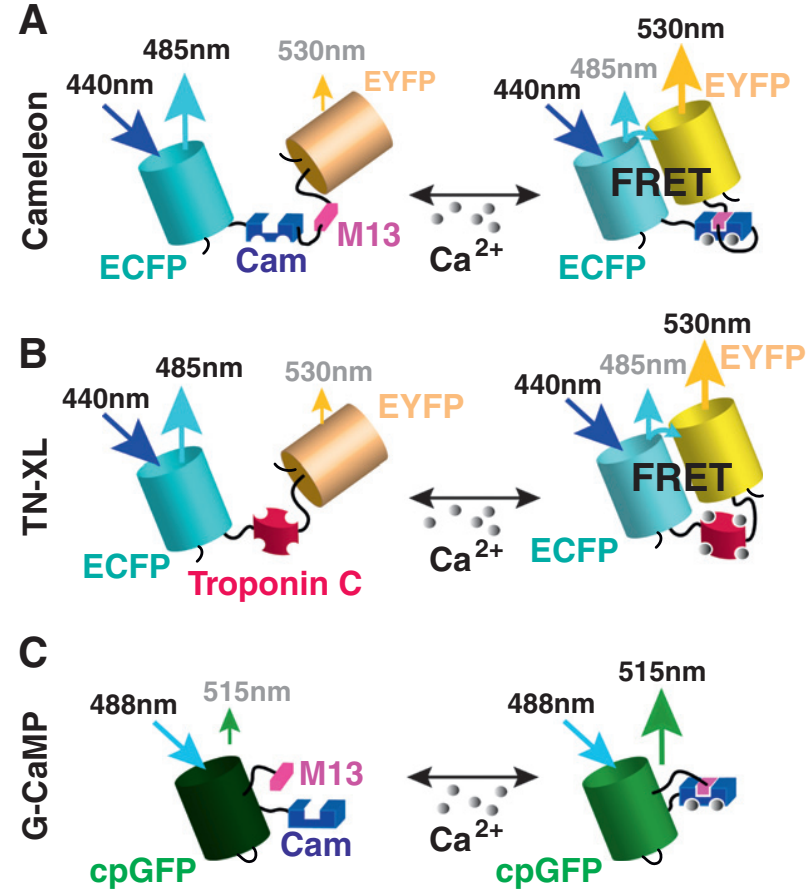

Fig. 1. Schematic depiction of different genetically encoded $\mathrm{Ca}^{2+}$ indicators (GECIs) used in Drosophila. GECIs can be divided into indicators based on a Förster resonance energy transfer (FRET) and those changing the fluorescence of a single fluorophore. A) In FRET based Cameleon-type sensors [28] a cyan fluorescence protein, e.g. ECFP and a yellow fluorescence protein, e.g. EYFP, are interconnected by a $\mathrm{Ca}^{2+}$-binding domain of calmodulin (Cam) and a calmodulin binding petide (M13). $\mathrm{Ca}^{2+}$ binding to Cam causes a conformational change bringing ECFP in closer proximity to EYFP which enhances FRET from ECFP to EYFP. B) Troponin-based sensors, like TN-XL [31], belong to the FRET-based GECI-family with a mode of function similar to Cameleon. However, the Cam-M13 sequences are replaced by the $\mathrm{Ca}^{2+}$ binding sequence troponin C. C) In GECIs that are based on circularly permutated GFP variants (cpGFP), like G-CaMP-type sensors [34], binding of $\mathrm{Ca}^{2+}$ to the Cam sequence results in a change within the barrel structure of the GFP, ultimately enhancing its fluorescence emission.

chromophore ( $535 \mathrm{~nm})$. In the Cameleon-type GECIs, first described by Miyawaki et al. [28] (Fig. 1A), $\mathrm{Ca}^{2+}$ binds to the $\mathrm{Ca}^{2+}$-binding domains of a calmodulin sequence, and a calmodulin-binding peptide subsequently mediates the conformational change of the whole molecule that brings the yellow fluorescing protein in closer proximity to the cyan fluorescing protein. An alternative strategy has been developed by Heim and Griesbeck [31] using a troponin C sequence as $\mathrm{Ca}^{2+}$ binding component that does not require an additional peptide (Fig. 1B).

Single chromophore GECIs, e.g. Camgaroo [32], Pericam [33] or G-CaMP [34], rely on a different mode of action. Here, modified GFP variants have been coupled to $\mathrm{Ca}^{2+}$-binding sequences. In the case of G-CamP and Pericam a GFP or YFP molecule has been circularly permuted such that $\mathrm{C}$ - and $\mathrm{N}$-termini have been fused and new $\mathrm{C}$ and $\mathrm{N}$-termini have been introduced (cpGFP) [34]. If the newly generated $\mathrm{N}$ - and $\mathrm{C}$-termini are linked to a calmodulin sequence and a calmodulin target peptide, binding of $\mathrm{Ca}^{2+}$ causes a conformational change of the entire molecule ultimately affecting the protonation state of the chromophore. An increase in intracellular $\mathrm{Ca}^{2+}$ can directly be detected as an increase in fluorescence emission (Fig. 1C). Several versions of Cameleons [28,35-41], troponin-based GECIs [31,42-44] and single chromophore sensors [32-34,45-48] have been engineered over recent years with improvements in various physical parameters of the protein, e.g. $\mathrm{Ca}^{2+}$ affinity, brightness, the dynamic range across $\mathrm{Ca}^{2+}$ concentrations, linearity and kinetics of fluorescence change as a function of $\mathrm{Ca}^{2+}$-binding, or insensitivity to environmental influences such as $\mathrm{pH}, \mathrm{Cl}^{-}$or $\mathrm{Mg}^{2+}$ (for comparative reviews on these aspects see [17-19]). 
Both single chromophore and FRET-based GECIs have been widely used to investigate neuronal activity in the Drosophila brain. Table 1 provides an overview over the GECIs for which transgenic fruit flies have been described. A rather comprehensive comparison of different GECI types in Drosophila has been performed by Reiff et al. [49] and Hendel et al. [50], providing helpful information about the relationship between action potential firing rates and fluorescence changes of diverse GECIs. In these studies GECIs were expressed in motor neurons of the neuromuscular junction at the larval body wall, and electrophysiological stimulation at different frequencies was correlated with fluorescence changes exhibited by the GECIs [49,50]. These comparative studies on the functionality of different GECIs under optimized and constant experimental conditions provide useful indications which GECI might be the best choice for a particular experiment. Parameters of importance are, for example, the dynamic range of the GECI used with respect to intracellular $\mathrm{Ca}^{2+}$ concentrations and the signal kinetics with respect to transient neuronal activity. However, the actual performance of a particular GECI in a brain depends also on particular experimental conditions which differ from those of cell cultures or tissue preparations. In the brain of a largely intact animal the neuronal activity evoked by physiological stimuli is often relatively small and movement of the structures

Table 1

Different GECIs used for optical $\mathrm{Ca}^{2+}$ experiments in the Drosophila nervous system.

\begin{tabular}{|c|c|c|c|}
\hline $\begin{array}{l}\text { Name and } \\
\text { reference } \\
\text { for GECI }\end{array}$ & $\begin{array}{l}\text { Reference for } \\
\text { Drosophila } \\
\text { strain }\end{array}$ & Investigated neurons & $\begin{array}{l}\text { Reference } \\
\text { for study }\end{array}$ \\
\hline $\begin{array}{l}\text { Cameleon } 2.0 \\
\text { [28] }\end{array}$ & [49] & $\begin{array}{l}\text { Neuromuscular junction } \\
\text { of the larval body wall }\end{array}$ & [49] \\
\hline \multirow[t]{4}{*}{$\begin{array}{l}\text { Cameleon } 2.1 \\
{[35]}\end{array}$} & {$[30,112]$} & $\begin{array}{l}\text { Olfactory sensory neurons and/ } \\
\text { or olfactory projection neurons }\end{array}$ & {$[30,90,118]$} \\
\hline & & $\begin{array}{l}\text { Mechanosensory neurons of } \\
\text { the Johnston's organ }\end{array}$ & {$[79,81]$} \\
\hline & & Femural chordotonal organ & {$[52]$} \\
\hline & & Dopaminergic neurons & [109] \\
\hline $\begin{array}{l}\text { Cameleon } 2.3 \\
\text { [36] }\end{array}$ & {$[49]$} & $\begin{array}{l}\text { Neuromuscular junction of } \\
\text { the larval body wall }\end{array}$ & [49] \\
\hline $\begin{array}{l}\text { Cameleon } 3.3 \\
{[36]}\end{array}$ & [49] & $\begin{array}{l}\text { Neuromuscular junction of } \\
\text { the larval body wall }\end{array}$ & {$[49,50]$} \\
\hline $\begin{array}{l}\text { Cameleon } 3.6 \\
\text { [38] }\end{array}$ & {$[50]$} & $\begin{array}{l}\text { Neuromuscular junction of } \\
\text { the larval body wall }\end{array}$ & {$[50]$} \\
\hline \multirow{2}{*}{$\begin{array}{l}\text { Camgaroo } 1 \\
\text { [32] }\end{array}$} & [119] & Mushroom bodies & [119] \\
\hline & & $\begin{array}{l}\text { Neuromuscular junction of } \\
\text { the larval body wall }\end{array}$ & [49] \\
\hline \multirow[t]{2}{*}{$\begin{array}{l}\text { Camgaroo } 2 \\
{[36]}\end{array}$} & [119] & $\begin{array}{l}\text { Neuromuscular junction of } \\
\text { the larval body wall }\end{array}$ & [49] \\
\hline & & Mushroom bodies & {$[119,120]$} \\
\hline \multirow[t]{6}{*}{$\begin{array}{l}\text { G-CaMP1.3 } \\
{[34]}\end{array}$} & {$[84,121]$} & $\begin{array}{l}\text { Olfactory sensory neurons and/ } \\
\text { or olfactory projection neurons }\end{array}$ & {$[84,88,89,121-127]$} \\
\hline & & Gustatory sensory neurons & {$[93,97,98]$} \\
\hline & & $\begin{array}{l}\text { Neuromuscular junction of } \\
\text { the larval body wall }\end{array}$ & [49] \\
\hline & & $\begin{array}{l}\text { GABAergic neurons of the } \\
\text { mushroom body }\end{array}$ & [128] \\
\hline & & Mushroom bodies & {$[100,101,102,121]$} \\
\hline & & Thermosensory neurons & {$[76,77]$} \\
\hline \multirow[t]{2}{*}{$\begin{array}{l}\text { G-CaMP } 1.6 \\
\text { [45] }\end{array}$} & {$[47,49]$} & $\begin{array}{l}\text { Neuromuscular junction of } \\
\text { the larval body wall }\end{array}$ & [49] \\
\hline & & Olfactory projection neurons & {$[47,129]$} \\
\hline \multirow[t]{4}{*}{$\begin{array}{l}\text { G-CaMP } 3 \\
\text { [47] }\end{array}$} & [47] & $\begin{array}{l}\text { Olfactory sensory neurons and/ } \\
\text { or olfactory projection neurons }\end{array}$ & {$[47,129]$} \\
\hline & & Mushroom bodies & [103] \\
\hline & & Lobula plate tangential neurons & {$[130,131]$} \\
\hline & & $\begin{array}{l}\text { Mushroom body extrinsic } \\
\text { neurons }\end{array}$ & {$[111]$} \\
\hline $\begin{array}{c}\text { TNL-15 } \\
{[31]}\end{array}$ & [49] & $\begin{array}{l}\text { Neuromuscular junction of } \\
\text { the larval body wall }\end{array}$ & [49] \\
\hline $\begin{array}{r}\mathrm{TN}-\mathrm{XL} \\
{[42]}\end{array}$ & [49] & $\begin{array}{l}\text { Neuromuscular junction of } \\
\text { the larval body wall }\end{array}$ & [49] \\
\hline \multirow[t]{2}{*}{$\begin{array}{c}\text { TN-XXL } \\
{[44]}\end{array}$} & {$[44]$} & $\begin{array}{l}\text { Neuromuscular junction of } \\
\text { the larval body wall }\end{array}$ & [44] \\
\hline & & Lamina monopolar cells & [99] \\
\hline
\end{tabular}

under investigation often cannot be completely avoided. Furthermore, depending on the preparation the optical resolution can be poor due to the small size of fine and spaciously arborizing structures and the hindered optical access through surrounding tissue. Depending on the parameters that are of most importance for the experimenter and the experimental situation, different GECIs provide advantages and disadvantages. A deciding factor for the quality of a $\mathrm{Ca}^{2+}$ imaging experiment is the signal-to-noise ratio which depends, of course, on the magnitude of relative fluorescence change as a function of intracellular calcium influx and on the variability of the signal. In general, the absolute signal intensity is much higher in singlechromophore GECIs, e.g. G-CaMP, when compared to FRET-based sensors. The overall noise present in the recording depends on which type of noise is dominating. Intrinsic fluctuations of the fluorescence emission (shot noise) are, at least from a theoretical point of view, more pronounced in ratiometric sensors because the emission intensities of two chromophores are divided by each other [19]. However, in the brain of a largely intact fruit fly noise is often added by small movements of the preparation. In this case noise can be eliminated to some degree by ratiometric measurements using FRET-based sensors because movement artifacts cause changes of emission of the two chromophores in the same direction, whereas $\mathrm{Ca}^{2+}$ signals are characterized by fluorescence changes in opposite directions [51]. In case of monitoring fluorescence through the pigmented cuticle of the fly [52] emission light is to some degree absorbed and scattered. Strong baseline fluorescence (brightness) is in this case advantageous. Typically, FRET-based sensors show a considerably stronger brightness (baseline fluorescence) when compared to single chromophore sensors [19]. However, recent versions of G-CaMP sensors have been greatly improved in this respect [47]. In the case of Drosophila the expression levels strongly determine baseline fluorescence, which can be influenced by the number of DNA insertions into the genome. Finally, technical considerations concerning the optical imaging setup might also be of importance. For FRET-based sensors, the simultaneous detection of two emission wavelengths is required, which complicates the optical imaging setup. On the contrary, G-CaMP can be employed with relatively basic microscopic methods. With the improvements made in the version G-CaMP3 [47] this GECI represents a good choice for most experiments.

\section{Binary expression systems in Drosophila}

The most important advantages of $D$. melanogaster for analyzing the neuronal basis underlying behavioral functions rely on the sophisticated genetic tools available. The Drosophila genome is fully sequenced [53] and germline transformation techniques to create transgenic animals [54] expressing certain genes represents nowadays a standard technique. However, a major strength of Drosophila relies on bipartite expression systems that uses two transgenic fly strains separating where the transgene of interest is expressed (driver strain) from which transgene is expressed (effector strain) in order to multiply possible combinations (Fig. 2). The Gal4-UAS-expression system $[55,56]$ represents the first and most commonly used tool. Here, the spatial and temporal expression of the yeast transcription factor Gal4 is determined by a specific genomic promoter or enhancer sequence present in the driver strain (Fig. 2A1). The expression of the $\mathrm{GECI}$ (or any other transgene) is achieved by crossing it to the effector strain that carries the DNA sequence of the GECI under control of an "upstream activator sequence" (UAS), a target sequence for Gal4. The F1 generation carries both transgenes, and the Gal4 expressed in dedicated neurons can bind to the UAS sequence, ultimately leading to a cell type-specific expression of the GECI (Fig. 2A1). Since many different Gal4 strains have been described, and electronic databases for thousands of Gal4-strains exist, e.g. at the Bloomington stock center at Indiana University [57] or the Drosophila Genetic Resource Center in Kyoto [58], GECIs can be easily expressed in a large 


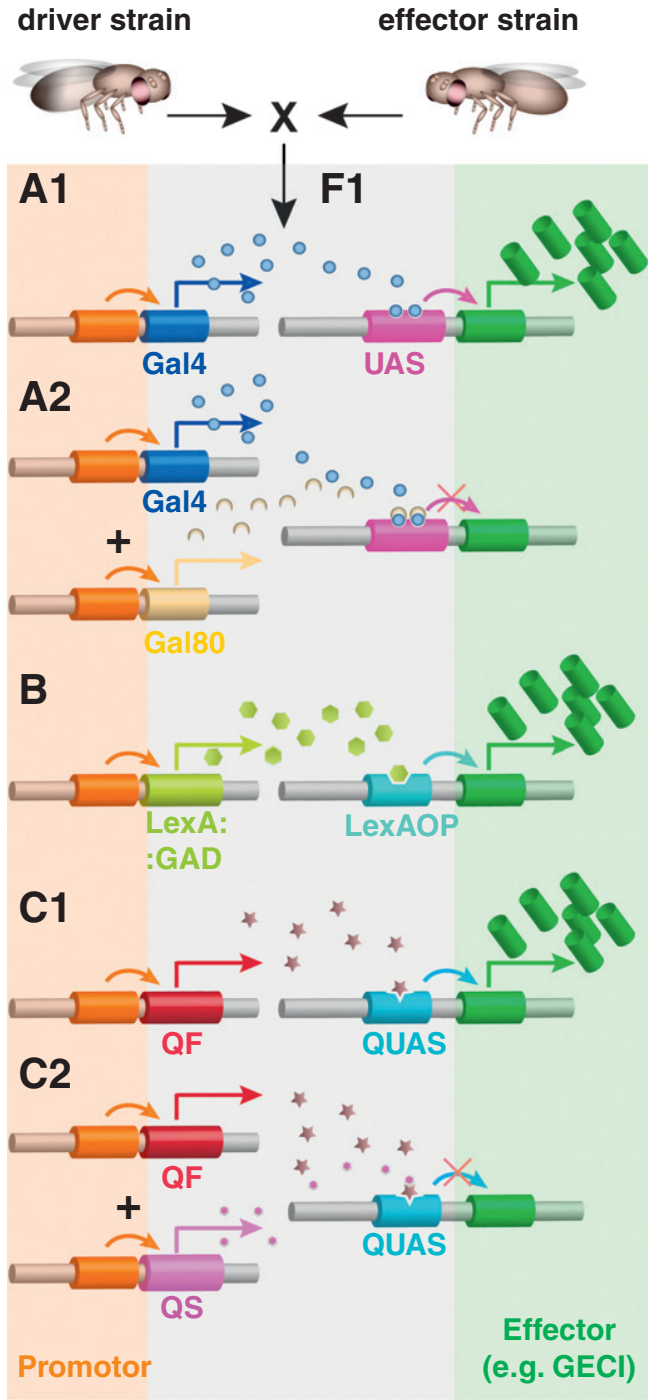

Fig. 2. Bipartite gene expression systems in Drosophila. A1) The most commonly used bipartite expression system in Drosophila is based on the yeast transactivator Gal4 [56], which is expressed in one fly strain (driver strain) under control of a genomic enhancer or promotor sequence. In a second fly strain (effector strain) the gene to be expressed e.g. a GECI, is coupled to an upstream activator sequence (UAS). If both fly strains are crossed, the F1 generation expresses the effector gene in those neurons determined by the Gal4 strain (adopted from [56]). A2) The expression of the effector gene can be repressed by the co-expression of Gal80, masking the activation domain of Gal4 [65] B) In the LexA system [62] the fusion of the GAL4 activation domain to the bacteria DNA binding domain of the LexA protein results in a Lex::GAD transactivator expressed in a driver strain. The desired effector gene is coupled to a LexAOP activation sequence which is inserted into the genome of an effector strain. Again, the F1 generation expresses the effector gene in a cell-type specific manner dependent on the driver strain used (adopted from [117]). C1) The repressible binary Q expression system consists, like the two other system, of a driver strain expressing the transcription factor QF and the effector strain with the desired effector under the control of a QUAS responsive transgene. When the QF and the QUAS-effector transgene are present in the same cell QF binds to QUAS and activates the transcription of the desired transgene. C2) Like in the UAS-Gal4 system in the $\mathrm{Q}$-system, expression can be modified through the presence of an inhibitor (QS) that represses QF $[63,64]$.

variety of diverse types of neurons. Variations of the original Gal4UAS-system with respect to temporally defined inducibility of gene expression (reviewed in [59]) and refinements of its molecular components [60] have further improved this very strong and versatile tool.

In some instances one would like to express two different transgenes in different neuronal populations. To accomplish that in a convenient way two alternative binary transcription systems have been described in addition to the Gal4-UAS system, the LexA-LexAop system [61,62] (Fig. 2B) and the Q system [63,64] (Fig. 2C1). Combinations of these binary transcription systems allow for the expression of multiple transgenes in combinations of neurons. This is of relevance since the expression of transactivating genes in driver strains, like Gal4 strains, is often determined by genomic enhancer elements, and the subsets of neurons expressing it often include many diverse types of cells. If one wishes to restrict the expression of GECIs to fewer neurons, repressors of transactivating proteins, e.g. the Gal4repressing protein Gal80 (Fig. 1A2) [65] or the QUAS repressing protein QS (Fig. 2C2) [63,64], can be targeted to a complementary subset of cells using a second binary transcription system. A variety of similar "intersectional strategies" to narrow down the transgene expression to very few neurons of interest has been described by now, which is comprehensively reviewed in [16].

\section{Optical calcium imaging in Drosophila}

Since the first description of optical $\mathrm{Ca}^{2+}$ imaging at the larval neuromuscular junction [66] and the central brain of Drosophila [30] this technique has evolved to a standard tool in Drosophila neurobiology. A variety of subgroups of neurons within the Drosophila central brain could be analyzed using GECIs, as schematically illustrated in Fig. 3. Optical $\mathrm{Ca}^{2+}$ imaging has been particularly useful to investigate mechanisms of sensory processing, a field of research in which our knowledge has expanded enormously in recent years. Olfactory and gustatory receptors of Drosophila have been characterized [67-71], TRP channels that mediate temperature sensations have been described [72-77], and neuronal sensory cells and signaling mechanisms mediating mechanosensory stimuli have been analyzed [78-81]. In the context of sensory processing $\mathrm{Ca}^{2+}$ imaging could in most instances be used to address two questions. First, what are the response spectra of particular sensory cells with respect to adequate stimulus parameters, and how are defined stimuli represented across populations of sensory cells? Second, where in the brain do these sensory cells project and is there a logical spatial organization in the internal representation of sensory stimuli? Whereas these questions could in principle be and in many cases have been addressed using sensillum recordings and neuroanatomical tracing of recorded cells, $\mathrm{Ca}^{2+}$ imaging across populations has added the possibility to visualize the activity of many neurons simultaneously.

The questions mentioned above have been most extensively studied in Drosophila in the olfactory system [82,83]. Fruit flies perceive odors by olfactory sensory neurons that are located on the third antennal segments and the maxillary palps $[82,83]$. Each olfactory sensory neuron expresses one or very few olfactory receptors, and those sensory cells expressing a common receptor project into the same specific glomeruli within the antennal lobes of the brain $[82,83]$. Olfactory projection neurons arborize in these glomeruli and convey olfactory information to more centrally located brain areas, namely the lateral horns and the mushroom bodies [82,83]. In this context optical $\mathrm{Ca}^{2+}$ imaging experiments have helped to determine the spatial logic of odor representation. Odor stimuli are encoded in the primary olfactory center of the fly's brain, the antennal lobe, in terms of combinatorial glomerular activity patterns [30,84], as has been shown earlier for other insects using bath-applied $\mathrm{Ca}^{2+}$ sensitive dyes (e.g. [85-87]). In further studies $\mathrm{Ca}^{2+}$ activity in first order sensory neurons, second order olfactory projection neurons and/or local interneurons interconnecting the antennal lobes' glomeruli was monitored. This enabled researchers to study more specific topics on olfactory processing, e.g. the representation of odor mixtures [88], odor intensity [89], a determination of similarity between odors [90], and even a change in olfactory representation caused by prolonged odor exposure [91].

In recent years, gustatory receptor genes encoded by the Drosophila genome have been characterized, which has been the starting point for an enormously fascinating field of research as more and more of these 
A

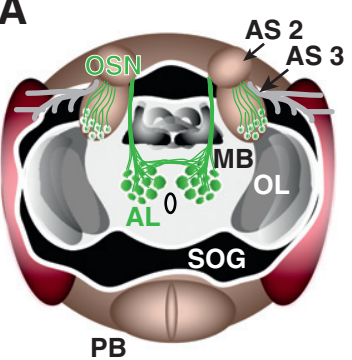

D

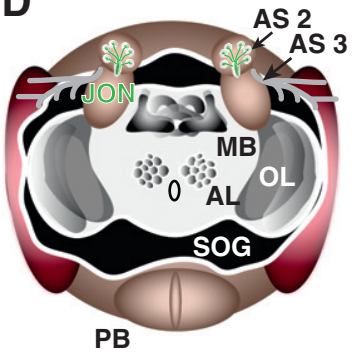

B

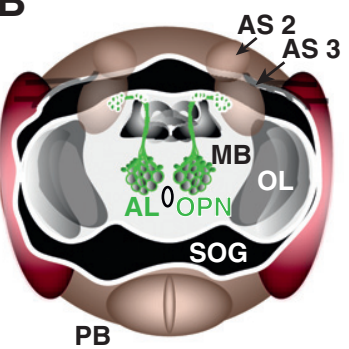

E

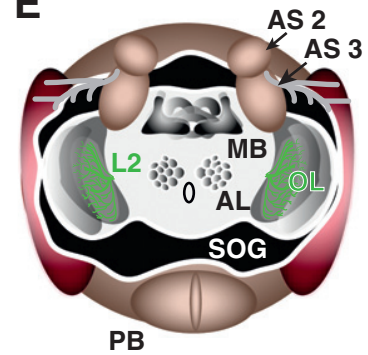

C

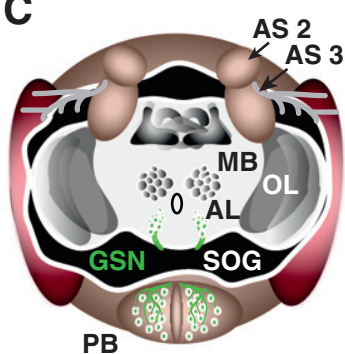

F

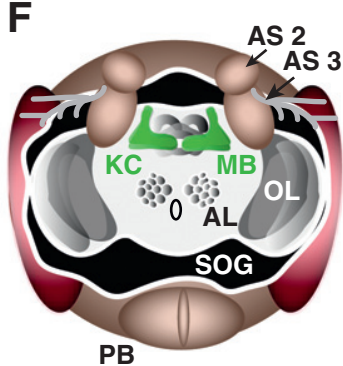

Fig. 3. In vivo $\mathrm{Ca}^{2+}$ imaging has been used in Drosophila to analyze diverse types of neurons, schematically depicted as frontal sections through a Drosophila brain. A) Odors are perceived by olfactory sensory neurons (OSN) located on the third antennal segments (A3) that project via the antennal nerves to the antennal lobes (AL) and terminate in spherical structures (glomeruli). $\mathrm{Ca}^{2+}$ imaging has been used to visualize the spatial representation of odors as glomerular activity patterns in the antennal lobes (e.g. [84,88-91]). B) This spatially encoded odor-information is conveyed by olfactory projection neurons (OPN) to higher brain areas. $\mathrm{Ca}^{2+}$ imaging has also been used to monitor the spatial representation of odors at this level of processing [30,84,88-91]. C) Gustatory stimuli are mediated by gustatory sensory neurons (GSN) located on the proboscis (PB) which project to the subesophageal ganglion (SOG) of the brain. The activation of different target areas by opposite taste categories in this region has been analyzed using Ca ${ }^{2+}$ imaging [93]. D) Mechanosensory information mediated by Johnston's organ neurons (JON) located on the second antennal segment (A2) has been analyzed using $\mathrm{Ca}^{2+}$ imaging [79]. E) Specific neurons located in the optical lobes (OL) of the Drosophila brain contribute to motion vision which has been analyzed using optical $\mathrm{Ca}^{2+}$ imaging [99]. F) Odor-evoked neuronal activity as reflected in Ca ${ }^{2+}$ dynamics has been analyzed using $\mathrm{Ca}^{2+}$ imaging also at the level of the intrinsic cells (Kenyon cells, $\mathrm{KC}$ ) of the mushroom body (MB) in the context of revealing coding principles [103] and associative memory formation [100-102].

receptors are being characterized with respect to their response spectra (reviewed in [82,92]). An interesting finding was that gustatory sensory cells responding to bitter stimuli and those responding to sugars show different target regions in the subesophageal ganglion of the fly's brain [93]. Here, $\mathrm{Ca}^{2+}$ imaging provided an addition to electrophysiological sensillum recordings (e.g. [94-96]), namely a tool to characterize response characteristics of gustatory sensory cells and to determine the representation of the sensory signals in the brain [93]. But also gustatory cells responsive to water [97] or carbonation of water [98] have been characterized using optical $\mathrm{Ca}^{2+}$ imaging, exemplifying how the adequate stimuli for sensory cells can be determined as an alternative method to electrophysiological recordings.

$\mathrm{Ca}^{2+}$ imaging has also proven to be of enormous value for the analysis of how auditory stimuli are encoded by neurons of the fruit fly's ear, the Johnston's organ located on the second antennal segment. Here, a spatial analysis of $\mathrm{Ca}^{2+}$ activity across Johnston's organ sensory cells through the cuticle of the intact animal has demonstrated that two groups of mechanosensory cells exist that either respond to sound-evoked stimuli or more stationary deflections of the mechanosensory organs required for gravity-detection $[79,80]$. Optical $\mathrm{Ca}^{2+}$ imaging provides here a powerful tool to further analyze the molecular basis of sound perception using specific mutations in genes that encode for proteins potentially involved in mechanosensory signal transduction [81].

Whereas chemosensory, mechanosensory or thermosensory stimuli do not directly interfere with the optical imaging readout, the investigation of neuronal representations of visual stimuli causes obvious problems for the experimenter. First, the excitation light is visually perceived by the animal, and second, applying visual stimuli may interfere with the detection system used to monitor emission light from the GECI. To address the question of how a distinct group of neurons within the optical lobe of the Drosophila brain, the lamina monopolar cells L2, contribute to motion vision, Reiff et al. [99] have developed a beautiful technique. Using a two-photon microscope the GECI, in this case the sensor TN-XXL [44], expressed in L2 cells was excited in the infrared range, which avoids a stimulation of the fly's photoreceptors. To be able to stimulate the retina with a moving optical stimulus without interfering with the optical readout the authors have applied a trick. Whenever the infrared laser has finished scanning a line across the specimen the visual stimulus was shortly applied, and since the switch between scanning and application of the visual stimulus was far below the temporal resolution of the fly's photoreceptors $(500 \mathrm{~Hz})$ visual stimulation and the detection could be separated. Thereby the authors have found that the $\mathrm{L} 2$ neurons under investigation transmit information about the brightness decrement occurring during the moving pattern [99]. Overall, optical $\mathrm{Ca}^{2+}$ imaging has been used to study neuronal representations of a variety of sensory modalities. It is not surprising that most studies employing $\mathrm{Ca}^{2+}$ imaging have focused on neuronal representations in primary sensory brain regions because sensory cells and directly coupled downstream neurons typically encode the sensory stimuli by membrane depolarizations and subsequent action potential frequencies as a function of stimulus intensity, reaching strong membrane depolarization, high firing frequencies and ultimately strong intracellular $\mathrm{Ca}^{2+}$ transients at high stimulus intensities. On the contrary, more centrally located neurons in higher brain regions often display only slight shifts in firing frequencies. However, optical $\mathrm{Ca}^{2+}$ imaging in third order olfactory neurons, the intrinsic neurons of the mushroom bodies (Kenyon cells), has also been successfully performed [100-103]. The mushroom body has been shown over many years to be a brain region necessary and sufficient for the association of odor stimuli with rewarding or punishing events through learning $[8,104,105]$. An interesting feature of the mushroom body is its proposed mode of encoding odors. Due to a high divergence from $\sim 150$ olfactory projection neurons onto 2500 Kenyon cells per brain hemisphere, a convergence of several projection neurons onto a given Kenyon cell and high firing thresholds of Kenyon cells, a sparse odor coding pattern is achieved $[103,106,107]$. That means, only very few 
out of a large array of Kenyon cells are selectively responding to any given odor stimulus $[103,106,107]$. Whereas this type of encoding information has been proposed because of computational considerations and electrophysiological recordings from individual cells, a more direct access would be to visualize the activity across larger ensembles of Kenyon cells. Indeed, this has been achieved recently using G-CaMP3 [47] genetically expressed in Kenyon cells, and the sparse activity of only a fraction out of hundreds of Kenyon cells evoked by a given odor has been visualized [103].

A sparse representation of odors across larger arrays of neurons would be, from a theoretical point of view, favorable to assign positive or negative values to specific odor signals through associative learning because of its less overlapping nature when compared to combinatorial coding scheme at the level of the antennal lobe $[8,104]$. The most commonly used associative learning procedure in Drosophila involves the presentation of an odor in temporal coincidence with electric shocks. A second odor is presented without any electric shock punishment. As a result, fruit flies strongly avoid the odor associated with and predicting the occurrence of the punitive stimulus [108]. In this context $\mathrm{Ca}^{2+}$ imaging using GECIs expressed in the mushroom bodies has been used to visualize changes in odor-evoked $\mathrm{Ca}^{2+}$ for the odor that has been temporally paired with an electric shock in comparison with the unpunished odor [100-102]. Therefore, $\mathrm{Ca}^{2+}$ imaging has been proven to be a valuable tool to determine neuronal correlates of associative learning and memory formation [100-102]. Moreover, modulatory neurons mediating the punishment information during this learning process [109], neurons mediating a consolidation process from shorter to longer lasting forms of olfactory memories [110], and mushroom body output neurons that are required for a behavioral readout of olfactory memories [111] have also been analyzed using $\mathrm{Ca}^{2+}$ imaging. Neuronal plasticity underlying learning and memory therefore represents another aspect for which optical $\mathrm{Ca}^{2+}$ imaging using GECIs has proven to be of enormous value. Lastly, we would like to note that this overview over $\mathrm{Ca}^{2+}$ imaging experiments in Drosophila is by far not all-encompassing, and the list of interesting investigations could certainly be substantially extended. In particular, $\mathrm{Ca}^{2+}$ imaging experiments at synaptic preparations, e.g. the larval neuromuscular junction, have not been addressed here.

\section{Optical calcium imaging of neuronal activity in Drosophila: practical information}

In the following paragraph we intend to illustrate how an optical $\mathrm{Ca}^{2+}$ imaging experiment can be performed with the help of two examples, first transcuticular optical $\mathrm{Ca}^{2+}$ imaging of olfactory sensory neurons in third instar larvae, and second optical $\mathrm{Ca}^{2+}$ imaging of olfactory neurons in the brain of adult animals. The first step is to create a preparation for microscopical access to the structures of interest in the brain or the sensory organs to be investigated. The simplest possibility is to excite the GECI and monitor its emitting light directly through the animal's cuticle without any surgical manipulation, provided the baseline fluorescence of the GECI is strong and the cells to be observed are located close to the cuticle [52]. The relative transparency of the Drosophila third instar larva allows for optical access to the olfactory sensory neurons located in the dorsal organs, the larval olfactory organs. Functional imaging in olfactory circuits of intact, living Drosophila larvae is, however, difficult due to the almost continuous peristaltic movements of the animals. Therefore, we have invented a method to immobilize the larva simply by placing the animal on a cover slip and attaching it with a transparent sticky tape such that its anterior sensory organs are exposed (Fig. 4A, B). This relatively simple preparation very efficiently reduces the animal's movements and allows reliable optical access to the somata and dendrites of larval olfactory sensory neurons (Fig. 4C). The FRET based GECI Cameleon $2.1[35,112]$ with its high baseline fluorescence was preferable for this approach. Furthermore, slight contractions or movements of the larval mouth hooks sometimes cannot be completely excluded, possibly leading to slight shifts in the focal plane and thus alterations in the fluorescence intensity. An internal control for these types of movement artifacts is advisable, and we therefore preferred in this case a ratiometric over a circularly permuted GECI. Using a fluorescence microscope equipped with a $20 \times$ air objective the preparation is illuminated at $440 \mathrm{~nm}$ wavelength, and the emitted light from ECFP and EYFP is projected onto the two halves
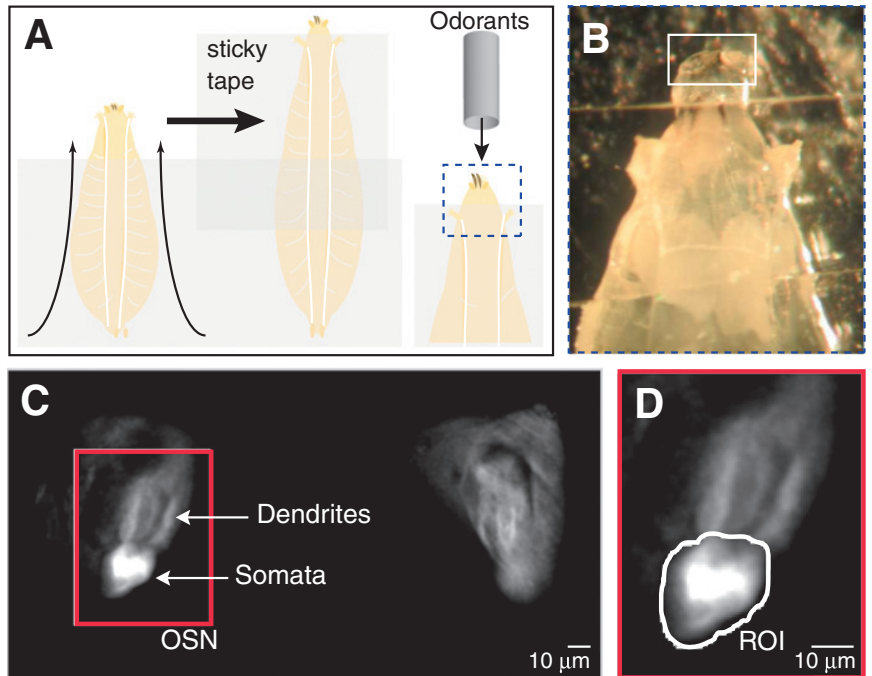

$\mathbf{E}$
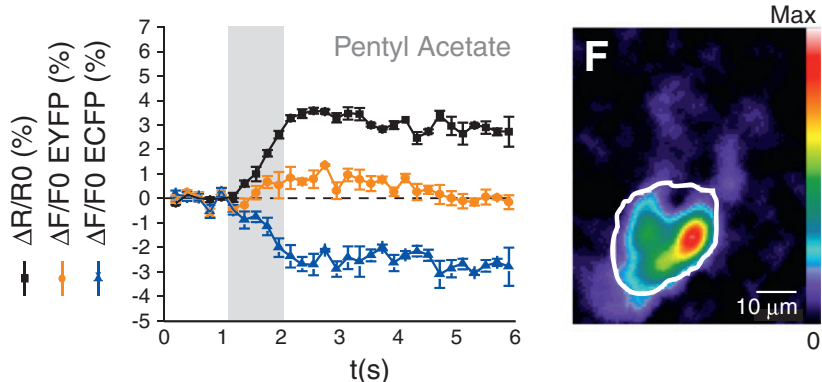

G
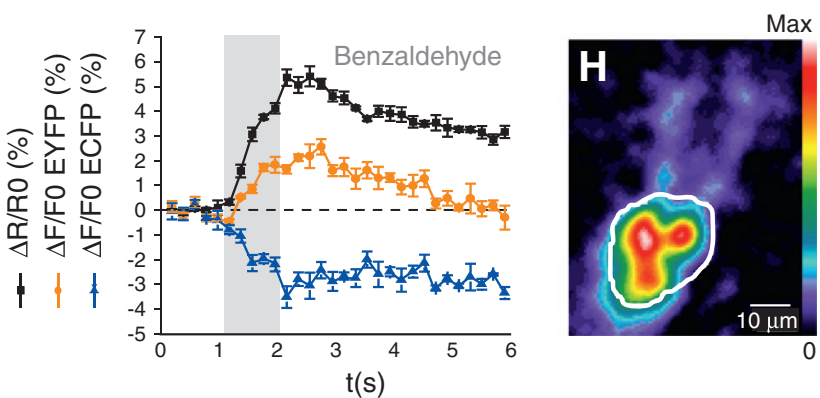

$\operatorname{Max}$

Fig 4. $\mathrm{Ca}^{2+}$ imaging of odor-evoked calcium dynamics in olfactory sensory neurons of third instar larvae using Cameleon 2.1 [35]. A) A Larva is immobilized with sticky tape to an object holder and slightly stretched. B) Magnification of an immobilized larva exposing the anterior region, indicated by the white rectangle, with the dorsal organs housing olfactory sensory neurons. C) Fluorescence image of Cameleon 2.1 in olfactory sensory neurons (OSN). D) The left dorsal organ shown in C) is in focus so that dendrites and somata can be differentiated. The white line indicates the region of interest (ROI) surrounding the somata in which changes in intracellular $\mathrm{Ca}^{2+}$ are determined. E-H) $\mathrm{Ca}^{2+}$ activity evoked by two different odorants in OSNs. E), G) Temporal dynamics $\mathrm{Ca}^{2+}$ activity in the ROI shown in D), evoked by pentyl acetate $(E)$ or benzaldehyde $(G)$, both diluted 1:10 in mineral oil. Relative changes in EYFP (yellow traces) and ECFP (blue traces) emission are indicated in addition to the ratio EYFP/ECFP (black traces). Traces indicate means \pm SEM of 3 odor stimulations within the same animal. The time of odor on- and offset is indicated as gray bars. F, H) False-color coded illustration of the spatial distribution of $\mathrm{Ca}^{2+}$ activity evoked by pentyl acetate $(\mathrm{F})$ and benzaldehyde $(\mathrm{H})$. Warm colors represent regions of high $\mathrm{Ca}^{2+}$ activity, cold colors regions of low or no $\mathrm{Ca}^{2+}$ increase. 
of a CCD camera chip. Two different odors, pentyl acetate (Fig. 4E, F) and benzaldehyde (Fig. 4G, H), were presented for $1 \mathrm{~s}$ each to the larva, and odor evoked $\mathrm{Ca}^{2+}$ responses of the olfactory sensory neurons were monitored at the level of the somata in the dorsal organ. $\mathrm{Ca}^{2+}$ activity elicited by these two odors differ in their spatial representation, as well as in the kinetics of the odor response.

Imaging calcium activities in the central nervous system of adult Drosophila requires a surgical intervention to achieve optical access to the brain. Here, we have anesthetized the flies briefly on ice and then restrained them with a transparent sticky tape covering a small chamber. From below the fly is restrained by a fine-meshed metal grid which slightly presses the fly against the sticky tape, but enables air exchange around the abdomen (Fig. 5A). A drop of a Ringer's solution is placed above the preparation, and a small hole is then cut through the sticky tape using a splint of a razor blade. The cuticle and tracheae and glands are carefully removed with fine forceps to expose the antennal lobes (Fig. 5B, C). In the example shown in Fig. 5 the ratiometric GECI Cameleon 2.1 is expressed in a large number of olfactory sensory neurons using the driver line Or83b-Gal4 [84]. The odors are then applied directly onto the fly's antennae using a controlled odor-delivery system and the temporal dynamics and spatial distribution of the calcium activities can be monitored using the imaging microscope equipped with a $20 \times$ water immersion objective. Here we demonstrate the responses of first order olfactory receptor neurons in different regions of the antennal lobes to odor stimulations of $2 \mathrm{~s}$ with methyl acetate (Fig. 5D, F) and pentanoic acid (Fig. 5E, G). Differential spatial and also temporal activity patterns in the antennal lobe of the fly can be determined.

We have used these experimental conditions to directly compare the FRET-based GECI Cameleon $2.1[35,112]$ and the latest innovation of the circularly permutated GECIs, G-CaMP3 [47]. Therefore, we expressed Cameleon 2.1 or G-CaMP3, respectively, under the control of the GH146-Gal4 driver line [113] in olfactory projection neurons and monitored $\mathrm{Ca}^{2+}$ dynamics evoked by $2 \mathrm{~s}$ odor stimulations with pentanoic acid in dendritic arborizations within the flies' antennal lobes. Both Cameleon 2.1 (Fig. 6A) and G-CaMP3 (Fig. 6B) show a well detectable baseline fluorescence, which facilitates the anatomical identification of the regions of interest, in this case the antennal lobes. Both Cameleon 2.1 (Fig. 6C) and G-CaMP3 (Fig. 6D) report clearly increases in intracellular $\mathrm{Ca}^{2+}$. However, the relative change in fluorescence using G-CaMP3 is much stronger when compared to Cameleon 2.1. Both GECIs show a very good signal to noise ratio, and both show similar dynamics of $\mathrm{Ca}^{2+}$ influx in olfactory sensory neurons in response to the odor (Fig. 6E, F).

\section{Summary and future directions}

Optical $\mathrm{Ca}^{2+}$ imaging using GECIs in Drosophila provides advantages over electrophysiological techniques when a spatial analysis of neuronal activity is required. First, the logic of stimulus representations across arrays of sensory and higher-order neurons can be analyzed, e.g. by the visualization of odor representations at the level of the primary olfactory neuropil (antennal lobe) with its highly overlapping, combinatorial coding scheme [30,84], or at the level of a higher order brain region (mushroom body) encoding information in terms of a sparse code principle [103]. Similarly, the logic of a differential representation of stimulus categories within the brain can be assessed, as in the case of different taste categories [93]. Second, spatio-temporal activity within individual cells can be visualized as in the case of the L2 cells of the motion vision system within the fly's optical lobes [99]. Third, changes in stimulus representations due to learning can be demonstrated across ensembles of neurons [100-102,109-111].
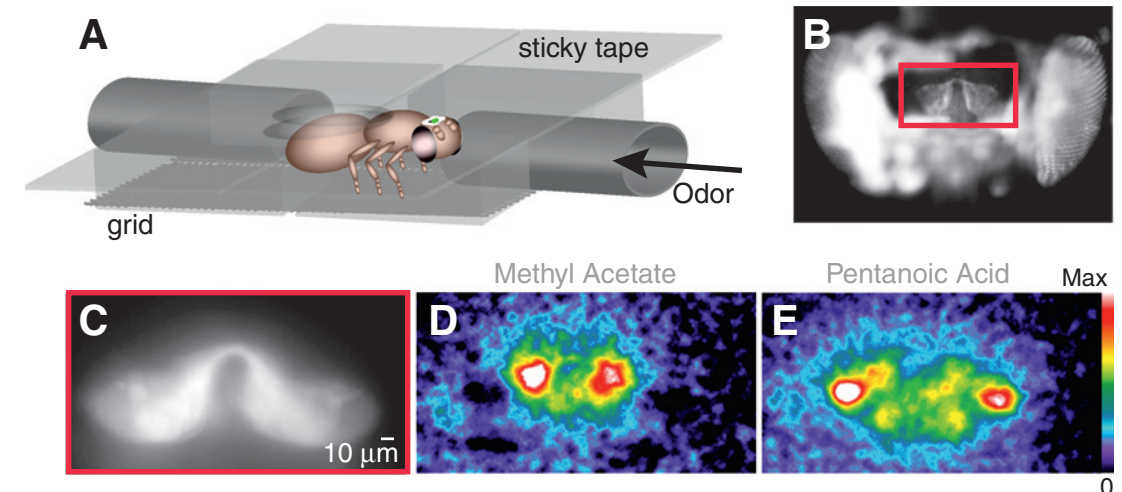

$\mathbf{F}$

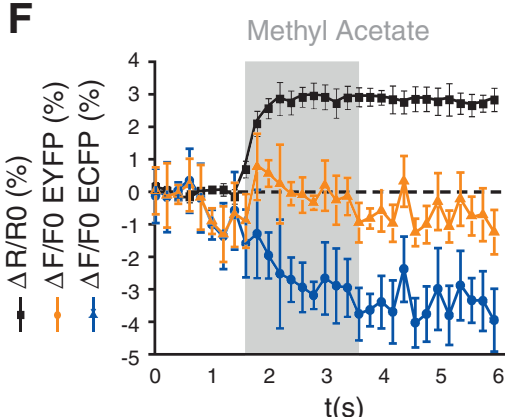

G

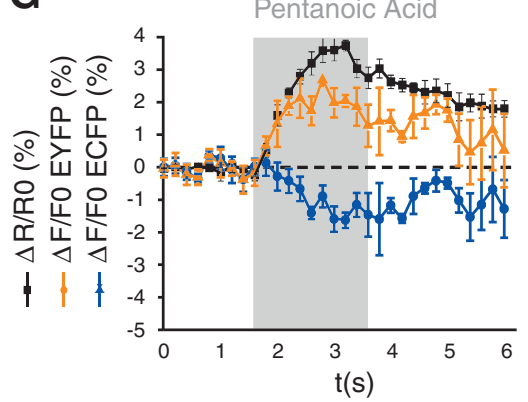

Fig. 5. $\mathrm{Ca}^{2+}$ imaging of odor-evoked calcium dynamics in olfactory sensory neurons in adult Drosophila using Cameleon 2.1 [35]. A) The fly's head is fixed to a thin sticky tape and restrained into a chamber with the antennae directed towards an air stream to which an odor can be applied. B) A window is cut through sticky tape and into the head capsule, thereby exposing the brain and the fluorescent antennal lobes. C) Fluorescence image Cameleon's EYFP emission in the two antennal lobes. D-E) False-color coded illustration of the spatial, glomerular distribution of $\mathrm{Ca}^{2+}$ activity in the antennal lobe evoked by $2 \mathrm{~s}$ of stimulation with methyl acetate (D) or pentanoic acid (E), diluted 1:5000 or 1:50 in mineral oil, respectively. Warm colors represent regions of high calcium activity, cold colors regions of low or no calcium increase. F, G) Temporal dynamics of intracellular Ca ${ }^{2+}$ activity in regions of high activity evoked by methyl acetate (F) or pentanoic acid $(G)$, indicated as gray bars. Relative changes in EYFP (yellow traces) and ECFP emission (blue traces) are indicated in addition to the ratio EYFP/ECFP (black traces). Traces indicate means \pm SEM of 3 odor stimulations within the same animal. 


\section{Cameleon 2.1}
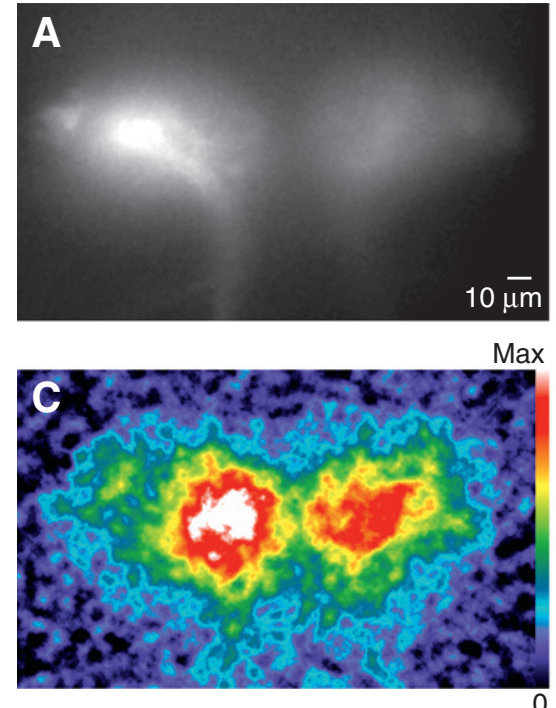

E

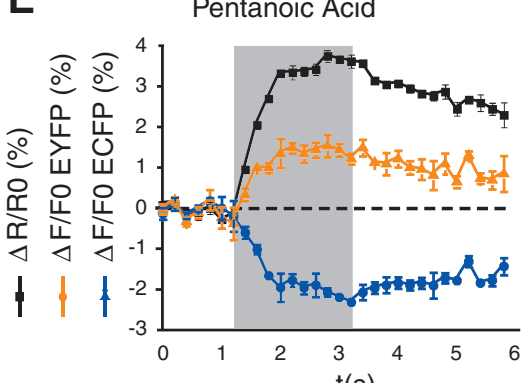

G-CaMP3

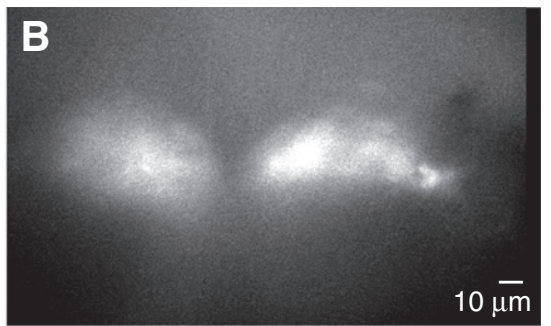

Max

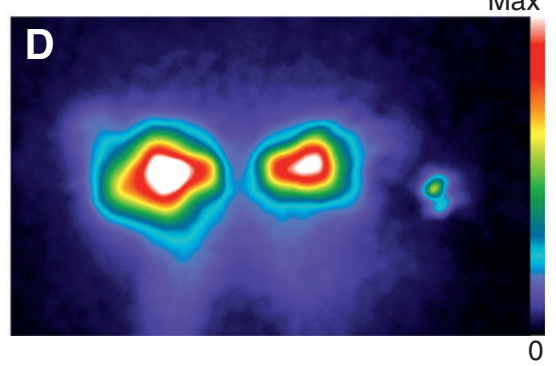

F

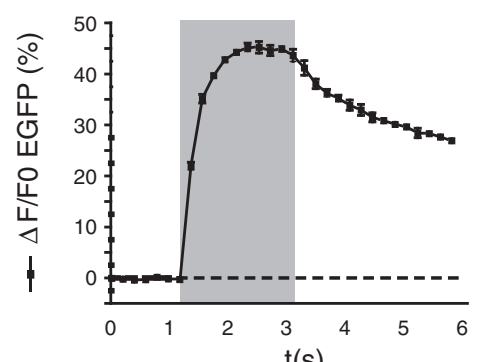

$t(s)$

Fig. 6. Comparison between the circularly permutated GECI G-CaMP3 [47] and the FRET-based sensor Cameleon 2.1 [35]. Cameleon 2.1 (A, C,E) and G-CaMP3 (B, D, F) are expressed in olfactory projection neurons and the odor response evoked by a $2 \mathrm{~s}$ stimulation with pentanoic acid, diluted 1:5000 in mineral oil, is measured at the level of the antennal lobes. A) and B) indicate the baseline fluorescence in both antennal lobes, either of the Cameleon's EYFP emission (A) or the G-CaMP emission (B). C, D) False-color coded illustration of the spatial distribution of odor-evoked calcium activity in Cameleon-expressing neurons (C) and G-CaMP expressing neurons (D). Warm colors represent regions of high calcium activity, cold colors regions of low or no calcium increase. E, F) Traces indicate means \pm SEM of 3 odor stimulations of three different animals each. For cameleon 2.1 (E) the relative changes in fluorescence emission is indicated for EYFP (yellow trace) and EYFP (blue trace) in addition to the ratio EYFP/ECFP (black trace).

Obviously, optical $\mathrm{Ca}^{2+}$ imaging represents only one physiological approach amongst others with certain advantages, but also inherent limitations. Most importantly, the spatial and the temporal resolution, both dependent on the microscope used, can be limited. The spatial resolution is often poor because of the sheer number of neurons that express the fluorescent probe, in particular when many neurons are densely packed in particular brain regions such that only sum activities can be observed. Several approaches have been pursuit in the field of Drosophila neurobiology to restrict the expression of genes for monitoring and manipulating neuronal activity to low numbers of identifiable neurons. First, more and more transgenic lines are being generated with exclusive and well described expression patterns (e.g. [114]). And second, strategies are being developed to combine different activators and inhibitors of gene expression to restrict the ultimate expression of genes to small subsets of neurons [summarized in 16]. Ultimately, these attempts will enable researchers to successively analyze many individual neurons. With respect to temporal resolution GECIs will have to be developed that will be sensitive enough to reliably detect very transient neuronal activity, e.g. $\mathrm{Ca}^{2+}$ dynamics accompanying single action potentials or subthreshold membrane potential fluctuations,. In addition, it is important to note that optical $\mathrm{Ca}^{2+}$ imaging can provide correlates for neuronal excitation. However, neuronal inhibition, typically mediated by chloride ions, represents a facet of neural coding that is equally important. Genetically encoded chloride-sensitive indicators [115,116] might, therefore, provide helpful tools.

\section{Acknowledgements}

This work has been supported by the German Science Foundation (SPP 1392 FI 821/2-1 and SFB 889/B04) and the German Federal Ministry for Education and Research via the Bernstein Center for Computational Neuroscience Göttingen.

\section{References}

[1] G.M. Rubin, Drosophila melanogaster as an experimental organism, Science 240 (1988) 1453-1459.

[2] K.F. Fischbach, M. Heisenberg, Structural brain mutant of Drosophila melanogaster with reduced cell number in the medulla cortex and with normal optomotor yaw response, Proc. Natl. Acad. Sci. U. S. A. 78 (1981) 1105-1109.

[3] M. Heisenberg, A. Borst, S. Wagner, D. Byers, Drosophila mushroom body mutants are deficient in olfactory learning, J. Neurogenet. 2 (1985) 1-30.

[4] R. Strauss, M. Heisenberg, A higher control center of locomotor behavior in the Drosophila brain, J. Neurosci. 13 (1993) 1852-1861.

[5] K.S. Broadie, Synaptogenesis in Drosophila: coupling genetics and electrophysiology, J. Physiol. Paris 88 (1994) 123-139.

[6] P. Clyne, A. Grant, R. O'Connell, J.R. Carlson, Odorant response of individual sensilla on the Drosophila antenna, Invert. Neurosci. 3 (1997) 127-135.

[7] R.I. Wilson, G.C. Turner, G. Laurent, Transformation of olfactory representations in the Drosophila antennal lobe, Science 303 (2004) 366-370.

[8] A. Fiala, Olfaction and olfactory learning in Drosophila: recent progress, Curr. Opin. Neurobiol. 17 (2007) 720-726.

[9] C. Montell, A taste of the Drosophila gustatory receptors, Curr. Opin. Neurobiol. 19 (2009) 345-353.

[10] A. Borst, Drosophila's view on insect vision, Curr. Biol. 19 (2009) 36-47.

[11] N. Peschel, C. Helfrich-Förster, Setting the clock-by nature: circadian rhythm in the fruitfly Drosophila melanogaster, FEBS Lett. 585 (2011) 1435-1442. 
[12] D.R. Nässel, A.M. Winther, Drosophila neuropeptides in regulation of physiology and behavior, Prog. Neurobiol. 92 (2010) 42-104.

[13] E. Buchner, S. Buchner, R. Hengstenberg, 2-Deoxy-D-glucose maps movementspecific nervous activity in the second visual ganglion of Drosophila, Science 205 (1997) 687-688.

[14] E. Buchner, S. Buchner, Mapping stimulus-induced nervous activity in small brains by [3H]2-deoxy-D-glucose, Cell Tissue Res. 211 (1980) 51-64.

[15] V. Rodrigues, E. Buchner, [3H]2-deoxyglucose mapping of odor-induced neuronal activity in the antennal lobes of Drosophila melanogaster, Brain Res. 324 (1984) 374-378.

[16] K.J. Venken, J.H. Simpson, H.J. Bellen, Genetic manipulation of genes and cells in the nervous system of the fruit fly, Neuron 72 (2011) 202-230.

[17] A.E. Palmer, R.Y. Tsien, Measuring calcium signaling using genetically targetable fluorescent indicators, Nat. Protoc. 1 (2006) 1057-1065.

[18] M. Mank, O. Griesbeck, Genetically encoded calcium indicators, Chem. Rev. 108 (2008) 1550-1564.

[19] S.A. Hires, L. Tian, L.L. Looger, Reporting neural activity with genetically encoded calcium indicators, Brain Cell Biol. 36 (2008) 69-86.

[20] G. Miesenböck, D.A. De Angelis, J.E. Rothman, Visualizing secretion and synaptic transmission with pH-sensitive green fluorescent proteins, Nature 394 (1998) 192-195.

[21] A.Y. Ting, K.H. Kain, R.L. Klemke, R.Y. Tsien, Genetically encoded fluorescent reporters of protein tyrosine kinase activities in living cells, Proc. Natl. Acad. Sci. U. S. A. 98 (2001) 15003-15008.

[22] J. Zhang, Y. Ma, S.S. Taylor, R.Y. Tsien, Genetically encoded reporters of protein kinase A activity reveal impact of substrate tethering, Proc. Natl. Acad. Sci. U. S. A. 98 (2001) 14997-15002.

[23] M. Sato, T. Ozawa, K. Inukai, T. Asano, Y. Umezawa, Fluorescent indicators for imaging protein phosphorylation in single living cells, Nat. Biotechnol. 20 (2002) 287-294.

[24] J.D. Violin, J. Zhang, R.Y. Tsien, A.C. Newton, A genetically encoded fluorescent reporter reveals oscillatory phosphorylation by protein kinase C, J. Cell Biol. 161 (2003) 899-909.

[25] D. Willoughby, D.M. Cooper, Live-cell imaging of cAMP dynamics, Nat. Methods 5 (2008) 29-36.

[26] H. Mutoh, A. Perron, W. Akemann, Y. Iwamoto, T. Knöpfel, Optogenetic monitoring of membrane potentials, Exp. Physiol. 96 (2011) 13-18.

[27] M.J. Berridge, Neuronal calcium signaling, Neuron 21 (1998) 13-26.

[28] A. Miyawaki, J. Llopis, R. Heim, J.M. McCaffery, J.A. Adams, M. Ikura, R.Y. Tsien, Fluorescent indicators for $\mathrm{Ca}^{2+}$ based on green fluorescent proteins and calmodulin, Nature 388 (1997) 882-887.

[29] V.A. Romoser, P.M. Hinkle, A. Persechini, Detection in living cells of $\mathrm{Ca}^{2+}$-dependent changes in the fluorescence emission of an indicator composed of two green fluorescent protein variants linked by a calmodulin-binding sequence. A new class of fluorescent indicators, J. Biol. Chem. 272 (1997) 13270-13274.

[30] A. Fiala, T. Spall, S. Diegelmann, B. Eisermann, S. Sachse, J.M. Devaud, E. Buchner, C.G. Galizia, Genetically expressed cameleon in Drosophila melanogaster is used to visualize olfactory information in projection neurons, Curr. Biol. 12 (2002) 1877-1884.

[31] N. Heim, O. Griesbeck, Genetically encoded indicators of cellular calcium dynamics based on troponin C and green fluorescent protein, J. Biol. Chem. 279 (2004) 14280-14286.

[32] G.S. Baird, D.A. Zacharias, R.Y. Tsien, Circular permutation and receptor insertion within green fluorescent proteins, Proc. Natl. Acad. Sci. U. S. A. 96 (1999) 11241-11246.

[33] T. Nagai, A. Sawano, E.S. Park, A. Miyawaki, Circularly permuted green fluorescent proteins engineered to sense $\mathrm{Ca}^{2+}$, Proc. Natl. Acad. Sci. U. S. A. 98 (2001) 3197-3202.

[34] J. Nakai, M. Ohkura, K. Imoto, A high signal-to-noise $\mathrm{Ca}\left({ }^{2+}\right)$ probe composed of a single green fluorescent protein, Nat. Biotechnol. 19 (2001) 137-141.

[35] A. Miyawaki, O. Griesbeck, R. Heim, R.Y. Tsien, Dynamic and quantitative $\mathrm{Ca}^{2+}$ measurements using improved cameleons, Proc. Natl. Acad. Sci. U. S. A. 96 (1999) 2135-2140.

[36] O. Griesbeck, G.S. Baird, R.E. Campbell, D.A. Zacharias, R.Y. Tsien, Reducing the environmental sensitivity of yellow fluorescent protein. Mechanism and applications, J. Biol. Chem. 276 (2001) 29188-29194.

[37] A.E. Palmer, C. Jin, J.C. Reed, R.Y. Tsien, Bcl-2-mediated alterations in endoplasmic reticulum $\mathrm{Ca}^{2+}$ analyzed with an improved genetically encoded fluorescent sensor, Proc. Natl. Acad. Sci. U. S. A. 101 (2004) 17404-17409.

[38] T. Nagai, S. Yamada, T. Tominaga, M. Ichikawa, A. Miyawaki, Expanded dynamic range of fluorescent indicators for $\mathrm{Ca}\left({ }^{2+}\right)$ by circularly permuted yellow fluorescent proteins, Proc. Natl. Acad. Sci. U. S. A. 101 (2004) 10554-10559.

[39] A.E. Palmer, M. Giacomello, T. Kortemme, S.A. Hires, V. Lev-Ram, D. Baker, R.Y. Tsien, $\mathrm{Ca}^{2+}$ indicators based on computationally redesigned calmodulinpeptide pairs, Chem. Biol. 13 (2006) 521-530.

[40] K. Truong, A. Sawano, H. Mizuno, H. Hama, K.I. Tong, T.K. Mal, A. Miyawaki, M. Ikura, FRET-based in vivo $\mathrm{Ca}^{2+}$ imaging by a new calmodulin-GFP fusion molecule, Nat. Struct. Biol. 8 (2001) 1069-1073.

[41] K. Horikawa, Y. Yamada, T. Matsuda, K. Kobayashi, M. Hashimoto, T. Matsu-ura, A. Miyawaki, T. Michikawa, K. Mikoshiba, T. Nagai, Spontaneous network activity visualized by ultrasensitive $\left.\mathrm{Ca}^{2+}{ }^{2+}\right)$ indicators, yellow Cameleon-Nano, Nat. Methods 7 (2010) 729-732.

[42] M. Mank, D.F. Reiff, N. Heim, M.W. Friedrich, A. Borst, O. Griesbeck, A FRET-based calcium biosensor with fast signal kinetics and high fluorescence change, Biophys. J. 90 (2006) 1790-1796.

[43] N. Heim, O. Garaschuk, M.W. Friedrich, M. Mank, R.I. Milos, Y. Kovalchuk, A. Konnerth, O. Griesbeck, Improved calcium imaging in transgenic mice expressing a troponin C-based biosensor, Nat. Methods 4 (2007) 127-129.
[44] M. Mank, A.F. Santos, S. Direnberger, T.D. Mrsic-Flogel, S.B. Hofer, V. Stein, T. Hendel, D.F. Reiff, C. Levelt, A. Borst, T. Bonhoeffer, M. Hübener, O. Griesbeck, A genetically encoded calcium indicator for chronic in vivo two-photon imaging, Nat. Methods 5 (2008) 805-811.

[45] M. Ohkura, M. Matsuzaki, H. Kasai, K. Imoto, J. Nakai, Genetically encoded brigh $\mathrm{Ca}^{2+}$ probe applicable for dynamic $\mathrm{Ca}^{2+}$ imaging of dendritic spines, Anal. Chem. 77 (2005) 5861-5869.

[46] Y.N. Tallini, M. Ohkura, B.R. Choi, G. Ji, K. Imoto, R. Doran, J. Lee, P. Plan, J. Wilson, H.B. Xin, A. Sanbe, J. Gulick, J. Mathai, J. Robbins, G. Salama, J. Nakai, M.I. Kotlikoff, Imaging cellular signals in the heart in vivo: cardiac expression of the high-signal $\mathrm{Ca}^{2+}$ indicator GCaMP2, Proc. Natl. Acad. Sci. U. S. A. 103 (2006) 4753-4758.

[47] L. Tian, S.A. Hires, T. Mao, D. Huber, M.E. Chiappe, S.H. Chalasani, L. Petreanu, J. Akerboom, S.A. McKinney, E.R. Schreiter, C.I. Bargmann, V. Jayaraman, K. Svoboda, L.L. Looger, Imaging neural activity in worms, flies and mice with improved GCaMP calcium indicators, Nat. Methods 6 (2009) 875-881.

[48] A. Muto, M. Ohkura, T. Kotani, S. Higashijima, J. Nakai, K. Kawakami, Genetic visualization with an improved GCaMP calcium indicator reveals spatiotemporal activation of the spinal motor neurons in zebrafish, Proc. Natl. Acad. Sci. U. S. A. 108 (2011) 5425-5430.

[49] D.F. Reiff, A. Ihring, G. Guerrero, E.Y. Isacoff, M. Joesch, J. Nakai, A. Borst, In vivo performance of genetically encoded indicators of neural activity in flies, J. Neurosci. 25 (2005) 4766-4778.

[50] T. Hendel, M. Mank, B. Schnell, O. Griesbeck, A. Borst, D.F. Reiff, Fluorescence changes of genetic calcium indicators and OGB-1 correlated with neural activity and calcium in vivo and in vitro, J. Neurosci. 28 (2008) 7399-7411.

[51] A. Fiala, T. Spall, In vivo calcium imaging of brain activity in Drosophila by transgenic cameleon expression, Sci. STKE 174 (2003) PL6.

[52] A. Kamikouchi, R. Wiek, T. Effertz, M.C. Göpfert, A. Fiala, Transcuticular optical imaging of stimulus-evoked neural activities in the Drosophila peripheral nervous system, Nat. Protoc. 5 (2010) 1229-1235.

[53] M.D. Adams, et al., The genome sequence of Drosophila melanogaster, Science 287 (2000) 2185-2195.

[54] G.M. Rubin, A.C. Spradling, Genetic transformation of Drosophila with transposable element vectors, Science 218 (1982) 348-353.

[55] J.A. Fischer, E. Giniger, T. Maniatis, M. Ptashne, GAL4 activates transcription in Drosophila, Nature 332 (1988) 853-856.

[56] A.H. Brand, N. Perrimon, Targeted gene expression as a means of altering cell fates and generating dominant phenotypes, Development 118 (1993) 401-415.

[57] http://flystocks.bio.indiana.edu/.

[58] http://www.dgrc.kit.ac.jp/en/index.html

[59] J.B. Duffy, GAL4 system in Drosophila: a fly geneticist's Swiss army knife, Genesis 34 (2002) 1-15.

[60] B.D. Pfeiffer, T.T. Ngo, K.L. Hibbard, C. Murphy, A. Jenett, J.W. Truman, G.M. Rubin, Refinement of tools for targeted gene expression in Drosophila, Genetics 186 (2010) 735-755.

[61] D. Szüts, M. Bienz, LexA chimeras reveal the function of Drosophila Fos as a context-dependent transcriptional activator, Proc. Natl. Acad. Sci. U. S. A. 97 (2000) 5351-5356

[62] S.L. Lai, T. Lee, Genetic mosaic with dual binary transcriptional systems in Drosophila, Nat. Neurosci. 9 (2006) 703-709.

[63] C.J. Potter, B. Tasic, E.V. Russler, L. Liang, L. Luo, The Q system: a repressible binary system for transgene expression, lineage tracing, and mosaic analysis, Cell 141 (2010) 536-548.

[64] C.J. Potter, L. Luo, Using the Q system in Drosophila melanogaster, Nat. Protoc. 6 (2011) 1105-1120.

[65] T. Lee, L. Luo, Mosaic analysis with a repressible neurotechnique cell marker for studies of gene function in neuronal morphogenesis, Neuron 22 (1999) 451-461.

[66] D.F. Reiff, P.R. Thiel, C.M. Schuster, Differential regulation of active zone density during long-term strengthening of Drosophila neuromuscular junctions, J. Neurosci. 22 (2002) 9399-9409.

[67] P.J. Clyne, C.G. Warr, M.R. Freeman, D. Lessing, J. Kim, J.R. Carlson, A novel family of divergent seven-transmembrane proteins: candidate odorant receptors in Drosophila, Neuron 22 (1999) 327-338.

[68] L.B. Vosshall, H. Amrein, P.S. Morozov, A. Rzhetsky, R. Axel, A spatial map of olfactory receptor expression in the Drosophila antenna, Cell 96 (1999) 725-736.

[69] R. Benton, K.S. Vannice, C. Gomez-Diaz, L.B. Vosshall, Variant ionotropic glutamate receptors as chemosensory receptors in Drosophila, Cell 136 (2009) $149-162$.

[70] P.J. Clyne, C.G. Warr, J.R. Carlson, Candidate taste receptors in Drosophila, Science 287 (2000) 1830-1834.

[71] K. Scott, R. Brady Jr., A. Cravchik, P. Morozov, A. Rzhetsky, C. Zuker, R. Axel, A chemosensory gene family encoding candidate gustatory and olfactory receptors in Drosophila, Cell 104 (2001) 661-673.

[72] W.D. Tracey Jr., R.I. Wilson, G. Laurent, S. Benzer, painless, a Drosophila gene essential for nociception, Cell 113 (2003) 261-273.

[73] Y. Lee, Y. Lee, J. Lee, S. Bang, S. Hyun, J. Kang, S.T. Hong, E. Bae, B.K. Kaang, J. Kim, Pyrexia is a new thermal transient receptor potential channel endowing tolerance to high temperatures in Drosophila melanogaster, Nat. Genet. 37 (2005) 305-310.

[74] M. Rosenzweig, K.M. Brennan, T.D. Tayler, P.O. Phelps, A. Patapoutian, P.A. Garrity, The Drosophila ortholog of vertebrate TRPA1 regulates thermotaxis, Genes Dev. 19 (2005) 419-424.

[75] M. Rosenzweig, K. Kang, P.A. Garrity, Distinct TRP channels are required for warm and cool avoidance in Drosophila melanogaster, Proc. Natl. Acad. Sci. U. S. A. (2008) 14668-14673. 
[76] F.N. Hamada, M. Rosenzweig, K. Kang, S.R. Pulver, A. Ghezzi, T.J. Jegla, P.A Garrity, An internal thermal sensor controlling temperature preference in Drosophila, Nature 454 (2008) 217-220.

[77] M. Gallio, T.A. Ofstad, L.J. Macpherson, J.W. Wang, C.S. Zuker, The coding of temperature in the Drosophila brain, Cell 144 (2011) 614-624.

[78] A. Kamikouchi, T. Shimada, K. Ito, Comprehensive classification of the auditory sensory projections in the brain of the fruit fly Drosophila melanogaster, J. Comp. Neurol. 499 (2006) 317-356

[79] A. Kamikouchi, H.K. Inagaki, T. Effertz, O. Hendrich, A. Fiala, M.C. Göpfert, K. Ito, The neural basis of Drosophila gravity-sensing and hearing, Nature 458 (2009) 165-171.

[80] S. Yorozu, A. Wong, B.J. Fischer, H. Dankert, M.J. Kernan, A. Kamikouchi, K. Ito, D.J. Anderson, Distinct sensory representations of wind and near-field sound in the Drosophila brain, Nature 458 (2009) 201-205.

[81] T. Effertz, R. Wiek, M.C. Göpfert, NompC TRP channel is essential for Drosophila sound receptor function, Curr. Biol. 21 (2011) 592-597.

[82] L.B. Vosshall, R.F. Stocker, Molecular architecture of smell and taste in Drosophila, Annu. Rev. Neurosci. 30 (2007) 505-533.

[83] C.G. Galizia, S. Sachse, Odor coding in insects, in: A. Menini (Ed.), The Neurobiology of Olfaction, CRC Press, Boca Raton (FL), 2010, chapter 2.

[84] J.W. Wang, A.M. Wong, J. Flores, L.B. Vosshall, R. Axel, Two-photon calcium imaging reveals an odor-evoked map of activity in the fly brain, Cell 112 (2003) 271-282.

[85] J. Joerges, A. Kuettner, C.G. Galizia, R. Menzel, Representations of odors and odor mixtures visualized in the honeybee brain, Nature 387 (1997) 285-288.

[86] C.G. Galizia, R. Menzel, B. Hölldobler, Optical imaging of odor-evoked glomerular activity patterns in the antennal lobes of the ant Camponotus rufipes, Naturwissenschaften 86 (1999) 533-537.

[87] C.G. Galizia, S. Sachse, H. Mustaparta, Calcium responses to pheromones and plant odours in the antennal lobe of the male and female moth Heliothis virescens, J. Comp. Physiol. A 186 (2000) 1049-1063.

[88] A.F. Silbering, C.G. Galizia, Processing of odor mixtures in the Drosophila antennal lobe reveals both global inhibition and glomerulus-specific interactions, J. Neurosci. 27 (2007) 11966-11977.

[89] A.F. Silbering, R. Okada, K. Ito, C.G. Galizia, Olfactory information processing in the Drosophila antennal lobe: anything goes? J. Neurosci. 28 (2008) 13075-13087.

[90] T. Niewalda, T. Völler, C. Eschbach, J. Ehmer, W.C. Chou, M. Timme, A. Fiala, B. Gerber, A combined perceptual, physico-chemical, and imaging approach to 'odour-distances' suggests a categorizing function of the Drosophila antennal lobe, PLoS One 6 (2011) e24300.

[91] S. Sachse, E. Rueckert, A. Keller, R. Okada, N.K. Tanaka, K. Ito, L.B. Vosshall, Activity-dependent plasticity in an olfactory circuit, Neuron 56 (2007) 838-850.

[92] E.A. Hallem, A. Dahanukar, J.R. Carlson, Insect odor and taste receptors, Annu. Rev. Entomol. 51 (2006) 113-135.

[93] S. Marella, W. Fischler, P. Kong, S. Asgarian, E. Rueckert, K. Scott, Imaging taste responses in the fly brain reveals a functional map of taste category and behavior, Neuron 49 (2006) 285-295

[94] M. Hiroi, N. Meunier, F. Marion-Poll, T. Tanimura, Two antagonistic gustatory receptor neurons responding to sweet-salty and bitter taste in Drosophila, J. Neurobiol. 61 (2004) 333-342.

[95] A. Dahanukar, Y.T. Lei, J.Y. Kwon, J.R. Carlson, Two Gr genes underlie sugar reception in Drosophila, Neuron 56 (2007) 503-516.

[96] L.A. Weiss, A. Dahanukar, J.Y. Kwon, D. Banerjee, J.R. Carlson, The molecular and cellular basis of bitter taste in Drosophila, Neuron 69 (2011) 258-272.

[97] P. Cameron, M. Hiroi, J. Ngai, K. Scott, The molecular basis for water taste in Drosophila, Nature 465 (2010) 91-95.

[98] W. Fischler, P. Kong, S. Marella, K. Scott, The detection of carbonation by the Drosophila gustatory system, Nature 448 (2007) 1054-1057.

[99] D.F. Reiff, J. Plett, M. Mank, O. Griesbeck, A. Borst, Visualizing retinotopic halfwave rectified input to the motion detection circuitry of Drosophila, Nat. Neurosci. 13 (2010) 973-978.

[100] Y. Wang, A. Mamiya, A.S. Chiang, Y. Zhong, Imaging of an early memory trace in the Drosophila mushroom body, J. Neurosci. 28 (2008) 4368-4376.

[101] D. Yu, D.B. Akalal, R.L. Davis, Drosophila alpha/beta mushroom body neurons form a branch-specific, long-term cellular memory trace after spaced olfactory conditioning, Neuron 52 (2006) 845-855.

[102] D.B. Akalal, D. Yu, R.L. Davis, A late-phase, long-term memory trace forms in the $\gamma$ neurons of Drosophila mushroom bodies after olfactory classical conditioning J. Neurosci. 30 (2010) 16699-16708.

[103] K.S. Honegger, R.A. Campbell, G.C. Turner, Cellular-resolution population imaging reveals robust sparse coding in the Drosophila mushroom body, J. Neurosci. 31 (2011) 11772-11785.

[104] M. Heisenberg, Mushroom body memoir: from maps to models, Nat. Rev. Neurosci. 4 (2003) 266-275.
[105] G.U. Busto, I. Cervantes-Sandoval, R.L. Davis, Olfactory learning in Drosophila Physiology (Bethesda) 25 (2010) 338-346.

[106] G.C. Turner, M. Bazhenov, G. Laurent, Olfactory representations by Drosophila mushroom body neurons, J. Neurophysiol. 99 (2008) 734-746.

[107] S.X. Luo, R. Axel, L.F. Abbott, Generating sparse and selective third-order responses in the olfactory system of the fly, Proc. Natl. Acad. Sci. U. S. A. 107 (2010) 10713-10718.

[108] T. Tully, W.G. Quinn, Classical conditioning and retention in normal and mutant Drosophila melanogaster, J. Comp. Physiol. A 157 (1985) 263-277.

[109] T. Riemensperger, T. Völler, P. Stock, E. Buchner, A. Fiala, Punishment prediction by dopaminergic neurons in Drosophila, Curr. Biol. 15 (2005) 1953-1960.

[110] D. Yu, A.C. Keene, A. Srivatsan, S. Waddell, R.L. Davis, Drosophila DPM neurons form a delayed and branch-specific memory trace after olfactory classical conditioning, Cell 123 (2005) 945-957.

[111] J. Séjourné, P.Y. Plaçais, Y. Aso, I. Siwanowicz, S. Trannoy, V. Thoma, S.R Tedjakumala, G.M. Rubin, P. Tchénio, K. Ito, G. Isabel, H. Tanimoto, T. Preat Mushroom body efferent neurons responsible for aversive olfactory memory retrieval in Drosophila, Nat. Neurosci. 14 (2011) 903-910.

[112] S. Diegelmann, A. Fiala, C. Leibold, T. Spall, E. Buchner, Transgenic flies expressing the fluorescence calcium sensor Cameleon 2.1 under UAS control, Genesis 34 (2002) 95-98.

[113] R.F. Stocker, G. Heimbeck, N. Gendre, J.S. de Belle, Neuroblast ablation in Drosophila P[GAL4] lines reveals origins of olfactory interneurons, J. Neurobiol. 32 (1997) 443-456

[114] B.D. Pfeiffer, A. Jenett, A.S. Hammonds, T.T. Ngo, S. Misra, C. Murphy, A. Scully, J.W. Carlson, K.H. Wan, T.R. Laverty, C. Mungall, R. Svirskas, J.T. Kadonaga, C.Q. Doe, M.B. Eisen, S.E. Celniker, G.M. Rubin, Tools for neuroanatomy and neurogenetics in Drosophila, Proc. Natl. Acad. Sci. U. S. A. 105 (2008) 9715-9720.

[115] T. Kuner, G.J. Augustine, A genetically encoded ratiometric indicator for chloride: capturing chloride transients in cultured hippocampal neurons, Neuron 27 (2000) 447-459.

[116] O. Markova, M. Mukhtarov, E. Real, Y. Jacob, P. Bregestovski, Genetically encoded chloride indicator with improved sensitivity, J. Neurosci. Methods 170 (2008) 67-76.

[117] S. Diegelmann, M. Bate, M. Landgraf, Gateway cloning vectors for the LexAbased binary expression system in Drosophila, Fly (Austin) 2 (2008) 236-239.

[118] D. Pelz, T. Roeske, Z. Syed, M. de Bruyne, C.G. Galizia, The molecular receptive range of an olfactory receptor in vivo (Drosophila melanogaster Or22a), J. Neurobiol. 66 (2006) 1544-1563.

[119] D. Yu, G.S. Baird, R.Y. Tsien, R.L. Davis, Detection of calcium transients in Drosophila mushroom body neurons with camgaroo reporters, J. Neurosci. 23 (2003) 64-72.

[120] V. Tsydzik, N.J. Wright, Dopamine modulation of the in vivo acetylcholine response in the Drosophila mushroom body, Dev. Neurobiol. 69 (2009) 705-714.

[121] Y. Wang, H.F. Guo, T.A. Pologruto, F. Hannan, I. Hakker, K. Svoboda, Y. Zhong, Stereotyped odor-evoked activity in the mushroom body of Drosophila revealed by green fluorescent protein-based $\mathrm{Ca}^{2+}$ imaging, J. Neurosci. 24 (2004) 6507-6514.

[122] G.S. Suh, A.M. Wong, A.C. Hergarden, J.W. Wang, A.F. Simon, S. Benzer, R. Axel, D.J. Anderson, A single population of olfactory sensory neurons mediates an innate avoidance behaviour in Drosophila, Nature 431 (2004) 854-859.

[123] V. Jayaraman, G. Laurent, Evaluating a genetically encoded optical sensor of neural activity using electrophysiology in intact adult fruit flies, Front. Neural Circuits 1 (2007) $1-9$

[124] C.M. Root, J.L. Semmelhack, A.M. Wong, J. Flores, J.W. Wang, Propagation of olfactory information in Drosophila, Proc. Natl. Acad. Sci. U. S. A. 104 (2007) $11826-11831$.

[125] C.M. Root, K. Masuyama, D.S. Green, L.E. Enell, D.R. Nässel, C.H. Lee, J.W. Wang, A presynaptic gain control mechanism fine-tunes olfactory behavior, Neuron 59 (2008) 311-321.

[126] K. Asahina, M. Louis, S. Piccinotti, L.B. Vosshall, A circuit supporting concentration-invariant odor perception in Drosophila, J. Biol. 8 (2009) 9.

[127] M. Ai, S. Min, Y. Grosjean, C. Leblanc, R. Bell, R. Benton, G.S. Suh, Acid sensing by the Drosophila olfactory system, Nature 468 (2010) 691-695.

[128] X. Liu, R.L. Davis, The GABAergic anterior paired lateral neuron suppresses and is suppressed by olfactory learning, Nat. Neurosci. 12 (2009) 53-59.

[129] G. Agarwal, E. Isacoff, Specializations of a pheromonal glomerulus in the Drosophila olfactory system, J. Neurophysiol. 105 (2011) 1711-1721.

[130] J.D. Seelig, M.E. Chiappe, G.K. Lott, A. Dutta, J.E. Osborne, M.B. Reiser, V. Jayaraman, Two-photon calcium imaging from head-fixed Drosophila during optomotor walking behavior, Nat. Methods 7 (2010) 535-540.

[131] M.E. Chiappe, J.D. Seelig, M.B. Reiser, V. Jayaraman, Walking modulates speed sensitivity in Drosophila motion vision, Curr. Biol. 20 (2010) 1470-1475. 
B. Contributions to other studies

\section{Mushroom body miscellanea: transgenic Drosophila strains expressing anatomical and physiological sensor proteins in Kenyon cells.}

My contribution :

- Created the mb247-Synapto-pHluorin strain.

- Performed and analyzed all in vivo optical imaging experiments. 


\title{
Mushroom body miscellanea: transgenic Drosophila strains expressing anatomical and physiological sensor proteins in Kenyon cells
}

\author{
Ulrike Pech ${ }^{1}$, Shubham Dipt ${ }^{1}$, Jonas Barth ${ }^{1}$, Priyanka Singh ${ }^{1}$, Mandy Jauch ${ }^{1}$, Andreas S. Thum ${ }^{2}$, \\ André Fiala $^{1 *}$ and Thomas Riemensperger ${ }^{1 *}$
}

Department of Molecular Neurobiology of Behavior, Georg-August-Universität Göttingen, Göttingen, Germany

${ }^{2}$ Department of Biology, Universität Konstanz, Konstanz, Germany

Edited by:

Claude Desplan, New York

University, USA

\section{Reviewed by:}

Michael Nitabach, Yale University

School of Medicine, USA

Bassem Hassan, Vlaams Instituut

voor Biotechnologie, Belgium

*Correspondence:

André Fiala and Thomas

Riemensperger,

Georg-August-Universität Göttingen,

Department of Molecular

Neurobiology of Behavior,

Julia-Lermontowa-Weg 3,

37077 Göttingen, Germany

e-mail: afiala@gwdg.de;

triemen@gwdg.de
The fruit fly Drosophila melanogaster represents a key model organism for analyzing how neuronal circuits regulate behavior. The mushroom body in the central brain is a particularly prominent brain region that has been intensely studied in several insect species and been implicated in a variety of behaviors, e.g., associative learning, locomotor activity, and sleep. Drosophila melanogaster offers the advantage that transgenes can be easily expressed in neuronal subpopulations, e.g., in intrinsic mushroom body neurons (Kenyon cells). A number of transgenes has been described and engineered to visualize the anatomy of neurons, to monitor physiological parameters of neuronal activity, and to manipulate neuronal function artificially. To target the expression of these transgenes selectively to specific neurons several sophisticated bi- or even multipartite transcription systems have been invented. However, the number of transgenes that can be combined in the genome of an individual fly is limited in practice. To facilitate the analysis of the mushroom body we provide a compilation of transgenic fruit flies that express transgenes under direct control of the Kenyon-cell specific promoter, mb247. The transgenes expressed are fluorescence reporters to analyze neuroanatomical aspects of the mushroom body, proteins to restrict ectopic gene expression to mushroom bodies, or fluorescent sensors to monitor physiological parameters of neuronal activity of Kenyon cells. Some of the transgenic animals compiled here have been published already, whereas others are novel and characterized here for the first time. Overall, the collection of transgenic flies expressing sensor and reporter genes in Kenyon cells facilitates combinations with binary transcription systems and might, ultimately, advance the physiological analysis of mushroom body function.

Keywords: Drosophila melanogaster, mushroom body, Kenyon cells, optical calcium imaging, GRASP, photoactivation, transgene expression, neuroanatomy

\section{INTRODUCTION}

The mushroom body of the arthropod brain is a prominent brain structure that has attracted the attention of neuroscientists for more than 160 years (Dujardin, 1850; Kenyon, 1896; Strausfeld et al., 1998; Fahrbach, 2006; Strausfeld et al., 2009; Pech et al., 2013). Functionally, the mushroom body has been implicated in a variety of adaptive behaviors, e.g., associative olfactory learning (Davis, 1993; Heisenberg, 2003; Fiala, 2007), locomotor activity (Martin et al., 1998), or sleep (Bushey and Cirelli, 2011). The information-processing properties of mushroom bodies remain, however, unclear and are the subject of much current research. The mushroom body of the fruit fly Drosophila melanogaster consists of $\sim 2000-2500$ intrinsic neurons per hemisphere called Kenyon cells (Technau, 1984; Aso et al., 2009). Kenyon cells extend their dendrites at the calyx, the main sensory input region of the mushroom body. Olfactory projection neurons originating from the antennal lobes, the primary olfactory neuropils of the insect brain, transmit odor information to the ipsilateral mushroom body calyx, where they form large presynaptic boutons
(Yasuyama et al., 2002; Leiss et al., 2009; Butcher et al., 2012). Kenyon cell dendrites contact these boutons and integrate odor information from many olfactory projection neurons (Caron et al., 2013). The Kenyon cells extend long axons into the protocerebrum, and the parallel bundles of axons together form the peduncle and the lobes of the mushroom body, the latter being both pre- and postsynaptic to mushroom body extrinsic neurons that provide afferent input to and/or efferent output from Kenyon cells (Ito et al., 1998; Tanaka et al., 2008). The mushroom body consists, however, not of an entirely homogeneous population of Kenyon cells, but rather one that can be subdivided into different subtypes according to different axonal projections (Yang et al., 1995; Crittenden et al., 1998; Aso et al., 2009). The $\alpha / \beta$-lobe and $\alpha^{\prime} / \beta^{\prime}$-lobe Kenyon cells bifurcate and extend one collateral into the dorsal-anterior direction and one toward the midline of the brain. The parallel, bundled axons collectively form the vertical $\alpha / \alpha^{\prime}$-lobes and the horizontal $\beta / \beta^{\prime}$-lobes. A third group of Kenyon cells does not divide their axons and form the $\gamma$-lobes that are positioned anterior to the $\beta / \beta^{\prime}$-lobes. These Kenyon cell 
subgroups can be further subdivided into those forming core and surface, posterior and anterior regions of the mushroom body (Tanaka et al., 2008; Aso et al., 2009).

Diverse aspects of the function of mushroom bodies have been investigated in a variety of insect species. Physiological properties of individual Kenyon cells and mushroom body extrinsic neurons have been analyzed in larger insects that are amenable to intracellular electrophysiological recordings, e.g., in cockroaches ( $\mathrm{Li}$ and Strausfeld, 1997; Mizunami et al., 1998; Li and Strausfeld, 1999; Demmer and Kloppenburg, 2009) or locusts (Stopfer et al., 2003; Cassenaer and Laurent, 2007). Honey bees represent excellent model organisms to study behavioral complexity and behavioral, experience-dependent plasticity in insects. In this context, the mushroom body and associated neurons have been investigated in detail, e.g., using pharmacological approaches (Hammer and Menzel, 1998; Louis et al., 2012), local anesthetics (Devaud et al., 2007), local cooling (Erber et al., 1980), optical $\mathrm{Ca}^{2+}$ imaging (Faber and Menzel, 2001; Szyszka et al., 2005), or electrophysiological recordings (Hammer, 1993; Strube-Bloss et al., 2011; Hussaini and Menzel, 2013).

In Drosophila, experimental approaches that involve physical intrusions, e.g., local injections, or the precise insertions of electrodes, are difficult due to the small size and fragility of the brain and its neurons. However, Drosophila melanogaster has been developed into an animal model system distinguishable from other insects by the feasibility to express transgenes in dedicated subpopulations of neurons (Olsen and Wilson, 2008; Venken et al., 2011). Transgenes that can help to analyze neuronal structure and/or function are, first, anatomical markers, e.g., cytosolic or subcellular anchored fluorescence proteins. Second, reporter proteins can be expressed to monitor physiological parameters of neuronal function, e.g., intracellular $\mathrm{Ca}^{2+}$ dynamics (Fiala et al., 2002; Riemensperger et al., 2012) or second-messenger signaling (Lissandron et al., 2007; Shafer et al., 2008). Third, effector proteins can be expressed to manipulate specific aspects of neuronal functioning. Membrane potentials can be affected through the expression of ion channels that are either constitutively in an open state (Nitabach et al., 2002), or dependent on external factors like temperature (Hamada et al., 2008) or light (Schroll et al., 2006). Likewise, chemical synaptic transmission can be prevented either constitutively (Sweeney et al., 1995; Baines et al., 2001) or reversibly (Kitamoto, 2001).

To target the expression of transgenes to specific neuronal populations, several bipartite expression systems have been invented for Drosophila, e.g., the Gal4-UAS-system (Brand and Perrimon, 1993), the lexA/lexAop-system (Lai and Lee, 2006), and the Q-system (Potter and Luo, 2011). These binary transcription systems typically divide into two transgenic fly strains-one for the desired transgene be expressed and the other for spatiotemporal control of the transgene. Large collections of "driver lines," e.g., Gal4 strains or lexA strains, have been assembled and made available so that a variety of neurons can be targeted, in some cases rather selectively (e.g., Jenett et al., 2012). Sophisticated additional genetic techniques have even upgraded the possibility of restricting transgene expression in space and time, e.g., with the help of heat-inducible promoters, the additional expression of repressors of gene expression, or through expression of recombinases (Duffy, 2002; Pfeiffer et al., 2008, 2010; Venken and Bellen, 2012). These multipartite expression strategies have helped to refine the expression of transgenes to very few neurons of interest. In addition, several binary transcription systems can be combined to express different transgenes in different neuronal subpopulations, e.g., to monitor the activity of a certain neuronal population while manipulating a different subset of neurons. Of course, the number of transgenes that can be simultaneously expressed in one individual fly is limited. To enhance the versatility of transgene expression in order to analyze the anatomy and/or function of the mushroom body we have created a number of flies that express transgenes under direct control of the promoter mb247 (Schulz et al., 1996; Zars et al., 2000). Two copies of the mb247 promoter drive gene expression in all types of Kenyon cells (Riemensperger et al., 2005; Pech et al., 2013) with relatively high specificity. Fluorescent markers, physiological sensor proteins, and effector proteins are expressed under control of the mb247 promoter. Binary transcription systems are, therefore, still available to express additional transgenes in complementary neuronal populations. The particular transgenes expressed are suitable to be combined with each other, thereby enabling analyzing distinct anatomical and functional neuronal parameters simultaneously.

\section{MATERIALS AND METHODS Drosophila STOCKS}

Flies were raised on standard cornmeal-agar food at $25^{\circ} \mathrm{C}$, $60 \%$ relative humidity and a $12 \mathrm{~h}$ light-dark cycle. The following published Drosophila strains were used: mb247-DsRed (Riemensperger et al., 2005), mb247-DsRed; mb247-splitGFP11, UAS-splitGFP1-10 (Pech et al., 2013), c305a-Gal4 (Krashes et al., 2007), TH-Gal4 (Friggi-Grelin et al., 2003), mb247-Gal4 (Zars et al., 2000), mb247-LexA::VP16 (Pitman et al., 2011), UASmcd8-GFP (Lee and Luo, 1999). UAS-FRT-Stop-FRT-mcd8-GFP (Yu et al., 2010), LexAop-GFP (Tamura et al., 2010), and actinFRT-Stop-FRT-Gal4; UAS-GFP; (Pignoni and Zipursky, 1997).

\section{GENERATION OF NOVEL DROSOPHILA STRAINS}

To generate mb247-C3paGFP flies the C3paGFP DNA was amplified from the genomic DNA of UAS-C3paGFP flies (Ruta et al., 2010) by linker PCR using the primers ATCAGATCT CAAAAATGGTGAGCAAGGGCGAGGA and AAGAAATG CGGCCGCTTACTTGTACAGCTCGTCC, producing BgIII and NotI restriction sites. For generating flies expressing the fluorescence $\mathrm{Ca}^{2+}$ indicators G-GECO1.1, G-GECO1.2, and R-GECO1.0 (Zhao et al., 2011) under control of the mb247 promoter, the DNA sequences from the original pCMV vectors (addgene \# 32444, 32445, 32446) were amplified by linker PCR using the primers ATCAGATCTCAAAAATGGTCGAC TCTTCACGTCG and AAGAAATGCGGCCGCCTACTTC GCTGTCATCATTT producing BglII and NotI restriction sites. To generate the mb247-flippase fly strain the flippaseIRES-flippase sequence was amplified, using linker PCR from the original vector (Bohm et al., 2010), using the primers GAAGATCTTCCACCATGCCACAATTTGGTATATTATG and GAAGGCCTTCTTATATGCGTCTATTTATGTAGG, producing 
BglII and StuI restriction sites, respectively. To drive expression of GCaMP3.0 (Tian et al., 2009) under control of the mb247 promoter, the 6xHis-tagged GCaMP3.0 DNA was amplified by linker PCR from the purified genomic DNA obtained from UAS-GCaMP3.0 flies (Tian et al., 2009) using the primers ATCAGATCTCAAAAATGGGTTCTCATCATCAT CATCATCATG and ATCGCGGCCGCTTACTTCGCTGT CATCATTTGTACAAACTCTTC, producing BglII and NotI restriction sites. For generating mb247-Synapto-pHluorin flies the Synapto-pHluorin DNA was amplified by linker PCR from the original vector (Miesenböck et al., 1998) using the primers GAAGATCTACGCGTGCCACCATGTCG and ATTTGCGGCCGCCTAGATTAACCGGTTTT, producing BglII and NotI restriction sites. All DNA constructs were inserted into the pCaSpeR vector containing two copies of the mb247 promoter fragment that was originally obtained from Martin Heisenberg's laboratory and fully sequenced in the course of this study. Germ-line transformation was performed by the BestGene company (Chino Hills, CA).

\section{IMMUNOHISTOCHEMISTRY}

Adult brains were dissected in ice-cold Ringer's solution containing $5 \mathrm{mM}$ Hepes, $130 \mathrm{mM} \mathrm{NaCl}, 5 \mathrm{mM} \mathrm{KCl}, 2 \mathrm{mM} \mathrm{MgCl}, 2 \mathrm{mM}$ $\mathrm{CaCl}_{2}, \mathrm{pH}=7.3$ (Estes et al., 1996), fixed for $2 \mathrm{~h}$ on ice in $4 \%$ paraformaldehyde dissolved in phosphate buffered saline (PBS), and washed three times in PBS containing $0.5 \%$ Triton X-100 (PBST) for 20 min each. After overnight pre-incubation in PBST containing $2 \%$ bovine serum albumin (blocking solution) at $4{ }^{\circ} \mathrm{C}$, brains were incubated for $5 \mathrm{~h}$ at room temperature with the primary antibodies diluted in blocking solution. For experiments with splitGFP the brains were pre-incubated in blocking solution containing $0.1 \%$ bovine serum albumin and $5 \%$ normal goat serum for $2 \mathrm{~h}$ at $4^{\circ} \mathrm{C}$. The following antibodies were used: mouse anti-nc82 against Bruchpilot (provided by Erich Buchner) diluted 1:5, rat anti-RFP (5F8, Chromotec) diluted 1:300, and rabbit anti-GFP (A6455, Invitrogen) diluted 1:200. Subsequently, brains were washed three times for $20 \mathrm{~min}$ each in PBST and incubated overnight at $4{ }^{\circ} \mathrm{C}$ with the secondary antibodies: goat anti-mouse conjugated with Cy3 (A1101, Invitrogen), goat anti-rabbit conjugated with Alexa Fluor 488 (A11034, Invitrogen) and goat anti-rat conjugated with Cy3 (A10522, Invitrogen), all diluted 1:300. To visualize reconstituted split-GFP, brains were incubated with antiGFP-20 (Sigma, G6539) diluted 1:200 in blocking solution at $4^{\circ} \mathrm{C}$ overnight and, after three washing steps at room temperature in PBST, with anti-mouse conjugated to Alexa488 diluted 1:250 in blocking solution at $4^{\circ} \mathrm{C}$ overnight. Afterwards, brains were washed three times in PBST for $20 \mathrm{~min}$ each, washed in PBS, overnight at $4^{\circ} \mathrm{C}$, embedded in Vectashield (Vector Laboratories) and images were acquired using a confocal laser scan microscope (SP2, Leica) equipped with an Apochromat $20 \times$ water immersion objective $(\mathrm{NA}=0.7)$. Images were analyzed using ImageJ.

\section{PHOTOACTIVATION OF PHOTOACTIVATABLE GFP}

For photoactivating photoactivatable GFP (paGFP) and the visualization of resulting in vivo fluorescence patterns, the brains of 7-day-old female flies were dissected and scanned using a Zeiss
LSM7 MP two-photon microscope equipped with a Zeiss wplan Apochromat $20 \times$ water immersion objective $(\mathrm{NA}=1.0)$, at an excitation wavelength of $950 \mathrm{~nm}$, a pixel dwell of $2.3 \mu \mathrm{s}$ and a line average of 4. PaGFP and red DsRed fluorescence were recorded simultaneously using a dichroic mirror in combination with 500-550 and 575-610 nm emission filters. To photoactivate paGFP, a small region within one of the two mushroom bodies was chosen, indicated by the mb247-DsRed fluorescence as a landmark, and subsequently this region was scanned at $760 \mathrm{~nm}$ with a laser power of $5 \%$ and $0.53 \mu$ s pixel dwell. Each pixel was excited 25 times in intervals of $\sim 1 \mathrm{~min}$ each. After $45 \mathrm{~min}$ the brains were scanned again as indicated above.

\section{In-vivo IMAGING}

To measure neuronal activity in the horizontal Drosophila mushroom body lobes, 3 to 6-day-old female flies expressing the respective sensors (GCaMP3.0, G-GECO1.1, G-GECO1.2, R-GECO1.0, or Synapto-pHluorin) were used. Flies were briefly anaesthetized on ice, immobilized in a small chamber with adhesive tape. A hole was cut through the head capsule for direct optical access. Tracheae were carefully removed and 1.5\% lowmelting agarose was injected into the head capsule to reduce the movement of the brain. The preparation was covered with Ringer's solution (Estes et al., 1996) and optical imaging was performed using a two-photon microscope (LSM7 MP, Zeiss) equipped with mode-locked Ti-sapphire Chameleon Vision II laser (Coherent) tuned to $690-1064 \mathrm{~nm}$, a $500-550 \mathrm{~m}$ band-pass filter for green fluorescent sensors and a $575-610 \mathrm{~nm}$ band-pass filter for R-GECO1.0, and a Zeiss w-plan Apochromat $20 \times$ water immersion objective $(\mathrm{NA}=1.0)$. Images were acquired at a frame rate of $5 \mathrm{~Hz}$ with an excitation wavelength of 920 and $950 \mathrm{~nm}$ for green or red fluorescent sensors, respectively. Odor stimuli (4methylcyclohexanol and 3-octanol, diluted in mineral oil 1:750 and 1:500, respectively) were applied in an air stream to the flies' antennae for $2 \mathrm{~s}$ each using a custom-built olfactometer at an air flow rate of $1 \mathrm{l} / \mathrm{min}$. Three stimulations with each odor were applied to each individual fly with an interstimulus interval of 20 s. Images were acquired using the Zeiss ZEN software and images were later aligned in the X-Y direction using a MatLab program to correct for slight movements of the preparation (Guizar-Sicairos et al., 2008). Changes in fluorescence emission were calculated within a region of interest covering the horizontal lobes as $\Delta F / F_{0}$ where $F$ is the fluorescence measured at each time point and $F_{0}$ the baseline fluorescence before odor stimulation. $F_{0}$ is calculated as the average of 5 frames before odor onset. For each fly the $\Delta F / F_{0}$ values of the 3 stimulations were averaged. To illustrate the spatial distribution of odor-evoked $\mathrm{Ca}^{2+}$ increases, false-color coded images were created. Three frames of baseline fluorescence directly preceding the odor onset were averaged and then subtracted from the average of 3 frames (400-1000 ms after stimulus onset) covering the peak of the fluorescence increase.

\section{RESULTS}

\section{EXPRESSION OF FLUORESCENCE SENSOR PROTEINS IN KENYON} CELLS USING THE DUPLICATED mb247 PROMOTER

We used a duplicated DNA construct of the promoter mb247 (Schulz et al., 1996; Zars et al., 2000) to direct the expression 
of transgenes to the mushroom bodies (Table 1). This strategy has been described before by Riemensperger et al. (2005), who expressed the red fluorescence protein DsRed (Matz et al., 1999) in Kenyon cells. Pitman et al. (2011) created a LexA::VP16 driver line using the same mb247 promoter construct. Using these published fly lines, we first confirmed that the mb247 promoterdriven transgene expression encompasses all types of Kenyon cells, i.e., covers all lobes. Indeed, as can be seen by the fluorescence of DsRed (Figures 1A1,A2), all mushroom body lobes express the fluorescence protein. Transgene expression driven by

Table 1 | Summary of fly strains expressing transgenes directly under control of the duplicated mb247-promoter.

\begin{tabular}{|c|c|c|c|}
\hline Fly strain & Application & References of fly strain & References of transgene \\
\hline mb247-DsRed & Anatomical landmark & Riemensperger et al., 2005 & Matz et al., 1999 \\
\hline mb247-LexA & Driver for lexA/lexAop binary expression system & Pitman et al., 2011 & Lai and Lee, 2006 \\
\hline mb247-Gal80 & Repression of Gal4 expression & Krashes et al., 2007 & Lee and Luo, 1999 \\
\hline mb247-GCaMP3.0 & $\mathrm{Ca}^{2+}$-imaging & This study & Tian et al., 2009 \\
\hline mb247-G-GECO1.1 & $\mathrm{Ca}^{2+}$-imaging & This study & Zhao et al., 2011 \\
\hline mb247-G-GECO1.2 & $\mathrm{Ca}^{2+}$-imaging & This study & Zhao et al., 2011 \\
\hline mb247-R-GECO1.0 & $\mathrm{Ca}^{2+}$-imaging & This study & Zhao et al., 2011 \\
\hline mb247-Synapto-pHluorin & $\mathrm{pH}$-dependent imaging of neurotransmission & This study & Miesenböck et al., 1998 \\
\hline mb247-splitGFP11 & Anatomical indicator of cell-cell contacts & Pech et al., 2013 & Feinberg et al., 2008 \\
\hline mb247-C3paGFP & Back-tracing of individual cells & This study & Ruta et al., 2010 \\
\hline mb247-flippase & Intersectional/mosaic targeting of cells & This study & Bohm et al., 2010 \\
\hline
\end{tabular}
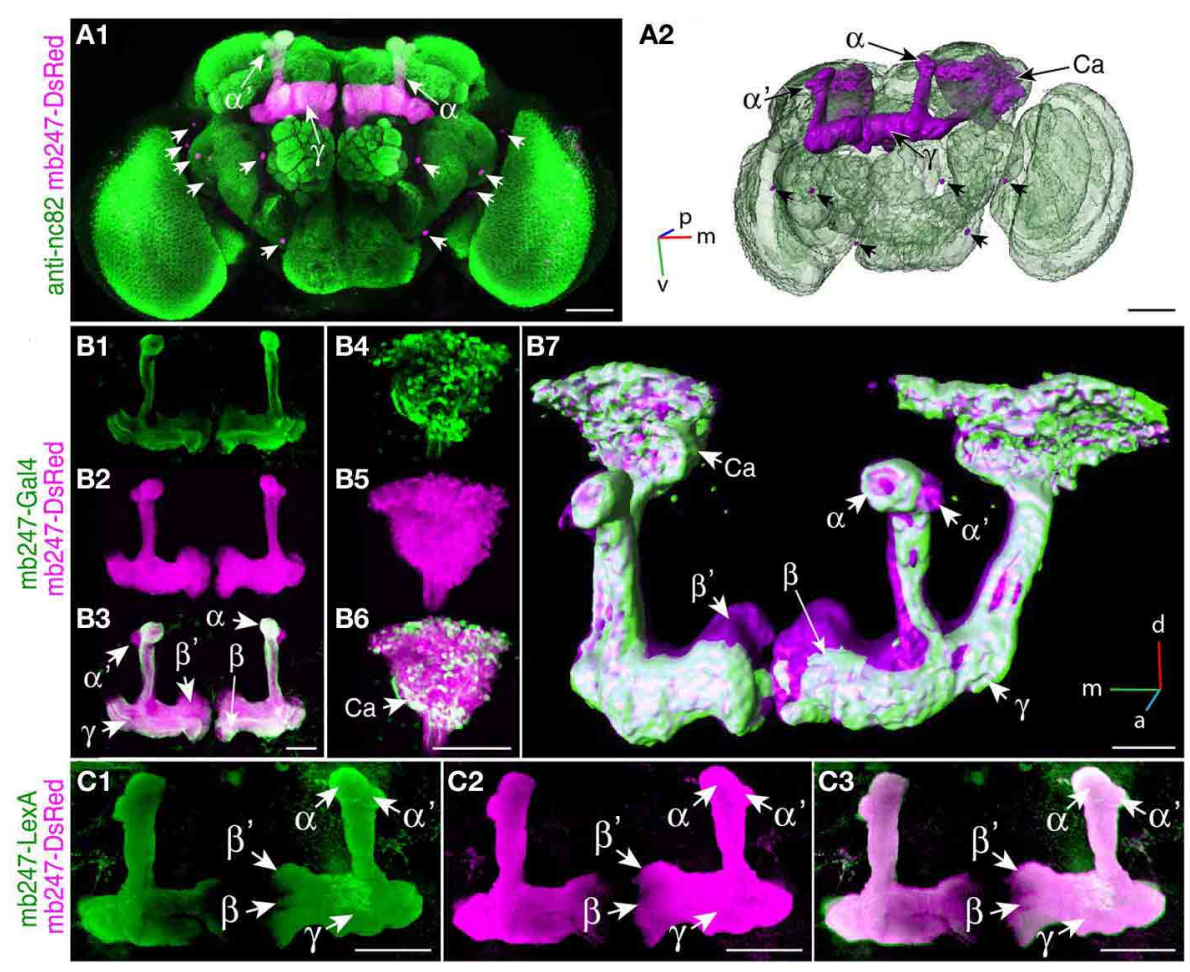

FIGURE 1 | Targeting the Drosophila mushroom body using a duplicated mb247 promoter construct. (A) Expression of DsRed in the mushroom bodies of a Drosophila brain (frontal view, A1). Expression in neurons outside the mushroom body is indicated by arrowheads. Neuropils are stained using an anti-bruchpilot-antibody (green) and the DsRed expression is shown in magenta. (A2) 3D reconstruction of the brain depicted in (A1). (B) Expression of mcd8-GFP under control of mb247-Gal4 is mainly confined to the $\alpha / \beta$ - and $\gamma$-lobes (B1). (B2) On the contrary, DsRed expression under control of the duplicated $\mathrm{mb} 247$ promoter is visible also in the $\alpha^{\prime} / \beta^{\prime}$-lobes. (B3) Overlay of
GFP expression shown in (B1) and DsRed expression shown in (B2). (B4-B6) Mushroom body calyx showing expression of GFP driven by mb247-Gal4 (B4), expression of DsRed driven by the mb247 promoter construct (B5), and an overlay of both (B6). (B7) 3D reconstruction of the brain depicted in (B1-B6). (C) GFP expression induced by mb247-LexA::VP16 (Pitman et al., 2011) is visible in $\alpha / \beta-, \alpha^{\prime} / \beta^{\prime}-$, and lobes $\gamma$-lobes (C1). (C2) DsRed expression under control of the mb247 promoter. (C3) Overlay of (C1) and (C2). Scale bars $=50 \mu \mathrm{m}$ in (A,B), and $100 \mu \mathrm{m}$ in (C). Ca, calyx; $\alpha, \alpha$-lobe; $\alpha^{\prime}$ $\alpha^{\prime}$-lobe; $\beta, \beta$-lobe; $\beta^{\prime}, \beta^{\prime}$-lobe; $\gamma, \gamma$-lobe; $p$, posterior; $m$, medial; $v$, ventral. 
the mb247 promoter is not completely restricted to the mushroom body and shows some "non-specific" expression. In addition to Kenyon cells, several scattered somata are visible in each hemisphere, located in the lateral suboesophageal ganglion and the protocerebrum (Figures 1A1,A2). Four to six cell somata are located directly below the $\gamma$-lobes of the mushroom bodies and form projections into the antennal lobes; $3-5$ somata are located in the dorsal protocerebrum, and their projections can be traced to the ipsi- and contralateral anterior lobe region and the medial protocerebrum. Mb247-promoter-driven DNA constructs are, therefore, not exclusively but very predominantly expressed in Kenyon cells. In contrast, expression directed to the mushroom body by the conventional mb247-Gal4 driver line (Zars et al., 2000) does not label $\alpha^{\prime} / \beta^{\prime}$-lobes, and the other lobes are not entirely labeled either (Figures 1B1-B7). The cores of the $\alpha / \beta$-lobes are, for example, less densely labeled (Figure 1B). The mb247-LexA::VP16 driver line described by Pitman et al. (2011), on the contrary, induces an expression pattern in Kenyon cells that completely overlaps with the expression of mb247-DsRed (Riemensperger et al., 2005) (Figures 1C1-C3), which confirms that the more encompassing expression pattern induced by the duplicated mb247 promoter construct is reproducible across transgenic fly lines.

We used the duplicated mb247 promoter to drive the expression of fluorescent $\mathrm{Ca}^{2+}$ sensor proteins. The intracellular $\mathrm{Ca}^{2+}$ level closely correlates with neuronal excitation (Berridge, 1998; Burgoyne, 2007). Optical $\mathrm{Ca}^{2+}$ imaging represents, therefore, a widely used technique to monitor the activity of neurons in general (Grienberger and Konnerth, 2012), and also in the central brain of Drosophila (Riemensperger et al., 2012). We first created transgenic flies expressing the widely-used fluorescence $\mathrm{Ca}^{2+}$ sensors GCaMP3.0 (Tian et al., 2009) under control of the mb247 promoter and performed two-photon optical $\mathrm{Ca}^{2+}$ imaging experiments with the focus on the horizontal lobes. Although the overall expression pattern induced by the promoter construct is recapitulated with this transgene as well, baseline fluorescence was more pronounced in the $\gamma$-lobes when compared to the $\beta^{\prime}$-lobes (Figure 2A1), which might reflect a higher tissue density or, alternatively, higher intracellular baseline $\mathrm{Ca}^{2+}$ levels. When the flies were stimulated with the odorants 3-octanol or 4-methylcyclohexanol, which are volatile chemicals that are often used for olfactory learning experiments in Drosophila, clear increases in intracellular $\mathrm{Ca}^{2+}$ were observed (Figures 2A1-A3). Relative fluorescence changes $\left(\Delta F / F_{0}\right)$ averaged across the entire horizontal lobes reached $23.9 \pm 4.4 \%$ for 3 -octanol and $21.8 \pm 4.3 \%$ for 4-methylcyclohexanol (mean \pm sem, $n=5$ each) (Figure 2A3). The time course of intracellular $\mathrm{Ca}^{2+}$ dynamics is characterized by a rapid increase in fluorescence emission starting with stimulus onset, a slight, adaptive decay during stimulation and a fast decay after stimulus offset. Recently, a novel subfamily of GCaMP-type $\mathrm{Ca}^{2+}$ sensor proteins has been engineered and named GECOs (Zhao et al., 2011). This development raised our attention because a red fluorescent version, R-GECO1.0, has been invented that can be combined with green fluorescent sensor or marker proteins (e.g., Tewson et al., 2012; Li et al., 2013). In addition, several green variants (G-GECOs) with different dissociation constants $\left(K_{d}\right)$ for $\mathrm{Ca}^{2+}$ have been created. We have created flies expressing G-GECO1.1 and G-GECO1.2 that differ in their $\mathrm{Ca}^{2+}$ affinities with $K_{d}$ values of $0.62 \mu \mathrm{M} \mathrm{Ca}^{2+}$ and $1.15 \mu \mathrm{M} \mathrm{Ca}^{2+}$ (Zhao et al., 2011) under control of the mb247 promoter. Flies expressing these sensors show detectable baseline fluorescence in Kenyon cells (Figures 2B1,C1), which we noticed to be clearly lower when compared to the baseline fluorescence of GCaMP3.0. Just as with GCaMP3.0, intracellular $\mathrm{Ca}^{2+}$ influx evoked by the two odors is detectable in the horizontal lobe regions of the mushroom bodies as a spatially distributed pattern (Figures 2A2,B2,C2). Relative maximum changes in G-GECO1.1 fluorescence $\left(\Delta F / F_{0}\right)$ evoked by the two odorants are similar to GCaMP3.0 with $22.7 \pm$ $3.0 \%$ for 3 -octanol and $18.7 \pm 3.3 \%$ for 4 -methylcyclohexanol (mean \pm sem, $n=5$ each). The kinetics of signal on- and offset are also comparable with GCaMP3.0 (Figure 2B3). Likewise, the G-GECO1.2 version shows equivalent changes in fluorescence emission intensity of $21.3 \pm 4.5 \%$ for 3 -octanol and $19.9 \pm$ $4.5 \%$ for 4 -methylcyclohexanol (mean \pm sem, $n=5$ each) (Figure 2C3). Drastic differences between the three types of sensor proteins were not observed under the experimental conditions used here, except for lower baseline fluorescence in the G-GECO type sensors. The red fluorescent version, R-GECO1.0 (Zhao et al., 2011), however, has the advantage that its emission wavelength is complementary to the green emission of many other transgenic tools relying on GFP variants. R-GECO1.0 is clearly expressed under control of the mb247 promoter (Figure 2D1). Baseline fluorescence is, however, under the conditions used here (two-photon excitation) drastically lower than that of GCaMP3.0. Relative changes in fluorescence elicited by the two odorants is also much smaller with $14.2 \pm 3.4 \%$ evoked by 3 -octanol and $9.7 \pm 1.6 \%$ evoked by 4 -methylcyclohexanol (mean \pm SEM, $n=5$ each) (Figures 2D2,D3). It must be noted that the lower baseline fluorescence and $\mathrm{Ca}^{2+}$-dependent increase in emission intensity might result from the two-photon excitation which is not optimized for exciting this red fluorescence protein. In fact, regular single-photon excitation at green light wavelengths causes stronger emission intensities. However, and also under our experimental conditions, the red fluorescence sensor is clearly functional in intrinsic mushroom body neurons. The time course of the odor-evoked change in fluorescence differs in the red R-GECO1.0 when compared to the green sensors. The $\Delta F / F_{0}$ signal decays back to baseline already during odor stimulation and shows a second peak following odor offset. This time course might more accurately reflect the sparse on- and offset action potential firing that has been described for Kenyon cells, e.g., using electrophysiological recordings in moths (Ito et al., 2008) or $\mathrm{Ca}^{2+}$ imaging with Fura-2 in honey bees (Szyszka et al., 2005).

Optical imaging of synaptic transmitter release using $\mathrm{pH}$ sensitive GFP variants targeted to the lumen of synaptic vesicles represents a further method used to monitor the effect of neuronal activity. We have expressed Synapto-pHluorin (Miesenböck et al., 1998) under direct control of the mb247 promoter (Figure 2E1). Odor-evoked increases in fluorescence elicited by odors are detectable in the mushroom body lobes, with relative changes in fluorescence of up to $3.2 \pm 0.7 \%$ evoked by 3 -octanol and $2.9 \pm 0.6 \%$ evoked by 4 -methylcyclohexanol (Figures 2E2,E3). The $\Delta F / F_{0}$ peak is followed by a slow decay 

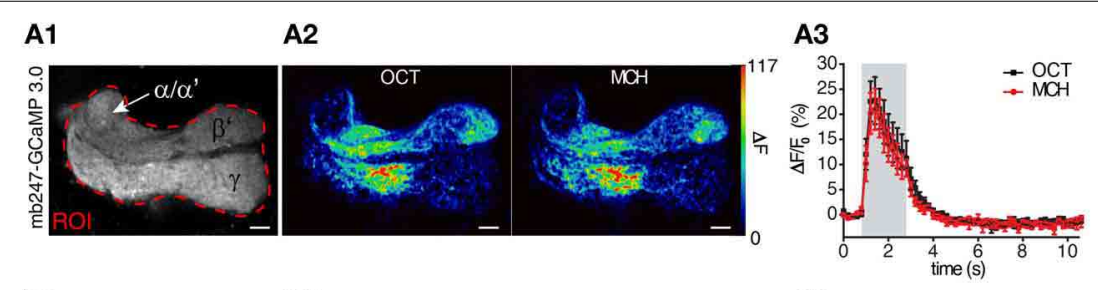

B1

B2
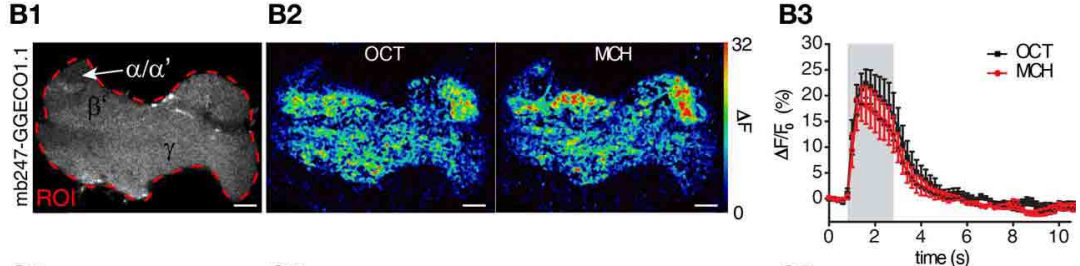

C1

C2

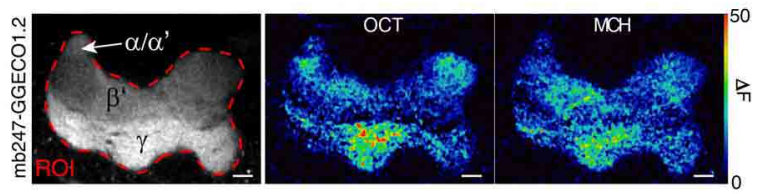

C3

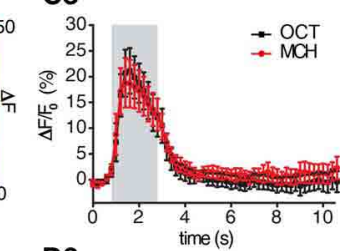

D1

D2

D3
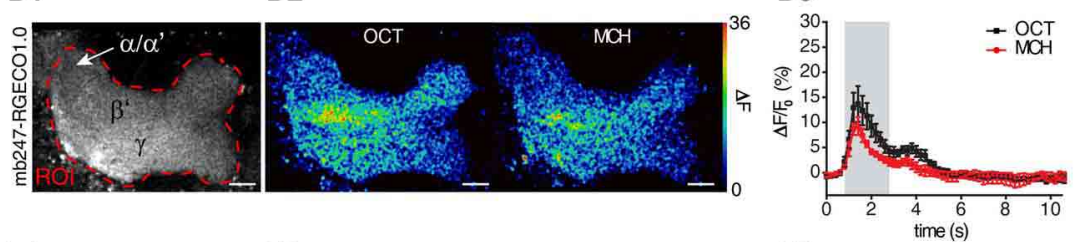

E1

E2

E3
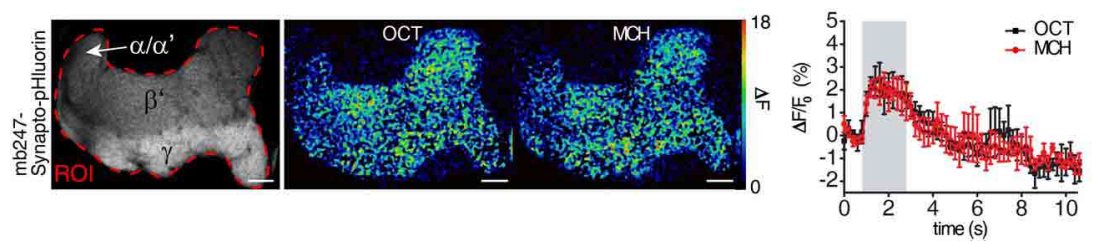

FIGURE 2 | Mb247-driven expression of fluorescent sensor proteins. Optical imaging of odor-evoked neuronal activity in Kenyon cells of the adult Drosophila mushroom body using different reporter proteins, i.e., (A1-A3) the $\mathrm{Ca}^{2+}$ sensor GCaMP3.0, (B1-B3) the $\mathrm{Ca}^{2+}$ sensor G-GECO1.1, (C1-C3) the $\mathrm{Ca}^{2+}$ sensor G-GECO1.2, (D1-D3) $\mathrm{Ca}^{2+}$ sensor R-GEC01.0, and (E1-E3) Synapto-pHluorin. The left row (A1-E1) illustrates the fluorescence protein expression in one focal plane covering the horizontal lobes of the mushroom body. The dashed red line indicates the region of interest (ROI) in which odor-evoked changes in fluorescence emission were monitored. The middle row (A2-E2) shows two false-color coded images illustrating the spatial distribution of fluorescence intensity changes with the ROI evoked by 3-octanol (OCT) and 4-mehylcyclohexanol (MCH). The right row (A3-E3) shows the temporal dynamics of relative changes in fluorescence within the $\mathrm{ROI}$ evoked by OCT (black line) and $\mathrm{MCH}$ (red line). The odor stimulus is indicated as gray bars. Relative changes in fluorescence are indicated as means $\pm \operatorname{SEM}$ ( $n=5$ animals each). $\alpha / \alpha^{\prime}$, part of the vertical $\alpha / \alpha^{\prime}$-lobes; $\beta^{\prime}, \beta^{\prime}$-lobe; $\gamma, \gamma$-lobes. Scale bars $=10 \mu \mathrm{m}$. below baseline $(\sim 2 \%)$ due to bleaching of the fluorophore. The signal-to-noise ratio is, under these experimental conditions, drastically lower than that of $\mathrm{Ca}^{2+}$ imaging, which results from the physical constraints of the physiological parameter that is measured here. The fly strain is, however, clearly functional in reporting synaptic vesicle release, and if this parameter needs to be recorded in subregions of the mushroom body, this fly strain might provide a helpful tool.

\section{MUSHROOM-BODY DIRECTED EXPRESSION OF splitGFP}

A prerequisite for understanding the function of the mushroom body circuitry is detailed knowledge of the contacts between mushroom body intrinsic neurons (Kenyon cells) and mushroom body extrinsic neurons. The recently described GRASP technique (Feinberg et al., 2008) that has been adapted to Drosophila by Gordon and Scott (2009), provides an attractive tool to visualize and pinpoint where exactly two cells contact each other in close proximity and might potentially form synapses. We have recently reported a transgenic fly that expresses one part of splitGFP targeted to the outer surface of the cell membrane under control of the mb247 promoter (Pech et al., 2013). In addition, the mb247-DsRed construct is expressed as a landmark. The second part of the splitGFP is expressed under UAS control. If a given transgenic Gal4 strain is crossed with this "MB-splitGFP" fly strain, reconstitution between the two membrane-bound splitGFP parts can be visualized (Pech et al., 2013). Again, we would 
like to underline that, in contrast to the widely used mb247-Gal4 line (Zars et al., 2000), the mb247 promoter constructs cover all types of Kenyon cells including $\alpha^{\prime} / \beta^{\prime}$-lobes (Riemensperger et al., 2005; Pech et al., 2013). First, the technique can be used to visualize Kenyon cells determined by a given Gal4 line. If both parts of the splitGFP are expressed in the same population of Kenyon cells, fluorescence is readily visible (Pech et al., 2013). This we exemplify here with the Drosophila line c305aGal4. This fly strain has been described as expressing Gal4 in the $\alpha^{\prime} / \beta^{\prime}$-lobes (Krashes et al., 2007), and in fact, the $\alpha^{\prime} / \beta^{\prime}$ lobes are labeled when c305a-Gal4 is crossed with MB-splitGFP (Figures 3A1-A5). In addition, faint splitGFP reconstitution is also observed in the $\gamma$-lobes (Figure 3A4). Secondly, the MBsplitGFP fly strain can also be used to visualize regions of close proximity between Kenyon cells and mushroom body extrinsic neurons (Pech et al., 2013). As an example, we have used the TH-Gal4 line (Friggi-Grelin et al., 2003), which covers a large proportion of tyrosine hydroxylase-immunoreactive neurons, i.e., several clusters of dopaminergic neurons in the Drosophila brain, in particular PPL1, PPL2ab, PPL2c, PAL, PPM1/2, and PPM3 clusters (Friggi-Grelin et al., 2003; Mao and Davis, 2009; Pech et al., 2013) (Figure 3B1). Reconstitution of a splitGFP signal at the contact points between these dopaminergic neurons and
Kenyon cells is clearly visible in the $\alpha$ - and $\gamma$-lobes and the heel of the horizontal lobes, as already described in Pech et al. (2013) (Figure 3B2).

\section{MUSHROOM BODY-SPECIFIC EXPRESSION OF PHOTOACTIVATABLE GFP}

The technique of photoactivating variants of GFP (Patterson and Lippincott-Schwartz, 2002) has been recently established in Drosophila to trace the neurites and projections of neurons from a particular point (Ruta et al., 2010; Pech et al., 2013). GFP-fluorescence is induced upon activation at $760 \mathrm{~nm}$, and the photoactivated GFP diffuses along the neurites (Figure 4A). A paGFP expression allows, therefore, the visualization of isolated neurons of interest against a background of dense populations of neurons. We used the mb247 promoter sequence to express the variant C3paGFP (Ruta et al., 2010) globally in Kenyon cells and activated paGFP in a small, defined region at the most posterior tips of the vertical $\alpha$-lobes (Figure 4A). The activated paGFP diffuses over time toward distal parts of the neurons and the cell bodies of the activated Kenyon cells. The paGFP signal can be traced from the activated region to the Kenyon cell somata at the level of the calyces and the tips of the posterior horizontal $\beta$-lobes (Figure 4B). If particular
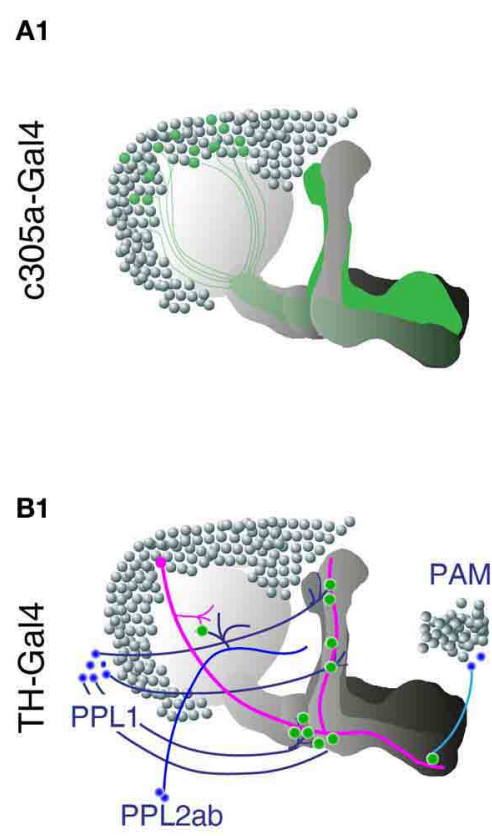

FIGURE 3 | Mushroom body-directed expression of reconstituted splitGFP to visualize cell-cell contacts within and between Kenyon cells. (A) The fly strain mb247-DsRed; mb247-splitGFP11, UAS-splitGFP1-10 is crossed with c305a-Gal4. The offspring shows reconstituted splitGFP fluorescence (rsGFP) in Kenyon cells forming $\alpha^{\prime} / \beta^{\prime}$ - and $\gamma$-lobes, in addition to the DsRed fluorescence. (A1) Schematic illustration of the splitGFP reconstitution between populations of intrinsic mushroom body neurons determined by the c305a-Gal4 line and the mb247-promoter line. (A2-A5) Immunhistochemical visualization of the splitGFP reconstitution in the brain (frontal view) at different optical sections, i.e., at the level of the $\alpha / \beta$ - and $\alpha^{\prime} / \beta^{\prime}$-lobes (A2), the Calyx (A3), the $\beta^{\prime}$-and $\gamma$-lobes (A4), and the peduncle (A5) DsRed fluorescence is shown in magenta, reconstituted GFP fluorescence in
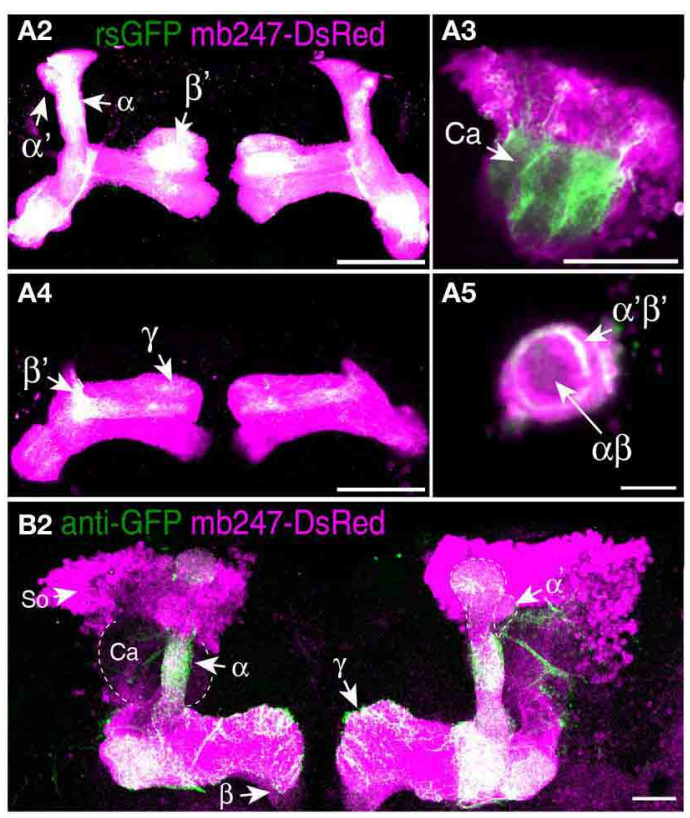

green. (B) The fly strain mb247-DsRed; mb247-splitGFP11, UAS-splitGFP1-10 is crossed with $\mathrm{TH}-\mathrm{Gal} 4$. The offspring shows reconstituted splitGFP

fluorescence at contact regions of close proximity between populations of dopaminergic neurons and Kenyon cells, in addition to the DsRed fluorescence. (B1) Schematic illustration of the splitGFP reconstitution between populations of intrinsic mushroom body neurons and dopaminergic neurons from the PAM cluster, PPL1 cluster, and PPL2ab cluster of dopaminergic neurons. (B2) Reconstituted splitGFP fluorescence between dopaminergic neurons and Kenyon cells indicates contacts of dopaminergic neurons predominantly in the $\alpha$ - and $\gamma$-lobes and the heel of the horizontal lobes. Mb247-DsRed fluorescence is shown in magenta and the reconstituted splitGFP labeled by anti-GFP in green. Ca, calyx; So, somata; Scale bars $=10 \mu \mathrm{m}$ in (A5), $40 \mu \mathrm{m}$ elsewhere. 

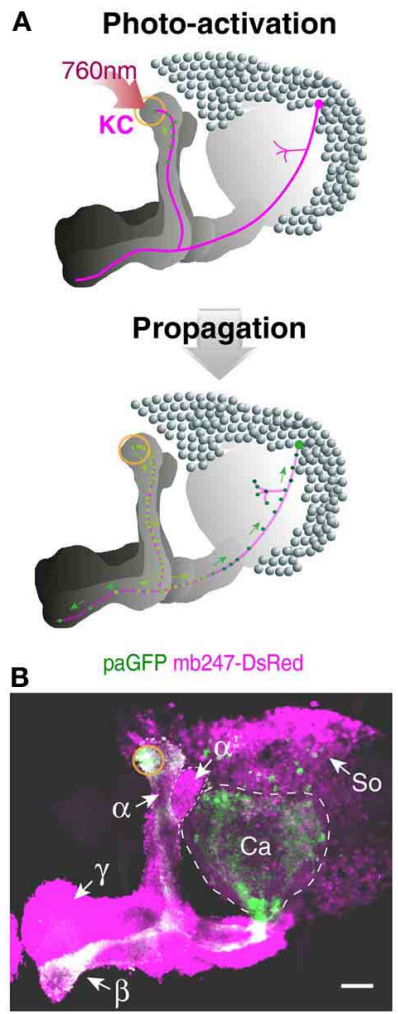

FIGURE 4 | Tracing Kenyon cells using photoactivatable GFP.

(A) Schematic depiction of photoactivated GFP (paGFP) signal (green) in defined populations of Kenyon cells in a frontal projection view. Mushroom body-localized paGFP is activated using a two-photon microscope at $760 \mathrm{~nm}$ in a region at the most posterior tip of the vertical $\alpha$-lobe (upper panel). The photactivated paGFP molecules diffuse over time along the neurites and toward the cell bodies of the respective Kenyon cells (lower panel).

(B) Fluorescence of paGFP in $\alpha$-lobe neurons. The region of the paGFP activation is indicated by an orange circle. $\alpha, \alpha$-lobe; $\alpha^{\prime}, \alpha^{\prime}$-lobe; $\beta, \beta$-lobe; $\gamma$, $\gamma$-lobe; Ca, calyx; So, somata; KC, Kenyon cell. Scale bars $=40 \mu \mathrm{m}$.

Kenyon cells need to be determined, e.g., by specific contacts with mushroom body extrinsic neurons, this fly strain might be helpful.

\section{INTERSECTIONAL EXPRESSION OF TRANSGENES IN THE MUSHROOM BODY USING mb247-FLIPPASE}

Many Gal4-driver lines that are potentially interesting for the investigation of mushroom bodies show not only expression in Kenyon cells, but also exhibit non-specific Gal4 expression in other neurons of the brain or thoracic ganglia (Aso et al., 2009). In order to specify and restrict expression to the mushroom bodies we have made use of an intersectional approach based on the yeast flippase recombinase (Golic and Lindquist, 1989; Struhl and Basler, 1993; Xu and Rubin, 1993; Bohm et al., 2010). We generated a fly line that permanently expresses the flippase protein in the Kenyon cells of the mushroom body, which can be combined with a DNA insertion carrying flippase recognition target sites (FRT). The constitutive flippase activity in Kenyon cells will induce the removal of any FRT-flanked DNA sequences. We first demonstrate the spatial specificity of the flippase activity by crossing mb247-FLP with a line that carries an actin-FRT-stopFRT-Gal4 sequence along with a UAS-GFP reporter (Pignoni and Zipursky, 1997). In the absence of flippase activity Gal4 expression, and therefore GFP expression, are prevented due to the preceding stop codon. Since the actin promoter drives expression ubiquitously in the brain, GFP expression reports, in this case, all cells in the brain that exhibit flippase activity; i.e., in the entire mushroom body (Figures 5A1-A3). This fly strain can be useful in multiple combinations. First, flippase activity in the mushroom body can be used to clip a stop codon that prevents transcription of a target gene, and thereby restrict gene expression to Kenyon cells. The intersectional logic of this "and" system is apparent when UAS-controlled transgenes are used that are preceded by a FRT-flanked stop cassette. UAS-induced expression is determined by a Gal4 driver pattern, but the transgenes are expressed only in these neurons that overlap with Kenyon cells. We show this by using the mb247-FLP line to restrict the expression pattern of a non-specific Gal4 line, c305a-Gal4 (Krashes et al., 2007) that drives Gal4 expression in a large number of neurons, e.g., in the antennal lobes or the suboesophageal ganglion of the Drosophila brain, in the mushroom body $\alpha^{\prime} / \beta^{\prime}$-lobes, and, albeit faintly, in the $\gamma$-lobes (Figures 5B1-B3). When a UAS-FRT-stop-FRT-GFP reporter is used (Yu et al., 2010) mb247-FLP restricts the expression of the transgene efficiently to Kenyon cells. Flies now show a strong expression of GFP in the $\alpha^{\prime} / \beta^{\prime}$-lobes and slight expression in the $\gamma$-lobes of the mushroom body, but no additional expression outside the mushroom bodies (Figures 5C1-C3). Of course, other applications of the mb247-FLP line are conceivable, e. g., removing a FRT-flanked Gal80 construct (Bohm et al., 2010) in order to restrict gene expression to all neurons except Kenyon cells, or to induce mitotic recombination during development (MARCM; Dang and Perrimon, 1992; Lee and Luo, 1999) in the mushroom body.

\section{DISCUSSION}

Discovering how brain circuits process and compute information and contribute to organizing behavior represents a key topic in current neuroscience. "Model animals" that can be genetically manipulated through the expression of transgenes, e.g., mice, zebrafish, C. elegans, or Drosophila melanogaster, are particularly favorable for this task. The mushroom body of the Drosophila brain provides a relatively delineated structure that can serve as a model circuitry to address fundamental aspects of neuronal processing in general. First, the mushroom body provides the possibility of analyzing how odor information is encoded and processed in central brain structures (Fiala, 2007; Masse et al., 2009). Fruit flies perceive odors with olfactory sensory neurons located on the third antennal segments and the maxillary palps (Vosshall and Stocker, 2007). These sensory neurons project to the antennal lobes, the structural and functional analogue of the olfactory bulb of vertebrates. Each olfactory sensory neuron expresses one or very few olfactory receptors, and those sensory neurons that express the same receptors target the same glomeruli in the antennal lobe (Vosshall and Stocker, 2007). As a consequence, odors are represented at the level of the antennal lobe in terms of overlapping, combinatorial activity of glomeruli 

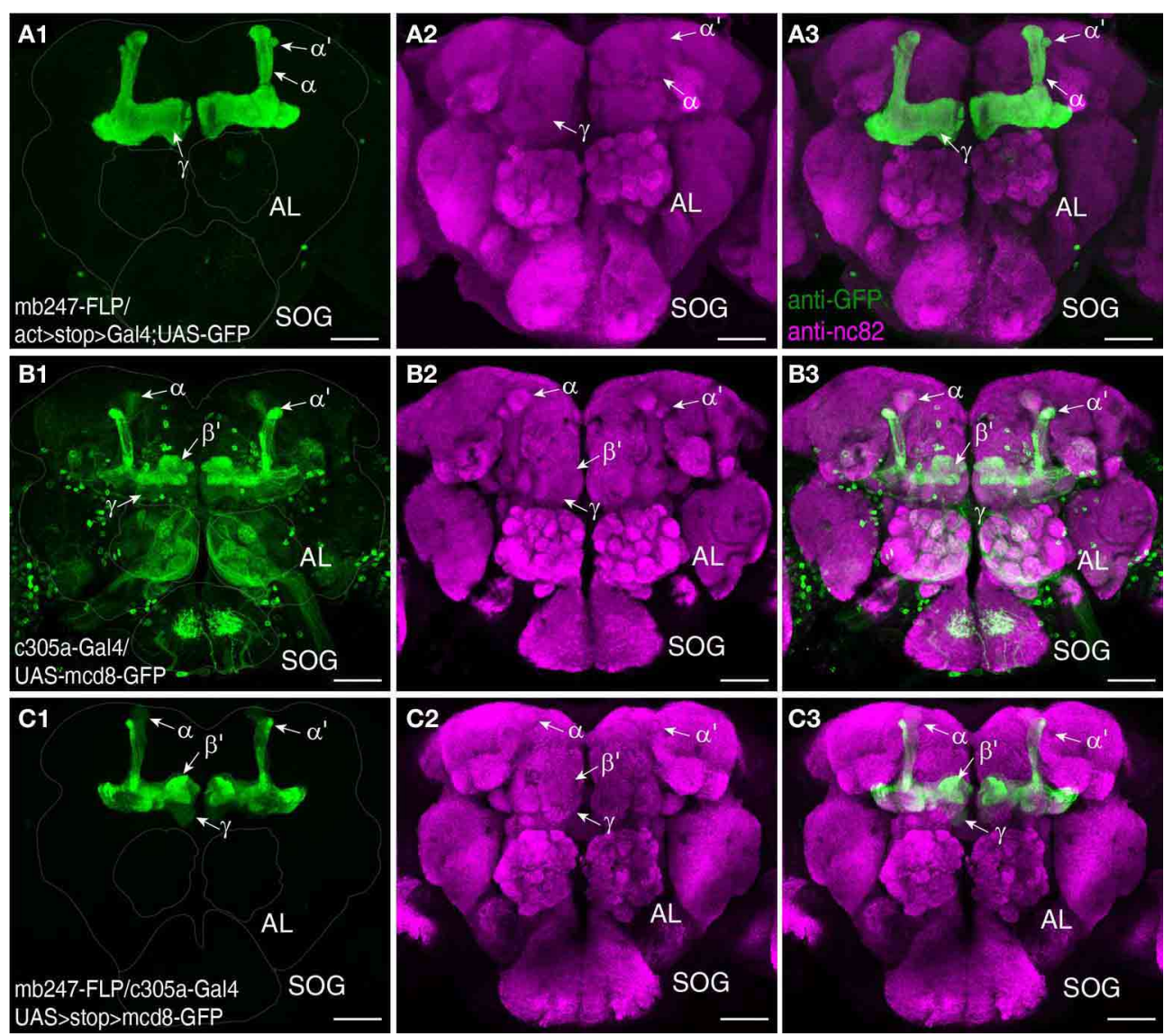

FIGURE 5 | Restriction of transgene expression to Kenyon cells using mb247-flippase. (A) As an indicator for flippase activity a fly strain is used that carries a ubiquitous Act-Gal4 driver, with a FRT-flanked stop cassette preceding the Gal4 driver sequence. The mb247 promoter-induced flippase expression mediates the excision of the FRT-flanked stop cassette and causes transcription of Gal4 exclusively in the mushroom bodies. GFP expression is visualized using an anti-GFP antibody (green) (A1), neuropils are visualized using an anti-bruchpilot antibody (magenta) (A2). (A3) shows the overlay of (A1,A2). (B) Expression pattern of c305a-Gal4 visualized by UAS:mcd8-GFP expression labeled by anti-GFP (green) (B1). Neuropils are visualized using an anti-bruchpilot antibody (magenta) (B2). (B3) shows the overlay of (B1,B2). Flies show strong expression of GFP in the $\alpha^{\prime} / \beta^{\prime}$ lobes and slight expression in the $\gamma$-mushroom body lobes and further strong expression outside the mushroom bodies predominantly in glomeruli of the antennal lobes (AL) and the subesophagial ganglion (SOG). (C) Flippase-mediated restriction of GFP expression labeled by an anti-GFP antibody (green) in c305a-Gal4 positive cells when UAS-FRT-stop-FRT-GFP is used. Flies show strong expression of GFP in the $\alpha / \beta$ - and slight expression in the $\gamma$-lobes, but no further expression outside the mushroom bodies (C1). Neuropils are visualized using an anti-bruchpilot antibody (magenta) (C2). (C3) shows the overlay of $(\mathbf{C 1}, \mathbf{C 2})$. Scale bars $=50 \mu \mathrm{m}$.
(Fiala et al., 2002; Wang et al., 2003). Olfactory projection neurons transfer olfactory information to the lateral horn and the mushroom body where they form large boutons (Tanaka et al., 2004). The mushroom body's Kenyon cells contact these boutons and receive input from many projection neurons (Caron et al., 2013). Odors are represented at the level of the mushroom body in terms of highly selective, sparse activity of very few ( $5 \%)$ Kenyon cells (Turner et al., 2008; Luo et al., 2010; Honegger et al., 2011). How the transformation from an overlapping, combinatorial odor code to a selective, sparse code is achieved, e.g., through the particular projection neuron to Kenyon cell connectivity, physiological properties of the respective neurons and inhibitory feedback loops, is subject to intense current research (Perez-Orive et al., 2002; Jortner et al., 2007; Turner et al., 2008; Luo et al., 2010; Honegger et al., 2011; Caron et al., 2013). The principle of converting spatio-temporal "codes" that reflect stimulus properties into "sparse codes" that do not directly reflect aspects of the original stimulus seems to be conserved across such evolutionarily distant species as mammals and insects (Leinwand and Chalasanim, 2011). Due to its relatively small number of neurons in comparison with cortical areas of the vertebrate brain, the mushroom body provides a favorable test system to analyze these topics. Second, the mushroom body also provides a favorable test system to investigate how experiencedependent changes in behavior caused by associative learning are mediated (Heisenberg, 2003; Davis, 2005; Fiala, 2007). Flies can be trained to associate an odor with a punishment or a reward (reviewed in Fiala, 2007), and synapses at the mushroom body lobes are believed to be a critical place for the coincidence of the two stimuli (Heisenberg, 2003). Particular dopaminergic neurons have been shown to mediate the punishment information during the associative learning (Schroll et al., 2006; Aso et al., 2010), whereas a different group of dopaminergic neurons mediates reward information (Liu et al., 2012). Octopaminergic neurons 
play an additional, modulatory role in reward learning (Hammer, 1993; Hammer and Menzel, 1998; Schroll et al., 2006; Burke et al., 2012). Investigating these two aspects of neuronal functioning requires appropriate experimental approaches.

Among all insects Drosophila melanogaster represents a favorable test organism because it is genetically manipulable and sophisticated genetic tools and expression systems have been invented to investigate the neuronal mechanisms underlying its behavior (Olsen and Wilson, 2008; Venken et al., 2011). These genetic techniques to dissect the structure and function of neuronal circuits encompass, first, anatomical methods to characterize the structure and connectivity of the constituting elements of the circuit, i.e., neurons and synapses. Second, genetic tools to monitor parameters of neuronal function have been engineered, e.g., $\mathrm{Ca}^{2+}$ sensors or fluorescence probes for synaptic vesicle release. Third, proteins to disrupt neuronal function or synaptic transmission can be expressed. And fourth, optogenetic and thermogenetic tools to artificially induce neuronal activity are available (Fiala et al., 2010; Riemensperger and Fiala, 2013). For analyzing specific parts of the mushroom body circuitry it is desirable to express combinations of transgenes in different populations of neurons. However, combinatorial expression of several transgenes is limited by the particular expression systems. One can combine the three binary expression systems that are available, i.e., the Gal4-UAS system (Brand and Perrimon, 1993), the LexA/LexAop system (Lai and Lee, 2006), and the Q system (Potter and Luo, 2011). Each expression system requires two genomic insertions, and the practical limitations in combining these transgenes are obvious. Mb247-driven expression of transgenes can be combined with one or two of the above-mentioned binary expression systems relatively simply (e.g., see Pech et al., 2013). The compilation of stable mushroom body-expressing transgenic Drosophila strains provided here might be of help in this regard.

\section{TARGETING AND VISUALIZING THE MUSHROOM BODY}

A visual landmark of the mushroom body helps to determine the spatial configuration of neurons relative to the mushroom body. The mb247-DsRed fly strain described by Riemensperger et al. (2005) has been used a number of times for that purpose (e.g., Lin et al., 2007; Tanaka et al., 2008; Claridge-Chang et al., 2009; Pech et al., 2013). As a prerequisite for physiological studies the visual landmark should ideally be detectable both in vivo and, for a post-hoc analysis, in fixed tissue, which is the case with DsRed. Here we describe two additional tools based on mb247-dependent transgene expression. Recently, Pech et al. (2013) adapted the GRASP technique (Feinberg et al., 2008; Gordon and Scott, 2009) and designed transgenic flies to visualize cell-to-cell contacts between Kenyon cells and mushroom body extrinsic neurons. It is often difficult to unambiguously identify and characterize putative innervations of potential mushroom body extrinsic neurons based on the expression of cytosolic or membrane-bound fluorescence proteins. The strategy of Pech et al. (2013) was to express one part of the membrane-bound split-GFP in the mushroom body and, in addition, a second part in a different subset of cells under UAS-control. This allows one to selectively visualize close proximity between intrinsic and extrinsic neurons. This tool can be combined with the expression of paGFP (Patterson and Lippincott-Schwartz, 2002; Ruta et al., 2010) under control of the mb247 promoter, which provides the possibility of tracking the anatomy of Kenyon cells from one particular Kenyon cell/extrinsic neuron contact up to the somata and axonal arborizations in different lobes. The combination of the mb247-DsRed, the mb247splitGFP, and/or the mb247-paGFP in combination with high resolution microscopy, e.g., two-photon microscopy (Denk et al., 1990), might be helpful for detailed anatomical studies in the intact Drosophila brain. Of course, these anatomical markers can also be combined with genetically encoded fluorescence sensors as reporters of neural activity. A third approach relies on the expression of the yeast-derived flippase, which can be used to restrict the expression of marker or effector genes to Kenyon cells with relatively high specificity. The repertoire of, among others, reporter and effector genes coupled to FRT-flanked stop cassettes is constantly growing. This will allow for a restricted visualization or manipulation of mushroom body intrinsic cells included in the often very non-specific enhancer trap lines.

\section{MONITORING NEURONAL ACTIVITY IN THE MUSHROOM BODY}

Since the very first description of genetically encoded $\mathrm{Ca}^{2+}$ sensors (Miyawaki et al., 1997; Romoser et al., 1997) and their first applications in Drosophila (Reiff et al., 2002; Fiala et al., 2002) continuous progress in their development has led to very improved versions of $\mathrm{Ca}^{2+}$ sensors. In particular, the invention of G-CaMP-type sensors (Nakai et al., 2001) has laid the foundation for engineering today's state-of-the art sensors (Tian et al., 2009; Zhao et al., 2011; Akerboom et al., 2012, 2013; Ohkura et al., 2012; Chen et al., 2013). Ratiometric FRET-based sensors, e.g., cameleon-type (Miyawaki et al., 1997) or troponin-based sensors like TN-XXL (Mank et al., 2008) are useful for particular applications. However, these sensors that are based on the simultaneous detection of YFP and CFP emission are difficult to be combined with the simultaneous detection of another wavelength. Therefore, we have chosen and directed four different single-wavelength $\mathrm{Ca}^{2+}$ reporters (GCaMP3.0, Tian et al., 2009; G-GECO1.1 and 1.2 and R-GECO1.0, Zhao et al., 2011) specifically to Kenyon cells under mb247 control. For a detailed and quantitative comparison of the four different $\mathrm{Ca}^{2+}$ reporters used, please refer to Walker et al. (2013) and Yamada and Mikoshiba (2012) The performance of the G-GECO1.1 and GGECO1.2 (Zhao et al., 2011) has been reported to be comparable to that of the GFP-based GCaMP 3.0, with varying dynamic ranges and kinetics, however. Our results on odor-evoked $\mathrm{Ca}^{2+}$ dynamics in Kenyon cells are in accordance with these reports, and all three green fluorescent sensors expressed in the mushroom body are functional and reliably detect odor responses of Kenyon cells. However, the functionality of R-GECO1.0 (Zhao et al., 2011) has been discussed controversially (Yamada and Mikoshiba, 2012). When expressed in the mushroom body, RGECO1.0 shows similar kinetics at signal onset as the two GGECO indicators. However, it shows much lower maximal signal intensity. This lower efficiency of the red fluorescent sensor in comparison with the green ones might be simply due to a lower excitability using two-photon excitation in the infrared range. That might also explain the critical evaluation of R-GECO1.0 
by Yamada and Mikoshiba (2012), who also used two-photon microscopy. An alternative red fluorescent $\mathrm{Ca}^{2+}$ sensor protein (RCaMP) has been described recently (Akerboom et al., 2013), which, on the one hand, appears favorable for twophoton excitation and simultaneous optogenetic activation of neurons using channelrhodopsin-2 (Akerboom et al., 2013). On the other hand, in a direct comparison (Akerboom et al., 2013) R-GECO1.0 shows higher sensitivity to detect action potentials, better signal to noise ratio and larger maximal fluorescence increase $\left(\Delta F / F_{0}\right)_{\max }$. We could confirm the high sensitivity of R-GECO1.0, as this was the only sensor that reported distinguishable on- and offset signals, mimicking the electrophysiological odor response of Kenyon cells (Ito et al., 2008). Further, RGECO1.0 has been used successfully in the olfactory system of the Drosophila brain in combination with the green sensor GCaMP3.0 (Li et al., 2013). The development of novel $\mathrm{Ca}^{2+}$ sensors progresses constantly, and improved GCaMP variants are published very frequently (Tian et al., 2009; Zhao et al., 2011; Akerboom et al., 2012, 2013; Chen et al., 2013). The pCaSpeRmb247 vector is available if one wishes to create additional fly strains that express novel sensors under mb247 promoter control.

However, $\mathrm{Ca}^{2+}$ imaging is not the only method of observing the activity of neurons. Sometimes it is advantageous to monitor synaptic transmitter release, in particular in the context of functional interactions between Kenyon cells and mushroom body extrinsic neurons. Therefore, we targeted Synapto-pHluorin (Miesenböck et al., 1998) to Kenyon cells. Compared to the $\mathrm{Ca}^{2+}$ sensors, Synapto-pHluorin shows a relatively small fluorescence increase in response to the odor onset. The low signal-to-noise ratio as can be estimated by the relation between its low signal and the relatively large standard errors. Only an estimated fraction of up to $5 \%$ of synaptic vesicles are used for release and reuptake at an active synapse (Denker et al., 2011), and Synapto-pHluorin is also expressed in the remaining $95 \%$ of vesicles of the reserve pool. The poor signal-to-noise ratio is, therefore, a result of the parameter measured.

In Drosophila, there is a fast-growing and impressive library of multipartite expression systems and their variants and modifications (Duffy, 2002; Pfeiffer et al., 2008, 2010; Bellen et al., 2011; Venken et al., 2011) to target more and more neuronal

\section{REFERENCES}

Akerboom, J., Carreras Calderón, N., Tian, L., Wabnig, S., Prigge, M., Tolö, J., et al. (2013). Genetically encoded calcium indicators for multi-color neural activity imaging and combination with optogenetics. Front. Mol. Neurosci. 6:2. doi: 10.3389/fnmol.2013.00002

Akerboom, J., Chen, T. W., Wardill, T.

J., Tian, L., Marvin, J. S., Mutlu,

S., et al. (2012). Optimization of a GCaMP calcium indicator for neural activity imaging. J. Neurosci. 32, 13819-13840. doi: 10.1523/JNEUROSCI.260112.2012
Aso, Y., Grübel, K., Busch, S., Friedrich, A. B., Siwanowicz, I., and Tanimoto, H. (2009). The mushroom body of adult Drosophila characterized by GAL4 drivers. J. Neurogenet. 23, 156-172. doi: 10.1080/01677060802471718

Aso, Y., Siwanowicz, I., Bräcker, L., Ito, K., Kitamoto, T., and Tanimoto, H. (2010). Specific dopaminergic neurons for the formation of labile aversive memory. Curr. Biol. 20, 1445-1451. doi: 10.1016/j.cub.2010.06.048

Baines, R. A., Uhler, J. P., Thompson, A., Sweeney, S. T., and Bate, M. (2001). Altered electrical properties

subsets or single neurons with greater precision. This study aims at complementing these genetic techniques for the specific application of analyzing a particular neuronal circuit, the mushroom body. If transgenes are expressed under direct control of the mb247 promoter the commonly used binary transcription systems are still available to express, in addition, marker proteins, fluorescence sensors, optogenetic, and thermogenetic actuator proteins. The broad palette of fly strains assembled here might be of help in this task.

\section{AUTHOR CONTRIBUTIONS}

Ulrike Pech created and characterized the mb247-splitGFP strain, the mb247-paGFP strain, and the mb247-GCaMP3.0 strain, performed immunohistochemistry and two-photon microscopy. Shubham Dipt created the mb247-Synapto-pHluorin strain and performed all in-vivo optical imaging experiments. Jonas Barth created the mb247-GECO strains, Priyanka Singh created the mb247-FLP strain, Mandy Jauch tested the mb247-lexA strain, Andreas S. Thum provided the DNA of the original pCaSpeRmb247-vector and created the mb247-Gal80 strain, André Fiala conceived the experiments, wrote the manuscript together with TR and Thomas Riemensperger created the mb247-DsRed strain, performed immunohistochemistry, analyzed data, supervised experiments and wrote the manuscript together with AF.

\section{ACKNOWLEDGMENTS}

We are grateful for having obtained the original pCaSpeRmb247 vector from Martin Heisenberg's laboratory. We thank Cori Bargman, Rudi Bohm, Erich Buchner, Robert E. Campbell, Gero Miesenböck, Hitoshi Okazawa, Silke Sachse, Kristin Scott, Stephan Sigrist, Takuya Tamura, Scott Waddell, Bing Zhang, and the Bloomington Stock Center for providing fly strains, antibodies, and DNA constructs. We are grateful to Stefan Dippel and David Vasmer for technical help. Funding was provided by the German Research Foundation (DFG), grants FI 821/2-1 (SPP 1392), FI 821/3-1, SFB 889/B04 and C02, and by the German Ministry for Research and Education (BMBF) via the Bernstein Center for Computational Neuroscience II (grant number 01GQ1005A). We acknowledge support by the German Research Foundation and the Open Access Publication Funds of the Göttingen University.

in Drosophila neurons developing without synaptic transmission. J. Neurosci. 21, 1523-1531.

Bellen, H. J., Levis, R. W., He, Y., Carlson, J. W., Evans-Holm, M., Bae, E., et al. (2011). The Drosophila gene disruption project: progress using transposons with distinctive site specificities. Genetics 188, 731-743. doi: 10.1534/genetics.111.126995

Berridge, M. J. (1998). Neuronal calcium signaling. Neuron 21, 13-26. doi: 10.1016/S08966273(00)80510-3

Bohm, R. A., Welch, W. P., Goodnight, L. K., Cox, L. W., Henry, L. G.,
Gunter, T. C., et al. (2010). A genetic mosaic approach for neural circuit mapping in Drosophila. Proc. Natl. Acad. Sci. U.S.A. 107, 16378-16383. doi: 10.1073/pnas.1004669107

Brand, A. H., and Perrimon, N. (1993). Targeted gene expression as a means of altering cell fates and generating dominant phenotypes. Development 118, 401-415.

Burgoyne, R. D. (2007). Neuronal calcium sensor proteins: generating diversity in neuronal $\mathrm{Ca} 2+$ signalling. Nat. Rev. Neurosci. 8, 182-193. doi: 10.1038/nrn2093

Burke, C. J., Huetteroth, W., Owald, D., Perisse, E., Krashes, M. J., 
Das, G., et al. (2012). Layered reward signalling through octopamine and dopamine in Drosophila. Nature 492, 433-437. doi: $10.1038 /$ nature1 1614

Bushey, D., and Cirelli, C. (2011). From genetics to structure to function: exploring sleep in Drosophila. Int. Rev. Neurobiol. 99, 213-244. doi: $\quad 10.1016 / \mathrm{B} 978-0-12-387003-$ 2.00009-4

Butcher, N. J., Friedrich, A. B., Lu, Z. Tanimoto, H., and Meinertzhagen, I. A. (2012). Different classes of input and output neurons reveal new features in microglomeruli of the adult Drosophila mushroom body calyx. J. Comp. Neurol. 520, 2185-2201. doi: 10.1002/cne.23037

Caron, S. J., Ruta, V., Abbott, L. F., and Axel, R. (2013). Random convergence of olfactory inputs in the Drosophila mushroom body. Nature 497, 113-117. doi: 10.1038/nature12063

Cassenaer, S., and Laurent, G. (2007) Hebbian STDP in mushroom bodies facilitates the synchronous flow of olfactory information in locusts. Nature 448, 709-713. doi: 10.1038/nature05973

Chen, T. W., Wardill, T. J., Sun, Y., Pulver, S. R., Renninger, S. L., Baohan, A., et al. (2013). Ultrasensitive fluorescent proteins for imaging neuronal activity. Nature 499, 295-300. doi: 10.1038/nature12354

Claridge-Chang, A., Roorda, R. D., Vrontou, E., Sjulson, L., Li, H., Hirsh, J., et al. (2009). Writing memories with lightaddressable reinforcement circuitry. Cell 139, 405-415. doi: 10.1016/j.cell.2009.08.034

Crittenden, J. R., Skoulakis, E. M., Hanm, K. A., Kalderon, D., and Davis, R. L. (1998). Tripartite mushroom body architecture revealed by antigenic markers. Learn. Mem. 5, $38-51$.

Dang, D. T., and Perrimon, N. (1992). Use of a yeast site-specific recombinase to generate embryonic mosaics in Drosophila. Dev. Genet. 13, 367-375. doi: 10.1002/dvg. 1020130507

Davis, R. L. (1993). Mushroom bodies and Drosophila learning. Neuron 11, 1-14. doi: 10.1016/0896-6273(93)90266-T

Davis, R. L. (2005). Olfactory memory formation in Drosophila: from molecular to systems neuroscience. Annu. Rev. Neurosci. 28, 275-302. doi: $\quad 10.1146 /$ annurev neuro 28 061604.13565

Demmer, H., and Kloppenburg, P. (2009). Intrinsic membrane properties and inhibitory synaptic input of kenyon cells as mechanisms for sparse coding. $J$ Neurophysiol. 102, 1538-1550. doi: 10.1152/jn.00183.2009

Denk, W., Strickler, J. H., and Webb W. W. (1990). Two-photon lase scanning fluorescence microscopy. Science 248, 73-76. doi: 10.1126/science. 2321027

Denker, A., Bethani, I., Kröhnert, K., Körber, C., Horstmann, H., Wilhelm, B. G. et al., (2011). A small pool of vesicles maintain synaptic activity in vivo. Proc. Natl. Acad. Sci. U.S.A. 108, 17177-17182. doi: $10.1073 /$ pnas.1112688108

Devaud, J. M., Blunk, A., Podufall,

J., Giurfa, M., and Grünewald,

B. (2007). Using local anaesthetics to block neuronal activity and map specific learning task to the mushroom bodies of an insect brain. Eur. J. Neurosci. 26 3193-3206. doi: 10.1111/j.14609568.2007.05904.x

Duffy, J. B. (2002). GAL4 system in Drosophila: a fly geneticist's Swiss army knife. Genesis 34, 1-15. doi: 10.1002/gene. 10150

Dujardin, F. (1850). Mémoire sur le système nerveux des insectes. Ann. Sci. Nat. Zool. 14, 195-206.

Erber, J., Masuhr, T., and Menzel, R. (1980). Localization of shortterm memory in the brain of the bee, Apis mellifera. Physiol. Entomol. 5, 343-358. doi: 10.1111/j.13653032.1980.tb00244.x

Estes, P. S., Roos, J., van der Bliek, A., Kelly, R. B., Krishnan, K. S., and Ramaswami, M. (1996). Traffic of dynamin within individual Drosophila synaptic boutons relative to compartment-specific markers. J. Neurosci. 16, 5443-5456.

Faber, T., and Menzel, R. (2001). Visualizing mushroom body response to a conditioned odor in honeybees. Naturwissenschaften $88,472-476$ doi: $10.1007 / \mathrm{s} 001140100263$

Fahrbach, S. E. (2006). Structure of the mushroom bodies of the insect brain. Annu. Rev. Entomol. 51, 209-232. doi: 10.1146/ annurev.ento.51.110104.150954

Feinberg, E. H., Vanhoven, M. K. Bendesky, A., Wang, G., Fetter, R. D., Shen, K., et al. (2008) GFP Reconstitution Across Synaptic Partners (GRASP) defines cell contacts and synapses in living nervous systems. Neuron 57, 353-363. doi: 10.1016/j.neuron.2007.11.030

Fiala, A. (2007). Olfaction and olfactory learning in Drosophila: recent progress. Curr. Opin.
Neurobiol. 17, 720-726. doi: 10.1016/j.conb.2007.11.009

Fiala, A., Spall, T., Diegelmann, S. Eisermann, B., Sachse, S., Devaud, J. M., et al. (2002). Genetically expressed cameleon in Drosophila melanogaster is used to visualize olfactory information in projection neurons. Curr. Biol. 12, 1877-1884. doi: 10.1016/S09609822(02)01239-3

Fiala, A., Suska, A., and Schlüter, O. M. (2010). Optogenetic approaches in neuroscience. Curr. Biol. 20, 897-903. doi 10.1016/j.cub.2010.08.053

Friggi-Grelin, F., Coulom, H., Meller M., Gomez, D., Hirsh, J., and Birman, S. (2003). Targeted gene expression in Drosophila dopaminergic cells using regulatory sequences from tyrosine hydroxyase. J. Neurobiol. 54, 618-627. doi $10.1002 /$ neu. 10185

Golic, K. G., and Lindquist, S. (1989). The FLP recombinase of yeast catalyzes site-specific recombination the Drosophila genome. Cell 59, 499-509. doi: 10.1016/00928674(89)90033-0

Gordon, M. D., and Scott, K. (2009). Motor control in a Drosophila taste circuit. Neuron 61, 373-384. doi 10.1016/j.neuron.2008.12.033

Grienberger, C., and Konnerth, A. (2012). Imaging calcium in neuNeuron 73, 862-885, doi: 10.1016/j.neuron.2012.02.011

Guizar-Sicairos, M., Thurman, S. T., and Fienup, J. R. (2008). Efficien subpixel image registration algorithms. Opt. Lett. 33, 156-158. doi 10.1364/OL.33.000156

Hamada, F. N., Rosenzweig, M. Kang, K., Pulver, S. R., Ghezzi A., Jegla, T. J., et al. (2008). An internal thermal sensor controlling temperature preference in Drosophila. Nature 454, 217-220. doi: $10.1038 /$ nature 07001

Hammer, M. (1993). An identified neuron mediates the unconditioned stimulus in associative olfactory learning in honeybees. Nature 366 59-63. doi: 10.1038/366059a0

Hammer, M., and Menzel, R. (1998) Multiple sites of associative odor learning as revealed by local brain microinjections of octopamine in honeybees. Learn Mem. 5, 146-156.

Heisenberg, M. (2003). Mushroom body memoir: from maps to models. Nat. Rev. Neurosci. 4, 266-275. doi: 10.1038/nrn1074

Honegger, K. S., Campbell, R. A., and Turner, G. C. (2011). Cellularresolution population imaging reveals robust sparse coding in the Drosophila mushroom body.
J. Neurosci. 31, 11772-11785. doi: 10.1523/JNEUROSCI.1099-11.2011 Hussaini, S. A., and Menzel, R. (2013). Mushroom body extrinsic neurons in the honeybee brain encode cues and contexts differently. J. Neurosci. 33, 7154-7164. doi: 10.1523/JNEUROSCI.1331-12.2013 Ito, I., Ong, R. C., Raman, B., and Stopfer, M. (2008). Olfactory learning and sipke timing dependent plasticity. Commun. Integr. Biol. 1, 170-181. doi: 10.4161/cib.1.2.7140

Ito, K., Suzuki, K., Estes, P., Ramaswami, M., Yamamoto, D., and Strausfeld, N. J. (1998). The organization of extrinsic neurons and their implications in the functional roles of the mushroom bodies in Drosophila melanogaster Meigen. Learn. Mem. 5, 52-77.

Jenett, A., Rubin, G. M., Ngo, T. T., Shepherd, D., Murphy, C., Dionne, H., et al. (2012). A GAL4-driver line resource for Drosophila neurobiology. Cell Rep. 2, 991-1001. doi: 10.1016/j.celrep.2012.09.011

Jortner, R. A., Farivar, S. S., and Laurent, G. (2007). A simple connectivity scheme for sparse coding in an olfactory system. J. Neurosci. 27, 1659-1669. doi: 10.1523/JNEUROSCI.4171-06.2007

Kenyon, F. (1896). The brain of the bee. A preliminary contribution to the morphology of the nervous system of the arthropoda. J. Comp. Neurol. 6, 133-210. doi: $10.1002 /$ cne. 910060302

Kitamoto, T. (2001). Conditional modification of behavior in Drosophila by targeted expression of a temperature-sensitive shibire allele in defined neurons. J. Neurobiol. 47, 81-92. doi: 10.1002/neu.1018

Krashes, M. J., Keene, A. C., Leung, B., Armstrong, J. D., and Waddell, S. (2007). Sequential use of mushroom body neuron subsets during drosophila odor memory processing. Neuron 53, 103-115. doi: 10.1016/j.neuron.2006.11.021

Lai, S. L., and Lee, T. (2006). Genetic mosaic with dual binary transcriptional systems in Drosophila. Nat. Neurosci. 9, 703-709. doi: 10.1038/nn1681

Lee, T., and Luo, L. (1999). Mosaic analysis with a repressible cell marker for studies of gene function in neuronal morphogenesis. Neuron 22, 451-461. doi: 10.1016/S08966273(00)80701-1

Leinwand, S. G., and Chalasanim, S. H. (2011). Olfactory networks: from sensation to perception. Curr. Opin. Genet. Dev. 21, 806-811. doi: 10.1016/j.gde.2011.07.006 
Leiss, F., Groh, C., Butcher, N. J., Meinertzhagen, I. A., and Tavosanis, G. (2009). Synaptic organization in the adult Drosophila mushroom body calyx. J. Comp. Neurol. 517, 808-824. doi: 10.1002/cne.22184

Li, H., Li, Y., Lei, Z., Wang, K., and Guo, A. (2013). Transformation of odor selectivity from projection neurons to single mushroom body neurons mapped with dual-color calcium imaging. Proc. Natl. Acad. Sci. U.S.A. 110, 12084-12109. doi: 10.1073/pnas. 1305857110

Li, Y., and Strausfeld, N. J. (1997) Morphology and sensory modality of mushroom body extrinsic neurons in the brain of the cockroach, Periplaneta americana. I. Comp. Neurol. 387, 631-650. doi: $\quad 10.1002 /($ SICI) 1096-9861 (19971103)387:4<631::AID-CNE9 $>3.0 . \mathrm{CO} ; 2-3$

Li, Y., and Strausfeld, N. J. (1999). Multimodal efferent and recurrent neurons in the medial lobes of cockroach mushroom bodies. J. Comp. Neurol. 409, 647-663. doi: 10.1002/(SICI) 1096-9861(19990712)409:4<647::A ID-CNE9>3.0.CO;2-3

Lin, H. H., Lai, J. S., Chin, A. L., Chen, Y. C., and Chiang, A. S. (2007). A map of olfactory representation in the Drosophila mushroom body. Cell 128, 1205-1217. doi: 10.1016/j.cell.2007.03.006

Lissandron, V., Rossetto, M. G., Erbguth, K., Fiala, A., Daga, A., and Zaccolo, M. (2007). Transgenic fruit-flies expressing a FRET-based sensor for in vivo imaging of cAMP dynamics. Cell Signal. 19, 2296-2303. doi: 10.1016/j.cellsig.2007.07.004

Liu, C., Plaçais, P. Y., Yamagata, N., Pfeiffer, B. D., Aso, Y., Friedrich, A. B., et al. (2012). A subset of dopamine neurons signals reward for odour memory in Drosophila. Nature 488, 512-516. doi: 10.1038/nature11304

Louis, T., Musso, P. Y., de Oliveira, S. B., Garreau, L., Giurfa, M., Raymond, V., et al. (2012). Amel $\alpha 8$ subunit knockdown in the mushroom body vertical lobes impairs olfactory retrieval in the honeybee, Apis mellifera. Eur. J. Neurosci. 36, 3438-3450. doi: 10.1111/j.14609568.2012.08261.x

Luo, S. X., Axel, R., and Abbott, L. F., (2010). Generating sparse and selective third-order responses in the olfactory system of the fly. Proc. Natl. Acad. Sci. U.S.A. 107, 10713-10718. doi: 10.1073/pnas. 1005635107
Mank, M., Santos, A. F., Direnberger, S., Mrsic-Flogel, T. D., Hofer, S. B. Stein, V., et al. (2008). A genetically encoded calcium indicator for chronic in vivo two-photon imaging. Nat. Methods 5, 805-811. doi: 10.1038/nmeth. 1243

Mao, Z., and Davis, R. L. (2009). Eight different types of dopaminergic neurons innervate the Drosophila mushroom body neuropil: anatomical and physiological heterogeneity. Front. Neural Circuits 3:5. doi: 10.3389/neuro.04.005.2009

Martin, J. R., Ernst, R., and Heisenberg, M. (1998). Mushroom bodies suppress locomotor activity in Drosophila melanogaster. Learn. Mem. 5, 179-191.

Masse, N. Y., Turner, G. C., and Jefferis, G. S. (2009). Olfactory information processing in Drosophila. Curr. Biol. 19, 700-713. doi: 10.1016/j.cub.2009.06.026

Matz, M. V., Fradkov, A. F., Labas, Y. A., Savitsky, A. P., Zaraisky, A. G., Markelov, M. L., et al. (1999). Fluorescent proteins from nonbioluminescent Anthozoa species. Nat. Biotechnol. 17, 969-973. doi: 10.1038/13657

Miesenböck, G., De Angelis, D. A., an Rothman, J. E. (1998). Visualizing secretion and synaptic transmission with $\mathrm{pH}$-sensitive green fluorescent proteins. Nature 394, 192-195. doi: $10.1038 / 28190$

Miyawaki, A., Llopis, J., Heim, R., McCaffery, J. M., Adams, J. A., Ikura, M., et al. (1997). Fluorescent indicators for $\mathrm{Ca} 2+$ based on green fluorescent proteins and calmodulin. Nature 388, 882-887. doi: $10.1038 / 42264$

Mizunami, M., Okada, R., Li, Y., and Strausfeld, N. J. (1998). Mushroom bodies of the cockroach: activity and identities of neurons recorded in freely moving animals. J. Comp. Neurol. 402, 501-519. doi: 10.1002/ (SICI) 1096-9861(19981228)402:4< 501::AID-CNE5>3.0.CO;2-M

Nakai, J., Ohkura, M., and Imoto, K. (2001). A high signal-to-noise $\mathrm{Ca}(2+)$ probe composed of a single green fluorescent protein. Nat. Biotechnol. 19, 137-141. doi: 10.1038/84397

Nitabach, M. N., Blau, J., and Holmes, T. C. (2002). Electrical silencing of Drosophila pacemaker neurons stops the free-running circadian clock. Cell 109, 485-495. doi: 10.1016/S0092-8674(02)00737-7

Ohkura, M., Sasaki, T., Sadakari, J., Gengyo-Ando, K., KagawaNagamura, Y., Kobayashi, C., et al. (2012). Genetically encoded green fluorescent $\mathrm{Ca} 2+$ indicators with improved detectability for neuronal Ca2+ signals. PLoS ONE 7:e51286. doi: 10.1371/journal.pone. 0051286

Olsen, S. R., and Wilson, R. I. (2008) Cracking neural circuits in a tiny brain: new approaches for understanding the neural circuitry of Drosophila. Trends Neurosci. 31, 512-520. doi: 10.1016/j.tins.2008.07.006

Patterson, G. H., and LippincottSchwartz, J. (2002). A photoactivatable GFP for selective photolabeling of proteins and cells. Science 297, 1873-1877. doi 10.1126/science. 1074952

Pech, U., Pooryasin, A., Birman, S., and Fiala, A. (2013). Localization of the contacts between Kenyon cells and aminergic neurons in the Drosophila melanogaster brain using splitGFP reconstitution. J. Comp. Neurol. doi: 10.1002/cne.23388.

Perez-Orive, J., Mazor, O., Turner G. C., Cassenaer, S., Wilson, R. I., and Laurent, G. (2002). Oscillations and sparsening of odor representations in the mushroom body. Science 297, 359-565. doi: 10.1126/science. 1070502

Pfeiffer, B. D., Jenett, A., Hammonds, A. S., Ngo, T. T., Misra, S., Murphy, C., et al. (2008). Tools for neuroanatomy and neurogenetics in Drosophila. Proc. Natl. Acad. Sci. U.S.A. 105, 9715-9720. doi: 10.1073/pnas.0803697105

Pfeiffer, B. D., Ngo, T. T., Hibbard, K. L., Murphy, C., Jenett, A., Truman J. W., et al. (2010). Refinement of tools for targeted gene expression in Drosophila. Genetics 186, 735-755. doi: 10.1534/genetics. 110.119917

Pignoni, and Zipursky. (1997) Induction of Drosophila eye development by decapentaplegic. Development 124, 271-278.

Pitman, J. L., Huetteroth, W., Burke, C. J., Krashes, M. J., Lai, S. L. Lee, T., et al. (2011). A pair of inhibitory neurons are required to sustain labile memory in the Drosophila mushroom body Curr. Biol. 21, 855-861. doi 10.1016/j.cub.2011.03.069

Potter, C. J., and Luo, L. (2011). Using the Q system in Drosophila melanogaster. Nat. Protoc. 6, 1105-1120. doi: 10.1038/nprot.2011.347

Reiff, D. F., Thiel, P. R., and Schuster C. M. (2002). Differential regulation of active zone density during long-term strengthening of Drosophila neuromuscular junctions. J. Neurosci. 22, 9399-9409.
Riemensperger, T., and Fiala, A. (2013). "Optophysiological approaches to learning and memory in Drosophila melanogaster," in Invertebrate Learning and Memory, eds $\mathrm{R}$. Menzel and P. R. Benjamin (London, San Diego: Academic Press), 59-66. doi: 10.1016/B978-012-415823-8.00006-X

Riemensperger, T., Pech, U., Dipt, S., and Fiala, A. (2012). Optical calcium imaging in the nervous system of Drosophila melanogaster. Biochim. Biophys. Acta 1820, 1169-1178. doi: 10.1016/j.bbagen.2012.02.013

Riemensperger, T., Völler, T., Stock, P., Buchner, E., and Fiala, A. (2005). Punishment prediction by dopaminergic neurons in Drosophila. Curr. Biol. 15, 1953-1960. doi: 10.1016/j.cub.2005.09.042

Romoser, V. A., Hinkle, P. M., and Persechini, A. (1997). Detection in living cells of $\mathrm{Ca} 2+$-dependent changes in the fluorescence emission of an indicator composed of two green fluorescent protein variants linked by a calmodulinbinding sequence. A new class of fluorescent indicators. J. Biol. Chem. 272, 13270-13274. doi: 10.1074/jbc.272.20.13270

Ruta, V., Datta, S. R., Vasconcelos, M. L., Freeland, J., Looger, L. L., and Axel, R. (2010). A dimorphic pheromone circuit in Drosophila from sensory input to descending output. Nature 468, 686-690. doi: 10.1038/nature09554

Schroll, C., Riemensperger, T., Bucher, D., Ehmer, J., Völler, T., Erbguth, K., et al. (2006). Light-induced activation of distinct modulatory neurons triggers appetitive or aversive learning in Drosophila larvae. Curr. Biol. 16, 1741-1747. doi: 10.1016/j.cub.2006. 07.023

Schulz, R. A., Chromey, C., Lu, M. F., Zhao, B., and Olson, E. N. (1996). Expression of the D-MEF2 transcription in the Drosophila brain suggests a role in neuronal cell differentiation. Oncogene 12, 1827-1831.

Shafer, O. T., Kim, D. J., DunbarYaffe, R., Nikolaev, V. O., Lohse, M. J., and Taghert, P. H. (2008). Widespread receptivity to neuropeptide PDF throughout the neuronal circadian clock network of Drosophila revealed by real-time cyclic AMP imaging. Neuron 58, 223-237. doi: 10.1016/j.neuron. 2008.02.018

Stopfer, M., Jayaraman, V., and Laurent, G. (2003). Intensity versus 
identity coding in an olfactory system. Neuron 39, 991-1004. doi: 10.1016/j.neuron.2003.08.011

Strausfeld, N. J., Hansen, L., Li, Y., Gomez, R. S., and Ito, K. (1998). Evolution, discovery, and interpretations of arthropod mushroom bodies. Learn. Mem. 5, 11-37.

Strausfeld, N. J., Sinakevitch, I., Brown, S. M., and Farris, S. M. (2009). Ground plan of the insect mushroom body: functional and evolutionary implications. J. Comp. Neurol. 513, 265-291. doi: $10.1002 / \mathrm{cne} .21948$

Strube-Bloss, M. F., Nawrot, M. P., and Menzel, R. (2011). Mushroom body output neurons encode odor-reward associations. J. Neurosci. 31, 3129-3140. doi: 10.1523/JNEUROSCI.2583-10.2011

Struhl, G., and Basler, K. (1993). Organizing activity of wingless protein in Drosophila. Cell 72, 527-540. doi: 10.1016/ 0092-8674(93)90072-X

Sweeney, S. T., Broadie, K., Keane, J., Niemann, H., and O'Kane, C. J. (1995). Targeted expression of tetanus toxin light chain in Drosophila specifically eliminates synaptic transmission and causes behavioral defects. Neuron 14, 341-351. doi: 10.1016/0896-6273(95)90290-2

Szyszka, P., Ditzen, M., Galkin, A., Galizia, C. G., and Menzel, R. (2005). Sparsening and temporal sharpening of olfactory representations in the honeybee mushroom bodies. J. Neurophysiol. 94, 3303-3313. doi: 10.1152/jn.00397.2005

Tamura, T., Horiuchi, D., Chen, Y C., Sone, M., Miyashita, T., Saitoe, M., et al. (2010). Drosophila PQBP1 regulates learning acquisition at projection neurons in aversive olfactory conditioning. J. Neurosci. 30, 14091-14101. doi: 10.1523/JNEUROSCI.131910.2010

Tanaka, N. K., Awasaki, T., Shimada, T., and Ito, K. (2004). Integration of chemosensory pathways in the Drosophila second-order olfactory centers. Curr. Biol. 14, 449-457. doi: 10.1016/j.cub. 2004.03.006

Tanaka, N. K., Tanimoto, H., and Ito, K. (2008). Neuronal assemblies of the Drosophila mushroom body. J. Comp. Neurol. 508, 711-755. doi: $10.1002 / \mathrm{cne} .21692$

Technau, G. M. (1984). Fiber number in the mushroom bodies of adult Drosophila melanogaster depends on age, sex and experience. J. Neurogenet. 1, 113-126. doi 10.3109/01677068409107077

Tewson, P., Westenberg, M., Zhao, Y., Campbell, R. E., Quinn, A. M., and Hughes, T. E. (2012). Simultaneous detection of $\mathrm{Ca} 2+$ and diacylglycerol signaling in living cells. PLoS ONE 7:e42791. doi: 10.1371/journal.pone.0042791

Tian, L., Hires, S. A., Mao, T., Huber, D., Chiappe, M. E. Chalasani, S. H., et al. (2009). Imaging neural activity in worms, flies and mice with improved GCaMP calcium indicators. Nat Methods 6, 875-881. doi: 10.1038/ nmeth.1398

Turner, G. C., Bazhenov, M., and Laurent, G. (2008). Olfactory representations by Drosophila mushroom body neurons. J. Neurophysiol. 99, 734-746 doi: 10.1152/jn.01283.2007

Venken, K. J., Simpson, J. H., and Bellen, H. J. (2011). Genetic manipulation of genes and cells in the nervous system of the fruit fly. Neuron 72, 202-230. doi: 10.1016/j.neuron.2011.09.021

Venken, K. J., and Bellen, H. J. (2012). Genome-wide manipulations of Drosophila melanogaster with transposons, Flp recombinase, and $\Phi C 31$ integrase. Methods Mol. Biol. 859, 203-228. doi: 10.1007/978-1-61779-603-6_12

Vosshall, L. B., and Stocker, R. F (2007). Molecular architecture of smell and taste in Drosophila. Annu. Rev. Neurosci. 30, 505-533. doi: 10.1146/annurev.neuro.30.051606. 094306

Walker, A. S., Burrone, J., and Meyer, M. P. (2013). Functional imaging in the zebrafish retinotectal system using RGECO. Front. Neural Circuits 7:34. doi: 10.3389/fncir.2013.00034

Wang, J. W., Wongm, A. M., Floresm, J., Vosshallm, L. B., and Axel, R (2003). Two-photon calcium imaging reveals an odor-evoked map of activity in the fly brain. Cell 112, 271-282. doi: 10.1016/S00928674(03)00004-7

$\mathrm{Xu}$, T., and Rubin, G. M. (1993). Analysis of genetic mosaics in developing and adult Drosophila tissues. Development 117 1223-1237.

Yamada, Y., and Mikoshiba, K. (2012). Quantitative comparison of novel GCaMP-type genetically encoded $\mathrm{Ca}(2+)$ indicators in mammalian neurons. Front. Cell. Neurosci. 6:41. doi: 10.3389/fncel.2012.00041

Yang, M. Y., Armstrong, J. D., Vilinsky, I., Strausfeld, N. J., and Kaiser, K. (1995). Subdivision of the Drosophila mushroom bodies by enhancer-trap expression patterns. Neuron 15, 45-54. doi: 10.1016/0896-6273(95)90063-2

Yasuyama, K., Meinertzhagen, I. A., and Schürmann, F. W. (2002). Synaptic organization of the mushroom body calyx in Drosophila melanogaster. J. Comp. Neurol. 445, 211-226. doi: 10.1002/cne.10155

Yu, J. Y., Kanaim, M. I., Demir E., Jefferis, G. S., and Dickson, B. J. (2010). Cellular organization of the neural circuit that drives Drosophila courtship behavior. Curr. Biol. 20, 1602-1614. doi: 10.1016/j.cub.2010.08.025

Zars, T., Fischer, M., Schulz, R., and Heisenberg, M. (2000). Localization of a short-term memory in Drosophila. Science 288, 672-675. doi: 10.1126/science.288.5466.672

Zhao, Y., Araki, S., Wu, J., Teramoto, T., Chang, Y. F., Nakano, M., et al. (2011). An expanded palette of genetically encoded $\mathrm{Ca}^{2}$ indicators. Science 333, 1888-1891. doi: 10.1126/science. 1208592

Conflict of Interest Statement: The authors declare that the research was conducted in the absence of any commercial or financial relationships that could be construed as a potential conflict of interest.

Received: 07 August 2013; paper pending published: 28 August 2013; accepted: 29 August 2013; published online: 23 September 2013.

Citation: Pech U, Dipt S, Barth J, Singh $P$, Jauch $M$, Thum AS, Fiala $A$ and Riemensperger $T$ (2013) Mushroom body miscellanea: transgenic Drosophila strains expressing anatomical and physiological sensor proteins in Kenyon cells. Front. Neural Circuits 7:147. doi: 10.3389/fncir.2013.00147

This article was submitted to the journal Frontiers in Neural Circuits.

Copyright (c) 2013 Pech, Dipt, Barth, Singh, Jauch, Thum, Fiala and Riemensperger. This is an openaccess article distributed under the terms of the Creative Commons Attribution License (CC BY). The use, distribution or reproduction in other forums is permitted, provided the original author(s) or licensor are credited and that the original publication in this journal is cited, in accordance with accepted academic practice. No use, distribution or reproduction is permitted which does not comply with these terms. 
B. Contributions to other studies

Restoring polyamines protects from age-induced memory impairment in an autophagy-dependent manner.

My contribution :

- Performed the experiments and analyzed the data for Figure 3. 


\title{
Restoring polyamines protects from age-induced memory impairment in an autophagy-dependent manner
}

\author{
Varun K Gupta $^{1,2}$, Lisa Scheunemann ${ }^{1}$, Tobias Eisenberg ${ }^{3}$, Sara Mertel ${ }^{1}$, Anuradha Bhukel ${ }^{1,2}$, Tom S Koemans ${ }^{4}$, \\ Jamie M Kramer ${ }^{4}$, Karen S Y Liu ${ }^{1,2}$, Sabrina Schroeder ${ }^{3}$, Hendrik G Stunnenberg ${ }^{5}$, Frank Sinner ${ }^{6,7}$, \\ Christoph Magnes ${ }^{6}$, Thomas R Pieber ${ }^{6,7}$, Shubham Dipt ${ }^{8}$, André Fiala ${ }^{8}$, Annette Schenck ${ }^{4}$, Martin Schwaerzel ${ }^{1}$, \\ Frank Madeo $^{3} \&$ Stephan J Sigrist ${ }^{1,2}$
}

Age-dependent memory impairment is known to occur in several organisms, including Drosophila, mouse and human. However, the fundamental cellular mechanisms that underlie these impairments are still poorly understood, effectively hampering the development of pharmacological strategies to treat the condition. Polyamines are among the substances found to decrease with age in the human brain. We found that levels of polyamines (spermidine, putrescine) decreased in aging fruit flies, concomitant with declining memory abilities. Simple spermidine feeding not only restored juvenile polyamine levels, but also suppressed age-induced memory impairment. Ornithine decarboxylase-1, the rate-limiting enzyme for de novo polyamine synthesis, also protected olfactory memories in aged flies when expressed specifically in Kenyon cells, which are crucial for olfactory memory formation. Spermidine-fed flies showed enhanced autophagy (a form of cellular self-digestion), and genetic deficits in the autophagic machinery prevented spermidine-mediated rescue of memory impairments. Our findings indicate that autophagy is critical for suppression of memory impairments by spermidine and that polyamines, which are endogenously present, are candidates for pharmacological intervention.

Age-induced memory impairment (AMI) is a common condition that is characterized by symptoms of cognitive decline that occur as part of the aging process. To date, molecular interventions to counteract AMI remain largely elusive, with the long lifespan of many animal models being a major limitation for studying age-related memory impairment. Drosophila, with its comparatively short lifespan and advanced genetics, is an ideal model system for unraveling the molecular mechanisms associated with AMI and testing putative means for preventing AMI. The age-dependent decline of (aversive) olfactory memory in Drosophila serves as an established model for AMI ${ }^{1-4}$. In this learning procedure, groups of flies are trained by presenting them with an odor that temporally coincides with the application of electric shocks and presenting a second odor without this punishment. Later on, flies can choose between the two arms of a T-maze containing either the odor that was paired with the electric shocks or the neutral odor. Learning is assessed by calculating the relative proportion of flies avoiding the punished odor. This type of associative olfactory memory is usually maintained over several hours ${ }^{5}$.

Studies in various model organisms have implicated autophagy as a crucial regulator of the aging process ${ }^{6}$. Autophagy is a process of cellular self-digestion in which portions of the cytoplasm are sequestered in double- or multi-membraned vesicles (autophagosomes) and then delivered to lysosomes for bulk degradation. In C. elegans, increased autophagy is necessary for lifespan extension by reduced insulin-like signaling ${ }^{7}$ and dietary restriction ${ }^{8}$. Similarly, increasing autophagy specifically in neurons can extend the lifespan of flies ${ }^{9}$, whereas reducing autophagy shortens lifespan and gives rise to neurodegeneration $^{9,10}$. Although autophagy is a key regulator of the aging process, its role in cognitive aging has not yet been addressed.

We found that the levels of simple polyamines (spermidine and putrescine) decreased in the heads of aging Drosophila, consistent with a decline in olfactory aversive memory in aging flies. Notably, restoration of polyamine levels by dietary spermidine supplementation suppressed AMI. Spermidine administration prevented the age-associated decrease of autophagy, and genetically induced deficits in the autophagic machinery occluded spermidinemediated protection of AMI. In addition, we found that the effects of spermidine on memory were not a result of generically improved health, but instead reflected neuron-intrinsic regulations. These results suggest that autophagy is important for cognitive aging and that polyamines, endogenous metabolites, are candidate substances for treating AMI.

${ }^{1}$ Institute for Biology/Genetics, Freie Universität Berlin, Berlin, Germany. ${ }^{2}$ NeuroCure, Charité, Berlin, Germany. ${ }^{3}$ Institute of Molecular Biosciences, University of Graz, Graz, Austria. ${ }^{4}$ Department of Human Genetics, Nijmegen Centre for Molecular Life Sciences, Donders Institute for Brain, Cognition and Behaviour, Radboud University Nijmegen Medical Centre, Nijmegen, The Netherlands. ${ }^{5}$ Department of Molecular Biology, Faculty of Science, Nijmegen Centre for Molecular Life Sciences, Radboud University Nijmegen, Nijmegen, The Netherlands. ${ }^{6}$ Health Institute for Biomedicine and Health Sciences, Joanneum Research Forschungs GesmBH, Graz, Austria. ${ }^{7}$ Department of Internal Medicine, Division of Endocrinology and Metabolism, Medical University of Graz, Austria. ${ }^{8}$ Georg-AugustUniversität Göttingen, Molecular Neurobiology of Behavior, Göttingen, Germany. Correspondence should be addressed to S.J.S. (stephan.sigrist@fu-berlin.de) or F.M. (frank.madeo@uni-graz). 
Figure 1 Spermidine feeding prevents the age-related decline of endogenous spermidine and putrescine levels. (a) Scheme illustrating de novo synthesis of polyamines from ornithine. * indicates the rate-limiting enzyme for de novo synthesis of polyamines. PMO, polyamine oxidase; SMO, spermine oxidase; SSAT, spermidine/spermine $\mathrm{N}(1)$ acetyltransferase. (b) Levels of spermidine in the heads of wild-type flies fed with food supplemented by 1 or $5 \mathrm{mM}$ spermidine (Spd $1 \mathrm{mM}+$ or $S p d^{5 \mathrm{mM}+}$, respectively) or food with no spermidine $\left(\mathrm{Spd}^{-}\right)$. Data are shown normalized to spermidine levels of 1-d-old (1 d) Spd- flies ( $n=4$ independent experiments; $F=14.08$ for Spd $^{-}$flies, $F=5.30$ for Spd $1 \mathrm{mM}+$ flies, $F=2.62$ for Spd $5 \mathrm{mM}+$ flies; one-way-ANOVA with Bonferroni correction). $15 \mathrm{~d}, 15 \mathrm{~d}$ old; 30 $\mathrm{d}, 30 \mathrm{~d}$ old. (c) Levels of putrescine in the heads of aged Spd $1 \mathrm{mM}+$ or Spd $5 \mathrm{mM}+$ flies compared with $\mathrm{Spd}^{-}$flies. Data are shown normalized to putrescine levels of 1 -d-old Spd- flies ( $n=4$

independent experiments; $F=24.31$ for Spd-flies, $F=0.22$ for Spd $1 \mathrm{mM}+$ flies, $F=6.67$ for Spd $5 \mathrm{mM}+$ flies; one-way-ANOVA with Bonferroni correction). ${ }^{*} P<0.05 ;{ }^{*} P<0.01 ;{ }^{* *} P<0.001 ;$ ns indicates not significant, $P>0.05$. Data are presented as mean \pm s.e.m.

\section{RESULTS}

\section{Polyamine levels decline in aging Drosophila brains}

A decrease in polyamine levels has been reported in aged brains of rodents and humans ${ }^{11,12}$. To investigate the role of polyamines in AMI, we began by measuring polyamine levels in heads of aged Drosophila, and analyzed the chemically related (and inter-convertible) species spermidine, putrescine and spermine (for a review, see ref. 13; Fig. 1a). In fact, both spermidine and putrescine levels were markedly reduced in the heads of 15 -d-old flies compared with young flies $\left(\mathrm{Spd}^{-} 1-3 \mathrm{~d}\right.$ old; Online Methods and Fig. 1b,c).
To determine whether simply feeding spermidine to flies would be sufficient to restore its expression in aged heads, we administered either 1 or $5 \mathrm{mM}$ spermidine to isogenized wild-type Drosophila $\left(w^{1118}\right)$ in standard fly food (Spd ${ }^{1 \mathrm{mM}+}$ or Spd ${ }^{5 \mathrm{mM}+}$, respectively). Spermidine levels (in fly heads) were largely protected by this treatment from age-dependent decline (Fig. 1b). Simultaneously, putrescine levels were greater than those in normal juvenile flies (Fig. 1c), indicating that the cellular uptake of spermidine is accompanied by either an increased conversion into putrescine or inhibition of the endogenous conversion of putrescine to spermidine (Fig. 1a).
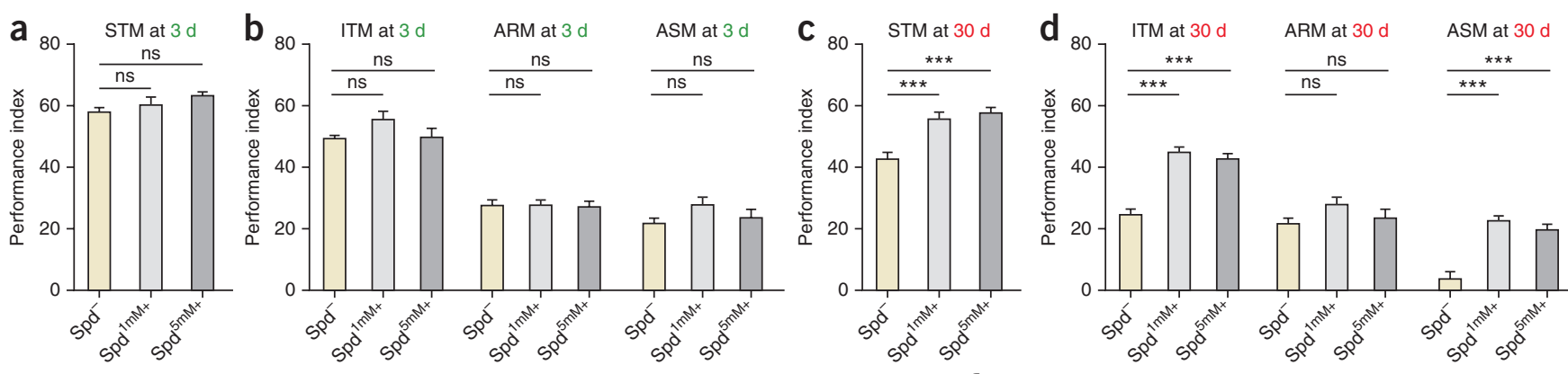

Figure 2 Spermidine-feeding rescues AMI. (a) Aversive associative memory performance at 3 min after training (STM) of 3-d-old (3 d) Spd $1 \mathrm{mM}+$ and Spd $5 \mathrm{mM}+$ flies in comparison to $\mathrm{Spd}^{-}$flies ( $n=8-9$ independent experiments, $F=2.28$, one-way-ANOVA with Bonferroni correction). (b) Aversive associative memory performance at $3 \mathrm{~h}$ after training (ITM), ARM and ASM of 3-d-old Spd $1 \mathrm{mM}+$ and $\mathrm{Spd}^{5 \mathrm{mM}+}$ compared with $\mathrm{Spd}^{-}$flies $(n=8-9$ independent experiments; $F=2.05$ for ITM, $F=0.02$ for ARM, $F=1.74$ for ASM; one-way ANOVA with Bonferroni correction). (c) STM of 30-d-old (30 d) Spd ${ }^{1 \mathrm{mM}+}$ and $\mathrm{Spd}^{5 \mathrm{mM}+}$ and $\mathrm{Spd}^{-}$flies ( $n=8-9$ independent experiments, $F=14.48$, one-way ANOVA with Bonferroni correction). (d) ITM, ARM and ASM

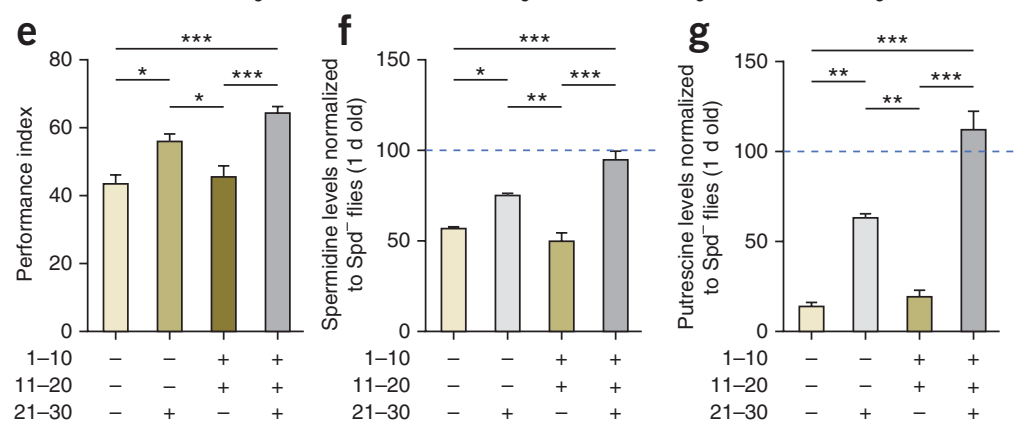
of 30-d-old Spd ${ }^{1 \mathrm{mM}+}$ and Spd ${ }^{5 \mathrm{mM}+}$ compared with $\mathrm{Spd}^{-}$flies $(n=7-9$ independent experiments; $F=43.26$ for ITM, $F=1.74$ for ARM, $F=26.12$ for ASM; one-way ANOVA with Bonferroni correction). (e) Spermidine feeding for $10 \mathrm{~d}$ before measuring memory was sufficient to suppress AMI in aged (30 d old) flies ( $n=9-11$ independent experiments, $F=12.32$, one-way ANOVA with Bonferroni correction). (f,g) Spermidine feeding on days 21-30 resulted in a significant increase in the levels of spermidine and putrescine in 30-d-old flies $(n=3$ independent experiments, $F=32.96$ ( $f$ ) and 64.77 (g), one-way ANOVA with Bonferroni correction). The feeding regime of the flies is presented beneath the graphs, with + and - indicating food with or without spermidine. ${ }^{*} P<0.05,{ }^{* *} P<0.01,{ }^{* *} P<0.001$; ns indicates not significant, $P>0.05$. Data are presented as mean \pm s.e.m. 
Table 1 Aversive olfactory avoidance and shock reactivity in aging wild-type flies with and without spermidine feeding

\begin{tabular}{|c|c|c|c|}
\hline & \multicolumn{2}{|c|}{ Olfactory avoidance } & \multirow[b]{2}{*}{ Shock reactivity } \\
\hline & OCT & $\mathrm{MCH}$ & \\
\hline$w^{1118} ; \mathrm{Spd}^{-} 3 \mathrm{~d}$ old & $65.5 \pm 4.2$ & $63.9 \pm 1.7$ & $70.5 \pm 2.0$ \\
\hline$w^{1118} ; \mathrm{Spd}^{1 \mathrm{mM}+} 3 \mathrm{~d}$ old & $71.1 \pm 3.2$ & $63.2 \pm 2.8$ & $64.1 \pm 2.9$ \\
\hline$w^{1118} ; \mathrm{Spd}^{5 \mathrm{mM}+} 3 \mathrm{~d}$ old & $62.1 \pm 2.9$ & $62.5 \pm 2.5$ & $63.1 \pm 2.4$ \\
\hline$w^{1118} ; \mathrm{Spd}^{-} 30 \mathrm{~d}$ old & $44.8 \pm 2.7$ & $45.2 \pm 3.3$ & $67.4 \pm 1.9$ \\
\hline$w^{1118} ; \mathrm{Spd}^{1 \mathrm{mM}+} 30 \mathrm{~d}$ old & $43.2 \pm 3.8$ & $43.0 \pm 3.5$ & $63.4 \pm 3.5$ \\
\hline$w^{1118} ; \mathrm{Spd}^{5 \mathrm{mM}+} 30 \mathrm{~d}$ old & $48.3 \pm 5.5$ & $41.8 \pm 5.1$ & $65.3 \pm 1.5$ \\
\hline
\end{tabular}

All values are mean \pm s.e.m.

This observation is consistent with the previously reported homeostatic control of polyamine levels ${ }^{14}$. On the other hand, levels of spermine declined considerably with age in both $\mathrm{Spd}^{+}$as well as $\mathrm{Spd}^{-}$flies (Supplementary Fig. 1).

\section{Spermidine feeding suppresses AMI}

We then asked whether this restoration of polyamine levels in aged flies could counter-act AMI. Young adult flies ( $3 \mathrm{~d}$ old) that were raised on food supplemented with spermidine (both Spd ${ }^{1 \mathrm{mM}+} \operatorname{or~Spd}^{5 \mathrm{mM}+}$ ) showed identical olfactory short-term memory (STM; memory tested immediately after odor conditioning) and intermediate-term memory performance (ITM; memory tested $3 \mathrm{~h}$ after odor conditioning) when compared to isogenic controls ( $\mathrm{Spd}^{-}$flies; Fig. 2a,b). Consistent with a previous report ${ }^{1}$, we also found substantial impairment in STM that first appeared in $10 \mathrm{~d}$-old flies and did not decrease any further during aging (Supplementary Fig. 2a). Likewise, ITM scores are also known to decline with age ${ }^{1,3}$. As anticipated, at $30 \mathrm{~d}$ of age, considerably reduced STM and ITM scores (Fig. 2c,d) were observed in control flies $\left(\mathrm{Spd}^{-}\right)$. In contrast, spermidine-fed $\left(\mathrm{Spd}^{+}\right)$flies showed higher STM and ITM scores than $\mathrm{Spd}^{-}$flies at $30 \mathrm{~d}$ of age (Fig. $\left.2 \mathbf{c}, \mathbf{d}\right)$. In fact, the performance of $30 \mathrm{~d} \mathrm{Spd}^{+}$flies (both Spd ${ }^{1 \mathrm{mM}+}$ and $\mathrm{Spd}^{5 \mathrm{mM}+}$ ) was comparable to that of young flies. In summary, simple spermidine feeding was sufficient to effectively protect both short- and intermediate-term olfactory memory from age-induced decline (Fig. 2c,d).

ITM can be dissected into anesthesia-sensitive memory (ASM) and anesthesia-resistant memory (ARM) components, which can be differentiated by distinct genetic mutants, as well as by specific pharmacological sensitivities ${ }^{1,2,4,15,16}$. ASM can be calculated by subtracting ARM scores, measured after amnestic cooling, from ITM. A previous study found that AMI has a strong influence on ASM, but not on $\mathrm{ARM}^{1}$. Consistently, we found that ARM was only slightly affected by aging, with spermidine feeding producing only a negligible effect (Fig. 2b,d). In contrast, ASM was nearly absent in control flies at

Figure $3 \mathrm{Ca}^{2+}$ imaging in the Kenyon cells in response to odors in aging wild-type flies. (a) Expression of GCaMP3.0 in the mushroom body of an individual fly, focused on the horizontal lobes. The red line indicates a region of interest used to determine changes in fluorescence emission. Scale bar represents $50 \mu \mathrm{m}$. (b) False color-coded image of $\mathrm{Ca}^{2+}$ activity in the horizontal mushroom body lobes shown in a. Warm colors indicate high levels, cold colors indicate low levels or no $\mathrm{Ca}^{2+}$ activity. The numbers indicate changes in fluorescence $\Delta F(\%)$. (c,d) Time course of $\mathrm{Ca}^{2+}$ increase in horizontal mushroom body lobes of 3-d-old and 30-d-old $\mathrm{Spd}^{-}$flies evoked by the odors 4-methyl-cyclohexanol $(\mathrm{MCH})$ or 3-octanol (OCT) in comparison with the diluent, mineral oil ( $n=5$ flies, nonparametric Mann-Whitney $U$ test found no substantial difference between the $\mathrm{Ca}^{2+}$ increase of 3-d-old and 30-d-old Spd- flies evoked by the odors). The gray bars indicate the duration of the odor stimuli. Data are presented as mean \pm s.e.m.
$30 \mathrm{~d}$ of age, but was preserved in age-matched $\mathrm{Spd}^{+}$flies (Fig. $\left.\mathbf{2 b}, \mathbf{d}\right)$. This specific effect of spermidine in protecting ASM without affecting ARM, together with the lack of any consequential effect of spermidine feeding on memory in young flies, argues against spermidine having a general, nonspecific role in memory consolidation.

The standard conditioning procedure that we used here included application of 12 electric shocks, a potentially saturating number for memory scores ${ }^{5}$, which might mask subtle spermidine-evoked changes in young flies. Thus, we also trained young flies under nonsaturating conditions, in which we only applied two electric shocks ${ }^{17}$. Again, we found no difference between the memory scores of young $(3 \mathrm{~d}$ old $)$ adult flies raised on either normal $\left(\mathrm{Spd}^{-}\right)$or spermidine supplemented food $\left(\mathrm{Spd}^{1 \mathrm{mM}+}\right.$ or $\mathrm{Spd}^{5 \mathrm{mM}+}$ ) (Supplementary Fig. 2b). This indicates that spermidine does not generally boost memory, but specifically protects aged flies from memory impairments.

\section{Spermidine-mediated effects are specific for memory}

Potentially, polyamine effects could be mediated through developmental changes, such as during critical periods in early adulthood. To address such putative developmental effects, we shifted flies between spermidine-containing and spermidine-free food. When tested on day 30 , in flies fed with spermidine only between days 0 and 20 (with spermidine supplementation being withdrawn for the last $10 \mathrm{~d}$ before testing; Fig. 2f,g), we found that the levels of polyamines (both spermidine and putrescine) declined to levels comparable to those of controls (no spermidine supplementation for days 1-30; Fig. 2f,g), as was the memory when tested at day 30 (Fig. 2e). In contrast, in flies fed with spermidine for the last $10 \mathrm{~d}$ before testing, levels of both polyamines (spermidine and putrescine) rose (Fig. 2f,g), and memory was partially, but substantially, restored (Fig. 2e). The fact that restoring polyamine levels during the $10 \mathrm{~d}$ before testing prevented AMI rules out the idea that the effects of spermidine-feeding on suppression of AMI are a result of affected developmental processes.

Given that spermidine feeding promotes longevity ${ }^{14}$, it might be argued that protection of memory is a byproduct of increased life expectancy and generally improved health. Thus, we asked whether spermidine feeding preserves the function of the fly nervous system in all respects or whether it rather has a more specific effect on memory. In our learning assay, we found that 30-d-old naive flies to exhibit decrease odor avoidance scores compared with 3-d-old flies, whereas the shock reactivity of these naive flies did not change (Table 1), consistent with previous AMI studies ${ }^{1,2}$. Notably, spermidine-feeding had no influence on this age-dependent decline in odor avoidance scores (Table 1).

Next, we wondered whether the lower memory scores in aged flies are a result of impaired processing of the olfactory information.
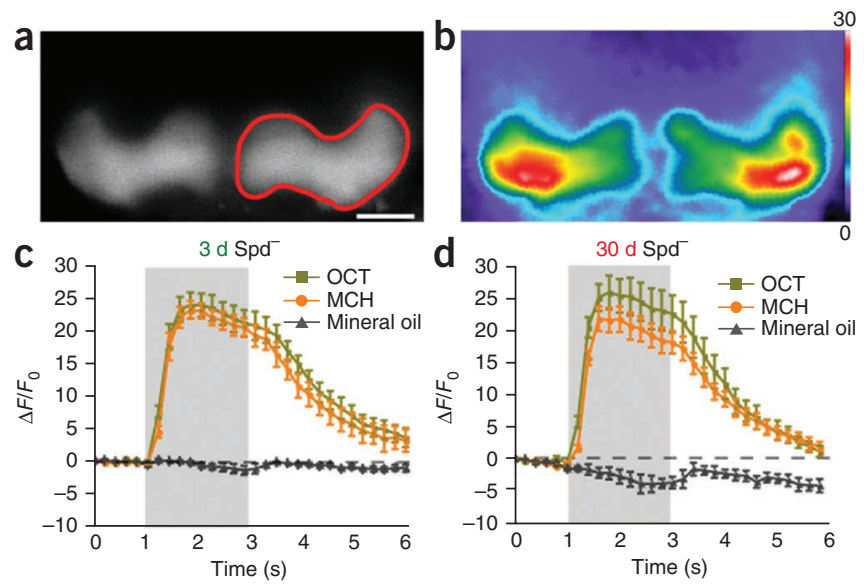
Figure 4 Spermidine feeding induces autophagy in the Drosophila brain. (a) Quantification of Atg8a proteins levels from head extracts normalized to $\alpha$-tubulin ( $n=17$ or $8-9$ technical replicates from two independent biological aging replicates for all data, $F=7.52$, one-way-ANOVA with Tukey post-test). The full-length blot is shown in Supplementary Figure 9a. (b) Western blot analysis of polyubiquitinated proteins (SDS-soluble protein fraction) from head extracts from $\mathrm{Spd}^{-}$( 3 and $30 \mathrm{~d}$ old) flies compared with $\mathrm{Spd}^{5 \mathrm{mM}+}$ (3 and $30 \mathrm{~d}$ old) flies. Right, quantification of polyubiquitinated proteins levels normalized to $\alpha$-tubulin ( $n=10$ or 5 technical replicates from two independent biological aging replicates for all data, $F=6.32$, one-way ANOVA with Tukey post-test). The full-length blot is shown in Supplementary Figure 9b. (c-f) Adult brains of 3-d-old and 30-d-old Spd- flies, as well as 3-d-old and 30-d-old Spd $5 \mathrm{mM}+$ flies

immunostained for ref(2)P. Scale bar represents $25 \mu \mathrm{m}$. (g) Quantification of ref(2)P intensity in the central brain region normalized to 3-d-old Spd ${ }^{-}$ ( $n=12-13$ independent brains for all data, $F=26.58$, one-way ANOVA with Bonferroni correction). ${ }^{*} P<0.05,{ }^{* *} P<0.01,{ }^{* * *} P<0.001 ; P>0.05$. Data are presented as mean \pm s.e.m.
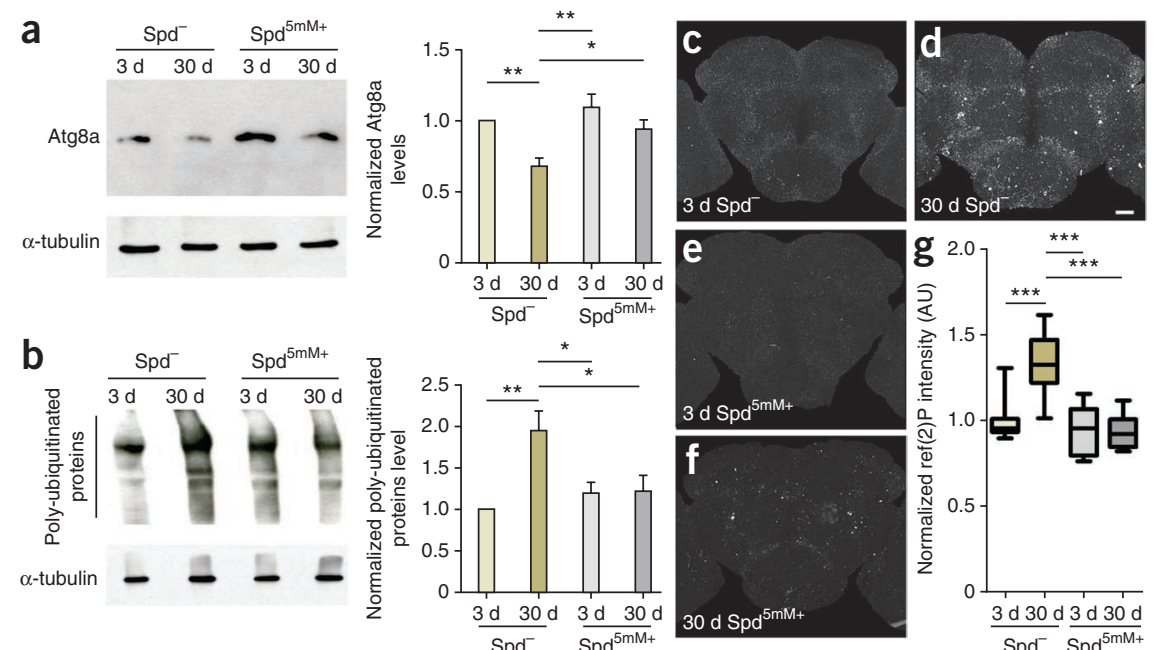

We measured changes in intracellular $\mathrm{Ca}^{2+}$ in the horizontal lobes of mushroom body in response to the two odors used in the learning assay using a fusion of the mushroom-specific enhancer mb247 to the $\mathrm{Ca}^{2+}$ indicator GCaMP3.0. We found no differences in the amplitude or time course of signals recorded in young ( $3 \mathrm{~d}$ old $)$ and aged $(30 \mathrm{~d}$ old $)$ flies (Fig. 3). Thus, it seems AMI is not caused by generic changes in odor information processing or in the intrinsic excitability of Kenyon cells. However, learning-associated $\mathrm{Ca}^{2+}$ transients in the dorsal paired medial neurons were recently found to decrease with aging ${ }^{3}$.

We also found an age-induced decrease in locomotor functions of flies, which was not rescued in 30-d-old flies by spermidine treatment (Supplementary Fig. 3). These results argue against a genuinely generic effect on nervous system function, but rather indicate that higher polyamine levels specifically influence associative olfactory learning abilities in aging flies.

Spermidine-mediated protection requires efficient autophagy What might be the mechanistic underpinnings of spermidinemediated protection from AMI? Spermidine administration induces autophagy in several model organisms ${ }^{14}$ and autophagy is crucially important for spermidine-mediated promotion of lifespan in yeast, C. elegans and Drosophila ${ }^{14}$. Notably, reduced basal autophagy in the nervous system of mice and flies has been shown to cause neurodegeneration ${ }^{9,18-20}$. In addition, the expression of several key genes in the autophagic pathway has been reported to decline with aging in the brain of humans and flies, potentially increasing neuronal vulnerability to the toxic effects of protein aggregates ${ }^{6,9}$.

Thus, we investigated whether autophagy might be critical for spermidine-mediated protection from AMI. To address this, we first assayed the levels of Atg8a (a widely used marker for autophagy) in western blots from fly head extracts ${ }^{21}$. Consistent with a previous report ${ }^{9}$, Atg8a protein levels were considerably reduced in heads from 30 -d-old $\mathrm{Spd}^{-}$flies compared with 3-d-old Spd ${ }^{-}$flies (Fig. 4a). Spermidine administration, however, blocked this age-related decline in Atg8a protein levels effectively (as seen by protein levels in the heads from Spd ${ }^{5 \mathrm{mM}+}$ flies; Fig. 4a). Suppression of autophagy has been associated with the accumulation of ubiquitinated protein aggregates ${ }^{9,10,22}$. In fact, we observed that the ageassociated increase in the amount of poly-ubiquitinated proteins was largely blocked in heads of spermidine-treated Drosophila (Spd ${ }^{5 \mathrm{mM}+}$; Fig. $\mathbf{4 b}$ ).
We next asked whether these ubiquitinated proteins were really being degraded by autophagy. The p62 family of proteins is closely associated with protein inclusions containing ubiquitin, as well as with key components of the autophagy pathway, thereby mediating autophagic clearance of ubiquitinated proteins ${ }^{19,23}$. In addition, ref(2)P, a Drosophila homolog of p62, was recently reported to accumulate with ubiquitinated neural protein aggregates in aged wild-type flies and autophagy mutants ${ }^{22}$. For a nervous systemspecific readout, we stained brains from flies of different ages with antibody to ref(2)P. As expected, ref(2)P levels increased with age, and spermidine administration suppressed this age-dependent increase (Fig. 4c-g). Thus, spermidine administration seems to prevent the accumulation of ubiquitinated proteins, most likely as a direct consequence of enhancing autophagy in aged Drosophila brains.

We then tested whether autophagy is functionally required for spermidine-mediated protection from AMI. Both Atg7 and Atg8 are essential for autophagy in Drosophila ${ }^{9,10,24-26}$. Given that $A \operatorname{tg} 7^{-1-}$ and Atg $8 a^{-1-}$ flies have a mean lifespan of only $30 \mathrm{~d}$ (ref. 10), we decided to test memory in both mutants at $20 \mathrm{~d}$ of age (Fig. 5). We found that $A \operatorname{tg} 7^{-1-}$ flies (Fig. 5a,c) showed reduced memory scores at a young age ( $3 \mathrm{~d}$ of age; Fig. 5a), which further declined relative to controls at later time points ( $20 \mathrm{~d}$ of age; Fig. $\mathbf{5 c}$ ). Notably, the memory-promoting effects of spermidine on STM were eliminated in $A \operatorname{tg} 7^{-1-}$ flies (Fig. 5a,c). We also tested the role of Atg8a using the hypomorphic allele Atg8a ${ }^{E P 362}$ of the X-chromosomal Atg8a. Female Atg8 $a^{-1-}$ flies also showed a reduced memory performance at both young ( $3 \mathrm{~d}$ old $)$ and old age $(20 \mathrm{~d}$ old), even with spermidine administration (Fig. 5b,d), indicating that spermidine failed to mediate AMI protection in $\mathrm{Atg} 8 \mathrm{a}^{-/-}$flies. Thus, the integrity of the autophagy system seems to be required for spermidine-mediated protection from AMI.

\section{Spermidine causes genome-wide transcriptional changes}

Taken together, our results indicate that spermidine administration results in nervous system-specific regulations that lead to the suppression of AMI, with the upregulation of autophagy being an essential component. However, spermidine has also been shown to cause major changes in the transcriptional status of yeast and cultivated human cells ${ }^{14,27}$. In addition, spermidine feeding in flies was recently 
Figure 5 Autophagy is required for spermidine's effects on AMI.

(a) Aversive associative memory performance 3 min after training (STM) was markedly reduced in 3-d-old Atg $^{-1}$ - flies (Atg ${ }^{d 14} / A^{A t g} 7^{d 77}$ ) compared with $A \operatorname{tg} 7^{-/+}$flies (Atg $7^{d 14} / C G 5335^{d 30}$ ) and wild-type flies (Atg $7^{+/+}$) ( $n=8-11$ independent experiments, $F=25.82$, one-way ANOVA with Bonferroni correction). The CG5335 ${ }^{d 30}$ line harbors an $A \operatorname{tg} 7^{+}$chromosome related to $A \operatorname{tg} 7^{d 14}$ and $A \operatorname{tg} 7^{d 77}$ that serves as a genetic background control. (b) Olfactory learning was disrupted in 3-d-old female flies homozygous for Atg8a hypomorph (Atg8a-/- or Atg8aEP362/Atg8aEP362) ( $n=8-9$ independent experiments, $F=22.8$, one-way ANOVA with Bonferroni correction). (c) STM is severely impaired in 20-d-old Atg $^{-1-}$ (Atg $7^{d 14} /$ Atg $^{d 77}$ ) flies and 20 -d-old Atg $^{-/+}$heterozygous (Atg7d14) CG5335 ${ }^{d 30}$ ) when compared to age-matched wild-type flies ( $n=8-9$ independent experiments, $F=18.02$, one-way ANOVA with Bonferroni correction). (d) Similarly, STM was markedly decreased in 20-d-old Atg8a $^{-1-}$ (Atg8aEP362/Atg8aEP362) mutant female flies compared with control flies $\left(\mathrm{Atg}_{\mathrm{tg}} \mathrm{a}^{+/+}\right.$) without any effect by spermidine feeding ( $n=8-9$ independent experiments, $F=68.43$, one-way ANOVA with Bonferroni correction). ${ }^{*} P<0.05,{ }^{*} P<0.01,{ }^{* * *} P<0.001$; ns indicates not significant, $P>0.05$. Data are presented as mean \pm s.e.m. The aversive olfactory avoidance and shock reactivity of different mutant are shown in Supplementary Table 5.

shown to protect from stress in both autophagy-dependent and autophagy-independent pathways ${ }^{28}$.

To explore the possibility that transcriptional modulation might be involved in spermidine-mediated suppression of AMI, we performed next generation mRNA sequencing (RNA-seq) in duplicate on head extracts prepared from 30 -d-old Spd ${ }^{5 \mathrm{mM}+}$ flies and compared them with extracts from 30 -d-old $\mathrm{Spd}^{-}$flies. RNA-seq, with its base pair-precise resolution allows quantitative global mapping of transcribed regions at superior levels of sensitivity and accuracy. Under our stringent conditions of analysis, only a few genes were found to be consistently either upregulated or downregulated in 30-d-old heads from spermidine-treated flies when compared with age-matched controls (data not shown).

a
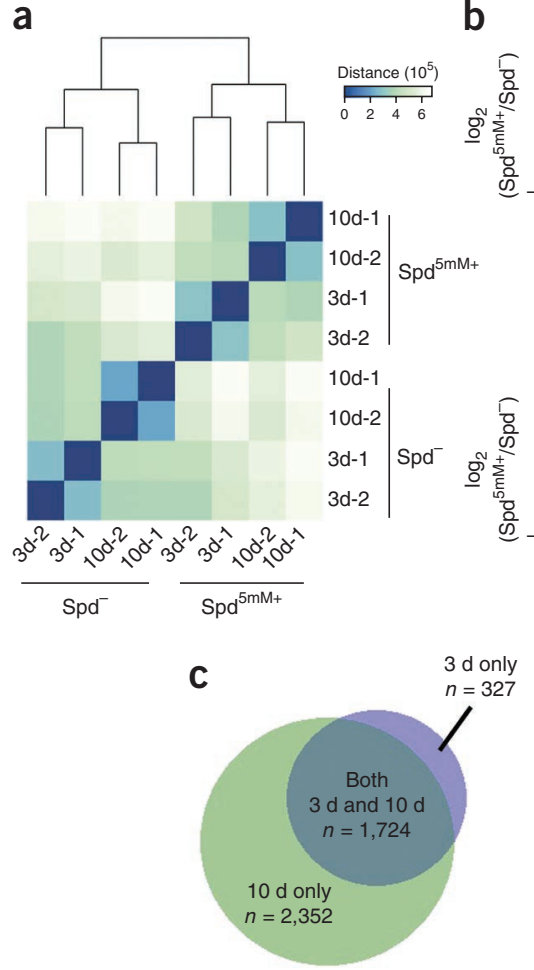
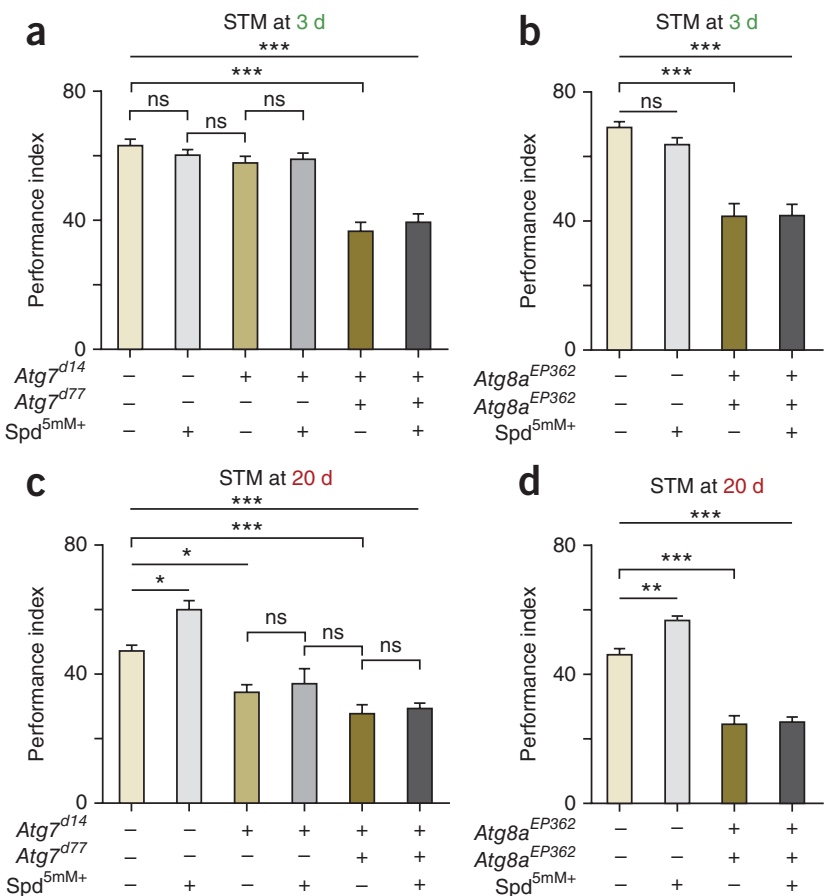

However, we reasoned that if transcriptional reprogramming is causally involved in the protective effects of polyamines, then it might precede, or at least be concomitant with, its effects on memory. In another experiment, we analyzed the kinetics of polyamine decline and memory. A substantial decline of both polyamines (spermidine and putrescine), together with memory, was observed by $10 \mathrm{~d}$ of age, which only slightly decreased further by $15 \mathrm{~d}$ (Supplementary Fig. 4).

We then carried out RNA-seq on head extracts prepared from 3-d-old and 10-d-old $\mathrm{Spd}^{5 \mathrm{mM}+}$ flies and compared them with age-matched $\mathrm{Spd}^{-}$flies. Sequenced reads were then aligned to the Drosophila genome with high stringency, allowing only one mismatch per read, and an average of $96 \%$ of aligned reads mapped to exons (Supplementary Table 1). Thereafter, the number of reads mapping to each gene was quantified and normalized for library size using DESeq $^{29}$. Hierarchical clustering of the normalized reads revealed a high degree of consistency between the biological replicates, clearly showing that spermidine-treated samples clustered away from untreated samples (Fig. 6a). Using an established statistical method based on the negative binomial distribution of sequenced reads ${ }^{29}$ (Supplementary Fig. 5), we then identified large global changes in gene expression induced by spermidine feeding, with 2,051 genes and 4,076 genes being modulated by a factor of 1.5 -fold $(P<0.05)$ in the heads of 3-d-old and 10-d-old flies. respectively (Fig. $6 \mathbf{b}$ and Supplementary Table 2). Notably, $84 \%$ of the genes that were differentially regulated after $3 \mathrm{~d}$ were also altered at $10 \mathrm{~d}$ of age (Fig. 6c).

Figure 6 Spermidine feeding induces widespread transcriptional changes in fly heads during aging. (a) Dendrogram and heat map illustrating Euclidean distances between gene expression profiles from 3-d-old and 10-d-old fly heads with or without spermidine feeding. (b) Differential expression analysis examining the effect of spermidine feeding on gene expression in heads of 3-d-old and 10-d-old flies using DESeq. Red and blue dots indicate a significant up- and down-regulation, respectively (fold change $>1.5$; adjusted $P<0.05$ ). The numbers of differentially regulated genes is indicated. Dark blue lines represent a 1.5 -fold change. (c) Venn diagram indicating the overlap in differentially expressed genes at 3 and $10 \mathrm{~d}$ of age under spermidine treatment. The raw sequence data for this figure are available in Supplementary Table 3. 
Figure 7 Brain-specific expression of Odc-1 is sufficient to suppress AMI. (a) Aversive associative memory performance in 3 min after training (STM) in 3-d-old female flies with pan-neuronal expression of UASOdc-1 ( $n=7$ independent experiments, $F=0.04$, one-way ANOVA with Bonferroni correction). (b) Aversive associative memory performance in $3 \mathrm{~h}$ after training (ITM), ARM and ASM of 3-d-old appl-driven UAS-Odc-1 female flies compared with control flies ( $n=7$ independent experiments; $F=1.95$ for ITM, $F=0.10$ for ARM, $F=1.05$ for ASM; one-way ANOVA with Bonferroni correction). (c) Pan-neuronal expression of the UASOdc-1 in a wild-type background suppressed AMI in 30-d-old female flies ( $n=7$ independent experiments, $F=21.35$, one-way-ANOVA with Bonferroni correction). (d) ITM, ARM and ASM of 30-d-old female flies with pan-neuronal expression of UAS-Odc-1 compared with their genetic controls ( $n=7-8$ independent experiments; $F=17.49$ for ITM, $F=1.58$ for ARM, $F=24.61$ for ASM; one-way ANOVA with Bonferroni correction). (e) Expressing Odc-1 in just the mushroom body was sufficient to protect from STM decline in 30-d-old flies ( $n=9-12$ independent experiments, $F=9.25$, one-way ANOVA with Bonferroni correction). (f) ITM, ARM and ASM of 30-d-old flies expressing mushroom body-specific Odc-1 compared with their genetic controls ( $n=8-9$ independent experiments; $F=11.73$ for ITM, $F=0.50$ for ARM, $F=16.6$ for ASM; one-way ANOVA with Bonferroni correction). ${ }^{* *} P<0.01,{ }^{* *} P<0.001$; ns indicates not significant, $P>0.05$. Data are presented as mean \pm s.e.m.

To provide insights into the function of the spermidineregulated brain transcriptome, we subjected all of the genes found to be either up- or downregulated by spermidine feeding to gene ontology enrichment analysis ${ }^{30}$ (Supplementary Table 3 ). The genes that were modulated by spermidine feeding in both 3 -d-old and 10 -d-old flies showed strong enrichment for GO terms, such as response to starvation, response to oxidative stress, phagocytosis and cellular homeostasis (Supplementary Table 3.3). This is consistent with the idea that general protective effects are mediated by spermidine administration. In addition, we also found over 2,000 transcriptional changes that were specific for 10-d-old flies (Supplementary Tables 2.1 and 2.2). On one hand, the GO term 'aging' was considerably enriched in $10-\mathrm{d}$-old $\mathrm{Spd}^{5 \mathrm{mM}+}$ flies, indicating that ageprotective gene functions are induced when polyamine levels are remain high during the onset of aging. On the other hand, transcriptional changes that are specific for neuronal genes appear to have a role in $\mathrm{Spd}^{5 \mathrm{mM}+}$ flies, with 'neuron differentiation' and 'neuron development' being the top two enriched GO terms (Supplementary Table 3.2). In addition, several genes ( 14 in total) with the GO annotation 'learning' were found to be specifically upregulated in 10-d-old $\mathrm{Spd}^{5 \mathrm{mM}+}$ flies when compared with untreated flies (Supplementary Tables 2.2 and 3.2). Notably, several of the learning-related genes (along with other genes) that were modulated by spermidine feeding were subsequently analyzed and validated by quantitative reversetranscription real-time PCR (Supplementary Fig. 6). Thus, learningassociated processes operating downstream of odor information processing could be a part of the polyamine-mediated protection of memory, indicating that brain-specific manipulation of polyamine synthesis might be sufficient for protecting against AMI.

\section{Mushroom body-specific Odc- 1 expression protects from AMI}

To determine whether nervous system-specific manipulations of polyamine levels are sufficient to protect against AMI, we genetically manipulated polyamine synthesis in specific brain regions. Ornithine decarboxylase-1 (Odc-1), which is highly conserved across evolution, is the rate-limiting enzyme for the de novo synthesis of polyamines (Fig. 1a); its activity is tightly regulated at all steps, starting from its initial synthesis continuing to its degradation ${ }^{31}$. We used the neuron-specific appl-Gal4 driver to express $O d c-1$ in the nervous system $(a p p l>O d c-1)$ and found that $a p p l>O d c-1$ flies
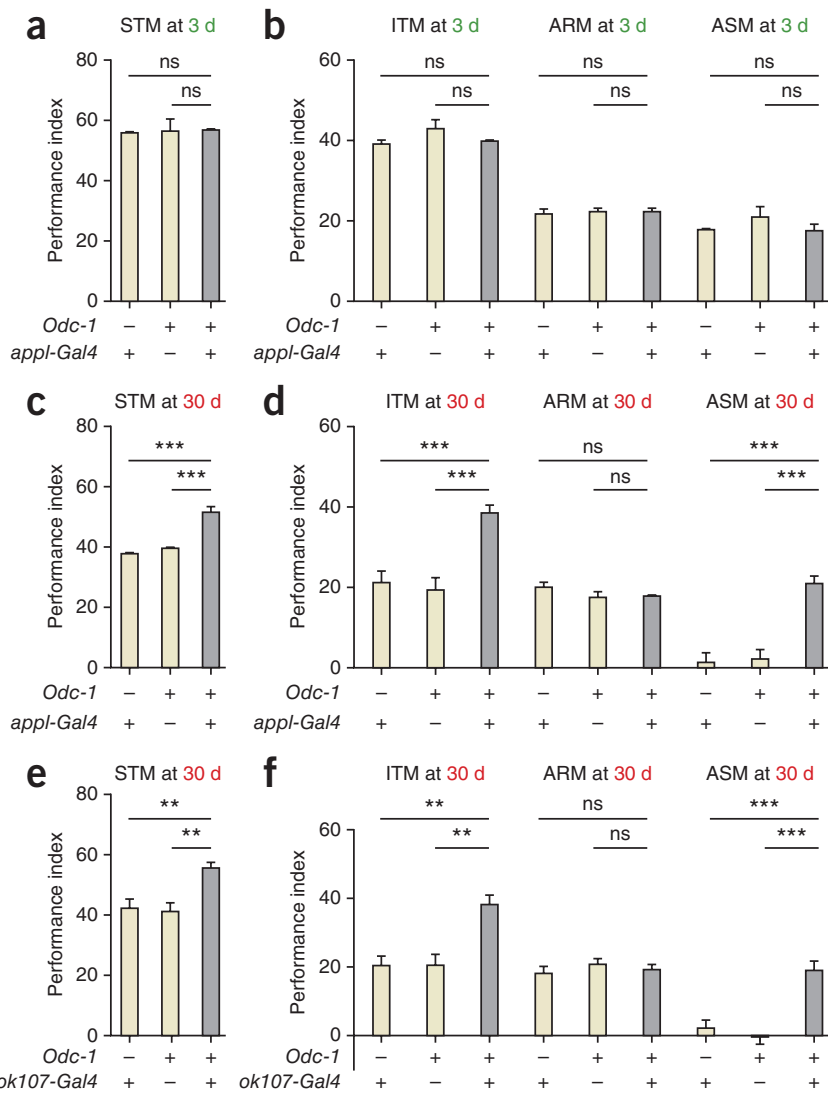

f

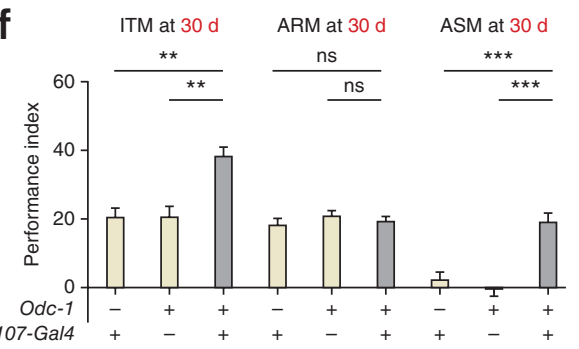

were effectively protected from AMI in both the STM and ITM assays (Fig. $7 \mathbf{a}-\mathbf{d}$ ). In fact, $a p p l>O d c-1$ flies showed almost identical test scores to aged flies in which AMI was suppressed by spermidine feeding (Fig. 2c,d). This finding suggests that promoting polyamine synthesis specifically in the nervous system is sufficient to suppress AMI.

Kenyon cells, which are neurons comprising the mushroom body of Drosophila brains, are known to be important for forming associative olfactory memories ${ }^{15,32}$. The re-expression of the memory gene rutabaga in Kenyon cells alone is sufficient to rescue the severe learning deficits of rutabaga mutant flies ${ }^{33}$. We expressed Odc-1 in mushroom body Kenyon cells (using ok107-Gal4) and found no effect on the memory scores (both STM and ITM) of young ok107>Odc-1 flies ( $3 \mathrm{~d}$ old) when compared with age-matched controls (Supplementary Fig. 7). Notably, aged $o k 107>O d c-1$ flies ( $30 \mathrm{~d}$ old) exhibited considerably higher STM and ITM scores when compared with genetic controls (Fig. 7e,f). Thus, promoting polyamine synthesis in a neuron population representing only about $2 \%$ of the Drosophila brain (Kenyon cells) was sufficient to protect from AMI. This finding confirms once more that polyamine restoration does not execute its effects on AMI via systemic regulations or generally improved health of the organism.

\section{DISCUSSION}

Aging is a multi-facet process that entails a decline of cognitive functions such as learning and memory. The proportion of older adults in our population is expected to grow rapidly over the next two decades. It is therefore increasingly important to advance research efforts for elucidating the mechanisms associated with cognitive aging to develop effective interventions and preventative therapies. We sought to understand the fundamental mechanisms of AMI. 
Polyamines (putrescine, spermidine and spermine; Fig. 1a) are among the substances that have been reported to decline with age. Putrescine shows an age-related decline in the CA1 region of hippocampus and the dentate gyrus region in rodents ${ }^{34}$, and the levels of spermidine and spermine have been shown to decrease with increasing age in rats ${ }^{11}$. Notably, levels of spermidine and spermine in basal ganglia also decrease with age in humans, suggesting that these polyamines are involved in white matter changes during aging 12 We found that the levels of all three polyamines (putrescine, spermidine and spermine) declined in the heads of aged flies relative to young flies (Fig. 1b,c). Although the decline of polyamines might be regarded as an established biochemical correlate of aging, the causal relationship to age-related deficits in cognitive functions has not been established. Simple dietary supplementation of spermidine allowed us to restore polyamine levels in the heads of aged flies (both spermidine and putrescine) to those seen in juveniles. This simple procedure was sufficient to effectively protect both short- and intermediate-term olfactory memory from age-induced decline.

The effects of spermidine to the protection of memories were specific in several regards. First, spermidine feeding had no effect on memory in young flies, either in terms of short or intermediateterm components, arguing against the possibilty that spermidine might function as a general memory enhancer (Fig. 2a,b). Second, ITM has two components, with aging strongly affecting ASM, but not $\mathrm{ARM}^{1}$. We found that spermidine feeding had only a negligible effect on ARM; instead, spermidine administration specifically prevented the age-related decline of ASM (Fig. 2b,d). Third, polyamine restoration appeared to specifically suppress AMIs, as olfactory avoidance scores of naive flies (Table 1) and locomotion activity (Supplementary Fig. 3) declined with age in both $\mathrm{Spd}^{+}$flies and age-matched $\mathrm{Spd}^{-}$ flies. Fourth, in flies fed with spermidine for the last $10 \mathrm{~d}$ before testing $\left(\mathrm{Spd}^{-}, 1-20+\mathrm{Spd}^{5 \mathrm{mM}+}, 21-30\right)$, polyamine levels (spermidine and putrescine) increased (Fig. 2f,g), and memory was considerably restored (Fig. 2e), indicating that AMI suppression by spermidine administration is not a result of altered development. Furthermore, expressing Odc-1 in just Kenyon cells was sufficient to ameliorate AMI (Fig. 7e,f). These findings indicate that spermidine-mediated suppression of AMI is not executed via its effects on systemic regulations or a generally improved health of the organism, but rather result from an intrinsic regulation of a small fraction of neurons.

The formation of memory requires dynamic changes in the neurons, including synapse formation and synaptic plasticity, steered by regulated protein synthesis and equally important protein degradation. In fact, the execution of effective quality control over proteins appears to be important for neurons to maintain proper neuronal physiology and functioning ${ }^{35}$. The process of autophagy is an important route for removing misfolded proteins and damaged organelles from cells via lysosomal-mediated bulk degradation ${ }^{20}$. Spermidine has been shown to operate as a natural inducer of autophagy in various model systems, including yeast, C. elegans, Drosophila and mice ${ }^{14,36}$. In fact, we found that spermidine feeding alleviated the age-induced dysfunction of autophagic machinery in flies, thereby preventing the accumulation of poly-ubiquitinated proteins and ref(2)P (Fig. 4).

What might be the mechanism by which spermidine administration prevents the decline of autophagy in aged Drosophila? Spermidine treatment induces autophagy in enucleated cells within a few hours as effectively as the well-known autophagy inducer rapamycin ${ }^{27}$, arguing for fast, post-transcriptional regulation. On the other hand, using RNA-seq, we found that positive regulators of autophagy (such as Atg1a) 37,38 were upregulated by spermidine feeding (Supplementary Fig. 6 and Supplementary Table 2). This is consistent with the transcriptional induction of autophagy genes that has been observed in spermidine-treated yeast ${ }^{14}$. Thus, it appears likely that polyamine restoration is associated with an integrated autophagy response on both short-term post-transcriptional and long-term transcriptional levels.

Several different studies in model organisms have suggested that autophagy is a crucial regulator of the age-associated pathologies 6,20 . Loss of autophagy has been shown to trigger neurodegeneration in mice $^{39}$, and autophagy-mediated clearance of TDP-43-positive inclusions has been reported to rescue learning impairment associated with a mouse model of neurodegenerative diseases involving TDP43 proteinopathies ${ }^{36}$. Similar to mice, reducing autophagy shortens lifespan and results in neurodegeneration in flies 9,10 . We found that the autophagic machinery was critical for amelioration of AMI, a per se nonpathological process, by spermidine feeding (Fig. 4).

The expression of Atg8a specifically in fly brains has been reported to prevent accumulation of ubiquitinated and oxidized proteins and to increase average adult lifespan ${ }^{9}$. Nevertheless, we found that overexpression of Atg8a with appl-Gal4 (atg8 $8^{E P 362}$ / appl-Gal4; Supplementary Fig. 8) had no effect on AMI, whereas spermidine feeding protected from AMI in this background. Thus, suppression of AMI seems to not be a byproduct of increased lifespan. Consistently, dietary restriction has been reported to increase the lifespan of Drosophila, but fails to protect cognitive functions with age ${ }^{40}$. In conclusion, spermidine feeding might trigger an integrated protection response, of which autophagy induction is one, but not the only, crucial component. In other words, several parallel pathways might be influenced by spermidine treatment that are, as a whole, responsible for suppression of AMI. Notably, both autophagy-dependent and autophagy-independent pathways were recently shown to be required for stress resistance by spermidine feeding in Drosophila ${ }^{28}$.

In addition, we found a broad transcriptional reprogramming in fly heads under spermidine treatment, with numerous GO terms related to neuron development and differentiation being highly enriched (Supplementary Table 3.2). Several genes that have been reported to function in memory were among those upregulated in response to spermidine treatment (Supplementary Tables $\mathbf{2}$ and $\mathbf{3}$ ). The adenylate cyclase rutabaga is essential for ASM formation ${ }^{41}$, and we found rut to be upregulated in $\mathrm{Spd}^{5 \mathrm{mM}+}$ flies (Supplementary Table 2). Furthermore, we found that a number of genes with conserved, central roles in memory and learning processes (stau, mnb, Nmdar2, $D o p R 2$ and $O a m b)^{42-46}$ were transcriptionally upregulated in $\mathrm{Spd}^{5 \mathrm{mM}+}$ flies (Supplementary Fig. 6 and Supplementary Table 2). In fact, it should be noted that the loss of spermine synthase is associated with changes in brain morphology and intellectual disability in humans (Synder-Robinson syndrome ${ }^{47}$ ). Similarly, among the most markedly downregulated genes following spermidine restoration was Glaz, the Drosophila homolog of Apolipoprotein D (ApoD; Supplementary Table 2). ApoD is one of the most upregulated proteins in the aged mammalian brain, and its increasing expression strongly correlates with aging-associated cognitive disorders ${ }^{48}$.

With regard to the specific transcriptional modulation resulting from enhanced spermidine levels, spermidine treatment in yeast modulates the activity of histone acetyl-transferases (HATs, such as Iki3p and Sas3p). This suggests that spermidine might actually affect epigenetic regulation, allowing (among other regulation) the induction of autophagy-relevant transcripts ${ }^{14}$. Notably, altered histone acetylation has been associated with age-dependent memory impairment and neurodegeneration in mice ${ }^{49,50}$.

Thus far, only a few substances have been found that protect from age-dependent memory decline, all of which are biologically 
exogenous compounds that can result in adverse side effects. Spermidine, however, is an endogenous metabolite and has the potential to become a high-value candidate for the treatment of age-induced dementia in humans.

\section{METHODS}

Methods and any associated references are available in the online version of the paper.

Accession codes. The raw sequence data obtained by RNA-seq are available at the NCBI Gene Expression Omnibus (http://www.ncbi. nlm.nih.gov/geo/) under series accession number GSE38998.

Note: Any Supplementary Information and Source Data files are available in the online version of the paper.

\section{ACKNOWLEDGMENTS}

We would like to thank T. Neufeld (University of Minnesota), L. Luo (Stanford University) and the Bloomington Stock Center for fly stocks, and S. Gaumer (University Versailles) for ref(2)P antibody. We are also grateful to M.G. Holt and B. Gerber for critically reading the manuscript. This work was supported by grants from the Deutsche Forschungsgemeinschaft to S.J.S. (Exc257, FOR1363), as well as A6/SFB 958 and DynAge Focus Area (Freie Universität Berlin) to S.J.S. the European Union (FP7 Gencodys HEALTH-241995) to H.G.S. and A.S., a VIDI grant from the Netherlands Organization for Scientific Research (917-96346 ) to A.S., the BMBF (BCCNII, grant number 01GQ1005A) to A.F., and the Emmy Noether Program to M.S. F.M. is grateful to the FWF for grants LIPOTOX, P23490, P24381 and I1000 (DACH). T.E. is recipient of an APART fellowship of the Austrian Academy of Sciences.

\section{AUTHOR CONTRIBUTIONS}

V.K.G., F.M. and S.J.S. designed the study. V.K.G., L.S., T.E., C.M., S.M., T.S.K., J.M.K., A.B., S.D., K.S.Y.L., S.S. and C.M. performed the experiments. V.K.G., L.S., T.E., T.S.K J.M.K., A.B., K.S.Y.L., S.S., S.D., C.M., F.M. and S.J.S. analyzed the data. F.S., M.S., T.R.P., A.F. and H.G.S. provided protocols, reagents and advice. All of the authors commented on the manuscript. V.K.G., A.S., F.M. and S.J.S. wrote the manuscript.

\section{COMPETING FINANCIAL INTERESTS}

The authors declare no competing financial interests.

Reprints and permissions information is available online at http://www. nature.com/ reprints/index.html.

1. Tamura, T. et al. Aging specifically impairs amnesiac-dependent memory in Drosophila. Neuron 40, 1003-1011 (2003).

2. Yamazaki, D. et al. The Drosophila DCO mutation suppresses age-related memory impairment without affecting lifespan. Nat. Neurosci. 10, 478-484 (2007).

3. Tonoki, A. \& Davis, R.L. Aging impairs intermediate-term behavioral memory by disrupting the dorsal paired medial neuron memory trace. Proc. Natl. Acad. Sci. USA 109, 6319-6324 (2012).

4. Saitoe, M., Horiuchi, J., Tamura, T. \& Ito, N. Drosophila as a novel animal mode for studying the genetics of age-related memory impairment. Rev. Neurosci. 16, 137-149 (2005)

5. Tully, T. \& Quinn, W.G. Classical conditioning and retention in normal and mutan Drosophila melanogaster. J. Comp. Physiol. A 157, 263-277 (1985).

6. Bishop, N.A., Lu, T. \& Yankner, B.A. Neural mechanisms of ageing and cognitive decline. Nature 464, 529-535 (2010).

7. Meléndez, A. et al. Autophagy genes are essential for dauer development and lifespan extension in C. elegans. Science 301, 1387-1391 (2003)

8. Hansen, M. et al. A role for autophagy in the extension of lifespan by dietary restriction in C. elegans. PLoS Genet. 4, e24 (2008)

9. Simonsen, A. et al. Promoting basal levels of autophagy in the nervous system enhances longevity and oxidant resistance in adult Drosophila. Autophagy 4 176-184 (2008).

10. Juhász, G., Erdi, B., Sass, M. \& Neufeld, T.P. Atg7-dependent autophagy promotes neuronal health, stress tolerance, and longevity but is dispensable for metamorphosis in Drosophila. Genes Dev. 21, 3061-3066 (2007).

11. Das, R. \& Kanungo, M.S. Activity and modulation of ornithine decarboxylase and concentrations of polyamines in various tissues of rats as a function of age. Exp. Gerontol. 17, 95-103 (1982).

12. Vivó, M. et al. Polyamines in the basal ganglia of human brain. Influence of aging and degenerative movement disorders. Neurosci. Lett. 304, 107-111 (2001).

13. Minois, N., Carmona-Gutierrez, D. \& Madeo, F. Polyamines in aging and disease. Aging (Albany, NY) 3, 716-732 (2011).
14. Eisenberg, T. et al. Induction of autophagy by spermidine promotes longevity. Nat. Cell Biol. 11, 1305-1314 (2009).

15. Heisenberg, M. Mushroom body memoir: from maps to models. Nat. Rev. Neurosci. 4, 266-275 (2003)

16. Quinn, W.G. \& Dudai, Y. Memory phases in Drosophila. Nature 262, 576-577 (1976).

17. Liu, X. \& Davis, R.L. The GABAergic anterior paired lateral neuron suppresses and is suppressed by olfactory learning. Nat. Neurosci. 12, 53-59 (2009).

18. Hara, T. et al. Suppression of basal autophagy in neural cells causes neurodegenerative disease in mice. Nature 441, 885-889 (2006).

19. Komatsu, M. et al. Homeostatic levels of p62 control cytoplasmic inclusion body formation in autophagy-deficient mice. Cell 131, 1149-1163 (2007).

20. Rubinsztein, D.C., Marino, G. \& Kroemer, G. Autophagy and aging. Cell 146 682-695 (2011).

21. Klionsky, D.J. et al. Guidelines for the use and interpretation of assays for monitoring autophagy in higher eukaryotes. Autophagy 4, 151-175 (2008).

22. Bartlett, B.J. et al. p62, Ref(2)P and ubiquitinated proteins are conserved markers of neuronal aging, aggregate formation and progressive autophagic defects. Autophagy 7, 572-583 (2011)

23. Pankiv, S. et al. p62/SQSTM 1 binds directly to Atg8/LC3 to facilitate degradation of ubiquitinated protein aggregates by autophagy. J. Biol. Chem. 282 24131-24145 (2007)

24. Mizushima, N. et al. A protein conjugation system essential for autophagy. Nature 395, 395-398 (1998).

25. Nakatogawa, H., Ichimura, Y. \& Ohsumi, Y. Atg8, a ubiquitin-like protein required for autophagosome formation, mediates membrane tethering and hemifusion. Cell 130, 165-178 (2007)

26. Xie, Z., Nair, U. \& Klionsky, D.J. Atg8 controls phagophore expansion during autophagosome formation. Mol. Biol. Cell 19, 3290-3298 (2008).

27. Morselli, E. et al. Spermidine and resveratrol induce autophagy by distinct pathways converging on the acetylproteome. J. Cell Biol. 192, 615-629 (2011).

28. Minois, N. et al. Spermidine promotes stress resistance in Drosophila melanogaster through autophagy-dependent and -independent pathways. Cell Death Dis. 3, e401 (2012)

29. Anders, S. \& Huber, W. Differential expression analysis for sequence count data. Genome Biol. 11, R106 (2010)

30. Huang, D.W., Sherman, B.T. \& Lempicki, R.A. Systematic and integrative analysis of large gene lists using DAVID bioinformatics resources. Nat. Protoc. 4, 44-57 (2009).

31. Pegg, A.E. Regulation of ornithine decarboxylase. J. Biol. Chem. 281 14529-14532 (2006)

32. Davis, R.L. Olfactory memory formation in Drosophila: from molecular to systems neuroscience. Annu. Rev. Neurosci. 28, 275-302 (2005).

33. McGuire, S.E., Le, P.T., Osborn, A.J., Matsumoto, K. \& Davis, R.L. Spatiotempora rescue of memory dysfunction in Drosophila. Science 302, 1765-1768 (2003).

34. Liu, P., Gupta, N., Jing, Y. \& Zhang, H. Age-related changes in polyamines in memory-associated brain structures in rats. Neuroscience 155, 789-796 (2008).

35. Lee, J.A. Neuronal autophagy: a housekeeper or a fighter in neuronal cell survival? Exp. Neurobiol. 21, 1-8 (2012).

36. Wang, I.F. et al. Autophagy activators rescue and alleviate pathogenesis of a mouse model with proteinopathies of the TAR DNA-binding protein 43. Proc. Natl. Acad. Sci. USA 109, 15024-15029 (2012)

37. Chang, Y.Y. \& Neufeld, T.P. An Atg1/Atg13 complex with multiple roles in TOR mediated autophagy regulation. Mol. Biol. Cell 20, 2004-2014 (2009).

38. Demontis, F. \& Perrimon, N. FOXO/4E-BP signaling in Drosophila muscles regulates organism-wide proteostasis during aging. Cell 143, 813-825 (2010).

39. Komatsu, M. et al. Loss of autophagy in the central nervous system causes neurodegeneration in mice. Nature 441, 880-884 (2006).

40. Burger, J.M., Buechel, S.D. \& Kawecki, T.J. Dietary restriction affects lifespan but not cognitive aging in Drosophila melanogaster. Aging Cell 9, 327-335 (2010).

41. Isabel, G., Pascual, A. \& Preat, T. Exclusive consolidated memory phases in Drosophila. Science 304, 1024-1027 (2004).

42. Dubnau, J. et al. The staufen/pumilio pathway is involved in Drosophila long-term memory. Curr. Biol. 13, 286-296 (2003).

43. Heisenberg, M., Borst, A., Wagner, S. \& Byers, D. Drosophila mushroom body mutants are deficient in olfactory learning. J. Neurogenet. 2, 1-30 (1985).

44. Keleman, K. et al. Dopamine neurons modulate pheromone responses in Drosophila courtship learning. Nature 489, 145-149 (2012).

45. Xia, S. et al. NMDA receptors mediate olfactory learning and memory in Drosophila. Curr. Biol. 15, 603-615 (2005).

46. Zhou, C. et al. Molecular genetic analysis of sexual rejection: roles of octopamine and its receptor OAMB in Drosophila courtship conditioning. J. Neurosci. 32 14281-14287 (2012).

47. Schwartz, C.E., Wang, X., Stevenson, R.E. \& Pegg, A.E. Spermine synthase deficiency resulting in $\mathrm{X}$-linked intellectual disability (Snyder-Robinson syndrome). Methods Mol. Biol. 720, 437-445 (2011).

48. Elliott, D.A., Weickert, C.S. \& Garner, B. Apolipoproteins in the brain: implications for neurological and psychiatric disorders. Clin. Lipidol. 51, 555-573 (2010).

49. Peleg, S. et al. Altered histone acetylation is associated with age-dependent memory impairment in mice. Science 328, 753-756 (2010).

50. Fischer, A., Sananbenesi, F., Wang, X., Dobbin, M. \& Tsai, L.H. Recovery of learning and memory is associated with chromatin remodeling. Nature 447, 178-182 (2007) 


\section{ONLINE METHODS}

Fly stocks and rearing conditions. All fly strains were reared under standard laboratory conditions $\left(25^{\circ} \mathrm{C}\right.$ with 12 -h: 12 -h light:dark cycle). Flies from an isogenized $w^{1118}$ strain were used as the wild-type control. $\operatorname{Atg} 7^{d 14}, \operatorname{Atg} 7^{d 77}$ and $C G 5335^{d 30}$ flies were kindly provided by T. Neufeld ${ }^{10}$ (University of Minnesota). These chromosomes were obtained from imprecise P-element using Atg7 ${ }^{E Y 10058}$ and $A \operatorname{tg} 7^{d 06996}$ in the $A \operatorname{tg} 7$ locus; $A \operatorname{tg} 7^{d 14}$ and $A \operatorname{tg} 7^{d 77}$ delete different exonic region of Atg7, thus trans-allellic combination of both creates an Atg7 null mutant situation. CG5335 ${ }^{\mathrm{d} 30}$ only deletes an intronic region in the Atg7 locus (not disturbing Atg7 function) without affecting any exon or Atg7 expression level, and serves as a background matched control here.

Atg8 $a^{E P 362}$ allele containing a P-element insertion in the Atg8a locus in the $w^{1118}$ background was obtained from the Bloomington Drosophila Stock Center, and appl-Gal4, appld(AGla) was a kind gift from L. Luo (Stanford University).

UAS-Odc-1 was constructed based on an Odc-1 cDNA clone (GH13851) obtained from the Berkeley Drosophila Genome Project. One fragment was obtained via PCR using 5'-CACCATGGCGGCCGCTACCCCTGAAAT-3' and $5^{\prime}$ - CTATATAGCTTGGAAGTACAGGGTCTTGGGG-3' primers and then ligated into pENTR-dTOPO (Invitrogen) according to the manufacturer's instructions. Finally, pENTR-dTOPO-Odc-1 was recombined with pUASTattB-rfa (kindly provided by C. Klämbt, University of Münster) using the Gateway System (Invitrogen) to yield pUAST-attB-Odc-1. After confirmation by doublestrand sequencing, transgenic flies were generated using the PhiC31 system with defined landing sites in the Drosophila genome was used ${ }^{51,52}$. For behavior experiments, transgenic UAS-Odc-1 flies were out-crossed to our wild-type $w^{1118}$ control for ten generations.

Spermidine (Sigma Aldrich) was prepared as a $2 \mathrm{M}$ stock solution in sterile distilled water, aliquoted in single-use portions and stored at $-20^{\circ} \mathrm{C}$. Fly food was prepared according to Bloomington media recipe (http://flystocks.bio.indiana.edu/ Fly_Work/media-recipes/media-recipes.htm) with minor modification, which we refer to as normal food. After food had cooled down to $40^{\circ} \mathrm{C}$, spermidine was added to normal food to a final concentration of $1 \mathrm{mM}$ or $5 \mathrm{mM}$, and called Spd ${ }^{1 \mathrm{mM}+}$ or $\mathrm{Spd}^{5 \mathrm{mM}+}$, respectively. For all experiments, parental flies mated on either normal or $\mathrm{Spd}^{+}\left(\mathrm{Spd}^{1 \mathrm{mM}+}\right.$ or Spd $\left.{ }^{5 \mathrm{mM}+}\right)$ food, and their progeny was allowed to develop on the respective food. Flies used in all experiments were F1 progeny. For aging, the flies were collected once a day, as a results specific age indicated will be age in days $\pm 24 \mathrm{~h}$. For time-shift experiments (Fig. 2e,f), the F1 progeny was allowed to age on one kind of food (normal or Spd $\mathrm{dmM}^{5 \mathrm{~m}}$ ) and then subsequently put on the other-kind of food (Spd ${ }^{5 \mathrm{mM}+}$ or normal) for specified time of time (for instance, days 1-20 on normal food and days 21-30 on $\mathrm{Spd}^{5 \mathrm{mM}+}$ food or vice-versa).

Extraction of polyamines for liquid chromatography-mass spectroscopy (LC-MS) measurements. $\mathrm{Spd}^{-}$and $\mathrm{Spd}^{+}$(both $\mathrm{Spd}^{1 \mathrm{mM}+}$ or $\mathrm{Spd}^{5 \mathrm{mM}+}$ ) flies were quick frozen in liquid nitrogen and stored at $-80^{\circ} \mathrm{C}$ until further processing. Subsequently, heads were collected after vortexing by mechanical separation. Extraction of polyamines from fly heads was performed according to the freeze-thaw method described previously ${ }^{14,53}$ with slight modifications. Briefly, about 20-30 mg of fly heads were semi-homogenized using Ultra-Turrax (IKA Laboratory Equipment) and polyamines extracted with $600 \mu 15 \%$ trichloroacetic acid (vol/vol) by three repeated freeze-thaw cycles. After extraction, ammonium formate $(0.4 \mathrm{M}$ final concentration) was added to supernatants and stored at $-80^{\circ} \mathrm{C}$ until polyamine measurements were performed using LC-MS.

Polyamine measurements using LC-MS. Polyamines were determined according to the method described previously ${ }^{54}$. Fly head extracts were diluted 1:20 in water and isotopically labeled internal standards for spermidine ${ }^{(13} \mathrm{C}_{4}$-spermidine) and putrescine $\left({ }^{2} \mathrm{H}_{8}\right.$-putrescine) were added. ${ }^{13} \mathrm{C}_{4}$-spermidine was also used for internal standardization of spermine. Calibration standards were prepared by spiking extraction buffer with specific concentrations of spermidine, putrescine, spermine and internal standards. Polyamines were derivatized to carbamyl-derivatives ${ }^{54}$ by adding $125 \mu \mathrm{l}$ of $1 \mathrm{M}$ carbonate buffer (pH 9), $800 \mu$ l of water and $20 \mu$ of isobutyl chloroformate to $100 \mu$ lof sample or calibration standard containing internal standards. All analysis were carried out on an Ultimate 3000 System (Dionex, LCPackings) coupled to a Quantum TSQ Ultra AM (Thermo Scientific) using an electrospray ion source. The system was controlled by Xcalibur Software 2.0. The stationary phase was a Kinetex 2.6- $\mu \mathrm{m} \mathrm{C18} \mathrm{100-A} \mathrm{50-mm} \times 2.1-\mathrm{mm}$ column (Phenomenex). $250 \mu \mathrm{l}$ of the derivatized samples were loaded on to an online-SPE column (Strata X, Phenomenex) using eluent A (flow rate $=1.5 \mathrm{ml} \mathrm{min}^{-1}$ ). After $2 \mathrm{~min}$, onlineSPE was switched to the analytical column and the polyamines were eluted and separated on the analytical column within 4 min using isocratic conditions $(80 \%$ eluent $\mathrm{B}$, flow rate $250 \mu \mathrm{l} \mathrm{min}^{-1}$ ). Polyamines were detected in multiple reaction monitoring mode using following transitions: Spermidine $(\mathrm{m} / z 446$-> 298V), putrescine $\left(\mathrm{m} / \mathrm{z} 289\right.$-> 115), spermine $(\mathrm{m} / \mathrm{z} 603 \rightarrow \mathrm{155}),{ }^{13} \mathrm{C}_{4}$-spermidine $(\mathrm{m} / \mathrm{z}$ 450 -> 302), ${ }^{2} \mathrm{H}_{8}$-putrescine $(\mathrm{m} / z 297$-> 123)

Behavioral assays. Standard single-cycle olfactory associative memory was performed as previously described ${ }^{1,5}$ with minor modifications. Briefly, about 60-80 flies received one training session, during which they were exposed sequentially to one odor (conditioned stimulus, $\mathrm{CS}^{+}, 3$-octanol or 4-methylcyclohexanol) paired with electric shock (unconditioned stimulus) and then to a second odor ( $\mathrm{CS}^{-}$, 4-methyl-cyclohexanol or 3-octanol) without the unconditioned stimulus for $60 \mathrm{~s}$ with a 30 -s rest interval between each odor presentation. For STM (memory tested immediately after odor conditioning), the conditioned odor avoidance was tested immediately after training. During testing, flies were exposed simultaneously to the $\mathrm{CS}^{+}$and $\mathrm{CS}^{-}$in a T maze for $30 \mathrm{~s}$. The flies were then trapped in either T maze arm, anesthetized and counted. From this distribution, a performance index was calculated as the number of flies avoiding the shocked odor minus the number avoiding the non-shocked odor divided by the total number of flies, multiplied by 100. A 50:50 distribution (no learning) yielded a performance index of zero and a 0:100 distribution away from the $\mathrm{CS}^{+}$yielded a performance index of 100. A final performance index was calculated by the average of both reciprocal indices for the two odors.

For ITM, flies were trained as described above, but tested $3 \mathrm{~h}$ after training. As a component of ITM, ARM was separated from ASM by cold-amnestic treatment, during which the trained flies were anesthetized $90 \mathrm{~s}$ on ice at $30 \mathrm{~min}$ before testing. In the end, ASM was calculated by subtracting the performance index of ARM from that of ITM for each training session on the same day, respectively.

Experiments were double blinded with respect to genotype and treatment and differently aged groups of various genotypes were measured side by side in randomized order.

Immunohistochemistry, confocal imaging and quantification. Adult brains were dissected in HL3 on ice and immediately fixed in cold $4 \%$ paraformaldehyde (vol/vol) for $20 \mathrm{~min}$ at $20-30^{\circ} \mathrm{C}$. After fixation, the samples were then incubated in $1 \%$ PBT (phosphate-buffered saline (PBS) containing $1 \%$ Triton $\mathrm{X}-100, \mathrm{vol} / \mathrm{vol}$ ) for $20 \mathrm{~min}$ and pre-incubated in $0.3 \% \mathrm{PBT}$ (PBS containing $0.3 \%$ Triton X-100) with $10 \%$ normal goat serum (vol/vol) for $2 \mathrm{~h}$ at $20-30{ }^{\circ} \mathrm{C}$. For primary antibody treatment, samples were incubated in $0.3 \% \mathrm{PBT}$ containing $5 \%$ normal goat serum and the primary antibodies for $48 \mathrm{~h}$ at $20-30^{\circ} \mathrm{C}$. After primary antibody incubation, brains were washed in $0.3 \% \mathrm{PBT}$, four times for 30 min at $20-30{ }^{\circ} \mathrm{C}$, and then overnight at $4{ }^{\circ} \mathrm{C}$. All samples were then incubated in $0.3 \%$ PBT with $5 \%$ normal goat serum containing the secondary antibodies for $24 \mathrm{~h}$ at $20-30^{\circ} \mathrm{C}$. Brains were washed four times for $30 \mathrm{~min}$ at $20-30^{\circ} \mathrm{C}$, then overnight at $4{ }^{\circ} \mathrm{C}$. Brains were finally mounted in Vectashield overnight before confocal scanning (Vector Laboratories). Antibodies were used at the following dilutions: rabbit antibody to $\operatorname{ref}(2) \mathrm{P}(1: 100)^{55}$ and Cy3-conjugated antibody to rabbit (1:400, Jackson ImmunoResearch, 111-165-006).

Image stacks of specimens were imaged on a Leica TCS SP 5 confocal microscope (Leica Microsystems) using a 20×, 0.7 NA oil objective for whole-brain imaging with voxel size of $361 \times 361 \times 200 \mathrm{~nm}$. Images were quantified using ImageJ software (http://rsbweb.nih.gov/ij/). Briefly, confocal stacks were merged into a single plane by using the maximum projection function. Subsequently, region of central brain was manually selected (using the free-hand function) and fluorescence intensity arbitrary units were measured and normalized to the area of the central brain for each brain.

$\mathrm{Ca}^{2+}$ imaging using GCaMP3.0. For $\mathrm{Ca}^{2+}$ imaging, GCaMP3.0 (ref. 56) was homozygously expressed under direct control of the $m b 247$ enhancer. 3-d-old or 30-d-old female flies were briefly anesthetized on ice and immobilized in a small chamber under thin sticky tape. A small window was cut through the sticky tape and the cuticle of the head capsule using a splint of a razor blade. Trachea were carefully removed and the brain was covered with Ringer's solution ${ }^{57}$ (which contains $5 \mathrm{mM}$ HEPES, $130 \mathrm{mM} \mathrm{NaCl}, 5 \mathrm{mM} \mathrm{KCl}, 2 \mathrm{mM} \mathrm{MgCl}_{2}, 2 \mathrm{mM} \mathrm{CaCl}_{2}$, 
$\mathrm{pH}=7.3$ ). Optical imaging was performed using a fluorescence microscope (Zeiss Axioscope 2 FS) equipped with a xenon lamp (Lambda DG-4, Shutter Instrument), a 14 bit CCD camera (Coolsnap HQ, Photometrics), a 20× waterimmersion objective and a GFP filter set. Image acquisition was controlled using the software Metafluor (Visitron Systems, Puchheim). Images were acquired at a frame rate of $5 \mathrm{~Hz}$, an illumination time of $80 \mathrm{~ms}$ and an excitation wavelength of $488 \mathrm{~nm}$. Odors (4-methyl-cyclohexanol or 3-octanol, diluted 1:100 or 1:150, respectively, in mineral oil, or pure mineral oil) were applied to the flies' antennae for $2 \mathrm{~s}$ each using a custom-built olfactometer at an air flow rate of $11 \mathrm{~min}^{-1}$. Four to five odor stimulations were applied to each individual fly. Acquired images were aligned using the ImageJ plugin TurboReg and a customized Java script. Fluorescence emission was determined within a region of interest covering the horizontal mushroom body lobes, and background fluorescence determined in a region of interest outside the labeled structure was subtracted. Changes in fluorescence emission were calculated as $\Delta F / F_{0}$ where $F$ is the fluorescence measured at each time point and $F_{0}$ the baseline fluorescence as the average of five frames before odor onset. The $\Delta F / F_{0}$ values of the four to five stimulations were averaged for each fly. For creating false color-coded images, five frames covering the peak of the increase in fluorescence were averaged and the average of five, preceding odor onset was subtracted.

Transcriptional profiling and Gene Ontology (GO) analysis. $\mathrm{Spd}^{-}$and $\mathrm{Spd}^{5 \mathrm{mM}+}$ flies of different ages ( 3 and $10 \mathrm{~d}$ ) were flash frozen in liquid nitrogen and heads were collected after vortexing by mechanical separation. Total RNA was extracted from approximately 100 heads per sample using the RNeasy Lipid tissue Mini kit (Qiagen). Biological duplicates were performed for all conditions. mRNA was purified using the Oligotex kit (Quiagen) and was reverse transcribed into a cDNA using the Superscript III Reverse Transcriptase (Invitrogen). Second strand synthesis was performed using the E. coli and T4 DNA polymerases (New England Biolabs). DNA end repair was performed on 9-20 ng of double stranded cDNA followed by ligation of Illumina sequencing adaptors and size selection for $300 \mathrm{bp}$. Fragments were amplified linearly (14 PCR cycles), as validated by quantitative real-time PCR (qPCR), and sample quality was assessed using the 2100 Agilent Bioanalyzer. Cluster generation and sequencing-by-synthesis (36 bp) was performed using the Illumina Genome Analyzer IIx according to standard protocols of the manufacturer, with the exception of one sample ( $\mathrm{Spd}^{-}$ 3 day, rep 2), which was sequenced on an Illumina HiSeq 2000. The image files generated by the Genome Analyzer IIx/Illumina HiSeq 2000 were processed to extract DNA sequence data. The raw sequence data from this study are available a the NCBI Gene Expression Omnibus (http://www.ncbi.nlm.nih.gov/geo/) under series accession number GSE38998. From the GAIIx, we obtained between 28 and 37 million reads, while the HiSeq produced 66 million reads (Supplementary Table 2). Sequenced reads were aligned to the Drosophila genome (NCBI build 5) using the Illumina Analysis Pipeline and the ELAND alignment software allowing one mismatch. Only tags that uniquely aligned to the genome were considered for further analysis and total alignment efficiency was between $70 \%$ and $82 \%$ (Supplementary Table 1), with the majority of unaligned reads mapping to repetitive sequences. The number of aligned reads mapping to gene coding sequences was counted using the python script htseq-count (http://www-huber.embl.de/ users/anders/HTSeq/doc/overview.html) with gene annotations extracted from the file Drosophila_melanogaster.BDGP5.70.gtf, available at http://www.ensembl. org. In all samples, over $95 \%$ of aligned reads mapped to exons and over $92 \%$ mapped unambiguously to one exon (Supplementary Table 1). The unambiguously mapped reads (ranging from 19-22 million reads for samples sequenced with the Illimina GAIIx and 45 million reads for the sample sequenced with the Illumina HiSeq) were used for further analysis of differential gene expression with the DESeq software ${ }^{29}$. The number of reads per gene was normalized using DESeq and hierarchical clustering on Euclidean distances was performed using the normalized values, revealing a high degree of similarity between biological replicates (Fig. 5a). DESeq uses variability between biological replicates in al conditions to estimate a dispersion value for each gene, which is essential for determining the statistical significance of differential expression. Notably, we observed a high degree of correlation between gene expression level (normalized number of reads) and dispersion, with genes having a low number of reads exhibiting higher dispersion (Fig. 5b). We then used DESeq to identify genes that were differentially expressed in response to spermidine based on the negative binomial distribution (adjusted $P<0.05$, fold change $>1.5$; Fig. 5b). Overlap of differentially expressed genes (Fig. 5b) was determined and visualized as a Venn diagram using BioVenn ${ }^{58}$, and GO analysis was performed using DAVID ${ }^{30}$. The full data set of differentially expressed transcripts and GO-terms are provided in Supplementary Tables 2 and 3 , respectively.

qPCR. For validation of differential expression, qPCR was performed using the GO-TaqQPCR master mix (Promega) and the 7900HT Fast Real Time PCR system (Applied Biosystems) according to the manufacturer's instructions. qPCR was performed using dilutions of sequenced cDNA libraries from heads of 10-d-old spermidine-treated and untreated flies. Gapdh1 and 14-3-3 epsilon were used as reference genes for normalization and calculation of fold change differences between spermidine-treated and untreated samples. Subsequently, the $\mathrm{Ct}$ values of spermidine-treated samples were subtracted from that of control samples, resulting in $-\Delta C^{\prime} \mathrm{T}$ and the relative concentrations were calculated as $2^{-} \Delta^{\mathrm{C}^{\prime} \mathrm{T}}$. All primers were tested for amplification efficiency according to standard methods (Supplementary Table 4).

Protein extraction, SDS-PAGE and western analysis. To detect polyubiquitinated proteins (PUPs), female fly heads (at least 60 of different age groups) were homogenized in $1 \%$ Triton X-100 (PBS containing protease inhibitors) on ice, the homogenates were centrifuged $(12,000 \mathrm{~g})$ for $10 \mathrm{~min}$ $\left(4{ }^{\circ} \mathrm{C}\right)$ and the supernatant (Triton X-100-soluble fraction) was collected. The remaining protein pellets were extracted with $2 \%$ SDS buffer (SDS fraction). In the case of Atg8a, the fly heads of females were homogenized in a $2 \%$ SDS buffer containing protease inhibitors. Protein concentrations were determined using BCA Assay Kit (Thermo Fischer) and $10 \mu \mathrm{g}$ of total protein per sample was loaded and resolved on 4-20\% gradient gels (BioRad) or 12\% gels for PUP and Atg8a, respectively, followed by electroblotting to nitrocellulose membranes (Millipore). Subsequently, blots were probed with monoclonal mouse antibody to tubulin (1:1,000, Sigma Aldrich), which served as loading control, together with polyclonal rabbit antibody to ubiquitin (1:1,000, Cell Signaling Technology) and polyclonal rabbit antibody to GABARAP (detects ATG8a, used 1:1,000, MBL) as previously described ${ }^{9}$. Immunoblots were scanned and intensity analysis was done using ImageJ software (using the gels and measurement function). The relative amounts of the PUP and Atg8a proteins from individual samples were quantified and corrected using antibody to tubulin as loading control. Statistical analysis was done in Microsoft Excel and GraphPad Prism software using one-way ANOVA and a Tukey post-test.

Climbing assay. Locomotor function of fruit flies was assessed using the climbing assay as previously reported ${ }^{59}$ with some slight modifications. Briefly, flies were previously sex-separated under mild $\mathrm{CO}_{2}$ anesthesia at days 1 and 28 . After recovery for $48 \mathrm{~h}$ (that is 3-d-old and 30-d-old flies), 10 flies were placed in a plastic vial, given $30 \mathrm{~s}$ to climb up. Each trial was video captured, and at the end of each trial the number of flies that climbed up to a vertical distance of $7 \mathrm{~cm}$ or above was recorded. Each trial was performed six times.

Statistics. Data were analyzed with Prism (GraphPad Software). No statistical methods were used to pre-determine sample sizes, but our sample sizes are similar to those reported in previous publications $1,3,9,14,60$. For the behavioral studies and polyamine estimation, the data were collected with the investigator blind to the genotypes, treatment and age of genotypes. There was no blinding in the other experiments. The data were collected and processed side by side in randomized order for all experiments. Data distribution was assumed to be normal but this was not formally tested. For comparison of more than two groups one-way ANOVA was used with either Bonferroni correction (except for Fig. 4a,b for which one-way ANOVA with Tukey correction was used. To compare two groups, non-parametric Mann-Whitney $U$ tests were used (in case of Fig. 3).

51. Bateman, J.R., Lee, A.M. \& Wu, C.T. Site-specific transformation of Drosophila via phic31 integrase-mediated cassette exchange. Genetics 173, 769-777 (2006)

52. Groth, A.C., Fish, M., Nusse, R. \& Calos, M.P. Construction of transgenic Drosophila by using the site-specific integrase from phage phic31. Genetics 166, 1775-1782 (2004). 
53. Minocha, R., Shortle, W.C., Long, S.L. \& Minocha, S.C. A rapid and reliable procedure for extraction of cellular polyamines and inorganic ions from plant tissues. J. Plant Growth Regul. 13, 187-193 (1994).

54. Byun, J.A. et al. Analysis of polyamines as carbamoyl derivatives in urine and serum by liquid chromatography-tandem mass spectrometry. Biomed. Chromatogr. 22, 73-80 (2008).

55. Nezis, I.P. et al. Ref(2)P, the Drosophila melanogaster homologue of mammalian p62, is required for the formation of protein aggregates in adult brain. J. Cell Biol. 180, 1065-1071 (2008).

56. Tian, L. et al. Imaging neural activity in worms, flies and mice with improved GCaMP calcium indicators. Nat. Methods 6, 875-881 (2009).
57. Estes, P.S. et al. Traffic of dynamin within individual Drosophila synaptic boutons relative to compartment-specific markers. J. Neurosci. 16, 5443-5456 (1996).

58. Hulsen, T., de Vlieg, J. \& Alkema, W. BioVenn: a web application for the comparison and visualization of biological lists using area-proportional Venn diagrams. BMC Genomics 9, 488 (2008)

59. Feany, M.B. \& Bender, W.W. A Drosophila model of Parkinson's disease. Nature 404, 394-398 (2000)

60. Zarnack, K. et al. Direct competition between hnRNP C and U2AF65 protects the transcriptome from the exonization of Alu elements. Cell 152, 453-466 (2013). 
B. Contributions to other studies

\section{Optical $\mathrm{Ca}^{2+}$ imaging using DNA-encoded fluorescence sensors in transgenic fruit flies, Drosophila melanogaster.}

My contribution :

- Developed the technique for the fly in vivo preparation (Figure 1).

- Performed the experiments and analyzed the data for Figure 2.

- Contributed in writing the manuscript. 


\title{
Optical Calcium Imaging Using DNA-Encoded Fluorescence Sensors in Transgenic Fruit Flies, Drosophila melanogaster
}

\author{
Shubham Dipt, Thomas Riemensperger, and André Fiala
}

\begin{abstract}
The invention of protein-based fluorescent biosensors has paved the way to target specific cells with these probes and visualize intracellular processes not only in isolated cells or tissue cultures but also in transgenic animals. In particular, DNA-encoded fluorescence proteins sensitive to $\mathrm{Ca}^{2+}$ ions are often used to monitor changes in intracellular $\mathrm{Ca}^{2+}$ concentrations. This is of particular relevance in neuroscience since the dynamics of intracellular $\mathrm{Ca}^{2+}$ concentrations represents a faithful correlate for neuronal activity, and optical $\mathrm{Ca}^{2+}$ imaging is commonly used to monitor spatiotemporal activity across populations of neurons. In this respect Drosophila provides a favorable model organism due to the sophisticated genetic tools that facilitate the targeted expression of fluorescent $\mathrm{Ca}^{2+}$ sensor proteins. Here we describe how optical $\mathrm{Ca}^{2+}$ imaging of neuronal activity in the Drosophila brain can be carried out in vivo using two-photon microscopy. We exemplify this technique by describing how to monitor odor-evoked $\mathrm{Ca}^{2+}$ dynamics in the primary olfactory center of the Drosophila brain.
\end{abstract}

Key words Optical $\mathrm{Ca}^{2+}$ imaging, Neuronal activity, Drosophila melanogaster, Two-photon microscopy, G-CaMP, Olfactory coding

\section{Introduction}

The invention of DNA-encoded fluorescence sensor proteins designed to report correlates of physiological processes within cells has brought forward physiological research remarkably [1,2]. This progress is most evident if one goes beyond the investigation of single cultured cells or tissue preparations and aims at analyzing cellular processes in tissues of intact, living animals, e.g., in correlation with behavior. For a long time, analyzing intracellular, biochemical processes, e.g., second messenger-mediated signaling, has relied on biochemical assays that require mechanical tissue preparation while monitoring changes in membrane potential typically involves electrophysiological techniques. In both cases one can either investigate only very few cells with high temporal preci- 
sion and high specificity with respect to cell types, or investigate larger pieces of tissue (e.g., parts of a brain) consisting of many diverse types of cells.

This situation has changed with the emergence of optical imaging techniques. The spatiotemporal dynamics of second messenger synthesis, kinase activity, vesicle release or membrane potential can now be monitored in real-time across larger arrays of cells using fluorescence dyes and appropriate microscopic setups [3]. The advantage of DNA-encoded fluorescence sensors over "conventional" synthetic fluorescence dyes is obvious. Perfusing tissues with a fluorescent dye relies on physical parameters, e.g., the diffusion properties of the dye or the tissue density at the site at which the dye is injected. The exact cell type to be monitored is, therefore, difficult to specify. Conversely, if the dye is injected directly into the cells under investigation, the number of cells that can be simultaneously analyzed remains limited. On the contrary, DNAencoded fluorescence proteins can be expressed by cells under the control of regulatory DNA elements (e.g., enhancers or promoters) that specify the cells with respect to a common genetic identity and, potentially, a common function. This advantage is most obvious in nervous tissue, as information processing is typically accomplished by networks of diverse types of interconnected nervous cells. Neuronal activity is primarily characterized by changes in membrane potential and subsequent transmitter release at chemical synapses, and the modulation of neuronal activity often involves second-messenger cascades. DNA-encoded fluorescence probes that are sensitive to these parameters indeed exist, which enables one to monitor dynamic changes in the synthesis of cyclic nucleotides [4], membrane potential [5] or intracellular $\mathrm{Ca}^{2+}$ [6]. Unfortunately, the DNA-encoded voltage sensor proteins designed for optically recording changes in membrane potential that are currently available do not yet provide sufficient signal-to-noise ratios for in vivo applications. On the contrary, sensors reporting synaptic vesicle release [7] have been very successfully used in the nervous system of largely intact animals. However, the most commonly measured parameter as a correlate of neuronal activity is the concentration of intracellular $\mathrm{Ca}^{2+}[6]$. Whereas $\mathrm{Ca}^{2+}$ serves as a signaling molecule in various neuronal subcompartments [8], its overall cytosolic concentration represents a good correlate of membrane depolarization, albeit with limited temporal resolution. Since the first descriptions of a genetically encoded calcium indicator (GECI) $[9,10]$, a large variety of GECIs have been created that differ in their principal mode of action (Förster resonance energy transfer between two chromophores or direct shifts in emission intensity of a single chromophore), their $\mathrm{Ca}^{2+}$ affinity and several physical parameters, e.g., sensitivity to environmental cellular conditions (for reviews see refs. 2, 3, 11). The DNA of these GECIs can be readily transferred into cells by electroporation, transfection 
reagents, or somatic transfections, e.g., mediated by viruses. The advantages of DNA-encoded sensor proteins are most evident in the context of transgenic animals. Indeed, germ line transformation can now be accomplished in a variety of organisms. Currently, a repertoire of sophisticated genetic techniques that enable researchers to restrict the expression of transgenes, in this case a $\mathrm{Ca}^{2+}$ sensor protein, to small and selected subsets of cells or at defined developmental stages are available for several model organisms, e.g., in the round worm Caenorhabditis elegans, the zebrafish Danio rerio, the house mouse Mus musculus or the fruit fly Drosophila melanogaster. Here we focus on Drosophila melanogaster as a model organism, and in particular on optical in vivo $\mathrm{Ca}^{2+}$ imaging in the brain [12]. For a protocol describing germ line transformation in Drosophila please refer to [13].

Besides the availability of versatile genetic tools by which transgenes can be selectively expressed [14], a prominent feature of Drosophila is its relatively large behavioral repertoire relative to $C$. elegans, despite having a relatively simple central nervous system (when compared to vertebrates). Technically, a major strength of Drosophila relies on bipartite expression systems by which the spatial and temporal expression of the GECI (or any other transgene) is achieved by crossing a "driver strain" expressing a transactivating molecule to a second transgenic "reporter strain" that carries the DNA sequence of the GECI whose expression is controlled by the transactivator [14]. The most commonly used binary expression system is the Gal4-UAS system [15], but several alternative systems have been described with a number of variations that help restrict the expression of the transgene precisely to neurons of interest $[12,14]$.

Here, we describe how optical $\mathrm{Ca}^{2+}$ imaging of neuronal activity in the intact brain of an entire transgenic animal can be practically performed using the olfactory system of the Drosophila brain as an example. Fruit flies perceive odors with $\sim 1,200$ olfactory sensory neurons located on the third antennal segments and the maxillary palps [16]. Each olfactory sensory neuron expresses one or very few olfactory receptors, each of which detects a variable range of odorants [17]. These sensory neurons project with their axons to the antennal lobe, the primary olfactory center of the fly's brain, and extend their terminal arborizations into spherical structures called glomeruli [16]. Since each sensory neuron expresses a limited number of olfactory receptors with a specific ligand-binding profile [17], odor information is subsequently represented within the antennal lobes as spatiotemporal patterns of glomerular activities [18]. To determine the spatiotemporal activation of neuronal structures in the Drosophila brain, we use twophoton microscopy because the application of wide-field microscopy is limited by light scattering across the $\mathrm{z}$-axis of extended pieces of tissue. The invention of two-photon micros- 
copy [19] has helped to improve spatial resolution by restricting the excitation of chromophores to defined focal planes. In summary, we intend to demonstrate how in vivo optical $\mathrm{Ca}^{2+}$ imaging in the brain of an intact transgenic animal can be achieved by providing a protocol for monitoring odor-evoked changes in $\mathrm{Ca}^{2+}$ concentration in olfactory sensory neurons at the level of the antennal lobes of Drosophila using two-photon microscopy.

\section{Materials}

\subsection{Materials for the In Vivo Preparation}

\subsubsection{Transgenic} Drosophila Strains

\subsubsection{Materials for the In Vivo Brain Preparation}

1. The "driver strain" Or83b-Gal4 [20].

2. The "reporter strain" UAS-G-CaMP 3.0 [21].

A variety of different transgenic fly lines carrying the DNA of diverse GECIs have been described (reviewed by 12, 23). For preparations in which slight movements of the specimen cannot be completely avoided, we prefer FRET-based sensors because shifts in fluorescence intensity caused by movements can be corrected through the intrinsic ratiometric properties of these sensors [12, $18,23,24]$. For other purposes, single chromophore sensors, e.g., G-CaMP 3.0 [21], are advantageous because only one emission wavelength needs to be detected. In order to express G-CaMP 3.0 in specific neuronal subsets, a "driver strain" that specifies the expression pattern must be chosen. Several binary expression systems have been described to date (reviewed by 12, 14, 23). The most commonly used system is the bipartite Gal4-UAS expression system [15], which we have used in our example here. To express G-CaMP 3.0 in olfactory sensory neurons, we have chosen a well described Gal4 driver line that expresses Gal4 under the control of a promoter fragment for the olfactory receptor co-receptor "Or83b" [20]. Both strains are available at the Bloomington stock center (http://flystocks.bio.indiana.edu/). The Fl progeny from the cross of these two strains will express G-CaMP 3.0 specifically in a large population of olfactory sensory neurons.

1. Microscope slides $(\sim 76 \mathrm{~mm} \times 26 \times \mathrm{mm} 1 \mathrm{~mm})$.

2. Fine stainless steel metal mesh (diameter of wire $\sim 0.3 \mathrm{~mm}$ ).

3. Regular transparent adhesive tape.

4. $0.1 \mathrm{~mm}$ breakable razor blades and blade holder.

5. A pair of very fine forceps (tip $<0.1 \mathrm{~mm}$ ).

6. A syringe with a very fine diameter $(0.5 \mathrm{~mm})$.

7. Ringer's solution: $5 \mathrm{mM}$ HEPES-NaOH, $\mathrm{pH}=7.3,130 \mathrm{mM}$ $\mathrm{NaCl}, 5 \mathrm{mM} \mathrm{KCl}$ [17], $2 \mathrm{mM} \mathrm{CaCl}_{2}, 2 \mathrm{mM} \mathrm{MgCl}_{2}$, and $36 \mathrm{mM}$ sucrose [22]. 
8. Binocular stereomicroscope.

9. Low melting agarose with a congealing temperature of $26-30{ }^{\circ} \mathrm{C}$.

First one has to build a preparation chamber in which the animal can be positioned and immobilized for the surgery and the optical imaging. To accomplish this, cut a piece of the metal mesh about the width of the objective slide. Put the metal mesh pieces over the microscope slide and fix it by wrapping it around the microscope slide using adhesive tape (Fig. la, step 1). Stick several layers of adhesive tape (about the thickness of a fly, i.e., $\sim 1.5 \mathrm{~mm}$ ) on top of the mesh. Then use a splint of a breakable razor blade and the blade holder to cut a small chamber of about the size of a fly $\left(\sim 3 \times 1 \times 1.5 \mathrm{~mm}^{3}\right)$ into the layers of adhesive tape (Fig. la, step 2). In front of the chamber, cut a passage through the layers of the adhesive tape for the odor application (Fig. la, step 2). The fly can now be positioned into this chamber for the preparation and optical imaging (Fig. 1a, steps 3-5).

\subsection{Equipment for Optical Calcium Imaging}

2.2.1 Optical Imaging Microscope
Optical $\mathrm{Ca}^{2+}$ imaging in the brain of Drosophila can be performed using a regular wide-field fluorescence microscope equipped with a sensitive CCD camera and appropriate filters. In fact, in many cases a wide-field microscope provides advantages over laser scanning microscopes if the $z$-axis resolution is not important $[23,24]$. If one intends to differentiate between focal planes in the $z$-axis, however, an upright two-photon microscope is beneficial. Userfriendly two-photon microscopes are available from several companies. In any case, the microscope and the laser should be installed on a well-damped vibration-isolated table on air supports. For two-photon excitation of the fluorophore, a mode-locked titanium:sapphire laser is needed as a light source (e.g., Chameleon lasers from Coherent or Mai Tai/Tsunami lasers from SpectraPhysics). These lasers generate femtosecond pulses of infrared light in the range between $\sim 700$ and 1,000 $\mathrm{nm}$. The microscope should be equipped with a sensitive photomultiplier for detection and an $X / \Upsilon$-scanning unit with high scanning speed (at least 5 frames $/ \mathrm{s}$ ). For using the $\mathrm{Ca}^{2+}$ indicator G-CaMP 3.0, an excitation wavelength of $920 \mathrm{~nm}$ is appropriate. To separate excitation and emission light, a dichroic mirror (690 nm long pass) is required. The G-CaMP emission can be detected using a regular GFP band-pass filter. Importantly, the microscope must be equipped with a water immersion objective appropriate for infrared imaging, in our case a $20 \times$ objective $(\mathrm{NA}=1.0)$. A xenon lamp as a conventional light source and a regular GFP filter set is helpful to locate the structures of interest. Software for data acquisition and the control of the components of the microscope is, of course, commercially available according to the microscope system used. Data analysis, however, is recommended to be done with specialized image processing software, e.g., MetaMorph (Molecular Devices) or ImageJ. 

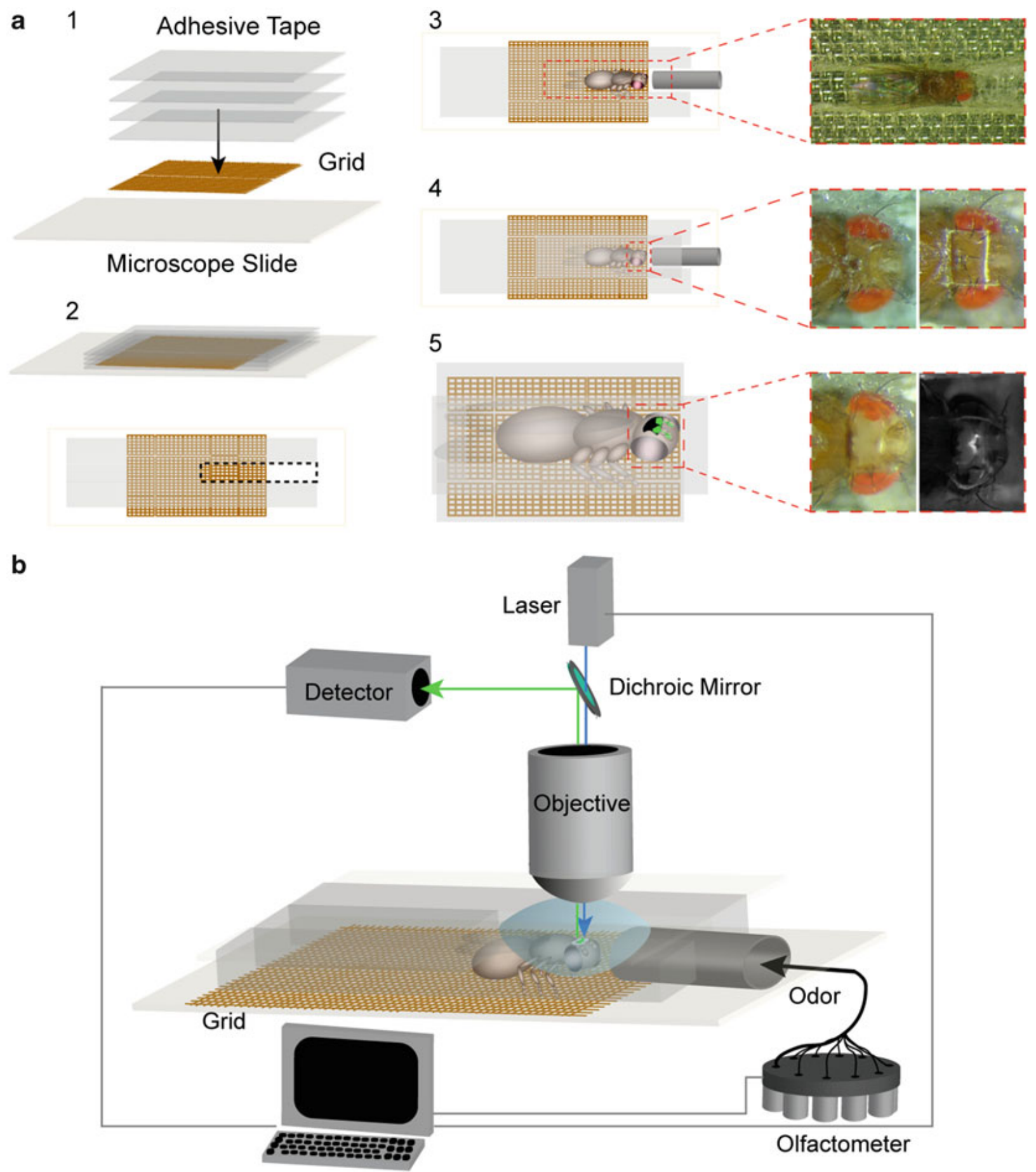

Fig. 1 (a) Schematic illustration of the fly brain in vivo preparation: (1) To restrain the fly in a small chamber, a metal grid is placed on a microscope slide and fixed with several layers of adhesive tape. Altogether the sticky tape layers should measure approximately the height of a fly. (2) A small passage is cut into the layers of the adhesive tape, leaving enough space for the fly and the tubing for the odor application. (3) The fly is placed into this corridor with the tubing directed towards its antennae. The chamber is then sealed from above with sticky tape. (4) In the next step a small window is cut into the adhesive tape, providing access to the head capsule such that the cuticle can be cautiously removed (5). (b) Schematic illustration of the optical imaging setup. The exposed brain is covered with Ringer's solution and placed under the microscope equipped with a water immersion objective. The $\mathrm{Ca}^{2+}$ sensor (e.g., G-CaMP 3.0) is excited by a 2-Photon laser, for G-CaMP at $920 \mathrm{~nm}$ with a maximum emission wavelength of $\sim 509 \mathrm{~nm}$. Odorants can be applied via a custom-built olfactometer that ensures a constant air flow rate for each odorant 
2.2.2 Device for Odor-Application (Only for Odor-Stimulation Experiments)
For odor stimulation, a custom-built stimulus device ("olfactometer") is used to apply the air-borne odors at constant airflow rates and the desired concentrations. In our case, the air is pumped by a regular aquarium pump into the olfactometer and separated into different channels controlled by computer-triggered electronic valves. The software controlling the microscope should be capable of controlling these additional devices. The individual airstreams are guided into Teflon tubings with a constant air flow rate of $\sim 1 \mathrm{ml} / \mathrm{s}$ and from there to small $(20 \mathrm{ml})$ odorant vials containing the odorants of choice. Under non-stimulation conditions, air is guided through a Teflon tube containing only the diluent, e.g., air or mineral oil. Airflows from different odor vials converge to a single outlet in front of the animal's antennae. To directly eliminate the odors from the experimental setup, an exhaust tube behind the animal is necessary to avoid odor contamination of the air. It is, of course, most important that contamination of all parts of the olfactometer with odorants is prevented and that any mechanosensory artifacts, such as might be caused by an air pressure decrease, are avoided. A variety of very similar olfactometer systems has been described (e.g., [25]).

\section{Methods}

\subsection{In Vivo Preparation of a Drosophila Brain}

1. Flies selectively expressing the G-CaMP 3.0 sensor [21] in neurons of interest can be generated by crossing the appropriate Gal4 line to the transgenic UAS-G-CaMP 3.0 line. In our experiments, we have crossed UAS-G-CaMP 3.0 with Or83bGal4 [20], which drives expression in a majority of olfactory sensory neurons ( see Note $\mathbf{1}$ ).

2. Immobilize the flies by cooling in an empty vial for no longer $\%$ min in ice (see Note 2 ).

3 . Use a small brush to gently put a single fly into the preparation chamber (see Fig. la step 3) described above and quickly seal it on top with a piece of adhesive tape before the animal recovers from anesthesia such that the fly remains restrained and the head capsule is in contact with the sticky surface of the adhesive tape.

4. Cut a small window through the adhesive tape and the cuticle of the head capsule with a splinter of a breakable razor blade. Appropriate blade holders are commercially obtainable (see Fig. la steps 4 and 5) (see Note 3).

5. To ameliorate optical access to the brain, remove cuticular structures as well as possible, then remove trachea and any accumulated fat from the head capsule using fine forceps. Proceed very carefully to avoid harming the brain tissue. 


\subsection{Optical $\mathrm{Ca}^{2+}$ Imaging}

6. Remove the hemolymph surrounding the brain carefully using small pieces of tissue paper and then inject $2 \%$ low melting agarose into the head capsule using a fine syringe of $0.5 \mathrm{~mm}$ diameter. Of course, the agarose should be close to the congealing point and not too warm. This step avoids desiccation and it physically immobilizes the tissue. The preparation is subsequently covered with Ringer's solution and the preparation chamber containing the fly is placed under the microscope.

1. Focus on the structure of interest (in our case the antennal lobes) using blue $(488 \mathrm{~nm}$ ) light from a regular light source, e.g., a xenon lamp, either by eye through the oculars or using a camera. Keep the illumination time as short as possible to avoid bleaching of the $\mathrm{Ca}^{2+}$ sensor.

2. Switch to the laser-scanning mode and refocus the specimen using the appropriate laser wavelength and band-pass filter (for G-CaMP 3.0 a two-photon excitation wavelength of $920 \mathrm{~nm}$ and a band-pass filter for GFP emission can be used). At this step fast scanning at low resolution is advisable to minimize bleaching.

3. Once the focus is set, choose a region of interest within which to scan the specimen. To maximize the scanning speed while maintaining the highest possible image resolution, the region of interest should be chosen as small as possible but large enough to cover all structures of interest.

4. Adjust the laser power and gain such that the laser power is low enough to avoid photo-toxicity, bleaching and heat production, but high enough that the baseline fluorescence is sufficiently visible.

5. Depending on the particular application and the expression level of the $\mathrm{Ca}^{2+}$ sensor, the scan speed may need to be adjusted. The adjustment of the frame rate may depend on the signal intensity of the baseline fluorescence, but frame rates from 3 to $10 \mathrm{~Hz}$ are reasonable for most physiological experiments. Lower frame rates increase photon collection per pixel at the cost of temporal resolution.

6. For the measurement itself, it is necessary to keep the number of acquired images as low as possible to avoid bleaching, but high enough to determine a reliable baseline as well as the onset and offset of the stimulus-evoked $\mathrm{Ca}^{2+}$ influx. For example, in the experiments shown in Fig. 2, a physiological stimulation with an odor of $2 \mathrm{~s}$ duration was used. The dynamics of intracellular $\mathrm{Ca}^{2+}$ concentration were observed using a recording protocol lasting for 85 frames at $5 \mathrm{~Hz}$ frame rate during which the odor stimulus was presented between frames 25-35. Between each measurement of physiological stimulations, a 


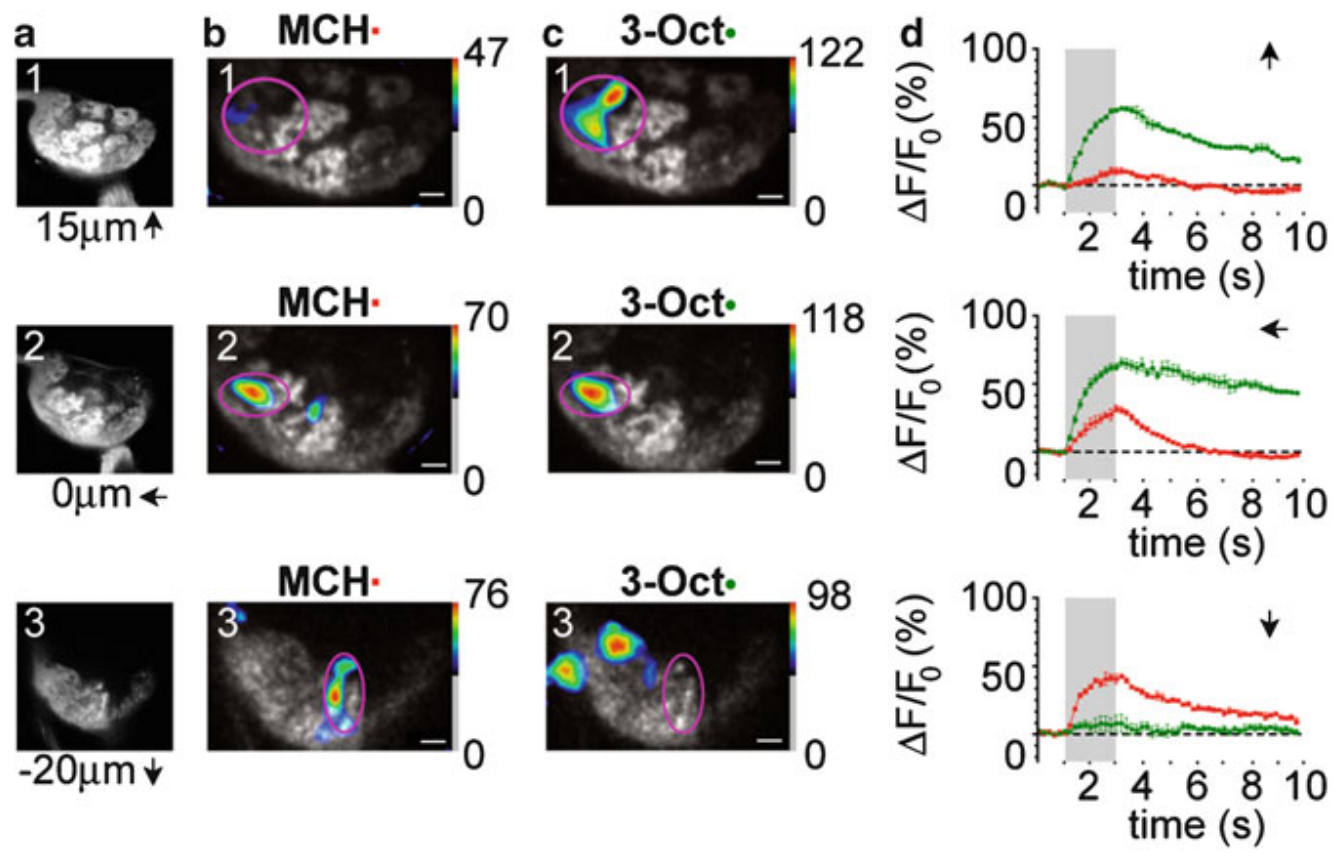

Fig. 2 Optical imaging of odor-evoked $\mathrm{Ca}^{2+}$ dynamics in olfactory sensory neurons of Drosophila at the level of the antennal lobe. (a) Baseline fluorescence of the G-CaMP 3.0 sensor expressed in a large population of sensory neurons. The numbers indicate the relative distances between different focal planes. Please note that olfactory glomeruli are very easily detectable. (b, c) False-color coded image of the spatial distribution of odor-evoked $\mathrm{Ca}^{2+}$ signals in the antennal lobe evoked by a 2 s stimulation with either 4-methylcyclohexanol ( $\mathrm{MCH}, 1: 750)$ (b) or 3-octanol (3-0ct, 1:500) (c) as odorants diluted in mineral oil. Recordings were taken at the three different focal planes of the antennal lobes shown in (a). Warm colors represent regions of high calcium activity while cold colors represent regions of low or no calcium increase. The false-color coded regions of $\mathrm{Ca}^{2+}$ increase are superimposed onto the grey scaled baseline fluorescence of the G-CaMP 3.0 sensor. The numbers indicate maximum relative changes in fluorescence $\left(F / F_{0}\right)$ across the entire image. The magenta circles indicate regions in which the temporal dynamics of $\mathrm{Ca}^{2+}$ changes is determined (d) Scale bars: $10 \mu \mathrm{m}$. (d) Temporal dynamics of intracellular $\mathrm{Ca}^{2+}$ increase in regions of high activity evoked by $\mathrm{MCH}$ (a) or 3-0ct (b). The duration of the odor stimulus is indicated as grey bars. Relative changes in fluorescence are indicated in red for $\mathrm{MCH}$ and in green for 3-0ct. Traces indicate means \pm SEM of three odor stimulations within the same animal. Note that the two odors evoke increases in intracellular $\mathrm{Ca}^{2+}$ in different regions of the antennal lobe and with different time courses

break period should be included to avoid continuous excitation of the fluorophore and potential photo-toxic effects. The acquisition is repeated several times at the desired focal planes.

\subsection{Data Analysis}

1. If there are slight movements of the structures under investigation during image acquisition, it is possible to align all the acquired images to reduce the movement effects using specialized algorithms (see Note 4 ).

2. In order to quantify potential dynamics in emission intensity reflecting intracellular $\mathrm{Ca}^{2+}$ dynamics using image processing software, arrange the acquired image files as a stack of images. Mark a region of interest (ROI) around the structure to be analyzed and obtain the raw grey values from the pixels within the ROI (see Fig. 2). 
3. Take the averaged values across the selected ROI from several images before the stimulus onset to obtain a reliable baseline fluorescence value $\left(F_{\mathrm{o}}\right)$.

4. Obtain the fluorescence values from the same ROI from all images before, during and after the stimulation and calculate $\left(F_{\mathrm{i}}-F_{\mathrm{o}}\right) / F_{\mathrm{o}}$, where $F_{\mathrm{i}}$ is the grey values reflecting the intensities from all images. As a result, the relative changes in emission intensity are obtained, representing the time course of stimulusevoked $\mathrm{Ca}^{2+}$ activity within the ROI (see Fig. $2 \mathrm{~d}$ ).

5. To visualize the spatial distribution of $\mathrm{Ca}^{2+}$ activity across the entire structure of interest (e.g., the antennal lobe), separately average several pre-stimulus images and post-stimulus images. The averaged pre-stimulus images reflect the baseline fluorescence intensity whereas the averaged post-stimulus images should indicate the time-point of maximum $\mathrm{Ca}^{2+}$ concentration evoked by the stimulation. In the case of Fig. $2 b, c$, three images before the odor onset were selected as pre-stimulus images $\left(F_{\mathrm{pre}}\right)$ and the images at around $2 \mathrm{~s}$ after the onset were chosen as poststimulus images $\left(F_{\text {post }}\right)$. The averages of both were subjected to a mean filter in which several pixels around each pixel as a center (in our case $5 \times 5$ ) are averaged to remove noise. $\left(F_{\text {post }} / F_{\text {pre }}\right)-1$ was calculated which equals $\left(F_{\text {post }}-F_{\text {pre }}\right) / F_{\text {pre }}$. To shift all pixels to positive values (negative values cannot be depicted in an image), an arbitrary number is added which then reflects the baseline. To present the data in percentage values, multiply it by a factor of 100. The resulting images are then shown in false-colors in which the different colors cover the range of signal intensities. One can superimpose these images onto the grey scaled baseline fluorescence images if one skips the lowest values, thereby showing only highly activated regions in correlation with the entire anatomy of the structure (Fig. 2b, c).

\section{Notes}

1. If expression level of the GECI is too low, it is helpful to create flies that are homozygous for both the UAS construct and the Gal4 construct. Raising the animals at higher temperatures (e.g., $29^{\circ} \mathrm{C}$ ) also may help to increase expression levels of the GECI.

2. Anesthetizing the animals using $\mathrm{CO}_{2}$ or chemicals is not recommended because it may affect the central nervous system.

3. Be careful that no Ringer's solution leaks through the window over the head capsule so that the antennae remain dry.

4. For aligning the images obtained by two-photon microscopy, we use a custom-written JAVA script executable by ImageJ which is based on the plugin "TurboReg" [26]. This rigid body algorithm uses landmarks present in a target image and aligns source images 
to target images such that the mean-squared difference between the source and the target images are minimized.

Acknowledgments

This work was supported by the Deutsche Forschungsgemeinschaft (SPP1392, FI 821/2-1, and SFB 889/B4) and the German Federal Ministry for Education and Research via the Bernstein Center for Computational Neuroscience Göttingen B01, grant number 01GQ1005A.

\section{References}

1. Miyawaki A (2003) Fluorescence imaging of physiological activity in complex systems using GFP-based probes. Curr Opin Neurobiol 13:591-596

2. Looger LL, Griesbeck O (2012) Genetically encoded neural activity indicators. Curr Opin Neurobiol 22:18-23

3. Miyawaki A (2005) Innovations in the imaging of brain functions using fluorescent proteins. Neuron 48:189-199

4. Willoughby D, Cooper DM (2008) Live-cell imaging of cAMP dynamics. Nat Methods 5:29-36

5. Mutoh H, Perron A, Akemann W, Iwamoto Y, Knöpfel T (2011) Optogenetic monitoring of membrane potentials. Exp Physiol 96:13-18

6. Tian L, Akerboom J, Schreiter ER, Looger LL (2012) Neural activity imaging with genetically encoded calcium indicators. Prog Brain Res 196:79-94

7. Dreosti E, Lagnado L (2011) Optical reporters of synaptic activity in neural circuits. Exp Physiol 96:4-12

8. Berridge MJ (1998) Neuronal calcium signaling. Neuron 21:13-26

9. Miyawaki A, Llopis J, Heim R, McCaffery JM, Adams JA, Ikura M, Tsien RY (1997) Fluorescent indicators for $\mathrm{Ca} 2+$ based on green fluorescent proteins and calmodulin. Nature 388:882-887

10. Romoser VA, Hinkle PM, Persechini A (1997) Detection in living cells of $\mathrm{Ca} 2+$-dependent changes in the fluorescence emission of an indicator composed of two green fluorescent protein variants linked by a calmodulinbinding sequence. A new class of fluorescent indicators. J Biol Chem 272:13270-13274

11. Mank M, Griesbeck O (2008) Genetically encoded calcium indicators. Chem Rev 108:1550-1564

12. Riemensperger $T$, Pech U, Dipt S, Fiala A (2012) Optical calcium imaging in the ner- vous system of Drosophila melanogaster. Biochim Biophys Acta 1820:1169-1178

13. Bachmann A, Knust E (2008) The use of P-element transposons to generate transgenic flies. Methods Mol Biol 420:61-77

14. Venken KJ, Simpson JH, Bellen HJ (2011) Genetic manipulation of genes and cells in the nervous system of the fruit fly. Neuron 72:202-230

15. Brand AH, Perrimon N (1993) Targeted gene expression as a means of altering cell fates and generating dominant phenotypes. Development 118:401-415

16. Vosshall LB, Stocker RF (2007) Molecular architecture of smell and taste in Drosophila. Annu Rev Neurosci 30:505-533

17. Hallem EA, Carlson JR (2006) Coding of odors by a receptor repertoire. Cell 125:143-160

18. Fiala A, Spall T, Diegelmann S, Eisermann B, Sachse S, Devaud JM, Buchner E, Galizia CG (2002) Genetically expressed cameleon in Drosophila melanogaster is used to visualize olfactory information in projection neurons. Curr Biol 12:1877-1884

19. Denk W, Strickler JH, Webb WW (1990) Two-photon laser scanning fluorescence microscopy. Science 248:73-76

20. Larsson MC, Domingos AI, Jones WD, Chiappe ME, Amrein H, Vosshall LB (2004) Or83b encodes a broadly expressed odorant receptor essential for Drosophila olfaction. Neuron 43:703-714

21. Tian L, Hires SA, Mao T, Huber D, Chiappe ME, Chalasani SH, Petreanu L, Akerboom J, McKinney SA, Schreiter ER, Bargmann CI, Jayaraman V, Svoboda K, Looger LL (2009) Imaging neural activity in worms, flies and mice with improved G-CaMP calcium indicators. Nat Methods 6:875-881

22. Estes PS, Roos J, van der Bliek A, Kelly RB, Krishnan KS, Ramaswami M (1996) Traffic of 
dynamin within individual Drosophila synaptic boutons relative to compartment-specific markers. J Neurosci 16:5443-5456

23. Strutz A, Völler T, Riemensperger T, Fiala A, Sachse S (2012) Calcium imaging of neural activity in the olfactory system of Drosophila. In: Martin JR (ed) Genetically encoded functional indicators. Springer Neuromethods 72:43-70

24. Fiala A, Spall T (2003) In vivo calcium imaging of brain activity in Drosophila by transgenic cameleon expression. Sci STKE (174):PL6

25. Olsson SB, Kuebler LS, Veit D, Steck K, Schmidt A, Knaden M, Hansson BS (2011) A novel multicomponent stimulus device for use in olfactory experiments. J Neurosci Methods 195:1-9

26. Thévenaz P, Ruttimann UE, Unser M (1998) A pyramid approach to subpixel registration based on intensity. IEEE Trans Image Process $7: 27-41$ 
B. Contributions to other studies

\section{Differential associative training enhances olfactory acuity in Drosophila melanogaster.}

My contribution :

- Designed and performed the experiments and analyzed the data for Figure 2, Figure 3, Figure 4, Figure 5, Figure 10 and Figure 11. 


\title{
Differential Associative Training Enhances Olfactory Acuity in Drosophila melanogaster
}

\author{
Jonas Barth, ${ }^{\star}$ Shubham Dipt, ${ }^{\star}$ Ulrike Pech, Moritz Hermann, Thomas Riemensperger, and André Fiala \\ Georg-August-University Göttingen, Johann-Friedrich-Blumenbach-Institute for Zoology and Anthropology, Molecular Neurobiology of Behavior, 37077 \\ Göttingen, Germany
}

Training can improve the ability to discriminate between similar, confusable stimuli, including odors. One possibility of enhancing behaviorally expressed discrimination (i.e., sensory acuity) relies on differential associative learning, during which animals are forced to detect the differences between similar stimuli. Drosophila represents a key model organism for analyzing neuronal mechanisms underlying both odor processing and olfactory learning. However, the ability of flies to enhance fine discrimination between similar odors through differential associative learning has not been analyzed in detail. We performed associative conditioning experiments using chemically similar odorants that we show to evoke overlapping neuronal activity in the fly's antennal lobes and highly correlated activity in mushroom body lobes. We compared the animals' performance in discriminating between these odors after subjecting them to one of two types of training: either absolute conditioning, in which only one odor is reinforced, or differential conditioning, in which one odor is reinforced and a second odor is explicitly not reinforced. First, we show that differential conditioning decreases behavioral generalization of similar odorants in a choice situation. Second, we demonstrate that this learned enhancement in olfactory acuity relies on both conditioned excitation and conditioned inhibition. Third, inhibitory local interneurons in the antennal lobes are shown to be required for behavioral fine discrimination between the two similar odors. Fourth, differential, but not absolute, training causes decorrelation of odor representations in the mushroom body. In conclusion, differential training with similar odors ultimately induces a behaviorally expressed contrast enhancement between the two similar stimuli that facilitates fine discrimination.

Key words: associative learning; Drosophila melanogaster; odor discrimination; olfaction; optical calcium imaging; sensory processing

\section{Introduction}

Most animals are able to detect, differentiate, and learn odors as indicators for food, mating partners, or hazardous substances. The fruit fly, Drosophila melanogaster, represents a key model organism for the analysis of genetic and neuronal mechanisms underlying odor detection and odor processing (Vosshall and Stocker, 2007). Moreover, Drosophila is widely used for investigating the neuronal basis of associative olfactory learning (Davis, 1993; Heisenberg, 2003; Fiala, 2007). Fruit flies detect odors with olfactory sensory neurons (OSNs) located on the third antennal segments and maxillary palps (Vosshall and Stocker, 2007). Each OSN expresses one or very few specific olfactory receptors, and

\footnotetext{
Received June 19, 2013; revised Dec. 13, 2013; accepted Dec. 17, 2013.

Author contributions: J.B., S.D., and A.F. designed research; J.B., S.D., U.P., M.H., and T.R. performed research; J.B., S.D., U.P., M.H., T.R., and A.F. analyzed data; J.B., U.P., T.R., and A.F. wrote the paper.

This work was supported by the German Research Foundation (FI 821/2-1, FI 821/3-1, SPP 1392, and SFB 889/B04 and (02), the German Ministry of Education and Research via the Bernstein Center for Computational Neuroscience Göttingen) Grant 01G01005A and the Göttingen Graduate School for Neurosciences, Biophysics and Molecular Biosciences. We thank Serge Birman, Silke Sachse, Hiromu Tanimoto, and the Bloomington stock center for providing fly strains; Veit Grabe and Silke Sachse for their help; and Hiromu Tanimoto and his laboratory members for technical advice.

The authors declare no competing financial interests.

*J.B. and S.D. contributed equally to this work.

Correspondence should be addressed to Dr. André Fiala, Georg-August-University Göttingen, Johann-FriedrichBlumenbach-Institute for Zoology and Anthropology, Molecular Neurobiology of Behavior, Julia-Lermontowa-Weg 3,37077 Göttingen, Germany. E-mail: afiala@gwdg.de.

DOI:10.1523/JNEUROSCI.2598-13.2014

Copyright $\odot 2014$ the authors $\quad$ 0270-6474/14/341819-19\$15.00/0
}

those OSNs that express the same receptors converge onto the same glomeruli in the antennal lobes (Vosshall and Stocker, 2007). The glomeruli are interconnected by local interneurons (Chou et al., 2010; Seki et al., 2010) that have been implicated in gain control (Olsen and Wilson, 2008; Root et al., 2008), temporal synchronization, and decorrelation of odor-evoked activity (Wilson et al., 2004; Wilson and Laurent, 2005). They have also been hypothesized to facilitate fine discrimination of similar odors (Wilson and Laurent, 2005). Olfactory projection neurons convey the odor information to the lateral horn and the mushroom body (Tanaka et al., 2004), a brain region critical for associative olfactory learning (Heisenberg, 2003).

Odor concentration and composition are often variable in time and space. This confronts the animal with a source of uncertainty about odor identity and, hence, possible relevance. To solve the problem of assigning relevance to variable stimuli, animals are able to generalize (i.e., to transfer a learned behavioral response to stimuli that are similar yet slightly different from a cue experienced as rewarding or punishing) (Pavlov, 1927; Guttman and Kalish, 1956; Ghirlanda and Enquist, 2003). The degree to which animals generalize across stimuli can be regarded as to which degree these are perceived as similar or distinct (Shepard, 1987; Pearce, 1994; Guerrieri et al., 2005; Niewalda et al., 2011). Perceptual similarity is, however, subject to experience. In some instances, it is advantageous for the animal to learn to differentiate precisely a relevant stimulus from a physically similar, but 
irrelevant, one. Enhanced behaviorally expressed discriminability between similar odors, which is referred to as olfactory acuity (Wilson and Stevenson, 2006), can be induced by differential associative learning in the course of which a conditioned stimulus $\left(\mathrm{CS}^{+}\right)$is reinforced by punishment or reward; a second, similar stimulus $\left(\mathrm{CS}^{-}\right)$is explicitly not reinforced (Pavlov, 1927; Hanson, 1959; Giurfa, 2004; Mishra et al., 2010). The ability to shift olfactory discrimination from generalizing across to differentiating between similar odors has been demonstrated in many species (Bitterman et al., 1983; Cleland et al., 2002; Fletcher and Wilson, 2002; Li et al., 2008; Mishra et al., 2010; Chapuis and Wilson, 2011; Chen CF et al., 2011). In Drosophila, however, it remains unclear whether differential training with similar odors enhances olfactory acuity as to facilitate distinction between them. We addressed this question using chemically similar odorants and analyzed the experience-dependent, relative change in perceptual similarity caused by differential training.

\section{Materials and Methods}

Drosophila strains and fly husbandry. Flies were raised on standard cornmeal medium at $25^{\circ} \mathrm{C}, 60 \%$ humidity, and a light/dark cycle of $12 \mathrm{~h} / 12 \mathrm{~h}$. The flies were raised in plastic vials containing $\sim 30 \mathrm{ml}$ medium with $\sim 200-300$ animals per vial and were transferred to fresh vials every 2-3 d. All behavioral experiments were performed between 1.5 and $10.5 \mathrm{~h}$ Zeitgebertime, ensuring controlled circadian conditions. Wild-type flies of the Canton-S strain were used if not stated otherwise, and the feeding status was equal throughout all experiments. For targeting local interneurons in the antennal lobe, the Gal4-enhancer-trap lines NP1227 and NP2426 (Sachse et al., 2007) were used. For visualizing these neurons, the flies were crossed with flies homozygous for both UAS-mCD8GFP and UAS-syb-GFP, kindly provided by S. Birman. For blocking synaptic transmission, a fly strain with two copies of the temperature sensitive shibire ${ }^{t s}$ allele on the $\mathrm{X}$-chromosome and the third chromosome under control of UAS was used (Kitamoto, 2001). For monitoring $\mathrm{Ca}^{2+}$ activity in olfactory sensory neurons or olfactory projection neurons, respectively, flies homozygous for Or83b-Gal4 (Wang et al., 2003) or GH146-Gal4 (Stocker et al., 1997) and UAS-GCaMP3.0 (Tian et al., 2009) were used. For monitoring $\mathrm{Ca}^{2+}$ activity in the mushroom body, GCaMP3.0 was expressed under direct control of the mb247-promoter (Pech et al., 2013).

Learning assays. For associative olfactory learning, groups of $\sim 100$ flies (5-9 d old) were trained as described by Tully and Quinn (1985) with modifications: four experiments were performed simultaneously in a modified learning apparatus described by Schwaerzel et al. (2002). A constant airflow of $\sim 167 \mathrm{ml} / \mathrm{min}$ in each training tube assured a constant odor flow inside the training tubes. The experiments were performed at diffuse light conditions and at a relative humidity of $60-80 \%$. Experimental groups were tested in parallel in a pseudo-randomized way, and flies were stringently controlled for their feeding status. To block synaptic transmission using UAS-shibire ${ }^{\text {ts }}$ flies, the temperature was adjusted to $31-33^{\circ} \mathrm{C}$. All other experiments were performed at $24-26^{\circ} \mathrm{C}$. Before each experiment, flies were transferred to empty vials and kept for $10 \mathrm{~min}$ at the respective temperature before the onset of the experiment. The odors 4-methylcyclohexanol (MCH), 3-octanol (3-Oct), 1-octen-3-ol (1-Oct), pentyl acetate (PA), and butyl acetate (BA) were purchased from SigmaAldrich and diluted in mineral oil. Training started 1 min after transferring the flies into training tubes each covered inside with an electrifiable grid. Each odor was presented for $1 \mathrm{~min}$ with a $1 \mathrm{~min}$ break between two odor applications. One odor $\left(\mathrm{CS}^{+}\right)$was temporally paired with 12 electric shocks of $90 \mathrm{~V} \mathrm{DC} \mathrm{(1.25} \mathrm{s} \mathrm{shock} \mathrm{and} 3.75 \mathrm{~s}$ interpulse intervals). The second odor $\left(\mathrm{CS}^{-}\right)$was presented without shocks. After another minute, the flies were transferred to the T-maze part of the apparatus with both odors presented from each side, and flies were tested for their odor preference for $2 \mathrm{~min}$. Subsequently, the flies were counted and a preference index was calculated by subtracting the number of flies on the side of the $\mathrm{CS}^{-}$from the number of flies on the side of the $\mathrm{CS}^{+}$, divided by the total number of flies. A learning index was calculated by averaging the absolute preference indices from two reciprocal experiments.

Climbing assay. For each trial, ten 6-8 d old flies were transferred into a $27-\mathrm{cm}$-long $10 \mathrm{ml}$ serological pipette without anesthesia and left to rest for $10 \mathrm{~min}$ at the appropriate temperature $\left(32-33^{\circ} \mathrm{C}\right.$ or $24.5-25.5^{\circ} \mathrm{C}$, respectively). After the resting period, the flies were tapped to the bottom of the pipette and were allowed to climb for $1 \mathrm{~min}$ before the number of flies was counted. A climbing index $(\mathrm{CI})$ was calculated as $\mathrm{CI}=0.5$ * $\left(n_{\text {total }}+n_{\text {up }}-n_{\text {down }}\right) / n_{\text {total }}$, where $n_{\text {total }}$ is the total number of flies, $\mathrm{n}_{\text {up }}$ is the number of flies in the uppermost $7 \mathrm{~cm}$ part of the pipette, and $n_{\text {down }}$ is the number of flies in the lowermost $7 \mathrm{~cm}$. The experiment was repeated three times per trial, and the mean CI was used as an indicator of locomotor performance.

Shock avoidance. Shock avoidance was assayed in the same training apparatus used for olfactory learning. One of the arms of the T-maze partition consisted of the training tube covered inside with the electrifiable grid. The flies were placed in the middle compartment between two arms of the T-maze. During the test of $1 \mathrm{~min}, 12$ electric shocks of $90 \mathrm{~V}$ DC (1.25 s shock and $3.75 \mathrm{~s}$ inter pulse intervals) were administered on one side and flies could distribute freely between the two sides. An avoidance index was calculated as the number of flies in the electrified tube minus the number of flies on the opposite side divided by the total number of flies.

Odor preference. Odor preference was assayed in the same training apparatus used for olfactory learning by placing the flies in the middle compartment between the two arms of the T-maze partition. One arm was equipped with an odor cup containing an odorant, whereas the odor cup on the other arm contained the solvent mineral oil. The flies were allowed to distribute for $2 \mathrm{~min}$. A preference index was calculated as the number of flies in the arm containing the odor minus the number of flies in the opposite arm of the T-maze divided by the total number of flies.

Functional calcium imaging. Female flies (3-5 d old) expressing the genetically encoded $\mathrm{Ca}^{2+}$ sensor GCaMP3.0 (Tian et al., 2009) were immobilized for $\sim 5$ min on ice. A single fly was subsequently placed in a custom-built fly holder and fixed using adhesive tape. A piece of a razor blade and a blade holder were used to cut a window through the tape and the cuticle of the fly's head. After careful removal of fat bodies and tracheae, $1.5 \%$ low melting agarose solution (Sigma-Aldrich) diluted in Ringer's solution ( 5 mm HEPES, pH 7.4, $130 \mathrm{~mm} \mathrm{NaCl}, 5 \mathrm{~mm} \mathrm{KCl,} 2 \mathrm{~mm}$ $\mathrm{CaCl}_{2}, 2 \mathrm{mM} \mathrm{MgCl}_{2}, 36 \mathrm{~mm}$ sucrose) of $<28^{\circ} \mathrm{C}$ was applied to the open head capsule. $\mathrm{Ca}^{2+}$ imaging was performed using an LSM 7 MP twophoton microscope (Carl Zeiss) equipped with a mode-locked Tisapphire Chameleon Vision II laser (Coherent), a 500-550 nm bandpass filter and a Plan-Apochromat $20 \times / 1.0 \mathrm{NA}$ water-immersion objective (Carl Zeiss). Attached to the microscope was a custom-built device to supply odorous air with a constant flow rate of $1 \mathrm{ml} / \mathrm{s}$ directly to the fly's antennae as described previously (Riemensperger et al., 2005.). Onset and duration of the odor stimulus were controlled using a customwritten LABVIEW program (National instruments). $\mathrm{Ca}^{2+}$ dynamics were monitored at an image acquisition rate of $5 \mathrm{~Hz}$ and an excitation wavelength of $920 \mathrm{~nm}$. For the measurements in olfactory sensory neurons, the odorants were presented three times each with a $20 \mathrm{~s}$ break between each stimulation, and data from three repetitive experiments were averaged for each fly. For measurements in olfactory projection neurons, which involved learning experiments, each odorant was presented only once with a $20 \mathrm{~s}$ break between each stimulation. In both olfactory sensory neurons and projection neurons, $\mathrm{Ca}^{2+}$ dynamics in the antennal lobe was measured in three different focal planes. Individual glomeruli were identified using optical sections and a 3D model of the glomerular structure of the antennal lobe (Laissue et al., 1999) available online in the fly brain atlas at http://www.flybrain.org (Armstrong et al., 1995) and by repeatedly comparing activity patterns across flies. Only those glomeruli that could be identified in all flies measured were further analyzed. The images were aligned to reduce small shifts in the X-Y direction using a custom written ImageJ plugin based on the TurboReg plugin (Thévenaz et al., 1998). The mean of five images before stimulus onset was used as baseline fluorescence $\left(\mathrm{F}_{0}\right)$. The difference in intensity $(\Delta \mathrm{F})$ was calculated by subtracting $\mathrm{F}_{0}$ from the fluorescence intensity value of each image $\left(\mathrm{F}_{\mathrm{i}}\right)$ and subsequently divided by the baseline fluo- 
rescence. Correlations between glomerular activity patterns evoked by odor pairs were calculated, and the maximal Pearson's correlation coefficient over the time course of the stimulation was determined for each fly and each odor pair. Measurements in the mushroom body were focused on the $\beta^{\prime}$ - and $\gamma$-lobe. The Amira 5.3.3 software (Visage Imaging) was used to reconstruct parts of the mushroom body from a $z$-stack covering several focal planes. For subjecting the animals to the training regimen under the microscope, flies were positioned onto two thin metal wires placed below the fly's thorax and subjected to 12 electric shock pulses of $90 \mathrm{~V}$ and $1.25 \mathrm{~s}$ with $3.75 \mathrm{~s}$ interpulse intervals. The temporal order of odor presentations and electric shocks was controlled using a customwritten LABVIEW program (National instruments). The images obtained from the mushroom body were aligned in the $\mathrm{X}-\mathrm{Y}$ direction using a MATLAB program (MathWorks) to correct for slight movements (Guizar-Sicairos et al., 2008). For the pixel-based analysis, a KalmanFilter (Kalman, 1960) was subsequently applied to efficiently remove noise without losing spatial information. It should be noted that the Kalman filter altered the temporal dynamics of displayed $\mathrm{Ca}^{2+}$ transients. False-color-coded images were obtained by subtracting the image directly before stimulus onset from the image at the maximum of the intensity difference (i.e., at $2 \mathrm{~s}$ after odor onset) and divided by the baseline fluorescence. Noise was reduced using a mean filter with a 5 pixel range for the antennal lobe and a 1 pixel range for the mushroom body.

Immunohistochemistry. Brains and thoracic ganglia of 7- to 10-d-old flies were dissected in ice-cold Ringer's solution, fixed in 4\% paraformaldehyde for $2 \mathrm{~h}$ on ice, and subsequently washed three times for 20-30 min each at room temperature in PBS, $\mathrm{pH} 7.4$, containing $0.6 \%$ Triton $\mathrm{X}-100$ (PBT). After blocking in 2\% BSA dissolved in PBT for $2 \mathrm{~h}$ at room temperature, the samples were incubated overnight at $4^{\circ} \mathrm{C}$ with mouse anti-brp antibody (Wagh et al., 2006), diluted 1:5, and rabbit anti-GFP, diluted 1:5000 (Jackson ImmunoResearch Laboratories). After three washing steps in PBT for 20-30 min each at room temperature, the samples were incubated in 3\% normal goat serum diluted in PBT for 30 min. Subsequently, the preparations were incubated for $2 \mathrm{~h}$ at room temperature with the secondary antibodies diluted in PBT containing 3\% normal goat serum and 2\% BSA. The anti-rabbit Alexa488-conjugated antibody (Invitrogen) was diluted 1:100, the anti-mouse Cy3-conjugated antibody (Invitrogen) 1:250. Afterward, the brains were washed three times for 20-30 min each and kept in PBS at $4^{\circ} \mathrm{C}$ until they were mounted in Vectashield (Vector Laboratories). Confocal laser scanning microscopy was done using a Leica SP2 microscope equipped with a PlanApochromat $20 \times / 0.7$ NA objective. Maximum value projections across the $z$-direction were calculated across image stacks.

Statistical analysis. Groups of data were tested for normal distribution using the Shapiro-Wilk test. For comparisons across two normally distributed groups of data, the two-sample Student's $t$ test was applied; for testing for statistical significant differences from 0 , the one-sample Student's $t$ test was used. For comparisons across more than two normally distributed groups of data, one-way ANOVA tests were used with subsequent Bonferroni-corrected post hoc comparisons. Dependent data were tested for significance using a one-way repeated-measures ANOVA and subsequent Bonferroni post hoc comparisons. For statistical comparisons between two non-normally distributed groups of data, the MannWhitney U test was used. Principal component analysis (PCA) was calculated using MATLAB (Mathworks). To quantify similarities between odor-evoked neuronal representations in the antennal lobe, Euclidean distances between odor-evoked, relative changes in fluorescence emission were calculated as follows: $\left(\sum\left(X_{i}-Y_{i}\right)^{2}\right)^{1 / 2}$, where $X_{i}$ and $Y_{i}$ are the fluorescence changes in the i-th glomerulus evoked by the odors $X$ and $Y$, respectively. Pearson's correlation coefficients between relative changes in fluorescence emission were determined as follows: $\operatorname{cov}(X, Y) /\left(\sigma_{x}, \sigma_{y}\right)$ where $X$ and $Y$ are two column vectors representing relative changes in fluorescence emission from all measured glomeruli evoked by two odors, cov is the covariance, and $\sigma_{x}$, and $\sigma_{y}$ are the standard deviations of $X$ and $Y$, respectively. In the mushroom body, Pearson's correlations between spatiotemporal $\mathrm{Ca}^{2+}$ dynamics evoked by odors were calculated based on pixels in defined regions of interest.

\section{Results \\ Generalization across two chemically and physiologically similar odors}

For our study, we chose two odorants, 3-Oct and 1-Oct, whose chemical structures differ only by one covalent bond (Fig. 1A). The odorant $\mathrm{MCH}$ serves as a chemically distinct odorant for comparison (Fig. 1A). We first determined the perceptual similarity between these odorants as expressed by the degree to which the animals generalize across them. Innate, naive preferences for the odors and their learnability are, however, often dependent on odorant concentration, and the flies' evaluation of odor quality can also be affected by the concentration (Masek and Heisenberg, 2008). To exclude potential effects of differential naive preference behavior, we first adjusted the respective odorant concentrations so that the flies' naive odor preferences were completely balanced. At the concentrations used throughout all experiments, the three odorants were slightly attractive to the animals, as indicated by positive preference indices (Fig. 1B). Because, in learning experiments, the animals were exposed to odors for $1 \mathrm{~min}$ with or without electric shock punishment, we tested whether the mere exposure to the odors would affect preference behavior, which was not the case $\left(F_{(2,45)}=0.03, p=0.96\right.$; one-way ANOVA). Behavioral adaptation due to $1 \mathrm{~min}$ odor exposure at the concentrations used could therefore be excluded. The diluent mineral oil did not evoke any behavioral response in a choice situation with blank air as alternative (Fig. 1B). In an absolute associative learning paradigm, we used either odor as a conditioned stimulus paired with electric shock punishment, or without any punishment, respectively. That is, the odor presentation was temporally paired with electric shocks $\left(\mathrm{CS}^{+}\right)$and the presentation of the diluent was not ("CS ${ }^{-}$"). In a reciprocal group the diluent served as "CS" " and the odor as $\mathrm{CS}^{-}$(Fig. 1C). The absolute training procedure was combined thereby with a reciprocal experimental design to exclude nonassociative effects (Dudai, 1977). The animals acquired an equally strong aversion to the $\mathrm{CS}^{+}$after training with either odor $\left(F_{(2,45)}=0.4, p=0.68\right.$; one-way ANOVA), which reflects an equal learnability of all three odors used (Fig. $1 D)$. However, when the learning indices obtained in these reciprocal experiments were separated for those animals exposed to the odors as a CS ${ }^{+}$and those that were exposed to the odors as a $\mathrm{CS}^{-}$, strong learned odor avoidance was observed in those animals in which the odor has been paired with the punishment, as indicated by negative preference indices (Fig. $1 E$ ). In contrast, those animals that perceived the odor as a $\mathrm{CS}^{-}$still showed an attraction toward the odorant that was even slightly higher than the innate preference, although the increase in odor preference is statistically significant only for the odorant 3 -Oct $\left(t_{(30)}=-5.1\right.$, $p<0.01)$, but not for 1-Oct $\left(t_{(30)}=-1.3, p=0.19\right)$ and $\mathrm{MCH}$ $\left(t_{(30)}=-1.75, p=0.09\right.$; two-sample $t$ test) (Fig. $\left.1 F\right)$. Pairing the diluent mineral oil with electric shocks did not induce any learning $\left(t_{(15)}=-0.04, p=0.97\right.$; one sample $t$ test) (Fig. $\left.1 F\right)$, confirming that the diluent did not cause a behavioral response and is not learned as a stimulus. After balancing the concentration of the three odorants such that they induced equal innate preference and showed equal learnability, we tested whether flies could generalize across the odors presented. To do so, we reciprocally trained them with one odor as $\mathrm{CS}^{+}$or $\mathrm{CS}^{-}$, respectively, and tested for their response to the same or a novel odor in a choice situation. This "recognition experiment" revealed that training with either 1-Oct (Fig. 1G) or 3-Oct (Fig. 1H) in a reciprocal, absolute learning paradigm caused avoidance not only of the trained odor (gray bars), but also of the chemically similar odor 
A<smiles>COCCOC(O)CO</smiles><smiles>C=CC(O)COCCOC</smiles><smiles></smiles>

4-methylcyclohexanol
B
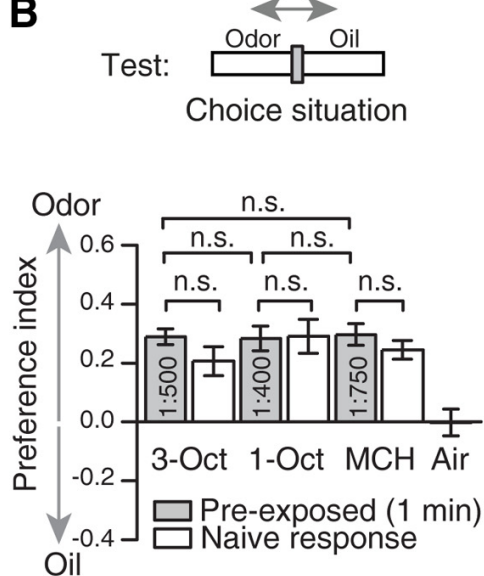

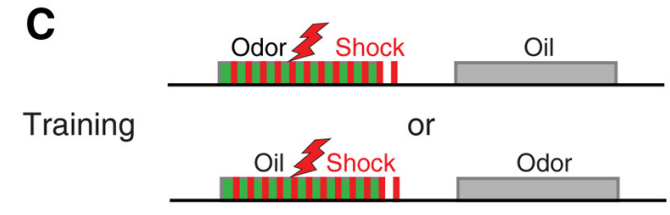

D

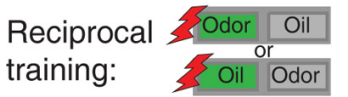

Test: Odor
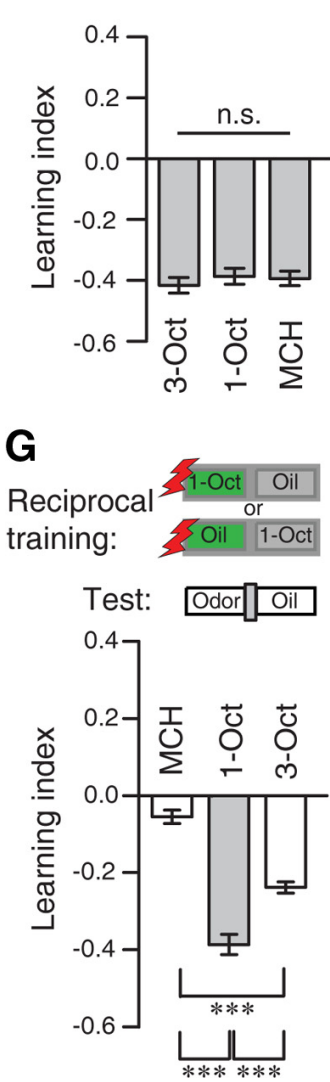

E

Training:

Test: Odor Oil

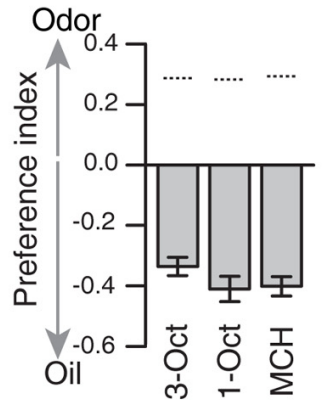

H

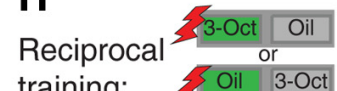
training:

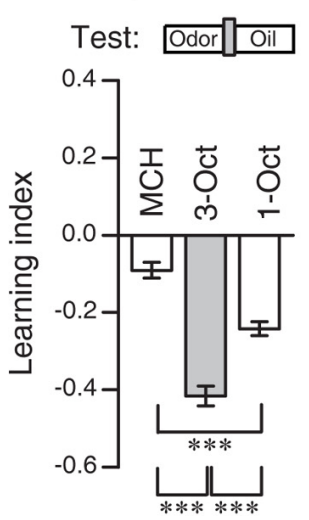

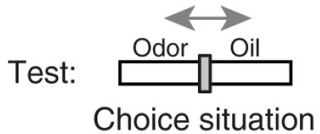

F

Training:

Test: Odor

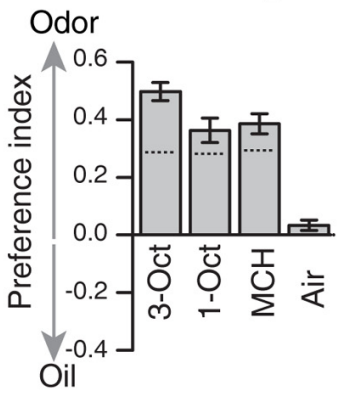

I
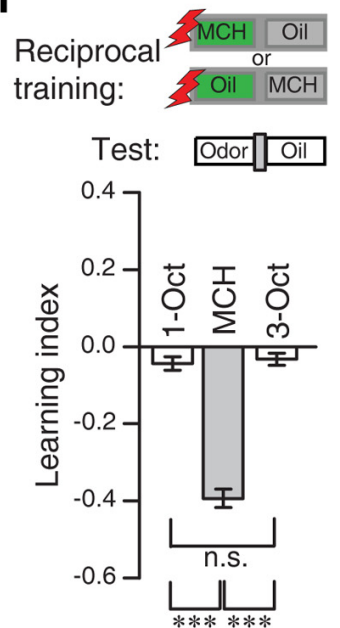

Figure 1. Generalization across similar odors. $\boldsymbol{A}$, Chemical structures of the three odorants used: $3-0 \mathrm{ct}, 1-0 \mathrm{ct}$, and $\mathrm{MCH}$. $\boldsymbol{B}, 0 \mathrm{dor}$ preference of flies for the odorants 3-0ct, 1-0ct, and MCH at the dilutions indicated within the bars in a T-maze choice situation against the diluent mineral oil ( $0 \mathrm{il})$. White bars represent the preferences of naive flies. Gray bars represent the responses of flies that were preexposed to the respective odor for $1 \mathrm{~min}$. No significant differences between preexposed and naive animals (twosample $t$ test), and no differences between the three odors at the indicated concentrations were observed. n.S., Not significant $(p>0.05$; one-way ANOVA). Bars indicate mean $\pm \mathrm{SEM} ; n=16$ for preexposed animals, $n=8$ for naive animals. No preference for the diluent mineral oil was detected when tested against blank air. C, Schematic illustration of the absolute associative conditioning regimen. Green box represents odor or diluent (mineral oil) that is temporally paired with electric shocks

to a high degree (Fig. 1G: 1-Oct vs $\mathrm{MCH}$ : $t_{(30)}=-11.6, p<0.01 ; 3$-Oct vs $\mathrm{MCH}$ : $t_{(30)}=-6.4, p<0.01 ; 3$-Oct vs 1-Oct: $t_{(30)}$ $=5.2, p<0.01$; Figure $1 H$ : 3 -Oct vs MCH: $t_{(30)}=-10.7, p<0.01 ; 1$-Oct vs $\mathrm{MCH}: t_{(30)}=-5.0, p<0.01 ; 1-$ Oct vs 3-Oct: $t_{(30)}=5.7, p<0.01$; one-way ANOVA with Bonferroni post hoc tests). The dissimilar odor, $\mathrm{MCH}$, was only slightly avoided after training with 1-Oct or 3-Oct. Conversely, associative training with $\mathrm{MCH}$ did not cause any generalization when 1-Oct or 3-Oct was presented in the test situation (1-Oct vs MCH: $t_{(30)}$ $=-12.9, p<0.01 ; 1$-Oct vs 3 -Oct: $t_{(30)}=$ $0.4, p=1 ; 3$-Oct vs MCH: $t_{(30)}=-13.3$, $p<0.01$; one-way ANOVA with Bonferroni post hoc tests) (Fig. 1I). The chemical similarity/dissimilarity of the three odorants is therefore reflected in equivalent degrees of generalization.

To test for a potential physiological manifestation of odor similarity as expressed in behavior, we performed optical $\mathrm{Ca}^{2+}$ imaging experiments (Riemensperger et al., 2012) and monitored odor-

\section{$\leftarrow$}

(red boxes). Gray box represents odor or diluent stimulation without punishment. During the test, the trained flies were subjected to a choice situation between odor and mineral oil. $D$, Equal learnability of the three odors after absolute, reciprocal training. n.s., Not significant ( $p>0.05$; one-way ANOVA). Bars indicate mean $\pm \mathrm{SEM} ; n=16$ for each experiment. Either the odor or the diluent was temporally paired with electric shocks (gray boxes in the schematic illustrations). Negative learning indices indicate learned avoidance of the stimulus previously paired with the electric shocks. $\boldsymbol{E}$, Preference indices of animals subjected to an odor simultaneously with the electric shocks and the diluent without electric shocks. For comparison, the dotted lines indicate odor preferences of untrained animals that have been preexposed to the odors as shown in $\boldsymbol{B}$. The associative training caused an aversive behavior, indicated by negative preference indices. Bars indicate mean $\pm \mathrm{SEM} ; n=16$ for each experiment. $\boldsymbol{F}$, Preference indices of animals that have received electric shock stimulation simultaneously with the presentation of the diluent, but not during odor presentation. The dotted lines indicate odor preferences of untrained animals that have been preexposed to the odors as shown in $\boldsymbol{B}$. The training caused a slight increase in attractiveness compared with the naive preference, which is statistically significant only for 3-0ct (3-0ct: naive vs trained, $p<0.001$, two-sample $t$ test). Bars indicate mean \pm SEM; $n=16$ for each experiment. $\mathbf{G}-\mathbf{I}$, Absolute, reciprocal training of 1-0ct $(\boldsymbol{G}), 3-0 \mathrm{ct}(\boldsymbol{H})$, and $\mathrm{MCH}(\boldsymbol{I})$ against the diluent mineral oil and test for the avoidance of each odor. In all cases, flies strongly avoided the trained odor (gray bars). Generalization was observed if flies were trained with either 1-0ct or 3-0ct and tested for the respective similar odor, but not if they were tested for the dissimilar odor $\mathrm{MCH}(\boldsymbol{G}, \boldsymbol{H})$. Flies did not generalize to 1-0ct or 3-0ct after training with MCH (I). n.s., Not significant ( $p>0.05$; one-way ANOVA with Bonferroni post hoc test). ${ }^{* * *} p<0.001$ (one-way ANOVA with Bonferroni post hoc test). $n=16$ for each experiment. Bars indicate mean \pm SEM. 
evoked $\mathrm{Ca}^{2+}$ activity in terminal arborizations of OSNs in the antennal lobes. Here, distinct OSNs expressing defined receptor proteins converge into identified glomeruli (Couto et al., 2005; Fishilevich and Vosshall, 2005; Vosshall and Stocker, 2007). Odor representations in the antennal lobes can therefore be described as overlapping, combinatorial glomerular activity patterns (Fiala et al., 2002; Wang et al., 2003). Using two-photon microscopy, we focused on three focal planes covering 29 of 51 olfactory glomeruli (Caron et al., 2013). Glomerular subdivisions of DA4 and VM5 could not be anatomically identified based on GCaMP3.0 expression, and VA1 was subdivided into three compartments: dorsal, lateral, and medial (Figs. 2 and 3). Stimulation with 1-Oct activated a glomerulus that could not be unambiguously identified and was therefore referred to as glomerulus X (Figs. 2C,D and $3 A$ ). 1-Oct and 3-Oct both evoked strong $\mathrm{Ca}^{2+}$ activity in a largely overlapping subset of glomeruli (Figs. $2 A-D$ and $3 A$ ). On the other hand, $\mathrm{MCH}$ induced a relatively more distinct activity pattern (Figs. 2C,D and $3 A$ ). Taking the representative odor responses measured in the three focal planes into account, 1-Oct and 3-Oct evoked more similar, overlapping neuronal activity in the antennal lobe compared with those evoked by $\mathrm{MCH}$ (Fig. $3 A$ ). To assess the degree of similarity between odor-evoked glomerular activities more quantitatively, we reduced the multidimensionality determined by the number of identified glomeruli using a PCA to illustrate the time course of glomerular $\mathrm{Ca}^{2+}$ dynamics. Figure $3 B$ shows that the $\mathrm{Ca}^{2+}$ dynamics across all measured glomeruli evoked by 3-Oct and 1-Oct are more similar to each other than to $\mathrm{MCH}$. When we calculated the Euclidean distances across the maximum $\mathrm{Ca}^{2+}$ activity in all optically recorded glomeruli, we found a significantly smaller distance between 3-Oct and 1-Oct to each other than between either of these and $\mathrm{MCH}\left(\mathrm{MCH} / 3-\mathrm{Oct}\right.$ vs $\mathrm{MCH} / 1-\mathrm{Oct}: t_{(10)}=1.1, p=0.85$; $\mathrm{MCH} / 3-O c t$ vs 1 -Oct/3-Oct: $t_{(10)}=5.3, p<0.01 ; \mathrm{MCH} / 1$-Oct vs 1-Oct/3-Oct: $t_{(10)}=4.2, p<0.01$; one-way repeated-measures ANOVA with Bonferroni post hoc test) (Fig. 3C). We additionally assessed the degree of similarity by calculating the Pearson's correlation between the $\mathrm{Ca}^{2+}$-activity patterns evoked by the three odorants at the time point of maximal $\mathrm{Ca}^{2+}$ amplitude. The correlation between the similar odors is significantly larger compared with the correlation between the dissimilar odor pairs $\left(\mathrm{MCH} / 3-\mathrm{Oct}\right.$ vs $\mathrm{MCH} / 1-\mathrm{Oct}: t_{(10)}=1.6, p=0.45 ; \mathrm{MCH} / 3-\mathrm{Oct}$ vs 1 -Oct/3-Oct: $t_{(10)}=3.9, p<0.01 ; \mathrm{MCH} / 1$-Oct vs 1 -Oct/3-Oct: $t_{(10)}=5.4, p=<0.01$; one-way repeated-measures ANOVA with Bonferroni post hoc test) (Fig. 3D). In conclusion, our experiments revealed that the similarity between the odors as observed in the animals' behavior is also reflected in the odor representation in OSNs within the antennal lobe.

OSNs synapse onto second-order olfactory projection neurons (OPNs) within the glomeruli of the antennal lobe. Because odor-evoked glomerular activity patterns are subject to processing by interneurons horizontally interconnecting the glomeruli, we asked whether the similarity between neuronal odor representations detected in OSNs is maintained at the level of OPNs. Therefore, we monitored odor-evoked $\mathrm{Ca}^{2+}$ activity in the arborizations of OPNs in the antennal lobes in three focal planes (Fig. $4 A-C$ ) that, together, captured glomeruli that partially overlapped with those analyzed in OSNs. Together, we recorded odor-evoked $\mathrm{Ca}^{2+}$ responses in OPNs within 18 identifiable glomeruli (Figs. $4 A-D$ and $5 A$ ), of which 12 were also monitored at the level of OSNs (Figs. $2 A-C$ and $3 A$ ). Compared with odorevoked $\mathrm{Ca}^{2+}$ transients in OSNs (Fig. $2 D$ ), those observed in OPNs are more variable in their dynamic structure (Fig. 4D), and activities of identified glomeruli (e.g., glomeruli DA2 or DM1) differ between the two neuronal populations (Figs. $3 A$ and $5 A$ ). However, when analyzing odor-induced $\mathrm{Ca}^{2+}$ activity patterns across all 18 glomeruli in OPNs, the two similar odors 3-Oct and 1-Oct are, again, more similar to each other in terms of neuronal representation than to the dissimilar odor $\mathrm{MCH}$ (Fig. 5A). In a PCA, the spatiotemporal activity of the dissimilar odor separates clearly from the similar ones (Fig. 5B). Just as it is the case in OSNs (Fig. 3C), we observed a significantly smaller distance between $\mathrm{Ca}^{2+}$ activity evoked by 3 -Oct and 1-Oct than between either of these and $\mathrm{MCH}$ when the maximal Euclidean distances across all optically recorded glomeruli during odor stimulation was calculated $\left(\mathrm{MCH} / 3\right.$-Oct vs MCH/1-Oct: $t_{(58)}=4.3, p<0.01$; $\mathrm{MCH} / 3$-Oct vs 1-Oct/3-Oct: $t_{(58)}=12.2, p<0.01 ; \mathrm{MCH} / 1-\mathrm{Oct}$ vs 1-Oct/3-Oct: $t_{(58)}=7.9, p<0.01$; one-way repeated-measures ANOVA with Bonferroni post hoc test) (Fig. 5C). In accordance, a significantly higher Pearson's correlation coefficient was found between $\mathrm{Ca}^{2+}$ patterns evoked by 3-Oct and 1-Oct than between either of these and signals evoked by $\mathrm{MCH}(\mathrm{MCH} / 3$-Oct vs $\mathrm{MCH} / 1-\mathrm{Oct}: t_{(58)}=3.9, p<0.01 ; \mathrm{MCH} / 3-\mathrm{Oct}$ vs 1 -Oct/3-Oct: $t_{(58)}=3.7, p<0.01 ; \mathrm{MCH} / 1$-Oct vs 1 -Oct/3-Oct: $t_{(58)}=7.6, p<$ 0.01 ; one-way repeated-measures ANOVA with Bonferroni post hoc test) (Fig. 5D). Conclusively, the similarity between the odors as observed in the animals' behavior is also reflected in the odor representation in second-order olfactory neurons.

\section{Local interneurons in the antennal lobe are required for discrimination between two similar odors}

The degree of generalization between stimuli is typically inversely related to their discriminability. However, generalization as an effect of transferring a learned response to a novel, similar stimulus requires that the two stimuli are discernable by an animal. Therefore, we asked whether the two similar odors could be discriminated by the animals at all. Indeed, they could, as the following experiment demonstrates. In a differential training procedure as illustrated in Figure 6A, either 3-Oct or 1-Oct was paired with electric shocks $\left(\mathrm{CS}^{+}\right)$, and the other, similar odor was explicitly presented without punishment $\left(\mathrm{CS}^{-}\right)$. In a subsequent choice situation with both odors as alternatives, the animals clearly avoided the $\mathrm{CS}^{+}$(Fig. $6 \mathrm{~B}$ ). Omission of the electric shocks resulted in indifferent behavior (Fig. $6 B$ ). Interestingly, the response was asymmetric for the two odors after conditioning. Training with 1-Oct as a $\mathrm{CS}^{+}$and 3-Oct as a $\mathrm{CS}^{-}$caused a strong aversion of the $\mathrm{CS}^{+}$in a subsequent choice situation between both odorants. The converse experiment, with 3-Oct as the $\mathrm{CS}^{+}$and 1 -Oct as the $\mathrm{CS}^{-}$, resulted in a much weaker avoidance of the $\mathrm{CS}^{+}$(untrained vs 3-Oct punished: $t_{(30)}=3.6, p<$ 0.01; 1-Oct punished vs 3-Oct punished: $t_{(30)}=13.8, p=<$ 0.01 ; 1-Oct punished vs untrained: $t_{(30)}=10.2, p=<0.01$; one-way ANOVA with Bonferroni post hoc test) (Fig. 6B, top). This demonstrates that an equal learnability of the two odors in an absolute training regimen does not imply equal discrimination learning in a differential conditioning paradigm. To correct for these odor-specific effects, we combined preference indices of two reciprocal experiments to a learning index (Fig. 6B, bottom graph). Clearly, 1-Oct and 3-Oct could be differentiated by the animals. However, compared with differential training of dissimilar odors, either 1-Oct versus $\mathrm{MCH}$ or 3-Oct versus $\mathrm{MCH}$, the learning index was significantly lower in magnitude, which confirmed the relatively lower discriminability of the two similar odors (3-Oct/1-Oct vs $\mathrm{MCH} / 3$ Oct: $t_{(30)}=3.2, p<0.01 ; 3$-Oct $/ 1$-Oct vs $\mathrm{MCH} / 1$-Oct: $t_{(30)}=$ 3.2, $p<0.01 ; \mathrm{MCH} / 1$-Oct vs MCH/3-Oct: $t_{(30)}=0.05, p=1$; one-way ANOVA with Bonferroni post hoc test). 
A
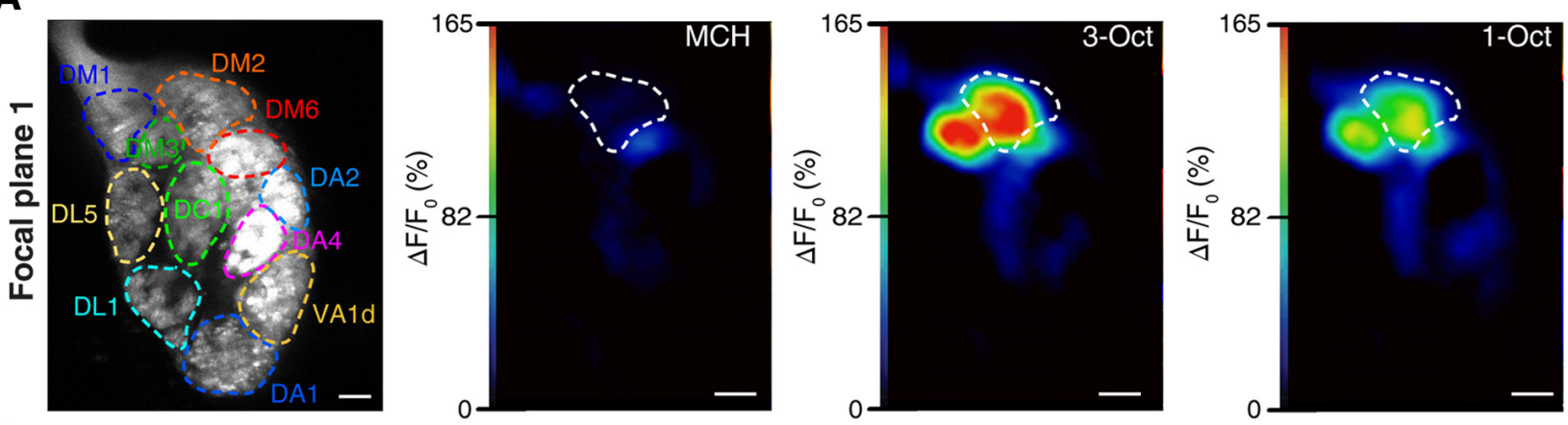

B
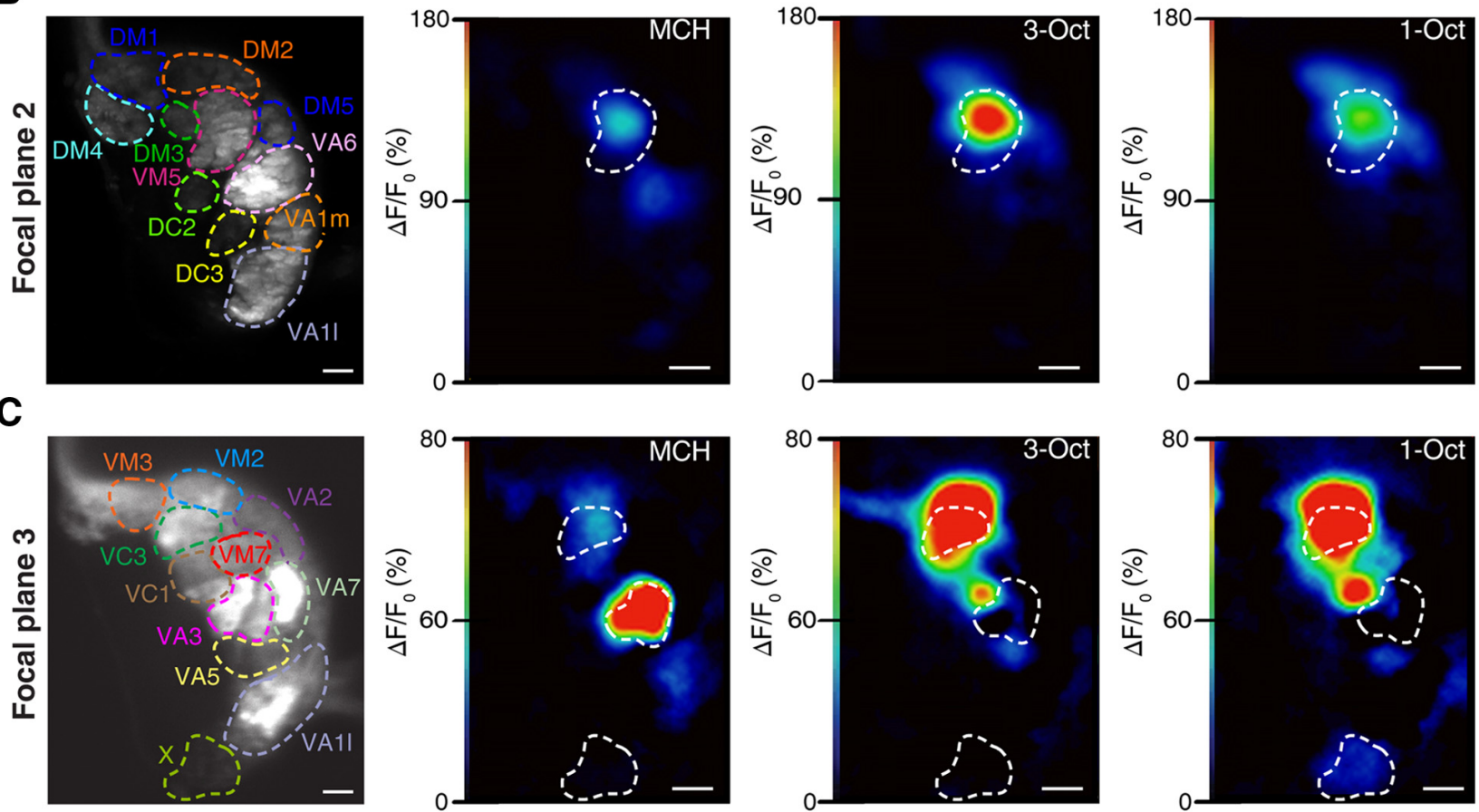

D
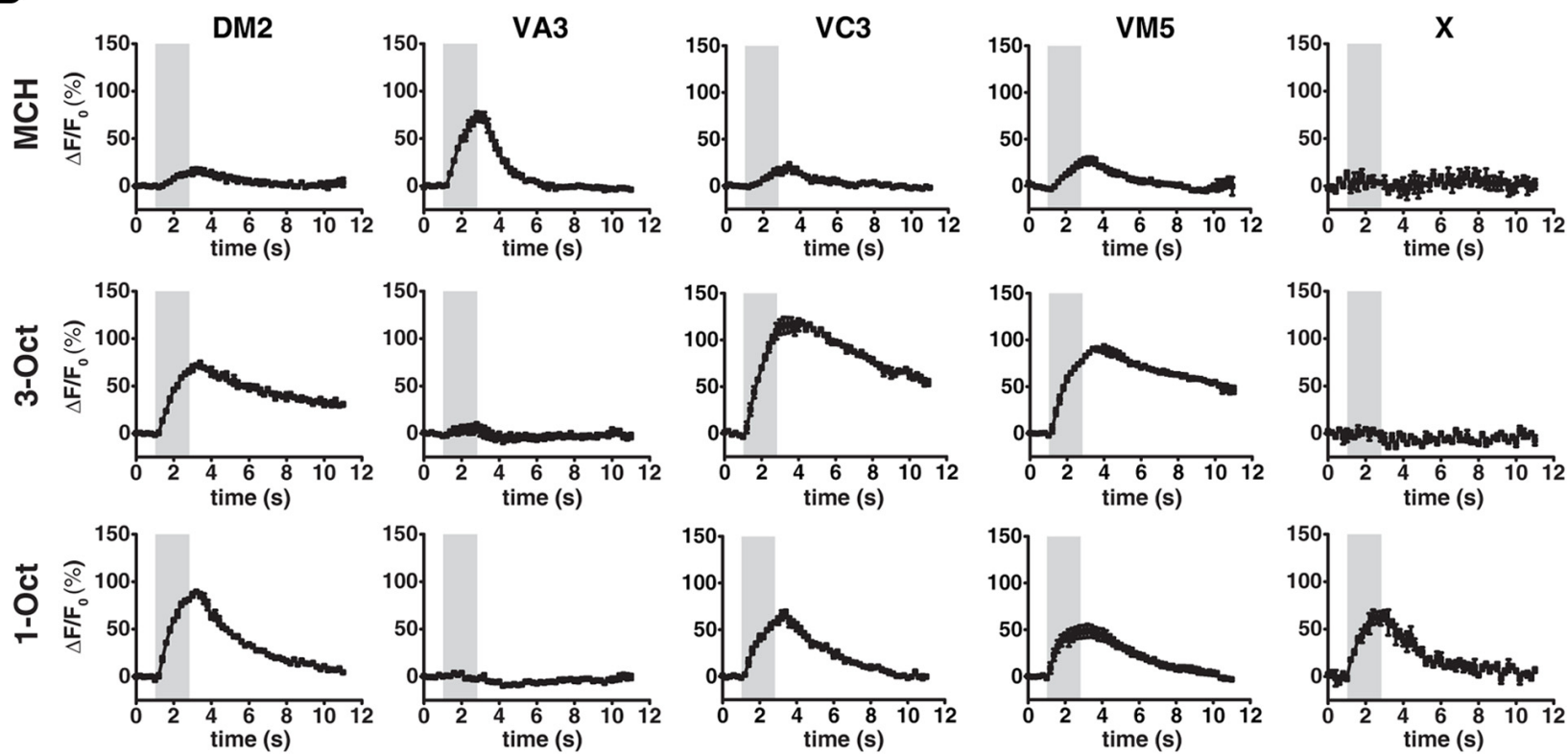

Figure 2. Two-photon $\mathrm{Ca}^{2+}$ imaging in olfactory sensory neurons in the antennal lobe reveals overlapping glomerular activity patterns evoked by similar odorants. $A-C$, Expression of the $\mathrm{Ca}^{2+}$ sensor $\mathrm{GCaMP3.0} \mathrm{in}$ olfactory sensory neurons visualized in three focal planes ( $A-C$ ) using two-photon microscopy shown for one representative animal. The outlines of identified olfactory glomeruli are indicated as dashed, colored lines. $\mathrm{Ca}^{2+}$ increase as relative change in fluorescence $\left(\Delta \mathrm{F} / \mathrm{F}_{0}\right)$ evoked by the odorants $\mathrm{MCH}, 3-0 \mathrm{ct}$, and 1-0ctis indicated as false colors. Scale bars, $10 \mu \mathrm{m}$. D, Time courses of $\mathrm{Ca}^{2+}$ changes in the antennal lobe of the fly shown in $A-C$ in the glomeruli DM2, VA3, VC3, VM5, and Xevoked by the odors $\mathrm{MCH}, 3-0 \mathrm{ct}$, and 1-0ct. The gray bars represent the time window of odor presentation. Graphs representmean $\pm \mathrm{SEM}$ of $\triangle \mathrm{F} / \mathrm{F}_{0}$ values acrossthreestimulations. 
A

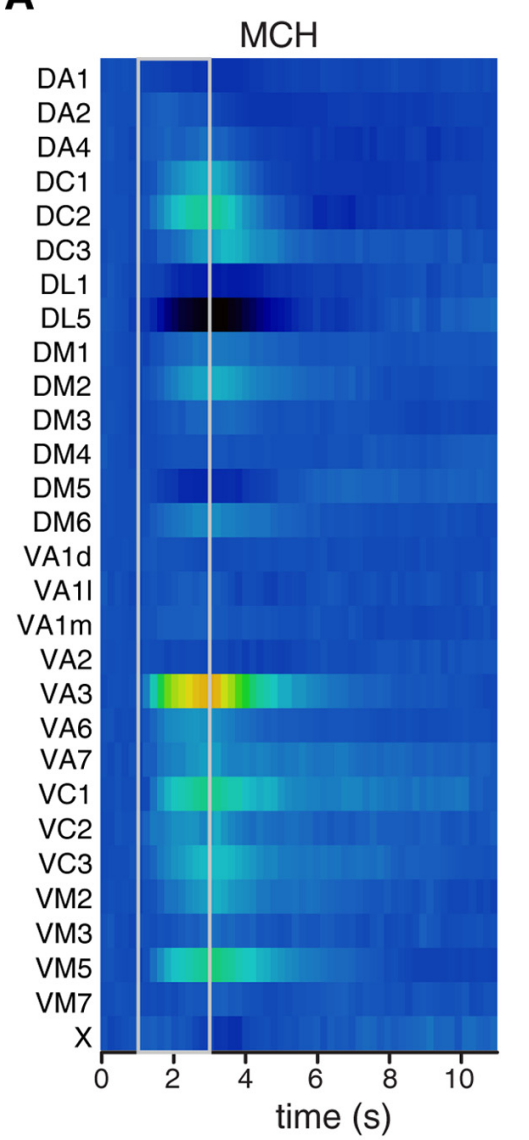

B

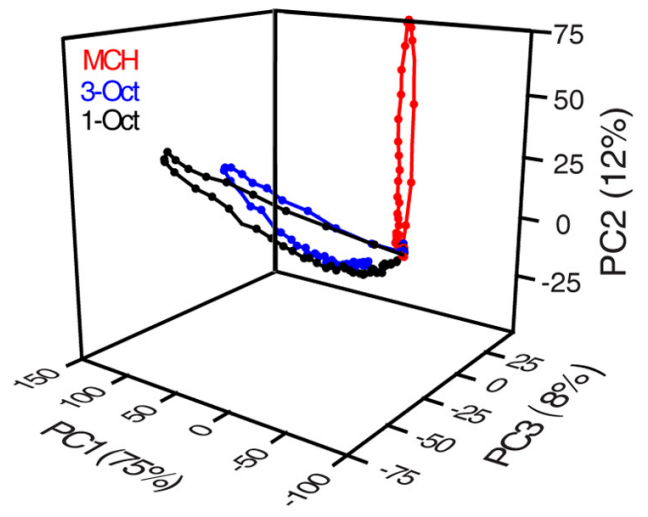

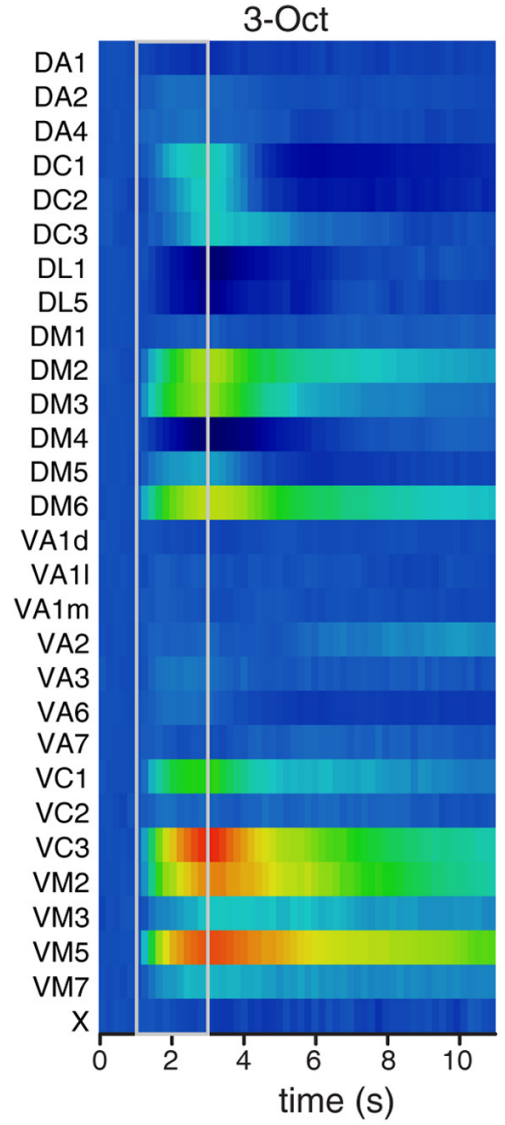

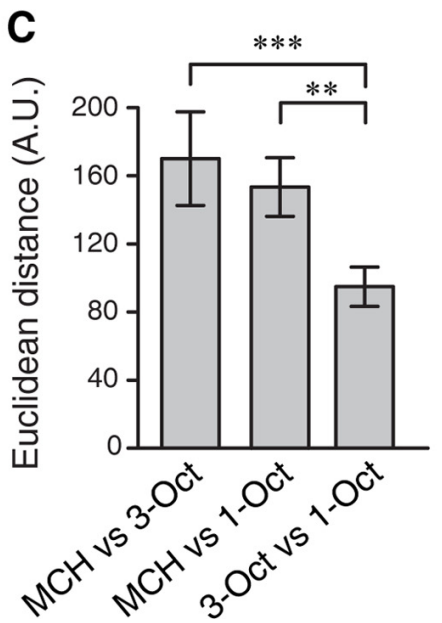

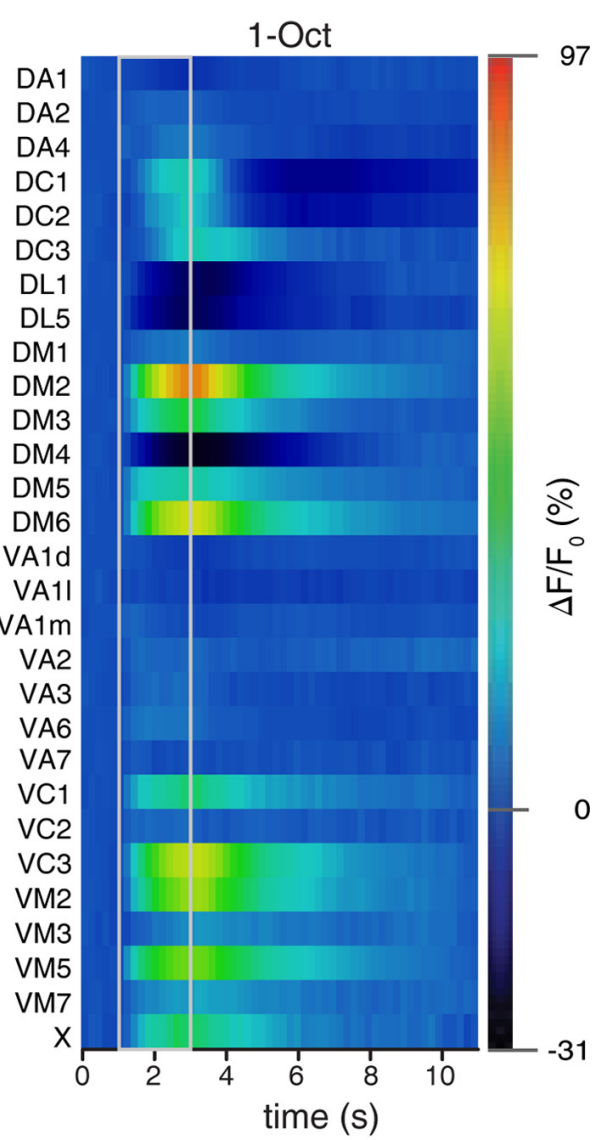

D

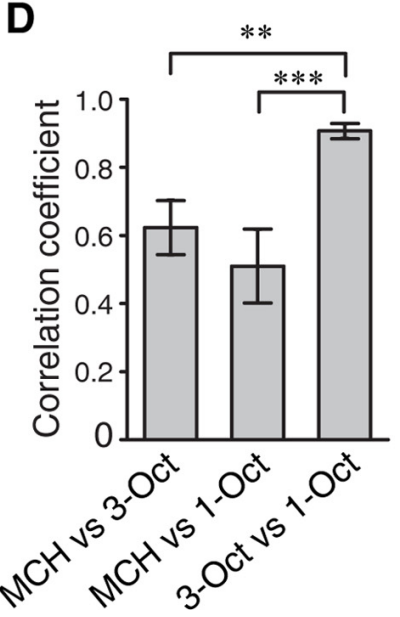

Figure 3. Dynamics and similarity of glomerular activity patterns in sensory neurons in the antennal lobe. $A, 0$ dor-evoked changes in fluorescence emitted by the $C^{2}{ }^{2+}$ sensor protein $\mathrm{GCaMP3} .0$ over time, shown as false colors in 29 identified glomeruli, in response to the odorants $\mathrm{MCH}, 3-0 \mathrm{ct}$, and 1-0ct. Gray lines indicate odor onset and offset. Values indicate mean; $n=6$ animals. $B, P C A$ of the time courses of the mean odor-evoked responses in olfactory sensory neurons across all 29 glomeruli. The first three principal components covered $>95 \%$ of the variance. $C$, Euclidean distances between the spatially distributed $\mathrm{Ca}^{2+}$ activity evoked by the three odorants at the time point of maximal $\mathrm{Ca}^{2+}$ change at odor offset. The Euclidean distance between the similar odors is significantly smaller than between dissimilar odors. D, Pearson's correlation between spatially distributed glomerular $\mathrm{Ca}^{2+}$ activity patterns evoked by the three odorants at the time point of maximal $\mathrm{Ca}^{2+}$ change (i.e., maximal correlation). The correlation coefficient is significantly higher between the similar odors than between the dissimilar odors. ${ }^{* * *} p<0.001$ (repeated measures one-way ANOVA with Bonferroni post hoc test). ${ }^{* *} p<0.01$ (repeated measures one-way ANOVA with Bonferroni post hoc test). $n=6$. Bars indicate mean \pm SEM.

We reasoned that the highly overlapping odor representation at the level of OSNs and OPNs for 1-Oct and 3-Oct compared with $\mathrm{MCH}$ might provide a physiological cause for their higher perceptual similarity. It has been suggested that inhibitory local interneurons in the antennal lobe contribute to a decorrelation of odor representations and, as a consequence, to fine discrimination of similar odors (Stopfer et al., 1997; Sachse and Galizia, 2002; Wilson and Laurent, 2005). To test whether local interneu- rons are indeed part of the neuronal circuitry contributing to olfactory acuity, we used temperature-sensitive shibire expression (Kitamoto, 2001) to selectively block synaptic transmission from subpopulations of inhibitory local interneurons during training and test. We focused on two groups of inhibitory local interneurons that had been described in detail previously (Chou et al., 2010; Seki et al., 2010): type I (LN1) and type II (LN2) interneurons, which are targeted by the two Gal4 enhancer trap lines, NP 
A
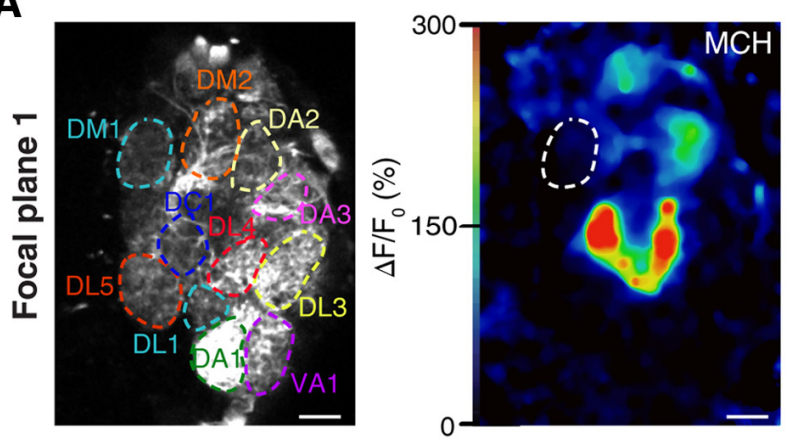

B

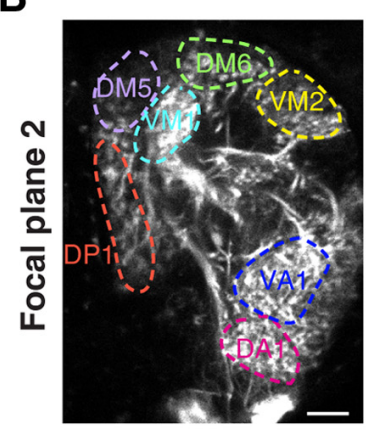

C

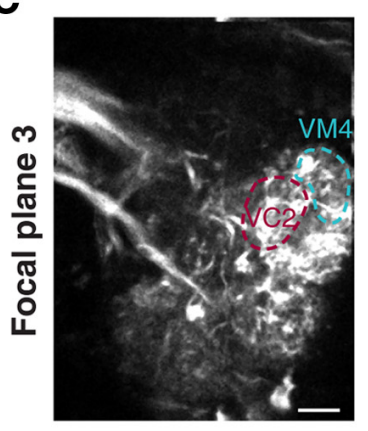

D
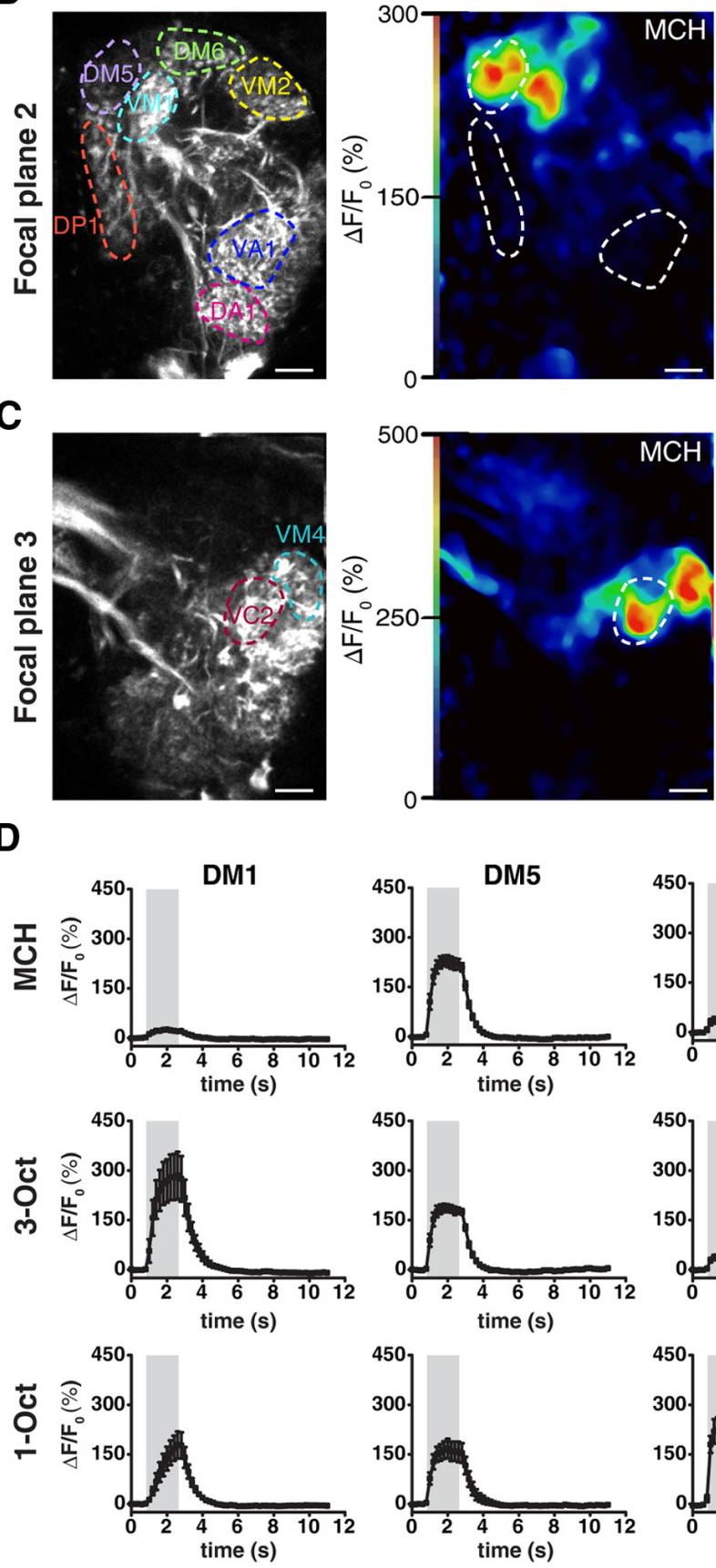

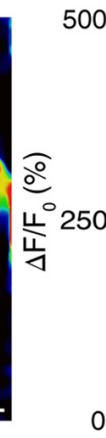

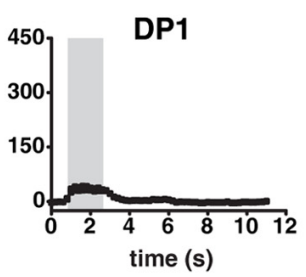
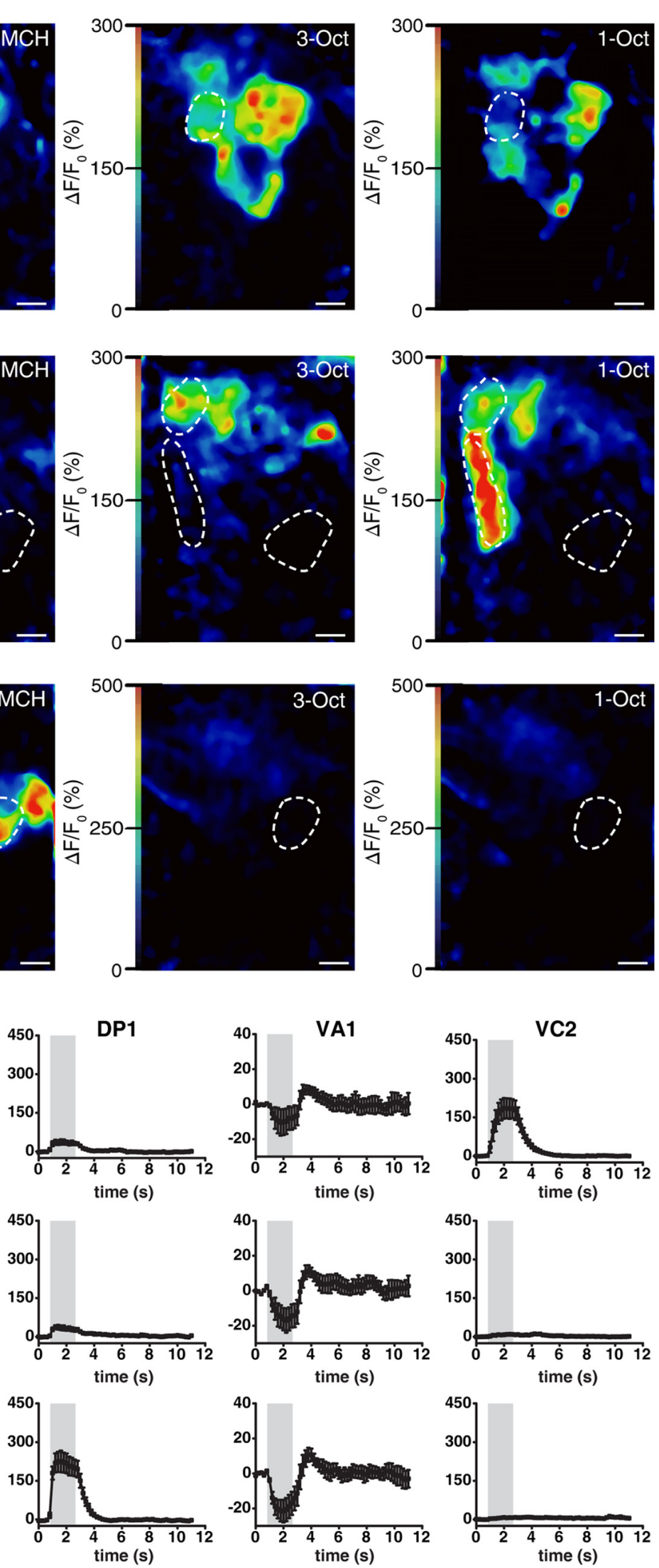

Figure 4. Two-photon $\mathrm{Ca}^{2+}$ imaging in olfactory projection neurons at the level of the antennal lobe. $\mathbf{A}-\mathbf{C}$, Expression of the $\mathrm{Ca}^{2+}$ sensor GCaMP3.0 in olfactory projection neurons visualized in three focal planes $(\mathbf{A - C})$ using two-photon microscopy for one representativeanimal. The outlines of identified olfactory glomeruliareindicatedas dashed, colored lines. $\mathrm{Ca}^{2+}{ }^{2}$ increaseas relativechangein fluorescence $\left(\Delta \mathrm{F} / \mathrm{F}_{0}\right)$ evoked by the odorants MCH,3-0ct, and 1-0ctisindicated as false colors. Scalebars, $10 \mu \mathrm{m}$. D, Time courses of mean $\mathrm{ad}^{2+}$ changes in the antennal lobe in the glomeruliDM1,DM5,DP1,VA1, and VC2 evoked bythe odors MCH, 3-0ct, and 1-0ct. Gray bars represent the time window of odor presentation. Graphs represent mean $\pm \mathrm{SEM}$. of $\Delta \mathrm{F} / \mathrm{F}_{0}$ values $(n=20$ animals $)$. 
A

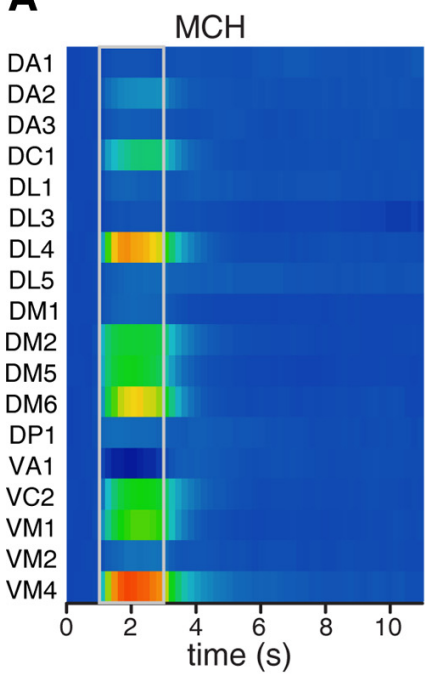

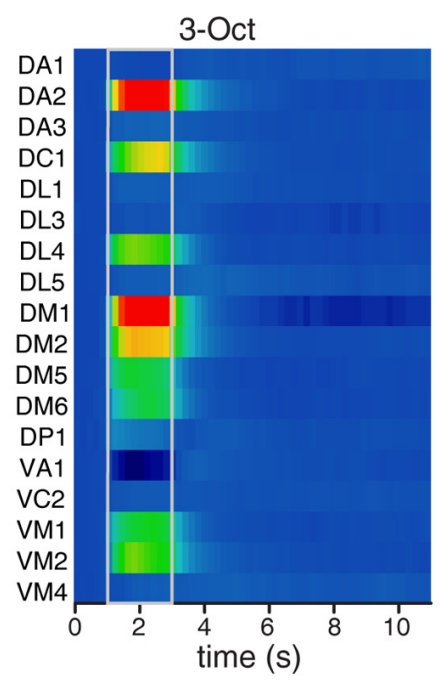

C

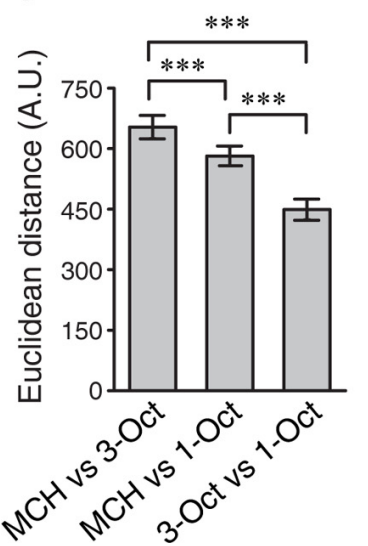

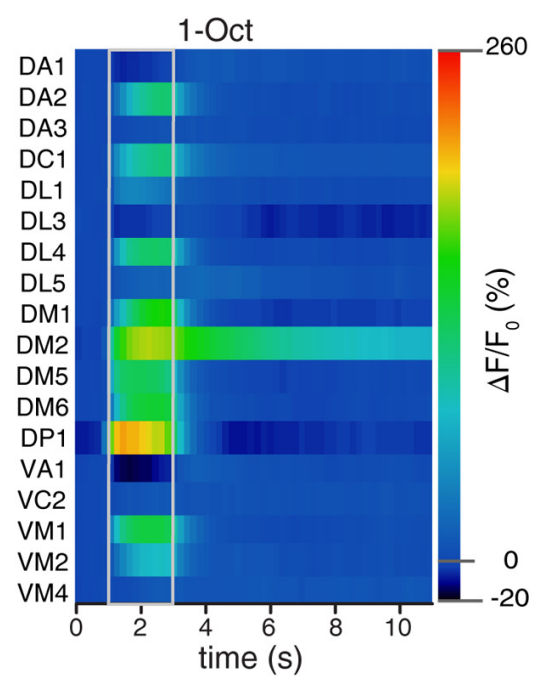

D

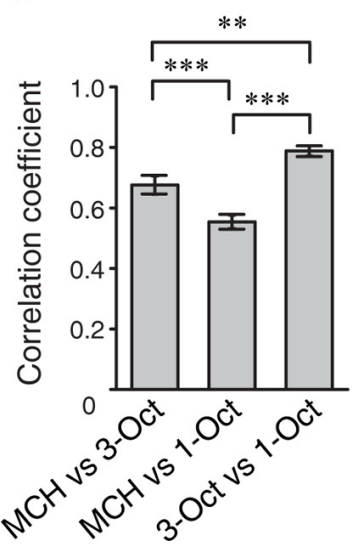

Figure 5. Dynamics and similarity of glomerular activity patterns in olfactory projection neurons in the antennal lobe. $A, 0$ dor-evoked changes in fluorescence emitted by the $C \mathrm{a}^{2+}$ sensor protein GCaMP3.0 over time, shown as false colors in 18 identified glomeruli, in response to the odorants MCH, 3-0ct, and 1-0ct. Odor onset and offset are indicated by gray lines. Values indicate mean; $n=$ 20 animals. $\boldsymbol{B}$, PCA of the time courses of the mean odor-evoked responses in olfactory projection neurons across all $18 \mathrm{glomeruli}$. The first three principal components covered $95 \%$ of the variance. C, Euclidean distances between the spatially distributed $\mathrm{Ca}^{2+}$ activity evoked by the three odorants at the time point of maximal $\mathrm{Ca}^{2+}$ change, which was variable across individuals. The Euclidean distance between the similar odors was significantly smaller than between dissimilar odors. $\boldsymbol{D}$, Pearson's correlation between spatially distributed glomerular $\mathrm{Ca}^{2+}$ activity patterns evoked by the three odorants at the time point of maximal correlation. The correlation coefficient was significantly higher between the similar odors than between the dissimilar odors. ${ }^{* *} p<0.001$ (repeated measures one-way ANOVA with Bonferroni post hoc test). ${ }^{* *} p<0.01$ (repeated measures one-way ANOVA with Bonferroni post hoc test). $n=20$. Bars indicate mean \pm SEM.

1227-Gal4 and NP 2426, respectively (Sachse et al., 2007). Type II interneurons could not be tested in learning assays because the respective Gal4 line (NP 2426) expresses Gal4 also in a large number of neurons located in the thoracic ganglia in a position similar to motor neurons (Fig. 6C), and flies of this Gal4 strain show locomotion deficits after expression of shibire ${ }^{t s}$ and at the restrictive temperature (Table 1). Type I local interneurons, however, could be tested, as we found the respective Gal4 line (NP 1227) to be specific for an expression in these neurons (Fig. 6D), and expression of shibire ${ }^{t s}$ did not alter locomotion, odor preferences, or electric shock avoidance at permissive or restrictive temperatures (Table 2). Of course, we cannot completely exclude Gal4 expression in neurons other than local interneurons that might be below our detection threshold. The temperaturedependent block of synaptic output from these neurons during both differential conditioning and the test situation significantly impaired discrimination between the two similar odors. Whereas at the permissive temperature of $25^{\circ} \mathrm{C}$ no significant difference between the three genotypes tested was observed $\left(F_{(2,25)}=3.13\right.$, $p=0.06$; one-way ANOVA), at the restrictive temperature of $32^{\circ} \mathrm{C}$ the learning index was significantly reduced in flies expressing shibire ${ }^{t s}$ in LN1-type interneurons (LN1 $>\mathrm{Shi}^{\text {ts }}$ vs LN1-Gal4: $t_{(24)}=3.1, p<0.05$; UAS:Shi ${ }^{\mathrm{ts}}$ vs LN1 $>$ Shi $^{\mathrm{ts}}: t_{(24)}=-3.5, p<$ 0.01; UAS:Shi ${ }^{\text {ts }}$ vs LN1-Gal4: $t_{(24)}=-0.5, p=1$; one-way ANOVA with Bonferroni post hoc test) (Fig. 6E). In contrast, performance after differential training of dissimilar odors (Fig. $6 F, G)$ was not affected by blocking synaptic transmission from type I local interneurons, independent of whether 1-Oct was trained against $\mathrm{MCH}\left(25^{\circ} \mathrm{C}: F_{(2,21)}=1, p=0.40 ; 32^{\circ} \mathrm{C}: F_{(2,21)}=\right.$ $0.1, p=0.92$; one-way ANOVA) (Fig. $6 F$ ) or 3-Oct was trained against $\mathrm{MCH}\left(25^{\circ} \mathrm{C}: F_{(2,21)}=0.7, p=0.49 ; 32^{\circ} \mathrm{C}: F_{(2,21)}=0.8\right.$, $p=0.46$; one-way ANOVA) (Fig. $6 G$ ). In addition, absolute training with only one odor, causing avoidance of this odor (Fig. $7 A, B$ ) and generalization across a similar odor (Fig. $7 C, D$ ), was not dependent on type I inhibitory local interneurons either at the permissive or at the restrictive temperature (Fig. $7 A ; 25^{\circ} \mathrm{C}$ : $F_{(2,24)}=0.8, p=0.46 ; 32^{\circ} \mathrm{C}: F_{(2,33)}=3.6, p=0.04$, post hoc pairwise comparison did not reveal any significance; Figure $7 B$; $25^{\circ} \mathrm{C}: F_{(2,23)}=0.8, p=0.47 ; 32^{\circ} \mathrm{C}: F_{(2,27)}=1.6, p=0.22$; Figure $7 C, 25^{\circ} \mathrm{C}: F_{(2,21)}=2.3, p=0.13 ; 32^{\circ} \mathrm{C}: F_{(2,21)}=0.5, p=0.63$; 
A

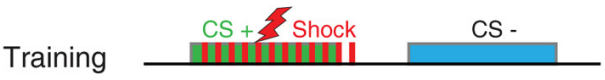

B
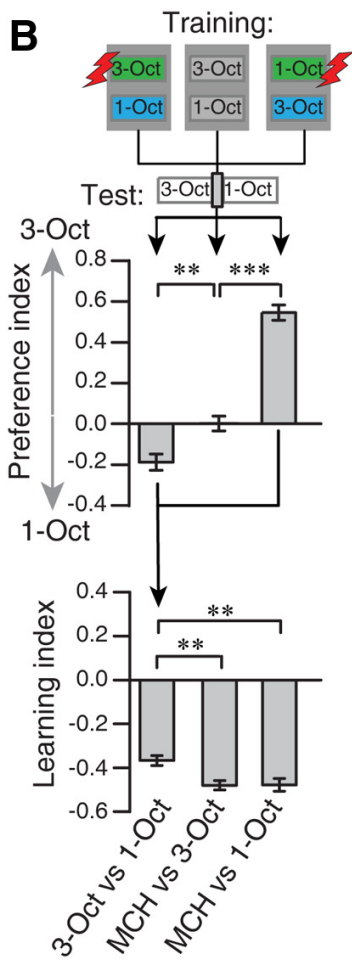

E

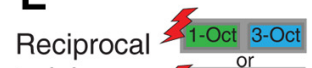
training:

Test: [1-Oct] 3 -Oct
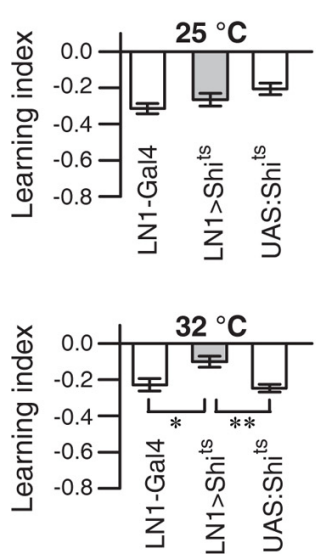

C

F training:
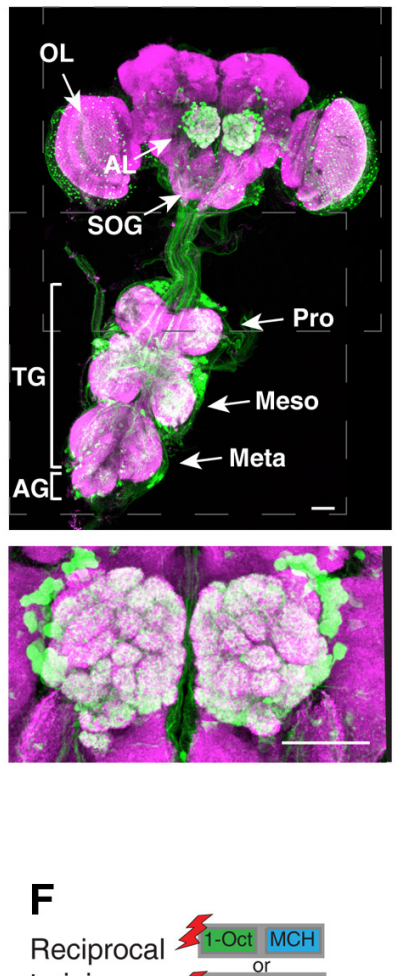

Test: 1-Oct
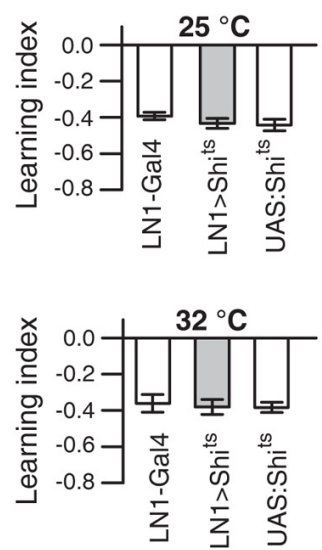
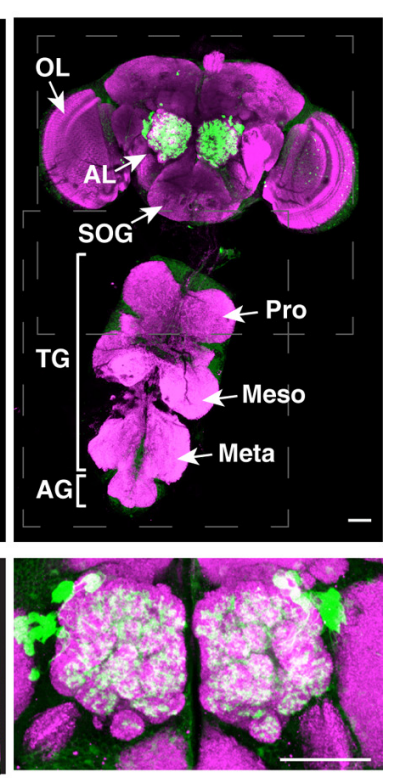

Test:

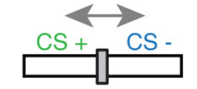

Choice situation

D

G

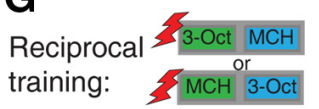

Test:
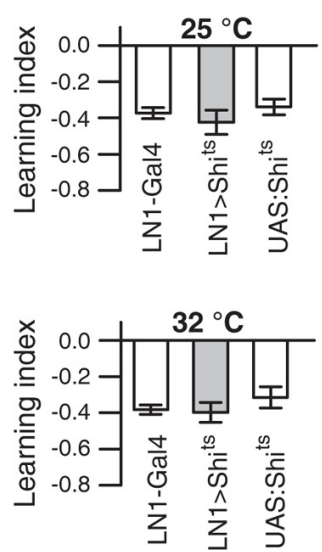

Figure 6. Local interneurons of the antennal lobes are required for fine discrimination of similar odors. $A$, Schematic illustration of the differential conditioning regimen. The green box represents the odor that is, as conditioned stimulus (CS $\left.{ }^{+}\right)$, temporally paired with electric shocks (red boxes). The blue box represents the odor presented without punishment (CS ${ }^{-}$). During the test, the trained flies are subjected to a choice situation between $C S^{+}$and $C S^{-}$. B. Differential training of either 3-0ct or 1-0ct resulted in avoidance of the odor temporally paired with the electric shock (as indicated as green boxes in the schematic illustration) in a choice situation for both odorants (test) (top). ${ }^{* * *} p<0.001$ (one-way ANOVA with Bonferroni post hoc test). ${ }^{*} p<0.05$ (one-way ANOVA with Bonferroni post hoc test). $n=8-13$ for each experiment. Bars indicate mean \pm SEM. When both odorants were presented without electric shocks, the response was indifferent. When the preference indices of the reciprocal training situations were combined (bottom), the resulting learning index was significantly lower compared with training with either 1-0ct or 3-0ct against the dissimilar odorant MCH (bottom). ${ }^{* *} p<0.01$ (one-way ANOVA with Bonferroni post hoc test). $n=8-13$ for each experiment. Bars indicate mean \pm SEM. C, GFP expression in the central brain and the thoracic ganglia under control of NP2426Gal4 (LN2-Gal4). D, GFP expression in the central brain and the thoracic ganglia under control of NP1227-Gal4 (LN1-Gal4). The expression is confined to local interneurons in the antennal lobe, as indicated in the magnified image. $\boldsymbol{C}, \boldsymbol{D}$, Green represents anti-GFP immune reactivity; magenta represents anti-nc 82 immune reactivity. Scale bars, $50 \mu \mathrm{m}$. AL, Antennal lobe; $0 \mathrm{~L}$, optic
Figure $7 D ; 25^{\circ} \mathrm{C}: F_{(2,22)}=0.1, p=0.90$; $32^{\circ} \mathrm{C}: F_{(2,21)}=0.1, p=0.95$; one-way ANOVA). These results demonstrate a role of inhibitory local interneurons for behaviorally expressed fine discrimination of similar odors in Drosophila. Moreover, chemical synaptic transmission from these neurons is dispensable for the discrimination of dissimilar odors and for odor generalization.

Differential training causes enhanced olfactory acuity due to conditioned excitation and conditioned inhibition The experiments reported so far show that flies generalize across chemically similar odors after absolute training, but they can discriminate between them after differential training. We asked next whether it is indeed the differential training procedure that enhances fine discrimination as observed in choice behavior. We therefore subjected flies to a choice test between 1-Oct and 3-Oct after either differential or absolute training (Fig. 8A). In differential training, one odor was presented as $\mathrm{CS}^{+}$, and the other as $\mathrm{CS}^{-}$, whereas in absolute training the $\mathrm{CS}^{-}$was replaced by the presentation of the diluent only. We found that avoidance of the $\mathrm{CS}^{+}$was much more pronounced after differential training than after absolute training (1-Oct absolute vs differential training: $t_{(30)}=-4.0$, $p<0.01 ; 3$-Oct absolute vs differential training: $t_{(30)}=-3.4, p<0.01$; twosample $t$ test) (Fig. 8A). This demonstrates that a differential training procedure can cause an enhancement in olfactory acuity. Again, we found asymmetric learning in the choice situation: learned responses were higher in the case of 1-Oct as $\mathrm{CS}^{+}$ compared with 3-Oct as $\mathrm{CS}^{+}$(Fig. 8A). The two training regimens, absolute and differential training, differed only in the

$\leftarrow$

lobe; SOG, suboesophageal ganglion; TG, thoracic ganglia; $A G$, abdominal ganglia; Pro, prothoracic ganglion; Meso, mesothoracic ganglion; Meta, metathoracic ganglion. $\boldsymbol{E}$, Differential, reciprocal training of 1-0ct and 3-0ct in flies expressing shibire $e^{\text {ts }}$ under control of LN1-Gal4 and the respective genetic controls. Training and test at the permissive temperature of $25^{\circ} \mathrm{C}$ did not result in different learning indices for all three genotypes. Significantly decreased learning was observed in flies expressing shibire ${ }^{t s}$ in local interneurons at the restrictive temperature of $32^{\circ} \mathrm{C} .{ }^{* *} p<0.01$ (one-way ANOVA with Bonferroni post hoc test). ${ }^{*} p<0.05$ (one-way ANOVA with Bonferroni post hoc test). $n=8-13$ for each experiment. Bars indicate mean \pm SEM. $\boldsymbol{F}, \boldsymbol{G}$, Differential, reciprocal training of the dissimilar odorants 1-0ct against MCH $(\boldsymbol{F})$ or 3-0ct against MCH (G) was not significantly $(p>0.05)$ affected by a temperature-dependent block of local interneurons. One-way ANOVA with Bonferroni post hoc test. $n=8-13$ for each experiment. Bars indicate mean $\pm \mathrm{SEM}$. 
Table 1. Blocking synaptic transmission in neurons targeted by NP2426-Gal4 results in locomotion deficits ${ }^{a}$

\begin{tabular}{lllll}
\hline Experiment & Temperature, $^{\circ} \mathrm{C}$ & LN2-Gal4 & LN2 $>$ Shi & UAS:Shi \\
\hline Climbing index & 25 & $0.77 \pm 0.02$ & $0.85 \pm 0.02$ & $0.68 \pm 0.03$ \\
& 32 & $0.60 \pm 0.03$ & $0.40 \pm 0.05$ & $0.73 \pm 0.05$ \\
\hline
\end{tabular}

${ }^{a}$ At the restrictive temperature, expression of shibire ${ }^{\text {ts }}$ under control of NP2426-Gal4 led to a decreased climbing index compared with the genetic controls, Values indicate mean $\pm S E M ; n=5$ for each experiment.

Table 2. Blocking synaptic transmission in neurons targeted by NP1227-Gal4 does not alter locomotion, shock avoidance, or odor preference ${ }^{a}$

\begin{tabular}{llrrr}
\hline Experiment & Temperature, $^{\circ} \mathrm{C}$ & \multicolumn{1}{l}{ LN1-Gal4 } & \multicolumn{1}{l}{ LN1 $>$ Shi } & \multicolumn{1}{c}{ UAS:Shi } \\
\hline Climbing index & 25 & $0.71 \pm 0.01$ & $0.71 \pm 0.04$ & $0.72 \pm 0.02$ \\
& 32 & $0.73 \pm 0.06$ & $0.71 \pm 0.04$ & $0.73 \pm 0.03$ \\
Shock avoidance & 25 & $-0.64 \pm 0.03$ & $-0.73 \pm 0.05$ & $-0.65 \pm 0.04$ \\
& 32 & $-0.63 \pm 0.08$ & $-0.57 \pm 0.10$ & $-0.45 \pm 0.10$ \\
3-0ct preference & 25 & $0.31 \pm 0.04$ & $0.38 \pm 0.09$ & $0.30 \pm 0.06$ \\
& 32 & $0.32 \pm 0.05$ & $0.38 \pm 0.05$ & $0.30 \pm 0.06$ \\
1-0ct preference & 25 & $0.49 \pm 0.05$ & $0.44 \pm 0.06$ & $0.32 \pm 0.06$ \\
& 32 & $0.26 \pm 0.03$ & $0.28 \pm 0.06$ & $0.21 \pm 0.06$ \\
\hline
\end{tabular}

${ }^{a}$ Comparable climbing indices were observed in flies expressing shibire ${ }^{\text {ts }}$ under control of NP1227-Gal4 at the permissive and the restrictive temperature. $n=5$ for each experiment. The shock avoidance index of flies expressing shibire ${ }^{t s}$ in LN1 neurons was comparable with the genetic controls at the permissive and the restrictive temperature. The behavior towards 1-octen-3-ol and 3-octanol in a T-maze choice test with the diluent was not significantly different in flies expressing shibire ${ }^{\text {ts }}$ under control of NP1227-Gal4 at both temperatures tested. Values indicate mean \pm SEM; $n=8$ for each experiment.

presentation of the $\mathrm{CS}^{-}$, and the temporal order of $\mathrm{CS}^{+}$and $\mathrm{CS}^{-}$ presentation was balanced in each experiment. We wondered whether the timing of the $\mathrm{CS}^{-}$might affect the learned discrimination. This is of importance as the enhanced discriminability of the two odors might have resulted from potential backward conditioning caused by the $\mathrm{CS}^{-}$after the $\mathrm{CS}^{+}$/unconditioned stimulus (US) stimulation. Indeed, inhibitory learning through backward US-CS paring has been described in Drosophila and honeybees (Hellstern et al., 1998; Tanimoto et al., 2004). However, when preference indices of the two groups of animals were separated post hoc, we did not find any significant difference between those animals that had perceived the $\mathrm{CS}^{+}$first and the $\mathrm{CS}^{-}$ afterward and those animals that had received $\mathrm{CS}^{+}$and $\mathrm{CS}^{-}$in the reverse order (1-Oct differential training $\mathrm{CS}^{+} \mathrm{vs} \mathrm{CS}^{-}: t_{(14)}=$ $1.1, p=0.3$; 1 -Oct absolute training $\mathrm{CS}^{+}$vs $\mathrm{CS}^{-}: t_{(14)}=-1.6$, $p=0.13$; 3 -Oct absolute training $\mathrm{CS}^{+}$vs CS $^{-}: t_{(14)}=-1.6, p=$ 0.13 ; 3-Oct differential training $\mathrm{CS}^{+}$vs $\mathrm{CS}^{-}: t_{(14)}=-0.9, p=$ 0.38 ; two-sample $t$ test) (Fig. $8 B$ ). The contribution of the $\mathrm{CS}^{-}$to enhanced olfactory acuity is therefore not equivalent to a US-CS backward pairing. However, its effect could not be attributed to a mere adaptation to the odorant used as $\mathrm{CS}^{-}$either, because exposure of the flies to either odor for $1 \mathrm{~min}$ did not alter their subsequent behavior toward the odor $(\mathrm{MCH}$ naive vs preexposed: $t_{(22)}=-0.9, p=0.37$; 1-Oct naive vs preexposed: $t_{(22)}=$ $0.1, p=0.92$; 3 -Oct naive vs preexposed: $t_{(22)}=-1.6, p=0.12$; two-sample $t$ test) (Fig. 1B). To further clarify the contribution of the $\mathrm{CS}^{-}$to the enhanced odor acuity, we asked whether the odor presented as a $\mathrm{CS}^{-}$induced conditioned inhibition independently of the temporal relation to the US. Therefore, we trained the animals again in either an absolute or a differential training regimen (i.e., with or without presenting a $\mathrm{CS}^{-}$), but we tested subsequently for conditioned inhibition in a choice situation between the $\mathrm{CS}^{-}$and a novel, dissimilar odorant (MCH) (Fig. 8C). In the absence of a $\mathrm{CS}^{-}$, the animals significantly avoided the odor that was similar to the $\mathrm{CS}^{+}$, which clearly reflected the generalization across similar odorants (Fig. 8C, white bars).
A

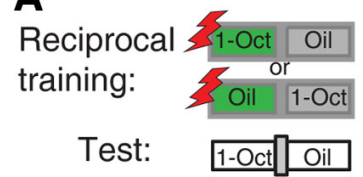

B
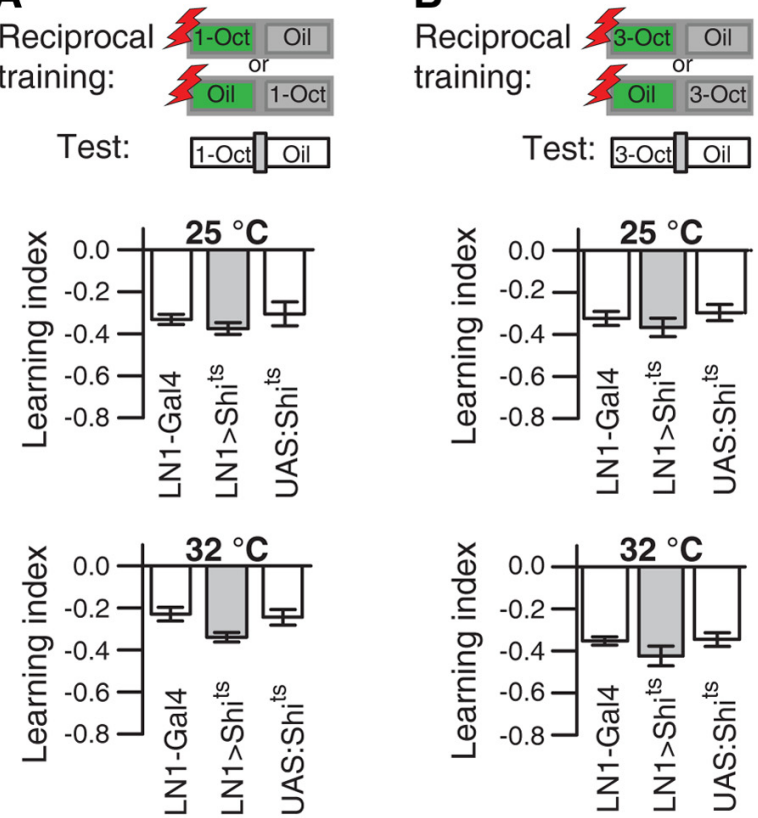

C

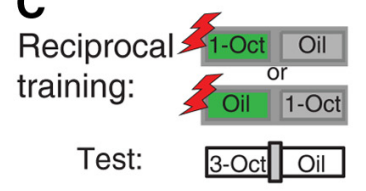

D
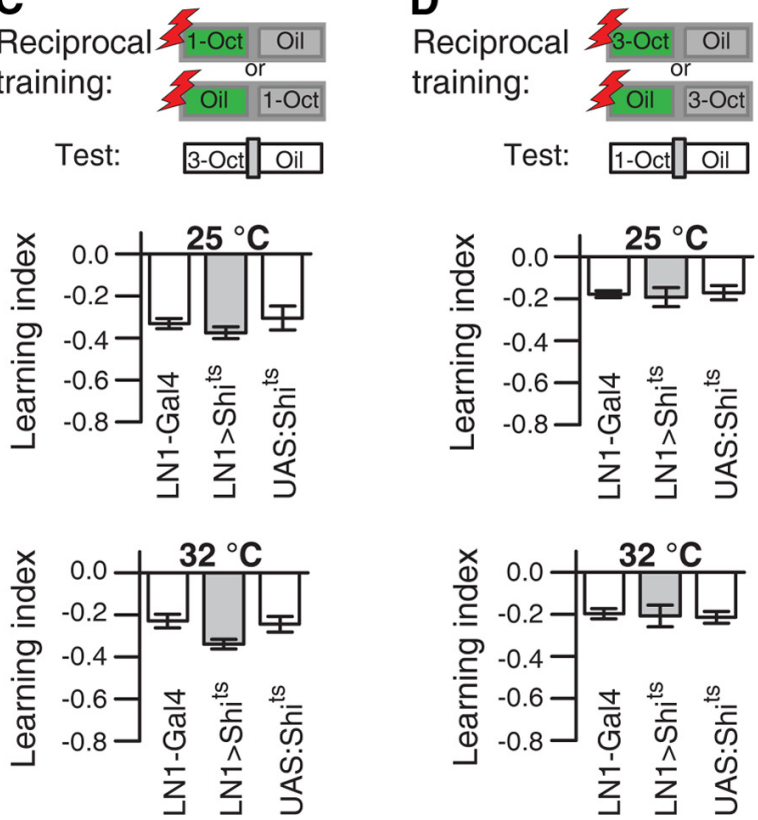

Figure 7. Synaptic output of type I local interneurons (LN1) is not necessary for the association of an odor with electric shocks and generalization across similar odorants. $\boldsymbol{A}, \boldsymbol{B}$, Absolute, reciprocal training with 1-0ct $(\boldsymbol{A})$ or 3-0ct $(\boldsymbol{B})$ against the diluent was not significantly affected by a temperature-dependent block of synaptic output from local interneurons. One-way ANOVA, $n=8-12$ for each experiment. Bars indicate mean \pm SEM. C, D, Generalization after absolute, reciprocal training of either $1-0 \mathrm{ct}(\boldsymbol{C})$ or 3-0ct $(\boldsymbol{D})$ to the similar, but not identical, odorant in a test situation was not significantly $(p>0.05)$ affected by a temperaturedependent block of synaptic output from local interneurons. One-way ANOVA, $n=8-12$ for each experiment. Bars indicate mean \pm SEM.

However, in differential training during which the similar odor was explicitly not paired with the punishment $\left(\mathrm{CS}^{-}\right)$, a significant decrease of this generalization was apparent (Fig. $8 C$, gray bars) (1-Oct absolute vs differential training: $t_{(30)}=-2.8, p<$ 0.01 ; 3 -Oct absolute vs differential training: $t_{(30)}=3.0, p<0.01$; two-sample $t$ test). Conclusively, differential training of similar odors comprises not only a conditioned excitation, but also a conditioned inhibition, which can contribute to an enhancement 
A
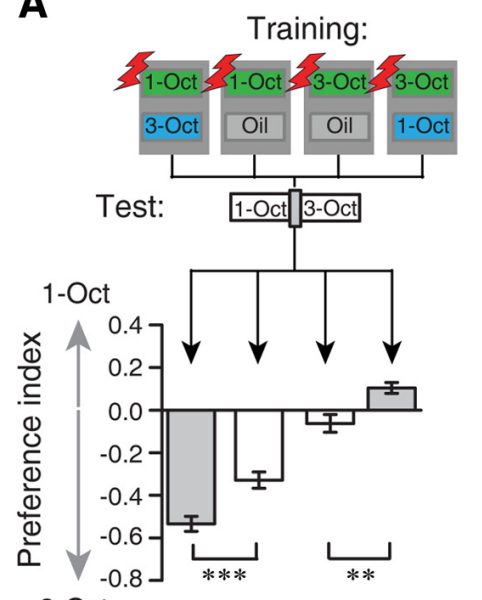

3-Oct

C

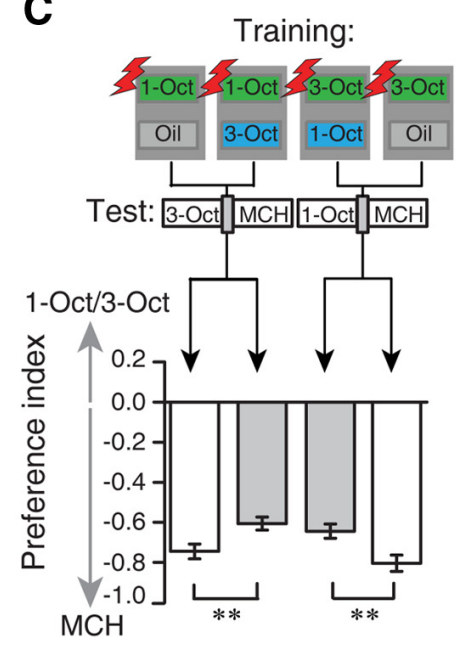

E

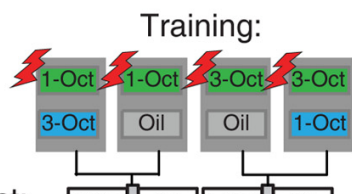

Test:

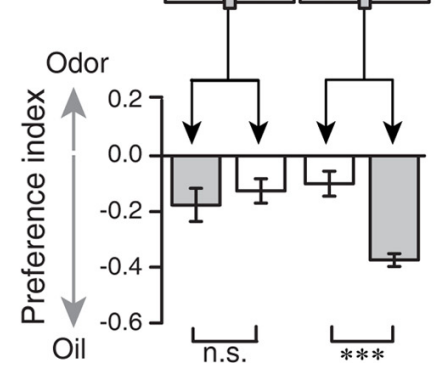

B

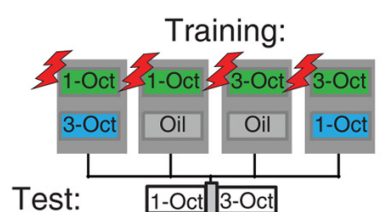

Test:
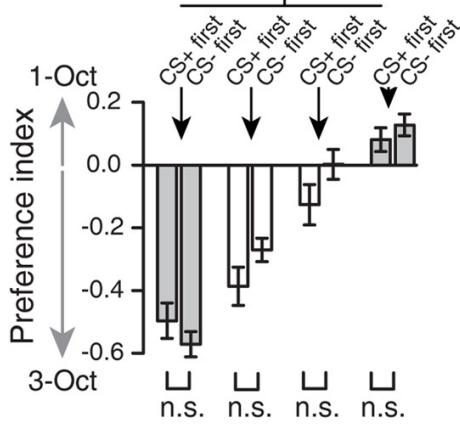

D

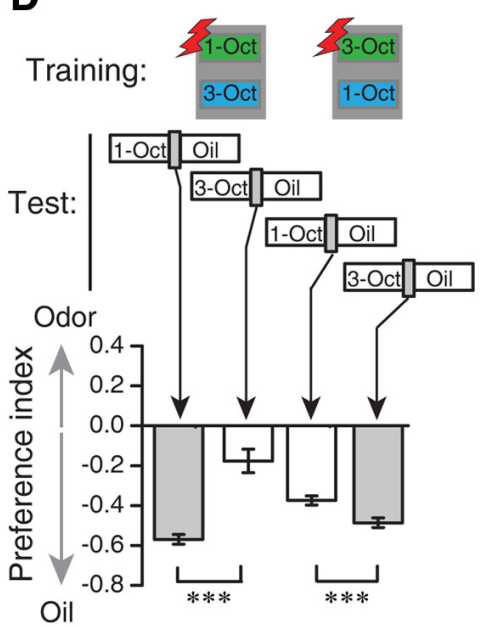

$\mathbf{F}$

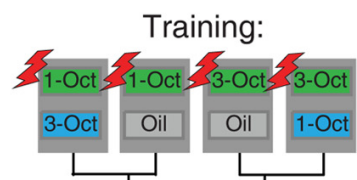

Test:

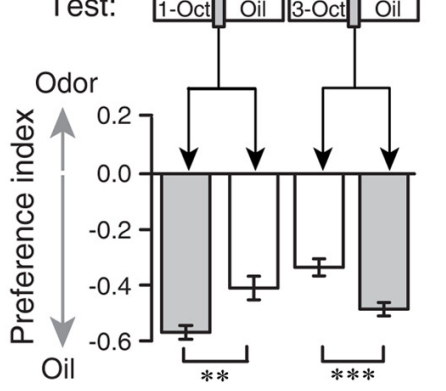

in learned odor discrimination. However, in this experiment, for conditioned inhibition the animals had no choice situation between the similar odors. We wondered whether differential training shifts the balance from generalization across to discrimination between them (Mishra et al., 2010). As an alternative hypothesis, the ability to discriminate in an appropriate choice situation between two similar odors might be independent of the ability to generalize across the similar odors. We subjected the animals to differential training between 1-Oct and 3-Oct and tested them afterward in a choice situation with the $\mathrm{CS}^{+}$versus the diluent only or the $\mathrm{CS}^{-}$versus the diluent, respectively. Not surprisingly, we found that animals strongly avoided the $\mathrm{CS}^{+}$if only this odor was present (Fig. $8 D$, gray bars). However, when tested for the $\mathrm{CS}^{-}$after differential training, the animals still showed a generalized response, although it is significantly smaller (Fig. $8 D$, white bars) (1-Oct CS+ vs $\mathrm{CS}^{-}: t_{(30)}=-6.1, p<0.01 ; 3$-Oct $\mathrm{CS}^{+}$ vs CS-: $t_{(30)}=-3.4, p<0.01$; two-sample $t$ test). This response is shown again in Figure $8 E$ (gray bars) in direct comparison with the half-scores after absolute training shown in Figure $1 G, H$. The generalization after differential training with 1-Oct as $\mathrm{CS}^{+}$was not weaker than after absolute training, and it was even stronger when 3 -Oct was used as $\mathrm{CS}^{+}$(1-Oct absolute vs differential training: $t_{(30)}=0.7, p=0.5$; 3-Oct absolute vs differential training: $t_{(30)}=5.5, p<0.01$; two-sample $t$ test) (Fig. $8 E$ ). This demonstrates that learned fine odor discrimination (i.e., enhanced olfactory acuity) is behaviorally expressed only if a choice situation between two odors for discrimination exists during the test situation. In other words, flies discriminate only if it is required in the test situation. In test situations in which only one odor is present, a generalized response is observed, independent of the

$\leftarrow$

and 3-0ct and a subsequent test for the nonreinforced odor against the diluent. Whereas the behavior was not different for 1-0ct as a $\mathrm{CS}^{+}$, pairing of 3-0ct with electric shocks resulted in a significantly stronger avoidance of 1-0ct after differential training. n.s., Not significant $(p>0.05$; two-sample $t$ test). ${ }^{* * *} p<0.001$ (two-sample $t$ tests). $n=16$ for each experiment. Bars indicate mean \pm SEM. $\boldsymbol{F}$, Comparison of absolute and differential training with 1-0ct and 3-0ct and a subsequent test for the $\mathrm{CS}^{+}$against the diluent. Differential training (gray bars) resulted in a significantly stronger avoidance of the $\mathrm{CS}^{+}$compared with absolute training (white bars). ${ }^{* *} p<0.001$ (two-sample $t$ test). ${ }^{* *} p<0.01$ (twosample $t$ test). $n=16$ for each experiment. Bars indicate mean \pm SEM. 
A<smiles>CCCCCOC(=O)OCCCCCCC(C)=O</smiles>

C

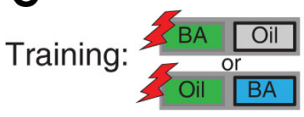

Test: Odor Oil

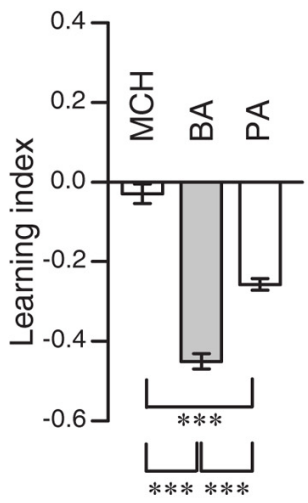<smiles>Oc1ccc(O)cc1</smiles>

4-methylcyclohexanol
B

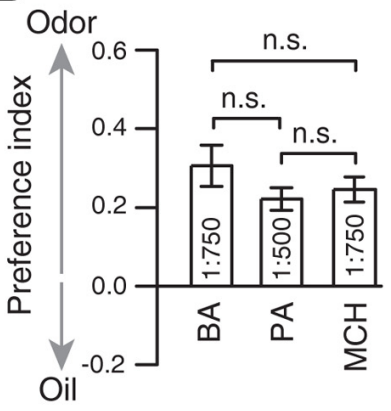

$E$

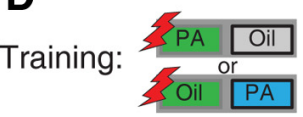

Training:

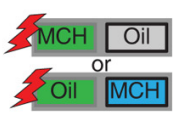

Test: Odor Oil

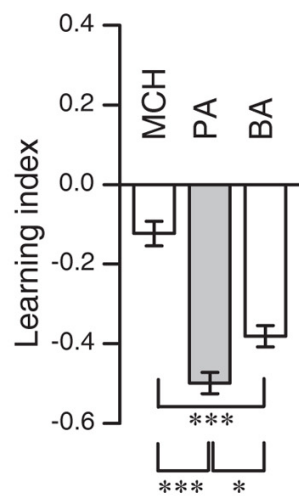

Test: Odor Oil

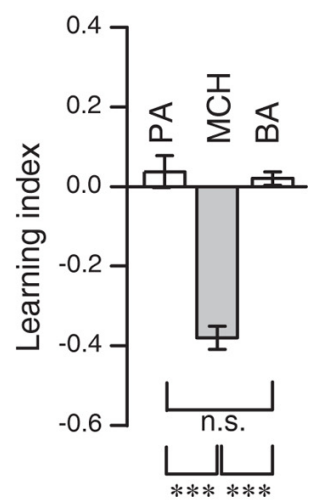

$\mathbf{F}$

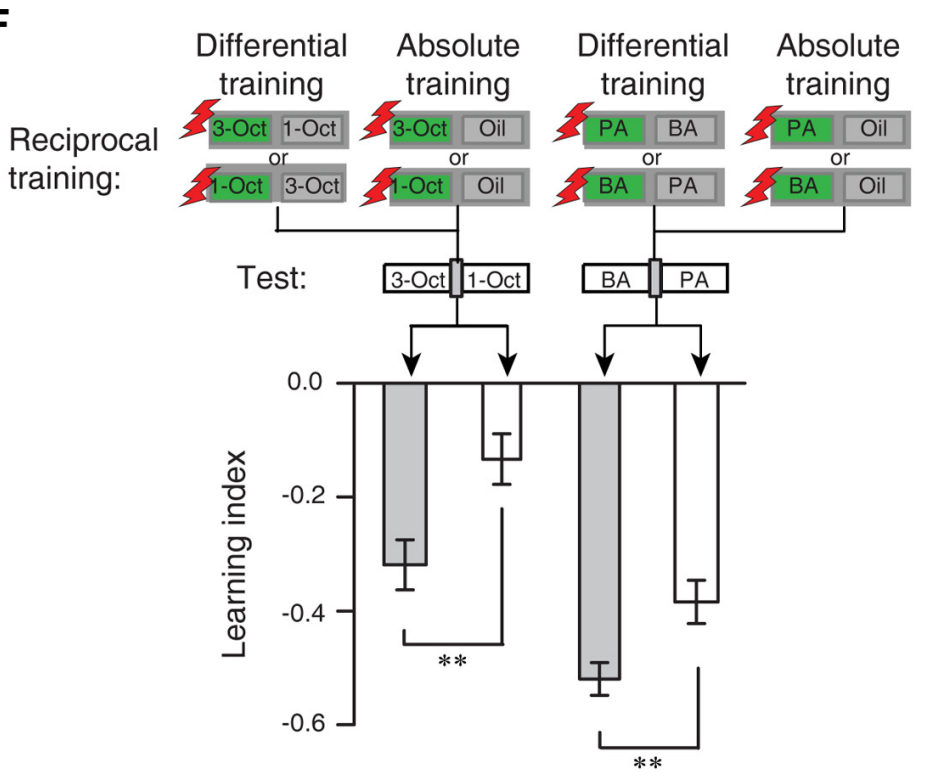

Figure 9. Generalization across a second pair of similar odors. $A$, Chemical structures of the three odorants used: $B A, P A$, and $M C H . B$, Odor preferences of naive flies for the odorants $\mathrm{BA}, \mathrm{PA}$, and $\mathrm{MCH}$ at the dilutions indicated within the bars in a T-maze choice situation against the diluent mineral oil (Oil). No difference between the three odors at the indicated concentrations were observed. n.s., Not significant ( $p>0.05$; one-way ANOVA with Bonferroni post hoc test). Bars indicate mean \pm SEM; $n=4-8$ for each experiment. C $\boldsymbol{E}$, Absolute, reciprocal training of $B A(C), P A(D)$, and $M C H(E)$ against the diluent and test for the avoidance of each odor. In all cases, flies strongly avoided the trained odor (gray bars). Generalization was observed if flies are trained with either BA or PA and tested for the respective similar odor, but not if they were tested for the dissimilar odor $\mathrm{MCH}(C, D)$. Flies did not generalize to $B A$ or $P A$ after training with $\operatorname{MCH}(\boldsymbol{E}): n=12$ for each experiment. Bars indicate mean \pm SEM; n.S., Not significant $(p>0.05) ;{ }^{*} p<0.05 ;{ }^{* * *} p<0.001$; one-way ANOVA with Bonferroni post hoc test. $\boldsymbol{F}$, Comparison between preference indices after absolute and differential training. Aversive learning was significantly higher after differential training compared with absolute training, both for the odor pairs 1-0ct/3-0ct (shown also in Fig. $8 A$ ) and BA/PA. ${ }^{* *} p<0.01$ (two-sample $t$ test). $n=32$ for each experiment.

type of previous training. This finding prompted us to analyze the collected data in the opposite context as well. We asked whether differential training, compared with absolute training, changes the response to the $\mathrm{CS}^{+}$if only the $\mathrm{CS}^{+}$is present in the test situation. Interestingly, animals that were subjected to a differential training procedure responded to the $\mathrm{CS}^{+}$with an increased avoidance compared with animals that had been subjected to an absolute training procedure (1-Oct absolute vs differential training: $t_{(30)}=3.3, p<0.01 ; 3$-Oct absolute vs differential training: $t_{(30)}=3.8 ; p<0.01$; two-sample $t$ test) (Fig. $8 F$ ). The enhanced olfactory acuity caused by differential training with similar odors is therefore not only based on conditioned inhibition. Rather, during training, the $\mathrm{CS}^{-}$also induces a behavioral contrast that enhances the efficiency of the conditioned excitation (Williams, 2002). As a net result, differential training improves the behavioral discrimination of very similar odors.

To confirm that the observed phenomenon of enhanced acuity caused by differential training of similar odors is not restricted to the single pair of similar odors that we have used throughout this study, we tested a second pair of chemically similar odors, PA and BA (Campbell et al., 2013) (Fig. 9A), at concentrations that evoked equally attractive, naive behavioral responses $\left(F_{(2,16)}=1.2, p=0.33\right.$; one-way ANOVA) (Fig. 9B). The learned behavioral response was strongly generalized to the similar odor in an absolute training regimen (Fig. $9 C-E$ ). When BA was trained, an aversive response was evoked also by PA $\left(\mathrm{MCH}\right.$ vs BA: $t_{(22)}=$ $-15.2, p<0.01$; $\mathrm{MCH}$ vs PA: $t_{(22)}=$ $-8.2, p<0.01$; BA vs PA: $t_{(22)}=7.0, p<$ 0.01 ; one-way ANOVA with Bonferroni post hoc test) (Fig. 9C). Conversely, when PA was trained, an aversive response was evoked also by BA (MCH vs PA: $t_{(22)}=$ $-9.3, p<0.01 ; \mathrm{MCH}$ vs BA: $t_{(22)}=-6.4$, $p<0.01$; BA vs PA: $t_{(22)}=2.9, p<0.05$; one-way ANOVA with Bonferroni post hoc test) (Fig. 9D). However, when $\mathrm{MCH}$ was trained, an aversive response was evoked neither by PA nor by BA ( $\mathrm{MCH}$ vs PA: $t_{(22)}=-9.8, p<0.01$; BA vs PA: $t_{(22)}=-0.4, p=1$; $\mathrm{MCH}$ vs BA: $t_{(22)}=$ $9.3, p<0.01$; one-way ANOVA with Bonferroni post hoc test) (Fig. 9E). However, also for this odor pair, the experience of the similar odor as a $\mathrm{CS}^{-}$in a differential training regimen strongly reduces behavioral generalization (Fig. 9F). Discriminability (i.e., odor acuity) between the 
two odors in the subsequent test situation is enhanced (1-Oct/3Oct absolute vs differential training: $t_{(62)}=-3.0, p<0.01$; BA/PA absolute vs differential training: $t_{(62)}=-2.9, p<0.01$; two-sample $t$ test) (Fig. 9F).

\section{Odor acuity learning causes decorrelation of olfactory representations in Kenyon cells but not in olfactory projection neurons}

After having shown that behaviorally expressed odor similarity is reflected in physiological odor responses in the antennal lobe and that odor acuity is enhanced after a differential conditioning regimen, we asked where in the brain this enhanced odor acuity might be reflected. Physiological changes in odor-evoked $\mathrm{Ca}^{2+}$ responses of OPNs in the antennal lobe of honey bees have been reported after long-term memory formation after differential associative training (Fernandez et al., 2009). This prompted us to investigate odor-evoked $\mathrm{Ca}^{2+}$ responses before and directly after conditioning at the level of the OPNs in the antennal lobe. Flies were subjected to either an absolute (Fig. 10A) or a differential (Fig. 10B) training regimen while fixed under the microscope, and the time courses of odor-evoked $\mathrm{Ca}^{2+}$-activity were measured before and after the training in each fly (Fig. $10 A, B$ ). To investigate possible changes in similarity of odor representations, we calculated the principal components of the mean odorevoked responses in olfactory projection neurons over time (Fig. $10 C$ ) and the Pearson's correlations during the stimulus (Fig. $10 D$ ) before and after training for absolute and differential conditioning. No significant differences between the correlation coefficients before and after training could be observed for either of the training paradigms (pretraining absolute vs differential training: $p=0.09 ; U=27 ; Z=-1.7$; post-training absolute vs differential training: $p=0.16 ; U=31 ; Z=-1.4$; Mann-Whitney $\mathrm{U}$ test).

We next asked whether the similarity between neuronal populations detected in the antennal lobe is maintained at higher olfactory processing centers. A broad wealth of data indicates that the coincidence detection between odor (CS) and punishment (US) as well as subsequent neuronal changes mediating the learned behavioral response can be, at least partially, localized to Kenyon cell output synapses (for review, see Heisenberg, 2003; Fiala, 2007). We first asked whether the similarity of the neuronal representation detected in the antennal lobe is maintained at the level of mushroom body lobes and, second, whether the similarity is altered by absolute or differential training. We expressed the $\mathrm{Ca}^{2+}$ sensor protein GCaMP3.0 (Tian et al., 2009) under direct control of the mushroom body-specific promoter mb247 (Pech et al., 2013). The expression induced by two copies of the mb247 promoter fragment covers all mushroom body lobes and subtypes of Kenyon cells (Fig. 11A,B). Two subpopulations of Kenyon cells in the mushroom body have been suggested as being particularly important for olfactory learning. On the one hand a change in neuronal activity due to learning has been visualized using $\mathrm{Ca}^{2+}$ imaging in the $\alpha^{\prime} / \beta^{\prime}$-lobes (Wang et al., 2008). On the other hand, a genetic rescue, mainly in the $\gamma$-lobes, of the rutabaga gene restores impaired short-term memory (Zars et al., 2000). Furthermore, a genetic rescue of the loss of a D1-like dopamine receptor in the $\gamma$-lobes likewise restores learning deficits (Qin et al., 2012). Therefore, we have chosen to focus on the $\beta$ 'and $\gamma$-lobes that could be observed simultaneously on the same focal plane (Fig. $11 A, B$ ). Odor-evoked $\mathrm{Ca}^{2+}$ transients in the bundled, axon-like neurites of Kenyon cells furnished with presynapses and postsynapses forming the horizontal lobes were reliably detectable. The three odorants 1-Oct, 3-Oct and $\mathrm{MCH}$ evoked activity patterns in Kenyon cell neurites constituting the $\gamma$ - and $\beta$-lobes (Fig. $11 C$ ). Odor-evoked $\mathrm{Ca}^{2+}$ activity in the $\gamma$-lobes was much weaker in amplitude than in the $\beta$ '-lobes (Fig. $11 C, D, G)$. This is in agreement with the report that $\gamma$-type Kenyon cells have higher firing thresholds at their input sites (Turner et al., 2008). The odor-evoked $\mathrm{Ca}^{2+}$ activity patterns in each fly were, however, not stereotypic or comparable in spatial distribution across animals, in agreement with the reported nonstereotypic odor representations in Kenyon cells (Murthy et al., 2008; Caron et al., 2013). Therefore, we performed a pixel-based, within-animal comparison to quantify how the spatial activity patterns evoked by the three odors correlated to each other. We found that the $\mathrm{Ca}^{2+}$ activity patterns within each measured fly induced by 1-Oct and by 3-Oct, respectively, correlated more strongly to each other than the responses to the pairs of dissimilar odors (i.e., 1-Oct and $\mathrm{MCH}$ or 3-Oct and $\mathrm{MCH}$, respectively), in both the $\beta^{\prime}$-lobes $\left(\mathrm{MCH} / 3\right.$-Oct vs $\mathrm{MCH} / 1$-Oct: $t_{(38)}=1.1, p=$ $0.84 ; \mathrm{MCH} / 3$-Oct vs 3-Oct/1-Oct: $t_{(38)}=9.2, p<0.01 ; \mathrm{MCH} / 1$ Oct vs 3-Oct/1-Oct: $t_{(38)}=8.1, p<0.01$; one-way repeatedmeasures ANOVA with Bonferroni post hoc test) (Fig. 11E) and the $\gamma$-shaft $\left(\mathrm{MCH} / 3\right.$-Oct vs MCH/1-Oct: $t_{(38)}=2.0, p=0.16$; $\mathrm{MCH} / 3$-Oct vs 3-Oct/1-Oct: $t_{(38)}=10.2, p<0.01 ; \mathrm{MCH} / 1$-Oct vs 3-Oct/1-Oct: $t_{(38)}=8.2, p<0.01$; one-way repeated-measures ANOVA with Bonferroni post hoc test) (Fig. 11H). In conclusion, the similarity/dissimilarity between odor representations manifested at the level of the antennal lobes is maintained at the level of the mushroom body lobes. We next asked whether the learned, enhanced odor acuity is reflected in the odor response pattern within the Kenyon cell neurites. Therefore, after initial odor responses were recorded, the flies were subjected to either absolute or differential conditioning directly under the microscope, and odor responses were recorded again afterward. Paralleling the behavioral experiments, half of the animals of each training group were trained using 3-Oct as $\mathrm{CS}^{+}$(and 1 -Oct as the $\mathrm{CS}^{-}$in the differential training regimen), the other half of the animals were trained using 1-Oct as the $\mathrm{CS}^{+}$. Again, the dissimilar odor $\mathrm{MCH}$ served as a control odor and was presented before and after, but not during, conditioning. We did not detect any significant difference in similarity of odor representations of the two similar odors, in the $\beta^{\prime}$-lobes, either when comparing initial odor responses or when comparing postconditioning responses of either absolutely or differentially trained flies, respectively (pretraining absolute vs differential training: $p=0.68 ; U=56$; $Z=0.4$; post-training absolute vs differential training: $p=$ 0.12; $U=71 ; Z=1.6$; Mann-Whitney U test) (Fig. $11 F$ ). However, when the same analysis of odor responses was performed for the $\gamma$-shaft and the correlation between the neuronal representations evoked by the trained, similar odors was calculated, we found a significant decorrelation after differential conditioning (pretraining absolute vs differential training: $p=0.24 ; U=66 ; Z=1.2$; post-training absolute vs differential training: $p<0.05 ; U=83 ; Z=2.5$; Mann-Whitney $U$ test) (Fig. 11I). The change in odor acuity cause by differential training is therefore reflected in the similarity of odor representations at the level of the $\gamma$-lobes of the mushroom body.

\section{Discussion}

\section{How is perceptual similarity between odors encoded?}

In fruit flies, several neuronal "labeled-line" pathways have evolved to detect, using narrow-tuned receptors, ecologically relevant odors exclusively and unambiguously, for instance, pheromones (Ha and Smith, 2006; Kurtovic et al., 2007), $\mathrm{CO}_{2}$ (Suh et 
A

Absolute Training

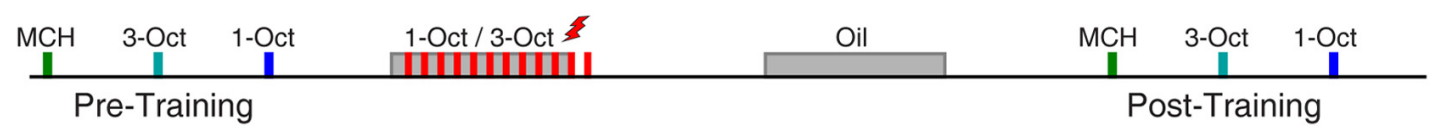
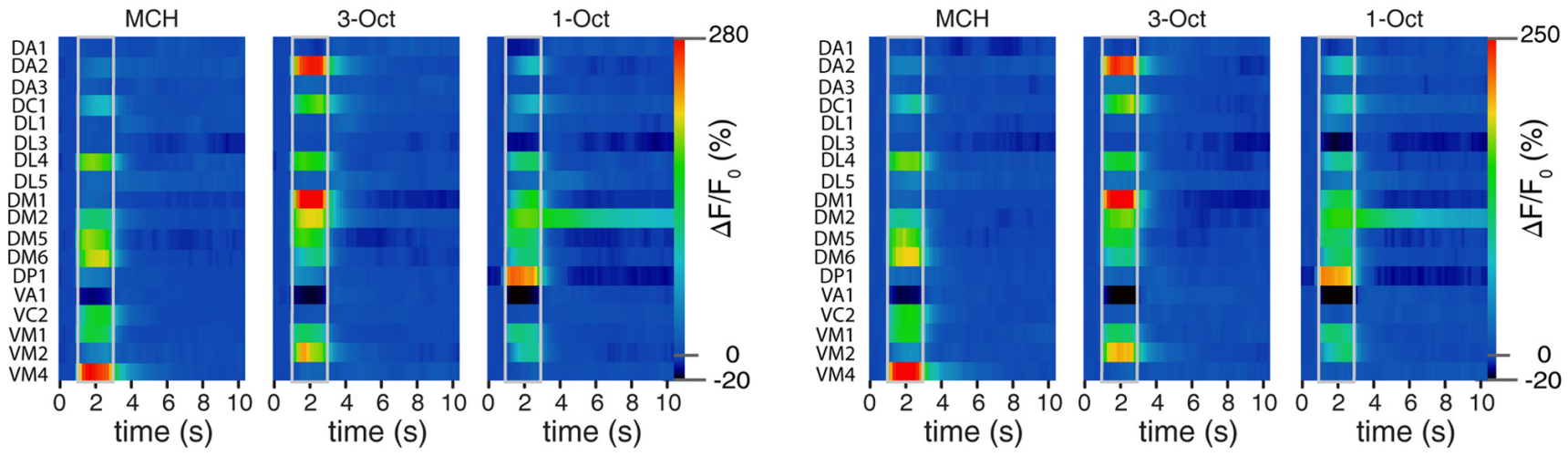

B

Differential Training
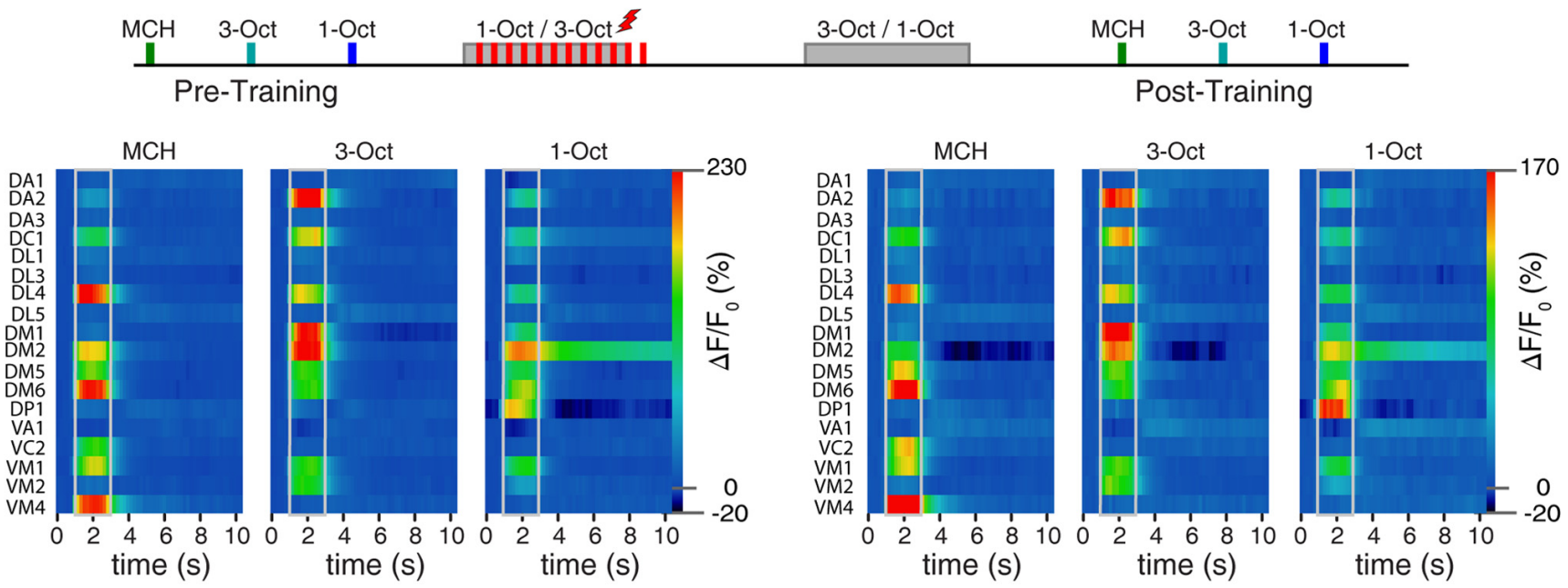

C

Absolute Training

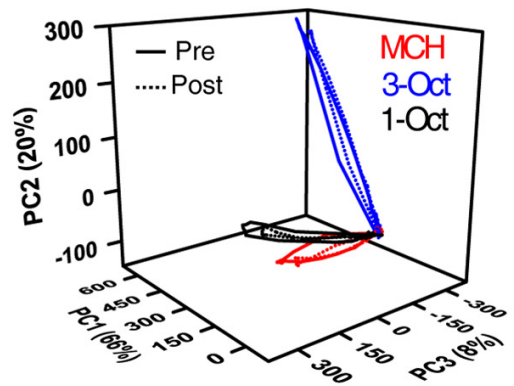

Differential Training

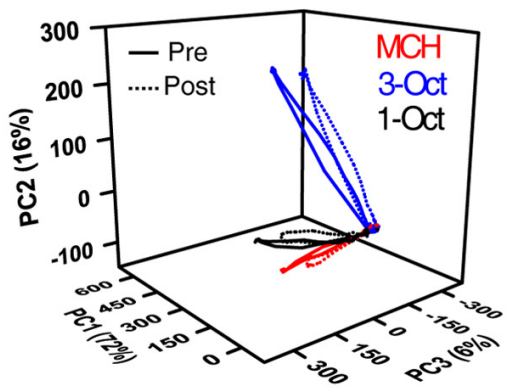

D

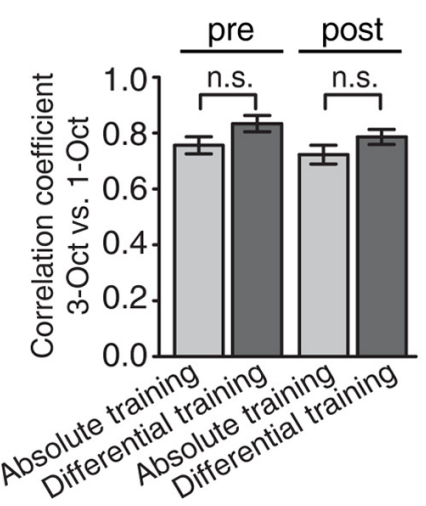

Figure 10. The spatiotemporal $\mathrm{Ca}^{2+}$ activity in olfactory projection neurons at the level of the antennal lobe evoked by two similar odors is not affected by differential or absolute conditioning. $A$, Schematic illustration of the absolute conditioning regimen applied to the prepared fly positioned under the microscope. Bottom, 0 dor-evoked changes $\mathrm{Ca}^{2+}$ activity over time are shown as false colors in 18 identified glomeruli in response to the odorants $\mathrm{MCH}, 3-0 \mathrm{ct}$, and 1-0ct before and after training, respectively. Left, Pretraining glomerular activation. Right, Post-training. Gray lines indicate odor onset and offset. Values indicate mean; $n=10$ animals. $\boldsymbol{B}$, Schematic illustration of the differential conditioning regimen applied to the prepared fly positioned under the microscope. Bottom, 0 dor-evoked changes $\mathrm{Ca}^{2+}$ activity over time are shown as false colors in 18 identified glomeruli in response to the odorants MCH, 3-0ct, and 1-0ct before and after training, respectively. Left, Pretraining glomerular activation. Right, Post-training. Gray lines indicate odor onset and offset. Values indicate mean; $n=10$ animals. C, PCA of the time courses of the mean odor-evoked responses in olfactory projection neurons before training (solid lines) and after training (dotted lines) for absolute conditioning (left) and for differential conditioning (right). The first three principal components covered $>94 \%$ of the variance. $D$, Pearson's correlation coefficients between the similar odors 1-0ct and 3-0ct before and after absolute or differential conditioning. No significant differences (n.s., Not significant; $p>0.05 ;$ Mann-Whitney U test, $n=10$ each) between absolute and differential training were observed. Bars indicate mean \pm SEM. 
A

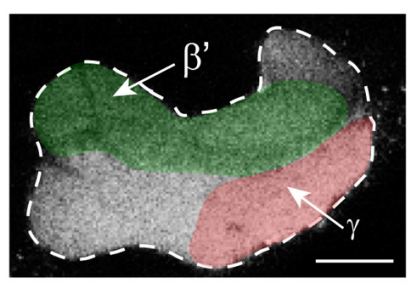

c

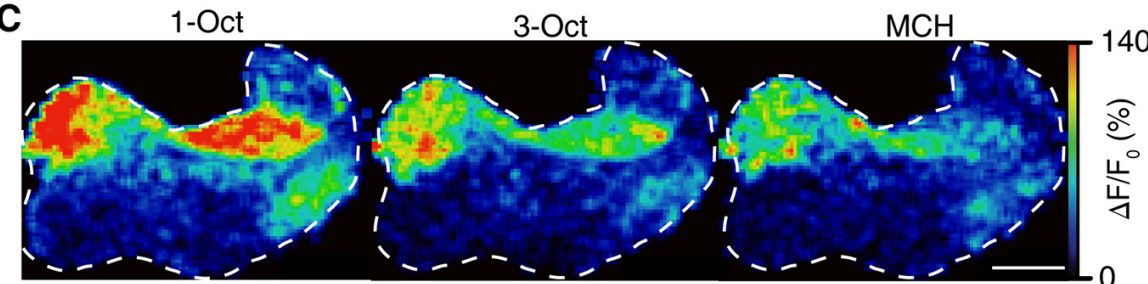

D

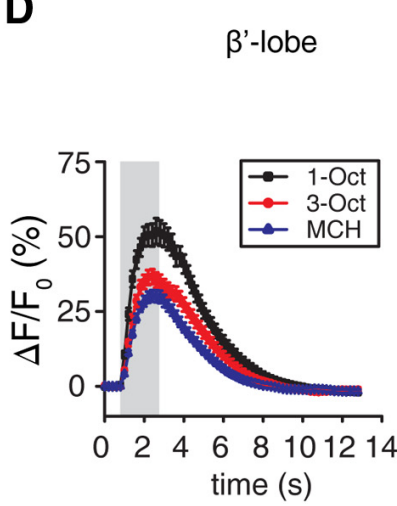

G

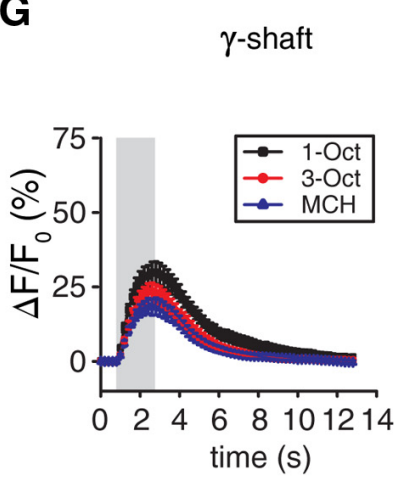

B

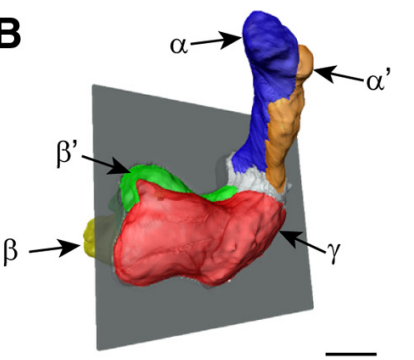

E

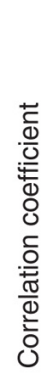

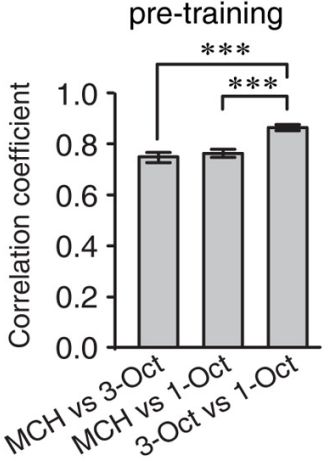

H

\section{$\gamma$-shaft pre-training}

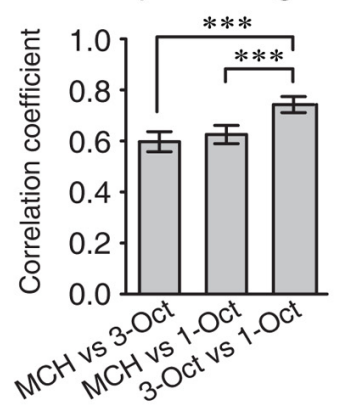

$F$

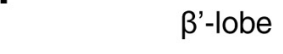

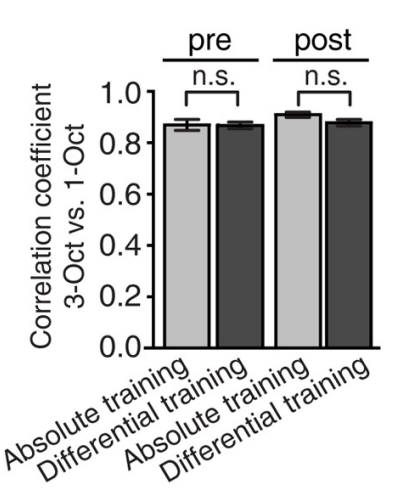

I

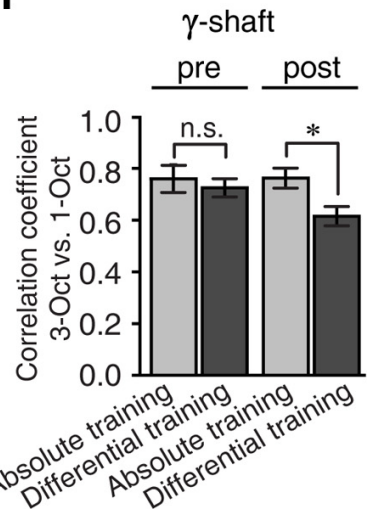

Figure 11. Dynamics and similarity of odor representations in the mushroom body. $\boldsymbol{A}$, Expression of the $\mathrm{Ca}^{2+}$ sensor GCaMP3.0 in the mushroom body shown in the focal plane chosen for imaging in the horizontal lobes of the mushroom body. The focal plane includes the $\gamma$-lobe and the $\beta^{\prime}$-lobe. Red represents the $\gamma$-shaft region; green represents the $\beta^{\prime}$-region. $B, 3 D$ reconstruction of parts of a mushroom body based on GCaMP3.0 expression in Kenyon cells of one fly used during an optical $\mathrm{Ca}^{2+}$ imaging experiment. Different colors represent distinct lobes. Gray represents the focal plane chosen for imaging. $C_{,} \mathrm{Ca}^{2+}$ increase in the focal plane shown in $\boldsymbol{A}$ and $\boldsymbol{B}$ is evoked by stimulation with 1-0ct, 3-0ct, and MCH, as indicated in false colors. Scale bars: $\boldsymbol{A}-\boldsymbol{C}, 20$ $\mu \mathrm{m}$. $\boldsymbol{D}$, Temporal dynamics of odor-evoked changes in fluorescence averaged over the $\beta^{\prime}$-lobe region in response to presentation of 1-0ct, 3-0ct, or MCH. The gray bar represents the duration of odor stimulation. Values indicate mean $\pm S E M ; n=20$. $E$, The $\mathrm{Ca}^{2+}$ signals evoked by the pair of similar odors in the $\beta^{\prime}$-lobe correlate significantly stronger than those evoked by the dissimilar odor pairs. Bars indicate mean \pm SEM of pixel-based Pearson's correlation coefficients. $n=20$. $^{* *} p<0.001$ (one-way repeatedmeasures ANOVA with Bonferroni post hoc test). $\boldsymbol{F}$, Pearson's correlation coefficients as measured in the $\beta^{\prime}$-lobe between the similar odors 1-0ct and 3-0ct before and after absolute or differential conditioning. No significant differences (n.s., Not significant; $p>0.05$; Mann-Whitney $\mathrm{U}$ test, $n=10$ each) between absolute and differential training were observed. $\mathbf{G}$, Temporal dynamics of odor-evoked changes in fluorescence averaged over the $\gamma$-shaft region in response to presentation of 1-0ct, 3-0ct, or MCH. The gray bar represents the duration of odor stimulation. Values indicate mean $\pm \mathrm{SEM} ; \boldsymbol{n}=20$. $\boldsymbol{H}, \mathrm{The} \mathrm{Ca}^{2+}$ signals evoked by the pair of similar odors in the $\gamma$-shaft correlate significantly stronger than those evoked by the dissimilar odor pairs. Bars indicate mean \pm SEM of pixel-based Pearson's correlation coefficients. $n=20{ }^{* * *} p<0.001$ (one-way repeated-measures ANOVA with Bonferroni al., 2004; Jones et al., 2007; Suh et al., 2007), or the mold odor, geosmin (Stensmyr et al., 2012), to elicit behavior. For all odors whose relevance is not innately predetermined, more plastic recognition mechanisms must exist that take stimulus variations into account. Learned relevance can be transferred to similarly perceived odors through generalization, a phenomenon that has been well described for olfactory learning in adult and larval Drosophila (Eschbach et al., 2011; Niewalda et al., 2011; Mishra et al., 2010; Chen YC, et al., 2011). We have chosen chemically similar odorants based on previous reports (Mishra et al., 2010; Campbell et al., 2013) and found a high degree of generalization between them. But how is perceived similarity manifested in the fly's brain? Odor-evoked glomerular activity is, at the level of the insect antennal lobe, rather stereotypic across individuals (e.g., Fiala et al., 2002; Couto et al., 2005; Fishilevich and Vosshall, 2005). The overlap in the activity pattern of glomeruli of the antennal lobe or the mammalian olfactory bulb, respectively, has been regarded as a correlate of chemical odorant similarity (Meister and Bonhoeffer, 2001; Belluscio and Katz, 2001; Johnson et al., 2009), and behaviorally determined odor similarity (Guerrieri et al., 2005; Kreher et al., 2008; Youngentob et al., 2006; Niewalda et al., 2011; Parnas et al., 2013), which is in accordance with our results. Interestingly, in Drosophila the spatial activity patterns of second-order OPNs in the antennal lobes match perceived odor similarity more closely than those of first-order OSNs (Niewalda et al., 2011), and the activity of OPNs correlates with innate odor discriminability (Parnas et al., 2013). In the course of transforming odor information from first- to second-order neurons, inhibitory local interneurons (LNs) have been suggested as a cause of fine-tuning of spatiotemporal activity patterns (Stopfer et al., 1997; Sachse and Galizia, 2002; Wilson et al., 2004; Wilson and Laurent, 2005), which might ultimately contribute to the discriminability between similar odors. In accordance with that idea, pharmacological interference with GABA receptors in the antennal lobes of honeybees disrupts local field potential oscillations caused by synchro-

post hoc test). I, Pearson's correlation coefficients as measured in the $\gamma$-shaft between the similar odors 1-0ct and 3-0ct before and after absolute or differential conditioning, respectively. A significant decorrelation between the two similar odors (n.s., Not significant; $p>0.05 ;{ }^{*} p<0.05$; Mann-Whitney $U$ test, $n=10$ each) was observed after differential training compared with absolute training. 
nized projection neurons and impairs fine discrimination between similar odors (Stopfer et al., 1997). We find that type I inhibitory LNs of the antennal lobe, which target OPNs and not OSNs (Tanaka et al., 2009), are required for fine discrimination of similar odors in Drosophila. However, in Drosophila, type I LNs are not required to maintain the synchrony of projection neurons, in contrast to type II LNs (Tanaka et al., 2009). In conclusion, the role of these inhibitory LNs for fine odor discrimination does not rely on synchronizing projection neuron activity. Lateral inhibition between glomeruli has also been suggested as a mechanism for fine odor discrimination (Sachse and Galizia, 2002; Wilson and Laurent, 2005), which can explain our results. Interestingly, the correlation between similar odorants, behaviorally expressed similarity, and odor-evoked spatial neuronal activity is maintained in the activity of intrinsic mushroom body neurons (Kenyon cells) in accordance with reports of $\mathrm{Ca}^{2+}$ transients detected in Kenyon cell somata (Campbell et al., 2013).

\section{What is learned in conditioned fine odor discrimination?}

Flies can clearly learn to differentiate various concentrations of odors (Masek and Heisenberg, 2008), combinations of binary odor mixtures (Eschbach et al., 2011; Borst, 1983), and a large number of diverse chemicals (Dudai, 1977). Olfactory responsiveness is modulated by several intrinsic or environmental factors, for instances, circadian rhythm (Krishnan et al., 1999), feeding status (Itskov and Ribeiro, 2013), or ambient temperature (Riveron et al., 2009), which all could lead to altered olfactory acuity. These influences can be experimentally distinguished from learned changes in olfactory acuity. It has been suggested that, in Drosophila, the presentation of the $\mathrm{CS}^{-}$has no influence during the formation of an associative odor memory (Borst, 1983; Masek and Heisenberg, 2008). Rather, only the exposure to the $\mathrm{CS}^{+}$simultaneously with the reinforcement has been proposed to cause learning, as reflected in respective cellular models of associative odor learning (Heisenberg, 2003; Fiala, 2007). We reasoned that neuronal mechanisms underlying learned fine odor discrimination might not be assessable using very high odor concentrations and chemically dissimilar odors. Here we show that, if two similar odors are used as $\mathrm{CS}^{+}$and $\mathrm{CS}^{-}$, respectively, exposure to the $\mathrm{CS}^{-}$causes conditioned inhibition that contributes to an enhanced discrimination (i.e., an improved olfactory acuity of initially confusable odors). Fruit flies can exhibit odorspecific learning strategies (e.g., Yarali et al., 2009). However, we have observed learned enhancement of odor acuity with two chemically similar pairs of odorants, suggesting that this phenomenon is not restricted to specific odorants. This new finding cannot be mechanistically explained by current cellular models of olfactory learning (Heisenberg, 2003; Busto et al., 2010). Interestingly, the effect of the $\mathrm{CS}^{-}$is not equivalent to inhibitory learning caused by backward conditioning (Tanimoto et al., 2004), as the conditioned inhibition found in our study is independent of the exact timing of the $\mathrm{CS}^{-}$presentation relative to the US. Conclusively, maintenance of odor representations over the training period has to be proposed. Indeed, trace conditioning experiments in flies and bees have demonstrated that odor representations can be maintained (Galili et al., 2011; Szyszka et al., 2011). Interestingly, the presence of the $\mathrm{CS}^{-}$during differential training not only induces a conditioned inhibition but enhances also the learned responsiveness to the $\mathrm{CS}^{+}$, a phenomenon known as behavioral contrast (Williams, 2002). Based on this finding, we have to assume an influence of the $\mathrm{CS}^{-}$on the $\mathrm{CS}^{+}$representation and/or reinforcement efficiency. A number of theoretical explanations for behavioral contrast have been formulated (Williams, 2002). The difference in learned behavior dependent on the presentation of a $\mathrm{CS}^{-}$can either be regarded as a consequence of the experience of the $\mathrm{CS}^{-}$in the differential training regimen, or, conversely, as a lack of experience in the absolute training regimen (i.e., a novelty effect). The genetic and physiological tools available in Drosophila might help to further dissect the neuronal basis for this phenomenon.

We find that generalization between the two similar odors is nearly symmetric in strength (i.e., absolute training of either odor results in similar avoidance of the other odor). However, discrimination of the two odorants after differential training is not symmetric: If 1-Oct is temporally paired with electric shocks and 3-Oct is not, 1-Oct is strongly avoided in a choice situation between the two odors. The reciprocal situation, however, is much less pronounced. This asymmetry might be explained by the neuronal, glomerular representations at the level of OSNs, as the representation of 3-Oct is "nested" within the 1-Oct representation. From a theoretical viewpoint, this can be regarded as a "negative patterning problem," which has been reported to be more difficult for flies to solve through associative learning than positive patterning in which the nonoverlapping representation is reinforced (Young et al., 2011).

\section{Neural systems for olfactory acuity learning: common principles across species?}

Decades of research have led to the current concept of a coincidence detection between odor (CS) information and punishment (US) information in Kenyon cells (Heisenberg, 2003; Fiala, 2007). Conclusively, the essential memory trace for associative odor learning can be localized in Kenyon cells. Here we show that acuity learning of similar odors causes decorrelation of odor representation in the $\gamma$-lobes of the mushroom body. Whereas learning-induced changes in synaptic transmission in the antennal lobe in Drosophila (Yu et al., 2004), and changes in $\mathrm{Ca}^{2+}$ dynamics correlating with long-term memory in bees (Fernandez et al., 2009) have been described, we do not find short-term changes in odor-evoked $\mathrm{Ca}^{2+}$ representations in OPNs resulting from differential training of similar odors.

Interestingly, neuronal mechanisms underlying olfactory acuity learning in Drosophila are similar to those in mammals. Associative learning of olfactory acuity in rodents changes response properties of piriform cortical neurons in such that discriminability of similar odors is, at a cellular level, enhanced (Litaudon et al., 1997; Kadohisa and Wilson, 2006; Chapuis and Wilson, 2011; Chen CF, et al., 2011). The mushroom body resembles the piriform cortex of mammals in several features. Kenyon cells each receive input from many different projection neurons (Caron et al., 2013; Gruntman and Turner, 2013), similarly to pyramidal cells of the anterior piriform cortex (Sosulski et al., 2011), thereby integrating information originating in many olfactory receptors. Furthermore, Kenyon cells respond to odors in a sparse, highly selective way, and any spatial, chemotopic information has been suggested to be eliminated at this level of processing (Murthy et al., 2008; Turner et al., 2008; Honegger et al., 2011), similarly to piriform cortex neurons (Stettler and Axel, 2009). Comparable functions of the mushroom body and the piriform cortex are therefore certainly conceivable. Our data demonstrate that differential associative training can affect olfactory acuity in a bidirectional way also in Drosophila and that this is not a property restricted to the brains of higher vertebrates.

\section{References}

Armstrong JD, Kaiser K, Müller A, Fischbach KF, Merchant N, Strausfeld NJ (1995) Flybrain, an on-line atlas and database of the Drosophila nervous system. Neuron 15:17-20. CrossRef Medline 
Belluscio L, Katz LC (2001) Symmetry, stereotypy, and topography of odorant representations in mouse olfactory bulbs. J Neurosci 21:2113-2122. Medline

Bitterman ME, Menzel R, Fietz A, Schäfer S (1983) Classical conditioning of proboscis extension in honeybees (Apis mellifera). J Comp Psychol 97: 107-119. CrossRef Medline

Borst A (1983) Computation of olfactory signals in Drosophila melanogaster. J Comp Physiol 152:373-383.

Busto GU, Cervantes-Sandoval I, Davis RL (2010) Olfactory learning in Drosophila. Physiology (Bethesda) 25:338-346. CrossRef Medline

Campbell RA, Honegger KS, Qin H, Li W, Demir E, Turner GC (2013) Imaging a population code for odor identity in the Drosophila mushroom body. J Neurosci 33:10568-10581. CrossRef Medline

Caron SJ, Ruta V, Abbott LF, Axel R (2013) Random convergence of olfactory inputs in the Drosophila mushroom body. Nature 497:113-117. CrossRef Medline

Chapuis J, Wilson DA (2011) Bidirectional plasticity of cortical pattern recognition and behavioral sensory acuity. Nat Neurosci 15:155-161. CrossRef Medline

Chen CF, Barnes DC, Wilson DA (2011a) Generalized vs stimulus-specific learned fear differentially modifies stimulus encoding in primary sensory cortex of awake rats. J Neurophysiol 106:3136-3144. CrossRef Medline

Chen YC, Mishra D, Schmitt L, Schmuker M, Gerber B (2011) A behavioral odor similarity "space" in larval Drosophila. Chem Senses 36:237-249. CrossRef Medline

Chou YH, Spletter ML, Yaksi E, Leong JC, Wilson RI, Luo L (2010) Diversity and wiring variability of olfactory local interneurons in the Drosophila antennal lobe. Nat Neurosci 13:439-449. CrossRef Medline

Cleland TA, Morse A, Yue EL, Linster C (2002) Behavioral models of odor similarity. Behav Neurosci 116:222-231. CrossRef Medline

Couto A, Alenius M, Dickson BJ (2005) Molecular, anatomical, and functional organization of the Drosophila olfactory system. Curr Biol 15:15351547. CrossRef Medline

Davis RL (1993) Mushroom bodies and Drosophila learning. Neuron 11:114. CrossRef Medline

Dudai Y (1977) Properties of learning and memory in Drosophila melanogaster. J Comp Physiol A Neuroethol Sens Neural Behav Physiol 114:6989. CrossRef

Eschbach C, Vogt K, Schmuker M, Gerber B (2011) The similarity between odors and their binary mixtures in Drosophila. Chem Senses 36:613-621. CrossRef Medline

Fernandez PC, Locatelli FF, Person-Rennell N, Deleo G, Smith BH (2009) Associative conditioning tunes transient dynamics of early olfactory processing. J Neurosci 29:10191-10202. CrossRef Medline

Fiala A (2007) Olfaction and olfactory learning in Drosophila: recent progress. Curr Opin Neurobiol 17:720-726. CrossRef Medline

Fiala A, Spall T, Diegelmann S, Eisermann B, Sachse S, Devaud JM, Buchner E, Galizia CG (2002) Genetically expressed cameleon in Drosophila melanogaster is used to visualize olfactory information in projection neurons. Curr Biol 12:1877-1884. CrossRef Medline

Fishilevich E, Vosshall LB (2005) Genetic and functional subdivision of the Drosophila antennal lobe. Curr Biol 15:1548-1553. CrossRef Medline

Fletcher ML, Wilson DA (2002) Experience modifies olfactory acuity: acetylcholine-dependent learning decreases behavioral generalization between similar odorants. J Neurosci 22:RC201. Medline

Galili DS, Lüdke A, Galizia CG, Szyszka P, Tanimoto H (2011) Olfactory trace conditioning in Drosophila. J Neurosci 31:7240-7248. CrossRef Medline

Ghirlanda S, Enquist M (2003) Reviews: a century of generalisation. Anim Behav 66:15-36. CrossRef

Giurfa M (2004) Conditioning procedure and color discrimination in the honeybee Apis mellifera. Naturwissenschaften 91:228-231. CrossRef Medline

Gruntman E, Turner GC (2013) Integration of the olfactory code across dendritic claws of single mushroom body neurons. Nat Neurosci 16: 1821-1829. CrossRef Medline

Guerrieri F, Schubert M, Sandoz JC, Giurfa M (2005) Perceptual and neura olfactory similarity in honeybees. PLoS Biol 3:e60. CrossRef Medline

Guizar-Sicairos M, Thurman ST, Fienup JR (2008) Efficient subpixel image registration algorithms. Opt Lett 33:156-158. CrossRef Medline

Guttman N, Kalish HI (1956) Discriminability and stimulus generalization. J Exp Psychol 51:79-88. CrossRef Medline
Ha TS, Smith DP (2006) A pheromone receptor mediates 11-cis-vaccenyl acetate-induced responses in Drosophila. J Neurosci 26:8727-8733. CrossRef Medline

Hanson HM (1959) Effects of discrimination training on stimulus generalization. J Exp Psychol 58:321-334. CrossRef Medline

Heisenberg M (2003) Mushroom body memoir: from maps to models. Nat Rev Neurosci 4:266-275. CrossRef Medline

Hellstern F, Malaka R, Hammer M (1998) Backward inhibitory learning in honeybees: a behavioral analysis of reinforcement processing. Learn Mem 4:429-444. CrossRef Medline

Honegger KS, Campbell RA, Turner GC (2011) Cellular-resolution population imaging reveals robust sparse coding in the Drosophila mushroom body. J Neurosci 31:11772-11785. CrossRef Medline

Itskov PM, Ribeiro C (2013) The dilemmas of the gourmet fly: the molecular and neuronal mechanisms of feeding and nutrient decision making in Drosophila. Front Neurosci 7:12. CrossRef Medline

Johnson BA, Xu Z, Ali SS, Leon M (2009) Spatial representations of odorants in olfactory bulbs of rats and mice: similarities and differences in chemotopic organization. J Comp Neurol 514:658-673. CrossRef Medline

Jones WD, Cayirlioglu P, Kadow IG, Vosshall LB (2007) Two chemosensory receptors together mediate carbon dioxide detection in Drosophila. Nature 445:86-90. CrossRef Medline

Kadohisa M, Wilson DA (2006) Separate encoding of identity and similarity of complex familiar odors in piriform cortex. Proc Natl Acad Sci U S A 103:15206-15211. CrossRef Medline

Kalman RE (1960) A new approach to linear filtering and prediction problems. J Basic Eng 82:35-45. CrossRef

Kitamoto T (2001) Conditional modification of behavior in Drosophila by targeted expression of a temperature-sensitive shibire allele in defined neurons. J Neurobiol 47:81-92. CrossRef Medline

Kreher SA, Mathew D, Kim J, Carlson JR (2008) Translation of sensory input into behavioral output via an olfactory system. Neuron 59:110-124. CrossRef Medline

Krishnan B, Dryer SE, Hardin PE (1999) Circadian rhythms in olfactory responses of Drosophila melanogaster. Nature 400:375-378. CrossRef Medline

Kurtovic A, Widmer A, Dickson BJ (2007) A single class of olfactory neurons mediates behavioural responses to a Drosophila sex pheromone. Nature 446:542-546. CrossRef Medline

Laissue PP, Reiter C, Hiesinger PR, Halter S, Fischbach KF, Stocker RF (1999) Three-dimensional reconstruction of the antennal lobe in Drosophila melanogaster. J Comp Neurol 405:543-552. CrossRef Medline

Li W, Howard JD, Parrish TB, Gottfried JA (2008) Aversive learning enhances perceptual and cortical discrimination of indiscriminable odor cues. Science 319:1842-1845. CrossRef Medline

Litaudon P, Mouly AM, Sullivan R, Gervais R, Cattarelli M (1997) Learning-induced changes in rat piriform cortex activity mapped using multisite recording with voltage sensitive dye. Eur J Neurosci 9:15931602. CrossRef Medline

Masek P, Heisenberg M (2008) Distinct memories of odor intensity and quality in Drosophila. Proc Natl Acad Sci US A 105:15985-15990. CrossRef Medline

Meister M, Bonhoeffer T (2001) Tuning and topography in an odor map on the rat olfactory bulb. J Neurosci 21:1351-1360. Medline

Mishra D, Louis M, Gerber B (2010) Adaptive adjustment of the generalization-discrimination balance in larval Drosophila. J Neurogenet 24:168-175. CrossRef Medline

Murthy M, Fiete I, Laurent G (2008) Testing odor response stereotypy in the Drosophila mushroom body. Neuron 59:1009-1023. CrossRef Medline

Niewalda T, Völler T, Eschbach C, Ehmer J, Chou WC, Timme M, Fiala A Gerber B (2011) A combined perceptual, physico-chemical, and imaging approach to 'odour-distances' suggests a categorizing function of the Drosophila antennal lobe. PLoS One 6:e24300. CrossRef Medline

Olsen SR, Wilson RI (2008) Lateral presynaptic inhibition mediates gain control in an olfactory circuit. Nature 452:956-960. CrossRef Medline

Parnas M, Lin AC, Huetteroth W, Miesenböck G (2013) Odor discrimination in Drosophila: from neural population codes to behavior. Neuron 79:932-944. CrossRef Medline

Pavlov IP (1927) Conditioned reflexes: an investigation of the physiological activity of the cerebral cortex. London: Oxford UP. 
Pearce JM (1994) Similarity and discrimination: a selective review and a connectionist model. Psychol Rev 101:587-607. CrossRef Medline

Pech U, Dipt S, Barth J, Singh P, Jauch M, Thum AS, Fiala A, Riemensperger T (2013) Mushroom body miscellanea: transgenic Drosophila strains expressing anatomical and physiological sensor proteins in Kenyon cells. Front Neural Circuits 7:147. CrossRef Medline

Qin H, Cressy M, Li W, Coravos JS, Izzi SA, Dubnau J (2012) Gamma neurons mediate dopaminergic input during aversive olfactory memory formation in Drosophila. Curr Biol 22:608-614. CrossRef Medline

Riemensperger T, Völler T, Stock P, Buchner E, Fiala A (2005) Punishment prediction by dopaminergic neurons in Drosophila. Curr Biol 15:19531960. CrossRef Medline

Riemensperger T, Pech U, Dipt S, Fiala A (2012) Optical calcium imaging in the nervous system of Drosophila melanogaster. Biochim Biophys Acta 1820:1169-1178. CrossRef Medline

Riveron J, Boto T, Alcorta E (2009) The effect of environmental temperature on olfactory perception in Drosophila melanogaster. J Insect Physio 55:943-951. CrossRef Medline

Root CM, Masuyama K, Green DS, Enell LE, Nässel DR, Lee CH, Wang JW (2008) A presynaptic gain control mechanism fine-tunes olfactory behavior. Neuron 59:311-321. CrossRef Medline

Sachse S, Galizia CG (2002) Role of inhibition for temporal and spatial odor representation in olfactory output neurons: a calcium imaging study. J Neurophysiol 87:1106-1117. Medline

Sachse S, Rueckert E, Keller A, Okada R, Tanaka NK, Ito K, Vosshall LB (2007) Activity-dependent plasticity in an olfactory circuit. Neuron 56: 838-850. CrossRef Medline

Schwaerzel M, Heisenberg M, Zars T (2002) Extinction antagonizes olfactory memory at the subcellular level. Neuron 35:951-960. CrossRef Medline

Seki Y, Rybak J, Wicher D, Sachse S, Hansson BS (2010) Physiological and morphological characterization of local interneurons in the Drosophila antennal lobe. J Neurophysiol 104:1007-1019. CrossRef Medline

Shepard RN (1987) Toward a universal law of generalization for psychological science. Science 237:1317-1323. CrossRef Medline

Sosulski DL, Bloom ML, Cutforth T, Axel R, Datta SR (2011) Distinct representations of olfactory information in different cortical centres. Nature 472:213-216. CrossRef Medline

Stensmyr MC, Dweck HK, Farhan A, Ibba I, Strutz A, Mukunda L, Linz J, Grabe V, Steck K, Lavista-Llanos S, Wicher D, Sachse S, Knaden M, Becher PG, Seki Y, Hansson BS (2012) A conserved dedicated olfactory circuit for detecting harmful microbes in Drosophila. Cell 151:1345-1357. CrossRef Medline

Stettler DD, Axel R (2009) Representations of odor in the piriform cortex. Neuron 63:854-864. CrossRef Medline

Stocker RF, Heimbeck G, Gendre N, de Belle JS (1997) Neuroblast ablation in Drosophila P[GAL4] lines reveals origins of olfactory interneurons. J Neurobiol 32:443-456. CrossRef Medline

Stopfer M, Bhagavan S, Smith BH, Laurent G (1997) Impaired odour discrimination on desynchronization of odour-encoding neural assemblies. Nature 390:70-74. CrossRef Medline

Suh GS, Wong AM, Hergarden AC, Wang JW, Simon AF, Benzer S, Axel R, Anderson DJ (2004) A single population of olfactory sensory neurons mediates an innate avoidance behaviour in Drosophila. Nature 431:854859. CrossRef Medline

Suh GS, Ben-Tabou de Leon S, Tanimoto H, Fiala A, Benzer S, Anderson DJ (2007) Light activation of an innate olfactory avoidance response in Drosophila. Curr Biol 17:905-908. CrossRef Medline

Szyszka P, Demmler C, Oemisch M, Sommer L, Biergans S, Birnbach B, Silbering AF, Galizia CG (2011) Mind the gap: olfactory trace conditioning in honeybees. J Neurosci 31:7229-7239. CrossRef Medline
Tanaka NK, Awasaki T, Shimada T, Ito K (2004) Integration of chemosensory pathways in the Drosophila second-order olfactory centers. Curr Biol 14:449-457. CrossRef Medline

Tanaka NK, Ito K, Stopfer M (2009) Odor-evoked neural oscillations in Drosophila are mediated by widely branching interneurons. J Neurosci 29:8595-8603. CrossRef Medline

Tanimoto H, Heisenberg M, Gerber B (2004) Experimental psychology: event timing turns punishment to reward. Nature 430:983. CrossRef Medline

Thévenaz P, Ruttimann UE, Unser M (1998) A pyramid approach to subpixel registration based on intensity. IEEE Trans Image Process 7:27-41. CrossRef Medline

Tian L, Hires SA, Mao T, Huber D, Chiappe ME, Chalasani SH, Petreanu L, Akerboom J, McKinney SA, Schreiter ER, Bargmann CI, Jayaraman V, Svoboda K, Looger LL (2009) Imaging neural activity in worms, flies and mice with improved GCaMP calcium indicators. Nat Methods 6:875881. CrossRef Medline

Tully T, Quinn WG (1985) Classical conditioning and retention in normal and mutant Drosophila melanogaster. J Comp Physiol A Neuroethol Sens Neural Behav Physiol 157:263-277. CrossRef Medline

Turner GC, Bazhenov M, Laurent G (2008) Olfactory representations by Drosophila mushroom body neurons. J Neurophysiol 99:734-746. CrossRef Medline

Vosshall LB, Stocker RF (2007) Molecular architecture of smell and taste in Drosophila. Annu Rev Neurosci 30:505-533. CrossRef Medline

Wagh DA, Rasse TM, Asan E, Hofbauer A, Schwenkert I, Dürrbeck H, Buchner S, Dabauvalle MC, Schmidt M, Qin G, Wichmann C, Kittel R, Sigrist SJ, Buchner E (2006) Bruchpilot, a protein with homology to ELKS/ CAST, is required for structural integrity and function of synaptic active zones in Drosophila. Neuron 49:833-844. CrossRef Medline

Wang JW, Wong AM, Flores J, Vosshall LB, Axel R (2003) Two-photon calcium imaging reveals an odor-evoked map of activity in the fly brain. Cell 112:271-282. CrossRef Medline

Wang Y, Mamiya A, Chiang AS, Zhong Y (2008) Imaging of an early memory trace in the Drosophila mushroom body. J Neurosci 28:4368-4376. CrossRef Medline

Williams BA (2002) Behavioral contrast redux. Anim Learn Behav 30:1-20. CrossRef Medline

Wilson DA, Stevenson RJ (2006) Learning to smell. Baltimore: Johns Hopkins UP.

Wilson RI, Laurent G (2005) Role of GABAergic inhibition in shaping odorevoked spatiotemporal patterns in the Drosophila antennal lobe. J Neurosci 25:9069-9079. CrossRef Medline

Wilson RI, Turner GC, Laurent G (2004) Transformation of olfactory representations in the Drosophila antennal lobe. Science 303:366-370. CrossRef Medline

Yarali A, Ehser S, Hapil FZ, Huang J, Gerber B (2009) Odour intensity learning in fruit flies. Proc Biol Sci 276:3413-3420. CrossRef Medline

Young JM, Wessnitzer J, Armstrong JD, Webb B (2011) Elemental and nonelemental olfactory learning in Drosophila. Neurobiol Learn Mem 96: 339-352. CrossRef Medline

Youngentob SL, Johnson BA, Leon M, Sheehe PR, Kent PF (2006) Predicting odorant quality perceptions from multidimensional scaling of olfactory bulb glomerular activity patterns. Behav Neurosci 120:1337-1345. CrossRef Medline

Yu D, Ponomarev A, Davis RL (2004) Altered representation of the spatial code for odors after olfactory classical conditioning: memory trace formation by synaptic recruitment. Neuron 42:437-449. CrossRef Medline

Zars T, Fischer M, Schulz R, Heisenberg M (2000) Localization of a shortterm memory in Drosophila. Science 288:672-675. CrossRef Medline 


\section{Abbreviations}

\begin{tabular}{ll} 
3-Oct & 3-octanol \\
AC & adenylat cyclase \\
AL & antennal lobe \\
ATP & adenosine triphosphate \\
Ca $^{2+}$ & calcium ion \\
cAMP & cyclic adenosine monophosphate \\
ddH ${ }_{2}$ O & doubled destilled water \\
DNA & deoxyribonucleic acid \\
dTRPA1 & Drosophila transient receptor potential channel A1 \\
ECFP & enhanced cyan fluorescent protein \\
eLN & excitatory local interneuron \\
et al. & et altri \\
EYFP & enhanced yellow fluorescent protein \\
GABA & $\gamma$-aminobutyric acid \\
GAD & GAL4 activation domain \\
GAL4 & galactosidase 4 \\
GAL80 & galactose/lactose metabolism regulatory protein \\
GECI & genetically encoded Ca \\
GFP indicator & green fluorescent protein \\
iLN & inhibitory local interneuron \\
KC & Kenyon cell \\
LH & lateral horn \\
LN & local interneuron \\
\hline
\end{tabular}


C. Abbreviations
LN1 type I inhibitory local interneurons
LN2 type II inhibitory local interneurons
MB mushroom body (corpora peduncula)
MCH 4-methylcyclohexanol
OR olfactory receptor
OSN olfactory sensory neurons
PA pentyl acetate
PBS phosphate buffered saline
PCA principal component analysis
PN projection neuron
QF transcription factor for activation of the $Q$ system for binary expression
QS inhibitor of the transcription factor QF in the Q system for binary expres- sion
QUAS binding region of the transcription factor QF in the $Q$ system for binary expression
Shi $^{\text {ts }} \quad$ temperature-sensitive shibire allele (dominant dynamin mutation) 


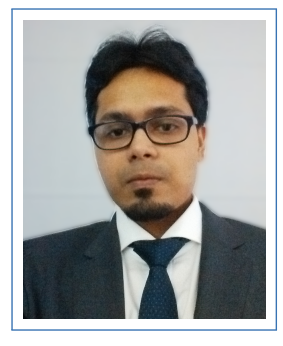

\section{Shubham Dipt}

Curriculum Vitae

\section{Education}

2002-2003 Secondary School (Matriculation), Indian Certificate of Secondary Education (ICSE), Don Bosco Academy, Patna, India.

2004-2005 Higher Secondary School, Central Board of Secondary Education (CBSE), Modi Public School, Kota, India.

2006 Indian Institute of Technology Joint Entrance Examination (IIT-JEE 2006), Qualified, All India Rank - 4871 out of 0.3 million applicants.

2006-2011 Bachelor and Masters Dual Degree Programme (Major: Biology), Indian Institute of Science Education and Research, Kolkata, India.

2011-2015 Doctor of Philosophy (Ph.D), Georg-August-Universität Göttingen \& Max Planck Institute for Dynamics and Self-Organization, Göttingen, Germany.

\section{Scholarships / Honours}

2006-2011 INSPIRE Scholarship from the Department of Science and Technology, India.

2010 Selected for Visiting Students Research Programme in TIFR-Centre for Applicable Mathematics, Bangalore, India under Dr. Seema Nanda

2009 Selected for Visiting Students Research Programme in Tata Institute of Fundamental Research, Mumbai, India under Professor Shobhona Sharma.

2009 Selected for Summer Research Fellowship Programme in Indian Academy of Sciences.

\section{Publications}

2014 Drep-2 is a novel synaptic protein important for learning and memory. eLife

2014 Differential Associative Training Enhances Olfactory Acuity in Drosophila melanogaster. The Journal of Neuroscience

2014 Optical Calcium Imaging Using DNA-Encoded Fluorescence Sensors in Transgenic Fruit Flies, Drosophila melanogaster. Methods in Molecular Biology

2013 Restoring polyamines protects from age-induced memory impairment in an autophagydependent manner. Nature Neuroscience

2013 Mushroom body miscellanea: transgenic Drosophila strains expressing anatomical and physiological sensor proteins in Kenyon cells. Frontiers in Neural Circuits

2012 Optical calcium imaging in the nervous system of Drosophila melanogaster. Biochimica et Biophysica Acta

Göttingen - Germany 


\section{Conferences}

2014 Poster at the European Computational Neuroscience Conference (BCCN 2014) in Göttingen, Germany

2014 Poster at the 107th Annual Meeting of the German Zoological Society (DZG 2014) in Göttingen, Germany

2012 Poster at the XIV European Drosophila Neurobiology Conference (NeuroFly 2012) in Padua, Italy 